Cochrane Database of Systematic Reviews

\title{
Interventions for preventing ophthalmia neonatorum (Review)
}

Kapoor VS, Evans JR, Vedula SS

Kapoor VS, Evans JR, Vedula SS.

Interventions for preventing ophthalmia neonatorum.

Cochrane Database of Systematic Reviews 2020, Issue 9. Art. No.: CD001862.

DOI: 10.1002/14651858.CD001862.pub4.

www.cochranelibrary.com 
TABLE OF CONTENTS

HEADER

ABSTRACT

PLAIN LANGUAGE SUMMARY

SUMMARY OF FINDINGS

BACKGROUND

OBJECTIVES

METHODS

RESULTS

Figure 1.

Figure 2.

Figure 3.

Figure 4.

Figure 5.

Figure 6.

Figure 7.

Figure 8.

Figure 9.

Figure 10.

Figure 11.

Figure 12.

DISCUSSION

Figure 13.

Figure 14.

Figure 15.

Figure 16.

Figure 17.

AUTHORS' CONCLUSIONS

ACKNOWLEDGEMENTS

REFERENCES

CHARACTERISTICS OF STUDIES

DATA AND ANALYSES

Analysis 1.1. Comparison 1: Any prophylaxis versus no prophylaxis, Outcome 1: Chlamydial conjunctivitis

Analysis 1.2. Comparison 1: Any prophylaxis versus no prophylaxis, Outcome 2: Bacterial conjunctivitis

Analysis 1.3. Comparison 1: Any prophylaxis versus no prophylaxis, Outcome 3: Any conjunctivitis of any aetiology ................

Analysis 1.4. Comparison 1: Any prophylaxis versus no prophylaxis, Outcome 4: Conjunctivitis of unknown aetiology ….........

Analysis 1.5. Comparison 1: Any prophylaxis versus no prophylaxis, Outcome 5: Nasolacrimal duct obstruction …..................

Analysis 1.6. Comparison 1: Any prophylaxis versus no prophylaxis, Outcome 6: Keratitis

Analysis 2.1. Comparison 2: Silver nitrate versus no prophylaxis, Outcome 1: Gonococcal conjunctivitis

Analysis 2.2. Comparison 2: Silver nitrate versus no prophylaxis, Outcome 2: Chlamydial conjunctivitis

Analysis 2.3. Comparison 2: Silver nitrate versus no prophylaxis, Outcome 3: Any conjunctivitis of any aetiology ...

Analysis 2.4. Comparison 2: Silver nitrate versus no prophylaxis, Outcome 4: Corneal abrasion

Analysis 2.5. Comparison 2: Silver nitrate versus no prophylaxis, Outcome 5: Nasolacrimal duct obstruction

Analysis 3.1. Comparison 3: Erythromycin versus no prophylaxis, Outcome 1: Gonococcal conjunctivitis

Analysis 3.2. Comparison 3: Erythromycin versus no prophylaxis, Outcome 2: Chlamydial conjunctivitis

Analysis 3.3. Comparison 3: Erythromycin versus no prophylaxis, Outcome 3: Bacterial conjunctivitis

Analysis 3.4. Comparison 3: Erythromycin versus no prophylaxis, Outcome 4: Any conjunctivitis of any aetiology

Analysis 3.5. Comparison 3: Erythromycin versus no prophylaxis, Outcome 5: Conjunctivitis of unknown aetiology ................

Analysis 3.6. Comparison 3: Erythromycin versus no prophylaxis, Outcome 6: Nasolacrimal duct obstruction ..........................

Analysis 4.1. Comparison 4: Tetracycline versus no prophylaxis, Outcome 1: Gonococcal conjunctivitis

Analysis 4.2. Comparison 4: Tetracycline versus no prophylaxis, Outcome 2: Chlamydial conjunctivitis

Analysis 4.3. Comparison 4: Tetracycline versus no prophylaxis, Outcome 3: Any conjunctivitis of any aetiology 
Analysis 5.1. Comparison 5: Povidone-iodine versus no prophylaxis, Outcome 1: Gonococcal conjunctivitis

Analysis 5.2. Comparison 5: Povidone-iodine versus no prophylaxis, Outcome 2: Chlamydial conjunctivitis

Analysis 5.3. Comparison 5: Povidone-iodine versus no prophylaxis, Outcome 3: Bacterial conjunctivitis

Analysis 5.4. Comparison 5: Povidone-iodine versus no prophylaxis, Outcome 4: Any conjunctivitis of any aetiology ...............

Analysis 5.5. Comparison 5: Povidone-iodine versus no prophylaxis, Outcome 5: Conjunctivitis of unknown aetiology ….........

Analysis 6.1. Comparison 6: Bacitracin-phenacaine versus no prophylaxis, Outcome 1: Gonococcal conjunctivitis ..................

Analysis 7.1. Comparison 7: Colostrum versus no prophylaxis, Outcome 1: Any conjunctivitis of any aetiology .......................

Analysis 8.1. Comparison 8: Erythromycin versus silver nitrate, Outcome 1: Chlamydial conjunctivitis

Analysis 8.2. Comparison 8: Erythromycin versus silver nitrate, Outcome 2: Bacterial conjunctivitis

Analysis 8.3. Comparison 8: Erythromycin versus silver nitrate, Outcome 3: Any conjunctivitis of any aetiology

Analysis 8.4. Comparison 8: Erythromycin versus silver nitrate, Outcome 4: Conjunctivitis of unknown aetiology ....................

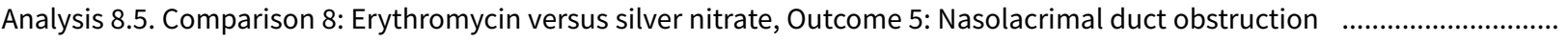

Analysis 9.1. Comparison 9: Tetracycline versus silver nitrate, Outcome 1: Chlamydial conjunctivitis

Analysis 9.2. Comparison 9: Tetracycline versus silver nitrate, Outcome 2: Nasolacrimal duct obstruction

Analysis 10.1. Comparison 10: Sulfacetamide versus silver nitrate, Outcome 1: Gonococcal conjunctivitis

Analysis 10.2. Comparison 10: Sulfacetamide versus silver nitrate, Outcome 2: Bacterial conjunctivitis

Analysis 10.3. Comparison 10: Sulfacetamide versus silver nitrate, Outcome 3: Any conjunctivitis of any aetiology .................. Analysis 10.4. Comparison 10: Sulfacetamide versus silver nitrate, Outcome 4: Conjunctivitis of unknown aetiology ................ Analysis 11.1. Comparison 11: Cetyl-pyridinium chloride versus silver nitrate, Outcome 1: Bacterial conjunctivitis .................. Analysis 11.2. Comparison 11: Cetyl-pyridinium chloride versus silver nitrate, Outcome 2: Any conjunctivitis of any aetiology .. Analysis 11.3. Comparison 11: Cetyl-pyridinium chloride versus silver nitrate, Outcome 3: Conjunctivitis of unknown aetiology Analysis 12.1. Comparison 12: Penicillin versus silver nitrate, Outcome 1: Gonococcal conjunctivitis ...................................... Analysis 12.2. Comparison 12: Penicillin versus silver nitrate, Outcome 2: Bacterial conjunctivitis Analysis 12.3. Comparison 12: Penicillin versus silver nitrate, Outcome 3: Any conjunctivitis of any aetiology .......................... Analysis 12.4. Comparison 12: Penicillin versus silver nitrate, Outcome 4: Conjunctivitis of unknown aetiology ...................... Analysis 13.1. Comparison 13: Penicillin IM versus silver nitrate, Outcome 1: Gonococcal conjunctivitis Analysis 13.2. Comparison 13: Penicillin IM versus silver nitrate, Outcome 2: Bacterial conjunctivitis ..................................... Analysis 13.3. Comparison 13: Penicillin IM versus silver nitrate, Outcome 3: Any conjunctivitis of any aetiology ..................... Analysis 13.4. Comparison 13: Penicillin IM versus silver nitrate, Outcome 4: Conjunctivitis of unknown aetiology ................... Analysis 14.1. Comparison 14: Povidone-iodine versus silver nitrate, Outcome 1: Gonococcal conjunctivitis

Analysis 14.2. Comparison 14: Povidone-iodine versus silver nitrate, Outcome 2: Chlamydial conjunctivitis ........................... Analysis 14.3. Comparison 14: Povidone-iodine versus silver nitrate, Outcome 3: Bacterial conjunctivitis

Analysis 14.4. Comparison 14: Povidone-iodine versus silver nitrate, Outcome 4: Any conjunctivitis of any aetiology .............. Analysis 14.5. Comparison 14: Povidone-iodine versus silver nitrate, Outcome 5: Conjunctivitis of unknown aetiology ........... Analysis 15.1. Comparison 15: Tetracycline versus erythromycin, Outcome 1: Chlamydial conjunctivitis

Analysis 15.2. Comparison 15: Tetracycline versus erythromycin, Outcome 2: Any conjunctivitis of any aetiology

Analysis 16.1. Comparison 16: Colostrum versus erythromycin, Outcome 1: Any conjunctivitis of any aetiology ....................... Analysis 17.1. Comparison 17: Povidone-iodine versus erythromycin, Outcome 1: Chlamydial conjunctivitis 
Analysis 22.1. Comparison 22: Povidone-iodine twice versus povidone-iodine once, Outcome 1: Gonococcal conjunctivitis .... 189 Analysis 22.2. Comparison 22: Povidone-iodine twice versus povidone-iodine once, Outcome 2: Chlamydial conjunctivitis .... 190 Analysis 22.3. Comparison 22: Povidone-iodine twice versus povidone-iodine once, Outcome 3: Bacterial conjunctivitis ........ 190 Analysis 22.4. Comparison 22: Povidone-iodine twice versus povidone-iodine once, Outcome 4: Any conjunctivitis of any 190 aetiology

Analysis 22.5. Comparison 22: Povidone-iodine twice versus povidone-iodine once, Outcome 5: Conjunctivitis of unknown aetiology

Analysis 23.1. Comparison 23: Penicillin IM versus topical penicillin, Outcome 1: Gonococcal conjunctivitis

HISTORY 
[Intervention Review]

\title{
Interventions for preventing ophthalmia neonatorum
}

\author{
Vimal Scott Kapoor ${ }^{1}$, Jennifer R Evans ${ }^{2}$, S Swaroop Vedula 3 \\ 1 University of Toronto, Toronto, Canada. ${ }^{2}$ Cochrane Eyes and Vision, ICEH, London School of Hygiene \& Tropical Medicine, London, UK. \\ 3Johns Hopkins University, Baltimore, Maryland, USA
}

Contact address: Vimal Scott Kapoor, vimal.kapoor@utoronto.ca,vimal.kapoor@mail.mcgill.ca.

Editorial group: Cochrane Eyes and Vision Group.

Publication status and date: New, published in Issue 9, 2020.

Citation: Kapoor VS, Evans JR, Vedula SS. Interventions for preventing ophthalmia neonatorum. Cochrane Database of Systematic Reviews 2020, Issue 9. Art. No.: CD001862. DOI: 10.1002/14651858.CD001862.pub4.

Copyright $@ 2020$ The Cochrane Collaboration. Published by John Wiley \& Sons, Ltd.

\begin{abstract}
A B S T R A C T
Background

Ophthalmia neonatorum is an infection of the eyes in newborns that can lead to blindness, particularly if the infection is caused by Neisseria gonorrhoeae. Antiseptic or antibiotic medication is dispensed into the eyes of newborns, or dispensed systemically, soon after delivery to prevent neonatal conjunctivitis and potential vision impairment.
\end{abstract}

\section{Objectives}

1. To determine if any type of systemic or topical eye medication is better than placebo or no prophylaxis in preventing ophthalmia neonatorum.

2. To determine if any one systemic or topical eye medication is better than any other medication in preventing ophthalmia neonatorum.

\section{Search methods}

We searched CENTRAL, MEDLINE, Embase, LILACS, and three trials registers, date of last search 4 October 2019. We also searched references of included studies and contacted pharmaceutical companies.

\section{Selection criteria}

We included randomised and quasi-randomised controlled trials of any topical, systemic, or combination medical interventions used to prevent ophthalmia neonatorum in newborns compared with placebo, no prophylaxis, or with each other.

\section{Data collection and analysis}

We used standard methods expected by Cochrane. Outcomes were: blindness or any adverse visual outcome at 12 months, conjunctivitis at 1 month (gonococcal (GC), chlamydial (CC), bacterial (BC), any aetiology (ACAE), or unknown aetiology (CUE)), and adverse effects.

\section{Main results}

We included 30 trials with a total of 79,198 neonates. Eighteen studies were conducted in high-income settings (the USA, Europe, Israel, Canada), and 12 were conducted in low- and middle-income settings (Africa, Iran, China, Indonesia, Mexico). Fifteen of the 30 studies were quasi-randomised. We judged every study to be at high risk of bias in at least one domain. Ten studies included a comparison arm with no prophylaxis. There were 14 different prophylactic regimens and 12 different medications in the 30 included studies.

Any prophylaxis compared to no prophylaxis

Unless otherwise indicated, the following evidence comes from studies assessing one or more of the following interventions: tetracycline $1 \%$, erythromycin $0.5 \%$, povidone-iodine $2.5 \%$, silver nitrate $1 \%$. None of the studies reported data on the primary outcomes: blindness 
or any adverse visual outcome at any time point. There was only very low-certainty evidence on the risk of GC with prophylaxis (4/5340 newborns) compared to no prophylaxis (5/2889) at one month (risk ratio (RR) $0.79,95 \%$ confidence interval (CI) 0.24 to $2.65,3$ studies). Lowcertainty evidence suggested there may be little or no difference in effect on CC (RR $0.96,95 \% \mathrm{Cl} 0.57$ to $1.61,4874$ newborns, 2 studies) and $\mathrm{BC}$ (RR $0.84,95 \% \mathrm{Cl} 0.37$ to $1.93,3685$ newborns, 2 studies). Moderate-certainty evidence suggested a probable reduction in risk of ACAE at one month (RR $0.65,95 \% 0.54$ to $0.78,9666$ newborns, 8 studies assessing tetracycline $1 \%$, erythromycin $0.5 \%$, povidone-iodine $2.5 \%$, silver nitrate $1 \%$, colostrum, bacitracin-phenacaine ointment). There was only very low-certainty evidence on $\mathrm{CUE}$ (RR $1.75,95 \% \mathrm{Cl} 0.37$ to 8.28, 330 newborns, 1 study). Very low-certainty evidence on adverse effects suggested no increased nasolacrimal duct obstruction (RR $0.93,95 \% \mathrm{Cl} 0.68$ to $1.28,404$ newborns, 1 study of erythromycin $0.5 \%$ and silver nitrate $1 \%$ ) and no increased keratitis (single study of 40 newborns assessing silver nitrate $1 \%$ with no events).

Any prophylaxis compared to another prophylaxis

Overall, evidence comparing different interventions did not suggest any consistently superior intervention. However, most of this evidence was of low-certainty and was extremely limited.

\section{Authors' conclusions}

There are no data on whether prophylaxis for ophthalmia neonatorum prevents serious outcomes such as blindness or any adverse visual outcome. Moderate-certainty evidence suggests that the use of prophylaxis may lead to a reduction in the incidence of ACAE in newborns but the evidence for effect on GC, CC or BC was less certain. Comparison of individual interventions did not suggest any consistently superior intervention, but data were limited. A trial comparing tetracycline, povidone-iodine (single administration), and chloramphenicol for GC and CC could potentially provide the community with an effective, universally applicable prophylaxis against ophthalmia neonatorum.

\section{PLAIN LANGUAGE SUMMARY}

\section{Medication to prevent infection of the eye in newborns}

\section{What was the aim of the review?}

The aim of this Cochrane Review was to determine if any medication is better than placebo or no preventive action in preventing ophthalmia neonatorum. Cochrane Review authors collected and analysed all relevant studies to answer this question and found 30 studies.

\section{Key messages}

There are no data on whether prophylaxis for ophthalmia neonatorum prevents serious outcomes such as blindness or visual impairment. Moderate-certainty evidence suggests that the use of prophylaxis may lead to a reduction in the incidence of any conjunctivitis of any cause in newborns but the evidence for effect on gonococcal or chlamydial conjunctivitis was of low to very-low certainty. Comparison of individual interventions did not suggest any consistently superior intervention, but data were limited.

\section{What was studied in the review?}

Ophthalmia neonatorum, also known as neonatal conjunctivitis, is an infection of the eye surface that affects newborn babies within the first month of life. It is usually caused by infection (bacterial or viral) picked up during birth. If left untreated, it can lead to blindness. The World Health Organization (WHO) recommends the following treatments to prevent ophthalmia neonatorum:

- tetracycline hydrochloride $1 \%$ eye ointment;

- erythromycin $0.5 \%$ eye ointment;

- povidone-iodine $2.5 \%$ solution (water-based);

- silver nitrate $1 \%$ solution;

- chloramphenicol $1 \%$ eye ointment.

Cochrane Review authors considered these treatments and others to prevent the development of conjunctivitis in newborns. They assessed the two main types of conjunctivitis separately - gonococcal conjunctivitis (caused by Neisseria gonorrhoeae) and chlamydial conjunctivitis (caused by Chlamydia trachomatis) - as well as conjunctivitis due to any bacteria (including Neisseria gonorrhoeae and Chlamydia trachomatis), conjunctivitis due to any cause or conjunctivitis of unknown cause.

\section{What are the main results of the review?}

Cochrane Review authors identified 30 studies with a total of 79,198 newborns. Eighteen studies took place in high-income settings (the USA, Europe, Israel, Canada), and 12 were conducted in low- and middle-income settings (Africa, Iran, China, Indonesia, Mexico). The main preventive medications evaluated in the included studies were: tetracycline $1 \%$, erythromycin $0.5 \%$, povidone-iodine $2.5 \%$, and silver nitrate $1 \%$.

Newborns given preventive medication are likely to have a lower chance of conjunctivitis within one month of birth compared with newborns not given preventive medication (moderate-certainty evidence). The evidence for specific causes of conjunctivitis (gonococcal, 
chlamydial) was less certain as these occurred less frequently in the included studies. None of the studies collected data on blindness or adverse vision outcomes.

\section{How up-to-date is the review?}

Cochrane Review authors searched for studies published up to 4 October 2019. 
SUMMARY OF FINDINGS

\section{Summary of findings 1. Any prophylaxis compared to no prophylaxis for the prevention of ophthalmia neonatorum in newborn children}

Any prophylaxis compared to no prophylaxis for the prevention of ophthalmia neonatorum in newborn children

Patient or population: newborn children

Setting: any maternity setting

Intervention: any prophylaxis, including povidone-iodine, erythromycin, tetracycline, silver nitrate, bacitracin-phenacaine, colostrum

Comparison: no prophylaxis (none of the studies used a placebo or sham treatment)

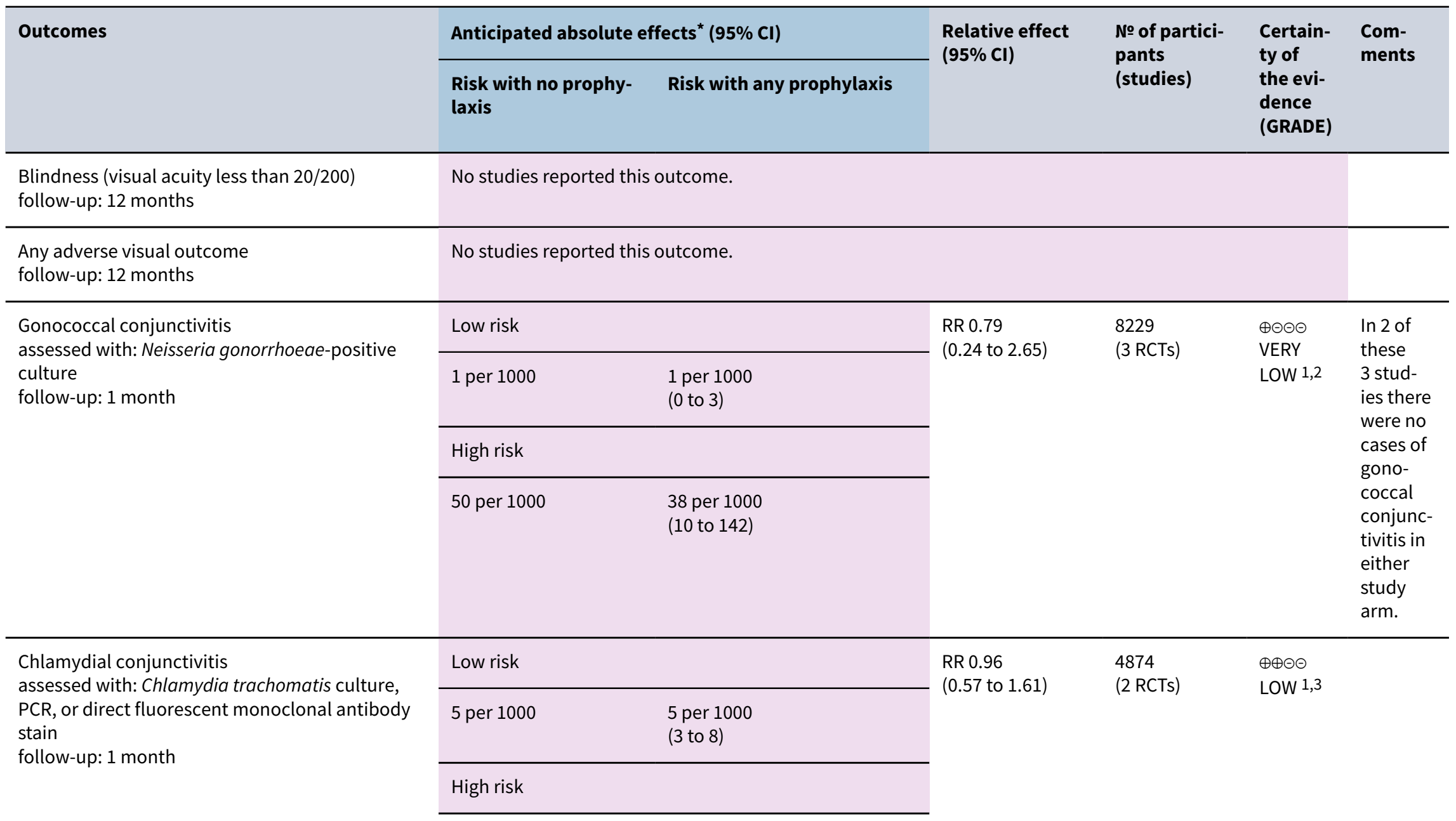




\begin{tabular}{|c|c|c|c|c|c|}
\hline \multirow{4}{*}{$\begin{array}{l}\text { Bacterial conjunctivitis } \\
\text { assessed with: any bacteria-positive culture, } \\
\text { smear, or Gram stain } \\
\text { follow-up: } 1 \text { month }\end{array}$} & \multirow{3}{*}{\multicolumn{2}{|c|}{$\begin{array}{l}100 \text { per } 1000 \\
\text { Low risk }\end{array}$}} & & & \\
\hline & & & & & \\
\hline & & & \multirow{4}{*}{$\begin{array}{l}\text { RR } 0.84 \\
(0.37 \text { to } 1.93)\end{array}$} & \multirow{4}{*}{$\begin{array}{l}3685 \\
\text { (2 RCTs) }\end{array}$} & \multirow{4}{*}{$\begin{array}{l}\oplus \oplus \ominus \ominus \\
\text { LOW } 1,3\end{array}$} \\
\hline & 3 per 1000 & $\begin{array}{l}3 \text { per } 1000 \\
(1 \text { to } 6)\end{array}$ & & & \\
\hline & \multicolumn{2}{|l|}{ High risk } & & & \\
\hline & 50 per 1000 & $\begin{array}{l}42 \text { per } 1000 \\
(19 \text { to } 97)\end{array}$ & & & \\
\hline \multirow{4}{*}{$\begin{array}{l}\text { Any conjunctivitis of any aetiology assessed with: } \\
\text { clinical assessment } \\
\text { follow-up: } 1 \text { month }\end{array}$} & \multicolumn{2}{|l|}{ Low risk } & \multirow{4}{*}{$\begin{array}{l}\text { RR } 0.65 \\
(0.54 \text { to } 0.78)\end{array}$} & \multirow{4}{*}{$\begin{array}{l}9666 \\
\text { (8 RCTs) }\end{array}$} & \multirow{4}{*}{$\begin{array}{l}\oplus \oplus \oplus \ominus \\
\text { MODER- } \\
\text { ATE } 1\end{array}$} \\
\hline & 3 per 1000 & $\begin{array}{l}2 \text { per } 1000 \\
(2 \text { to } 2)\end{array}$ & & & \\
\hline & \multicolumn{2}{|l|}{ High risk } & & & \\
\hline & 300 per 1000 & $\begin{array}{l}195 \text { per } 1000 \\
(162 \text { to } 134)\end{array}$ & & & \\
\hline \multirow{2}{*}{$\begin{array}{l}\text { Conjunctivitis of unknown aetiology assessed } \\
\text { with: culture negative } \\
\text { follow-up: } 1 \text { month }\end{array}$} & \multicolumn{2}{|c|}{ Study population } & \multirow{2}{*}{$\begin{array}{l}\text { RR } 1.75 \\
(0.37 \text { to } 8.28)\end{array}$} & \multirow{2}{*}{$\begin{array}{l}330 \\
(1 \mathrm{RCT})\end{array}$} & \multirow{2}{*}{$\begin{array}{l}\oplus \odot \Theta \odot \\
\text { VERY } \\
\text { LOW } 1,2\end{array}$} \\
\hline & 20 per 1000 & $\begin{array}{l}35 \text { per } 1000 \\
\text { ( } 7 \text { to } 166)\end{array}$ & & & \\
\hline Adverse effects & \multicolumn{4}{|c|}{$\begin{array}{l}\text { In a single study (Bell 1993), any prophylaxis (erythromycin } 0.5 \% \text { or silver nitrate } 1 \% \text { ) did not } \\
\text { appear to be associated with an increased risk of nasolacrimal duct obstruction compared } \\
\text { with no prophylaxis (RR } 0.93,95 \% \mathrm{Cl} 0.68 \text { to } 1.28 \text { ). } \\
\text { A single study of } 40 \text { newborns comparing silver nitrate } 1 \% \text { with control reported that no events } \\
\text { of keratitis were observed in the prophylaxis and no-prophylaxis groups (Graf 1994). }\end{array}$} & $\begin{array}{l}\oplus \odot \Theta \Theta \\
\text { VERY } \\
\text { LOW } 1,2\end{array}$ \\
\hline
\end{tabular}

*The risk in the intervention group (and its $95 \%$ confidence interval) is based on the assumed risk in the comparison group and the relative effect of the intervention (and its $95 \% \mathrm{Cl}$ ). The assumed risks (low and high) in the comparison group were estimated from relevant prevalence studies (Appendix 8).

Cl: confidence interval; PCR: polymerase chain reaction; RCT: randomised controlled trial; RR: risk ratio

\section{GRADE Working Group grades of evidence}

High-certainty: We are very confident that the true effect lies close to that of the estimate of the effect.

Moderate-certainty: We are moderately confident in the effect estimate: the true effect is likely to be close to the estimate of the effect, but there is a possibility that it is substantially different.

Low-certainty: Our confidence in the effect estimate is limited: the true effect may be substantially different from the estimate of the effect. 
Very low-certainty: We have very little confidence in the effect estimate: the true effect is likely to be substantially different from the estimate of effect.

1Downgraded $(-1)$ for risk of bias: studies were at high or unclear risk of bias.

2Downgraded (-2) for imprecision: sparse data.

${ }^{3}$ Downgraded (-1) for imprecision: 95\% confidence interval includes no effect. 


\section{B A C K G R O U N D}

\section{Description of the condition}

Ophthalmia neonatorum, also called neonatal conjunctivitis, is an inflammatory disorder of the eye surface in newborns in the first month of life (WHO 1986). In Europe in the late 1800s, a significant percentage of blind people were blind due to gonococcal ophthalmia neonatorum. Specifically, it was reported that the percentage of blindness from ophthalmia neonatorum was $8 \%$ in Copenhagen, 20\% in Berlin, 30\% in Vienna, and 45\% in Paris (Buller 1900; Haussman 1895; Milot 2008). It has been estimated that during the same period $20 \%$ to $80 \%$ of children in blind institutions in Germany were blind due to ophthalmia neonatorum caused by Neisseria gonorrhoeae (Konigstein 1882). In the USA, amongst new admissions into schools for the blind between 1906 and 1911, approximately $24 \%$ were due to ophthalmia neonatorum caused by Ngonorrhoeae, with a range of $8 \%$ to $45 \%$ (Barsam 1966). In 1918, St Margaret's Hospital in London (UK) was opened specifically for the treatment of neonates with ophthalmia neonatorum (Anonymous 1918; Anonymous 1919).

To this day, ophthalmia neonatorum remains a significant cause of childhood corneal blindness in low- and middleincome countries, mainly from $N$ gonorrhoeae (Whitcher 2001). There are major epidemiological challenges in determining the prevalence and incidence of blindness from ophthalmia neonatorum. Notwithstanding these limitations, cross-sectional surveys have estimated the percentage of blindness or severe visual impairment due to ophthalmia neonatorum to be 3\% in Ethiopia (Kello 2003), 0.7\% in Bangladesh (Muhit 2007), 0.4\% in Malaysia (Koay 2015), 5\% in East Africa (Foster 1991), 0.8\% in India (Rahi 1995), and 2\% in Tanzania (Foster 1987).

Gonococcal ophthalmia neonatorum is associated with the most severe consequences. Gonococcal ophthalmia neonatorum is mainly contracted from the mother's infected birth canal during delivery, but can also be contracted in utero by ascending infections. Neonates born to gonorrhoea-infected mothers have a $30 \%$ to $50 \%$ risk of developing gonococcal conjunctivitis (Laga 1989). Untreated or inappropriately treated gonococcal conjunctivitis can result in corneal perforation and vision loss in 24 hours (Donham 2008; Duke-Elder 1965). In one case series, the mean duration of corneal perforation from untreated gonococcal conjunctivitis was 11 days (Kawashima 2009). In areas with low incidence of gonococcal ophthalmia neonatorum or limited access to appropriate health care, appropriate clinical diagnosis and appropriate therapy may be delayed, which can lead to loss of vision (Bastion 2006; McElnea 2015; Schwab 1985; Wan 1986). After historical declines in rates of gonorrhoea, it made a resurgence in some high-income countries In 2012, amongst adults aged 15 to 49 years, it is estimated there were 27 million cases of gonorrhoea globally (Newman 2015). Furthermore, there is increasing incidence of drug-resistant strains of $N$ gonorrhoeae globally (Martin 2015; Van de Laar 2012; WHO 2012). The pooled mean prevalence of $N$ gonorrhoeae was estimated at 3.7\% $(95 \%$ confidence interval (Cl) $2.8 \%$ to $4.6 \%$ ) in pregnant women in Eastern and Southern Africa, and $2.7 \%(95 \% \mathrm{Cl} 1.7 \%$ to $3.7 \%)$ in pregnant women in West and Central Africa (Chico 2012).

Chlamydial ophthalmia neonatorum is also associated with a high risk of corneal and conjunctival scarring, haemorrhagic conjunctivitis, and rarely, loss of vision if left untreated (Chang
2006; Darville 2015; Whitcher 2001). It is caused by transmission of Chlamydia trachomatis from the mother to the newborn during delivery. The risk of chlamydial transmission from an infected mother to newborns is $15 \%$ on average (range $=8 \%$ to $44 \%$; Rosenman 2003). Furthermore, an increased prevalence of chlamydial infection in some high-income countries is associated with a commensurate rise in risk of chlamydial conjunctivitis (Quirke 2008). Chlamydial ophthalmia neonatorum is much more prevalent than gonococcal ophthalmia neonatorum, and has historically been underdiagnosed due to lack of accurate diagnostic techniques (Darville 2015; Yip 2008). Pooled mean prevalence of $C$ trachomatis was higher at $6.9 \%(95 \% \mathrm{Cl} 5.1 \%$ to $8.6 \%)$ in pregnant women in Eastern and Southern Africa, and $6.1 \%(95 \% \mathrm{Cl} 4.0 \%$ to $8.3 \%$ ) in pregnant women in West and Central Africa (Chico 2012).

Ophthalmia neonatorum may be caused by bacteria other than Neisseria and Chlamydia. The relative frequencies of bacterial causes of ophthalmia neonatorum reported in the literature vary by study and geographic location (Amini 2008; Chhabra 2008; Di Bartolomeo 2001; Di Bartolomeo 2005; Hammerschlag 1993; Mohile 2002; Sandström 1984). Even though certain bacteria are frequently cultured from neonates with conjunctivitis (e.g. Staphylococcus aureus), their role as the causative agent of conjunctivitis is uncertain because these bacteria may be frequently cultured from the eyes of asymptomatic neonates (Amini 2008; Fransen 1987; Krohn 1993).

Finally, some viruses such as herpes simplex and adenovirus can cause ophthalmia neonatorum (Albert 1994). Often, no causative pathogen can be found in newborns with ophthalmia neonatorum due to methods for obtaining and culturing for bacteria, or due to causes other than bacteria such as chemical conjunctivitis or nasolacrimal duct obstruction (Sandström 1987).

While most micro-organisms that cause ophthalmia neonatorum are acquired during passage through the birth canal, others are acquired after birth from caregivers or the nasopharyngeal passages of the newborn (Krohn 1993). The relative importance of intrapartum versus postpartum sources of infection varies based on extraneous factors such as socioeconomic status of mothers (Isenberg 1995; Vedantham 2004; Verma 1994).

\section{Description of the intervention}

There are four strategies to achieve the public health goal of eliminating ophthalmia neonatorum and its adverse vision consequences:

1. preventing spread of sexually transmitted infections;

2. screening women who are pregnant for genital infection;

3. administering prophylaxis to newborns soon after birth;

4. diagnosing and treating eye infections in newborns at an early stage (Foster 1995; Laga 1989).

German-born obstetrician and gynaecologist Carl Siegmund Franz Credé introduced the third strategy, ophthalmia neonatorum prophylaxis (Crede 1884; Dunn 2000; Oriel 1991). In a seminal study, albeit a case series, Credé showed that silver nitrate administered to newborns reduced the incidence of ophthalmia neonatorum from $13.6 \%$ to $0.05 \%$ (Crede 1881). The relevance of prophylaxis for ophthalmia neonatorum and debate about the optimal medication has since evolved in response to the discovery of new antibiotics; concerns about side effects with silver nitrate such as chemical 
conjunctivitis and impact on maternal-infant bonding; and the declining prevalence of $N$ gonorrhoeae in some countries and increased reporting rates of chlamydial ophthalmia neonatorum in others (Napchan 2005; Wahlberg 1982).

Initially, studies looking for alternatives to silver nitrate mainly examined penicillin as ophthalmia neonatorum prophylaxis. Later, erythromycin and tetracycline were studied in comparison to silver nitrate, and more recently, povidone-iodine. There have been studies of other prophylactic agents, but the majority of jurisdictions in the world today appear to use either erythromycin or povidone-iodine as ophthalmia neonatorum prophylaxis. However, there remains a high degree of variability in the agents used for ophthalmia neonatorum prophylaxis, with some jurisdictions using prophylactic medications that are uncommon or not well-studied (Guala 2005; Zloto 2016).

Erythromycin and tetracycline gained acceptance as prophylactic agents in the 1980s because of their allegedly superior activity against $C$ trachomatis and because they lacked some of the side effects of silver nitrate, such as chemical conjunctivitis (Isenberg 1994a). However, it remains unresolved whether these antibiotic agents are, in fact, any more effective than silver nitrate in preventing chlamydial conjunctivitis. Furthermore, the emergence of beta-lactamase-producing $N$ gonorrhoeae has reduced the prophylactic effectiveness of erythromycin and tetracycline (Ison 1998; Martin 2015; Van de Laar 2012; WHO 2012). Povidone-iodine, introduced in studies in the 1960s as a surgical antiseptic and disinfectant agent, has been used relatively more recently as a candidate for ophthalmia neonatorum prophylaxis. It allegedly has many advantages over silver nitrate, erythromycin, and tetracycline, including economic feasibility, broader antibacterial spectrum, lack of generation of bacterial resistance, and no reports of anaphylaxis (Grzybowski 2018; Isenberg 1994b). Other prophylactic measures that have been used beyond antimicrobial or antiseptic agents include cleansing the eyelids with sterile swabs; cleansing the eyes with distilled water and wiping dry; and physiological saline.

Credés original procedure for ophthalmic prophylaxis called for administration "directly after birth" (Crede 1881). Timing of prophylaxis of ophthalmia neonatorum after birth has been addressed by one small study (Muhe 1986). This study suggested that increasing delay in administration of prophylaxis after birth can lead to a trend to increasing failure of the intervention (Muhe 1986). This study also suggested that a delay in prophylaxis greater than four hours can lead to a four- to five-fold risk of gonococcal ophthalmia neonatorum (Laga 1989; Muhe 1986). Three guidelines have suggested optimal timing of prophylaxis, but cited no evidence: In 2002, the Canadian Pediatric Society Guideline suggested prophylaxis administration within one hour after birth (CPS 2002); in 2011, the United States Preventive Services Task Force recommendation suggested timing of prophylaxis administration no later than 24 hours after birth (USPSTF 2011); and in 2017, the World Health Organization (WHO) suggested timing of prophylaxis to be "immediately after birth" (WHO 2017).

\section{How the intervention might work}

Ophthalmia neonatorum prophylaxis agents used around the world are antimicrobial or antiseptic agents, which, when administered topically, or rarely systemically, destroy or inhibit micro-organisms in the eye to prevent conjunctivitis and keratitis
(Kramer 2002). The micro-organisms may be acquired from the mother's infected birth canal, in utero by ascending infections, or from the hospital or home environment.

\section{Why it is important to do this review}

Launched in 1999, Vision 2020 is a global initiative of the WHO and the International Agency for the Prevention of Blindness with the goal to eliminate avoidable blindness by 2020 (WHO 1999). Vision 2020 was updated by the WHO in 2013 to develop a Global Action Plan from 2014 to 2019 "to reduce the prevalence of avoidable visual impairment by $25 \%$ by 2019" (WHO 2013). Controlling childhood blindness is a high priority of this plan, as it has been estimated that $4 \%$ of all global blindness is due to childhood blindness, and that $45 \%$ of all childhood blindness is avoidable. Corneal scarring is one of five childhood blindness conditions prioritised for control. While vitamin A and measles are responsible for the majority of corneal scarring, ophthalmia neonatorum is a significant cause of corneal blindness, mainly in low- and middle-income countries such as those in sub-Saharan Africa (Gilbert 2012; Robaei 2014; Whitcher 2001; WHO 2013).

In sub-Saharan Africa, the two major agents responsible for corneal blindness and scarring, $N$ gonorrhoeae and $C$ trachomatis, have high prevalence in pregnant women.

The WHO, in conjunction with the United Nations Children's Fund and the United Nations Population Fund, has developed guidelines through its Integrated Management of Pregnancy and Childbirth (IMPAC) strategy to reduce child and maternal mortality and morbidity. The IMPAC approach includes preventative and curative elements, targeting health systems, health workers, families, and communities. The Pregnancy, Childbirth, Postpartum and Newborn Care guide's evidence-based recommendations include eye prophylaxis for prevention of ophthalmia neonatorum. Evidence on this intervention and the relative effectiveness of different prophylactic regimens is therefore essential to this intervention (WHO 2015).

There is considerable global variability in recommendations on whether to use ophthalmia neonatorum prophylaxis, and the prophylactic agent used. Certain jurisdictions still carry out ophthalmia neonatorum prophylaxis, including Brazil (Caligaris 2010), the USA (USPSTF 2011), Italy (Guala 2005), Spain (Luna 2009), Canada (Moore 2015), Slovenia (Jug Došler 2015), France (Dageville 2015), Turkey (Eser 2009), certain areas of Central America, some countries in Africa, parts of the Far East, areas of the Middle East, and sections of Central Asia (Zloto 2016). Norway, Great Britain, Sweden, the Netherlands (Rours 2008; Volksgezondheid 1980), Australia (Shaw 1977), Belgium (Tribolet 2016), and Denmark (Pande 2006), discontinued ophthalmia neonatorum prophylaxis several years ago (Kramer 2002). As recently as 2010, England and Wales removed ophthalmia neonatorum from the list of notifiable diseases, even though there is some evidence of significant underreporting of the incidence of ophthalmia neonatorum (Department of Health, UK 2010; Dharmasena 2015). In Canada, there have been recent recommendations that ophthalmia neonatorum be discontinued, although no legislative changes have been made in the country as yet (Moore 2015). Some groups in Canada oppose this recommendation (Mulholland 2015), and others question whether the alternative strategy of prenatal screening is an optimal sole substitute for prophylaxis (Poliquin 2015). The Canadian recommendation to discontinue prophylaxis has been 
made in spite of the fact that the rate of chlamydia in Canada has increased $57.6 \%$ (Totten 2015a), and the rate of gonorrhoea has increased 38.9\% from 2003 to 2012, mainly in women (Totten 2015b). In France, ophthalmia neonatorum prophylaxis is no longer universally recommended. Ocular prophylaxis is only recommended for neonates where there is a risk of sexually transmitted infections in the mother, and where the mother has had poor prenatal care (AFSSAPS 2010). Still, other jurisdictions are looking to implement ophthalmia neonatorum prophylaxis (Pastor 2015).

The global variability in practices regarding prophylaxis for ophthalmia neonatorum may be explained by the following:

1. there is uncertainty about the evidence of effectiveness and risk-benefit ratio of the various prophylactic agents, particularly against $C$ trachomatis and $N$ gonorrhoeae;

2. the prevalence and distribution of $N$ gonorrhoeae and $C$ trachomatis is variable, and has evolved over time, raising the possibility that universal prophylaxis may no longer be justified;

3. the relative effectiveness of different medications for prophylaxis of ophthalmia neonatorum remains to be determined.

In this systematic review, we aimed to synthesise the available evidence to inform care and policy regarding prophylaxis for ophthalmia in the newborn.

\section{OB JECTIVES}

1. To determine if any type of systemic or topical eye medication is better than placebo or no prophylaxis in preventing ophthalmia neonatorum.

2. To determine if any one systemic or topical eye medication is better than any other medication in preventing ophthalmia neonatorum.

\section{METHODS}

\section{Criteria for considering studies for this review}

\section{Types of studies}

We considered randomised and quasi-randomised controlled trials.

\section{Types of participants}

Trials that enrolled newborn infants were eligible for inclusion in the review.

\section{Types of interventions}

We included trials comparing any topical, systemic, or combination medical interventions with placebo, no prophylaxis, or with each other.

\section{Types of outcome measures}

We considered the following outcomes:

\section{Primary outcomes}

1. Proportion of infants developing blindness, defined as a visual acuity of 20/200 or worse measured, e.g., using a Teller visual acuity card at 12 months.

2. Proportion of infants developing any adverse visual outcome measured, e.g., with a Teller visual acuity card at 12 months.

3. Proportion of neonates developing gonococcal conjunctivitis (GC) within 28 days of birth, where diagnosis was made with a laboratory-based method to identify the infecting organism. We anticipated that most studies would not have studied blindness as an outcome. Because severe GC is associated with a substantial risk for loss of vision, we considered this outcome as a substitute for the more important measure of blindness.

\section{Secondary outcomes}

1. Proportion of neonates developing chlamydial conjunctivitis (CC) within 28 days of birth.

2. Proportion of neonates developing bacterial conjunctivitis (BC) within 28 days of birth: this includes cases of conjunctivitis confirmed to be of bacterial origin by culture or Gram stain, or both. In addition to conjunctivitis cases of other bacterial aetiology, this category includes GC and CC.

3. Proportion of neonates developing any clinical conjunctivitis within 28 days of birth, referred to as any conjunctivitis cases of any aetiology (ACAE): this includes all cases of conjunctivitis clinically diagnosed, irrespective of aetiology. This would include infectious and non-infectious conjunctivitis. Infectious conjunctivitis includes BC, mycoplasma conjunctivitis, chlamydial or viral conjunctivitis. Non-infectious conjunctivitis includes chemical, toxic, or mechanical conjunctivitis. In cases where there was selective outcome reporting, and all cases of clinical conjunctivitis were not reported, this outcome was not included in comparisons.

4. Proportion of neonates developing conjunctivitis of unknown aetiology (CUE) within 28 days of birth: this includes cases of conjunctivitis that are culture-negative, where the aetiology is unknown. These may be infectious, but showing no growth of pathogenic agents on culture media, or on other methods to identify microbiologic aetiology. This may include noninfectious conjunctivitis, such as chemical conjunctivitis. Finally, it may be a mix of the aforementioned causes of conjunctivitis. In many cases, it is calculated by subtracting the total conjunctivitis cases of any aetiology from the conjunctivitis cases proven to be of bacterial origin.

5. Proportion of neonates developing the following adverse effects of ophthalmia neonatorum prophylaxis:

a. keratitis within 28 days of birth;

b. nasolacrimal duct obstruction within 60 days of birth.

\section{Search methods for identification of studies}

\section{Electronic searches}

The Cochrane Eyes and Vision Information Specialist conducted systematic searches in the following databases for randomised controlled trials and controlled clinical trials. There were no restrictions to language or year of publication. The databases were last searched on 4 October 2019.

- Cochrane Central Register of Controlled Trials (CENTRAL; 2019, Issue 10) (which contains the Cochrane Eyes and Vision Trials 
Register) in the Cochrane Library (searched 4 October 2019) (Appendix 1).

- MEDLINE Ovid (1946 to 4 October 2019) (Appendix 2).

- Embase Ovid (1980 to 4 October 2019) (Appendix 3).

- LILACS (Latin American and Caribbean Health Science Information database (1982 to 4 October 2019) (Appendix 4).

- ISRCTN registry (www.isrctn.com/editAdvancedSearch; searched 4 October 2019) (Appendix 5).

- US National Institutes of Health Ongoing Trials Register ClinicalTrials.gov (www.clinicaltrials.gov/; searched 4 October 2019) (Appendix 6).

- World Health Organization (WHO) International Clinical Trials Registry Platform (ICTRP) (www.who.int/ictrp/en/; searched 4 October 2019) (Appendix 7).

\section{Searching other resources}

We checked the reference lists of identified trial reports and existing review articles and contacted pharmaceutical companies to locate additional trials.

\section{Data collection and analysis}

\section{Selection of studies}

Two review authors independently screened titles and abstracts of records retrieved from the searches, categorising each record as either include, exclude, or unclear. We retrieved full-text articles of records that any of the review authors marked as include or unclear. Two review authors independently assessed the full-text articles and marked them as include or exclude. We reported reasons for full-text articles excluded in this process. The review authors resolved disagreements through discussion and consensus. In cases where additional information was needed before a decision could be made on the eligibility of full-text articles, we attempted to obtain this information from the study investigator.

\section{Data extraction and management}

For each eligible study (using all reports from the study), two review authors independently extracted information on methods, participants, interventions, outcomes, and funding sources using data forms developed for this review. We contacted the study authors for information missing from available reports.

\section{Assessment of risk of bias in included studies}

Two review authors independently assessed risk of bias in each included study according to methods described in Chapter 8 of the Cochrane Handbook for Systematic Reviews of Interventions (Higgins 2017). We assessed risk of bias for generation and concealment of the allocation sequence; masking of participants, caregivers/study personnel, and outcome assessors; completeness of follow-up; reporting biases (selective outcome reporting); and other sources of potential bias such as funding.

The review authors judged the risk of bias for each item as high, low, or unclear. We contacted study authors if the information in the available reports was insufficient to make an assessment. We used the available information if study authors did not respond within six weeks.

\section{Measures of treatment effect}

We computed the risk ratio for dichotomous outcomes.

\section{Unit of analysis issues}

We considered the individual as the unit of analysis. The assigned intervention was typically administered to both eyes, and we considered infants to be infected if at least one eye was affected. We excluded studies in which each eye within an infant was randomised to a different intervention, even if data were reported separately for each eye. No cluster-randomised or cross-over randomised controlled trials were identified and indeed they are not anticipated in this topic area.

\section{Dealing with missing data}

We contacted authors of included trials where we identified missing data on risk of bias or outcomes. If authors provided information on risk of bias that was not described in the trial reports, then this information was marked as such in the review. If missing data on an outcome were not available from study authors, then we assessed whether a meta-analysis was possible using an intention-to-treat or available-case approach.

\section{Assessment of heterogeneity}

We assessed heterogeneity in effect estimates from the included trials through a visual examination of the forest plot and based on the $1^{2}$ statistic. We considered $1^{2}$ values of $60 \%$ or greater to indicate substantial heterogeneity. We did not perform a metaanalysis when we found substantial heterogeneity, and instead provided a narrative summary of the findings. We also considered the nature of interventions and the patient population to evaluate clinical heterogeneity in the included trials.

\section{Assessment of reporting biases}

For meta-analyses in which we included more than 10 trials, we planned to construct funnel plots to assess the potential for publication bias. We planned that if a trial protocol was available, we would assess whether all outcomes relevant to this review that were specified in the protocol were also described in the published reports.

\section{Data synthesis}

We performed a meta-analysis for comparisons where we found minimal or no clinical heterogeneity and without substantial statistical heterogeneity. We used a random-effects model, except for comparisons with two eligible studies, when we used a fixedeffect model. For trials with more than one comparison group, we included the trial in relevant non-overlapping comparisons. In addition, we collapsed data across intervention groups to include such trials in the comparison of any prophylaxis versus no prophylaxis. We used the number allocated in the denominator for our calculations in all cases except when these data were not available in the trial reports. We did not explicitly consider risk of bias as a factor when determining whether to include eligible trials in meta-analyses. We considered sensitivity analyses based on risk of bias as discussed below.

For outcomes where at least one trial explicitly reported that no events were observed in either the treatment or control arm, we performed a meta-analysis using the Mantel-Haenszel method and a continuity correction proportional to the inverse of the opposite arm (Sweeting 2004). Specifically, the continuity correction we used was $1 /(r+1)$ in the treatment arm and $r /(r+1)$ in the control group, where $r$ is the ratio of sample sizes in the two arms. We 
used R (version 3.3.2) to conduct meta-analyses that included the continuity correction (R Core Team 2013).

\section{Subgroup analysis and investigation of heterogeneity}

We planned to consider subgroups by cause of infection, however the available data were insufficient to permit such analyses. We also planned to separately analyse studies conducted in high-income versus low-income countries using the classification specified by the United Nations (WESP2016). However, this was not possible given the diversity in settings in which the included trials were conducted.

\section{Sensitivity analysis}

Where data were available, we excluded studies with high risk of selection bias in sensitivity analyses.

\section{Summary of findings and assessment of the certainty of the evidence}

We prepared 'Summary of findings' tables using the GRADE approach for the following eight outcomes (Langendam 2013).

1. Blindness

2. Any adverse visual outcome
3. Gonococcal conjunctivitis (GC)

4. Chlamydial conjunctivitis (CC)

5. Bacterial conjunctivitis (BC)

6. Any conjunctivitis of any aetiology (ACAE)

7. Conjunctivitis of unknown aetiology (CUE)

8. Adverse effects

\section{RES U L T S}

\section{Description of studies}

\section{Results of the search}

The electronic searches yielded a total of 498 records (Figure 1). After removal of 32 duplicates, we screened the remaining 466 records. We obtained the full-text reports of 105 records for further assessment. Of these, we included 34 reports of 30 studies (see Characteristics of included studies for details). We excluded 70 reports of 63 studies (see Characteristics of excluded studies for details). One study (Matinzadeh 2007)is currently awaiting classification (see Characteristics of studies awaiting classification); if we receive further information on this study we will assess it for inclusion in future updates of this review. 


\section{Figure 1. PRISMA flow diagram}

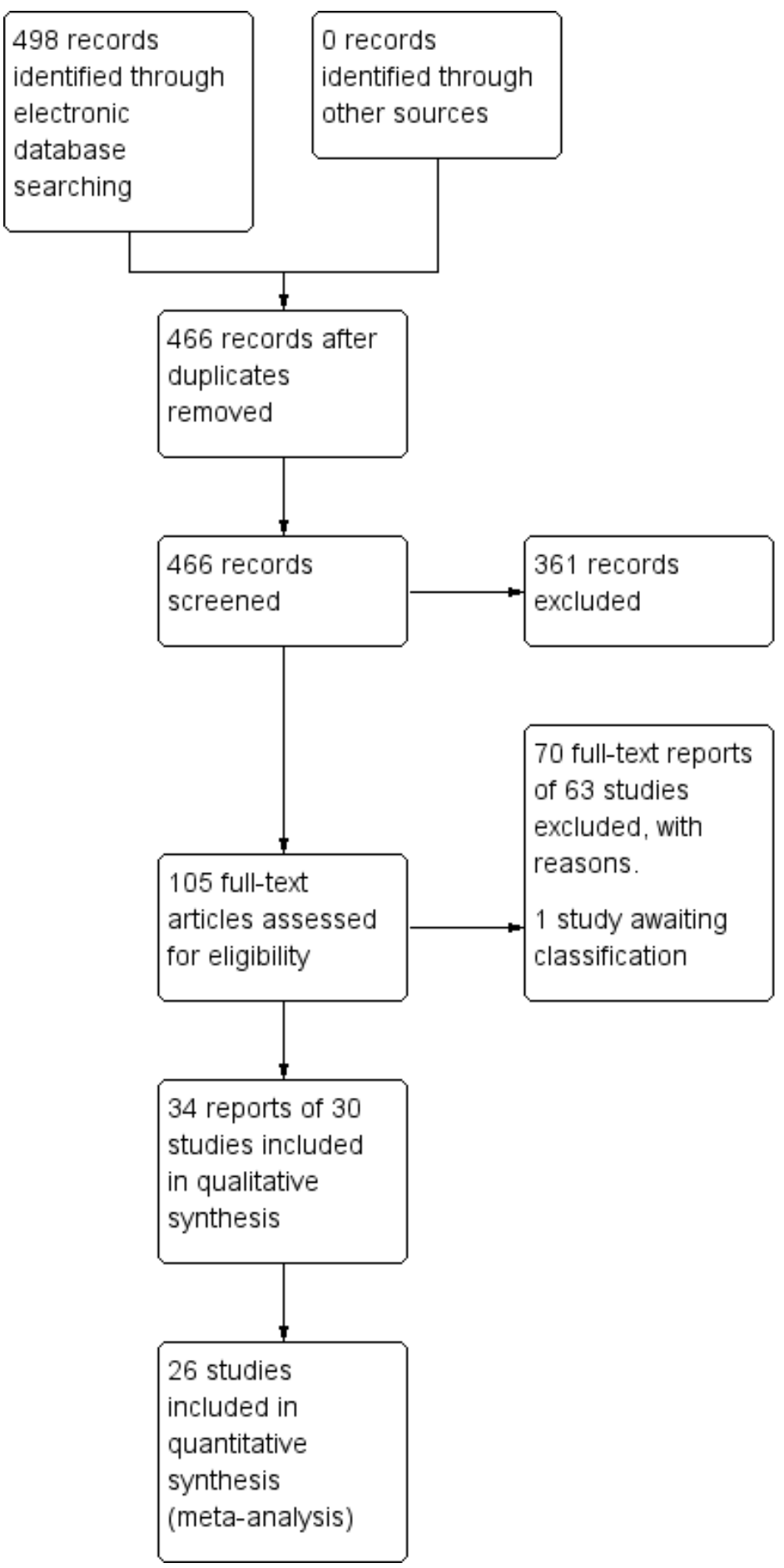

\section{Included studies}

\section{Setting and participants}

Specific details on each included study are shown in the Characteristics of included studies table.

\section{Design}

Half of the included studies were quasi-randomised (15/30; 50\%), and half were randomised (15/30; 50\%); see Figure 2 . 
Figure 2. Table of Trial Settings

INTERVENTIONS FOR PROPHYLAXIS OF OPHTHALMIA NEONATORUM - TRIAL SETTINGS

\begin{tabular}{|c|c|c|c|c|c|}
\hline No. & STUDY & $\begin{array}{l}\text { NUMBER OF } \\
\text { ARMS }\end{array}$ & \begin{tabular}{|} 
RANDOMISED OR \\
QUASI-RANDOMISED
\end{tabular} & COUNTRY & COUNTRY CLASSIFICATION* \\
\hline 1 & Davidson 1951 & 2 & QR & USA & HIGH INCOME \\
\hline 2 & Cousineau 1952 & 2 & QR & Canada & HIGH INCOME \\
\hline 3 & Harris 1957 & 2 & QR & USA & HIGH INCOME \\
\hline 4 & Posner 1959 & 2 & QR & USA & HIGH INCOME \\
\hline 5 & Christian 1960 & 2 & QR & USA & HIGH INCOME \\
\hline 6 & Kaivonen I 1965 & 2 & QR & Finland & HIGH INCOME \\
\hline 7 & Kaivonen II 1965 & 2 & QR & Finland & HIGH INCOME \\
\hline 8 & Hammerschlag 1980 & 2 & $\mathbf{R}$ & USA & HIGH INCOME \\
\hline 9 & Wahlberg 1982 & 3 & $\mathbf{R}$ & Sweden & HIGH INCOME \\
\hline 10 & Siegel 1982 & 2 & QR & USA & HIGH INCOME \\
\hline 11 & Hick 1985 & 2 & $\mathbf{R}$ & USA & HIGH INCOME \\
\hline 12 & Fischer 1988 & 2 & QR & Zaire & LOW INCOME \\
\hline 13 & Laga 1988 & 2 & QR & Kenya & LOW INCOME \\
\hline 14 & Hammerschlag 1989 & 3 & QR & USA & HIGH INCOME \\
\hline 15 & Brussieux 1991 & 2 & QR & France & HIGH INCOME \\
\hline 16 & Chen 1992 & 4 & QR & China & LOW INCOME \\
\hline 17 & Bell 1993 & 3 & $\mathbf{R}$ & USA & HIGH INCOME \\
\hline 18 & Graf 1994 & 2 & $\mathbf{R}$ & Germany & HIGH INCOME \\
\hline 19 & Isenberg 1995 & 3 & QR & Kenya & LOW INCOME \\
\hline 20 & Isenberg 2003 & 2 & QR & Kenya & LOW INCOME \\
\hline 21 & Zbojan 2004 & 2 & $\mathbf{R}$ & Slovakia & UPPER MIDDLE INCOME \\
\hline 22 & Richter 2006 & 2 & $\mathbf{R}$ & Germany & HIGH INCOME \\
\hline 23 & Ali 2007 & 3 & $\mathbf{R}$ & Iran & LOWER MIDDLE INCOME \\
\hline 24 & Ghahramani 2007 & 2 & $\mathbf{R}$ & Iran & LOWER MIDDLE INCOME \\
\hline 25 & Ramirez-Ortiz 2007 & 2 & $\mathbf{R}$ & Mexico & UPPER MIDDLE INCOME \\
\hline 26 & David 2011 & 2 & $\mathbf{R}$ & Israel & HIGH INCOME \\
\hline 27 & Ghotbi 2012 & 2 & $\mathbf{R}$ & Iran & UPPER MIDDLE INCOME \\
\hline 28 & Ghaemi 2014 & 3 & $\mathbf{R}$ & Iran & UPPER MIDDLE INCOME \\
\hline 29 & Bramantyo 2015 & 2 & $\mathbf{R}$ & Indonesia & LOWER MIDDLE INCOME \\
\hline 30 & Pastor 2015 & 2 & $\mathbf{R}$ & Angola & UPPER MIDDLE INCOME \\
\hline
\end{tabular}

\section{Sample sizes}

The total number of neonates included in the review across all 30 studies was 79,198 . The sample size in individual trials ranged from 40 to 32,058 neonates. The average number of neonates in the included studies was 2988, with a median of 654.5 .

\section{Setting}

Of the 30 trials, 18 studies (60\%) were conducted in high-income economies ( 9 in the USA, 7 in Europe, 1 each in Canada and Israel), and $12(40 \%)$ were conducted in low- and middle-income economies ( 3 in Kenya, 4 in Iran, 1 each in Zaire, Mexico, Indonesia, China, and Angola). Two studies explicitly reported recruiting participants at high risk, for example inner-city populations (Figure 2) (Hammerschlag 1980; Hammerschlag 1989).

\section{Time period of trials}

A significant number of trials were conducted more than 50 years ago. Of the 30 included trials, seven (23\%) trials were conducted between 1940 and 1960, with most of these trials (six) taking place between 1950 and 1960. Four trials (13\%) were conducted between 1960 and 1980; eight trials (27\%) between 1980 and 2000; seven trials (23\%) between 2000 and 2010; and four trials (13\%) from 2010 to the present.

\section{Interventions}

Fourteen different prophylactic regimens and 12 different prophylactic interventions were studied across the 30 included trials (Figure 3). Silver nitrate was used in the majority of trials (18 out of 30); mainly in older trials up to the early 1990s. Erythromycin was used in 10 trials; tetracycline in 9 trials; and povidone-iodine in 9 trials. Povidone-iodine was used mainly in more recent trials from the 1990s to the present. The route of delivery for medications was topical ocular administration, with the exception of two trials that used intramuscular penicillin. No prophylaxis was used as one arm of the study in 10 of the 30 trials. Of all trials that included no prophylaxis in one arm of the study, placebo was used in only one trial: Wahlberg 1982 used physiological normal saline in one arm of the trial. 


\section{Figure 3. Table of Trials, Interventions, Method of Allocation, Settings}

\section{INTERVENTIONS FOR PROPHYLAXIS OF OPHTHALMIA NEONATORUM - TABLE OF TRIALS, METHOD OF ALLOCATION \& INTERVENTIONS}

\begin{tabular}{|c|c|c|c|c|c|c|c|c|c|c|c|c|c|c|c|c|c|c|}
\hline \multirow[b]{2}{*}{ No. } & \multirow{2}{*}{ STUDY } & \multirow{2}{*}{\begin{tabular}{|l} 
NUMBER \\
OF ARMS
\end{tabular}} & \multirow{2}{*}{$\begin{array}{l}\text { R or aR } \\
\text { TRIAL: }\end{array}$} & \multicolumn{15}{|c|}{ TRIAL ARMS } \\
\hline & & & & siver nemane & ERrmpources & Terpacracawe & Pevorone rome & 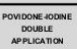 & нескавамим & Pencoums & pexacumam & 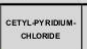 & Matracen & susicetrmose & 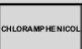 & cocostrom & ceroumoe & Mopropouruavis \\
\hline 1 & Davidson 1951 & 2 & QR & $\mathrm{SN}$ & & & & & & PEN & PENIM & & & & & & & \\
\hline 2 & Cousineau 1952 & 2 & QR & $\mathrm{SN}$ & & & & & & & & & & $\mathrm{SCM}$ & & & & \\
\hline 4 & Posner 1959 & 2 & QR & & & & & & & & & & BC-P & & & & & N.P. \\
\hline 5 & Christian 1960 & 2 & QR & SN & ERY & & & & & & & & & & & & & \\
\hline 6 & Kaivonen I 1965 & 2 & QR & SN & & & & & & & & CPC & & & & & & \\
\hline 9 & Wahlberg 1982 & 3 & $\mathbf{R}$ & SN & & & & & HEX & & & & & & & & & N.P. \\
\hline 10 & Siegel 1982 & 2 & QR & & & TET & & & & & PENGIM & & & & & & & \\
\hline 11 & Hick 1985 & 2 & $\mathbf{R}$ & $\mathrm{SN}$ & & TET & & & & & & & & & & & & \\
\hline 12 & Fischer 1988 & 2 & QR & SN & & TET & & & & & & & & & & & & \\
\hline 13 & Laga 1988 & 2 & QR & $\mathrm{SN}$ & & TET & & & & & & & & & & & & \\
\hline 14 & Hammerschlag 1989 & 3 & QR & $\mathrm{SN}$ & ERY & TET & & & & & & & & & & & & \\
\hline 19 & Isenberg 1995 & 3 & QR & $\mathrm{SN}$ & ERY & & PI & & & & & & & & & & & \\
\hline 20 & Isenberg 2003 & 2 & QR & & & & PI & $P 1 \times 2$ & & & & & & & & & & \\
\hline 21 & Zbojan 2004 & 2 & $\mathrm{R}$ & & & & PI & & & & & & & & & & G.BROMIDE & \\
\hline 22 & Richter 2006 & 2 & $\mathbf{R}$ & $\mathrm{SN}$ & & & PI & & & & & & & & & & & \\
\hline 23 & Ali 2007 & 3 & $\mathbf{R}$ & & ERY & & PI & & & & & & & & & & & N.P. \\
\hline 24 & Ghahramani 2007 & 2 & $R$ & & ERY & & & & & & & & & & & & & N.P. \\
\hline 25 & \begin{tabular}{|l|} 
Ramirez-Ortiz 2007 \\
\end{tabular} & 2 & $R$ & & & & PI & & & & & & & & $\mathrm{CPh}$ & & & \\
\hline 26 & David 2011 & 2 & $\mathbf{R}$ & & & TET & PI & & & & & & & & & & & \\
\hline 27 & Ghotbi 2012 & 2 & $R$ & & ERY & TET & & & & & & & & & & & & N.P. \\
\hline 28 & Ghaemi 2014 & 3 & $\mathbf{R}$ & & ERY & & & & & & & & & & & COLOSTRUM & & N.P. \\
\hline 29 & Bramantyo 2015 & 2 & $\mathbf{R}$ & & & & PI & & & & & & & & $\mathrm{CPh}$ & & & \\
\hline 30 & Pastor 2015 & 2 & $\mathbf{R}$ & & & & PI & & & & & & & & & & & N.P. \\
\hline
\end{tabular}

The full list of interventions used in the included studies is as follows.

1. Silver nitrate solution (18 studies: all $1 \%$ )

2. Erythromycin ointment (10 studies: all $0.5 \%$, except for one study in which concentration was not specified)

3. Tetracycline ( 9 studies: all 1\%; 2 studies used solution instead of ointment)

4. Povidone-iodine:
a. solution (9 studies: all $2.5 \%$ )

b. double application (1 study).

5. Hexarginum (1 study: $10 \%$ solution contains $1 \mathrm{~g} \mathrm{AgNO} 3+36 \mathrm{~g}$ $\mathrm{CH} 3 \mathrm{NH} 2$ dissolved in $63 \mathrm{~g}$ sterile water)

6. Penicillin:

a. ointment ( 2 studies: 1 study: penicillin G 1\% ointment; 1 study: penicillin ointment 100,000 units/g);

b. intramuscular injection (IM) (2 studies: 1 study: penicillin 10,000 units per IM injection; 1 study: penicillin G 25,000 to 50,000 units per IM injection depending on birthweight)

7. Cetyl-pyridinium chloride solution (2 studies: 1 study: $0.1 \% ; 1$ study: $0.05 \%)$

8. Bacitracin-phenacaine ointment (1 study: bacitracin 500 units/ g; $2 \%$ phenacaine hydrochloride)
9. Sulphacetimide ointment (1 study: $10 \%$ ointment)

10.Chloramphenicol solution ( 2 studies)

11.Carbethopendecinium bromide solution (1 study)

12. Colostrum ( 1 study: 2 drops of mother's colostrum in each eye)

\section{Follow-up time}

The included trials varied widely in duration of follow-up and the time at which outcomes were analysed and reported. Eleven out of $30(37 \%)$ studies followed up neonates for one month. Four trials (13\%) followed up neonates for more than one month. In 12 trials (40\%), follow-up was less than one month. In three trials (10\%), no follow-up period was specified. Of the four trials that followed up neonates for more than one month, two trials followed up neonates for three to five months, and two trials for 60 days. Of the two trials that followed up neonates for 60 days, we were able to extract $30-$ day data from one trial, and in the other trial, follow-up was only for the outcome of nasolacrimal duct obstruction. Of the 12 trials that followed up neonates for less than a month, nine trials followed neonates for 10 days or less, and three trials followed neonates for two weeks. 


\section{Outcomes}

Eighteen of the 30 included trials (60\%) reported the outcome of gonococcal conjunctivitis (GC). Thirteen of these 18 trials (43\%) reported no actual cases of GC in any arm of the study. Consequently, only five studies out of the 30 included trials (17\%) reported any cases of neonates with GC.

Thirteen of the 30 included studies (43\%) reported the outcome of chlamydial conjunctivitis (CC). One of these 13 studies found no cases of CC in either arm of the study. Seven of the 30 trials (23\%) were conducted between 1940 to 1960, when methods to detect $C$ trachomatis were not readily available. Ten out of 30 trials (33\%) reported rates of bacterial conjunctivitis (BC). Twenty-four out of 30 trials $(80 \%)$ reported the outcome of total number of clinical conjunctivitis cases. In 10 of 30 trials (33\%), we were able to determine rates of conjunctivitis that were culture-negative.

We were unable to extract outcome data from four studies. In Richter 2006, the outcomes of conjunctivitis were not well defined, such that they could not be extracted. In Wahlberg 1982, the outcomes of total conjunctivitis, culture-negative conjunctivitis, BC, GC, and CC were not presented by allocation group; the denominators did not correspond to allocation groups; and the data were presented as percentages. In Bramantyo 2016, there were no conjunctivitis cases; follow-up time was only 24 hours; and conjunctivitis was not specified in the methods as an outcome. Finally, in Pastor 2015, there was a high loss to follow-up, and repeated communications with the study authors failed to clarify confusion over the remaining data, so that we felt the data could not be extracted.

\section{Excluded studies}

We excluded 63 studies (see Figure 1, Characteristics of excluded studies)

\section{Risk of bias in included studies}

The risk of bias is summarised in Figure 4 and Figure 5. 
Figure 4. Risk of bias summary: review authors' judgements about each risk of bias item for each included study.

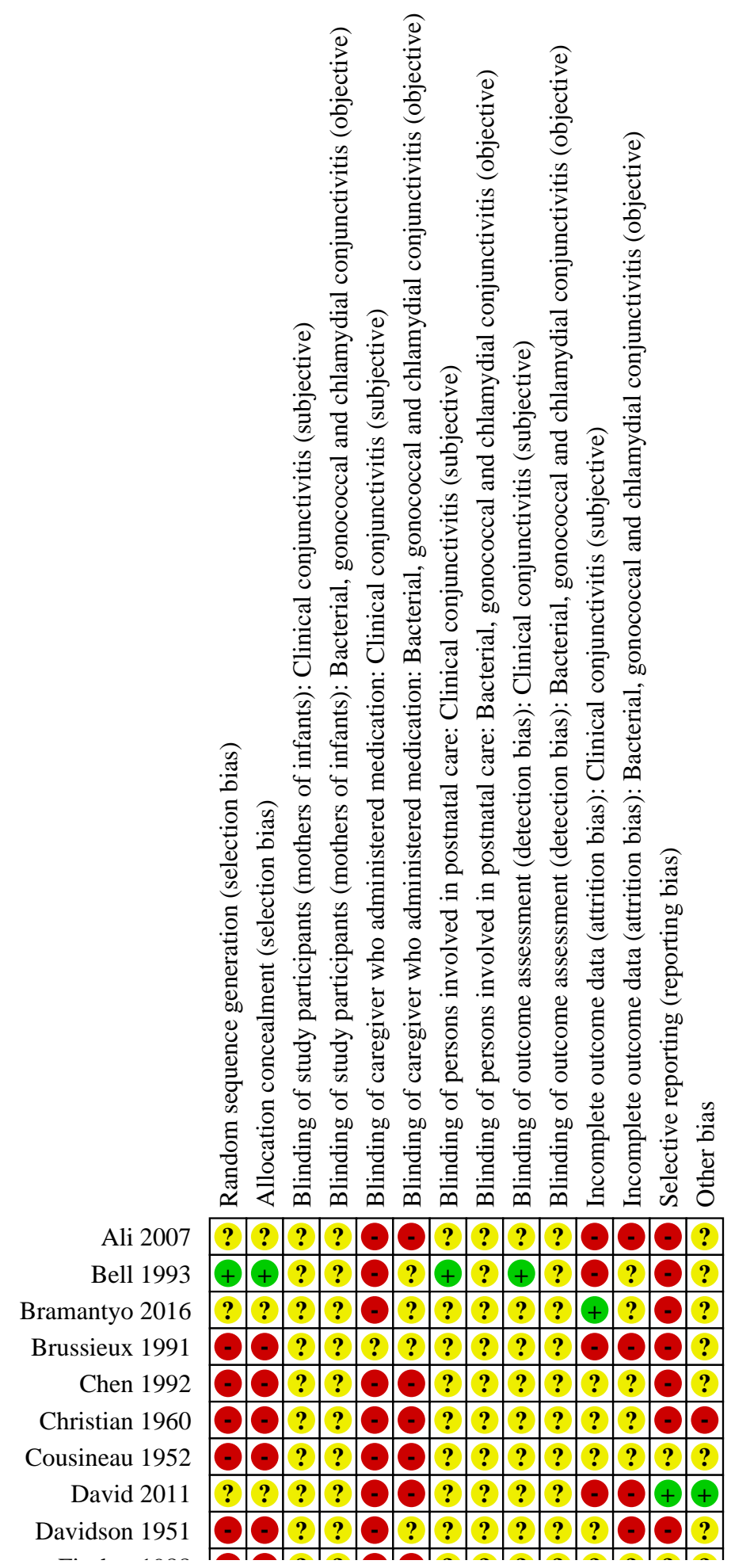


Figure 4. (Continued)

\begin{tabular}{|c|c|c|c|c|c|c|c|c|c|c|c|c|c|c|}
\hline Davidson 1951 & & 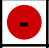 & $?$ & $?$ & - & $?$ & $?$ & $?$ & $?$ & $?$ & $?$ & & - & ? \\
\hline Fischer 1988 & & - & $?$ & $?$ & & 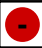 & $?$ & $?$ & $?$ & $?$ & $?$ & $?$ & $?$ & $?$ \\
\hline Ghaemi 2014 & + & $?$ & $?$ & $?$ & $ت$ & $?$ & $?$ & $?$ & $\odot$ & $?$ & - & $?$ & - & $?$ \\
\hline Ghahramani 2007 & $?$ & $?$ & $?$ & $?$ & 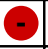 & $?$ & $?$ & $?$ & $?$ & $?$ & + & $?$ & 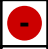 & $?$ \\
\hline Ghotbi 2012 & $?$ & $?$ & $?$ & $?$ & - & $?$ & $?$ & $?$ & $?$ & $?$ & $?$ & $?$ & $?$ & $?$ \\
\hline Graf 1994 & $?$ & $?$ & $?$ & $?$ & $?$ & $?$ & $?$ & $?$ & $?$ & $?$ & + & $?$ & + & $?$ \\
\hline Hammerschlag 1980 & $?$ & $?$ & $?$ & $?$ & $?$ & - & $?$ & $?$ & $?$ & $?$ & $?$ & 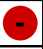 & - & $?$ \\
\hline ammerschlag 1989 & E & 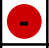 & $?$ & $?$ & $?$ & $=$ & $?$ & $?$ & $?$ & $?$ & $?$ & - & - & $?$ \\
\hline Harris 1957 & E & E & $?$ & $?$ & - & $?$ & $?$ & $?$ & $?$ & $?$ & $?$ & $?$ & - & $?$ \\
\hline Hick 1985 & + & + & $?$ & $?$ & $?$ & $?$ & $?$ & $?$ & $?$ & $?$ & $?$ & $?$ & $?$ & $?$ \\
\hline Isenberg 1995 & & & $?$ & $?$ & 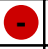 & $=$ & - & - & $?$ & + & $?$ & $?$ & + & $?$ \\
\hline Isenberg 2003 & & - & $?$ & $?$ & $?$ & $?$ & $?$ & $?$ & $?$ & $?$ & $?$ & $?$ & + & \pm \\
\hline Kaivonen 1965a & 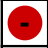 & E & $?$ & $?$ & $?$ & $?$ & $?$ & $?$ & $?$ & $?$ & $?$ & $?$ & - & E \\
\hline Kaivonen 1965b & & $=$ & $?$ & $?$ & $?$ & $?$ & $?$ & $?$ & $?$ & $?$ & $?$ & $?$ & - & $?$ \\
\hline Laga 1988 & & 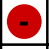 & $?$ & $?$ & - & $=$ & $?$ & $?$ & $?$ & $?$ & $=$ & 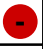 & $?$ & $?$ \\
\hline Pastor 2015 & + & $?$ & $?$ & $?$ & - & $?$ & $?$ & $?$ & $?$ & $?$ & E & $?$ & 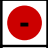 & $?$ \\
\hline Posner 1959 & & $\Theta$ & $?$ & $?$ & $?$ & $?$ & $?$ & $?$ & $?$ & $?$ & $?$ & $?$ & - & $?$ \\
\hline Ramirez-Ortiz 2007 & + & + & $?$ & $?$ & $?$ & - & $?$ & $?$ & $?$ & $?$ & $?$ & E & & + \\
\hline Richter 2006 & $?$ & $?$ & $?$ & $?$ & $?$ & $?$ & $?$ & $?$ & $?$ & $?$ & $?$ & $?$ & $\Theta$ & $?$ \\
\hline Siegel 1982 & E & 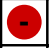 & $?$ & $?$ & $?$ & 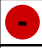 & $?$ & $?$ & $?$ & $?$ & $?$ & $?$ & $?$ & $?$ \\
\hline Wahlberg 1982 & $?$ & $?$ & $?$ & $?$ & $?$ & $?$ & $?$ & $?$ & $?$ & $?$ & E & $?$ & - & $?$ \\
\hline Zbojan 2004 & $?$ & $?$ & $?$ & $?$ & & $?$ & $?$ & $?$ & $?$ & $?$ & $?$ & $?$ & $?$ & $?$ \\
\hline
\end{tabular}

Figure 5. Risk of bias graph: review authors' judgements about each risk of bias item presented as percentages across all included studies.

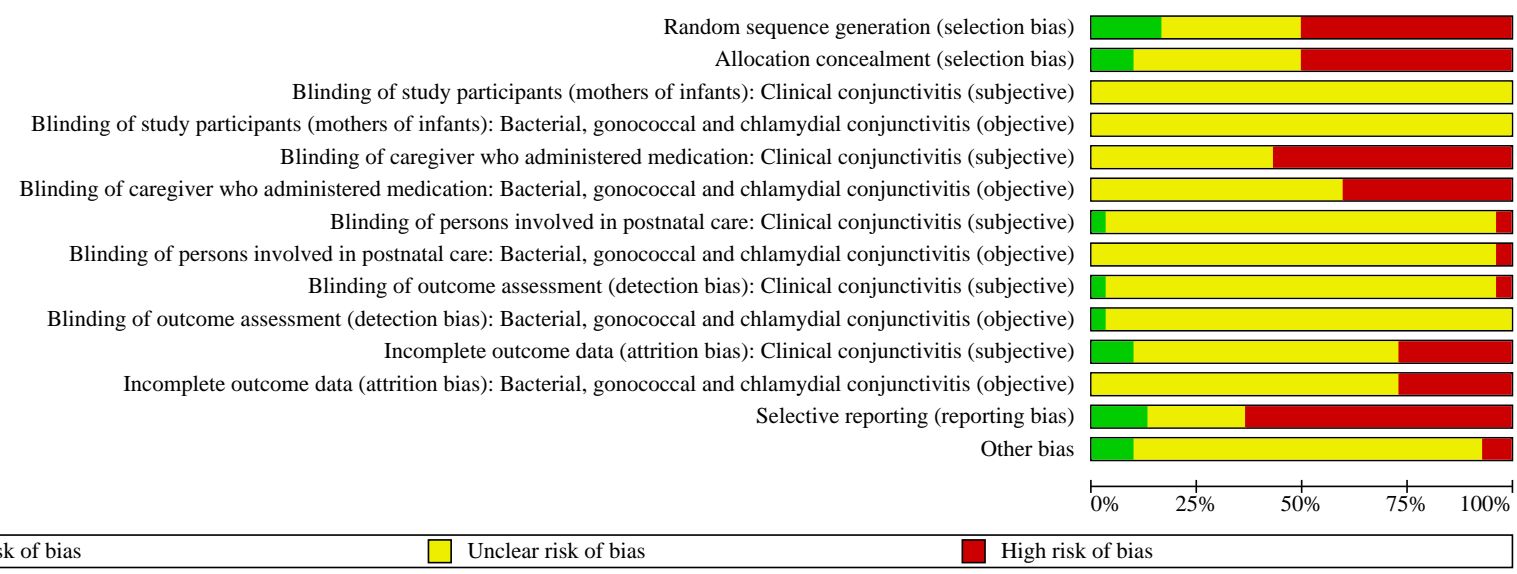

\section{Allocation}

Of the 15 quasi-randomised trials, three trials alternated by day, six trials alternated by week, two trials alternated by month, and three trials applied prophylaxis to alternate neonates. In the remaining quasi-randomised trial, the prophylaxis for each day was selected from a previously prepared random sequence of assignments. Amongst the 15 randomised trials, only four trials reported the method of randomisation; the remaining 11 trials described allocation using the word "random" or "randomised" but did not provide any further information on how the random sequence was generated.

We assessed 15 out of the 30 included trials (50\%) as having a high risk of selection bias based on random sequence generation. These 15 trials were generally quasi-randomised studies using alternation by neonate or by day, week, or month. Eleven of the 30 included trials (37\%) used the word "random" or "randomised" in 
the methods, but provided no further information on the random sequence generation process. We found only four trials (13\%) to have a low risk of selection bias based on random sequence generation.

Similarly, amongst the 30 included trials, we assessed 15 trials $(50 \%)$ as having a high risk of selection bias based on inadequate concealment of allocations prior to assignment. Again, these 15 trials were generally quasi-randomised studies as mentioned above. Twelve of the 30 included trials $(40 \%)$ used the word "random" or "randomised" in the methods, but provided no further information on the allocation concealment. We found only five trials (17\%) to have a low risk of selection bias based on the fact that participants or investigators could not foresee the assignment.

\section{Blinding}

We assessed masking (blinding) of participants, personnel, and outcome assessors for studies in this review. We further categorised "personnel" into three subcategories: 1 . mothers of infants; 2. people administering prophylaxis; and 3 . people involved in postnatal care.

In none of the trials was there any explicit mention of any attempt to make the interventions look the same, or dispense them from containers that made them indistinguishable from the other interventions. In only one trial, Wahlberg 1982, it may have been the case that the interventions looked the same, and were dispensed from containers that looked the same, but this was not explicit. The ophthalmic medications used in each of the included studies did not look the same, except possibly in Wahlberg 1982. For instance, silver nitrate is a clear solution; erythromycin is an ointment; and povidone-iodine is an orange-red solution. The physical characteristics of the interventions studied in the included trials are described in Table 1.

In all but five of the 30 included trials, the medications would have looked strikingly different, either because of colour or consistency. The difference in appearance of the medication would prevent masking for the person administering the medication and could lead to bias, which could influence the outcome. In fact, one study, Fischer 1988, demonstrated lack of adherence to application of medication secondary to lack of masking, which influenced rates of conjunctivitis in one arm of the study.

Masking is further affected by the fact that the majority of the medications leave a stain on the eyes after dispensing. Silver nitrate leaves lid stains that may last 30 to 48 hours. Povidoneiodine may stain periocular skin for minutes to hours. Antibiotic ointments such as erythromycin, tetracycline, and penicillin can leave a residue that may last for hours. In four of the five abovementioned trials in which the medications may have looked the same, one of the interventions was silver nitrate, which would have affected masking of mothers and people involved in postnatal care due to silver nitrate's propensity to cause lid stains. In the remaining trial in which the medications may have looked the same, there were three intervention arms, with two arms using erythromycin and tetracycline ointment, and the last arm having no prophylaxis. In this trial the ointment allocation groups could therefore be distinguished from the no-prophylaxis group. In fact, in only one of the 10 trials where no prophylaxis was one of the arms of the study was any placebo used; hence, there was lack of masking for the person administering the medication in the remaining nine trials with a no-prophylaxis arm.

To summarise, in no studies was it possible to mask the person administering the prophylaxis for ophthalmia neonatorum. There are also risks of compromising masking in mothers of neonates and people involved in postnatal care due to the presence of prophylaxis staining and residue. Finally, for outcome assessments conducted soon after prophylaxis administration, particularly for the interventions of silver nitrate, antibiotic ointments, and povidone-iodine, masking could be compromised.

In spite of the performance bias and detection bias introduced by the appearance and residual staining of the prophylaxis medications, trials were scored on these categories, by subjective and objective outcome. We classified clinical conjunctivitis as a subjective outcome, and BC, CC and GC as an objective outcome. We considered CUE, which was essentially culturenegative conjunctivitis, as objective, given that it was derived from subtracting BC cases from total clinical conjunctivitis cases. Our 'risk of bias' assessment for masking essentially found that there was much similarity in the classifications between the subjective and objective outcomes of conjunctivitis. In summary, the vast majority of studies had a high or unclear risk of performance bias, and unclear risk of detection bias, across most outcomes.

There is an additional aspect of silver nitrate that introduces a form of detection bias. Sixteen of the 30 included trials (53\%) used silver nitrate. In 13 of these trials, there was an outcome derived from eye culture results. In any trial with silver nitrate, there could be biased outcome assessment. Silver nitrate causes a chemical conjunctivitis in the first 72 hours. As a result, more neonates in the silver nitrate allocation group could be referred for culture in the first 72 hours. Finding bacteria in the culture does not necessarily prove that the bacteria caused the conjunctivitis. The conjunctivitis could be chemical, but growing normal flora of the eye. Alternatively, the conjunctivitis could be chemical with a chlamydial carrier. Finally, the conjunctivitis could very well be caused by the bacteria or chlamydia. Considerations about incubation periods, and assessing for carriers and normal flora with asymptomatic cases, could have assisted with differential diagnosis, but no trial made an attempt to distinguish these possible outcomes.

\section{Incomplete outcome data}

We assessed eight of the 24 studies that reported the outcome of total clinical conjunctivitis cases as at high risk of bias due to the high proportion of missing outcome data in proportion to event rates. We judged 13 of these 24 studies to be at unclear risk of bias due to poor reporting in the study. We graded only three studies as at low risk of attrition bias. Of the 20 studies that reported $B C, C C$ or GC we assessed 10 as at high risk and 10 as at unclear risk of attrition bias. The two studies reporting nasolacrimal duct obstruction as an outcome had a high risk of attrition bias. We graded the single study that reported the outcome of keratitis as at low risk of attrition bias.

\section{Selective reporting}

We did not have access to the protocols for any of the included studies, therefore we compared the outcomes listed in the methods section of the trial with those reported in the results. We judged 19 of the 30 included trials to be at high risk of bias for selective 
outcome reporting. We assessed seven studies as at unclear risk and only four studies as at low risk of reporting bias.

\section{Other potential sources of bias}

More than half of the included studies (18/30; 60\%) did not specify a source of funding. Of the remaining 12 studies $(40 \%)$, nine studies were funded by a non-governmental organisation, charitable foundation, government agency, hospital, or medical school; funding sources that would seem unlikely to have biased the methodology or results. Three studies were funded by pharmaceutical companies, which supplied one of the interventions in the trial (Davidson 1951; Hammerschlag 1980; Posner 1959). In all three studies, outcomes favoured the intervention supplied and funded by the pharmaceutical company. We were unable to determine if any aspect of the methodology may have been affected to the point of risk of bias by any pharmaceutical funding.

Only five of the 30 included trials (17\%) made a declaration of interest, specifying there was no conflict of interest. The remaining 25 trials (83\%) made no reference to any declaration of interest.

Only three of the 30 included trials (10\%) provided sufficiently detailed information in the study report to enable ruling out other potential sources of bias.

We contacted the study authors if the information in the available reports was insufficient to permit assessment. Some authors were not contacted due to the age of the studies. We contacted the study authors of 14 of the 30 included trials, of which authors of nine studies provided a response.

\section{Effects of interventions}

See: Summary of findings 1 Any prophylaxis compared to no prophylaxis for the prevention of ophthalmia neonatorum in newborn children
None of the trials reported any data on two of the primary outcomes specified for this review: incidence of blindness and visual impairment. Data on the remaining outcomes are discussed below (Table 2). In this review, we did not attempt to rank the relative effectiveness of the various interventions; this has to be addressed in a subsequent network meta-analysis. Instead, we discuss our findings for important pairwise comparisons of interventions along with an overall comparison of any prophylaxis versus no prophylaxis. The individual pairwise comparisons are organised as follows: interventions compared with silver nitrate; interventions compared with erythromycin; interventions compared with tetracycline; and other comparisons.

\section{Any prophylaxis versus no prophylaxis}

See Summary of findings 1.

Any prophylaxis was associated with a statistically significant reduction in risk for any conjunctivitis of any aetiology (ACAE) but not for GC, BC, CC, or conjunctivitis of unknown aetiology (CUE). The certainty of evidence was moderate for ACAE but low for all other outcomes. For the sake of clarity in the narrative, within each outcome under the overall comparison of any prophylaxis versus no prophylaxis, we will describe effects for individual medications versus no prophylaxis (see Table 3; Table 4; Table 5; Table 6; Table 7; Table 8).

\section{Gonococcal conjunctivitis}

There was only very low-certainty evidence on the risk of GC with prophylaxis (4/5340 newborns) compared to no prophylaxis (5/2889) at one month (risk ratio (RR) $0.79,95 \%$ confidence interval (CI) 0.24 to $2.65,3$ studies, $\mathrm{I}^{2}=0 \%$; Figure 6 ).

\section{Figure 6. Forest plot of comparison: 1 Any prophylaxis versus no prophylaxis, outcome: 1.1 Gonococcal conjunctivitis}

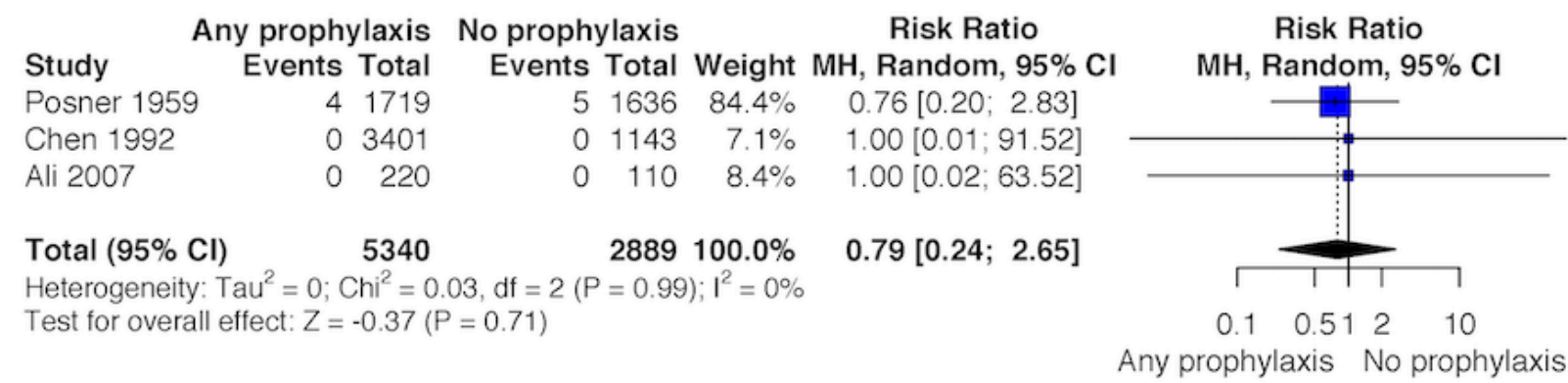

Data on GC were available for the following medications individually compared with no prophylaxis: silver nitrate, erythromycin, tetracycline, povidone-iodine, and bacitracin. For all these medications, a single trial provided data on comparison with no prophylaxis except erythromycin for which data were provided by two trials. No events of GC were observed when silver nitrate (Chen 1992), erythromycin (Ali 2007; Chen 1992), tetracycline (Chen 1992), or povidone-iodine (Ali 2007), were compared with no prophylaxis. It was thus not possible to estimate the effect of prophylaxis with these medications against GC. Bacitracin was associated with a RR of 0.76 for GC but with very wide Cls (RR 0.76 , $95 \% \mathrm{Cl} 0.20$ to 2.83 ) (Posner 1959).

\section{Chlamydial conjunctivitis}

In a meta-analysis of two trials, any prophylaxis did not appear to reduce the incidence of CC (RR $0.96,95 \% \mathrm{Cl} 0.57$ to $1.61,4874$ 
participants, 2 studies; $12=0 \%$; Analysis 1.1). GRADE certainty of the evidence was low.

Data on CC were available for the following medications individually compared with no prophylaxis: silver nitrate, erythromycin, tetracycline, and povidone-iodine. For all these medications, a single trial provided data on comparison with no prophylaxis except erythromycin for which data were provided by two trials. There was little or no difference with the risk of CC for silver nitrate (RR $1.06,95 \% \mathrm{Cl} 0.55$ to 2.02 ; Chen 1992). Povidoneiodine was associated with an increased risk of CC (RR 2.00, 95\% $\mathrm{Cl} 0.18$ to 22.74; Ali 2007), but this association was not statistically significant. There was little or no difference in CC with erythromycin (RR $0.93,95 \% \mathrm{Cl} 0.49$ to $1.77,2526$ participants, 2 studies; $12=$ $0 \%$; Analysis 3.2) and tetracycline (RR $0.82,95 \% \mathrm{Cl} 0.42$ to 1.63 ) compared with no prophylaxis (Chen 1992).

\section{Bacterial conjunctivitis}

In a meta-analysis of two trials, any prophylaxis did not appear to reduce the incidence of $\mathrm{BC}$ (RR $0.84,95 \% \mathrm{Cl} 0.37$ to $1.93,3685$ participants, 2 studies; $1^{2}=0 \%$; Analysis 1.2). GRADE certainty of the evidence was low.

Data on BC were available for the following medications individually compared with no prophylaxis: erythromycin and povidone-iodine. A single trial provided data on $\mathrm{BC}$ for both these medications (Ali 2007). There was no evidence that erythromycin was associated with a reduced risk of $\mathrm{BC}$ compared with no prophylaxis (RR $0.80,95 \% \mathrm{Cl} 0.22$ to 2.90 ) (Ali 2007). Povidoneiodine did not appear to reduce the incidence of $\mathrm{BC}$ compared with no prophylaxis (RR 1.00, 95\% Cl 0.30 to 3.36) (Ali 2007).

\section{Any conjunctivitis of any aetiology}

In a meta-analysis of eight trials, any prophylaxis was associated with a $35 \%(95 \% \mathrm{Cl} 22 \%$ to $46 \%)$ reduction in risk of ACAE compared with no prophylaxis (RR $0.65,95 \% \mathrm{Cl} 0.54$ to 0.78 , 9666 participants, 8 studies; $\left.\right|^{2}=11 \%$; Analysis 1.3 ). GRADE certainty of the evidence was moderate.

Data on ACAE were available for the following medications individually compared with no prophylaxis: silver nitrate, erythromycin, tetracycline, povidone-iodine, and colostrum. In a meta-analysis of three trials, silver nitrate was associated with a reduced risk of ACAE compared with no prophylaxis (RR 0.67, $95 \% \mathrm{Cl} 0.52$ to $0.87,2713$ participants, 3 studies; $12=0 \%$; Analysis 2.3). In a meta-analysis of six trials, erythromycin was associated with a reduced risk of ACAE compared with no prophylaxis (RR $0.68,95 \% \mathrm{Cl} 0.51$ to $0.89,3509$ participants, 6 studies; $\left.\right|^{2}=38 \%$; Analysis 3.4). Similarly, in a meta-analysis of two trials, tetracycline was associated with a reduced risk of ACAE compared with no prophylaxis (RR $0.72,95 \% \mathrm{Cl} 0.55$ to $0.94,2519$ participants, 2 studies; $1^{2}=0 \%$; Analysis 4.3 ). Data from a single trial suggest that povidone-iodine reduces the risk of ACAE compared with no prophylaxis (RR $0.38,95 \% \mathrm{Cl} 0.18$ to 0.77 ) (Ali 2007). Finally, a single trial indicates colostrum is associated with a reduction in risk of ACAE compared with no prophylaxis that was not statistically significant (RR $0.72,95 \% \mathrm{Cl} 0.45$ to 1.14 ) (Ghaemi 2014).

\section{Conjunctivitis of unknown aetiology}

In a single trial (Ali 2007), any prophylaxis was associated with an increased risk of CUE compared with no prophylaxis, but the $\mathrm{Cls}$ were very wide (RR $1.75,95 \% \mathrm{Cl} 0.37$ to 8.28 ). GRADE certainty of the evidence was very low.

\section{Adverse events}

In a single trial (Bell 1993), any prophylaxis (erythromycin 0.5\% or silver nitrate $1 \%$ ) did not appear to be associated with an increased risk of nasolacrimal duct obstruction compared with no prophylaxis (RR $0.93,95 \% \mathrm{Cl} 0.68$ to 1.28). GRADE certainty of the evidence was very low. A single trial of silver nitrate $1 \%$ in 40 newborn children reported that no events of keratitis were observed in the prophylaxis and no-prophylaxis groups (Graf 1994).

\section{Erythromycin versus silver nitrate}

See summary of findings in Table 9.

\section{Gonococcal conjunctivitis}

In a meta-analysis of four trials, two of which reported no incidence of GC, erythromycin was associated with a 2.28 -fold increase in risk of GC compared with silver nitrate (RR $2.28,95 \% \mathrm{Cl} 0.88$ to 5.90 , 14,855 participants, 4 studies; $12=0 \%$; Figure 7 ). GRADE certainty of the evidence was very low. The wide $\mathrm{Cl}$ indicate that the estimate is compatible with a $12 \%$ reduction in risk and a 5.9 -fold increase in risk.

Figure 7. Forest plot of comparison: 8 Erythromycin versus silver nitrate, outcome: 8.1 Gonococcal conjunctivitis.

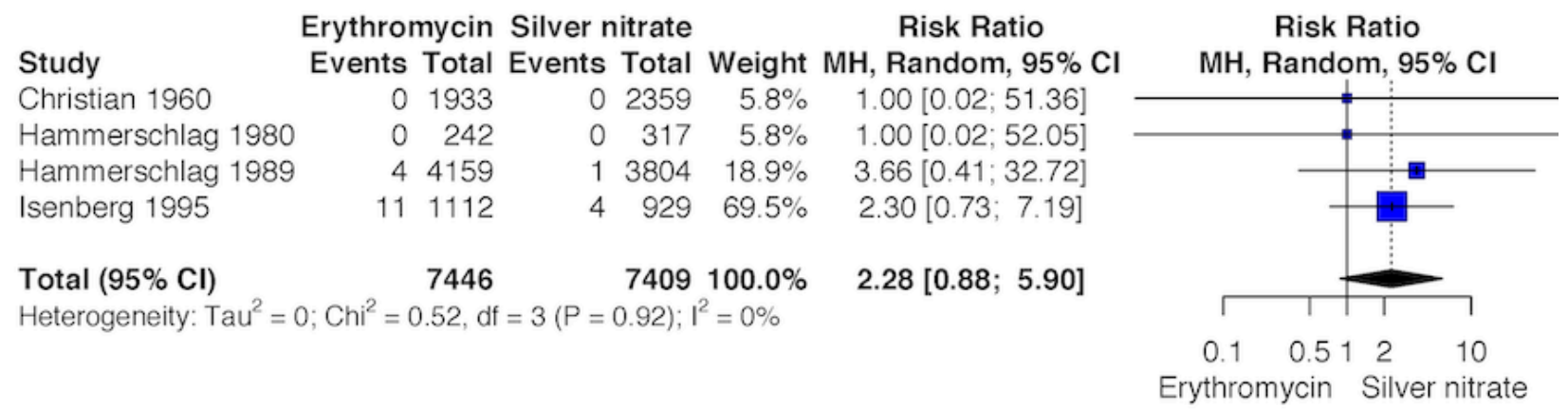




\section{Chlamydial conjunctivitis}

In a meta-analysis of four trials, erythromycin was associated with a $25 \%$ reduction ( $95 \% \mathrm{Cl} 49 \%$ reduction to $9 \%$ increase) in risk of
CC compared with silver nitrate (RR $0.75,95 \% \mathrm{Cl} 0.51$ to $1.09,13,472$ participants, 4 studies; $I^{2}=30 \%$; Analysis 8.1 ; Figure 8$)$. GRADE certainty of the evidence was low.

Figure 8. Forest plot of comparison: 8 Erythromycin versus silver nitrate, outcome: 8.2 Chlamydial conjunctivitis.

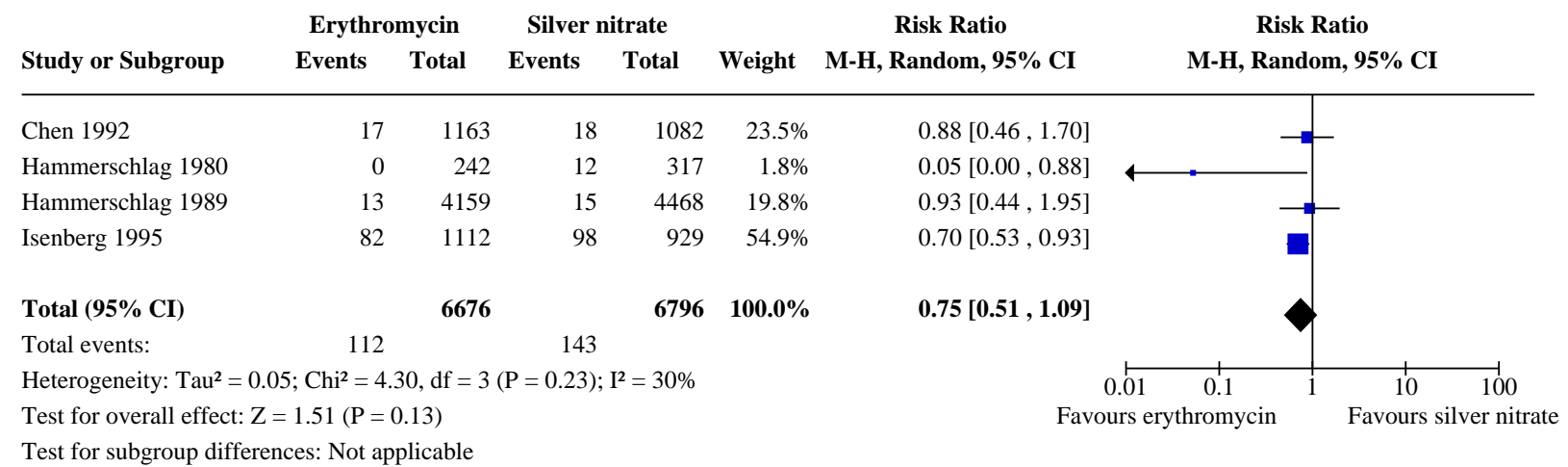

\section{Bacterial conjunctivitis}

In a meta-analysis of two trials, erythromycin was associated with a lower incidence of BC compared with silver nitrate (RR 0.83, 95\% $\mathrm{Cl} 0.69$ to 1.01, 6333 participants; 2 studies; $\mathrm{I}^{2}=55 \%$; Analysis 8.2). GRADE certainty of the evidence was low. The two trials had considerable differences in study design, which explains the observed $1^{2}$ of $55 \%$. The trials were conducted about 35 years apart. While both trials allocated infants by alternation and were thus at high risk of selection bias, Christian 1960 alternated infants and reported inadequate alternation for the first two months of the trial.

\section{Any conjunctivitis of any aetiology}

There was considerable statistical heterogeneity $(12=90 \%)$ amongst the four trials that compared erythromycin versus silver nitrate and reported data for this outcome (Analysis 8.3), thus we did not conduct a meta-analysis of the four trials. The protective effect of erythromycin in Christian 1960 was about three orders of magnitude higher than that seen in the remaining three trials, which may be explained by its high risk of selection bias. GRADE certainty of the evidence was very low. In a sensitivity analysis excluding Christian 1960, which was at a high risk of selection bias, there was no evidence that erythromycin reduced the risk of ACAE any more than silver nitrate (RR $1.02,95 \% \mathrm{Cl} 0.80$ to 1.30). GRADE certainty of the evidence was low.

\section{Conjunctivitis of unknown aetiology}

In a single trial (Isenberg 1995), there was little evidence of any difference between erythromycin and silver nitrate for risk of CUE
(RR $0.96,95 \% \mathrm{Cl} 0.77$ to 1.19 ). GRADE certainty of the evidence was low (Analysis 8.4).

\section{Adverse events}

In one trial, erythromycin was associated with a reduced risk of nasolacrimal duct obstruction compared with silver nitrate. This association was not statistically significant (RR $0.81,95 \% \mathrm{Cl} 0.55$ to 1.20; Analysis 8.5) (Bell 1993). GRADE certainty of the evidence was low.

Overall, we rated the certainty of the evidence for erythromycin versus silver nitrate to be moderate for GC, CC, and BC (see Table 9). We considered the certainty of the evidence for ACAE to be very low, owing to heterogeneous estimates in the included studies.

\section{Tetracycline versus silver nitrate}

See summary of findings in Table 10.

\section{Gonococcal conjunctivitis}

In a meta-analysis of five trials, tetracycline was associated with a $34 \%$ reduction in risk of GC when compared with silver nitrate, but this effect was consistent with both a $79 \%$ reduction in risk and a 2.05-fold increase in risk (RR $0.66,95 \% \mathrm{Cl} 0.21$ to 2.05, 14,501 participants, 5 studies; $I^{2}=0 \%$; Figure 9). GRADE certainty of the evidence was very low. While the statistical heterogeneity was not high, all five trials were at high or unclear risk of bias for all 'Risk of bias' domains assessed. Furthermore, three of the five trials reported no events of GC. We thus considered the overall certainty of evidence for this analysis to be low. 
Figure 9. Forest plot of comparison: 9 Tetracycline versus silver nitrate, outcome: 9.1 Gonococcal conjunctivitis.

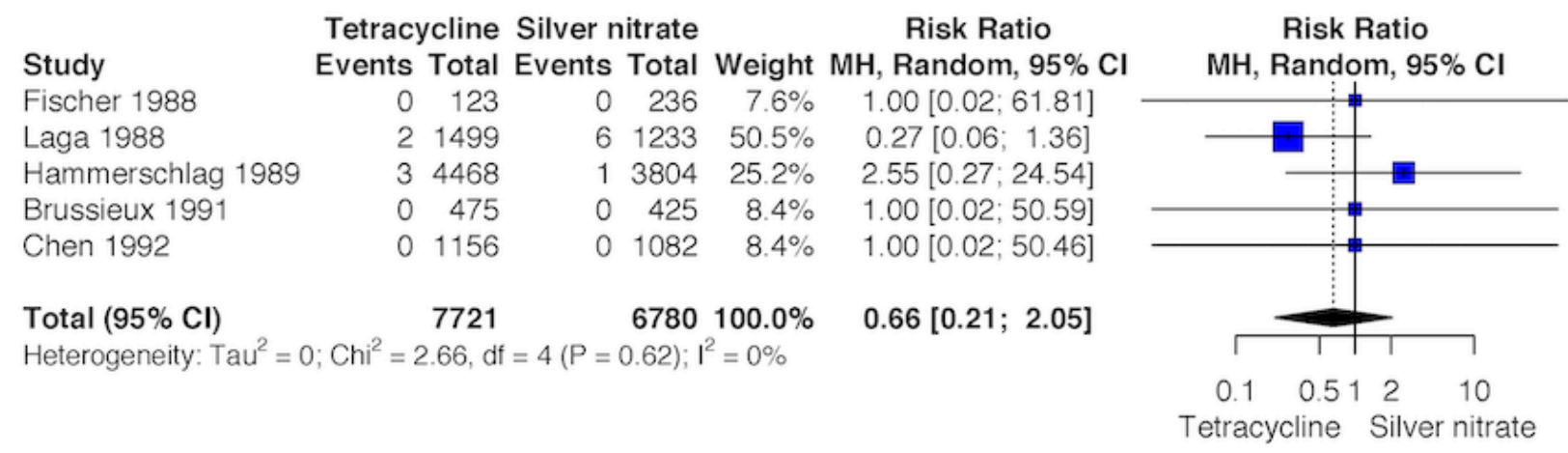

\section{Chlamydial conjunctivitis}

In a meta-analysis of four trials, tetracycline was associated with a reduced risk of $\mathrm{CC}$, but the effect was not statistically significant
(RR $0.64,95 \% \mathrm{Cl} 0.40$ to $1.02,14,142$ participants, 4 studies; $\mathrm{I}^{2}=$ $0 \%$; Analysis 9.1; Figure 10). GRADE certainty of the evidence was low.

Figure 10. Forest plot of comparison: 9 Tetracycline versus silver nitrate, outcome: 9.2 Chlamydia conjunctivitis.

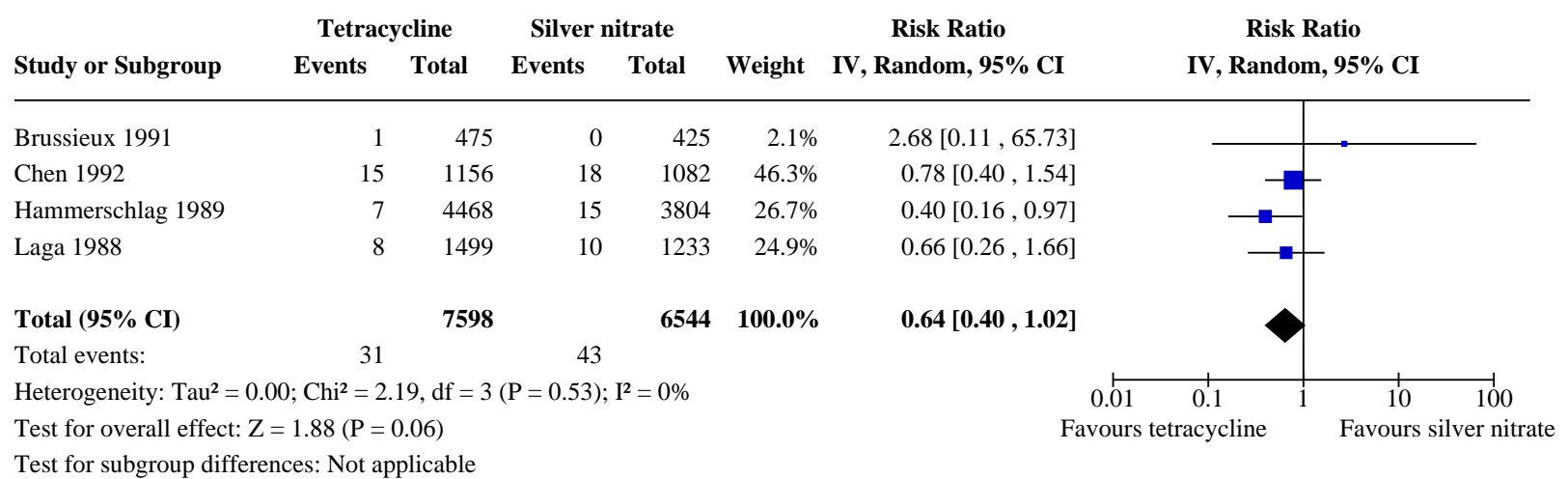

\section{Bacterial conjunctivitis}

No data were available for $\mathrm{BC}$ comparing tetracycline versus silver nitrate.

\section{Any conjunctivitis of any aetiology}

In a meta-analysis of four trials, tetracycline was associated with a lower risk of ACAE compared with silver nitrate (RR $0.80,95 \% \mathrm{Cl}$
0.66 to $0.98,6229$ participants, 4 studies; $\left.\right|^{2}=0 \%$; Figure 11 ). One of the four trials included in this meta-analysis reported no events in infants treated with silver nitrate. GRADE certainty of the evidence was moderate. 
Figure 11. Forest plot of comparison: 9 Tetracycline versus silver nitrate, outcome: 9.3 Any conjunctivitis of any aetiology

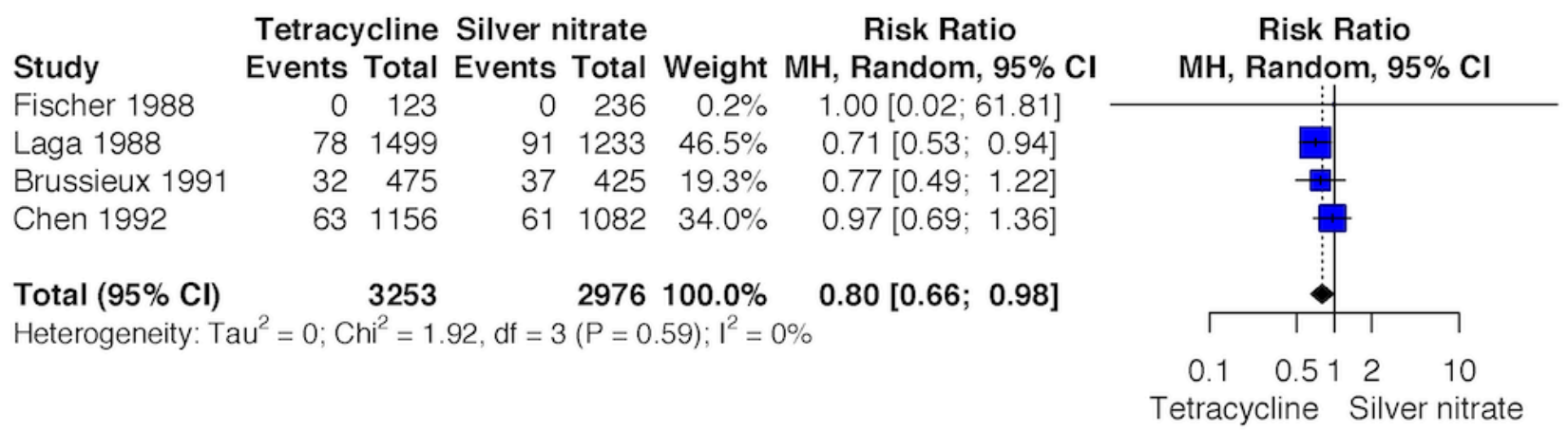

\section{Conjunctivitis of unknown aetiology}

No data were available for CUE comparing tetracycline versus silver nitrate.

\section{Adverse events}

In one trial, tetracycline appeared to be associated with a higher risk of nasolacrimal duct obstruction, but the variance in the estimate was high (RR $1.57,95 \% \mathrm{Cl} 0.63$ to $3.91,145$ participants, 1 study) (Analysis 9.2). GRADE certainty of the evidence was low.

\section{Sulfacetamide versus silver nitrate}

See summary of findings in Table 11.

\section{Gonococcal conjunctivitis}

No events of $G C$ were reported in one trial comparing sulfacetamide versus silver nitrate (Cousineau 1952). GRADE certainty of the evidence was very low.

\section{Chlamydial conjunctivitis}

No data on CC were available in one trial comparing sulfacetamide versus silver nitrate (Cousineau 1952).

\section{Bacterial conjunctivitis}

In one trial (Cousineau 1952), sulfacetamide was associated with little or no difference in risk of $\mathrm{BC}$ compared with silver nitrate (RR $0.88,95 \% \mathrm{Cl} 0.45$ to 1.74 ). This association was not statistically significant. GRADE certainty of the evidence was low.

\section{Any conjunctivitis of any aetiology}

In one trial (Cousineau 1952), sulfacetamide was associated with a lower risk of ACAE than silver nitrate (RR $0.54,95 \% \mathrm{Cl} 0.32$ to 0.89 ). GRADE certainty of the evidence was moderate.

\section{Conjunctivitis of unknown aetiology}

In one trial (Cousineau 1952), sulfacetamide was associated with a lower risk of CUE than silver nitrate (RR $0.27,95 \% \mathrm{Cl} 0.11$ to 0.66 ). GRADE certainty of the evidence was moderate.

\section{Adverse events}

No adverse events data were available comparing sulfacetamide versus silver nitrate.

\section{Cetyl-pyridinium chloride versus silver nitrate}

See summary of findings in Table 12.

\section{Gonococcal conjunctivitis}

No data were available on GC comparing cetyl-pyridinium chloride versus silver nitrate.

\section{Chlamydial conjunctivitis}

No data were available on CC comparing cetyl-pyridinium chloride versus silver nitrate.

\section{Bacterial conjunctivitis}

In a meta-analysis of two trials, cetyl-pyridinium chloride was associated with a higher risk of $\mathrm{BC}$ compared with silver nitrate; this association was not statistically significant (RR $1.79,95 \% \mathrm{Cl} 0.59$ to $5.41,599$ participants, 2 studies; $I^{2}=15 \%$ ). GRADE certainty of the evidence was low.

\section{Any conjunctivitis of any aetiology}

In a meta-analysis of two trials, cetyl-pyridinium chloride was associated with a higher risk of ACAE compared with silver nitrate; this association was not statistically significant (RR 1.08, 95\% Cl 0.40 to $2.90,599$ participants, 2 studies; $12=60 \%)$. GRADE certainty of the evidence was very low.

\section{Conjunctivitis of unknown aetiology}

In a meta-analysis of two trials, cetyl-pyridinium chloride was associated with a lower risk of CUE compared with silver nitrate; this association was not statistically significant (RR $0.14,95 \% \mathrm{Cl}$ 0.01 to 2.71, 599 participants, 2 studies; $12=60 \%$ ) (Kaivonen 1965a; Kaivonen 1965b). GRADE certainty of the evidence was low.

\section{Adverse events}

No adverse events data were available comparing cetyl-pyridinium chloride versus silver nitrate.

\section{Penicillin versus silver nitrate}

See summary of findings in Table 13 and Table 14. 


\section{Gonococcal conjunctivitis}

One trial reported no events of GC comparing topical and intramuscular (IM) penicillin versus silver nitrate (Davidson 1951). GRADE certainty of the evidence was very low.

\section{Chlamydial conjunctivitis}

No data were available on CC comparing topical and IM penicillin versus silver nitrate.

\section{Bacterial conjunctivitis}

In one trial, topical penicillin was associated with a reduced risk of $\mathrm{BC}$ compared with silver nitrate (RR $0.34,95 \% \mathrm{Cl} 0.18$ to 0.65 ) (Davidson 1951). GRADE certainty of the evidence was moderate.

In one trial, IM penicillin was associated with a reduced risk of $B C$ compared with silver nitrate; this association was not statistically significant (RR $0.75,95 \% \mathrm{Cl} 0.46$ to 1.24) (Davidson 1951). GRADE certainty of the evidence was very low.

\section{Any conjunctivitis of any aetiology}

Two trials compared topical penicillin versus silver nitrate for ACAE. While both trials reported that penicillin was associated with a lower risk of ACAE compared with silver nitrate, their estimates were statistically heterogeneous ( $I^{2}=93 \%$; Analysis 12.3), precluding a meta-analysis. The RR for ACAE was $0.15(95 \% \mathrm{Cl}$ 0.12 to 0.20 ) in Davidson 1951 and 0.78 (95\% Cl 0.35 to 1.70$)$ in Harris 1957. GRADE certainty of the evidence was very low. Both trials were reported in the 1950s, and were at high risk of selection bias and unclear risk of performance, detection, and attrition biases. Multiple factors may explain the heterogeneity observed in this analysis, including lack of a specific definition of conjunctivitis in Davidson 1951, requiring interpretation by the review authors; and use of wax ampoules that the study authors reported were sometimes defective, leading to evaporation and potentially increased concentration of silver nitrate, and eventually frequency of chemical conjunctivitis.

In one trial, IM penicillin was associated with a reduced risk of ACAE compared with silver nitrate (RR $0.26,95 \% \mathrm{Cl} 0.21$ to 0.32 ) (Davidson 1951). GRADE certainty of the evidence was moderate.

\section{Conjunctivitis of unknown aetiology}

In one trial, topical penicillin was associated with a reduced risk of CUE compared with silver nitrate (RR $0.13,95 \% \mathrm{Cl} 0.10$ to 0.18 ) (Davidson 1951). GRADE certainty of the evidence was low.

In one trial, IM penicillin was associated with a reduced risk of CUE compared with silver nitrate (RR $0.21,95 \% \mathrm{Cl} 0.17$ to 0.27 ) (Davidson 1951). GRADE certainty of the evidence was moderate.

\section{Adverse events}

No adverse events data were available comparing penicillin versus silver nitrate.

\section{Povidone-iodine versus silver nitrate}

See summary of findings in Table 15.

\section{Gonococcal conjunctivitis}

In a single trial (Isenberg 1995), povidone-iodine was associated with a higher risk of GC than silver nitrate, which was not statistically significant (RR $1.94,95 \% \mathrm{Cl} 0.60$ to 6.29). GRADE assessment of the evidence was low.

\section{Chlamydial conjunctivitis}

In a single trial (Isenberg 1995), povidone-iodine was associated with a lower risk of CC than silver nitrate (RR $0.52,95 \% \mathrm{Cl} 0.38$ to 0.71 ). GRADE certainty of the evidence was moderate.

\section{Bacterial conjunctivitis}

In a single trial (Isenberg 1995), povidone-iodine was associated with a lower risk of $\mathrm{BC}$ than silver nitrate (RR $0.75,95 \% \mathrm{Cl} 0.61$ to 0.92). GRADE certainty of the evidence was moderate.

\section{Any conjunctivitis of any aetiology}

In a single trial (Isenberg 1995), povidone-iodine was associated with a lower risk of ACAE than silver nitrate (RR $0.72,95 \% \mathrm{Cl} 0.63$ to $0.84)$. GRADE certainty of the evidence was moderate.

\section{Conjunctivitis of unknown aetiology}

In a single trial (Isenberg 1995), povidone-iodine was associated with a lower risk of CUE than silver nitrate (RR $0.70,95 \% \mathrm{Cl} 0.55$ to 0.89). GRADE certainty of the evidence was moderate.

\section{Adverse events}

No adverse events data were available comparing povidone-iodine versus silver nitrate.

\section{Tetracycline versus erythromycin}

See summary of findings in Table 16.

\section{Gonococcal conjunctivitis}

In a meta-analysis of two trials, there was no evidence that tetracycline was associated with a statistically significant reduction in risk of GC compared with erythromycin (RR $0.73,95 \% \mathrm{Cl} 0.18$ to $2.95,10,946$ participants, 2 studies; $I^{2}=0 \%$ ). GRADE certainty of the evidence was low.

\section{Chlamydial conjunctivitis}

In a meta-analysis of two trials, tetracycline was associated with a lower risk of CC than erythromycin, but the reduction in risk was not statistically significant (RR $0.72,95 \% \mathrm{Cl} 0.42$ to $1.25,10,946$ participants, 2 studies; $1^{2}=0 \%$ ). GRADE certainty of the evidence was low.

\section{Bacterial conjunctivitis}

No data were available on $\mathrm{BC}$ comparing tetracycline versus erythromycin.

\section{Any conjunctivitis of any aetiology}

There was significant heterogeneity $\left(1^{2}=69 \%\right)$ in estimates for risk of ACAE from two trials comparing tetracycline and erythromycin, which precluded a meta-analysis (Analysis 15.2). A $25 \%$ risk reduction $(95 \% \mathrm{Cl} 46 \%$ reduction to $2 \%$ increase) was observed in Chen 1992 (RR $0.75,95 \% \mathrm{Cl} 0.54$ to 1.02), whilst a $38 \%$ increase in risk $(95 \% \mathrm{Cl} 24 \%$ reduction to 2.47 fold increase) was observed in Ghotbi 2012 (RR 1.38, 95\% Cl 0.76 to 2.47 ). GRADE certainty of the evidence was very low. The heterogeneity observed between the two trials may be explained 
by methodological and clinical factors. Ghotbi 2012 reported randomly allocating neonates to interventions, whilst Chen 1992 alternated the interventions monthly. Furthermore, Ghotbi 2012 was described as "single-blind", whilst Chen 1992 did not involve any masking. Clinically, the causative agents of conjunctivitis in neonates could differ between the two trials. However, only Chen 1992 reported culture results, and we are unable to definitively ascertain diversity in aetiology of conjunctivitis as a source of heterogeneity. Separately, the ointments used in the two studies were of identical concentrations, but they were manufactured by different companies. This difference may explain heterogeneous outcomes to the extent that the manufacturing protocols contributed to differential efficacy of the antibiotics.

\section{Conjunctivitis of unknown aetiology}

No data were available on CUE comparing tetracycline versus erythromycin.

\section{Adverse events}

No adverse events data were available comparing tetracycline versus erythromycin.

\section{Colostrum versus erythromycin}

See summary of findings in Table 17.

\section{Gonococcal conjunctivitis}

No data were available on GC comparing colostrum versus erythromycin.

\section{Chlamydial conjunctivitis}

No data were available on CC comparing colostrum versus erythromycin.

\section{Bacterial conjunctivitis}

No data were available on BC comparing colostrum versus erythromycin.

\section{Any conjunctivitis of any aetiology}

In a single trial (Ghaemi 2014), colostrum was associated with a higher risk of ACAE compared with erythromycin, but the effect was not statistically significant (RR $1.49,95 \% \mathrm{Cl} 0.80$ to 2.78). The trial was at low risk of selection bias but at high risk of attrition bias. GRADE certainty of the evidence was low.

\section{Conjunctivitis of unknown aetiology}

No data were available on CUE comparing colostrum versus erythromycin.

\section{Adverse events}

No adverse events data were available comparing colostrum versus erythromycin.

\section{Povidone-iodine versus erythromycin}

See summary of findings in Table 18.

\section{Gonococcal conjunctivitis}

Two trials compared povidone-iodine with erythromycin and reported data on GC; one of the trials reported no events in both groups (Ali 2007). The second trial (Isenberg 1995) was larger by about 10 times; only a few events of GC were observed, resulting in high variance in the estimate. Povidone-iodine was associated with a lower risk of GC compared with erythromycin, but the effect was not statistically significant (RR $0.85,95 \% \mathrm{Cl} 0.36$ to $2.01,2408$ participants, 2 studies; $\mathrm{I}^{2}=0 \%$; Figure 12 ). GRADE certainty of the evidence was low.

Figure 12. Forest plot of comparison: 17 Povidone-iodine versus erythromycin, outcome: 17.1 Gonococcal conjunctivitis

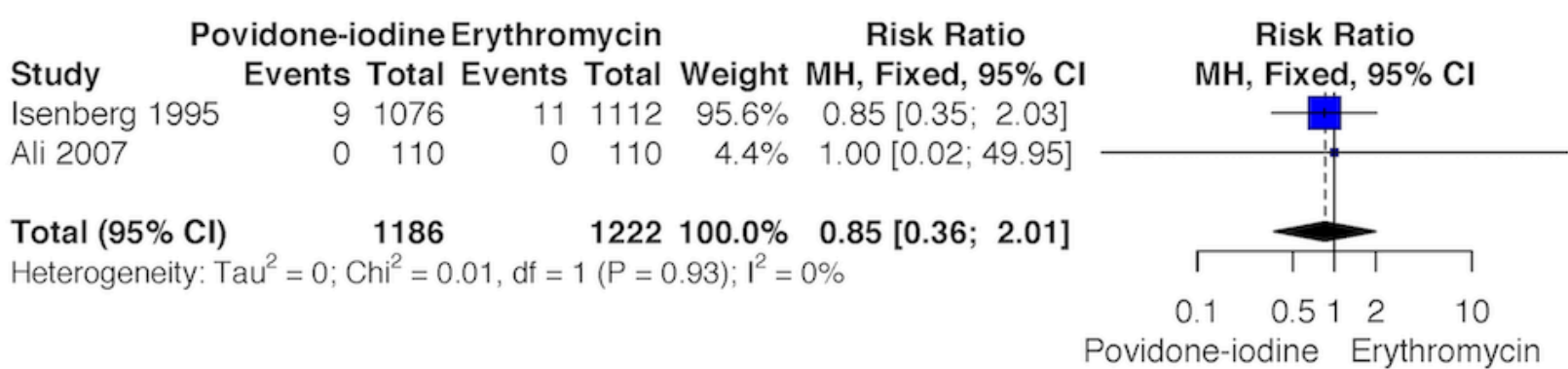

\section{Chlamydial conjunctivitis}

In a meta-analysis of two trials, povidone-iodine was associated with a lower risk of CC compared with erythromycin, but the effect was not statistically significant (RR $0.74,95 \% \mathrm{Cl} 0.54$ to $1.02,2408$ participants, 2 studies; $1^{2}=0 \%$; Analysis 17.1). GRADE certainty of the evidence was low.

\section{Bacterial conjunctivitis}

Povidone-iodine was associated with a lower risk of $\mathrm{BC}$ compared with erythromycin, but the effect was not statistically significant
(RR $0.87,95 \% \mathrm{Cl} 0.71$ to 1.07 ; Analysis 17.2 ). GRADE certainty of the evidence was low.

\section{Any conjunctivitis of any aetiology}

In a meta-analysis of two trials, povidone-iodine was associated with a lower risk of ACAE compared with erythromycin; there was some statistical heterogeneity $\left(\mathrm{RR} 0.78,95 \% \mathrm{Cl} 0.68\right.$ to $0.90 ; \mathrm{I}^{2}=$ $45 \%$; Analysis 17.3). GRADE certainty of the evidence was moderate. 


\section{Conjunctivitis of unknown aetiology}

In a meta-analysis of two trials, povidone-iodine was associated with a lower risk of CUE compared with erythromycin (RR 0.74, 95\% $\mathrm{Cl} 0.58$ to 0.93 ; Analysis 17.4). GRADE certainty of the evidence was low.

\section{Adverse events}

No adverse events data were available comparing povidone-iodine versus erythromycin.

\section{Penicillin IM versus tetracycline}

See summary of findings in Table 19.

\section{Gonococcal conjunctivitis}

None of the 32,058 participants in a single trial comparing IM penicillin versus tetracycline developed GC (Siegel 1982). GRADE certainty of the evidence was moderate.

\section{Chlamydial conjunctivitis}

In a single trial (Siegel 1982), IM penicillin was associated with a lower risk of CC compared with tetracycline, but the effect was not statistically significant (RR $0.75,95 \% \mathrm{Cl} 0.48$ to 1.17 ). GRADE certainty of the evidence was moderate.

\section{Bacterial conjunctivitis}

No data were available on $\mathrm{BC}$ comparing IM penicillin versus tetracycline.

\section{Any conjunctivitis of any aetiology}

No data were available on ACAE comparing IM penicillin versus tetracycline.

\section{Conjunctivitis of unknown aetiology}

No data were available on CUE comparing IM penicillin versus tetracycline.

\section{Adverse events}

No adverse events data were available comparing IM penicillin versus tetracycline.

\section{Povidone-iodine versus tetracycline}

See summary of findings in Table 20.

\section{Gonococcal conjunctivitis}

No incidence of GC was reported in a single trial of 410 infants (David 2011). GRADE certainty of the evidence was low.

\section{Chlamydial conjunctivitis}

No incidence of CC was reported in a single trial of 410 infants (David 2011). GRADE certainty of the evidence was low.

\section{Bacterial conjunctivitis}

In a single trial (David 2011), povidone-iodine was associated with a higher risk of $\mathrm{BC}$ compared with tetracycline; the association was not statistically significant (RR 2.04, 95\% Cl 0.99 to 4.22). GRADE certainty of the evidence was low.

\section{Any conjunctivitis of any aetiology}

In a single trial (David 2011), povidone-iodine was associated with a higher risk of ACAE compared with tetracycline; the association was not statistically significant (RR $3.01,95 \% \mathrm{Cl} 1.52$ to 5.98 ). GRADE certainty of the evidence was low.

\section{Conjunctivitis of unknown aetiology}

In a single trial comparing povidone-iodine versus tetracycline (David 2011), 10 events of CUE were reported with povidone-iodine but no events of CUE were reported with tetracycline, thus the effect was not estimable. GRADE certainty of the evidence was low.

\section{Adverse events}

No adverse events data were available comparing povidone-iodine versus tetracycline.

\section{Povidone-iodine versus chloramphenicol}

See summary of findings in Table 21.

\section{Gonococcal conjunctivitis}

None of the 2004 infants in a single trial comparing povidoneiodine versus chloramphenicol developed GC (Ramirez-Ortiz 2007). GRADE certainty of the evidence was low.

\section{Chlamydial conjunctivitis}

In a single trial (Ramirez-Ortiz 2007), povidone-iodine was associated with a higher risk of CC compared with chloramphenicol, but the association was not statistically significant (RR $1.77,95 \% \mathrm{Cl}$ 0.97 to 3.22). GRADE certainty of the evidence was low.

\section{Bacterial conjunctivitis}

No data were available on $\mathrm{BC}$ for povidone-iodine versus chloramphenicol.

\section{Any conjunctivitis of any aetiology}

No data were available on ACAE for povidone-iodine versus chloramphenicol.

\section{Conjunctivitis of unknown aetiology}

No data were available on CUE for povidone-iodine versus chloramphenicol.

\section{Adverse events}

No adverse events data were available for povidone-iodine versus chloramphenicol.

\section{Povidone-iodine versus carbethopendecinium bromide}

See summary of findings in Table 22.

\section{Gonococcal conjunctivitis}

No data were available on GC for povidone-iodine versus carbethopendecinium bromide.

\section{Chlamydial conjunctivitis}

No data were available on CC for povidone-iodine versus carbethopendecinium bromide. 


\section{Bacterial conjunctivitis}

No data were available on BC for povidone-iodine versus carbethopendecinium bromide.

\section{Any conjunctivitis of any aetiology}

In a single trial (Zbojan 2004), povidone-iodine was associated with a lower risk of ACAE, but the effect was not statistically significant (RR $0.44,95 \% \mathrm{Cl} 0.15$ to 1.35 ). GRADE certainty of the evidence was low.

\section{Conjunctivitis of unknown aetiology}

No data were available on CUE for povidone-iodine versus carbethopendecinium bromide.

\section{Adverse events}

No adverse events data were available for povidone-iodine versus carbethopendecinium bromide.

\section{Povidone-iodine twice versus povidone-iodine once}

See summary of findings in Table 23.

\section{Gonococcal conjunctivitis}

No incidence of GC was reported amongst 719 infants in a single trial comparing povidone-iodine administered twice versus once (Isenberg 2003). GRADE certainty of the evidence was very low.

\section{Chlamydial conjunctivitis}

In a single trial (Isenberg 2003), povidone-iodine administered twice was associated with a higher risk of CC compared with a single dose of povidone-iodine, but the variance in the estimate was large (RR $1.27,95 \% \mathrm{Cl} 0.26$ to 6.24 ). GRADE certainty of the evidence was very low.

\section{Bacterial conjunctivitis}

In a single trial (Isenberg 2003), povidone-iodine administered twice was associated with a higher risk of $\mathrm{BC}$ compared with a single dose of povidone-iodine, but the effect was not statistically significant (RR $1.69,95 \% \mathrm{Cl} 0.59$ to 4.82 ). GRADE certainty of the evidence was very low.

\section{Any conjunctivitis of any aetiology}

In a single trial (Isenberg 2003), povidone-iodine administered twice was associated with a higher risk of ACAE compared with a single dose of povidone-iodine, but the effect was not statistically significant (RR 1.32, 95\% Cl 0.99 to 1.75 ). GRADE certainty of the evidence was low.

\section{Conjunctivitis of unknown aetiology}

In a single trial (Isenberg 2003), povidone-iodine administered twice was associated with a higher risk of CUE compared with a single dose of povidone-iodine, but the effect was not statistically significant (RR $1.29,95 \% \mathrm{Cl} 0.95$ to 1.74 ). GRADE certainty of the evidence was low.

\section{Adverse events}

No adverse events data were available for povidone-iodine administered twice versus once.

\section{Penicillin IM versus topical penicillin}

See summary of findings in Table 24.

\section{Gonococcal conjunctivitis}

No incidence of GC was reported amongst 2795 infants in a single trial comparing IM versus topical penicillin (Davidson 1951). GRADE certainty of the evidence was very low.

\section{Chlamydial conjunctivitis}

No incidence of CC was reported amongst 2795 infants in a single trial comparing IM versus topical penicillin (Davidson 1951).

\section{Bacterial conjunctivitis}

In a single trial (Davidson 1951), IM penicillin was associated with a higher risk of $\mathrm{BC}$ compared with topical penicillin (RR 2.19, 95\% Cl 1.14 to 4.24$)$. GRADE certainty of the evidence was low.

\section{Any conjunctivitis of any aetiology}

In a single trial (Davidson 1951), IM penicillin was associated with a higher risk of ACAE compared with topical penicillin (RR 1.71, 95\% $\mathrm{Cl} 1.26$ to 2.32 ). GRADE certainty of the evidence was low.

\section{Conjunctivitis of unknown aetiology}

In a single trial (Davidson 1951), IM penicillin was associated with a higher risk of CUE compared with topical penicillin (RR 1.58, 95\% Cl 1.12 to 2.25 ). GRADE certainty of the evidence was low.

\section{Adverse events}

No adverse events data were available for penicillin IM versus topical penicillin.

\section{DISCUSSION}

\section{Summary of main results}

No data were available from the included trials for the primary vision outcomes of blindness and any adverse visual outcome. Gonococcal conjunctivitis (GC) may be considered a surrogate for vision outcomes because it is associated with a high risk of blindness.

For prophylaxis of GC, the protective effect of none of the interventions we examined was statistically significant, but in general studies were underpowered for this rare outcome and we judged the evidence to be low-certainty. Based on effect estimates, silver nitrate appeared to be more effective for prophylaxis of GC than erythromycin or povidone-iodine (Figure 13). A similar protective effect with silver nitrate was not observed against tetracycline, however. 


\section{Figure 13. Prophylaxis of GC}

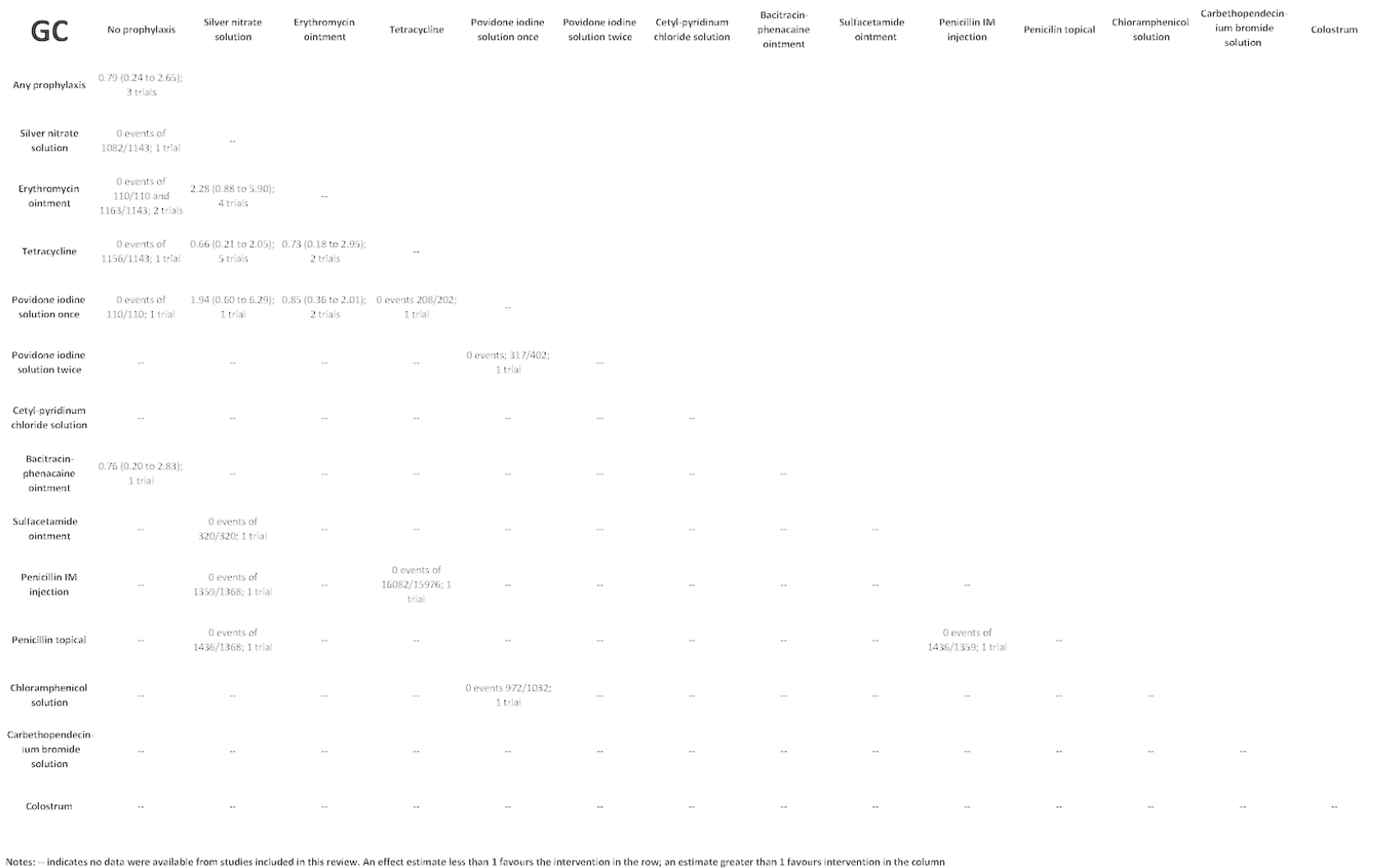

For prophylaxis of chlamydial conjunctivitis (CC), the protective effect of povidone-iodine relative to silver nitrate was statistically significant, and a consistent effect was seen relative to erythromycin (Figure 14). There were limited data against tetracycline. However, povidone-iodine was associated with a higher risk of CC compared with chloramphenicol, which was not statistically significant. 
Figure 14. Prophylaxis of CC

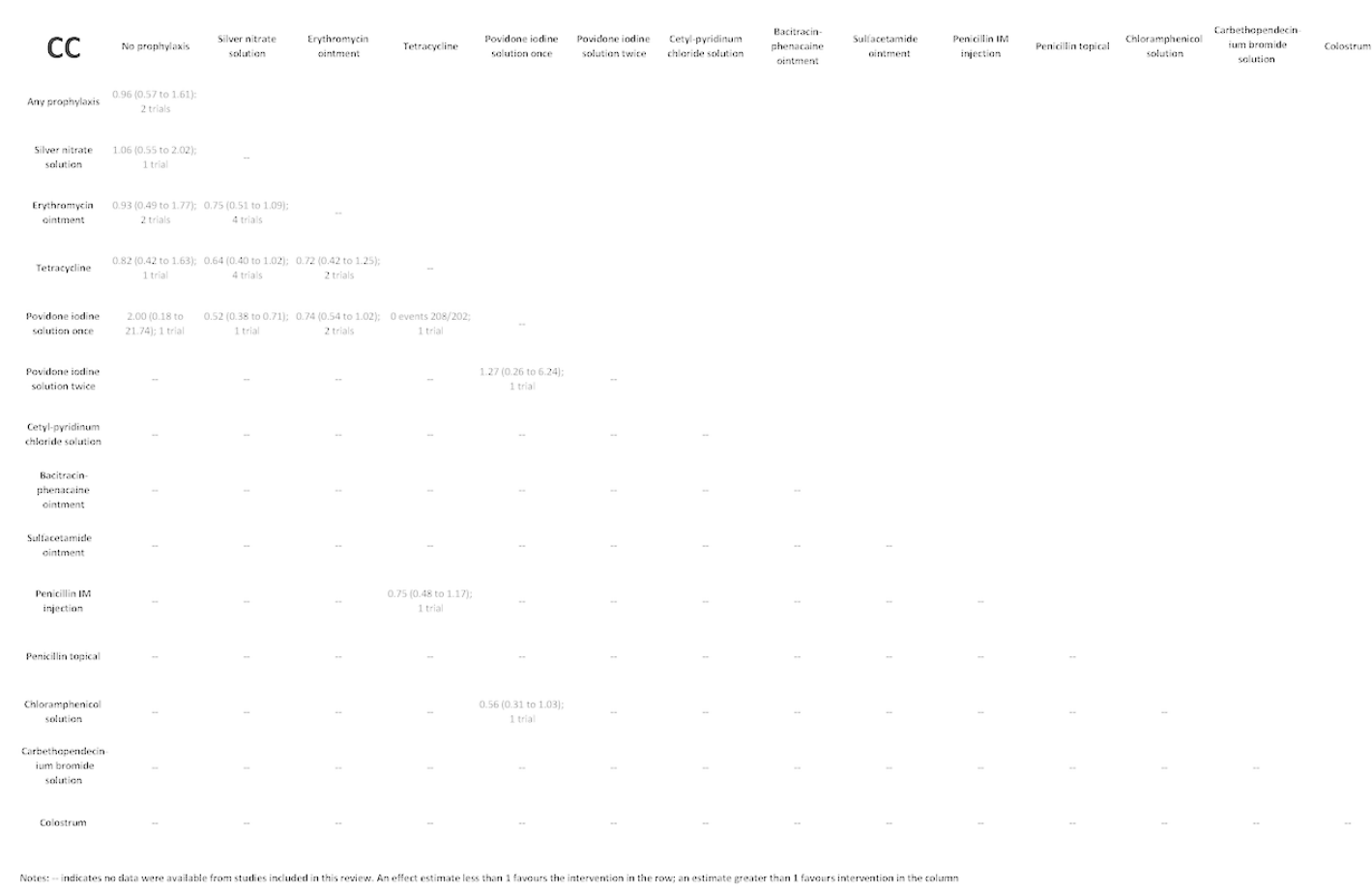

For prophylaxis of bacterial conjunctivitis (BC), povidone-iodine appeared to be more effective than silver nitrate and erythromycin but less effective than tetracycline (Figure 15). Furthermore, erythromycin, sulfacetamide, and penicillin were associated with a protective effect against BC compared with silver nitrate; only the effect of topical penicillin relative to silver nitrate was statistically significant. Our findings also indicate that administering povidoneiodine twice was associated with a higher risk of $\mathrm{CC}, \mathrm{BC}$, and any conjunctivitis of any aetiology (ACAE) compared with single administration of povidone-iodine. 


\section{Figure 15. Prophylaxis of $B C$}

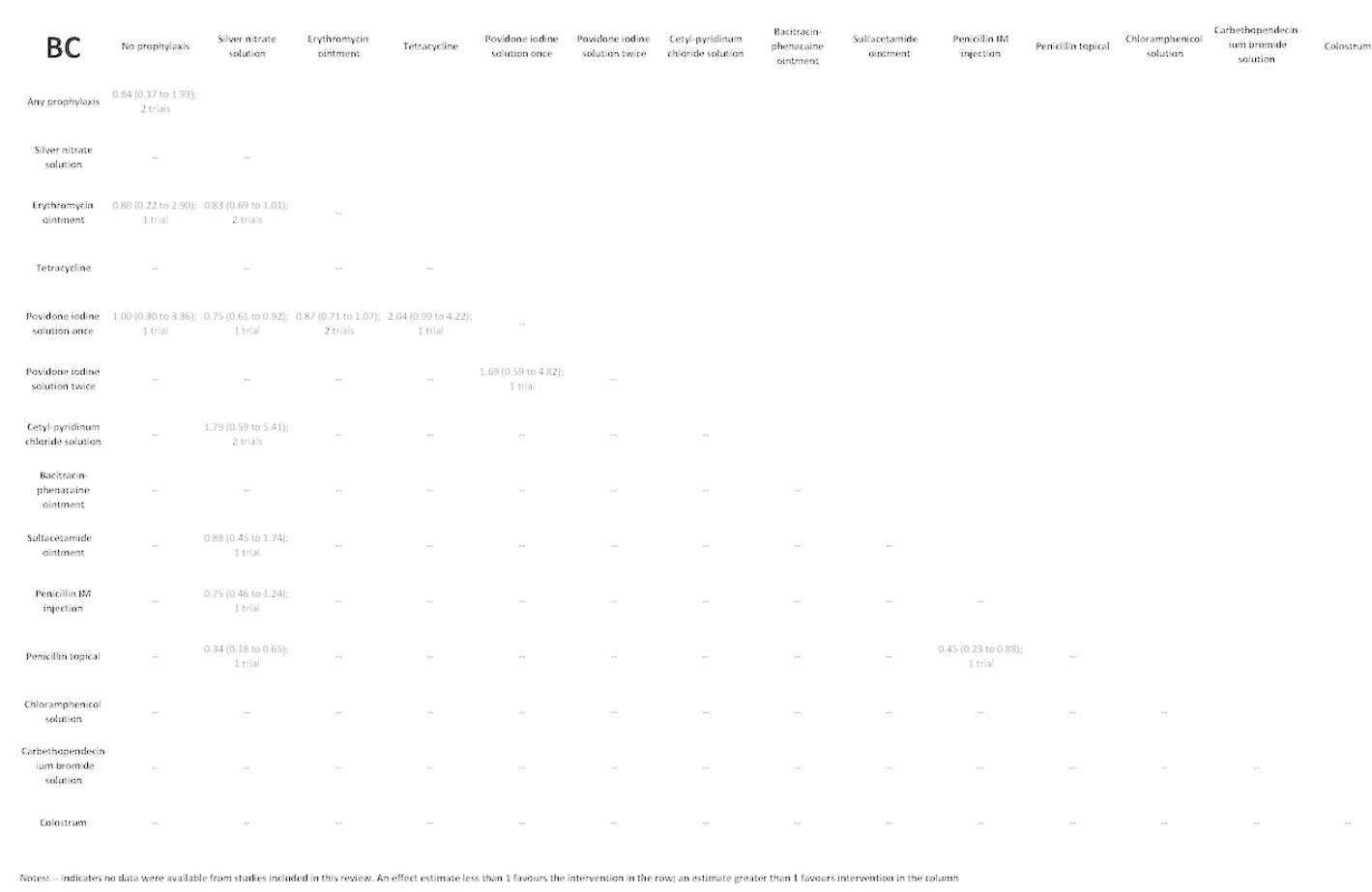

For prophylaxis of ACAE, any prophylaxis was associated with a lower incidence (Figure 16). Povidone-iodine, erythromycin, silver nitrate, and tetracycline had a statistically significant protective effect against ACAE compared with no prophylaxis. However, colostrum did not show a statistically significant protective effect against ACAE. Furthermore, povidone-iodine, erythromycin, tetracycline, penicillin, and sulfacetamide seemed to be more protective against ACAE compared with silver nitrate and povidoneiodine appeared to be less protective against ACAE compared with tetracycline. 
Figure 16. Prophylaxis of ACAE

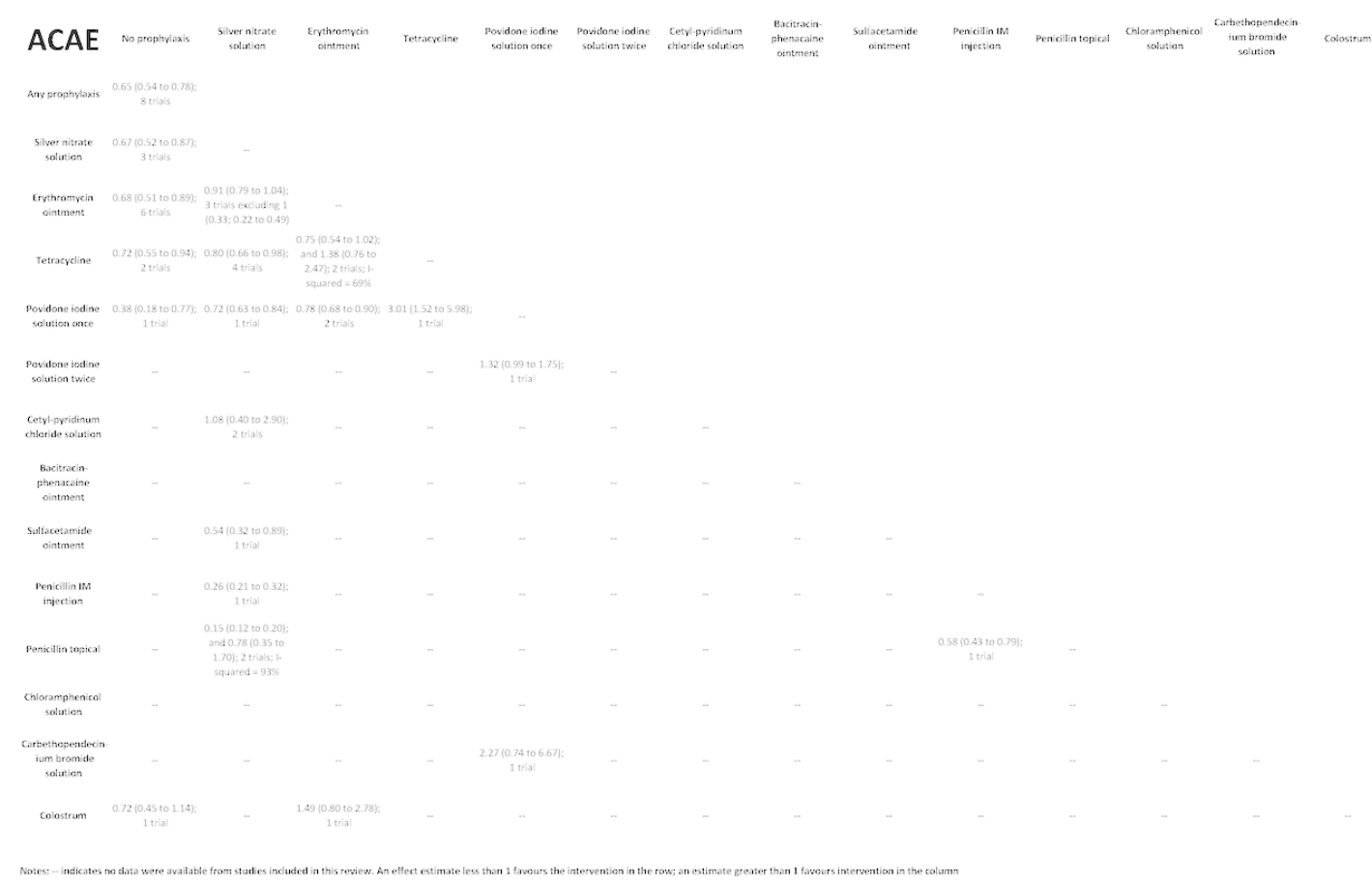

Finally, our findings demonstrate that silver nitrate is associated with a statistically significant increased risk of chemical conjunctivitis when compared with povidone-iodine, cetylpyridinium chloride, sulfacetamide, and penicillin (Figure 17). Povidone-iodine was associated with a reduced risk of chemical conjunctivitis when compared with erythromycin. There was high variance in the estimates showing an apparent increased risk of chemical conjunctivitis observed with povidone-iodine relative to no prophylaxis and to tetracycline. 
Figure 17. Prophylaxis of CUE

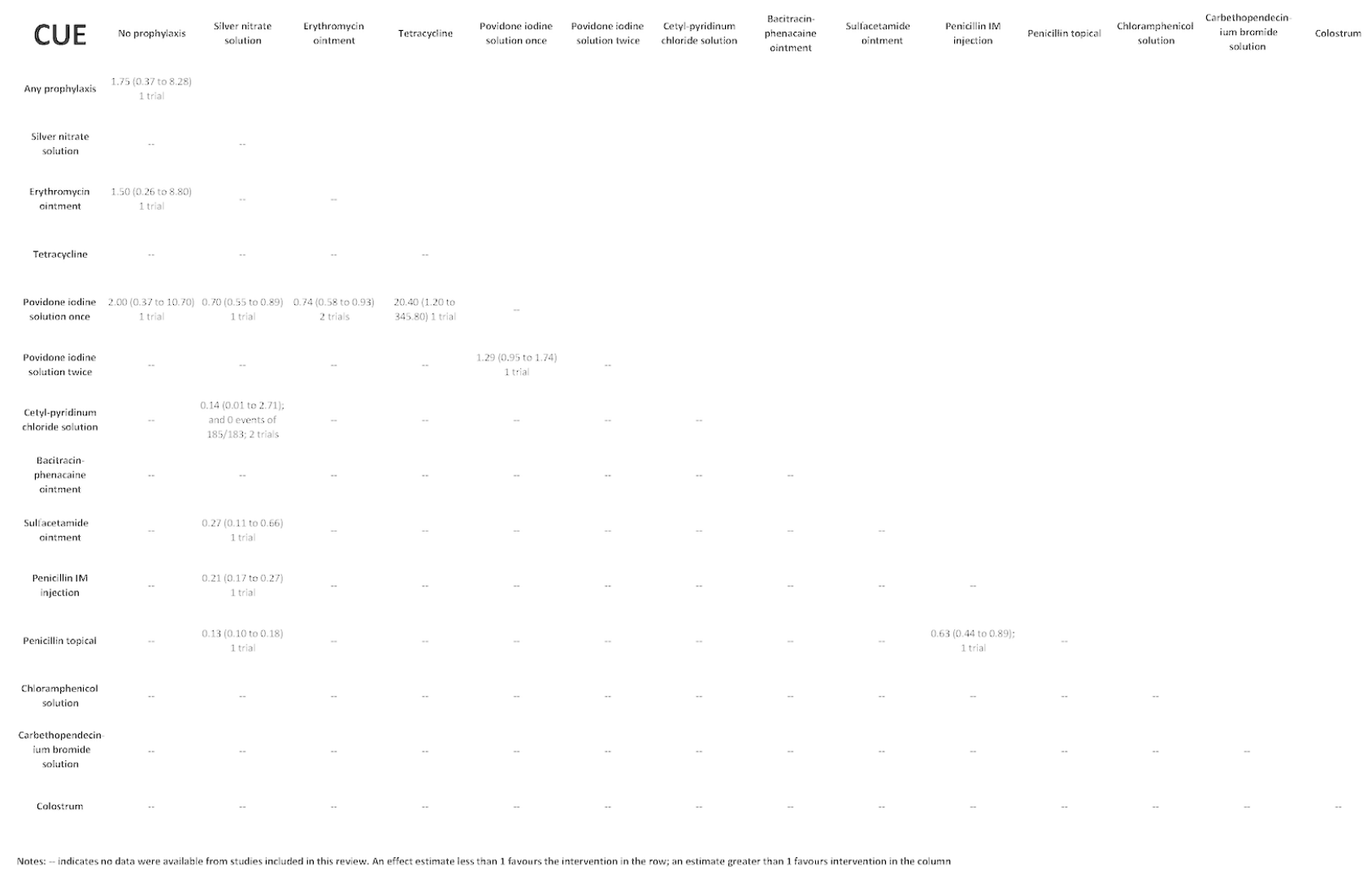

\section{Overall completeness and applicability of evidence}

No trials included the primary outcome of blindness or any adverse visual outcome. There could be ethical and logistical barriers to conducting such a trial. The very low incidence of blindness following ophthalmia neonatorum means trials must be very large to have adequate power to detect clinically meaningful differences in treatment effects. The low event rate makes it difficult to determine if prophylaxis is effective for GC, and difficult to determine the relative effect of the various prophylactic medications for the critical outcome of GC.

This review included studies conducted all over the world, in low-, middle- and high-income countries, and various settings within countries, with high and low baseline prevalence of sexually transmitted and other infections that may be causal agents of ophthalmia neonatorum. Twelve (40\%) of the 30 studies were conducted in low- and middle-income economies, with the majority in Iran and Kenya. Three studies took place in Kenya, four studies in Iran and one each in Zaire, Mexico, Indonesia, China, and Angola). Eighteen studies (60\%) were conducted in high-income countries, with the majority in the USA and Europe. Nine studies were conducted in the USA, seven in European countries, one in Canada, and one in Israel.

More than $50 \%$ of the trials were conducted more than 20 years ago, and about a third of them were conducted more than 40 years ago. Healthcare systems, the epidemiology of infectious diseases, and drug resistance have changed during this time in many settings, which makes generalisability difficult.
The majority of trials studied silver nitrate (61\%), erythromycin $(38 \%)$, tetracycline $(35 \%)$, povidone-iodine $(27 \%)$, and no prophylaxis (31\%). Silver nitrate does not appear to be manufactured anymore, and, a limited global survey of agents used for ophthalmia neonatorum prophylaxis found that silver nitrate is no longer used in the world (Zloto 2016). However, a variation containing organically bound silver, and alleged to have a similar effect to silver nitrate, called Targesin ( $1 \%$ silver protein acetyl tannate eye drops) is still used in Slovenia (Jug Došler 2015). The majority of the world seems to use erythromycin or povidoneiodine for ophthalmia neonatorum prophylaxis.

The practice of ophthalmia neonatorum prophylaxis varies globally, with many countries no longer considering it to be an important public health intervention, and some countries continuing with prophylaxis. This review provides some moderatecertainty evidence on the use of prophylaxis to prevent all-cause cases of ophthalmia neonatorum, but not specifically gonococcal ophthalmia neonatorum, which has a high risk of blindness, and was the original purpose of prophylaxis. The results provide data on the relative effectiveness of the predominant medications used in the world for ophthalmia neonatorum prophylaxis, but no conclusions on which prophylaxis is most effective for the critical outcome of GC, which leads to an adverse visual outcome.

\section{Quality of the evidence}

The certainty of evidence available to address objectives specified for this review was at best moderate, and was low or very low for most of the comparisons studied. Major factors that affected our assessment of the certainty of the evidence included 
high potential for selection bias (inadequate randomisation), performance/detection bias due to masking that was either insufficient or impossible, and attrition bias. Our assessment of the certainty of the evidence was affected by heterogeneity across trials in only a few instances.

\section{Potential biases in the review process}

We conducted an extensive search of the literature; our search may not have identified studies presented at conferences in paediatrics and neonatal medicine. We specified a broad inclusion criteria to encompass quasi-randomised and randomised studies, therefore we rated several of the included studies to be at high risk of bias and the certainty of the evidence as low. Furthermore, several trials included in this review were published over a period of about six decades. Trial reporting has significantly varied over time. Consequently, there was insufficient information to assess the risk of bias for many of the included trials, which we judged as unclear and moderated our evaluation of the certainty of the evidence. The duration of follow-up and time at which outcomes were assessed were highly varied in the included trials. Whilst our approach to combine data across trials, which assessed outcomes at different times in the 28-day period after birth, may have added uncertainty to our estimates, it is unlikely that our approach would have led to biased estimates. We used an intention-to-treat analysis for all but three trials. This approach is likely to have led to an underestimation of effect in some instances of our meta-analyses. We chose this approach because an available-case analysis would have limited the data only to the subgroup of trial participants who were followed up.

\section{Agreements and disagreements with other studies or reviews}

Another detailed systematic review on the subject of ophthalmia neonatorum has been published (Darling 2010). This review was conducted with methods similar to Cochrane methodology, and the inclusion criteria were similar to those specified for our review. However, Darling 2010 included trials only reporting data on gonococcal and chlamydial ophthalmia neonatorum. In contrast, our systematic review included these outcomes, but also included the outcomes of $\mathrm{BC}$, culture-negative conjunctivitis, and all clinical conjunctivitis cases irrespective of aetiology. The data reported by Darling 2010 for the outcomes of CC and GC were similar to those identified in our review. Darling 2010 concluded that the evidence supports the use of ophthalmia prophylaxis where there is high prevalence of maternal gonorrhoeal and chlamydial infection at birth. However, the review questioned the current evidence for North American laws mandating universal neonatal eye prophylaxis and suggested a "reexamination of this policy". The Darling 2010 review served as a major catalyst reference for the revised 6 March 2015 (and reaffirmed in 28 February 2018) Canadian Pediatric Society Position Statement on Preventing Ophthalmia Neonatorum, which states: "Paediatricians and other physicians caring for newborns should advocate to rescind ocular prophylaxis regulations in jurisdictions in which this is still legally mandated".

The findings of our review are consistent with the evidence review supporting the recommendations provided by the World Health Organization (WHO 2017). This evidence review only examined the outcomes of CC and GC. This review also included randomised and non-randomised studies. For all neonates, the May 2017 World Health Organization Recommendations on Newborn Health, and the 2016 World Health Organization Sexually Transmitted Infection guidelines "recommend topical ocular prophylaxis for the prevention of gonococcal and chlamydial ophthalmia neonatorum". The WHO based this recommendation on lowcertainty evidence, although categorised the recommendation as strong.

The findings of our review are less consistent with the evidence review supporting the January 2019 updated recommendation published by the US Preventive Services Task Force (USPSTF) (Guirguis-Blake 2019; USPSTF 2019). The USPSTF recommendations were based upon a systematic review that only reported data on the outcome of gonococcal ophthalmia neonatorum. In this review, the USPSTF concluded "with high certainty that the net benefit of topical ocular prophylaxis of all newborns to prevent gonococcal ophthalmia neonatorum is substantial". Our review did not find high-certainty evidence for prevention of gonococcal ophthalmia neonatorum but moderatecertainty evidence of an effect on clinical conjunctivitis more generally.

\section{AUTHORS' CONCLUSIONS}

\section{Implications for practice}

Overall, evidence comparing different interventions did not suggest any consistently superior intervention as most of the available evidence was of low-certainty and was limited.

Our findings lead to the following implications for practice.

1. Prophylaxis for ophthalmia neonatorum reduces risk of neonatal clinical conjunctivitis (any conjunctivitis of any aetiology (ACAE), based on moderate-certainty evidence.

2. Our analyses did not identify any medication as effective against gonococcal conjunctivitis (GC), but in general studies were underpowered for this rare outcome and we judged the evidence to be low-certainty. Thus practice should be based on knowledge about known sensitivity of Neisseria gonorrhoeae to antibiotics. This is especially relevant in the context of worldwide concern regarding anti-microbial resistance.

3. Silver nitrate and tetracycline may be considered for prophylaxis of GC, although it seems that silver nitrate is no longer manufactured. Low-certainty evidence including data from all available comparisons (i.e., povidone-iodine versus any prophylaxis, silver nitrate, and erythromycin), suggests that povidone-iodine appears to be effective for prophylaxis against chlamydial conjunctivitis (CC). Although some have suggested that povidone-iodine may be associated with an elevated risk of chemical conjunctivitis (Moore 2015), our findings indicate that it is in fact associated with a lower risk of chemical conjunctivitis.

4. While chloramphenicol appeared to be more effective than povidone-iodine for prophylaxis against CC based on lowcertainty evidence, data on its effectiveness for preventing GC were limited. Similarly, tetracycline may be an alternative for povidone-iodine against CC based on low-certainty evidence, but the data were insufficient in this regard.

5. Finally, there are no data on whether prophylaxis for ophthalmia neonatorum prevents serious outcomes such as blindness or any adverse visual outcome. 
Although moderate-certainty statistically significant evidence in this review suggests that prophylaxis with antibiotics is more effective than silver nitrate for neonatal clinical conjunctivitis (ACAE), instituting antibiotic prophylaxis may be erroneous. Studies demonstrate an increasing prevalence of antibiotic resistance across the globe, specifically for $N$ gonorrhoeae (Lewis 2014). Furthermore, this resistance appeared to be specific against antibiotics found to be more effective than silver nitrate for ACAE in our review, including erythromycin, tetracycline, sulfacetamide, and penicillin (Unemo 2016). Policy regarding prophylaxis should thus also be informed by the prevalence of Neisseria and Chlamydia, their resistance profile against specific antibiotics, as well as access to prenatal screening and care. In areas of high prevalence of $N$ gonorrhoeae infection in pregnant women, low access to antenatal maternal screening, and low access to high-quality care, ophthalmia neonatorum prophylaxis may be considered an effective strategy to prevent blindness. A recent systematic review has found that $N$ gonorrhoeae prevalence in pregnancy can be as high as $4.6 \%(95 \% \mathrm{Cl} 4.0 \%$ to $5.2 \%)$ in low-income countries (Davey 2016). UNICEF statistics further indicate that only $42 \%$ of pregnant women in least-developed countries attended at least four antenatal care visits, and only $77 \%$ attended at least one visit, suggesting that $23 \%$ women did not receive any antenatal care (UNICEF ). Considering that neonates born to untreated $N$ gonorrhoeae-infected mothers have a $30 \%$ to $50 \%$ risk of developing gonococcal ophthalmia, the risk of blindness in endemic areas is therefore high (Laga 1989). In areas where rates of $N$ gonorrhoeae are low in pregnancy, rates of antenatal screening are high, and there is good access to highquality care, resource utilisation concerns may render ophthalmia neonatorum prophylaxis a relatively less attractive strategy to prevent blindness.

\section{Implications for research}

Our findings lead to the following implications for research.

1. A trial comparing tetracycline, povidone-iodine (single administration), and chloramphenicol for GC and CC is likely to provide the community with an effective, universally applicable prophylaxis against ophthalmia neonatorum. Our findings suggest that silver nitrate is more effective than erythromycin and povidone-iodine for $\mathrm{GC}$ with limited evidence against tetracycline and chloramphenicol (Figure 13). In addition, povidone-iodine appeared to be more effective than silver nitrate and erythromycin but less effective than chloramphenicol for CC (Figure 14). Well-designed trials to determine whether povidone-iodine is more effective than tetracycline and whether chloramphenicol is more effective than povidone-iodine for GC and CC will thus yield a universally applicable prophylaxis for ophthalmia neonatorum.

2. Although the eventual goal for prophylaxis for ophthalmia neonatorum is to prevent vision loss and blindness, it is unlikely that trials may be designed to address these outcomes. This is because the outcomes are rare, necessitating large sample sizes to detect meaningful effects. It is also imperative that infections such as gonorrhoea and chlamydia be effectively treated when prenatally diagnosed in the mother. A realistic approach to determine the effect of prophylaxis for preventing GC, and the relative effectiveness of medications to do so, is thus to conduct well-designed randomised controlled trials in targeted settings, for example in populations with high risk or prevalence of infections such as gonorrhoea and chlamydia. The design of such trials should emphasise procedures to minimise losses to follow-up and prompt treatment of positive gonococcal or chlamydial cultures with the goal of avoiding severe adverse visual outcomes.

3. A control group with no intervention or placebo is not acceptable in future trials on prophylaxis for ophthalmia neonatorum.

4. Any future trials on the effectiveness of colostrum for prophylaxis of ophthalmia neonatorum must adequately justify the rationale for why colostrum may potentially prevent ophthalmia neonatorum, particularly GC and CC.

5. Gonococcal and chlamydial infections in the newborn may affect and manifest within organs other than the eye. It is possible that prophylaxis for ophthalmia neonatorum may inadvertently mask such infection in non-ocular sites, but subsequently manifest with non-ocular symptoms and complications. This hypothesis may be addressed in future trials on prophylaxis for ophthalmia neonatorum by following up neonates for non-ocular manifestations of gonococcal and chlamydial infections despite successful prevention of ophthalmia neonatorum.

6. Finally, in this review, we did not address the relative effectiveness of available medications as prophylaxis for ophthalmia neonatorum. Whilst we included some pairwise comparisons, a network meta-analysis is needed to address the relative effects of different interventions using both direct and indirect evidence. Our findings suggest that the data available from the trials identified in this systematic review may be sufficient for a network meta-analysis for some outcomes such as ACAE, but not for others such as GC.

Finally, some have suggested that strategies other than prophylaxis at birth may be effective to prevent ophthalmia neonatorum, for example screening and treating pregnant women for gonococcal or chlamydial infections (Moore 2015). However, randomised controlled trials may not be the optimal study design to obtain evidence on the relative effects of different strategies for preventing ophthalmia neonatorum due to ethical reasons, for example withholding treatment from women infected with chlamydia or gonorrhoea. Other study designs such as interrupted time series may be optimal to determine the relative effect of different strategies to prevent ophthalmia neonatorum in different settings, for example defined by levels of prenatal screening, baseline risk of maternal gonorrhoeal and chlamydial infections, or access to prenatal care and treatment.

\section{ACK N O WLEDGEMENTS}

Thank you to Karen Neves at the Kellogg Health Sciences Library and Chris Emeneau at Medical Computing, both at Dalhousie University, Halifax, Nova Scotia.

We would like to express our gratitude to $\mathrm{Dr} G$ Robert LaRoche, MD, FRCSC, Professor of Ophthalmology, Dalhousie University, Head, Division of Pediatric Ophthalmology and Oculomotility for providing the idea for this review, assisting with the past version of the protocol, and early stages of the review.

We thank Dr Robin Whyte, MD, FRCPC, post-retiree Professor in the Division of Neonatal-Perinatal Medicine, Department of Pediatrics, Faculty of Medicine, Dalhousie University for his contribution to the published protocol and early stages of the review. 
We are grateful to Haroon Saloojee and Prof Kay Dickersin for peer review comments on an earlier version of the protocol for this review. We thank Clare Gilbert, M Qureshi, and Kerry Dwan for their comments on the review.

We thank the following people for providing translations of studies in the following languages: Czech - Myroslava Tataryn, Farsi Mohammed Ziaei, French - Michel Paques, Japanese - Michiyo Hirose and Norwegian - Tero Kivela.

Gratitude is also extended to the 1999 Staff of the Systematic Reviews Training Unit at the Institute of Child Health in London, UK.

At McMaster University, Nancy Fowler at the Department of Family Medicine, McMaster University, Hamilton, Ontario is much appreciated for granting time for the review.

At the University of Toronto, we would like to acknowledge Bart Harvey, Fran Scott, and Michael Finkelstein for also providing time to work on the review.

Of course, this review would not be possible without the helpful advice and encouragement of Cochrane Eyes and Vision: Anupa Shah, Catey Bunce, Katherine Henshaw, Karen Blackhall, Shona Burman-Roy, Jennifer Evans, and Richard Wormald and also from Julian PT Higgins,
A special thank you to $\mathrm{Dr}$ Gordon Guyatt and $\mathrm{Dr}$ Romina Brignardello Petersen for assisting with learning and applying GRADE.

We would like thank $\mathrm{Dr}$ Roger F Soll, MD, H. Wallace Professor of Neonatology, University of Vermont College of Medicine and Co-ordinating Editor of Cochrane Neonatal, and Dr Kamiar Mireskandari MBChB, FRCSEd, FRCOphth, PhD, Staff Ophthalmologist and Associate Professor, Department of Ophthalmology and Visual Sciences, Hospital for Sick Children and University of Toronto for assistance with defining visual outcomes.

Thank you to Dr Raj Rathee, childhood friend, and now Staff Ophthalmologist at the North York General Hospital, for his assistance with clinical questions.

I am forever indebted to my mother, Sudershan Kapoor, and my wife, Seema Rawla, and children, Kabir Mohan Kapoor and Ajouni Mayael Kapoor, for their enduring love, patience, and support.

This review is dedicated to my father, the late Dr Brij Mohan Kapoor, Professor Emeritus, Saint Mary's University, Halifax, Nova Scotia, who had great respect for those who dedicated their careers to research. 
REFE RE N CES

\section{References to studies included in this review}

\section{Ali 2007 \{published data only\}}

* Ali Z, Khadije D, Elahe A, Fateme N, Mohammad M, Fateme Z, et al. Prophylaxis of ophthalmia neonatorum comparison of Betadine, erythromycin and no prophylaxis. Journal of Tropical Pediatrics 2007;53(6):388-92.

Bell 1993 \{published data only\}

* Bell TA, Grayston JT, Krohn MA, Kronmal RA. Randomized trial of silver nitrate, erythromycin, and no eye prophylaxis for the prevention of conjunctivitis among newborns not at risk for gonococcal ophthalmitis. Eye Prophylaxis Study Group. Pediatrics 1993;92(6):755-60.

Krohn MA, Hillier SL, Bell TA, Kronmal RA, Grayston JT. The bacterial etiology of conjunctivitis in early infancy. Eye Prophylaxis Study Group. American Journal of Epidemiology 1993;138(5):326-32.

\section{Bramantyo 2016 \{published data only\}}

Bramantyo T, Roeslani RD, Andriansjah A, Sitorus RS. The efficacy of $1 \%$ chloramphenicol eye ointment versus $2.5 \%$ povidone-iodine ophthalmic solution in reducing bacterial colony in newborn conjunctivae. Asia-Pacific Journal of Ophthalmology 2015;4(3):180-3.

\section{Brussieux 1991 \{published data only\}}

* Brussieux J, Boisivon A, Theron HP, Faidherbe C, Machado N, Michelon B. Prevention of neonatal conjunctivitis. A comparative clinical and bacteriologic study of 2 eyedrops: silver nitrate and oxytetracycline chlorhydrate [Prevention des conjonctivites neonatales. Etude comparative clinique et bacteriologique de deux collyres: nitrate d' argent et chlorhydrate d' oxytetracycline]. Annales de Pediatrie 1991;38(9):637-41.

\section{Chen 1992 \{published data only\}}

* Chen JY. Prophylaxis of ophthalmia neonatorum: comparison of silver nitrate, tetracycline, erythromycin and no prophylaxis. Pediatric Infectious Disease Journal 1992;12(12):1026-30.

\section{Christian 1960 \{published data only\}}

* Christian JR. Comparison of ocular reactions with the use of silver nitrate and erythromycin ointment in ophthalmia neonatorum prophylaxis. Journal of Pediatrics 1960;57(1):55-60.

Cousineau 1952 \{published data only\}

* Cousineau GG, Lloyd LA. Preliminary report on prophylaxis of ophthalmia neonatorum. Transactions of the Canadian Ophthalmological Society 1952;5:75-80.

\section{David 2011 \{published data only\}}

* David M, Rumelt S, Weintraub Z. Efficacy comparison between povidone iodine $2.5 \%$ and tetracycline $1 \%$ in prevention of ophthalmia neonatorum. Ophthalmology 2011;118(7):1454-8.
Davidson 1951 \{published data only\}

* Davidson HH, Hill JH. Penicillin in the prophylaxis of ophthalmia neonatorum. Journal of the American Medical Association 1951;145(14):1052-5.

Fischer 1988 \{published data only\}

* Fischer PR, Reta BB. Prevention of neonatal conjunctivitis in Zaire. Annals of Tropical Paediatrics 1988;8(2):85-6.

Ghaemi 2014 \{published data only\}

* Ghaemi S, Navaei P, Rahmirad S, Behjati M, Kelishadi R. Evaluation of preventive effects of colostrum against neonatal conjunctivitis: a randomized clinical trial. Journal of Education and Health Promotion 2014;3:63. [DOI: 10.4103/2277-9531.134776]

Ghahramani 2007 \{published data only\}

* Ghahramani M, Ghahramani AA. Epidemiologic study of ophthalmia neonatorum and impact of prophylaxis on its incidence. Acta Medica Iranica 2007;45(5):361-4.

\section{Ghotbi 2012 \{published data only\}}

Ghotbi N. Comparison of the effect of tetracycline $1 \%$ and erythromycin $0.5 \%$ ophthalmic ointments for prophylaxis of ophthalmia neonatorum. Scientific Journal of Kurdistan University of Medical Sciences 2012;17(3):20-5.

\section{Graf 1994 \{published data only\}}

* Graf H, Retke U, Schilling C, Schmidt M. Reaction of the ophthalmic front region to the Crede-prophylaxis [Die reakton des vorderen augenabschnittes auf die Crede-prophylaxe]. Zentralblatt fur Gynakologie 1994;116(11):639-42.

Hammerschlag 1980 \{published data only\}

* Hammerschlag MR, Chandler JW, Alexander ER, English M, Chiang WT, Koutsky L, et al. Erythromycin ointment for ocular prophylaxis of neonatal chlamydial infection. JAMA 1980;244(20):2291-3.

Hammerschlag 1989 \{published data only\}

* Hammerschlag MR, Cummings C, Roblin PM, Williams TH, Delke I. Efficacy of neonatal ocular prophylaxis for the prevention of chlamydial and gonococcal conjunctivitis. New England Journal of Medicine 1989;320(12):769-72.

Harris 1957 \{published data only\}

* Harris ST. Silver nitrate in ophthalmia neonatorum prophylaxis. Sight Saving Review 1957;27(3):152-3.

Hick 1985 \{published data only\}

* Hick JF, Block DJ, Ilstrup DM. A controlled study of silver nitrate prophylaxis and the incidence of nasolacrimal duct obstruction. Journal of Pediatric Ophthalmology and Strabismus 1985;22(3):92-3.

Isenberg 1995 \{published data only\}

* Isenberg SJ, Apt L, Wood M. A controlled trial of povidoneiodine as prophylaxis against ophthalmia neonatorum. New England Journal of Medicine 1995;332(9):562-6. 
Isenberg SJ, Apt L, Wood M. The influence of perinatal infective factors on ophthalmia neonatorum. Journal of Pediatric Ophthalmology and Strabismus 1996;33(3):185-8.

Isenberg 2003 \{published data only\}

Isenberg SJ, Apt L, Del Signore M, Gichuhi S, Berman NG. A double application approach to ophthalmia neonatorum prophylaxis. British Journal of Ophthalmology 2003;87(12):1449-52.

Kaivonen 1965a \{published data only\}

* Kaivonen M. Prophylaxis of ophthalmia neonatorum. A clinical and bacteriologic study of ophthalmia neonatorum and conjunctival bacterial flora of the newborn. Acta Ophthalmologica 1965;79(Suppl):9-70.

Kaivonen 1965b \{published data only\}

* Kaivonen M. Prophylaxis of ophthalmia neonatorum. A clinical and bacteriologic study of ophthalmia neonatorum and conjunctival bacterial flora of the newborn. Acta Ophthalmologica 1965;79(Suppl):9-70.

Laga 1988 \{published data only\}

* Laga M, Plummer FA, Piot P, Datta P, Namaara W, Ndinya-Achola JO, et al. Prophylaxis of gonococcal and chlamydial ophthalmia neonatorum. A comparison of silver nitrate and tetracycline. New England Journal of Medicine 1988;318(11):653-7.

Pastor 2015 \{published data only (unpublished sought but not used)\}

Alexandre I, Justel M, Martinez P, Ortiz de Lejarazu R, Pastor JC. First attempt to implement ophthalmia neonatorum prophylaxis in Angola: microorganisms, efficacy, and obstacles. Journal of Ophthalmology 2015;2015:Article ID 326526. [DOI: $10.1155 / 2015 / 326526]$

\section{Posner 1959 \{published data only\}}

Posner AC, Anderson GD, Prigot A. Observations on the prophylaxis of ophthalmia neonatorum in a municipal hospital. In: Antibiotics Annual 1958-59. New York: Medical Encyclopedia Inc, 1958:552-5.

\section{Ramirez-Ortiz 2007 \{published data only\}}

* Ramirez-Ortiz MA, Rodriguez-Almaraz M, Ocha-Diaz Lopez H, Diaz-Prieto P, Rodriguez-Suarez RS. Randomised equivalency trial comparing $2.5 \%$ povidone-iodine eye drops and ophthalmic chloramphenicol for preventing neonatal conjunctivitis in a trachoma endemic area in southern Mexico. British Journal of Ophthalmology 2007;91(11):1430-4.

\section{Richter 2006 \{published data only\}}

Richter R, Below H, Kadow I, Kramer A, Müller C, Fusch C. Effect of topical $1.25 \%$ povidone-iodine eyedrops used for prophylaxis of ophthalmia neonatorum on renal iodine excretion and thyroid-stimulating hormone level. Journal of Pediatrics 2006;148(3):401-3.

\section{Siegel 1982 \{published data only\}}

* Siegel JD, McCracken GH, Threlkeld N, DePasse BM, Rosenfeld CR. Single dose penicillin prophylaxis in neonatal
group-B streptococcal disease. Conclusion of a 41 month controlled trial. Lancet 1982;1(8287):1426-30.

Siegel JD, McCracken GH, Threlkeld N, Milvenan B, Rosenfeld CR. Single dose penicillin prophylaxis against neonatal group-B streptococcal infections. New England Journal of Medicine 1980;303(14):769-75.

Wahlberg 1982 \{published and unpublished data\}

Wahlberg V. Crede's prevention method has lost its justification today [Credeprofylax - en metod som idag saknar berattigande]. Nordisk Medicin 1982;97(2):36-7.

Wahlberg V. Reconsideration of Crede prophylaxis. A study of maternity and neonatal care. Acta Paediatrica Scandinavica 1982;295(Suppl):1-73.

\section{Zbojan 2004 \{published data only\}}

Zbojan J, Dluholucky S, Zacharovska M. Credeization with povidone-iodine - efficiency and a possible influence on thyroid gland in newborns [Kredeizacia povidon-jodidom - ucinnost a mozny vplyv na stitnu zlazu novorodenca]. Cesko Slovenska Pediatrie 2004;59(1):11-3.

\section{References to studies excluded from this review}

\section{Assadian 2002 \{published data only\}}

Assadian O, Assadian A, Aspock C, Hahn D, Koller W. Prophylaxis of ophthalmia neonatorum - a nationwide survey of the current practice in Austria. Wiener Klinische Wochenschrift 2002;114(5-6):194-9.

\section{Bailey 1993 \{published data only\}}

Bailey RL, Arullendran P, Whittle HC, Mabey DC. Randomised controlled trial of single-dose azithromycin in treatment of trachoma. Lancet 1993;342(8869):453-6.

Baptista 1968 \{published data only\}

Baptista OR. Ophthalmia neonatorum. Prophylaxis with 5nitro-2-furaldehyde-semicarbazone (Furacin). British Journal of Venereal Diseases 1968;44(2):189.

* Baptista OR. Ophthalmia neonatorum. Prophylaxis with 5nitro-2-furaldehyde-semicarbazone [Oftalmia neonatorum. Prevencao com 5 nitro 2 furaldeido semicarbazona (Furacin)]. Hospital 1967;71(1):187-93.

\section{Baveja 1997 \{published data only\}}

Baveja UK, Hiranandani MK, Talwar P, Sen DK. Laboratory techniques for diagnosis of chlamydial infections of the eye. Journal of Communicable Diseases 1997;29(3):247-53.

Bobo 1997 \{published data only\}

Bobo LD, Novak N, Munoz B, Hsieh YH, Quinn TC, West S. Severe disease in children with trachoma is associated with persistent Chlamydia trachomatis infection. Journal of Infectious Disease 1997;176(6):1524-30.

Brady 1997 \{published data only\}

Brady RC. Index of suspicion. Case 2. Diagnosis: gonorrhea conjunctivitis. Pediatrics in Review 1997;18(5):175-7. 
Burr 2017 \{published data only\}

Burr SE, Camara B, Oluwalana C, Bojang E, Bottomley C, Bojang $A$, et al. Does azithromycin given to women in labour decrease ocular bacterial infection in neonates? A double-blind, randomized trial. BMC Infectious Diseases 2017;17(1):799.

\section{Candano 1951 \{published data only\}}

Candano A, Avila Cisneros I. Prophylaxis against ophthalmia neonatorum. Penicillin as a substitute for Crede's method [Profilaxis de la oftalmia neonatorum. Utilizacion de la penicilina en substitucion del metodo Crede]. Boletin de la Sociedad Medica Del Centro, Materno Infantil General Maximino Avila Camacho 1950;1:57-61.

Candano A, Avila Cisneros I. Prophylaxis against ophthalmia neonatorum. Penicillin as a substitute for Crede's method. British Journal of Venereal Diseases 1951;27(3):167.

\section{CDC 1998 \{published data only\}}

* Centers for Disease Control and Prevention (CDC). Acute Hemorrhagic Conjunctivitis -- St. Croix, U.S. Virgin Islands, September-October 1998. Morbidity and Mortality Weekly Report (MMWR) 1998;47(42):899-901.

\section{Clark 1951 \{published data only\}}

* Clark SG, Culler AM. Aureomycin as prophylaxis against ophthalmia neonatorum. American Journal of Ophthalmology 1951;34:840-7.

Clark SG, Culler AM. Aureomycin as prophylaxis against ophthalmia neonatorum. British Journal of Venereal Diseases 1951;27(4):209.

\section{Cohen 1990 \{published data only\}}

Cohen R, de la Rocque F, Bouhanna A, Boucherat M, Elbez A, Lecompte MD, et al. Randomized study of cefatrizine versus cefaclor in conjunctivitis otitis syndrome [Etude randomisee cefatrizine versus cefaclor dans le syndrome otite-conjonctivite]. Pathologie Biologie Paris 1990;38(5 Part 2):517-20.

Darling 2010 \{published data only\}10.1016/j.jmwh.2009.09.003 Darling EK, McDonald H. A meta-analysis of the efficacy of ocular prophylactic agents used for the prevention of gonococcal and chlamydial ophthalmia neonatorum. Journal of Midwifery and Women's Health 2010;55(4):319-27.

\section{Darougar 1977 \{published data only\}}

Darougar S, Viswalingam M, Treharne JD, Kinnison JR, Jones BR. Treatment of TRIC infection of the eye with rifampin or chloramphenicol. British Journal of Ophthalmology 1977;4(4):255-9.

\section{Dhir 1967 \{published data only\}}

Dhir SP, Agarwal LP, Detels R, Wang SP, Grayston JT. Field trial of two bivalent trachoma vaccines in children of Punjab Indian villages. American Journal of Ophthalmology 1967;63(5 Suppl):1639-44.
Drago 1998 \{published data only\}

Drago F, La-Manna C, Marino V, Marino A, Emmi I, Reibaldi A. Norfloxacin exhibits negligible serum concentrations after repeated conjunctival instillation in children. British Journal of Clinical Pharmacology 1998;46(3):272-4.

Fok 1995 \{published data only\}

Fok TF, Wong W, Cheng AF. Use of eyepatches in phototherapy: effects on conjunctival bacterial pathogens and conjunctivitis. Pediatric Infectious Disease Journal 1995;14(12):1091-4.

\section{Fransen 1984 \{published data only\}}

Fransen L, Nsanze H, D'Costa L, Brunham RC, Ronald AR, Piot P. Single-dose kanamycin therapy of gonococcal ophthalmia neonatorum. Lancet 1984;2(8414):1234-7.

\section{Gross 1997 \{published data only\}}

Gross RD, Hoffman RO, Lindsay RN. A comparison of ciprofloxacin and tobramycin in bacterial conjunctivitis in children. Clinical Pediatrics 1997;36(8):435-44.

\section{Hammerschlag 1997 \{published data only\}}

Hammerschlag MR, Roblin PM, Gelling M, Tsumura N, Jule JE, Kutlin A. Use of polymerase chain reaction for the detection of Chlamydia trachomatis in ocular and nasopharyngeal specimens from infants with conjunctivitis. Pediatric Infectious Disease Journal 1997;16(3):293-7.

\section{Hammerschlag 1998 \{published data only\}}

Hammerschlag MR, Gelling M, Roblin PM, Kutlin A Jule JE. Treatment of neonatal chlamydial conjunctivitis with azithromycin. Pediatric Infectious Disease Journal 1998;17(11):1049-50.

\section{Heggie 1985 \{published data only\}}

Heggie AD, Jaffe AC, Stuart LA, Thombre PS, Sorensen RU. Topical sulfacetamide vs oral erythromycin for neonatal chlamydial conjunctivitis. American Journal of Diseases of Children 1985;139(6):564-6.

\section{Horven 1993 \{published data only\}}

Horven I. Acute conjunctivitis. A comparison of fusidic acid viscous eye drops and chloramphenicol. Acta Ophthalmologica 1993;71(2):165-8.

Iroha 1998 \{published data only\}

Iroha EO, Kesah CN, Egri-Okwaji MT, Odugbemi TO. Bacterial eye infection in neonates, a prospective study in a neonatal unit. West African Journal of Medicine 1998;17(3):168-72.

\section{Isenberg 1994 \{published data only\}}

Isenberg SJ, Apt L, Yoshimori R, Leake RD, Rich R. Povidoneiodine for ophthalmia neonatorum prophylaxis. American Journal of Ophthalmology 1994;118(6):701-6.

\section{Jacobson 1988 \{published data only\}}

Jacobson JA, Call NB, Kasworm EM, Dirks MS, Turner RB. Safety and efficacy of topical norfloxacin versus tobramycin in the treatment of external ocular infections. Antimicrobial Agents and Chemotherapy 1988;32(12):1820-4. 
Keenan 2010 \{published data only\}10.1001/ archophthalmol.2009.365.

Keenan JD, Eckert S, Rutar T. Cost analysis of povidoneiodine for ophthalmia neonatorum prophylaxis. Archives of Ophthalmology 2010;128(1):136-7.

Khan 2016 \{published data only\}

Khan FA, Hussain MA, Niazi SPK, Haq ZU, Akhtar N. Efficacy of $2.5 \%$ and $1.25 \%$ povidone-iodine solution for prophylaxis of ophthalmia neonatorum. Journal of the College of Physicians and Surgeons Pakistan 2016;26(2):121-4.

Klein 1997 \{published data only\}

Klein JO. History of macrolide use in pediatrics. Pediatric Infectious Disease Journal 1997;16(4):427-31.

Kramer 1997 \{published data only\}

Kramer A, Behrens-Baumann W. Prophylactic use of topical anti-infectives in ophthalmology. Ophthalmologica 1997;211(Suppl 1):68-76.

\section{Laga 1986 \{published data only\}}

Laga M, Naamara W, Brunham RC, D'Costa LJ, Nsanze H, Piot $P$, et al. Single dose therapy of gonococcal ophthalmia neonatorum with ceftriaxone. New England Journal of Medicine 1986;315(22):1382-5.

Lietman 1998 \{published data only\}

* Lietman T, Dawson C, Osaki S. Ocular chlamydial infections. International Ophthalmology Clinics 1998;38(4):125-35.

Maharajan 1997 \{published data only\}

* Maharajan S, Thomas R, Kuriakose T. Conjunctivitis and red eye. National Medical Journal of India 1997;10(3):130-3.

\section{Mani 1997 \{published data only\}}

Mani VR, Vidya KC. A microbiological study of ophthalmia neonatorum in hospital-born babies. Journal of the Indian Medical Association 1997;95(7):416-7.

\section{Mann 1954 \{published data only\}}

* Mann I. Clinical observations on the prophylaxis of ophthalmia neonatorum. British Journal of Ophthalmology 1954;38:734-41.

Mann I. Clinical observations on the prophylaxis of ophthalmia neonatorum. British Journal of Venereal Diseases 1955;31(3):199.

\section{Margileth 1957 \{published data only\}}

Margileth AM. Comparison of ocular reaction using penicillin and bacitracin ointments in ophthalmia neonatorum prophylaxis. British Journal of Venereal Diseases 1958;34(3):266.

* Margileth AM. Comparison of ocular reaction using penicillin and bacitracin ointments in ophthalmia neonatorum prophylaxis. Journal of Pediatrics 1957;51:646-51.
Markham 1994 \{published data only\}

Markham A, Faulds D. Roxithromycin. An update of its antimicrobial activity, pharmacokinetic properties and therapeutic use. Drugs 1994;48(2):297-326.

\section{McAuley 1994 \{published data only\}}

McAuley J, Boyer KM, Patel D, Mets M, Swisher C, Roizen N, et al. Early and longitudinal evaluations of treated infants and children and untreated historical patients with congenital toxoplasmosis: the Chicago Collaborative Treatment Trial. Clinical Infectious Diseases 1994;18(1):38-72.

Mets 1997 \{published data only\} Mets MB, Holfels E, Boyer KM, Swisher CN, Roizen N, Stein L, et al. Eye manifestations of congenital toxoplasmosis. American Journal of Ophthalmology 1997;123(1):1-16.

Meyer 1997 \{published data only\}

Meyer D. Ophthalmia neonatorum prophylaxis - can we do it more cost-effectively. South African Medical Journal 1997;87(4):76-7.

Nakagawa 1997 \{published data only\}

Nakagawa H. Treatment of chlamydial conjunctivitis. Ophthalmologica 1997;211(Suppl 1):25-8.

Nichols 1966 \{published data only\}

Nichols RL, Bell SD Jr, Murray ES, Haddad NA, Bobb AA. Studies on trachoma. V. Clinical observations in a field trial of bivalent trachoma vaccine at three dosage levels in Saudi Arabia. American Journal of Tropical Medicine and Hygiene 1966;15(4):639-47.

\section{Normann 2002 \{published data only\}}

Normann EK, Bakken O, Peltola J, Andreasson B, Buhl S, Sigg P, et al. Treatment of acute neonatal bacterial conjunctivitis: a comparison of fucidic acid to chloramphenicol drops. Acta Ophthalmologica Scandinavica 2002;80(2):183-7.

Peters 1992 \{published data only\}

Peters DH, Clissold SP. Clarithromycin. A review of its antimicrobial activity, pharmacokinetic properties and therapeutic potential. Drugs 1992;44(1):117-64.

Ratelle 1997 \{published data only\}

Ratelle S, Keno D, Hardwood M, Etkind PH. Neonatal chlamydial infections in Massachusetts, 1992-1993. American Journal of Preventive Medicine 1997;13(3):221-4.

\section{Reimer 1997 \{published data only\}}

Reimer K, Fleischer W, Brogmann B, Schreier H, Burkhard P, Lanzendorfer A, et al. Povidone-iodine liposomes - an overview. Dermatology 1997;1995(Suppl 2):93-9.

Rivlin 1997 \{published data only\}

Rivlin ME, Morrison JC, Grossman JH 3rd. Comparison of pregnancy outcome between treated and untreated women with chlamydial cervicitis. Journal of the Mississippi State Medical Association 1997;38(11):404-7. 
Rosenman 2003 \{published data only\}

Rosenman MB, Mohan BE, Downs SM, Kleiman MB. Oral erythromycin prophylaxis vs watchful waiting in caring for newborns exposed to Chlamydia trachomatis. Archives of Pediatrics and Adolescent Medicine 2003;157(6):565-71.

\section{Schaller 1997 \{published data only\}}

Schaller U, Mino de Kaspar H, Schriever S, Klauss V. Ophthalmia neonatorum caused by Chlamydia trachomatis. Rapid diagnosis and therapy [Ophthalmia neonatorum durch Chlamydia trachomatis. Schnelldiagnostik und therapie]. Ophthalmologe 1997;94(5):317-20.

\section{Seiga 1993 \{published data only\}}

* Seiga K, Shoji T. Chemoprophylaxis of ophthalmia neonatorum through vertical infection. Evaluation of Crede's method using norfloxacin and gentamicin. Japanese Journal of Antibiotics 1993;4(4):331-6.

\section{Silva 2008 \{published data only\}}

* Silva LR, Gurgel RQ, Ribeiro D, Lima R, Cuevas LE. Current usefulness of Crede's method of preventing neonatal ophthalmia. Annals of Tropical Paediatrics 2008;28(1):45-8.

Sorsby 1949 \{published data only\}

Sorsby A, Kane I. Optimal methods in the treatment of ophthalmia neonatorum. British Medical Journal 1949;2:562-5.

\section{Stenberg 1991 \{published data only\}}

Stenberg K, Mardh PA. Treatment of chlamydial conjunctivitis in newborns and adults with erythromycin and roxithromycin. Journal of Antimicrobial Chemotherapy 1991;28(2):301-7.

Sud 1995 \{published data only\}

Sud RN, Greval RS, Bajwa RS. Topical flurbiprofen therapy in vernal keratoconjunctivitis. Indian Journal of Medical Sciences 1995;49(9):205-9.

\section{Sung 1998 \{published data only\}}

Sung L, MacDonald NE. Gonorrhea: a pediatric perspective. Pediatrics in Review 1998;19(1):13-6.

\section{van Bogaert 1997 \{published data only\}}

van Bogaert LJ. Controversies in the approach to ophthalmia neonatorum. South African Medical Journal 1997;87(1):76-7.

\section{Wallace 1998 \{published data only\}}

Wallace DK, Steinkuller PG. Ocular medications in children. Clinical Pediatrics 1998;37(11):645-52.

\section{West 1995 \{published data only\}}

West S, Munoz B, Lynch M, Kayongoya A, Chilangwa Z, Mmbaga BB, et al. Impact of face washing on trachoma in Kongwa, Tanzania. Lancet 1995;345(8943):155-8.

\section{Winceslaus 1987 \{published data only\}}

Winceslaus J, Goh BT, Dunlop EM, Mantell J, Woodland RM, Forsey $\mathrm{T}$, et al. Diagnosis of ophthalmia neonatorum. British Medical Journal Clinical Research Edition 1987;295(6610):1377-9.
Woolridge 1967 \{published data only\}

Woolridge RL, Grayston JT, Chang IH, Yang CY, Cheng KH. Longterm follow-up of the initial (1959-1960) trachoma vaccine field trial on Taiwan. American Journal of Ophthalmology 1967;63(Suppl 5):1650-5.

Wu 2003 \{published data only\}

Wu SX, Yang J, Liu G. A clinical study in China of neonatal conjunctivitis caused by Chlamydia trachomatis. Clinical Pediatrics 2003;42(1):83-4.

\section{Yasunaga 1977 \{published data only\}}

Yasunaga S, Kean E. Effect of three ophthalmic solutions on chemical conjunctivitis in the neonate. Obstetrical and Gynecological Survey 1977;32(7):593-4.

* Yasunaga S, Kean EH. Effect of three ophthalmic solutions on chemical conjunctivitis in the neonate. American Journal of Diseases of Children 1977;131(2):159-61.

\section{Yetman 1997 \{published data only\}}

Yetman RJ, Coody DK. Conjunctivitis: a practice guideline. Journal of Pediatric Health Care 1997;11(5):238-41.

\section{Zanoni 1992 \{published data only\}}

Zanoni D, Isenberg SJ, Apt L. A comparison of silver nitrate with erythromycin for prophylaxis against ophthalmia neonatorum Clinical Pediatrics 1992;5(5):295-8.

\section{References to studies awaiting assessment}

Matinzadeh 2007 \{published data only\}10.1258/004947507779951880

Matinzadeh ZK, Beiragdar F, Kavemanesh Z, Abolgasemi H, Amirsalari S. Efficacy of topical ophthalmic prophylaxis in prevention of ophthalmia neonatorum. Tropical Doctor 2007;37(1):47-9.

\section{Additional references}

\section{Adachi 2016}

Adachi K, Nielsen-Saines K, Klausner JD. Chlamydia trachomatis infection in pregnancy: the global challenge of preventing adverse pregnancy and infant outcomes in sub-Saharan Africa and Asia. BioMed Research International 2016;2016:9315757. [DOI: 10.1155/2016/9315757]

\section{AFSSAPS 2010}

Agence Française de Sécurité Sanitaire. Prophylaxis of conjunctival infections of newborn children [Prophylaxie des infections conjonctivales du nouveauné]. ansm.sante.fr/var/ansm_site/storage/original/ application/8d7b81471c088327d5343c5c102feafa.pdf (accessed 23 March 2016).

\section{Albert 1994}

Albert DM, Jakobiec FA (editors). Principles and Practice of Ophthalmology: Clinical Practice. Philadelphia: W.B. Saunders Company, 1994. 
Amini 2008

Amini E, Ghasemi M, Daneshjou K. A five-year study in Iran of ophthalmia neonatorum: prevalence and etiology. Medical Science Monitor 2008;14(2):90-6.

\section{Anonymous 1918}

Anonymous. Hospital for ophthalmia neonatorum. British Journal of Ophthalmology 1918;2(11):574.

\section{Anonymous 1919}

Anonymous. The early treatment of ophthalmia neonatorum. British Journal of Ophthalmology 1919;3(8):365.

\section{Barsam 1966}

Barsam PC. Specific prophylaxis of gonorrheal ophthalmia neonatorum. A review. New England Journal of Medicine 1966;274(13):731-4.

\section{Bastion 2006}

Bastion ML, Prakash K, Siow YC, Loh SS. Bilateral corneal perforation in a sexually active adult male with gonococcal conjunctivitis. Medical Journal of Malaysia 2006;61(3):366-8.

\section{Blatt 2012}

Blatt AJ, Lieberman JM, Hoover DR, Kaufman HW. Chlamydial and gonococcal testing during pregnancy in the United States. American Journal of Obstetrics and Gynecology 2012;207(1):55.e1-8.

\section{Buller 1900}

Buller F. Gonorrhoea in its relation to diseases of the eye. Montreal Medical Journal 1900;29(3):185-7.

\section{Caligaris 2010}

Caligaris LS, Medina NH, Durkin SR, Haro-Munoz E, Chinen NH. Assessment of the current ocular health practices within neonatal units in the City of São Paulo, Brazil. Ophthalmic Epidemiology 2010;17(5):333-7.

\section{Chang 2006}

Chang K, Cheng VY, Kwong NS. Neonatal haemorrhagic conjunctivitis: a specific sign of chlamydial infection. Hong Kong Medical Journal 2006;12(1):27-32.

\section{Chhabra 2008}

Chhabra MS, Motley WW, Mortensen JE. Eikenella corrodens as a causative agent for neonatal conjunctivitis. Journal of AAPOS 2008;12(5):524-5.

\section{Chico 2012}

Chico RM, Mayaud P, Ariti C, Mabey D, Ronsmans C, Chandramohan D. Prevalence of malaria and sexually transmitted and reproductive tract infections in pregnancy in sub-Saharan Africa: a systematic review. JAMA 2012;307(19):2079-86.

\section{CPS 2002}

Canadian Paediatric Society. Recommendations for the prevention of neonatal ophthalmia. Paediatric Child Health 2002;7(7):480-8

\section{Crede 1881}

Crede CS. Ophthalmia neonatorum in newborn children [Die verhürtung der augenentzündung der neugeborenen]. Archiv fur Gynaekologie 1881;17:50-3.

\section{Crede 1884}

Crede CSF. Die Verhütung der Augenentzündung der Neugeborenen (Ophthalmoblennorrhoea neonatorum) der häufigsten und wichtigsten Ursache der Blindheit. Berlin: Hirschwald, 1884.

\section{Dageville 2015}

Dageville $C$. Is prophylaxis against ophthalmia neonatorum justified in French maternity wards? Archives of Pediatrics 2015;22(2):128-9.

\section{Darville 2015}

Darville T. Chlamydia trachomatis infections in neonates and young children. Seminars in Pediatric Infectious Diseases 2005;16(4):235-44.

\section{Davey 2016}

Davey DL, Shull HI, Billings JD, Wang D, Adachi K, Klausner JD. Prevalence of curable sexually transmitted infections in pregnant women in low- and middle-income countries from 2010 to 2015. A systematic review. Sexually Transmitted Diseases 2016;43(7):450-8.

\section{Department of Health, UK 2010}

Department of Health. Explanatory memorandum to the health protection (part 2a orders) regulations, 2010. No. 658. www.legislation.gov.uk/uksi/2010/658/pdfs/ uksiem_20100658_en.pdf (accessed 25 November 2013).

\section{Dharmasena 2015}

Dharmasena A, Hall N, Goldacre R, Goldacre MJ. Time trends in ophthalmia neonatorum and dacryocystitis of the newborn in England, 2000-2011: database study. Sexually Transmitted Infections 2015;91(5):342-5.

\section{Di Bartolomeo 2001}

Di Bartolomeo S, Mirta DH, Janer M, Rodríguez Fermepin MR, Sauka D, Magariños F, et al. Incidence of Chlamydia trachomatis and other potential pathogens in neonatal conjunctivitis. International Journal of Infectious Diseases 2015;5(3):139-43.

\section{Di Bartolomeo 2005}

Di Bartolomeo S, Higa M, Janer M, Pennisi A, Balbin G, Priore G. Neonatal conjunctivitis in a hospital at Gran Buenos Aires. Last 5 years update. Revista Argentina de Microbiologia 2005;37(3):139-41.

\section{Donham 2008}

Donham BP, Gibler WB. Images in emergency medicine. Gonococcal conjunctivitis. Annals of Emergency Medicine 2008;52(1):11.

\section{Duke-Elder 1965}

Duke-Elder S. Diseases of the outer eye. In: System of Ophthalmology. Vol. 8 (Part 1). St Louis: CV Mosby Co, 1965:167-74. 


\section{Dunn 2000}

Dunn PM. Dr. Carl Credé (1819-1892) and the prevention of ophthalmia neonatorum. Archives of Disease in Children. Fetal and Neonatal Edition 2000;83(2):F158-9.

\section{Eser 2009}

Eser I, Bozaci M. A nationwide survey of prophylaxis against ophthalmia neonatorum in Turkey. Turkish Journal of Medical Sciences 2009;39(5):771-4.

\section{Foster 1987}

Foster A, Sommer A. Corneal ulceration, measles, and childhood blindness in Tanzania. British Journal of Ophthalmology 1987;71(5):331-43.

\section{Foster 1991}

Foster A, Gilbert C. Community efforts in the reduction of corneal blindness in developing countries. Refractive and Corneal Surgery 1991;7(6):445-8.

\section{Foster 1995}

Foster A, Volker K. Ophthalmia neonatorum in developing countries. New England Journal of Medicine 1995;332(9):600-1.

\section{Fransen 1987}

Fransen L, van den Berghe P, Mertens A. Incidence and bacterial aetiology of neonatal conjunctivitis. European Journal of Pediatrics 1987;146(2):152-5.

\section{Gilbert 2012}

Gilbert C, Rahi J. Visual impairment and blindness in children - magnitude and causes. In: Johnson G, Minassian D, Weale W, West S, editors(s). Epidemiology of Eye Disease. London: Imperial College Press, 2012.

\section{Glanville 2006}

Glanville JM, Lefebvre C, Miles JN, Camosso-Stefinovic J. How to identify randomized controlled trials in MEDLINE: ten years on. Journal of the Medical Library Association 2006;94(2):130-6.

\section{Grzybowski 2018}

Grzybowski A, Kanclerz P, Myers WG. The use of povidoneiodine in ophthalmology. Current Opinion in Ophthalmology 2018;29(1):19-32.

\section{Guala 2005}

Guala A, Guarino R, Zaffaroni M, Martano C, Fabris C, Pastore G, et al. The impact of national and international guidelines on newborn care in the nurseries of Piedmont and Aosta Valley. BMC Pediatrics 2005;5:45. [DOI: 10.1186/1471-2431-5-45]

\section{Guirguis-Blake 2019}

Guirguis-Blake JM, Evans CV, Rushkin M. Ocular Prophylaxis for Gonococcal Ophthalmia Neonatorum: Updated Evidence Report and Systematic Review for the US Preventive Services Task Force. JAMA 2019;321(4):404-6.

\section{Hammerschlag 1993}

Hammerschlag MR. Neonatal conjunctivitis. Pediatric Annals 1993;22(6):346-51.

\section{Haussman 1895}

Haussman. Hygiene and public health. Ophthalmia neonatorum. Canadian Practitioner Journal 1895;20:697.

\section{Higgins 2017}

Higgins JPT, Altman DG, Sterne JAC (editors). Chapter 8: Assessing risk of bias in included studies. In: Higgins JPT, Churchill R, Chandler J, Cumpston MS (editors), Cochrane Handbook for Systematic Reviews of Interventions version 5.2.0 (updated June 2017). The Cochrane Collaboration, 2017. Available from www.training.cochrane.org/handbook.

\section{Isenberg 1994a}

Isenberg SJ (editor). The Eye in Infancy. 2nd edition. St Louis: Mosby, 1994.

\section{Isenberg 1994b}

Isenberg SJ, Apt L, Yoshimori R, Leake R, Rich R. Povidoneiodine for ophthalmia neonatorum prophylaxis. American Journal of Ophthalmology 1994;118(6):701-6.

\section{Ison 1998}

Ison A, Dillon JR, Tapsall JW. The epidemiology of global antibiotic resistance among Neisseria gonorrhoeae and Haemophilus ducreyi. Lancet 1998;351(Suppl):SM8-11.

\section{Jug Došler 2015}

Jug Došler A, Petročnik P, Mivšek AP, Zakšek T, Skubic M. Neonatal prophylaxis: prevention of Vitamin K deficiency haemorrhage and neonatal ophthalmia. Zdravstveno Varstvo 2015;54(3):184-93.

\section{Kawashima 2009}

Kawashima M, Kawakita T, Den S, Tomita M, Shimazaki J. Surgical management of corneal perforation secondary to gonococcal keratoconjunctivitis. Eye 2009;23(2):339-44.

\section{Kello 2003}

Kello AB, Gilbert CE. Causes of severe visual impairment and blindness in schools for the blind in Ethiopia. British Journal of Ophthalmology 2003;87(5):526-30.

\section{Koay 2015}

Koay CL, Patel DK, Tajunisah I, Subrayan V, Lansingh VC. A comparative analysis of avoidable causes of childhood blindness in Malaysia with low income, middle income and high income countries. International Ophthalmology 2015;35(2):201-7.

\section{Konigstein 1882}

Konigstein L. For the prophylaxis of blenorrhea neonatorum [Zur Prophylaxe der blenorrhea neonatorum]. Arch Kinderheik 1882;2:341-69.

\section{Kramer 2002}

Kramer A, Behrens-Baumann W (editors). Antiseptic Prophylaxis and Therapy in Ocular Infections. Vol. 33. Developments in Ophthalmology. Basel: Karger, 2000. 


\section{Krohn 1993}

Krohn MA, Hillier SL, Bell TA, Kronmal RA, Grayston JT. The bacterial etiology of conjunctivitis in early infancy. American Journal of Epidemiology 1993;138(5):326-32.

\section{Laga 1989}

Laga M, Meheus A, Plot P. Epidemiology and control of gonococcal ophthalmia neonatorum. Bulletin of the World Health Organization 1989;67(5):471-7.

\section{Langendam 2013}

Langendam MW, Akl EA, Dahm P, Glasziou P, Guyatt G, Schunemann HJ. Assessing and presenting summaries of evidence in Cochrane Reviews. Systematic Reviews 2013;2:81. [DOI: 10.1186/2046-4053-2-81]

\section{Lewis 2014}

Lewis DA. Global resistance of Neisseria gonorrhoeae: when theory becomes reality. Current Opinion in Infectious Disease 2014;27(1):62-7.

\section{Luna 2009}

Luna MS, Alonso CR, Mussons FB, Urcelay IE, Conde JR, Narbona E, et al. Recommendations for the care of the healthy normal newborn at delivery and during the first postnatal hours. Anales de Pediatria 2009;71(4):349-61.

\section{Martin 2015}

Martin I, Sawatzky P, Liu G, Mulvey MR. Antimicrobial resistance to Neisseria gonorrhoeae in Canada: 2009-2013. Canadian Communicable Diseases Report 2015;41(2):35-41.

\section{McElnea 2015}

McElnea E, Stapleton P, Khan S, Stokes J, Higgins G. Challenges in the management of Neisseria gonorrhoeae keratitis. International Ophthalmology 2015;35(1):135-40.

\section{Milot 2008}

Milot J. Ophthalmia neonatorum of the newborn and its treatments in Canadian medical publications: 1872-1985. Canadian Bulletin of Medical History 2008;25(2):499-514.

\section{Mohile 2002}

Mohile M, Deorari AK, Satpathy G, Sharma A, Singh M. Microbiological study of neonatal conjunctivitis with special reference to Chlamydia trachomatis. Indian Journal of Ophthalmology 2002;50(4):295-9.

\section{Moore 2015}

Moore DL, MacDonald NE, Canadian Paediatric Society, Infections Diseases and Immunization Committee. Preventing ophthalmia neonatorum. Paediatric Child Health 2015;20(2):93-6.

\section{Muhe 1986}

Muhe L, Tafari N. Is there a critical time for prophylaxis against neonatal gonococcal ophthalmia? Genitourinary Medicine 1986;62(5):356-7.

\section{Muhit 2007}

Muhit MA, Shah SP, Glibert CE. Causes of severe visual impairment and blindness in Bangladesh: a study of 1935 children. British Journal of Ophthalmology 2007;91(8):1000-4.

\section{Mulholland 2015}

Mulholland C, Gardiner J. Ophthalmia neonatorum prophylaxis. Canadian Journal of Ophthalmology 2015;50(4):328-9.

\section{Mullick 2005}

Mullick S, Watson-Jones D, Beksinska M, Mabey D. Sexually transmitted infections in pregnancy: prevalence, impact on pregnancy outcomes, and approach to treatment in developing countries. Sexually Transmitted Infections 2005;81(4):294-302.

\section{Napchan 2005}

Napchan BM, Morales RP, Carvalho ML, Cunha KV, Figueras A. From suspicion to action: the chemical conjunctivitis and silver nitrate connexion example in Brazilian hospitals. Pharmacoepidemiology and Drug Safety 2005;14(8):555-9.

\section{Newman 2015}

Newman L, Rowley J, Vander Hoorn S, Wijesooriya NS, Unemo M, Low N, et al. Global estimates of the prevalence and incidence of four curable sexually transmitted infections in 2012 based on systematic review and global reporting. PLOS ONE 2015;10(12):e0143304.

\section{Oriel 1991}

Oriel JD. Eminent venereologists 5: Carl Credé. Genitourin Medicine 1991;67(1):67-9.

\section{Pande 2006}

Pande $\mathrm{H}$. When the custom goes blind -25 years without Credé's prophylaxis [Når sedvane gjør blind - 25 år uten Credés lapisprofylakse]. Tidsskr Nor Lægeforen 2006;8(126):1087-90.

\section{Poliquin 2015}

Poliquin V, Wylie J, Cole R, Yudin MH, Van Caesseele P. Preparedness for implementing change in neonatal ocular prophylaxis policies. Journal of Obstetrics and Gynaecology Canada 2016;38(1):7-8.

\section{Quirke 2008}

Quirke M, Cullinane A. Recent trends in chlamydial and gonococcal conjunctivitis among neonates and adults in an Irish hospital. International Journal of Infectious Diseases 2008;12(4):371-3.

\section{R Core Team 2013 [Computer program]}

R Foundation for Statistical Computing R: A language and environment for statistical computing. R Core Team. Vienna, Austria: R Foundation for Statistical Computing, 2013. www.Rproject.org.

\section{Rahi 1995}

Rahi JS, Sripathi S, Gilbert CE, Foster A. Childhood blindness in India: causes in 1318 blind school students in nine states. Eye 1995;9(5):545-50. 


\section{Robaei 2014}

Robaei D, Watson S. Corneal blindness: a global problem. Clinical and Experimental Ophthalmology 2014;42(3):213-4.

\section{Rours 2008}

Rours IG, Hammerschlag MR, Ott A, De Faber TJ, Verbrugh HA, de Groot R, et al. Chlamydia trachomatis as a cause of neonatal conjunctivitis in Dutch infants. Pediatrics 2008;121(2):e321-6.

\section{Sandström 1984}

Sandström KI, Bell TA, Chandler JW, Kuo CC, Wang SP, Grayston JT, et al. Microbial causes of neonatal conjunctivitis. Journal of Pediatrics 1984;105(5):706-11.

\section{Sandström 1987}

Sandström I. Etiology and diagnosis of neonatal conjunctivitis. Acta Paediatrica Scandinavica 1967;76(2):221-7.

\section{Schwab 1985}

Schwab L, Tizazu T. Destructive epidemic of Neisseria gonorrhea in African adults. British Journal of Ophthalmology 1985;69(7):525-8.

\section{Shaw 1977}

Shaw EB. Questions need for prophylaxis with silver nitrate. Pediatrics 1977;59(5):792.

\section{Sweeting 2004}

Sweeting MJ, Sutton AJ, Lambert PC. What to add to nothing? Use and avoidance of continuity corrections in meta-analysis of sparse data. Statistics in Medicine 2004;23(9):1351-75.

\section{Totten 2015a}

Totten S, MacLean R, Payne E, Severini A. Chlamydia and lymphogranuloma venereum in Canada: 2003-2012 Summary Report. Canadian Communicable Diseases Report 2015;41(2):20-25.

\section{Totten 2015b}

Totten S, MacLean R, Payne E. Gonorrhea in Canada: 2003-2012. Canadian Communicable Diseases Report 2015;41(2):26-29.

\section{Tribolet 2016}

Tribolet S, Gillard P, Lefèvre A, Rigo V. Gonococcal ophthalmia neonatorum: clinical illustration, prevention, and future prospects. Archives of Pediatrics 2016;23(3):297-300.

\section{Unemo 2016}

Unemo M, Del Rio C, Shafer WM. Antimicrobial resistance expressed by Neisseria gonorrhoeae: a major global public health problem in the 21st century. Microbiology Spectrum 2016;4(3):10.1128/microbiolspec. El10-0009-2015.

\section{UNICEF}

UNICEF. Antenatal care. data.unicef.org/maternal-health/ antenatal-care.html (accessed 13 December 2019).

\section{USPSTF 2011}

US Preventive Services Task Force (USPSTF). Ocular prophylaxis for gonococcal ophthalmia neonatorum: U.S. Preventive
Services Task Force reaffirmation recommendation statement. Agency for Healthcare Research and Quality 2011.

\section{USPSTF 2019}

US Preventive Services Task Force. Ocular Prophylaxis for Gonococcal Ophthalmia Neonatorum: US Preventive Services Task Force Reaffirmation Recommendation Statement. JAMA 2019;321(4):394-8

\section{Van de Laar 2012}

Van de Laar M, Spiteri G. Increasing trends of gonorrhoea and syphilis and the threat of drug-resistant gonorrhoea in Europe. European Surveillance 2012;17(29):202-25.

\section{Vedantham 2004}

Vedantham V. Prophylaxis of ophthalmia neonatorum. British Journal of Ophthalmology 2004;88(10):1352.

\section{Verma 1994}

Verma M, Chhatwal J, Varughese PV. Neonatal conjunctivitis: a profile. Indian Pediatrics 1994;31(11):1357-61.

\section{Volksgezondheid 1980}

Volksgezondheid (Leidschendam) Geneeskundige Hoofdinspectie van de. Methode van Crede, ja of nee?: staatstoezicht op de volksgezondheid. Geneeskundigen Hoofdinspectie van de Volksgezondheid, 1980.

\section{Wan 1986}

Wan WL, Farkas GC, May WN. The clinical characteristics and course of adult gonococcal conjunctivitis. American Journal of Ophthalmology 1986;102(5):575-83.

\section{WESP2016}

United Nations. World economic situation and prospects. www.un.org/en/development/desa/policy/wesp/ wesp_current/2016wesp_full_en.pdf (accessed 10 April 2019).

\section{Whitcher 2001}

Whitcher JP, Srinivasan M, Upadhyay MP. Corneal blindness: a global perspective. Bulletin of the World Health Organization 2001;79(3):214-21.

\section{WHO 1986}

World Health Organization. Conjunctivitis of the newborn: prevention and treatment at the primary health care level. apps.who.int/iris/ bitstream/10665/39482/1/9241560886_eng.pdf (accessed 23 March 2016).

\section{WHO 1999}

World Health Organization. What is VISION 2020? www.who.int/ blindness/partnerships/vision2020/en/ (accessed 24 March 2016).

\section{WHO 2012}

World Health Organization. Baseline report on global sexually transmitted infection surveillance. apps.who.int/iris/ bitstream/10665/85376/1/9789241505895_eng.pdf (accessed 24 March 2016). 


\section{WHO 2013}

World Health Organization. Universal eye health: a global action plan 2014-2019. www.who.int/blindness/actionplan/en/ (accessed 23 March 2016).

\section{WHO 2015}

WHO, UN Population Fund, UNICEF. Integrated Management of Pregnancy and Childbirth. Pregnancy, Childbirth, Postpartum and Newborn Care: a Guide for Essential Practice. 3rd edition. Geneva: WHO, 2015.

\section{WHO 2017}

World Health Organization. WHO recommendations on newborn health: guidelines approved by the WHO Guidelines Review Committee. Vol. WHO/MCA/17.07. Geneva: World Health Organization, 2017. [CC BY-NC-SA 3.0 IGO]

\section{Yip 2008}

Yip PP, Chan WH, Yip KT, Que TL, Kwong NS, Ho CK. The use of polymerase chain reaction assay versus conventional methods in detecting neonatal chlamydial conjunctivitis. Journal of Pedatric Ophthalmology and Strabismus 2008;45(4):234-9.

\section{Zloto 2016}

Zloto O, Gharaibeh A, Mezer E, Stankovic B, Isenberg S, Wygnanski-Jaffe T. Ophthalmia neonatorum treatment and prophylaxis: IPOSC global study. Graefe's Archive for Clinical and Experimental Ophthalmology 2016;254(3):577-82.

\section{References to other published versions of this review \\ Kapoor 2016}

Kapoor VS, Whyte R, Vedula SS. Interventions for preventing ophthalmia neonatorum. Cochrane Database of Systematic Reviews 2016, Issue 9. Art. No: CD001862. [DOI: 10.1002/14651858.CD001862.pub3]

* Indicates the major publication for the study

\section{CHARACTERISTICS OF STUDIES}

Characteristics of included studies [ordered by study ID]

Ali 2007

\section{Study characteristics}

\section{Methods}

Parallel-group, single-centre trial.

Paper states randomised by "simple randomization technique". No further comments on method of randomisation.

Unit of randomisation: neonate.

Losses to follow-up:

1. unclear how many neonates did not self-report back to the clinic with conjunctivitis;

2. unclear how many neonates in each group were examined on a weekly basis;

3 . in the erythromycin group, $7 / 19$ with conjunctivitis (37\%) reported to the lab for cultures;

4 . in the control group, $7 / 24$ with conjunctivitis $(29 \%)$ attended the laboratory for cultures.

Exclusions after allocation: 10 newborns in povidone-iodine (Betadine) group; 7 neonates in erythromycin group; and 3 neonates in placebo group for "various reasons including infection, lack of cooperation or failure to return for follow-up". Total of 20 were excluded.

No discussion on how missing data were handled in the papers.

No reported power calculation.

Participants

Setting: Vali-e-Asr Hospital in Tehran, Iran.

Number allocated: 330.

Age: neonates.

Sex: M:F 171 (55\%):139 (45\%).

Inclusion criteria:

1. all babies born from January 2004 to August 2005 at the Vali-e-Asr Hospital;

2. neonates without congenital eye abnormalities; 
Ali 2007 (Continued)

3. neonates of mothers who had not used any form of antibiotics within the last 48 hours;

4. absence of rupture of membranes for more than $18 \mathrm{~h}$;

5. absence of meconium aspiration.

Exclusion criteria: none specified.

No comment on equivalence of baseline criteria.

- Intervention 1: 2.5\% sterile povidone-iodine (Betadine) ophthalmic drops (number of drops not specified) $(n=110)$.

- Intervention 2: 0.5\% erythromycin ophthalmic ointment (amount not specified) ( $n=110)$.

- Intervention 3: no intervention; no placebo used $(n=110)$.

Time to intervention: "During the first few hours after birth".

Pre-intervention manoeuvres: none specified.

Postintervention manoeuvres: none specified.

Outcomes

1. Infants with clinical conjunctivitis confirmed by paediatrician.

2. Infants with positive culture results of eye swabs.

3. Infants with chlamydial conjunctivitis diagnosed by PCR.

4. Infants with gonococcal conjunctivitis (none found in study).

Follow-up: within a month of birth.

Intervals at which outcomes assessed:

1. examined weekly;

2. parents advised to report to clinic if clinical signs of conjunctivitis within $24 \mathrm{~h}$ of birth to 1 month after birth.

Methods indicate follow-up was 1 month, but results seem to suggest follow-up of 2 weeks. A sentence in the results states: "Nine subjects from group A (18.4\%), 19 from group B and 24 (22.4\%) from group C visited the clinic due to conjunctival redness and tearing or [serious] or purulent discharge during the first $24 \mathrm{~h}$ through 2 weeks of birth...".

Notes on definition of conjunctivitis:

Redness and hyperaemia or purulent ocular discharge.

Amongst neonates returning for assessment, unclear how many actually had red eyes and hyperaemia and how many had purulent ocular discharge.

No adverse events reported.

Notes

Date study conducted: June 2004 to August 2005.

Source of funding not specified.

No declaration of interest found in paper.

No reported subgroup analysis.

Trial investigators were not contacted.

\section{Risk of bias}


Ali 2007 (Continued)

Random sequence genera- Unclear risk tion (selection bias)
Paper states randomised by "simple randomization technique". No further details on methods of randomisation. Concerns about randomisation, as exactly 110 in each of the 3 groups after allocation.

Insufficient information about the sequence generation process to permit judgement of 'Yes' or 'No'

Allocation concealment $\quad$ Unclear risk Insufficient information to permit judgement of risk of bias
(selection bias)

(selection bias)

Blinding of study partici- Unclear risk pants (mothers of infants) Clinical conjunctivitis (subjective)
The 2 interventions differ in colour and consistency. Povidone-iodine is a red solution that leads to transient residual staining of the eye that the mother would notice. Erythromycin is a translucent ointment that the mother would initially notice in the infant. There was no placebo in the allocation group that received no prophylaxis. The mothers of neonates with noticeable medication may handle the eyes of the infant more than mothers of neonates with no prophylaxis. This could lead to differential introduction of pathogenic bacteria into the eyes of these neonates. Masking of the mother was not addressed in this paper.
Blinding of study participants (mothers of infants) Bacterial, gonococcal and chlamydial conjunctivitis (objective)
Unclear risk

The study does not comment on whether the mothers were masked as to the intervention dispensed in the neonate's eyes. As mentioned, the mother can identify what was dispensed in the infant's eyes, as povidone-iodine causes lid staining and erythromycin is an ointment that is noticeable. The mothers of neonates with noticeable medication may handle the eyes of the infant more than mothers of neonates with no prophylaxis. This could lead to differential introduction of pathogenic bacteria into the eyes of these neonates. Lack of masking of medication appearance may therefore lead to bias in bacterial conjunctivitis cases, but unlikely in chlamydia cases.

\author{
Blinding of caregiver who \\ administered medication \\ Clinical conjunctivitis \\ (subjective)
}

High risk

The 2 interventions differ in colour and consistency. Povidone-iodine is a red solution that leads to transient residual staining of the eye. Erythromycin is a translucent ointment that the mother would initially notice in the infant. No placebo was used in the allocation group that received no prophylaxis. There appears to have been no attempt to mask the appearance of the medication. The study makes no comment as to whether the person administering the medication was masked.
Blinding of caregiver who administered medication Bacterial, gonococcal and chlamydial conjunctivitis (objective)
High risk

No placebo was used in the no-prophylaxis group, therefore the neonates in the povidone-iodine group and erythromycin group had handling of the eyes by the person who administers medication, but not in the no-prophylaxis group. Handling of the eyes by the person who administers medication could introduce pathogenic medication into the eyes of the neonates and affect bacterial conjunctivitis cases. It is uncertain if this could affect chlamydial conjunctivitis cases, as it was unclear if the person who administered the medication could also have been involved in the birth process, and if there were measures to protect hand hygiene.

\author{
Blinding of persons in- \\ volved in postnatal care \\ Clinical conjunctivitis \\ (subjective)
}

Unclear risk Since povidone-iodine causes staining of the eye, and erythromycin ointment may leave temporary residual medication, those involved in postnatal care were likely not initially masked to the medication used as prophylaxis. Furthermore, there is no indication in the paper that an attempt was made to mask those involved in postnatal care. Masking not addressed in the paper.
Blinding of persons in-
Unclear risk
The 2 interventions differ in colour and consistency. Povidone-iodine is a red volved in postnatal care solution that leads to transient residual staining of the eye. Erythromycin is a Bacterial, gonococcal and chlamydial conjunctivitis translucent ointment that the mother would initially notice in the infant. No placebo was used in the allocation group that received no prophylaxis. There (objective) appears to have been no attempt to mask the appearance of the medication. 
The study makes no comment as to whether the person administering the medication was masked.

Blinding of outcome as- Unclear risk sessment (detection bias)

Clinical conjunctivitis

(subjective)
COMMENT: The study does not mention masking of outcome assessors. In the first hours of clinical assessment for conjunctivitis, there may be lack of masking as erythromycin is an ointment that leaves residue, and povidone-iodine is orange-red and leads to eye staining. It is unclear how many cases of conjunctivitis were diagnosed during this time period. In

ambiguous cases of clinical conjunctivitis, whether identified by personnel, mother, or outcome assessor, there may be differential group behaviour to include or exclude cases of clinical conjunctivitis. In this paper, conjunctivitis was defined as redness and hyperaemia OR the presence of purulent ocular discharge, which leaves open further subjectivity in the diagnosis of conjunctivitis.

\section{Blinding of outcome as- Unclear risk} sessment (detection bias) Bacterial, gonococcal and chlamydial conjunctivitis (objective)
COMMENT: The study does not mention masking of outcome assessors. Any bias in the clinical diagnosis of conjunctivitis will impact the rates of bacterial and chlamydial conjunctivitis if one knows which prophylaxis has been given to a neonate.

In ambiguous cases of clinical conjunctivitis, whether identified by personnel, mother, or outcome assessor, there may be differential assessor behaviour to include or exclude cases of clinical conjunctivitis, thereby increasing or decreasing likelihood of swabbing the neonate's eye for bacterial or chlamydial conjunctivitis. Presence of bacteria or chlamydia on a swab does not necessarily prove that the bacteria caused the conjunctivitis, as the bacteria could be part of the normal flora of the eye, or the chlamydia could be a carrier.

The effect of lack of masking on the outcome of bacterial conjunctivitis, and, in particular, chlamydial conjunctivitis, is less as compared to the effect on clinical conjunctivitis. PCR was used to diagnosis of chlamydia, which is objective, but can also be sensitive enough to detect a possible carrier.

However, event rates for chlamydial conjunctivitis were very low in the paper, therefore the impact of any bias could be large.
Incomplete outcome data High risk

(attrition bias)

Clinical conjunctivitis

(subjective)
QUOTE: "Ten newborns from group A, seven from group B, and three from group $C$, were excluded from the study for various reasons including infection, lack of cooperation, and failure to return for follow-up."

COMMENT: Differential exclusion, reasons not all clearly outlined. High rates of exclusion in relation to event rate.

QUOTE: "Only 23 (44\%) of the neonates of with conjunctivitis who were referred to the laboratory actually did so. However, all 9 subjects in group A, attended the laboratory. .... In group $B$, among the 19 cases with clinical conjunctivitis, only seven (37\%) attended the laboratory,... In group C, only seven $(29 \%)$ newborns with conjunctivitis attended the laboratory,..."

COMMENT: Loss to follow-up was very high in relation to event rate and was uneven amongst groups.

QUOTE: "The neonates were then examined on a weekly basis and the parents were advised to bring the child to the hospital clinic in case such findings were observed within 24 h to one month after birth."

"Nine subjects from group A, 19 from group B (18.4\%) , and 24 (22.4\%) from group $C$ visited the clinic due to conjunctival redness and tearing and or serious purulent discharge during the first 24 h through 2 weeks of birth..." 
COMMENT: The methods indicate that follow-up was 1 month, but the results indicate that follow-up was 2 weeks. We also have no data on the distribution of neonates with purulent discharge.

COMMENT: 1 or more outcomes of interest in the review are reported incompletely so that they cannot be entered in a meta-analysis.

\begin{tabular}{lll}
\hline Other bias $\quad$ Unclear risk Insufficient information to assess whether an important risk of bias exists \\
\hline
\end{tabular}

\section{Bell 1993}

\section{Study characteristics}

Methods

Parallel-group RCT.

Randomised by computer algorithm using a permuted block design with block size of 18; envelopes of consecutively ordered random assignments selected by ward clerk and assigned by primary care nurse.

Unit of randomisation: neonate.

Exclusions after allocation: none specified.

Losses to follow-up: 39 women not available for personal observation during the study.

No reported power calculation.

Recruited women from University of Washington Medical Center-associated obstetric clinics.

Number allocated: 669:

- silver nitrate: 221;

- erythromycin: 222;

- none: 226.

Age: neonates.

Sex: M:F unknown.

Inclusion criteria: women attending the University of Washington Medical Center-associated obstetric clinics.

Exclusion criteria: women with the following characteristics:

1. younger than 16 years old;

2. with gonorrhoea in the current pregnancy;

3. who lived more than $50 \mathrm{~km}$ from the medical centre or had plans to move within 2 months of delivery;

4. with significant social problems;

5. who planned to be in hospital less than $48 \mathrm{~h}$ after delivery;

6. who understood English poorly;

7. who lacked telephone access;

8. with positive Neisseria gonorrhoeae culture within $48 \mathrm{~h}$ of delivery;

9. with antimicrobials within $48 \mathrm{~h}$ of delivery.

Equivalence of baseline characteristics: yes.

Ethnic minorities included but specific ethnicity not reported. Data provided by ethnic group:

- silver nitrate group: $25 \%$ ethnic minorities; 
Bell 1993 (Continued)

- erythromycin group: $15 \%$ ethnic minorities;

- no-prophylaxis group: $19 \%$ ethnic minorities.

Interventions

Number of interventions: 3 .

- Intervention 1: erythromycin $0.5 \%$ ointment applied to eyes 1 time allocated to 222 neonates (amount not specified).

- Intervention 2: silver nitrate $1 \%$ solution applied to eyes 1 time allocated to 221 neonates (number of drops not specified).

- Intervention 3: no prophylaxis (no placebo or sham was used) allocated to 226 neonates.

Time to intervention: not specified.

Pre-intervention manoeuvres: none specified.

Postintervention manoeuvres: none specified.

\section{Outcomes}

1. Infants with conjunctivitis.

2. Infants with nasolacrimal duct impatency.

3. Infants with epiphora.

Follow-up: up to 60 days.

Enrolled women were telephoned by study staff at day 4 or 5 , day 8 or 9 , day 30, and day 60 .

Examined at age 30 to $48 \mathrm{~h}$, and day 13 to 15 and any time parent requested exam for conjunctivitis.

Definition of conjunctivitis: determined by telephone interview and clinical examination. Parents were asked to telephone if they noticed discharge from the infant's eyes that was more than normal.

Clinical score from 0 to 3 given for each of the following 4 signs (but not reported):

1. lid oedema and erythema;

2. purulent discharge;

3. conjunctival hyperaemia;

4. bleeding.

Tear fluid samples, conjunctival smears, and specimens for bacterial isolation were obtained.

Conjunctivitis was defined as:

- the presence of at least 3 leukocytes on a Gram-stained smear;

- staining for Chlamydia trachomatis on a smear for direct fluorescent antibody staining; or

- the presence of leukocyte esterase in a specimen of tears.

Adverse events of epiphora and nasolacrimal duct patency reported.

Notes on definition of conjunctivitis: The clinical definition of conjunctivitis was not specified. Furthermore, it was not specified if all the neonates were examined, smeared, and tear sampled, or only those with epiphora and/or conjunctivitis. Finally, if only those neonates with a specific eye sign score were smeared or only those neonates with epiphora were tear sampled, this was not specified.

No declaration of interest specified.

Trial investigators were contacted and reply received.

Subgroup analysis: Cox survival analysis by follow-up times:

1. 0 to 60 days; 
Bell 1993 (Continued)
2. 0 to 14 days;
3. 15 to 60 days.

\section{Risk of bias}

\begin{tabular}{lll}
\hline Bias & Authors' judgement & Support for judgement \\
\hline $\begin{array}{ll}\text { Random sequence genera- } \\
\text { tion (selection bias) }\end{array}$ & Low risk & $\begin{array}{l}\text { QUOTE: "At delivery, neonates were randomised with equal frequency to sil- } \\
\text { ver nitrate, erythromycin, or no ocular prophylaxis." }\end{array}$ \\
& $\begin{array}{l}\text { QUOTE: "The random allocations were assigned by computer algorithm using } \\
\text { a permuted block design with a block size of 18." }\end{array}$
\end{tabular}

\begin{tabular}{ll}
\hline $\begin{array}{l}\text { Allocation concealment } \\
\text { (selection bias) }\end{array}$ & Low risk \\
& $\begin{array}{l}\text { QUOTE: "When a woman was admitted to the labor and delivery suite, the ran- } \\
\text { dom assignment was determined by a ward clerk who took an envelope from } \\
\text { ried out by the primary care nurse." }\end{array}$ \\
\hline
\end{tabular}

\section{Blinding of study partici- Unclear risk} pants (mothers of infants) Clinical conjunctivitis (subjective)
COMMENT: The study does not mention whether mothers were masked to the intervention. No placebo was used in the allocation group that received no prophylaxis. The other 2 interventions differ in colour and consistency. Silver nitrate is a clear solution, and erythromycin is a translucent ointment. Furthermore, silver nitrate sometimes causes eyelid stain that can last for 30 to 48 hours and a chemical conjunctivitis that can last up to 72 hours. Erythromycin is a translucent ointment that the mother would initially notice in the infant, therefore it would be apparent which medication was being dispensed in a particular infant's eyes in the first 3 days for silver nitrate. The mothers of neonates with noticeable medication may handle the eyes of the infant more than mothers of neonates with no prophylaxis. This could lead to a differential introduction of pathogenic bacteria into the eyes of these neonates.
Blinding of study partici- Unclear risk pants (mothers of infants) Bacterial, gonococcal and chlamydial conjunctivitis (objective)
The study did not report bacterial, gonococcal, or chlamydial conjunctivitis as outcomes, therefore there was no assessment of bias for this category with these outcomes.
Blinding of caregiver who administered medication Clinical conjunctivitis (subjective)

High risk

QUOTE: "The assignment was carried out by the primary care nurse."

COMMENT: No placebo was used in the allocation group that received no prophylaxis. The other 2 interventions differ in colour and consistency. Silver nitrate is a clear solution, and erythromycin is a translucent ointment. Furthermore, silver nitrate sometimes causes eyelid stain that can last for 30 to 48 hours and a chemical conjunctivitis that can last up to 72 hours.

The person administering the medication would handle the eyes of neonates with erythromycin and silver nitrate, but not those of neonates with no prophylaxis as there was no placebo. This could lead to differential introduction of pathogenic bacteria into the eyes of these neonates.

Blinding of caregiver who administered medication Bacterial, gonococcal and chlamydial conjunctivitis (objective)
Unclear risk

The study did not report bacterial, gonococcal, or chlamydial conjunctivitis as outcomes, therefore there was no assessment of bias for this category with these outcomes.

\section{Blinding of persons in- Low risk} volved in postnatal care Clinical conjunctivitis (subjective)
COMMENT: Silver nitrate is a clear solution, and erythromycin is a translucent ointment. Furthermore, silver nitrate sometimes causes eyelid stain that can last for 30 to 48 hours and a chemical conjunctivitis that can last up to 72 hours. 
Bell 1993 (Continued)

QUOTE: "Prophylaxis notation in the medical record was covered to keep study and other clinical personnel masked as to the assignment." QUOTE: "I can assure you that all persons providing care were unaware of the assigned treatment" (letter from author)

QUOTE: "When asked to guess the type of prophylaxis used, the clinicians exceeded chance guessing by $21 \%$ for silver nitrate, $2 \%$ for erythromycin, and $9 \%$ for no prophylaxis."

COMMENT: This guessing was conducted $30 \mathrm{~h}$ and $48 \mathrm{~h}$ after birth. The percentage of prophylaxis correctly guessed by examiner was $54 \%, 31 \%$, and $42 \%$ for silver nitrate, erythromycin, and no prophylaxis, respectively. This was a statistically significant difference at $<0.001$.

COMMENT: Silver nitrate is a clear solution, and erythromycin is a translucent ointment. Furthermore, silver nitrate sometimes causes eyelid stain that can last for 30 to 48 hours and a chemical conjunctivitis that can last up to 72 hours.

COMMENT: Even though masking could be compromised for up to 48 hours, efforts were made to keep study and clinical personnel masked to the assignment.

\begin{tabular}{|c|c|c|}
\hline $\begin{array}{l}\text { Blinding of persons in- } \\
\text { /olved in postnatal care } \\
\text { Bacterial, gonococcal and }\end{array}$ & Unclear risk & $\begin{array}{l}\text { The study did not report bacterial, gonococcal, or chlamydial conjunctivitis } \\
\text { as outcomes, therefore there was no assessment of bias for this category with } \\
\text { these outcomes. }\end{array}$ \\
\hline
\end{tabular}

chlamydial conjunctivitis (objective)

\section{Blinding of outcome as- Low risk} sessment (detection bias) Clinical conjunctivitis (subjective)
QUOTE: "The clinical study personnel were not told of the assignment of individual subjects and were not informed of the study's findings until after the observation of subjects had ended."

COMMENT: Some outcome assessments for conjunctivitis would have likely been conducted at the $30 \mathrm{~h}$ and $48 \mathrm{~h}$ of birth, considering that the median age of infants at diagnosis were 9 to 14 days. It was established that at $30 \mathrm{~h}$ and 48 $\mathrm{h}$ of birth, it was possible to identify the type of prophylaxis an infant received (see above). However, these likely represent a small proportion of cases in relation to event rate.

\section{Blinding of outcome as- sessment (detection bias) Bacterial, gonococcal and chlamydial conjunctivitis (objective)}

Unclear risk

The study did not report bacterial, gonococcal, or chlamydial conjunctivitis as outcomes, therefore there was no assessment of bias for this category with these outcomes.

\section{Incomplete outcome data High risk} (attrition bias)

Clinical conjunctivitis

(subjective)
QUOTE: "Among 669 randomised women, 39 were not available for personal observation during the study period. These 39 were almost equally distributed among the three prophylaxis groups: 16(7\%) among 221 randomised to silver nitrate, 12 (5\%) among 222 randomised to erythromycin, and 11 (5\%) among 226 randomised to no prophylaxis."

COMMENT: Despite incomplete outcome data being approximately equally distributed across intervention groups, the reasons for why these infants were not available for outcome assessment were not provided by the study authors. Furthermore, these are high losses in follow-up in relation to event rates. QUOTE: "Among the 521 infants who did not develop conjunctivitis, 10 received care from another physician for signs of conjunctivitis without the parents' first notifying study personnel. These infants are included with those without conjunctivitis because study personnel could not verify the case by using study criteria or obtain laboratory specimens from the infant." QUOTE: "Inclusion of the 10 suspected cases on conjunctivitis who were known to study personnel only by parental report did not change the hazard ratios for silver nitrate and erythromycin prophylaxis (data not shown)." 

also unaware of the distribution amongst the intervention groups of these 10 infants.

\begin{tabular}{|c|c|c|}
\hline $\begin{array}{l}\text { Incomplete outcome data } \\
\text { (attrition bias) } \\
\text { Bacterial, gonococcal and }\end{array}$ & Unclear risk & $\begin{array}{l}\text { The study did not report bacterial, gonococcal, or chlamydial conjunctivitis } \\
\text { as outcomes, therefore there was no assessment of bias for this category with } \\
\text { these outcomes. }\end{array}$ \\
\hline
\end{tabular}
chlamydial conjunctivitis (objective)

Selective reporting (re- High risk porting bias)
QUOTE: "An examination for suspected conjunctivitis began by observing the severity of lid edema, erythema, conjunctival hyperemia, purulent discharge, and bleeding when the mucosa was swabbed. Each sign was graded 0 to 3 according to predetermined criteria; the scores were summed for each eye, with a maximum of 12 possible."

COMMENT: The clinical scores for suspected conjunctivitis were not reported in the paper. What clinical score defined conjunctivitis is also not reported.

QUOTE: "Silver nitrate eye prophylaxis caused no sustained deleterious effects and even some benefit to infants born to women without Neisseria gonorrhoeae. However, the effect was modest and against microorganisms of low virulence."

QUOTE: “...the microorganisms found in association with the conjunctivitis were for the most part common flora of the upper respiratory tract and uncommon vaginal flora." (unpublished observation)

COMMENT: The authors apparently collected data on the micro-organisms that infected the infants' eyes, but did not mention this in the methods, or report any of the results of these data.

QUOTE: "The primary outcome measured was conjunctivitis. This was determined by examination of the infant and cytologic or biochemical confirmation. Conjunctivitis was defined as the presence of at least three leukocytes on a Gram-stained smear or on a smear made for direct fluorescent antibody staining for $\mathrm{C}$ trachomatis or by detection of any amount of leukocyte esterase in a specimen of tears..."

COMMENT: The authors did not report the number of infants, if any, that were positive for direct fluorescent antibody staining for $C$ trachomatis.

QUOTE: "Among the 758 women enrolled, 89 were not randomised." COMMENT: It is unclear why the 89 women who were enrolled after the 28th week of pregnancy were then subsequently not randomised.

QUOTE: "The 29 infants who were treated with antimicrobials for medical conditions other than conjunctivitis before age 60 days were not censored from the study."

COMMENT: There is postrandomisation administration of an intervention that could enhance the effect of the prophylactic regimen. The distribution of these infants across the intervention groups is also unclear.

\section{Study characteristics}


Bramantyo 2016 (Continued)

Method of allocation: "randomly"; no other information provided.

Unit of randomisation: neonate.

Exclusions after allocation: none specified.

Losses to follow-up: none specified.

Number randomised: 60 .

No reported power calculation.

Unusual study design: main outcome was bacterial colonies.

\section{Participants}

Country: Jakarta, Indonesia.

Setting: Cipto Mangunkusumo Hospital, Jakarta.

Ethnic group: not specified.

Total number of participants: 60 .

Sex: M:F 29 (48\%):31 (52\%).

Average age range: not specified.

Inclusion criteria:

1. normal babies born by vaginal delivery at full-term birth.

Exclusion criteria:

1. babies with congenital eye malformation;

2. babies whose parents refused to take part in the study;

3. equivalence of baseline characteristics: yes.

Interventions

Number of interventions: 2 .

- Intervention: povidone-iodine $2.5 \%$ ophthalmic solution applied to eye conjunctiva ( $n=30$ ).

- Comparator: chloramphenicol $1 \%$ eye ointment applied to eye conjunctiva $(n=30)$.

Time to intervention: 1 hour after birth.

Pre-intervention manoeuvres: conjunctival swabs were taken from the inferior fornix of the right eyes before application of prophylaxis.

Postintervention manoeuvres: conjunctival swabs were retaken 2 hours after the prophylactic agent had been applied.

1. Reduction in bacterial colony-forming units before and after prophylaxis.

2. Comparison of reduction of bacterial colony-forming units between 2 prophylactic agents.

In the results section, but not in the methods, the following outcomes were specified:

1. "...no newborns experienced toxic conjunctivitis or corneal opacities caused by the toxic effects of the $2.5 \%$ povidone-iodine ophthalmic solution or the $1 \%$ chloramphenicol eye ointment."

2. "...no clinical evidence of conjunctivitis was found."

Adverse events reported: yes; "...no newborns experienced toxic conjunctivitis or corneal opacities caused by the toxic effects of the $2.5 \%$ povidone-iodine ophthalmic solution or the $1 \%$ chloramphenicol eye ointment." 
Follow-up time: not specified. At least up to $24 \mathrm{~h}$ : "In our study, there was no toxic conjunctivitis reaction or corneal opacities found $24 \mathrm{~h}$ after the administration of the prophylactic $1 \%$ chloramphenicol eye ointment or the $2.5 \%$ povidone-iodine ophthalmic solution."

Notes

Date conducted: October to December 2012.

Sources of funding: not specified.

Declaration of interest: "The authors have no funding or conflicts of interest to declare."

No reported subgroup analysis.

Trial investigators were contacted but no response was received.

\section{Risk of bias}

\begin{tabular}{|c|c|c|}
\hline Bias & Authors' judgement & Support for judgement \\
\hline $\begin{array}{l}\text { Random sequence genera- } \\
\text { tion (selection bias) }\end{array}$ & Unclear risk & QUOTE: "A total of 60 samples were divided randomly into 2 groups." \\
\hline $\begin{array}{l}\text { Allocation concealment } \\
\text { (selection bias) }\end{array}$ & Unclear risk & COMMENT: Not described \\
\hline $\begin{array}{l}\text { Blinding of study partici- } \\
\text { pants (mothers of infants) } \\
\text { Clinical conjunctivitis } \\
\text { (subjective) }\end{array}$ & Unclear risk & $\begin{array}{l}\text { COMMENT: The study is described as "double-blind", but masking of mothers } \\
\text { of the intervention was not specifically addressed. Furthermore, the } 2 \text { inter- } \\
\text { ventions differ in colour and consistency. Povidone-iodine is an orange-red so- } \\
\text { lution that can lead to periocular stains that last minutes to hours. Chloram- } \\
\text { phenicol is an ointment that can leave a residue in the eyes that could be no- } \\
\text { ticed for hours. }\end{array}$ \\
\hline
\end{tabular}

Blinding of study participants (mothers of infants) Bacterial, gonococcal and chlamydial conjunctivitis (objective)
Unclear risk

The study did not report bacterial, gonococcal, or chlamydial conjunctivitis as outcomes, therefore there was no assessment of bias for this category with these outcomes.
Blinding of caregiver who administered medication Clinical conjunctivitis (subjective)
High risk

COMMENT: The study is described as "double-blind", but masking of the person administering the medication was not addressed. The 2 interventions differ in colour and consistency. Povidone-iodine is an orange-red solution that can lead to periocular stains that last minutes to hours. Chloramphenicol is an ointment that may leave a residue in the eyes that can be noticed for hours. The 2 interventions are therefore readily identifiable. Any bias on the part of the person administering the medication could affect adherence or compliance with the application method of the medication, which, in turn, could affect the medication's prophylactic effect.

\begin{tabular}{|c|c|c|}
\hline $\begin{array}{l}\text { Blinding of caregiver who } \\
\text { administered medication } \\
\text { Bacterial, gonococcal and } \\
\text { chlamydial conjunctivitis } \\
\text { (objective) }\end{array}$ & Unclear risk & $\begin{array}{l}\text { The study did not report bacterial, gonococcal, or chlamydial conjunctivitis } \\
\text { as outcomes, therefore there was no assessment of bias for this category with } \\
\text { these outcomes. }\end{array}$ \\
\hline $\begin{array}{l}\text { Blinding of persons in- } \\
\text { volved in postnatal care } \\
\text { Clinical conjunctivitis } \\
\text { (subjective) }\end{array}$ & Unclear risk & $\begin{array}{l}\text { COMMENT: The study is described as "double-blind", but masking of people in- } \\
\text { volved in postnatal care was not addressed. The } 2 \text { interventions differ in colour } \\
\text { and consistency. Povidone-iodine is an orange-red solution that can lead to } \\
\text { periocular stains that last minutes to hours. Chloramphenicol is an ointment } \\
\text { that may leave a residue in the eyes that can be noticed for hours. }\end{array}$ \\
\hline
\end{tabular}


It is uncertain in this study if there were cases of conjunctivitis identified in the time period when masking would be affected. However, the follow-up time could be as short as 24 hours. It is uncertain if people involved in postnatal care were also involved in identification of cases of conjunctivitis. If they were, and were unmasked, this could influence decisions to identify clinical conjunctivitis cases.

\begin{tabular}{|c|c|c|}
\hline $\begin{array}{l}\text { Blinding of persons in- } \\
\text { volved in postnatal care } \\
\text { Bacterial, gonococcal and }\end{array}$ & Unclear risk & $\begin{array}{l}\text { The study did not report bacterial, gonococcal, or chlamydial conjunctivitis } \\
\text { as outcomes, therefore there was no assessment of bias for this category with } \\
\text { these outcomes. }\end{array}$ \\
\hline
\end{tabular}
these outcomes.

(objective)

\begin{tabular}{|c|c|c|}
\hline $\begin{array}{l}\text { Blinding of outcome as- } \\
\text { sessment (detection bias) } \\
\text { Clinical conjunctivitis } \\
\text { (subjective) }\end{array}$ & Unclear risk & $\begin{array}{l}\text { COMMENT: The study is described as "double-mask", but masking of the per- } \\
\text { son who was involved in outcome assessment of conjunctivitis was not specif- } \\
\text { ically addressed in this paper. The } 2 \text { interventions differ in colour and consis- } \\
\text { tency. Povidone-iodine is an orange-red solution that can lead to periocular } \\
\text { stains that last minutes to hours. Chloramphenicol is an ointment that may } \\
\text { leave a residue in the eyes that can be noticed for hours. }\end{array}$ \\
\hline
\end{tabular}

Conjunctivitis cases appear to be diagnosed in the first $24 \mathrm{~h}$, therefore masking could have been affected and influenced the outcome.

\begin{tabular}{|c|c|c|}
\hline $\begin{array}{l}\text { Blinding of outcome as- } \\
\text { sessment (detection bias) } \\
\text { Bacterial, gonococcal and } \\
\text { chlamydial conjunctivitis } \\
\text { (objective) }\end{array}$ & Unclear risk & $\begin{array}{l}\text { The study did not report bacterial, gonococcal, or chlamydial conjunctivitis } \\
\text { as outcomes, therefore there was no assessment of bias for this category with } \\
\text { these outcomes. }\end{array}$ \\
\hline
\end{tabular}

\begin{tabular}{ll}
\hline $\begin{array}{l}\text { Incomplete outcome data } \\
\text { (attrition bias) }\end{array}$ & Low risk \\
$\begin{array}{l}\text { Clinical conjunctivitis } \\
\text { (subjective) }\end{array}$ & $\begin{array}{l}\text { low-up time appears to be 24 h. Any losses to follow-up were therefore likely to } \\
\text { be small. }\end{array}$
\end{tabular}

\begin{tabular}{ll}
\hline $\begin{array}{l}\text { Incomplete outcome data } \\
\text { (attrition bias) }\end{array}$ & $\begin{array}{l}\text { The study did not report bacterial, gonococcal, or chlamydial conjunctivitis } \\
\text { as outcomes, therefore there was no assessment of bias for this category with } \\
\text { Bacterial, gonococcal and }\end{array}$ \\
$\begin{array}{l}\text { chlamydial conjunctivitis } \\
\text { (objective) }\end{array}$ &
\end{tabular}

\begin{tabular}{lll}
\hline $\begin{array}{l}\text { Selective reporting (re- } \\
\text { porting bias) }\end{array}$ & High risk & $\begin{array}{l}\text { COMMENT: A primary outcome of conjunctivitis was not prespecified, and is } \\
\text { reported incompletely. }\end{array}$ \\
\hline Other bias & Unclear risk & Insufficient information to assess whether an important risk of bias exists \\
\hline
\end{tabular}

Brussieux 1991

\title{
Study characteristics
}

Methods

\author{
Parallel-group, single-centre trial. \\ All infants born in a particular day were randomised to the same prophylaxis using a serially numbered \\ envelope (daily randomisation as opposed to infant randomisation). \\ Unit of randomisation: neonate. \\ Exclusions after randomisation: no comment.
}


Brussieux 1991 (Continued)
Losses to follow-up:
1. tetracycline group: $55 \%$;
2. silver nitrate: $54 \%$.
No comment on how missing data were handled.
No reported power calculation.
Unusual study design: none.

\section{Participants}

Setting: Saint-Germain-en-Laye, France

Number allocated: 900 neonates:

- oxytetracycline: 475 ;

- silver nitrate: 425 .

Age: neonates.

Sex: M:F 488 (54\%): 412 (46\%).

Inclusion criteria:

- infants born at the maternity ward of Saint-Germain Hospital from February to September 1989.

Exclusion criteria:

- newborns with "disease" or "pathology" were excluded from the study; unclear if this was specified a priori or after allocation.

No comment on equivalence of baseline criteria.

- Intervention 1: 1\% aqueous oxytetracycline hydrochlorate ophthalmic drops (number of drops not specified) $(n=475)$.

- Intervention 2: $1 \%$ silver nitrate ophthalmic drops (number of drops not specified) $(n=425)$.

Time to intervention: not specified, according to translation.

Pre-intervention manoeuvres: not specified, according to translation.

Postintervention manoeuvres: not specified, according to translation.

Sum of the number of neonates with bacteria on Gram stain or culture from ocular specimens at day 7 with the number of neonates with bacteria on Gram stain or culture from ocular specimens on day 30.

1 case of chlamydia on bacterial culture.

2 forms were completed:

1 form was completed during the infant's stay at the maternity hospital in the first week. It is unclear if the infant was examined daily, by referral by a healthcare provider or parent, or at random during the first week.

The eye was graded as:

1. normal;

2. minimal signs - palpebral oedema or tearing;

3. pathological signs. 
Any abnormality noted by a paediatrician, either minimal or pathological, was referred to an ophthalmologist.

Only infants with pathological signs had their eyes cultured.

Another form was completed apparently by the mother of the infant at any time upon date of discharge until day 30. It is unclear who completed the form.

An attempt was made to examine the infant once at the end of the first month of age by a general practitioner or paediatrician.

It is unclear when these cultures were done in relation to the development of the pathological signs.

Follow-up: 2 examinations were performed on each child: Exam 1 any time from day 1 to day 7 while in hospital by paediatrician. Exam 2 any time from day 7 to day 30 by paediatrician or general practitioner.

Definition of conjunctivitis or ophthalmia neonatorum: not explicitly defined in paper, according to translation.

No adverse events reported.

Date of study: February to September 1989

No declaration of interest specified.

No reported subgroup analysis.

Trial investigators were contacted and reply received.

Article translated from French to English.

High loss to follow-up on day 30.

\section{Risk of bias}

\begin{tabular}{lll}
\hline Bias & Authors' judgement & $\begin{array}{l}\text { Support for judgement } \\
\begin{array}{l}\text { Random sequence genera- } \\
\text { tion (selection bias) }\end{array}\end{array}$ \\
\hline $\begin{array}{l}\text { Allocation concealment } \\
\text { (selection bias) }\end{array}$ & High risk & $\begin{array}{l}\text { QUOTE: (translated from French) "the topical medication... was randomized } \\
\text { every day at 13:00 to be given during } 24 \text { hours to all newborns" } \\
\text { COMMENT: Sequence generated by some rule based on day. }\end{array}$ \\
& $\begin{array}{l}\text { QUOTE: "A serially numbered envelope sealed was taken to determine the } \\
\text { treatment of the day which changed every day at } 1 \text { pm" (letter from author) } \\
\text { QUOTE: (translated from French) "The nurses, who knew which drop was giv- } \\
\text { en, wrote down on a notebook what medication was given on that day, and } \\
\text { the name... of the child who received them..." } \\
\text { COMMENT: It is unclear what role the nurses had in enrolling participants. Ran- } \\
\text { domisation was by day, therefore whilst there may be allocation concealment } \\
\text { by day, there was no allocation concealment by the next intervention for the } \\
\text { next neonate that same day. }\end{array}$
\end{tabular}

\begin{tabular}{|c|c|c|}
\hline $\begin{array}{l}\text { Blinding of study partici- } \\
\text { pants (mothers of infants) }\end{array}$ & Unclear risk & $\begin{array}{l}\text { QUOTE: "The parents were aware of the protocol but the treatment was } \\
\text { known only at the end of the study." (letter from author) }\end{array}$ \\
\hline $\begin{array}{l}\text { Clinical conjunctivitis } \\
\text { (subjective) }\end{array}$ & & $\begin{array}{l}\text { COMMENT: The } 2 \text { interventions were silver nitrate and tetracycline. The oxyte- } \\
\text { tracycline hydrochloride used in the study was not ointment but aqueous, } \\
\text { quite possibly making it similar in appearance to silver nitrate. However, sil- } \\
\text { ver nitrate sometimes causes a chemical conjunctivitis that can last up to } 72 \\
\text { hours, and silver nitrate causes lid stains that can last } 30 \text { to } 48 \text { hours. It is un- }\end{array}$ \\
\hline
\end{tabular}


clear if oxytetracycline chloride leads to lid stains. The mothers of neonates with noticeable medication may handle the eyes of the infant more, potentially affecting the outcome.
Blinding of study participants (mothers of infants) Bacterial, gonococcal and chlamydial conjunctivitis (objective)
Unclear risk

QUOTE: "The parents were aware of the protocol but the treatment was known only at the end of the study." (letter from author)

COMMENT: The 2 interventions were silver nitrate and tetracycline. The oxytetracycline hydrochloride used in the study was not ointment but aqueous, quite possibly making it similar in appearance to silver nitrate. However, silver nitrate sometimes causes a chemical conjunctivitis that can last up to 72 hours, and silver nitrate causes lid stains that can last 30 to 48 hours. It is unclear if oxytetracycline chloride leads to lid stains. The mothers of neonates with noticeable medication may handle the eyes of the infant more, potentially affecting the outcome of bacterial conjunctivitis, and even chlamydial conjunctivitis, depending on hygiene measures.

Lack of masking, with concomitant bias, could influence which neonates are brought forward by parents for bacteriological analysis. This is important considering chlamydial conjunctivitis exists as an asymptomatic carrier.
Blinding of caregiver who administered medication Clinical conjunctivitis (subjective)
Unclear risk

QUOTE: (translated from French) "The nurses, who knew which drop was given, wrote down on a notebook what medication was given on that day, and the name... of the child who received them..."

COMMENT: The study does not state whether the person administering the medication was masked, or if the vials holding the different medications were made to look the same or kept in their original labelled vials.

Knowledge of the medication being dispensed, along with any concomitant bias, could affect adherence and differential application of prophylaxis, which, in turn, could affect preventive effect of the development of conjunctivitis.
Blinding of caregiver who administered medication Bacterial, gonococcal and chlamydial conjunctivitis (objective)
Unclear risk

QUOTE: (translated from French) "The nurses, who knew which drop was given, wrote down on a notebook what medication was given on that day, and the name... of the child who received them..."

COMMENT: The study does not state whether the person administering the medication was masked, or if the vials holding the different medications were made to look the same or kept in their original labelled vials.

Knowledge of the medication being dispensed, along with any concomitant bias, could affect adherence and differential application of prophylaxis, which, in turn, could affect preventive effect of the development of chlamydial conjunctivitis.

$\begin{array}{ll}\begin{array}{l}\text { Blinding of persons in- } \\ \text { volved in postnatal care }\end{array} & \text { QUNClear risk: "The nurse providing care after the birth room did not know which } \\ \text { Clinical conjunctivitis } & \text { treatment had been given" (letter from author) } \\ \text { (subjective) } & \text { QUOTE: (translated from French) "The nurses, who knew which drop was giv- } \\ & \text { en, wrote down on a notebook what medication was given on that day, and } \\ \text { the name... of the child who received them..." } & \text { COMMENT: Since silver nitrate causes lid stains that can last up to } 30 \text { to } 48 \\ & \text { hours, those involved in postnatal care were likely not masked to the medica- } \\ & \text { tion used until this time period. It is unclear if the nurses who wrote down the } \\ \text { medication administered had a specific communication barrier with those in- } & \text { volved in postnatal care. }\end{array}$

Blinding of persons involved in postnatal care Bacterial, gonococcal and chlamydial conjunctivitis (objective)
Unclear risk QUOTE: "The nurse providing care after the birth room did not know which
treatment had been given" (letter from author)
QUOTE: (translated from French) "The nurses, who knew which drop was giv-
en, wrote down on a notebook what medication was given on that day, and
the name... of the child who received them..."
COMMENT: Since silver nitrate causes lid stains that can last up to 30 to 48
hours, those involved in postnatal care were likely not masked to the medica-
tion used until this time period. It is unclear how many cases of conjunctivitis
were identified for referral for bacteriological analysis during this time period. 
Lack of masking could differentially lead to referral of clinical cases with "minimal" ocular signs for bacteriological analysis, which could, in turn, falsely and differentially identify chlamydial carriers as opposed to true cases of chlamydial conjunctivitis. Considering the low event rate of chlamydial conjunctivitis, this could be important.

COMMENT: It is unclear if the nurses who wrote down the medication administered had a specific communication barrier with those involved in postnatal care.

Blinding of outcome assessment (detection bias) Clinical conjunctivitis (subjective)
Unclear risk

QUOTE: "The investigators did not have access to the notebook until the end of the study."

QUOTE: "Only the midwives knew the result and noted a number on the infant case history." (letter from author)

COMMENT: These statements appear to infer that the outcome assessors were masked, but this is not entirely clear. As silver nitrate creates lid stains that last 30 to 48 hours, outcome assessments during this time were likely not masked. It is uncertain what efforts were undertaken to overcome this. There is no formal clinical conjunctivitis definition in the paper, only grading of clinical ocular signs, which creates more subjectivity. Any lack of masking could therefore influence the outcome more so.
Blinding of outcome assessment (detection bias) Bacterial, gonococcal and chlamydial conjunctivitis (objective)
Unclear risk

QUOTE: "The investigators did not have access to the notebook until the end of the study."

QUOTE: "Only the midwives knew the result and noted a number on the infant case history." (letter from author)

COMMENT: These statements appear to infer that the outcome assessors were masked, but this is not entirely clear. As silver nitrate creates lid stains that last 30 to 48 hours, outcome assessments during this time were likely not masked. It is uncertain what efforts were undertaken to overcome this. There is no formal clinical conjunctivitis definition in the paper, only grading of clinical ocular signs, which creates more subjectivity. Any lack of masking could therefore influence the outcome more so.

Neonates' ocular signs were graded as normal, minimal, and pathological. It appears that all neonates, possibly including those with minimal ocular signs, were sent for bacteriological analysis. This widens diagnostic ambiguity, therefore any lack of masking by the outcome assessors could significantly bias those chosen for bacteriological analysis.

"Minimal" eyes signs were defined in the paper as "simple palpebral edema, more or less important, on one or both sides with no or minimal tearing".

Chlamydia found on bacteriological analysis with minimal eye signs, as defined in the paper, may not be chlamydial conjunctivitis, but could also be identification of ocular chlamydial carrier.
Incomplete outcome data High risk (attrition bias)

Clinical conjunctivitis

(subjective)
QUOTE: (translated from paper) " 1128 children were born in the nursery during the study period...All newborns that had disease were excluded from the study, even if that disease did not motivate the transfer to a neonatal department: 228 charts were excluded"

COMMENT: What type of disease motivated exclusion is not specified, and exclusions by allocation group are not provided.

QUOTE: (translated from paper) " 407 forms... among the 900 charts have been sent by the practitioners who performed the first month visit."

COMMENT: 2 examinations were performed for each neonate. The first was performed during the stay at the maternity hospital, which was about 1 week. The second exam was conducted outside the hospital by the neonate's general practitioner or paediatrician at the end of the first month of age. At day 30 , there was 55\% loss in the tetracycline group, and 54\% loss in the silver nitrate group. Although balanced, the losses to follow-up are high relative to event rate. 
Brussieux 1991 (Continued)

Incomplete outcome data (attrition bias)

Bacterial, gonococcal and chlamydial conjunctivitis (objective)
High risk

QUOTE: (translated from paper) " 1128 children were born in the nursery during the study period...All newborns that had disease were excluded from the study, even if that disease did not motivate the transfer to a neonatal department: 228 charts were excluded"

COMMENT: What type of disease motivated exclusion is not specified, and exclusions by allocation group are not provided.

QUOTE: (translated from paper) " 407 forms... among the 900 charts have been sent by the practitioners who performed the first month visit."

COMMENT: 2 examinations were performed for each neonate. The first was performed during the stay at the maternity hospital, which was about 1 week. The second exam was conducted outside the hospital by the neonate's general practitioner or paediatrician at the end of the first month of age. At day 30 , there was $55 \%$ loss in the tetracycline group, and $54 \%$ loss in the silver nitrate group. Although balanced, the losses to follow-up are very high relative to event rate for chlamydial conjunctivitis.

\section{Selective reporting (re- High risk} porting bias)
COMMENT: We cannot extract the outcome of bacterial conjunctivitis from this study, as it appears that "minimal" and "pathological" ocular signs were swabbed for bacteriological analysis. Neonates with "minimal" ocular signs is inconsistent with a definition of conjunctivitis. Swabbing eyes with minimal signs may over-represent neonates with growth of normal flora of the eye, and may not necessarily indicate that the isolated organism caused the eye signs. As a result, as an outcome of interest in the review it is reported incompletely and so cannot be entered in the meta-analysis.

COMMENT: It is also unclear if the organisms recovered represent individual neonates or the same neonate having multiple different organisms.

Other bias Unclear risk

COMMENT: In any trial with silver nitrate, there could be diagnostic bias. Silver nitrate causes a chemical conjunctivitis in the first 72 hours. As a result, more neonates in the silver nitrate allocation group could be referred for culture in the first 72 hours. Finding bacteria in the culture does not necessarily prove that the bacteria caused the conjunctivitis. The conjunctivitis could be chemical, but growing normal flora of the eye. Alternatively, the conjunctivitis could be chemical with a chlamydial carrier. Finally, the conjunctivitis could very well be caused by the bacteria or chlamydia. Consideration of incubation periods, and assessing for carriers and normal flora with asymptomatic cases, could assist with differential diagnosis.

\section{Study characteristics}

Methods

Parallel-group, single-centre trial.

Method of allocation: rotated monthly.

Unit of randomisation: neonate.

Rates of follow-up in comparison groups: not reported.

Analysis by intention-to-treat: unclear.

Exclusions after allocation: none specified.

Losses to follow-up: none specified.

Unusual study design: none identified.

No comment on how missing data handled. 
Chen 1992 (Continued)

No reported power calculation.

Participants

Setting: Taichung, Taiwan, Republic of China.

Number allocated: 4544:

- silver nitrate: 1082 ;

- tetracycline: 1156;

- erythromycin:1163;

- no prophylaxis: 1143 .

Age: neonates.

Sex: M:F unknown.

Inclusion criteria: neonates born at Chung Shan Medical and Dental College Hospital.

Exclusion criteria: none specified.

No comment on equivalence of baseline characteristics.

- Intervention 1: erythromycin $0.5 \%$ ointment, 1 dose applied to eyes (dose amount not specified) (n = 1163).

- Intervention 2: tetracycline $1 \%$ ointment, 1 dose applied to eyes (dose amount not specified) ( $\mathrm{n}=$ 1156).

- Intervention 3: silver nitrate $1 \%$ solution, 1 dose applied to eyes (dose amount not specified) ( $n=1082)$.

- Intervention 4: no prophylaxis (no sham or placebo) $(n=1143)$.

Time to intervention: immediately after birth.

Pre-intervention manoeuvres: none specified.

Postintervention manoeuvres: none specified.

Outcomes

1. Infants with any conjunctivitis.

2. Infants with bacterial conjunctivitis.

3. Infants with non-bacterial conjunctivitis.

4. Infants with chlamydial conjunctivitis.

5. Infants with non-chlamydial conjunctivitis.

Follow-up: up to 4 weeks of age.

Infants examined in nursery. Also, infants examined 1 week after discharge and at 4 weeks of age, or when infants developed conjunctivitis.

Definition of conjunctivitis: purulent conjunctival discharge and conjunctival hyperaemia for infants meeting clinical criteria: aerobic and anaerobic bacterial cultures, and monoclonal antibody stain to detect Chlamydia trachomatis were obtained.

No adverse events reported in study.

No source of funding specified.

No declaration of interest specified.

No reported subgroup analysis.

We contacted authors for clarifications on masking, but have received no reply to date. 
Chen 1992 (Continued)

Risk of bias

\begin{tabular}{|c|c|c|}
\hline Bias & Authors' judgement & Support for judgement \\
\hline $\begin{array}{l}\text { Random sequence genera- } \\
\text { tion (selection bias) }\end{array}$ & High risk & $\begin{array}{l}\text { QUOTE: "The neonatal ocular prophylactic preparations... were rotated } \\
\text { monthly." } \\
\text { COMMENT: Non-random component in the sequence generation process }\end{array}$ \\
\hline $\begin{array}{l}\text { Allocation concealment } \\
\text { (selection bias) }\end{array}$ & High risk & $\begin{array}{l}\text { QUOTE: "The neonatal ocular prophylactic preparations... were rotated } \\
\text { monthly." } \\
\text { COMMENT: Participants or investigators enrolling participants could foresee } \\
\text { assignments. }\end{array}$ \\
\hline $\begin{array}{l}\text { Blinding of study partici- } \\
\text { pants (mothers of infants) } \\
\text { Clinical conjunctivitis } \\
\text { (subjective) }\end{array}$ & Unclear risk & $\begin{array}{l}\text { COMMENT: The study did address masking by the study participants. The } 4 \text { in- } \\
\text { terventions differ in colour and consistency. Silver nitrate is a clear solution. } \\
\text { Also, silver nitrate sometimes causes a chemical conjunctivitis that can last up } \\
\text { to } 72 \text { hours, and silver nitrate causes lid stains that can last } 30 \text { to } 48 \text { hours. Ery- } \\
\text { thromycin is a translucent ointment that may leave residue that can be notice- } \\
\text { able for hours. Tetracycline is a light-yellow ointment that may be difficult to } \\
\text { distinguish from erythromycin, but that also leaves a residue in the eyes that } \\
\text { can last for hours. There was no placebo in the allocation group that received } \\
\text { no prophylaxis. The mothers of neonates with noticeable medication of the } \\
\text { eyes may handle the eyes of the infant more than mothers of neonates with no } \\
\text { prophylaxis, potentially affecting the outcome of clinical conjunctivitis. }\end{array}$ \\
\hline
\end{tabular}

Blinding of study partici- Unclear risk pants (mothers of infants) Bacterial, gonococcal and chlamydial conjunctivitis (objective)
COMMENT: The study did address masking by the study participants. The 4 interventions differ in colour and consistency. Silver nitrate is a clear solution. Also, silver nitrate sometimes causes a chemical conjunctivitis that can last up to 72 hours, and silver nitrate causes lid stains that can last 30 to 48 hours. Erythromycin is a translucent ointment that may leave residue that can be noticeable for hours. Tetracycline is a light-yellow ointment that may be difficult to distinguish from erythromycin, but that also leaves a residue in the eyes that can last for hours. There was no placebo in the allocation group that received no prophylaxis. The mothers of neonates with noticeable medication of the eyes may handle the eyes of the infant more than mothers of neonates with no prophylaxis, potentially affecting the outcome of clinical conjunctivitis through introduction of pathogenic material into the eyes.

Lack of masking may lead the mothers of neonates with noticeable medication of the eyes to handle the eyes of the infant more than mothers of neonates with no prophylaxis, potentially affecting the outcome of bacterial conjunctivitis, and even chlamydial conjunctivitis, depending on hygiene measures. Lack of masking, with concomitant bias, could influence which neonates are brought forward by parents for bacteriological analysis. This is important considering that chlamydial conjunctivitis can exist as an asymptomatic carrier, or be the cause of chlamydial conjunctivitis.

\footnotetext{
Blinding of caregiver who administered medication

High risk

COMMENT: The study did not address masking by the person who administers the medication.

Clinical conjunctivitis

(subjective)

The 4 interventions differ in colour and consistency. Silver nitrate is clear solution. Erythromycin is a translucent ointment that may leave residue that can be noticeable for hours. Tetracycline is a light-yellow ointment that may be difficult to distinguish from erythromycin. There was no placebo in the allocation group that received no prophylaxis.

Lack of masking of the person who administers the medication could influence the outcome of clinical conjunctivitis, if preferences affected adherence to application. Furthermore, there was no placebo. Therefore, in 3 arms of the study, there was handling of the eyes by the person who administers medication, and no handling of the eyes in the remaining arm. Handling of eyes for
} 
Blinding of caregiver who administered medication Bacterial, gonococcal and chlamydial conjunctivitis (objective)
High risk

COMMENT: The study did not address masking by the person who administers the medication.

The 4 interventions differ in colour and consistency. Silver nitrate is clear solution. Erythromycin is a translucent ointment that may leave residue that can be noticeable for hours. Tetracycline is a light-yellow ointment that may be difficult to distinguish from erythromycin. There was no placebo in the allocation group that received no prophylaxis.

Lack of masking of the person who administers the medication could influence the outcome of clinical conjunctivitis, if preferences affected adherence to application. Furthermore, there was no placebo. Therefore, in 3 arms of the study, there was handling of the eyes by the person who administers medication, and no handling of the eyes in the remaining arm. Handling of eyes for application of prophylaxis could differentially introduce pathogenic organisms, depending on hygiene practices. This could differentially affect the rates of conjunctivitis, and consequent pick-up of more chlamydial carriers, which are not true cases of chlamydial conjunctivitis.

\section{Blinding of persons in- Unclear risk} volved in postnatal care Clinical conjunctivitis (subjective)
COMMENT: The 4 interventions differ in colour and consistency. Silver nitrate is clear solution. Also, silver nitrate sometimes causes a chemical conjunctivitis that can last up to 72 hours, and silver nitrate causes lid stains that can last 30 to 48 hours. Erythromycin is a translucent ointment that may leave residue that can be noticeable for hours. Tetracycline is a light-yellow ointment that may be difficult to distinguish from erythromycin, but that also leaves a residue in the eyes that can last for hours. There was no placebo in the allocation group that received no prophylaxis, therefore the eyes of neonates in the no-prophylaxis arm would differ from the eyes of the neonates in the other arms. There is certainly the possibility that in the first 72 hours people involved in postnatal care could identify the medication dispensed.

\section{Blinding of persons in- \\ Unclear risk} volved in postnatal care Bacterial, gonococcal and chlamydial conjunctivitis (objective)
COMMENT: The study did not address masking of people involved postnata care. The 4 interventions differ in colour and consistency. Silver nitrate is a clear solution. Also, silver nitrate sometimes causes a chemical conjunctivitis that can last up to 72 hours, and silver nitrate causes lid stains that can last 30 to 48 hours. Erythromycin is a translucent ointment that may leave residue that can be noticeable for hours. Tetracycline is a light-yellow ointment that may be difficult to distinguish from erythromycin, but that also leaves a residue in the eyes that can last for hours. There was no placebo in the allocation group that received no prophylaxis, therefore the eyes of neonates in the no-prophylaxis arm would differ from the eyes of neonates in the other arms. There is certainly the possibility that in the first 72 hours people involved in postnatal care could identify the medication dispensed.

Lack of masking of people involved in postnatal care could lead to selection or reporting bias of cases referred for further clinical assessment, and this could vary by treatment arm, depending on the bias of the person involved in postnatal care. This would, in turn, bias rates of conjunctivitis by treatment arm.
Blinding of outcome as- Unclear risk sessment (detection bias) Clinical conjunctivitis (subjective)
QUOTE: "The infants were examined in the nursery. The parents were advised to bring their infants to the pediatric clinic of our hospital for follow-up 1 week after discharge and at 4 weeks of age or when the infants developed conjunctivitis within the first month of life."

COMMENT: The study does not mention masking of outcome assessors. The 3 interventions differ in colour or consistency, or both. No placebo was used. Silver nitrate sometimes causes a chemical conjunctivitis that can last up to 72 hours, and lid stains that can last 30 to 48 hours. Tetracycline is a yellowish ointment that may leave an ocular residue for hours. Erythromycin is a translucent ointment that may leave residue that can be noticeable for hours. It is un- 
known how many cases of conjunctivitis were identified during the time period when these stains remained.

Blinding of outcome assessment (detection bias) Bacterial, gonococcal and chlamydial conjunctivitis (objective)
Unclear risk

COMMENT: The study does not mention masking of outcome assessors. Silver nitrate creates lid stains that last 30 to 48 hours and chemical conjunctivitis that can last 72 hours, therefore outcome assessments during this time period were likely not masked. Although the mean age of onset of chlamydial conjunctivitis was 6.4 days with a range of 3 to 18 days, which is likely beyond the period of residual lid stains, the study authors do not provide the range of onset times for other forms of conjunctivitis.

\section{Incomplete outcome data Unclear risk} (attrition bias)

Clinical conjunctivitis

(subjective)
COMMENT: The study authors do not provide any information on the presence or absence of incomplete outcome data. Attritions and exclusions are not reported. Although it is possible there were no attritions or exclusions, this is unlikely. For example, the infant mortality rate for Taiwan for 2009 is estimated to be 5.35 ( 5.35 deaths/1000 live births). In this study, which was conducted from 1989 to 1991, 4544 infants were included in the study. Therefore, we would expect at least a few infants excluded by death within the observation period of 4 weeks.

\section{Incomplete outcome data Unclear risk} (attrition bias)

Bacterial, gonococcal and

chlamydial conjunctivitis

(objective)
COMMENT: The study authors do not provide any information on the presence or absence of incomplete outcome data. Attritions and exclusions are not reported. Although it is possible there were no attritions or exclusions, this is unlikely. For example, the infant mortality rate for Taiwan for 2009 is estimated to be 5.35 ( 5.35 deaths/1000 live births). In this study, which was conducted from 1989 to 1991, 4544 infants were included in the study. Therefore, we would expect at least a few infants excluded by death within the observation period of 4 weeks.

QUOTE: "Schulz observed that the apparent lack of exclusions was associated with more 'beneficial' effect sizes as well as with less likelihood of adequate allocation concealment (Schulz 1996). Hence, failure to report exclusions in trials in Schulz's study may have been a marker of poor trial conduct rather than true absence of any exclusions." (from the Cochrane Handbook)

Selective reporting (re- High risk porting bias)
COMMENT: The study authors report the microbial agents recovered from conjunctival cultures, but do not report the results by intervention group, except for chlamydia. They also create a category of "nonchlamydial conjunctivitis", which would have been better categorised as bacterial conjunctivitis and no growth on culture conjunctivitis, for inclusion in the meta-analysis. Therefore, 1 or more outcomes of interest in the review are reported incompletely so that they cannot be entered in a meta-analysis.

Other bias Unclear risk

COMMENT: In any trial with silver nitrate, there could be diagnostic bias. Silver nitrate causes a chemical conjunctivitis in the first 72 hours. As a result, in the first 72 hours, more neonates in the silver nitrate allocation group could be referred for culture in the first 72 hours. Finding bacteria in the culture does not necessarily prove that the bacteria caused the conjunctivitis. The conjunctivitis could be chemical, but growing normal flora of the eye. Alternatively, the conjunctivitis could be chemical with a chlamydial carrier. Finally, the conjunctivitis could very well be caused by the bacteria or chlamydia. Consideration of incubation periods, and assessing for carriers and normal flora with asymptomatic cases, could assist with differential diagnosis.

\section{Study characteristics}


Christian 1960 (Continued)

Methods
Parallel-group, single-centre trial.

Allocation was by alternate infants.

Unit of randomisation: neonate.

Rates of follow-up in comparison groups: unclear.

Exclusions after allocation: none specified or addressed in paper.

Losses to follow-up: : none specified or addressed in paper.

Unusual study design: Difference in allocation group sizes due to "difficulty in initiating the routine of alternating patients during the first 2 months of the study".

\section{Participants}

Setting: Chicago, IL, USA.

Newborn Nurseries of Lewis Memorial Maternity Hospital, Chicago.

Ethnic group: $56 \%$ of neonates were African-American.

Number allocated: 4292 neonates:

- silver nitrate: 2359;

- erythromycin: 1933.

Age: neonates.

Sex: M:F 2214:2178.

Inclusion criteria: all infants born June 1958 to June 1959 at the Newborn Nurseries of Lewis Memorial Maternity Hospital, Chicago.

Exclusion criteria: none specified.

No comment on equivalence of baseline characteristics.
Number of interventions: 2 .

- Intervention 1: erythromycin ointment 1-centimetre strip $5 \mathrm{mg} / \mathrm{g}$ wax ampoule $(\mathrm{n}=1933)$.

- Intervention 2: silver nitrate $1 \%$ solution wax ampoule, 1 to 2 drops $(n=2359)$.

Time to intervention: no later than 10 minutes after delivery.

Pre-intervention manoeuvres: eyes of the newborn were wiped thoroughly with absorbent cotton.

Postintervention manoeuvres: none specified.

\section{Reaction grades:}

- 0 - no reaction;

- I - redness and swelling;

- II - redness, swelling, and discharge;

- III - redness, swelling, and copious purulent discharge.

2. Infants with positive bacterial culture of those eyes having reaction grades I, II, or III.

3. Infants with gonococcal conjunctivitis.

Follow-up: 4 to 5 days postpartum in methods, but results reported up to day 8 .

Eyes of each infant were examined daily by a paediatric resident; methods indicate cultures were only taken on those having reactions II or III, but in practice, it appears that cultures were taken in those having reactions I, II, and III. 


Dotes of study: June 1958 to June 1959.
Funding source not specified.
No declaration of interest.
No reported subgroup analysis.
Trial investigators not contacted.

\section{Risk of bias}

\begin{tabular}{|c|c|c|}
\hline Bias & Authors' judgement & Support for judgement \\
\hline $\begin{array}{l}\text { Random sequence genera- } \\
\text { tion (selection bias) }\end{array}$ & High risk & $\begin{array}{l}\text { QUOTE: "An attempt was made to alternate infants irrespective of type of de- } \\
\text { livery, prematurity, race, etc. A total of } 4292 \text { infants were observed during a pe- } \\
\text { riod of } 1 \text { year" } \\
\text { QUOTE: "The discrepancy in the two groups resulted from difficulty in initiat- } \\
\text { ing the routine of alternating the routine of alternating patients during the first } \\
\text { two months of the study." } \\
\text { COMMENT: Non-random process in the sequence generation }\end{array}$ \\
\hline
\end{tabular}

Allocation concealment High risk
(selection bias)
(selection bias)

QUOTE: "An attempt was made to alternate infants irrespective of type of delivery, prematurity, race, etc. A total of 4292 infants were observed during a period of 1 year"

QUOTE: "The discrepancy in the two groups resulted from difficulty in initiating the routine of alternating the routine of alternating patients during the first two months of the study."

COMMENT: Participants or investigators enrolling participants could possibly have foreseen assignments, thus introducing selection bias.

\begin{tabular}{|c|c|c|}
\hline $\begin{array}{l}\text { Blinding of study partici- } \\
\text { pants (mothers of infants) } \\
\text { Clinical conjunctivitis } \\
\text { (subjective) }\end{array}$ & Unclear risk & $\begin{array}{l}\text { COMMENT: The study does not mention whether mothers were masked to } \\
\text { the intervention. Silver nitrate sometimes causes a chemical conjunctivitis } \\
\text { that can last up to } 72 \text { hours, and silver nitrate causes lid stains that can last } 30 \\
\text { to } 48 \text { hours. Erythromycin ointment leaves a ocular residue that can last for } \\
\text { hours. The mother may be able to identify the medication; the impact on per- } \\
\text { formance bias is unknown. }\end{array}$ \\
\hline
\end{tabular}

\begin{tabular}{|c|c|c|}
\hline $\begin{array}{l}\text { Blinding of study partici- } \\
\text { pants (mothers of infants) } \\
\text { Bacterial, gonococcal and } \\
\text { chlamydial conjunctivitis } \\
\text { (objective) }\end{array}$ & Unclear risk & $\begin{array}{l}\text { COMMENT: The study does not mention whether mothers were masked on } \\
\text { the intervention. Silver nitrate sometimes causes a chemical conjunctivitis } \\
\text { that can last up to } 72 \text { hours, and silver nitrate causes lid stains that can last } 30 \\
\text { to } 48 \text { hours. Erythromycin ointment leaves a ocular residue that can last for } \\
\text { hours. The mother may be able to identify the medication; the impact on per- } \\
\text { formance bias is unknown. } \\
\text { Mothers may differentially refer neonates with chemical conjunctivitis or lid } \\
\text { stains for assessment for conjunctivitis, which could lead to systematic bias } \\
\text { on swabbing neonates for bacterial assessment. The presence of bacteria on } \\
\text { swab may indicate pathogenic cause of conjunctivitis, presence of chemical } \\
\text { conjunctivitis with normal bacterial flora, or carrier state with chemical con- } \\
\text { junctivitis. }\end{array}$ \\
\hline
\end{tabular}

Blinding of caregiver who administered medication Clinical conjunctivitis (subjective)
High risk

COMMENT: The study does not mention whether the person administering the medication is masked, but the medications appear different.

The 2 interventions differ in colour and consistency. Silver nitrate is a clear solution. Erythromycin is a translucent ointment that leaves an ocular residue 
Blinding of caregiver who administered medication Bacterial, gonococcal and chlamydial conjunctivitis (objective)
High risk

COMMENT: The study does not mention whether the person administering the medication is masked, but the medications appear different.

The 2 interventions differ in colour and consistency. Silver nitrate is a clear solution. Erythromycin is a translucent ointment that leaves an ocular residue that can last for hours. Therefore, it would be apparent which medication was being dispensed in a given infant's eyes.

Lack of masking, combined with bias on the part of the person administering medication, could influence adherence to administration. This could selectively predispose 1 allocation group to obtain bacterial conjunctivitis over the other, and therefore affect the bacterial conjunctivitis cases differentially by allocation group.
Blinding of persons involved in postnatal care Clinical conjunctivitis (subjective)
Unclear risk

COMMENT: The study does not mention whether those involved in postnatal case are masked to the intervention. Silver nitrate sometimes causes a chemical conjunctivitis that can last up to 72 hours, and silver nitrate causes lid stains that can last 30 to 48 hours. Erythromycin is a translucent ointment that leaves an ocular residue that can last for hours. Therefore, people involved in postnatal care may not be masked as to the medication used during this initial time period.

\section{Blinding of persons in- Unclear risk} volved in postnatal care Bacterial, gonococcal and chlamydial conjunctivitis (objective)
COMMENT: The study does not mention whether those involved in postnatal case are masked to the intervention. Silver nitrate sometimes causes a chemical conjunctivitis that can last up to 72 hours, and silver nitrate causes lid stains that can last 30 to 48 hours. Erythromycin is a translucent ointment that leaves an ocular residue that can last for hours. Therefore, people involved in postnatal care may not have been masked to the medication used during this initial time period.

We are uncertain of the participation of people involved in postnatal care in identifying conjunctivitis cases for bacterial swabbing. Bias and lack of masking could lead to systematic differential rates of conjunctivitis cases referred for swabbing for bacterial assessment. Considering the follow-up of the study is only 5 to 8 days, lack of masking could have a significant effect on the event rates.

The presence of bacteria on swab may indicate pathogenic cause of conjunctivitis, presence of chemical conjunctivitis with normal bacterial flora, or carrier state with chemical conjunctivitis.

\begin{tabular}{|c|c|c|}
\hline $\begin{array}{l}\text { Blinding of outcome as- } \\
\text { sessment (detection bias) } \\
\text { Clinical conjunctivitis } \\
\text { (subjective) }\end{array}$ & Unclear risk & $\begin{array}{l}\text { COMMENT: The study does not mention masking of outcome assessors. As sil- } \\
\text { ver nitrate creates lid stains that last } 30 \text { to } 48 \text { hours, and tetracycline ointment } \\
\text { may leave residue for hours, outcome assessments during this time were likely } \\
\text { not masked. Considering that neonates were only followed up } 4 \text { to } 5 \text { days post } \\
\text { partum, there is greater potential for bias on outcome assessments. } \\
\text { Furthermore, there is no formal clinical conjunctivitis case definition, only } \\
\text { grading the eyes by varying amounts of redness and discharge. This makes the } \\
\text { definition of conjunctivitis more subjective, adding further possible bias. }\end{array}$ \\
\hline
\end{tabular}

Blinding of outcome as- Unclear risk sessment (detection bias) Bacterial, gonococcal and chlamydial conjunctivitis (objective)
COMMENT: The study does not mention whether outcome assessors and those assessing for bacterial culture were masked as the allocation group. As silver nitrate creates lid stains that last 30 to 48 hours, and tetracycline ointment may leave residue for hours, outcome assessments during this time were likely not masked. Considering that neonates were only followed up 4 to 5 days postpartum, there is greater potential for bias on outcome assessments.

The data further show that most of the ocular reactions and positive bacterial cultures were obtained on day 1 and day 2 , when there would be the greatest potential to identify allocation group based on ocular staining.

In ambiguous cases of clinical conjunctivitis, there may be differential assessor behaviour to include or exclude cases of clinical conjunctivitis, thereby in- 
Christian 1960 (Continued)

creasing or decreasing likelihood of swabbing the neonate's eye for bacterial conjunctivitis. Presence of bacteria on a swab does not necessarily prove that the bacteria caused the conjunctivitis, as the bacteria could be a carrier but the conjunctivitis caused by chemical conjunctivitis. This possible bias is further magnified by the fact that the swabs for culture were not reserved for those conjunctivitis cases with purulent discharge, as specified in the methods, but were taken from red eyes with no discharge. This widened definition of clinical conjunctivitis allows for more subjective ambiguous cases of conjunctivitis, which are more prone to bias when masking is absent.

Incomplete outcome data Unclear risk (attrition bias)

Clinical conjunctivitis

(subjective)
QUOTE: "The period of observation of all infants except premature or full term infants with some disturbance requiring prolonged hospitalization, was 4-5 days postpartum."

COMMENT: The study authors do not provide any information on the presence or absence of incomplete outcome data. It appears that all infants were followed in hospital, and likely loss to follow-up was low, but this unclear from the study.
Incomplete outcome data Unclear risk (attrition bias)

Bacterial, gonococcal and chlamydial conjunctivitis (objective)
COMMENT: As above, the study authors do not provide any information on the number of infants with clinical conjunctivitis who were not assessed for bacterial conjunctivitis due to loss of follow-up. Selective reporting (re- High risk
porting bias)
COMMENT: The authors specified in the methods that cultures would be taken from eyes graded II and III, but in the results cultures were taken from eyes graded I, II, III, without explanation. Therefore, a primary outcome is reported using measurements not prespecified.
COMMENT: In any trial with silver nitrate, there could be differential diagnostic activity. This could lead to increased diagnosis of true but harmless cases of disease. For example, silver nitrate induces a chemical conjunctivitis. This chemical conjunctivitis could lead to increased selective bacterial cultures of infants' eyes in the silver nitrate group. A positive bacterial culture found from a swab of conjunctivitis due to chemical conjunctivitis does not necessarily mean that the bacteria caused the conjunctivitis. The bacteria could be part of the normal flora of the eye, with an associated chemical conjunctivitis, or the bacteria could be the causal agent of the conjunctivitis.

In addition to a high risk of bias for method of allocation, there was a discrepancy in the number of infants in the 2 groups due to failure of alternation in the first 2 months of the 1-year study, leading to 2359 in the group receiving erythromycin and 1933 in the group receiving silver nitrate.

\section{Study characteristics}

Methods
Method of allocation: alternation.
Unit of randomisation: neonate.
Exclusions after allocation: none specified and issue not addressed in paper.
Losses to follow-up: none specified and issue not addressed in paper.
No comment on how missing data were handled.


Cousineau 1952 (Continued)

No reported power calculation.

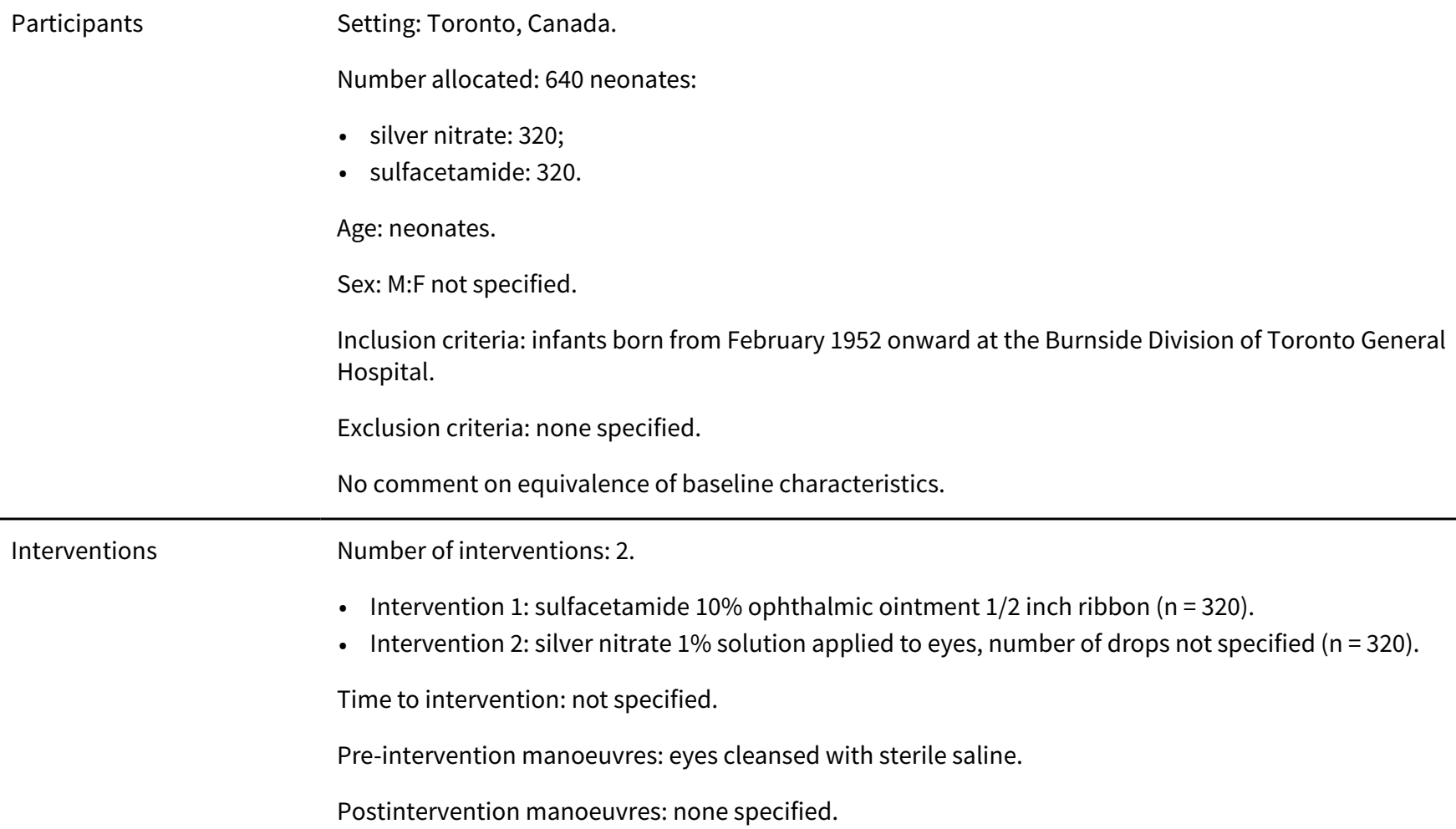

- Intervention 1: sulfacetamide $10 \%$ ophthalmic ointment $1 / 2$ inch ribbon $(n=320)$.

- Intervention 2: silver nitrate $1 \%$ solution applied to eyes, number of drops not specified $(n=320)$.

Time to intervention: not specified.

Pre-intervention manoeuvres: eyes cleansed with sterile saline.

Postintervention manoeuvres: none specified.

1. Infants with purulent discharge from eyes.

2. Infants with purulent discharge from eyes due to drug reaction, defined as any discharge from eyes in the first 3 days following birth.

3. Infants with purulent discharge from eyes due to infection, defined as eye discharge from which organisms were recovered after the third postnatal day.

4. Infants with gonococcal conjunctivitis.

Follow-up: at least 3 days and likely 9 days; frequency of follow-up not specified, but likely daily.

Definition of conjunctivitis: purulent discharge from 1 or both eyes.

Cultures were obtained from infants with purulent discharge after the third postnatal day.

No adverse events reported.

\begin{tabular}{|c|c|}
\hline \multirow[t]{5}{*}{ Notes } & No sources of funding specified. \\
\hline & No declaration of interest specified. \\
\hline & Date of study: February 1952; no end date specified. \\
\hline & No reported subgroup analysis. \\
\hline & Trial investigators were not contacted. \\
\hline
\end{tabular}

\section{Risk of bias}

\begin{tabular}{lll}
\hline Bias & Authors' judgement & Support for judgement \\
\hline $\begin{array}{ll}\text { Random sequence genera- } \\
\text { tion (selection bias) }\end{array}$ & High risk & $\begin{array}{l}\text { QUOTE: “...alternate babies received, as prophylaxis, 1 per cent silver nitrate } \\
\text { solution and } 10 \text { percent sulphacetimide ointment,...” }\end{array}$ \\
\hline
\end{tabular}




\begin{tabular}{|c|c|c|}
\hline $\begin{array}{l}\text { Allocation concealment } \\
\text { (selection bias) }\end{array}$ & High risk & $\begin{array}{l}\text { COMMENT: Participants or investigators enrolling participants could possibly } \\
\text { have foreseen assignments, thus introducing selection bias. }\end{array}$ \\
\hline
\end{tabular}

\begin{tabular}{|c|c|c|}
\hline $\begin{array}{l}\text { Blinding of study partici- } \\
\text { pants (mothers of infants) }\end{array}$ & Unclear risk & $\begin{array}{l}\text { COMMENT: The study does not mention whether mothers were masked to the } \\
\text { intervention. }\end{array}$ \\
\hline $\begin{array}{l}\text { Clinical conjunctivitis } \\
\text { (subjective) }\end{array}$ & & $\begin{array}{l}\text { Silver nitrate sometimes causes a chemical conjunctivitis that can last up to } \\
72 \text { hours, and silver nitrate causes lid stains that can last } 30 \text { to } 48 \text { hours. Sulfac- } \\
\text { etamide ointment leaves an ocular residue that can last for hours. The mother } \\
\text { may be able to identify the medication; the impact on performance bias is un- } \\
\text { known. }\end{array}$ \\
\hline
\end{tabular}

Blinding of study partici- Unclear risk pants (mothers of infants) Bacterial, gonococcal and chlamydial conjunctivitis (objective)

COMMENT: The study does not mention whether mothers were masked to the intervention.

Silver nitrate sometimes causes a chemical conjunctivitis that can last up to 72 hours, and silver nitrate causes lid stains that can last 30 to 48 hours. Sulfacetamide ointment leaves an ocular residue that can last for hours. The mother may be able to identify the medication; the impact on performance bias is unknown.

The mothers of neonates with ocular signs of prophylaxis may handle the eyes of the infant differently, which could, in turn, introduce pathogenic bacteria into the eyes differently, and differentially affect bacterial conjunctivitis cases in the allocation groups.

Mothers may be more or less likely to refer the neonate for assessment of clinical conjunctivitis if they are aware of the prophylaxis administered. If the parent is aware of the intervention, this may influence whether the neonate is swabbed or not for conjunctivitis in ambiguous cases. Note that a positive bacterial culture of a conjunctivitis case does not confirm the bacteria as the cause of the conjunctivitis. Conjunctivitis could be chemical in nature with normal flora bacteria on culture, or it could be true bacterial conjunctivitis.

Blinding of caregiver who administered medication Clinical conjunctivitis (subjective)
High risk

COMMENT: The study does not mention whether the person administering the medication was masked, but the medications appear different.

The 2 interventions differ in colour and consistency. Silver nitrate is a clear solution, and sulfacetamide is an ointment.

Lack of masking of the person who administers the medication could influence the outcome of clinical conjunctivitis through differential handling of the eyes.

Blinding of caregiver who administered medication High risk COMMENT: The study does not mention whether the person administering the medication was masked, but the medications appear different.

Bacterial, gonococcal and chlamydial conjunctivitis (objective)
The 2 interventions differ in colour and consistency. Silver nitrate is a clear solution, and sulfacetamide is an ointment.

Lack of masking of the person who administers the medication could influence the outcome of clinical conjunctivitis through differential handling of the eyes. Differential handling of the eyes may lead to differential introduction of bacteria into the eyes of neonates by allocation group. Therefore, lack of masking of the person who administers medication may influence the outcome of bacterial conjunctivitis.

COMMENT: The study does not mention whether the person involved in postnatal care was masked.

The 2 interventions differ in colour and consistency. Silver nitrate sometimes causes a chemical conjunctivitis that can last up to 72 hours, and silver nitrate
Blinding of persons in-
volved in postnatal care Clinical conjunctivitis (subjective)
Unclear risk

Interventions for preventing ophthalmia neonatorum (Review)

Copyright @ 2020 The Cochrane Collaboration. Published by John Wiley \& Sons, Ltd. 

ocular residue that can last for hours.

The person involved in postnatal care may be able to identify the medication; the impact on performance bias is unknown. This bias could be significant, as follow-up time was 3 to 9 days in this study, and it appears that neonates were kept in hospital in the nursery throughout this time. Therefore, people involved in postnatal care may not be masked as to the medication used as prophylaxis during this initial time period. Lack of masking of the people involved in postnatal care could differentially affect the identification of clinical conjunctivitis cases, and handling of the eyes, both of which could influence the outcome of clinical conjunctivitis.

\section{Blinding of persons in- Unclear risk} volved in postnatal care Bacterial, gonococcal and chlamydial conjunctivitis (objective)
COMMENT: The study does not mention whether the person involved in postnatal care was masked.

The 2 interventions differ in colour and consistency. Silver nitrate sometimes causes a chemical conjunctivitis that can last up to 72 hours, and silver nitrate causes lid stains that can last 30 to 48 hours. Sulfacetamide ointment leaves an ocular residue that can last for hours.

The person involved in postnatal care may be able to identify the medication; the impact on performance bias is unknown. This bias could be significant, as follow-up time was 3 to 9 days in this study, and it appears that neonates were kept in hospital in the nursery throughout this time. Therefore, people involved in postnatal care may not be masked as to the medication used as prophylaxis during this initial time period. Lack of masking of the people involved in postnatal care could differentially affect the identification of clinical conjunctivitis cases that may be referred for bacterial analysis, and the handling of the eyes, both of which could influence the outcome of bacterial conjunctivitis.

COMMENT: The study does not mention masking of outcome assessors. Silver nitrate sometimes causes a chemical conjunctivitis that can last up to 72 hours, and silver nitrate causes lid stains that can last 30 to 48 hours. Sulfacetamide ointment leaves an ocular residue that can last for hours. Considering that neonates were only followed up 3 to 9 days postpartum, and the low event rate, there is a greater potential for bias on outcome assessments.
Blinding of outcome assessment (detection bias) Bacterial, gonococcal and chlamydial conjunctivitis (objective)
Unclear risk

COMMENT: The study does not mention masking of outcome assessors. Silver nitrate sometimes causes a chemical conjunctivitis that can last up to 72 hours, and silver nitrate causes lid stains that can last 30 to 48 hours. Sulfacetamide ointment leaves an ocular residue that can last for hours. Considering that neonates were only followed up 3 to 9 days postpartum, and the low event rate, there is a greater potential for bias on outcome assessments.

In ambiguous cases of clinical conjunctivitis, there may be differential assessor behaviour to include or exclude cases of clinical conjunctivitis, thereby increasing or decreasing likelihood of swabbing the neonate's eye for bacterial conjunctivitis. Presence of bacteria on a swab does not necessarily prove that the bacteria caused the conjunctivitis, as the bacteria could be a carrier, but the conjunctivitis caused by chemical conjunctivitis. 


\section{Cousineau 1952 (Continued)}

Incomplete outcome data (attrition bias)

Bacterial, gonococcal and chlamydial conjunctivitis (objective)
Unclear risk

COMMENT: The study authors do not provide any information on the presence or absence of incomplete cases of bacterial conjunctivitis. It appears that most infants were followed in hospital for 3 to 9 days, and loss to follow-up was likely low, but this is unclear from the study. Small losses to follow-up could significantly influence the results considering the low event rate and small sample size.

Selective reporting (re- Unclear risk
porting bias)

COMMENT: The study is not clear as to whether any neonate had gonococcal conjunctivitis or inclusion conjunctivitis. Although other studies in the same paper mention this outcome, and it is possible there were simply no cases of gonococcal or inclusion conjunctivitis, this is not clear.

Other bias Unclear risk

COMMENT: In any trial with silver nitrate, there could be differential diagnostic activity, which could lead to increased diagnosis of true but harmless cases of disease. For example, silver nitrate induces a chemical conjunctivitis. This chemical conjunctivitis could lead to increased selective bacterial cultures of infants' eyes in the silver nitrate intervention group. A positive bacterial culture found from a swab of conjunctivitis due to chemical conjunctivitis does not necessarily mean that the bacteria caused the conjunctivitis. The bacteria could be part of the normal flora of the eye, with an associated chemical conjunctivitis, or the bacteria could be the causal agent of the conjunctivitis.

David 2011

\section{Study characteristics}

Methods

Parallel-group, single-centre RCT.

Method of allocation: "random". No other details provided on method of allocation.

Unit of randomisation: neonate.

Losses to follow-up: in the povidone-iodine group, 7 (3.4\%) were lost to follow-up, and in the tetracycline group, 9 (4.4\%) were lost to follow-up.

Exclusions after allocation: none specified in paper.

Handling of missing data not discussed in paper.

Reported power calculation: yes. 410 neonate sample size had $80 \%$ power to detect $20 \%$ difference.

Unusual study design: none.

Participants

Setting: not explicitly specified, likely Nahariya, Israel.

Number allocated: 410 .

Age: neonates.

Sex: M:F 52.5\%:47.5\%.

Inclusion criteria:

1. full-term healthy neonates born after 37 weeks.

Exclusion criteria:

1. infants with ocular malformations.

2. mothers who were treated with systemic or local antibiotics 1 week before delivery. 
David 2011 (Continued)

Equivalence of baseline characteristics: yes.

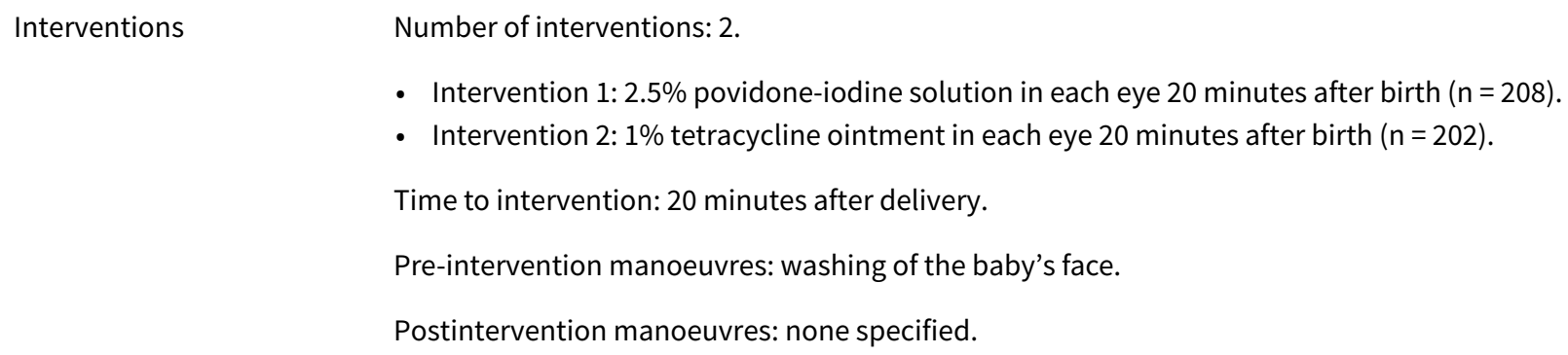

- Intervention $1: 2.5 \%$ povidone-iodine solution in each eye 20 minutes after birth $(n=208)$.

- Intervention 2: 1\% tetracycline ointment in each eye 20 minutes after birth $(n=202)$.

Time to intervention: 20 minutes after delivery.

Pre-intervention manoeuvres: washing of the baby's face.

Postintervention manoeuvres: none specified.

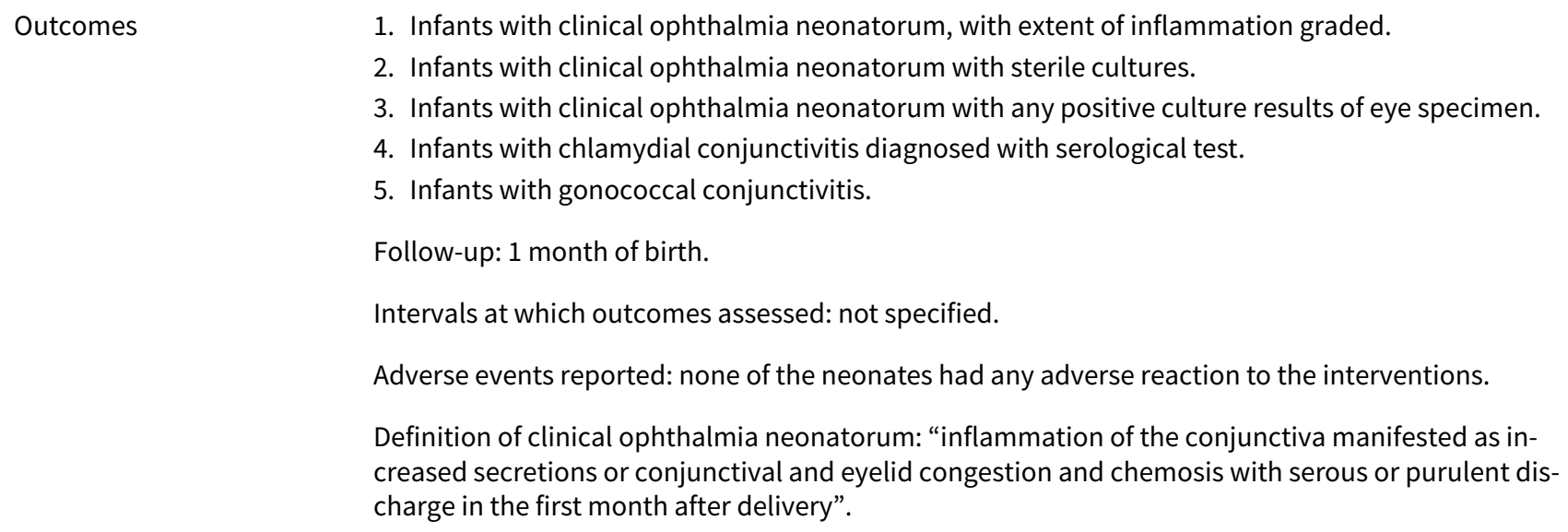

Sources of funding: not identified.

Declaration of interest: "The author(s) have no proprietary or commercial interest in any materials discussed in this article."

Trial investigators have not been contacted.

No reported subgroup analysis.

\section{Risk of bias}

\begin{tabular}{|c|c|c|}
\hline Bias & Authors' judgement & Support for judgement \\
\hline $\begin{array}{l}\text { Random sequence genera- } \\
\text { tion (selection bias) }\end{array}$ & Unclear risk & $\begin{array}{l}\text { COMMENT: Paper states "random". Method of allocation not specified. } \\
\text { Insufficient information about the sequence generation process to permit } \\
\text { judgement of low risk of high risk. }\end{array}$ \\
\hline $\begin{array}{l}\text { Allocation concealment } \\
\text { (selection bias) }\end{array}$ & Unclear risk & Insufficient information to permit judgement \\
\hline $\begin{array}{l}\text { Blinding of study partici- } \\
\text { pants (mothers of infants) } \\
\text { Clinical conjunctivitis } \\
\text { (subjective) }\end{array}$ & Unclear risk & $\begin{array}{l}\text { Masking of the mother was not addressed in the paper. } \\
\text { COMMENT: The } 2 \text { interventions differ in colour and consistency. Povidone-io- } \\
\text { dine is an orange-red solution that leads to residual staining of the eye and } \\
\text { surrounding periocular skin can last minutes to hours, which the mother } \\
\text { would notice. Tetracycline is a light-yellow ointment that leaves a residue that } \\
\text { can last for hours in the eyes of the infant. The mothers may handle the eyes } \\
\text { of neonates differently by allocation group, based on eye staining and residue. }\end{array}$ \\
\hline
\end{tabular}


This could lead to differential introduction of pathogenic bacteria into these neonates' eyes, causing clinical conjunctivitis.

Blinding of study participants (mothers of infants) Bacterial, gonococcal and chlamydial conjunctivitis (objective)

Unclear risk

COMMENT: The study does not mention whether the mothers were masked as to the intervention dispensed in the neonate's eyes. As mentioned, the mother may be able to identify what was dispensed in the infant's eyes, as povidone-iodine can lead to periocular staining for minutes to hours, and tetracycline is an ointment that leaves residue that can last hours. The mothers may handle the eyes of neonates differently by allocation group, based on eye staining and residue. This could lead to differential introduction of pathogenic bacteria into the eyes of these neonates, causing bacterial conjunctivitis. Although this is less likely to occur with Neisseria gonorrhoeae and Chlamydia trachomatis, it is still possible in situations of poor hygiene. Furthermore, chlamydia can cause asymptomatic infection, not necessarily conjunctivitis; Furthermore, chlamydia can cause asymptomatic infection, not necessarily conjunctivitis; therefore, selective bias of conjunctivitis cases sent for bacteriological analysis (cases caused by maternal contamination due to differential eye handling) may actually identify chlamydial carriers rather than true chlamydial conjunctivitis.

Blinding of caregiver who administered medication Clinical conjunctivitis (subjective)
High risk

COMMENT: The study does not mention whether the person administering the medication was masked.

The 2 interventions differ in colour and consistency. Povidone-iodine is an orange-red solution. Tetracycline is a light-yellow ointment. There is no reported attempt to mask the appearance of the medication.

Lack of masking could influence the outcome of clinical conjunctivitis if there was bias on the part of the person who administered the medication.
Blinding of caregiver who administered medication Bacterial, gonococcal and chlamydial conjunctivitis (objective)
High risk

COMMENT: The study does not mention whether the person administering the medication was masked.

The 2 interventions differ in colour and consistency. Povidone-iodine is an orange-red solution. Tetracycline is a light-yellow ointment. There is no reported attempt to mask the appearance of the medication.

Lack of masking could influence the outcome of bacterial conjunctivitis if knowledge of the prophylaxis, say, systematically influenced adherence to dispensing of the person who administered the medication.

It is uncertain if lack of masking could influence the outcomes of chlamydial and gonococcal conjunctivitis cases. If the person who administered the medication could also have been involved in the birth process, or if there were differential hand-washing techniques by allocation group, this is a possibility.

\begin{tabular}{|c|c|c|}
\hline $\begin{array}{l}\text { Blinding of persons in- } \\
\text { volved in postnatal care } \\
\text { Clinical conjunctivitis } \\
\text { (subjective) }\end{array}$ & Unclear risk & $\begin{array}{l}\text { COMMENT: Masking of people involved in postnatal care was not addressed } \\
\text { in this paper. Since povidone-iodine causes staining of the eye that can last } \\
\text { minutes to hours, and tetracycline ointment can leave residual medication for } \\
\text { hours, those involved in postnatal care may not be masked to the medication } \\
\text { used as prophylaxis initially. Furthermore, there is no indication in the paper } \\
\text { that an attempt was made to mask those involved in postnatal care. }\end{array}$ \\
\hline
\end{tabular}

Blinding of persons in- Unclear risk
volved in postnatal care Bacterial, gonococcal and chlamydial conjunctivitis (objective)
COMMENT: Masking of people involved in postnatal care was not addressed in this paper. Since povidone-iodine causes staining of the eye that can last minutes to hours, and tetracycline ointment can leave residual medication for hours, those involved in postnatal care may not be masked to the medication used as prophylaxis initially.

Lack of masking could influence case finding of conjunctivitis in ambiguous cases, and subsequent referral for culture. This could affect identifica- 
David 2011 (Continued)

tion of bacterial conjunctivitis cases, and bring about spurious identification of chlamydial carriers as opposed to true chlamydial conjunctivitis. Lack of masking of people providing postnatal care is likely be at low risk of bias for the outcome of gonococcal conjunctivitis.

\begin{tabular}{|c|c|c|}
\hline $\begin{array}{l}\text { Blinding of outcome as- } \\
\text { sessment (detection bias) } \\
\text { Clinical conjunctivitis } \\
\text { (subjective) }\end{array}$ & Unclear risk & $\begin{array}{l}\text { COMMENT: The study does not mention masking of outcome assessors. In the } \\
\text { first hours of clinical assessment for conjunctivitis, there may be lack of mask- } \\
\text { ing as tetracycline is an ointment that leaves residue, and povidone-iodine is } \\
\text { orange-red and leads to eye staining. It is unclear how many cases of conjunc- } \\
\text { tivitis were diagnosed during this time period. In ambiguous cases of clinical } \\
\text { conjunctivitis, with lack of masking, there may be differential group behaviour } \\
\text { to include or exclude cases of clinical conjunctivitis. In this paper, conjunctivi- } \\
\text { tis was defined as inflammation of the conjunctiva with discharge, which pro- } \\
\text { vides less subjectivity in the diagnosis of conjunctivitis. }\end{array}$ \\
\hline
\end{tabular}

Blinding of outcome as- Unclear risk sessment (detection bias) Bacterial, gonococcal and chlamydial conjunctivitis (objective)
COMMENT: The study does not mention masking of outcome assessors. In the first hours of clinical assessment for conjunctivitis, there may be lack of masking as tetracycline is an ointment that leaves residue, and povidone-iodine is orange-red and leads to eye staining. It is unclear how many cases of conjunctivitis were diagnosed during this time period. In ambiguous cases of clinical conjunctivitis, with lack of masking, there may be differential group behaviour to include or exclude cases of clinical conjunctivitis. In this paper, conjunctivitis was defined as inflammation of the conjunctiva with discharge, which provides less subjectivity in the diagnosis of conjunctivitis.

With lack of masking, in ambiguous cases of clinical conjunctivitis, whether identified by personnel, mother, or outcome assessor, there may be differential assessor behaviour to include or exclude cases of clinical conjunctivitis, thereby increasing or decreasing the likelihood of swabbing the neonate's eye for bacterial or chlamydial conjunctivitis. Presence of bacteria or chlamydia on a swab does not necessarily prove that the bacteria caused the conjunctivitis, as the bacteria could be part of the normal flora of the eye, or the chlamydia could be a carrier.

PCR was used for the diagnosis of chlamydia, which is objective, but is also sensitive enough to detect a possible carrier.

The outcome of gonococcal conjunctivitis would likely be less influenced by lack of masking.

\section{Incomplete outcome data High risk} (attrition bias)

Clinical conjunctivitis

(subjective)

QUOTE: Sixteen newborns did not complete the 1 month follow-up and were omitted from the study; 7 (3.4\%) of them received povidone-iodine and 9 $(4.4 \%)$ of them received tetracycline

COMMENT: Although there are small losses to follow-up and these are roughly symmetrical, they are high in relation to event rates.

$\begin{array}{ll}\begin{array}{l}\text { Incomplete outcome data } \\ \text { (attrition bias) }\end{array} & \text { High risk } \\ \text { Bacterial, gonococcal and } & \begin{array}{l}\text { QUOTE: Sixteen newborns did not complete the } 1 \text { month follow-up and were } \\ \text { omitted from the study; } 7 \text { (3.4\%) of them received povidone-iodine and } 9\end{array} \\ \text { (4.4\%) of them received tetracycline }\end{array}$

COMMENT: Although there are small losses to follow-up and these are roughly (objective) symmetrical, they are high in relation to event rates of bacterial conjunctivitis.

Selective reporting (re- Low risk It is highly likely that all prespecified outcomes were reported.
porting bias)

Other bias Low risk No other potential sources of bias were identified.


Davidson 1951

\section{Study characteristics}

\section{Methods}

Parallel-group, single-centre trial.

Method of allocation: alternation by week.

Unit of randomisation: neonate.

Exclusions after allocation: none specified and issue not addressed in paper.

Losses to follow-up: none specified.

No comment on handling of missing data.

No reported power calculation.

Unusual study design: methods state that cultures were taken from neonates with ocular discharge. The numbers that had discharge and the numbers that were cultured do not match. In some cases the numbers cultured were less than those with discharge, but in 1 group, the number cultured was greater than the number with discharge.

\section{Participants} Setting: Baltimore, USA.

Number randomised: 4163 neonates:

- penicillin ointment: 1436;

- penicillin IM: 1359;

- silver nitrate: 1368 .

Age: neonates.

Sex: M:F not specified.

Inclusion criteria: infants born from 1 May 1948 to 20 March 1950.

Exclusion criteria: none specified.

- Intervention 1: penicillin 100,000 units/gram ophthalmic ointment, per eye $1 / 2$ inch length $=7000$ units or $0.07 \mathrm{~g}(\mathrm{n}=1436)$.

- Intervention 2: aqueous penicillin 10,000 units per IM injection ( $n=1359)$.

- Intervention 3: silver nitrate $1 \%$ solution 2 drops per eye $(n=1368)$.

Time to intervention: immediately after birth.

Pre-intervention manoeuvres: none identified.

Postintervention manoeuvres: after administration of silver nitrate, eyes flushed out with normal saline.

No comment on equivalence of baseline characteristics.

Outcomes

1. Infants with 1 or more signs of ocular irritation, such as redness, oedema, and/or discharge.

2. Infants with conjunctivitis where infection was "proved or probable" from ocular discharge.

3. Infants with clinical ophthalmia neonatorum.

4. Infants with gonococcal conjunctivitis.

5. Infants with ocular redness.

6. Infants with ocular oedema.

7. Infants with ocular discharge. 
Follow-up: 10 days postpartum.

Adverse events reported 1 episode of penicillin sensitisation for neonate given IM injection of penicillin, but unclear if in this particular study.

Definition of conjunctivitis: not defined.

Definition of bacterial conjunctivitis: cultured neonates' eyes with any discharge and categorised results as:

- no infection;

- infection doubtful;

- infection proved or probable.

Infection "proved or probable" included those eye discharge specimens that had organisms in both smears and cultures and excluded diphtheroids and Micrococcus pyogenes.

Definition of clinical ophthalmia neonatorum: not defined.

Source of funding: "All penicillin preparations used in this investigation were supplied by E.R. Squibb \& Sons."

Source of funding: "This project was supported by the United States Public Health Service, Division of Venereal Diseases."

No declaration of interest report was made in this study.

Subgroup analysis of neonates born via caesarean section.

Trial investigators were not contacted.

\section{Risk of bias}

Bias Authors' judgement Support for judgement

Random sequence genera- High risk tion (selection bias)
QUOTE: "Three schemes of prophylaxis were used in rotation.... During one week all newborns had penicillin ointment....The following week every infant was given 10,000 units by intramuscular injection. During the third week silver nitrate prophylaxis was carried out ..."

COMMENT: Non-random process in the sequence generation

\begin{tabular}{ll}
\hline $\begin{array}{l}\text { Allocation concealment } \\
\text { (selection bias) }\end{array}$ & High risk
\end{tabular}$\quad \begin{aligned} & \text { COMMENT: Participants or investigators enrolling participants could possibly } \\
& \text { have foreseen assignments, thus introducing selection bias. }\end{aligned}$

Blinding of study partici- Unclear risk pants (mothers of infants)

COMMENT: The study does not mention whether mothers were masked to the Clinical conjunctivitis (subjective)
Silver nitrate sometimes causes a chemical conjunctivitis that can last up to 72 hours, and silver nitrate causes lid stains that can last 30 to 48 hours. Penicillin ointment leaves an ocular residue that can last for hours. The penicillin given IM could leave a needle mark on the neonate. The mother may be able to identify the medication; the impact on performance bias is unknown.

COMMENT: The study does not mention whether mothers were masked to the intervention.

Silver nitrate sometimes causes a chemical conjunctivitis that can last up to 72 hours, and silver nitrate causes lid stains that can last 30 to 48 hours. Penicillin ointment leaves an ocular residue that can last for hours. The penicillin given 
IM could leave an needle mark on the neonate. The mothers may handle the eyes of neonates differently by allocation group, based on ocular signs of prophylaxis, which could, in turn, introduce pathogenic bacteria into the eyes differently, and differentially affect bacterial conjunctivitis cases in the allocation groups.

Mothers may be more or less likely to refer the neonate for assessment of clinical conjunctivitis if they are aware of the prophylaxis administered. If the parent is aware of the intervention, it may influence whether the neonate is swabbed or not for conjunctivitis in ambiguous cases. Note that positive bacterial culture of a conjunctivitis case does not confirm the bacteria as the cause of the conjunctivitis. Conjunctivitis could be chemical in nature with normal flora bacteria on culture, or it could true bacterial conjunctivitis.

Blinding of caregiver who administered medication Clinical conjunctivitis (subjective)
High risk

COMMENT: The study does not mention whether the person administering the medication was masked, but the medications appear different.

The 3 interventions differ in colour, consistency, and route of delivery. Silver nitrate is a clear solution. Penicillin $\mathrm{G}$ ointment is a clear or white ointment. Penicillin G IM is an aqueous solution administered intramuscularly as opposed to topically. Lack of masking could influence the outcome.
Blinding of caregiver who administered medication Bacterial, gonococcal and chlamydial conjunctivitis (objective)
Unclear risk

COMMENT: The study does not mention whether the person administering the medication was masked, but the medications appear different.

The 3 interventions differ in colour, consistency, and route of delivery. Silver nitrate is a clear solution. Penicillin G ointment is a clear or white ointment. Penicillin G IM is an aqueous solution administered intramuscularly as opposed to topically. Lack of masking could influence the outcome. No ocular or IM injection placebo was used, therefore in 2 allocation arms the neonate's eyes are handled by the person who administers the intervention, and in the remaining arm, there is no ocular handling. This could influence the outcome of bacterial conjunctivitis.
Blinding of persons in- Unclear risk volved in postnatal care Clinical conjunctivitis (subjective)
COMMENT: The study does not mention whether those involved in postnatal care were masked to the intervention. Silver nitrate sometimes causes a chemical conjunctivitis that can last up to 72 hours, and silver nitrate causes lid stains that can last 30 to 48 hours. Penicillin ointment leaves an ocular residue that can last for hours. Post-injection of penicillin IM, there could be a residual needle mark. The person involved in postnatal care may be able to identify the medication; the impact on performance bias is unknown. This bias could be significant, as follow-up time was only 10 days, and it appears neonates were kept in hospital in the nursery throughout this time. Therefore, people involved in postnatal care may not be masked as to the medication used as prophylaxis during this initial time period.

\section{Blinding of persons in- Unclear risk} volved in postnatal care Bacterial, gonococcal and chlamydial conjunctivitis (objective)
COMMENT: The study does not mention whether those involved in postnatal care were masked to the intervention. Silver nitrate sometimes causes a chemical conjunctivitis that can last up to 72 hours, and silver nitrate causes lid stains that can last 30 to 48 hours. Penicillin ointment leaves an ocular residue that can last for hours. Post-injection of penicillin IM, there could be a residual needle mark. The person involved in postnatal care may be able to identify the medication; the impact on performance bias is unknown. This bias could be significant, as follow-up time was only 10 days, and it appears neonates were kept in hospital in the nursery throughout this time, therefore people involved in postnatal care may not be masked as to the medication used as prophylaxis during this initial time period.

If people involved in postnatal care were also involved in identification of conjunctivitis cases for swabbing, lack of masking could influence the outcome of bacterial conjunctivitis. People involved in postnatal care not masked to 
Blinding of outcome as- Unclear risk sessment (detection bias)

Clinical conjunctivitis

(subjective)

COMMENT: The study does not mention masking of outcome assessors. Silver nitrate sometimes causes a chemical conjunctivitis that can last up to 72 hours, and silver nitrate causes lid stains that can last 30 to 48 hours. Penicillin ointment leaves an ocular residue that can last for hours. Post-injection of penicillin IM, there could be a residual needle mark. Considering that neonates were only followed up 10 days postpartum, there is greater potential for bias on outcome assessments.

Furthermore, there is no formal clinical conjunctivitis case definition, only grading the eyes by the presence of redness, oedema, or discharge.

Blinding of outcome assessment (detection bias) Bacterial, gonococcal and chlamydial conjunctivitis (objective)
Unclear risk

COMMENT: The study does not mention masking of outcome assessors. Silver nitrate sometimes causes a chemical conjunctivitis that can last up to 72 hours, and silver nitrate causes lid stains that can last 30 to 48 hours. Penicillin ointment leaves an ocular residue that can last for hours. Post-injection of penicillin IM, there could be a residual needle mark. Considering that neonates were only followed up 10 days postpartum, there is greater potential for bias on outcome assessments.

Furthermore, there is no formal clinical conjunctivitis case definition, only grading the eyes by the presence of redness, oedema, or discharge.

In ambiguous cases of clinical conjunctivitis, there may be differential assessor behaviour to include or exclude cases of clinical conjunctivitis, thereby increasing or decreasing the likelihood of swabbing the neonate's eye for bacterial conjunctivitis. Presence of bacteria on a swab does not necessarily prove that the bacteria caused the conjunctivitis, as the bacteria could be a carrier but the conjunctivitis caused by chemical conjunctivitis. Although the study indicated that cultures were only taken from neonates with discharge, reducing ambiguity, the number of neonates cultured does not match the number of neonates with discharge.
Incomplete outcome data Unclear risk (attrition bias)

Clinical conjunctivitis

(subjective)
COMMENT: The study authors do not provide any information on the presence or absence of incomplete outcome data. It appears that most infants were followed in hospital, and loss to follow-up was likely low, but this unclear from the study. The authors do not define clinical conjunctivitis.
Incomplete outcome data High risk (attrition bias)

Bacterial, gonococcal and

chlamydial conjunctivitis

(objective)
QUOTE: "A specimen for culture and smear was taken from every eye in which a discharge developed..."

COMMENT: As above, the study authors do not provide any information on the number of infants with clinical conjunctivitis who were not assessed for bacterial conjunctivitis due to loss of follow-up. Furthermore, although the study indicated that cultures were only taken from neonates with discharge, the number of neonates cultured does not match the number of neonates with ocular discharge. This difference could be accounted for by the fact that the unit for amount of discharge cases was the eye, whilst the unit for the amount of positive cultures was the neonate.

QUOTE: "Eleven unsatisfactory cultures from two infants treated with penicillin ointment, two given intramuscularly, and seven treated with silver nitrate were omitted."

COMMENT: Considering event rates of bacterial conjunctivitis, these losses to follow-up are high, and also asymmetrical.

Selective reporting (re- High risk $\quad$ COMMENT:
porting bias)


Davidson 1951 (Continued)

1. Although the study indicated that cultures were only taken from neonates with discharge, the number of neonates cultured does not match the number of neonates with ocular discharge.

2. There was no formal diagnosis of conjunctivitis provided in the study.

Other bias Unclear risk

COMMENT: In any trial with silver nitrate, there could be differential diagnostic activity. This could lead to increased diagnosis of true but harmless cases of disease. For example, silver nitrate induces a chemical conjunctivitis. This chemical conjunctivitis could lead to increased selective bacterial cultures of infants' eyes in the silver nitrate intervention group. A positive bacterial culture found from a swab of conjunctivitis due to chemical conjunctivitis does not necessarily mean that the bacteria caused the conjunctivitis. The bacteria could be part of the normal flora of the eye, with an associated chemical conjunctivitis, or the bacteria could be the causal agent of the conjunctivitis. This study, however, appeared to attempt to distinguish normal flora of the eye and pathogenic bacteria in those cases of ocular reactions with discharge.

Fischer 1988

\section{Study characteristics}

Methods

Parallel-group, single-centre trial.

Alternation by week.

Unit of randomisation: neonate.

Exclusions after randomisation: none specified.

Losses to follow-up: none specified.

No comment on handling of missing data.

No reported power calculation.

Unusual study design and issues: 91 neonates, the majority of whom were allocated to tetracycline, did not receive any prophylaxis as delivery room staff "forgot". They were followed up.

The paper states that "a total of 16 more children were born during the weeks designated for silver nitrate than during the weeks designated for tetracycline". Considering there were 450 allocated, this suggests that there were 233 in the silver nitrate group and 217 in the erythromycin group. The paper also states that "several of the staff admitted that they tended to 'forget' the tetracycline most often because it was 'messy'". Therefore, the 91 with no prophylaxis were likely in the tetracycline group. It appears that 3 allocated to tetracycline crossed over into silver nitrate.

Participants

Setting: Nyankunde, Zaire.

Number allocated: 450:

- silver nitrate: 233 (233 allocated but 236 received silver nitrate);

- tetracycline: 217 (217 allocated, but 214 received tetracycline - amongst 214, 123 received tetracycline, and 91 "forgotten").

Age: neonates.

Sex: M:F unknown.

Inclusion criteria: babies born at Evangelical Medical Center between 10 November 1986 and 27 January 1987. 
Fischer 1988 (Continued)

Exclusion criteria: none specified.

No comment of equivalence of baseline characteristics.
Number of interventions: 2 .

- Intervention 1: tetracycline $1 \%$ ophthalmic ointment, dose not specified ( $\mathrm{n}=217$ allocated) (233 allocated but 236 received silver nitrate).

- Intervention 2: silver nitrate $1 \%$ ophthalmic solution, dose not specified ( $n=233$ allocated) ( 217 allocated, but 214 received tetracycline - among 214, 123 received tetracycline, and 91 "forgotten").

Time to intervention: 1 dose "as soon as conveniently possible after birth".

Pre-intervention manoeuvres: none specified.

Postintervention manoeuvres: none specified.

Notes

1. Infants with conjunctivitis.

2. Infants with gonococcal conjunctivitis.

Follow-up: not specified, but cases found were diagnosed in first week of life.

Definition of conjunctivitis: conjunctival inflammation and discharge. Gram stain and culture of conjunctival secretions.

Date of recruitment was 10 November 1986 to 27 January 1987.

No source of funding was specified.

No declaration of interest was made.

No reported subgroup analysis.

Authors were contacted for clarifications on masking, but no response received.

\section{Risk of bias}

\section{Bias \\ Authors' judgement Support for judgement}

Random sequence genera- High risk tion (selection bias)

QUOTE: "Treatment was alternated during week-long periods between a solution of $1 \%$ silver nitrate and an ointment containing $1 \%$ tetracycline"

QUOTE: "A total of 450 newborns were entered into the study. Of these 236 received silver nitrate, 123 received tetracycline, and 91 were 'forgotten'"

Allocation concealment High risk (selection bias)

QUOTE: "Treatment was alternated during week-long periods between a solution of $1 \%$ silver nitrate and an ointment containing $1 \%$ tetracycline"

QUOTE: "The name and treatment received were noted for each patient."

COMMENT: Participants or investigators enrolling participants could possibly foresee assignments, thus introducing selection bias.

Blinding of study partici- Unclear risk pants (mothers of infants)

Clinical conjunctivitis

(subjective)
COMMENT: Masking of mothers of the intervention was not addressed in this study. Furthermore, the 2 interventions differ in colour and consistency. Silver nitrate is a clear solution. Also, silver nitrate sometimes causes a chemical conjunctivitis that can last up to 24 hours, and lid stains that can last 30 to 48 hours. Tetracycline is a light-yellow ointment that can leave a residue that can last hours and be noticed by the mother. It is unknown how this could affect performance bias. 
Fischer 1988 (Continued)

Blinding of study participants (mothers of infants) Bacterial, gonococcal and chlamydial conjunctivitis (objective)
Unclear risk

COMMENT: Masking of mothers to the intervention was not addressed in this study. Furthermore, the 2 interventions differ in colour and consistency. Silver nitrate is a clear solution. Also, silver nitrate sometimes causes a chemical conjunctivitis that can last up to 24 hours, and lid stains that can last 30 to 48 hours. Tetracycline is a light-yellow ointment that can leave a residue that can last hours and be noticed by the mother. It is unknown how this could affect performance bias.

The mothers may differentially handle the eyes of neonates based on the visible signs of prophylaxis. This could lead to differential introduction of pathogenic bacteria into the eyes of these neonates. Therefore, the lack of masking of medication appearance may lead to bias in the bacterial or gonococcal conjunctivitis cases, depending on hygiene measures. Considering the low event rates of gonococcal conjunctivitis, this could introduce important bias. It is unknown how many neonates developed conjunctivitis in the time period when the medication could be identified, but follow-up time was only 1 week.
Blinding of caregiver who administered medication Clinical conjunctivitis (subjective)
High risk

QUOTE: "...several of the staff admitted that they tended to 'forget' the tetracycline most often because it was 'messy'"

COMMENT: Masking of the person who administers the medication was not specifically addressed in this study. As mentioned, the 2 interventions differ in colour and consistency. Silver nitrate is a clear solution. Also, silver nitrate sometimes causes a chemical conjunctivitis that can last up to 24 hours, and lid stains that can last 30 to 48 hours. Tetracycline is a light-yellow ointment that can leave a residue that can last hours. It appears that the nurses knew which medication was tetracycline and that it affected adherence, and consequently the outcome.
Blinding of caregiver who administered medication Bacterial, gonococcal and chlamydial conjunctivitis (objective)
High risk

QUOTE: "...several of the staff admitted that they tended to 'forget' the tetracycline most often because it was "messy'"

COMMENT: Masking of the person who administers the medication was not specifically addressed in this study. As mentioned, the 2 interventions differ in colour and consistency. Silver nitrate is a clear solution. Also, silver nitrate sometimes causes a chemical conjunctivitis that can last up to 24 hours, and lid stains that can last 30 to 48 hours. Tetracycline is a light-yellow ointment that can leave a residue that can last hours.

With lack of masking, the person administering the medication could dispense the medication differently, or there could be differential adherence problems, thereby altering the bactericidal effect.

It appears that the nurses knew which medication was tetracycline and that it affected adherence, and consequently the outcome.

\section{Blinding of persons in- Unclear risk} volved in postnatal care Clinical conjunctivitis (subjective)
COMMENT: Masking of people involved in postnatal care was not addressed in this study. Silver nitrate sometimes causes a chemical conjunctivitis that can last up to 24 hours, and lid stains that can last 30 to 48 hours. Tetracycline is a light-yellow ointment that can leave a residue that can last hours and be noticed by those involved in postnatal care.

It is unclear how long the neonates were involved in postnatal care, and how many cases of conjunctivitis were identified at this time. It is also unclear if those involved in postnatal care were also involved in identifying cases of conjunctivitis, and to what extent.

It is unknown how this could affect performance bias, however follow-up time was only 1 week, and the event rates were very low. Therefore, minor bias would significantly affect outcomes. For instance, people involved in postnatal care may differentially handle the eyes of neonates based on the visible signs 
Fischer 1988 (Continued)

of prophylaxis. This could lead to differential introduction of pathogenic bacteria into the eyes of these neonates.

Blinding of persons involved in postnatal care Bacterial, gonococcal and chlamydial conjunctivitis (objective)
Unclear risk

COMMENT: Masking of those involved in postnatal care was not addressed in this study. Silver nitrate sometimes causes a chemical conjunctivitis that can last up to 24 hours, and lid stains that can last 30 to 48 hours. Tetracycline is a light-yellow ointment that can leave a residue that can last hours and be noticed by those involved in postnatal care.

It is unclear how long the neonates were involved in postnatal care, and how many cases of conjunctivitis were identified at this time. It is also unclear if those involved in postnatal care were also involved in identifying cases of conjunctivitis, and to what extent.

It is unknown how this could affect performance bias, however follow-up time was only 1 week, and the event rates were very low. Therefore, minor bias would significantly affect outcomes. For instance, those involved in postnatal care may differentially handle the eyes of neonates based on the visible signs of prophylaxis. This could lead to differential introduction of pathogenic bacteria into the eyes of these neonates, including Neisseria gonorrhoeae, in settings where hygiene may not be ideal, such as the setting where this trial was held, Zaire.

Any bias in identification of conjunctivitis cases could influence cases referred for swabbing for $\mathrm{N}$ gonorrhoeae. For instance, if the people involved in postnatal care were aware that neonates were given silver nitrate, and were aware of the concomitant chemical conjunctivitis, in ambiguous cases, the nurse may erroneously ignore cases of 'true' gonococcal conjunctivitis.

The incubation period of gonococcal conjunctivitis is likely outside the period of time at which the people involved in postnatal care would be influencing identification and care. Furthermore, these cases are likely more clinically severe, eliminating ambiguity. Therefore, the lack of masking of those involved in postnatal care will likely introduce less bias for the outcome of gonococcal conjunctivitis. Still, the low event rate of gonococcal conjunctivitis could make minor bias important and clinically significant.

\section{Blinding of outcome as- \\ sessment (detection bias) \\ Clinical conjunctivitis}

(subjective)

\section{Unclear risk}

QUOTE: "The name and treatment received were noted for each patient."

COMMENT: From the statement above, it appears there was no attempt to mask outcome assessors, however we cannot be certain of this. The 2 interventions differ in colour and consistency. Silver nitrate is a clear solution. Also, silver nitrate sometimes causes a chemical conjunctivitis that can last up to 24 hours, and lid stains that can last 30 to 48 hours. Tetracycline is a light-yellow ointment that can leave a residue that can last hours.

It is unknown how many cases of conjunctivitis were identified during the time when these stains remained, but the follow-up was only 1 week in this study.
Blinding of outcome assessment (detection bias) Bacterial, gonococcal and chlamydial conjunctivitis (objective)
Unclear risk

QUOTE: "The name and treatment received were noted for each patient."

COMMENT: From the statement above, it appears there was no attempt to mask outcome assessors, however we cannot be certain of this. The 2 interventions differ in colour and consistency. Silver nitrate is a clear solution. Also, silver nitrate sometimes causes a chemical conjunctivitis that can last up to 24 hours, and lid stains that can last 30 to 48 hours. Tetracycline is a light-yellow ointment that can leave a residue that can last hours.

It is unknown how many cases of conjunctivitis were identified during the time when these stains remained, but the follow-up was only 1 week in this study. 
The incubation period of gonococcal conjunctivitis is likely outside the period of time at which these stains would remain. Furthermore, gonococcal conjunctivitis is likely more clinically severe, reducing ambiguity in diagnosis. In the silver nitrate arm, if masking was compromised, any cases presenting within the chemical conjunctivitis period could be ignored by the assessor aware of chemical conjunctivitis, and possibly miss a 'true' gonococcal conjunctivitis case. Gonococcal conjunctivitis has a very low event rate, so minor bias could significantly alter outcomes.

Incomplete outcome data Unclear risk (attrition bias)

Clinical conjunctivitis

(subjective)
QUOTE: "Conjunctivitis was diagnosed during the first week of life in each case."

QUOTE: "A total of 450 newborns were entered into the study. Of these 236 received silver nitrate, 123 received tetracycline, and 91 were 'forgotten'"

COMMENT: The "forgotten" cases received no prophylaxis. The follow-up time is not prespecified in the study. It was at least 1 week, as the study authors state that the conjunctivitis cases were diagnosed in the first week. It is unknown if any neonates allocated to prophylaxis were subsequently lost to follow-up. It is unclear if neonates were in hospital throughout this time, or when, and if they were discharged from hospital during this time. If they were discharged from hospital, it is unclear how they were followed up. It appears that some of the 91 infants who were "forgotten" were followed up, as all the gonococcal conjunctivitis cases were in this group.

COMMENT: The paper states that "a total of 16 more children were born during the weeks designated for silver nitrate than during the weeks designated for tetracycline". Considering there were 450 allocated, this therefore suggests that there were 233 in the silver nitrate group and 217 in the erythromycin group. The paper also states that "several of the staff admitted that they tended to 'forget' the tetracycline most often because it was 'messy'". Therefore, the 91 with no prophylaxis were likely in the tetracycline group. It appears that 3 allocated to tetracycline crossed over into silver nitrate. This is not explicit in the paper, but had to be calculated.
Incomplete outcome data

(attrition bias)

Bacterial, gonococcal and

chlamydial conjunctivitis

(objective)
Unclear risk

QUOTE: "Conjunctivitis was diagnosed during the first week of life in each case.”

QUOTE: "A total of 450 newborns were entered into the study. Of these 236 received silver nitrate, 123 received tetracycline, and 91 were 'forgotten'"

COMMENT: The "forgotten" cases received no prophylaxis. The follow-up time is not prespecified in the study. It was at least 1 week, as the study authors state that the conjunctivitis cases were diagnosed in the first week. It is unknown if any neonates allocated to prophylaxis were subsequently lost to follow-up. It is unclear if neonates were in hospital throughout this time, or when, and if they were discharged from hospital during this time. If they were discharged from hospital, it is unclear how they were followed up. It appears that some of the 91 infants who were "forgotten" were followed up, as all the gonococcal conjunctivitis cases were in this group.

COMMENT: The paper states that "a total of 16 more children were born during the weeks designated for silver nitrate than during the weeks designated for tetracycline". Considering there were 450 allocated, this therefore suggests that there were 233 in the silver nitrate group and 217 in the erythromycin group. The paper also states that "several of the staff admitted that they tended to 'forget' the tetracycline most often because it was 'messy'". Therefore, the 91 with no prophylaxis were likely in the tetracycline group. It appears that 3 allocated to tetracycline crossed over into silver nitrate. This is not explicit in the paper, but had to be calculated. 
Fischer 1988 (Continued)

$\begin{array}{ll}\begin{array}{l}\text { Selective reporting (re- } \\ \text { porting bias) }\end{array} & \text { Unclear risk } \\ \end{array}$

Other bias Unclear risk In any trial with silver nitrate, there could be diagnostic bias.

Silver nitrate causes a chemical conjunctivitis in the first 72 hours. As a result, in the first 72 hours, more neonates in the silver nitrate allocation group could be referred for culture in the first 72 hours. Finding bacteria in the culture does not necessarily prove that the bacteria caused the conjunctivitis. The conjunctivitis could be chemical, but growing normal flora of the eye. Alternatively, the conjunctivitis could be chemical with a chlamydial carrier. Finally, the conjunctivitis could very well be caused by the bacteria or chlamydia. Consideration of incubation periods, and assessing for carriers and normal flora with asymptomatic cases, could assist with differential diagnosis.

Ghaemi 2014

\section{Study characteristics}

Methods

Parallel-group, single-centre RCT.

Unit of allocation: neonate.

Losses to follow-up: 300 allocated, but follow-up data on 268 only. Uncertain if 32 for which no follow-up data were available were losses to follow-up, exclusions before or after randomisation, or missing data.

Missing data handling: available-case analysis.

Power calculation: based on between-group difference of one SD or less, with $90 \%$ power and an error of less than 0.05 . A minimum of 80 participants was calculated for each group.

Unusual study design: excluded neonates with culture-negative eye swabs before prophylaxis.

Participants Setting: University of Medical Sciences, Isfahan, Iran.

Number allocated: 300; unknown allocation by intervention; only numbers followed up available; losses by allocation group unavailable.

Age: neonates.

Sex: M:F not available.

Inclusion criteria:

1. preterm neonates;

2. preterm neonates with culture-negative eye swabs.

Exclusion criteria:

1. neonates with positive culture of eye swabs, apparently taken before application of prophylaxis.

There was no comment on equivalence of baseline characteristics.

Interventions Number of interventions: 3.

- Intervention 1: 2 drops of mother's colostrum in each eye ( $n=89$ followed up).

- Intervention 2: $0.5 \%$ erythromycin ophthalmic ointment in each eye ( $n=82$ followed up).

- Intervention 3: no intervention ( $n=97$ followed up). 
Ghaemi 2014 (Continued)

Time to intervention: "Immediately after birth".

Pre-intervention manoeuvres: eyes of neonates swabbed for bacterial culture.

Postintervention manoeuvres: none specified.

Outcomes
$\begin{aligned} & \text { 2. Infants with clinical conjunctivitis. } \\ & \text { ture of eye swabs are pre-prophylaxis or postprophylaxis, therefore this outcome was not entered into } \\ & \text { the review, pending author clarification. }\end{aligned}$

Follow-up: 28 days.

Frequency of follow-up: weekly or at the time of occurrence of symptoms of neonatal conjunctivitis.

Notes on definition of conjunctivitis: the clinical definition of conjunctivitis was not specified. Only infants with a diagnosis of clinical conjunctivitis were referred to eye swab and bacterial culture/PCR.

No adverse events reported.

Notes Study was conducted from November 2011 to July 2012, and published in 2014.

Source of funding: Medical School, Isfahan University of Medical Sciences, Isfahan, Iran

Authors indicated no conflict of interest.

No reported subgroup analysis.

Trial investigators were contacted, but no response received.

\section{Risk of bias}

\begin{tabular}{|c|c|c|}
\hline Bias & Authors' judgement & Support for judgement \\
\hline $\begin{array}{l}\text { Random sequence genera- } \\
\text { tion (selection bias) }\end{array}$ & Low risk & $\begin{array}{l}\text { QUOTE: "....and then by using the table of random numbers, they were ran- } \\
\text { domly assigned into three groups" }\end{array}$ \\
\hline $\begin{array}{l}\text { Allocation concealment } \\
\text { (selection bias) }\end{array}$ & Unclear risk & Insufficient information to permit judgement \\
\hline $\begin{array}{l}\text { Blinding of study partici- } \\
\text { pants (mothers of infants) } \\
\text { Clinical conjunctivitis } \\
\text { (subjective) }\end{array}$ & Unclear risk & $\begin{array}{l}\text { QUOTE: "The study was double-masked, the parents and the person who fol- } \\
\text { lowed up the patients were not aware about the treatment used" } \\
\text { COMMENT: The study does not note specifically what was done to mask par- } \\
\text { ents. } \\
\text { Erythromycin is a translucent ointment that the mother would initially notice } \\
\text { in the infant. Colostrum is yellowish or creamy in colour. There was no place- } \\
\text { bo in the allocation group that received no prophylaxis. The mothers of the } \\
\text { neonates with noticeable residual ointment or colostrum of the eyes may han- } \\
\text { dle the eyes of the infant more than mothers of neonates with no prophylaxis. } \\
\text { This could lead to differential introduction of pathogenic bacteria into the eyes } \\
\text { of these neonates. It is unclear what degree of bias these factors could intro- } \\
\text { duce to the study. }\end{array}$ \\
\hline
\end{tabular}

Blinding of study partici- Unclear risk pants (mothers of infants) Bacterial, gonococcal and chlamydial conjunctivitis (objective)
See support for judgement above for masking of study participants for clinical conjunctivitis. 
Ghaemi 2014 (Continued)

Blinding of caregiver who administered medication Clinical conjunctivitis (subjective)
High risk

COMMENT: Masking of the person who administers the medication was not addressed in this paper. Erythromycin is a translucent ointment. Colostrum is yellowish or creamy in colour. There was no placebo in the allocation group that received no prophylaxis. Those who administer the medication would handle the eyes of neonates with erythromycin and colostrum, but not the eyes of the neonates with no prophylaxis, as there was no placebo. This could lead to differential introduction of pathogenic bacteria into the eyes of these neonates.
Blinding of caregiver who administered medication Bacterial, gonococcal and chlamydial conjunctivitis (objective)
Unclear risk

The study did not report bacterial, gonococcal, or chlamydial conjunctivitis as outcomes, or this information could not be extracted from the study. Consequently, there was no assessment of bias for this category with these outcomes.
Blinding of persons involved in postnatal care Clinical conjunctivitis (subjective)
Unclear risk

QUOTE: "The study was double-masked, the parents and the person who followed up the patients were not aware about the treatment used"

COMMENT: Masking of the person who was involved in postnatal care was not addressed in this paper.

Erythromycin is a translucent ointment.

Colostrum is yellowish or creamy in colour.

There was no placebo in the allocation group that received no prophylaxis.

\begin{tabular}{|c|c|c|}
\hline $\begin{array}{l}\text { Blinding of persons in- } \\
\text { volved in postnatal care } \\
\text { Bacterial, gonococcal and } \\
\text { chlamydial conjunctivitis }\end{array}$ & Unclear risk & $\begin{array}{l}\text { The study did not report bacterial, gonococcal, or chlamydial conjunctivitis } \\
\text { as outcomes, or this information could not be extracted from the study. Con- } \\
\text { sequently, there was no assessment of bias for this category with these out- } \\
\text { comes. }\end{array}$ \\
\hline
\end{tabular}
(objective)

\section{Blinding of outcome as- High risk} sessment (detection bias) Clinical conjunctivitis (subjective)
QUOTE: "The study was double-masked, the parents and the person who followed up the patients were not aware about the treatment used"

COMMENT: It is unclear if the person who followed up the participant was the actual outcome assessor. In the initial stages of clinical assessment for conjunctivitis, there may be a lack of masking, as erythromycin is an ointment that leaves residue, and colostrum can leave stains. It is unclear how many cases of conjunctivitis were diagnosed during this early period of time. There was no placebo in the no-prophylaxis group. In

ambiguous cases of clinical conjunctivitis, whether identified by personnel, mother, or outcome assessor, there may be differential group behaviour to include or exclude cases of clinical conjunctivitis with lack of masking. Conjunctivitis was not defined in this paper to permit a determination of how subjective diagnosis could have been.

\begin{tabular}{|c|c|c|}
\hline $\begin{array}{l}\text { Blinding of outcome as- } \\
\text { sessment (detection bias) } \\
\text { Bacterial, gonococcal and } \\
\text { chlamydial conjunctivitis } \\
\text { (objective) }\end{array}$ & Unclear risk & $\begin{array}{l}\text { The study did not report bacterial, gonococcal, or chlamydial conjunctivitis } \\
\text { as outcomes, or this information could not be extracted from the study. Con- } \\
\text { sequently, there was no assessment of bias for this category with these out- } \\
\text { comes. }\end{array}$ \\
\hline $\begin{array}{l}\text { Incomplete outcome data } \\
\text { (attrition bias) } \\
\text { Clinical conjunctivitis } \\
\text { (subjective) }\end{array}$ & High risk & $\begin{array}{l}\text { COMMENT: } 32 \text { of } 300(10.6 \%) \text { neonates had no data reported. Only } 268 \text { had } \\
\text { follow-up data. It is unclear why there was no follow-up data for the other } 32 \\
\text { neonates. There is no further information. This number is high relative to event } \\
\text { rates. }\end{array}$ \\
\hline
\end{tabular}


Ghaemi 2014 (Continued) (attrition bias)

Bacterial, gonococcal and chlamydial conjunctivitis (objective)

Selective reporting (reporting bias)
Unclear risk

The study did not report bacterial, gonococcal, or chlamydial conjunctivitis as outcomes, or this information could not be extracted from the study. Consequently, there was no assessment of bias for this category with these outcomes.

QUOTE: "Overall, 300 preterm neonates, with culture negative eye swabs, were enrolled..."

QUOTE: "Using swab, ocular culture from each participant was examined and neonates with positive eye cultures were excluded from the study."

COMMENT: It appears that neonates with positive eye cultures on delivery, before application of prophylaxis, were excluded from the study for unexplained reasons. The primary initial purpose of the neonatal ophthalmia prophylaxis was to prevent transmission of pathogens from the mother's birth canal to the neonate's eyes. Therefore, this study appears to be evaluating the effect of neonatal prophylaxis on infections acquired from sources other than the mother.

Other bias Unclear risk

QUOTE: "The results of eye swab culture of all patients reported Staphlyococcus aureus"

COMMENT: It is unusual for all 66 neonates to have culture-positive conjunctivitis and all growing the same organism. Authors have been contacted for clarification.

\section{Study characteristics}

Methods $\quad$ Parallel-group, single-centre RCT.

Neonates "randomly divided". No further information on method of allocation provided.

Unit of randomisation: neonate.

Losses to follow-up: not discussed in paper.

Missing data handling: not discussed in paper.

Exclusions after allocation: none specified in paper.

Reported power calculation: no.

Unusual study design: none identified.

Participants

Setting: Gonabad, Iran.

Number allocated: unknown, but 130 were included in the study. Unknown if there were exclusions after allocation.

Age: neonates.

Sex: M:F 54.6\%:45.4\% of all neonates. M:F ratio: tetracycline group: 1.9; no-prophylaxis group: 1.3.

Inclusion criteria: full-term neonates born through the birth canal.

Exclusion criteria: infants born through caesarean section. Unclear if this exclusion occurred before or after allocation. 
Ghahramani 2007 (Continued)

Equivalence of baseline characteristics: yes; no statistically significant difference in the following characteristics:

1. birthweight;

2. M:F ratio;

3. gestational age.

Interventions

Number of interventions: 2 .

- Intervention 1: $0.5 \%$ erythromycin ointment into both eyes; dose not specified $(n=65)$.

- Intervention 2: no prophylaxis $(n=65)$.

Time to intervention: not specified.

Pre-intervention manoeuvres: none specified.

Postintervention manoeuvres: none specified.

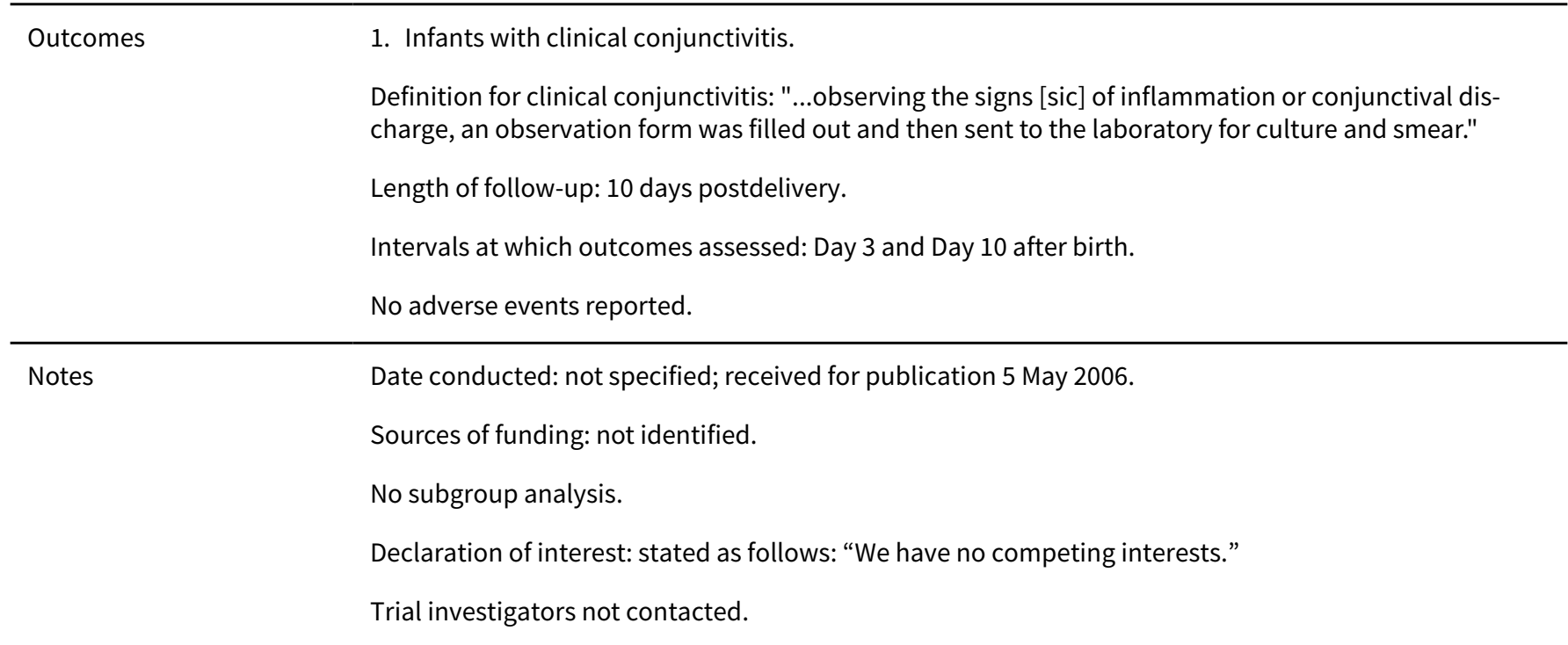

\section{Risk of bias}

\begin{tabular}{|c|c|c|}
\hline Bias & Authors' judgement & Support for judgement \\
\hline \multirow[t]{3}{*}{$\begin{array}{l}\text { Random sequence genera- } \\
\text { tion (selection bias) }\end{array}$} & Unclear risk & $\begin{array}{l}\text { COMMENT: Paper states "randomly divided". No further information on } \\
\text { method of allocation. }\end{array}$ \\
\hline & & Exact same number in each group: 65 and 65. \\
\hline & & $\begin{array}{l}\text { Insufficient information about the sequence generation process to permit } \\
\text { judgement. }\end{array}$ \\
\hline $\begin{array}{l}\text { Allocation concealment } \\
\text { (selection bias) }\end{array}$ & Unclear risk & Insufficient information to permit judgement. \\
\hline \multirow{2}{*}{$\begin{array}{l}\text { Blinding of study partici- } \\
\text { pants (mothers of infants) } \\
\text { Clinical conjunctivitis } \\
\text { (subjective) }\end{array}$} & Unclear risk & COMMENT: Masking of the mother was not addressed in this paper. \\
\hline & & $\begin{array}{l}\text { Erythromycin is a translucent ointment that the mother would initially notice } \\
\text { in the infant. There was no placebo in the allocation group that received no } \\
\text { prophylaxis. The mothers of neonates with noticeable medication of the eyes } \\
\text { may handle the eyes of the infant more than mothers of neonates with no pro- } \\
\text { phylaxis. This could lead to differential introduction of pathogenic bacteria in- } \\
\text { to the eyes of these neonates. }\end{array}$ \\
\hline
\end{tabular}


Ghahramani 2007 (Continued)

Blinding of study partici- Unclear risk The study did not report bacterial, gonococcal, or chlamydial conjunctivitis pants (mothers of infants) Bacterial, gonococcal and chlamydial conjunctivitis (objective) as outcomes, therefore there was no assessment of bias for this category with these outcomes.
Blinding of caregiver who administered medication Clinical conjunctivitis (subjective)
High risk

\section{COMMENT:}

Masking of the person who administers medication was not addressed in this paper.

Erythromycin is a translucent. There was no placebo in the allocation group that received no prophylaxis. Those who administer the medication would handle the eyes of neonates with erythromycin, but not neonates with no prophylaxis. This could lead to differential introduction of pathogenic bacteria into the eyes of these neonates.

Blinding of caregiver who

Unclear risk

The study did not report bacterial, gonococcal, or chlamydial conjunctivitis as outcomes, therefore there was no assessment of bias for this category with these outcomes.

Bacterial, gonococcal and

chlamydial conjunctivitis

(objective)

\begin{tabular}{|c|c|c|}
\hline Blinding of persons in- & Unclear risk & COMMENT: \\
\hline $\begin{array}{l}\text { Clinical conjunctivitis } \\
\text { (subjective) }\end{array}$ & & $\begin{array}{l}\text { Masking of the person who was involved in postnatal care was not addressed } \\
\text { in this paper. }\end{array}$ \\
\hline
\end{tabular}

Erythromycin ointment may leave a residual medication for a temporary period, such that those involved in postnatal care were likely not masked to the medication used as prophylaxis in the initial few hours.

Blinding of persons in- $\quad$ Unclear risk
volved in postnatal care
Bacterial, gonococcal and
chlamydial conjunctivitis
(objective)

The study did not report bacterial, gonococcal, or chlamydial conjunctivitis as outcomes, therefore there was no assessment of bias for this category with these outcomes.

Blinding of outcome as- Unclear risk sessment (detection bias) Clinical conjunctivitis (subjective)

COMMENT: The study does not mention masking of outcome assessors. In the
first hours of clinical assessment for conjunctivitis, there may be lack of masking, as erythromycin is an ointment that leaves residue. It is unclear how many cases of conjunctivitis were diagnosed during this time period. In

ambiguous cases of clinical conjunctivitis, whether identified by personnel, mother, or outcome assessor, there may be differential group behaviour to include or exclude cases of clinical conjunctivitis with lack of masking. In this paper, conjunctivitis was defined as signs of inflammation OR conjunctival discharge, which permits further subjectivity in the diagnosis of conjunctivitis.

\begin{tabular}{|c|c|c|}
\hline $\begin{array}{l}\text { Blinding of outcome as- } \\
\text { sessment (detection bias) } \\
\text { Bacterial, gonococcal and }\end{array}$ & Unclear risk & $\begin{array}{l}\text { The study did not report bacterial, gonococcal, or chlamydial conjunctivitis } \\
\text { as outcomes, therefore there was no assessment of bias for this category with } \\
\text { these outcomes. }\end{array}$ \\
\hline
\end{tabular}
chlamydial conjunctivitis (objective)
COMMENT: No reporting of incomplete outcome data. See Table 2 in paper where data appear to be present on all neonates.

(attrition bias)

Low risk

Clinical conjunctivitis

(subjective) 
Ghahramani 2007 (Continued)

Incomplete outcome data (attrition bias)

Bacterial, gonococcal and chlamydial conjunctivitis (objective)
Unclear risk

The study did not report bacterial, gonococcal, or chlamydial conjunctivitis as outcomes, therefore there was no assessment of bias for this category with these outcomes.
Selective reporting (re- High risk porting bias)
QUOTE: "Thus, all the neonates were examined on the third and tenth days after birth, and in case of observing signs of inflammation or conjunctival discharge, an observation form was filled out and then sent to the laboratory for culture and smear."

COMMENT: The paper did not report culture results despite sending neonates for culture and smear.

COMMENT: The study did not follow neonates for 28 days.

COMMENT: We ranked this study as at unclear risk of bias as there is a significant amount of information not reported in the study. Consequently, there is insufficient information to assess whether an important risk of bias exists.

\section{Ghotbi 2012}

\section{Study characteristics}

Methods

Parallel-group, single-centre RCT.

Unit of randomisation: neonate.

No losses to follow-up specified.

No exclusions after randomisation specified.

No comment on missing data.

No reported power calculation.

Participants

Setting: Medical Science University Pediatric Ward, Kurdistan, Sanandaj, Iran.

Number allocated: 330 .

Age: neonates.

Sex: M:F 143 (43\%):187 (57\%).

Inclusion criteria:

1. term neonates born by vaginal or C-section in the obstetrical ward of Sanandaj spring of 2011 to spring of 2012.

Exclusion criteria:

1. neonates with congenital ophthalmic anomalies;

2. neonates whose mothers had antibiotic treatment during the 48 hours before delivery;

3. neonates whose mothers had ruptured membranes during the 18 hours prior to delivery;

4. neonates with meconium aspiration.

Baseline characteristics reported. Equivalence for birthweight, type of delivery, level of literacy, age of the mother, parity of mother, vaginal infection. 
Ghotbi 2012 (Continued)

- Intervention 1: tetracycline ointment, $1 \mathrm{~cm}$; administered into eyes $(\mathrm{n}=110)$.

- Intervention 2: 0.5\% erythromycin ointment, $1 \mathrm{~cm}$; administered into eyes $(n=110)$.

- Intervention 3: no intervention $(n=110)$.

Time to intervention: 1 hour after birth.

Pre-intervention manoeuvres: cleaning, washing, and drying the infant, and after feeding breast milk.

Postintervention manoeuvres: none specified.

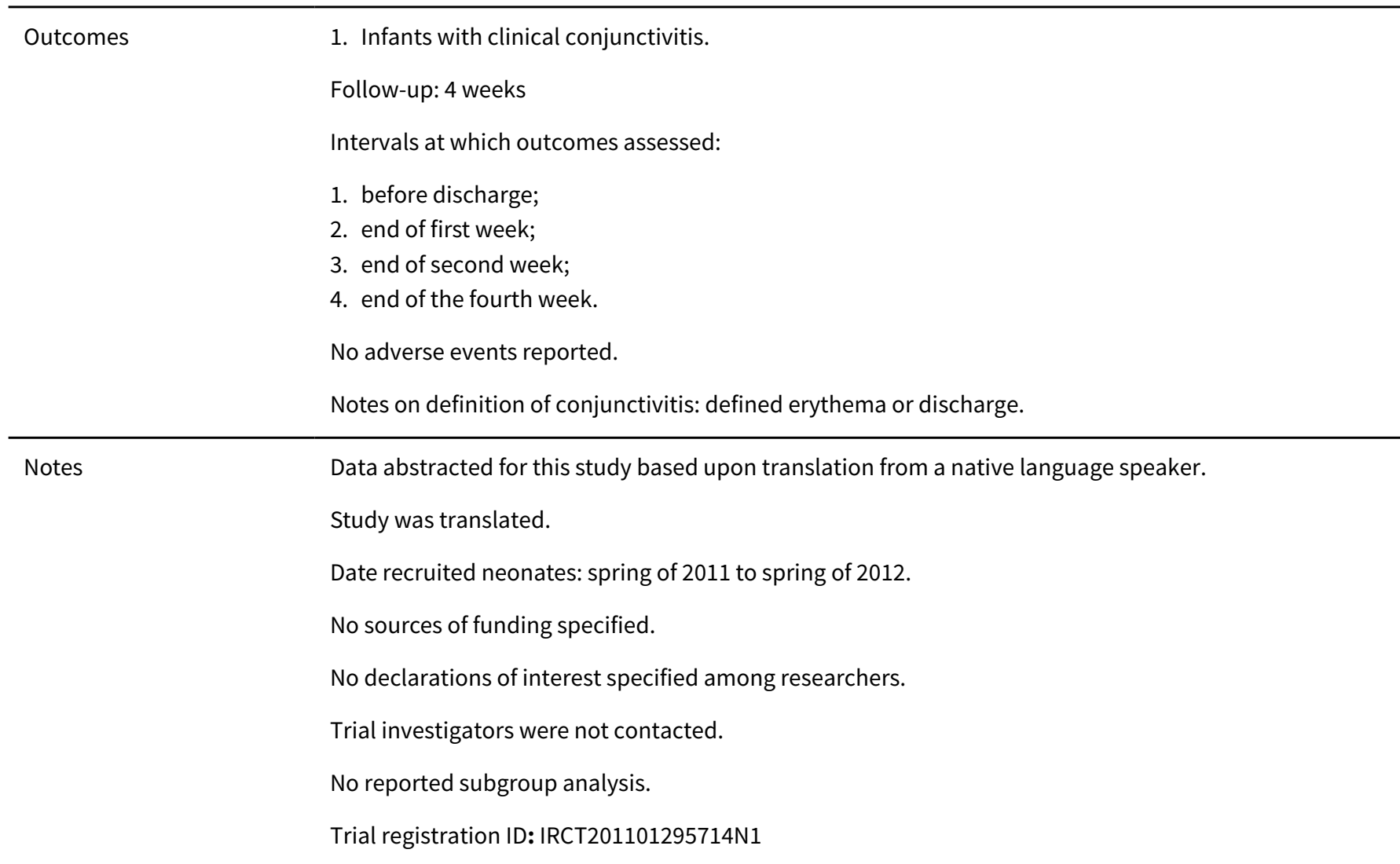

\section{Risk of bias}

\begin{tabular}{lll}
\hline Bias & Authors' judgement & Support for judgement \\
\hline $\begin{array}{ll}\text { Random sequence genera- } \\
\text { tion (selection bias) }\end{array}$ & Unclear risk & $\begin{array}{l}\text { QUOTE: (translated) "This study was a randomized controlled and single blin } \\
\text { ed in one center with no placebo". }\end{array}$ \\
& $\begin{array}{l}\text { COMMENT: No other information was provided on the random sequence } \\
\text { generation process. Insufficient information about the sequence generation } \\
\text { process to permit judgement. }\end{array}$
\end{tabular}

\begin{tabular}{lll}
\hline $\begin{array}{l}\text { Allocation concealment } \\
\text { (selection bias) }\end{array}$ & Unclear risk & Insufficient information to permit judgement. \\
\hline $\begin{array}{l}\text { Blinding of study partici- } \\
\text { pants (mothers of infants) }\end{array}$ & Unclear risk & $\begin{array}{l}\text { COMMENT: Masking of the mother was not addressed in this paper. Ery- } \\
\text { thromycin is a translucent ointment. Tetracycline is a light-yellow ointment. }\end{array}$ \\
$\begin{array}{l}\text { Clinical conjunctivitis } \\
\text { (subjective) }\end{array}$ & $\begin{array}{l}\text { Both medications leave a residue in the eyes that can be noticed for hours. } \\
\text { There was no placebo in the allocation group that received no prophylaxis. }\end{array}$ \\
& $\begin{array}{l}\text { The mothers of neonates with noticeable medication of the eyes may handle } \\
\text { the eyes of the infant more than mothers of neonates with no prophylaxis. This }\end{array}$
\end{tabular}


could lead to differential introduction of pathogenic bacteria into the eyes of these neonates, depending on hygiene measures.

\begin{tabular}{|c|c|c|}
\hline $\begin{array}{l}\text { Blinding of study partici- } \\
\text { pants (mothers of infants) } \\
\text { Bacterial, gonococcal and }\end{array}$ & Unclear risk & $\begin{array}{l}\text { The study did not report bacterial, gonococcal, or chlamydial conjunctivitis } \\
\text { as outcomes, therefore there was no assessment of bias for this category with } \\
\text { these outcomes. }\end{array}$ \\
\hline
\end{tabular}
chlamydial conjunctivitis (objective)

\section{Blinding of caregiver who High risk} administered medication Clinical conjunctivitis (subjective)
COMMENT: Masking of the person who administers medication was not addressed in this paper. Erythromycin is a translucent ointment. Tetracycline is a light-yellow ointment. There was no placebo in the allocation group that received no prophylaxis. Those who administer the medication would handle the eyes of neonates with erythromycin and tetracycline, but not neonates with no prophylaxis. This could lead to differential introduction of pathogenic bacteria into the eyes of these neonates, depending on hygiene measures. There may also be differences in adherence to medication administration of the person administering the medication, if there was no masking and there was bias.
Unclear risk The study did not report bacterial, gonococcal, or chlamydial conjunctivitis as outcomes, therefore there was no assessment of bias for this category with these outcomes.
Blinding of caregiver who administered medication Bacterial, gonococcal and chlamydial conjunctivitis (objective)

$\begin{array}{ll}\begin{array}{l}\text { Blinding of persons in- } \\ \text { volved in postnatal care }\end{array} & \text { Unclear risk } \\ \begin{array}{l}\text { Clinical conjunctivitis } \\ \text { (subjective) }\end{array} & \begin{array}{l}\text { COMMENT: Masking of the person who was involved in postnatal care was not } \\ \text { addressed in this paper. }\end{array} \\ & \begin{array}{l}\text { Erythromycin is a translucent ointment. Tetracycline is a light-yellow oint- } \\ \text { ment. There was no placebo in the allocation group that received no prophy- } \\ \text { laxis. }\end{array} \\ \begin{array}{l}\text { The ointments may leave a residual medication for a temporary period, such } \\ \text { that those involved in postnatal care were possibly not masked to at least no } \\ \text { prophylaxis and ointments in the initial few hours. }\end{array}\end{array}$

\begin{tabular}{|c|c|c|}
\hline $\begin{array}{l}\text { Blinding of persons in- } \\
\text { volved in postnatal care } \\
\text { Bacterial, gonococcal and }\end{array}$ & Unclear risk & $\begin{array}{l}\text { The study did not report bacterial, gonococcal, or chlamydial conjunctivitis } \\
\text { as outcomes, therefore there was no assessment of bias for this category with } \\
\text { these outcomes. }\end{array}$ \\
\hline
\end{tabular}
Bacterial, gonococcal and (objective)

\begin{abstract}
as outcomes, therefore there was no assessment of bias for this category with these outcomes.
\end{abstract}

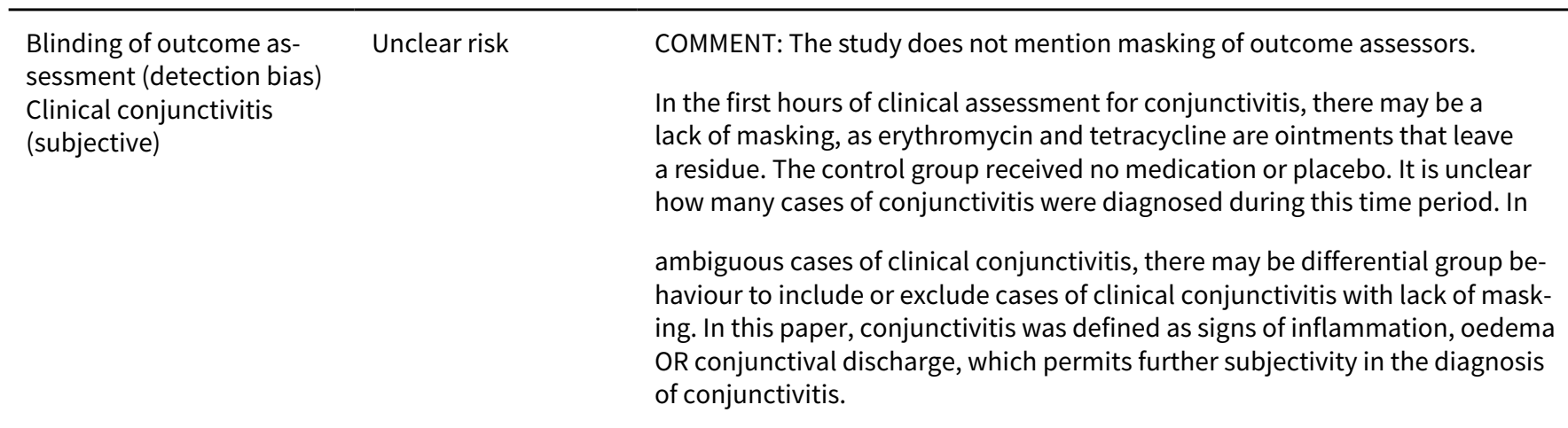

Blinding of outcome as-
sessment (detection bias) $\quad$ Unclear risk

The study did not report bacterial, gonococcal, or chlamydial conjunctivitis as outcomes, therefore there was no assessment of bias for this category with these outcomes. 
Ghotbi 2012 (Continued)

Bacterial, gonococcal and

chlamydial conjunctivitis

(objective)

Incomplete outcome data Unclear risk COMMENT: The study did not address incomplete outcome data.
(attrition bias)

(attrition bias)

Clinical conjunctivitis

(subjective)

\section{Incomplete outcome data Unclear risk}

(attrition bias)

Bacterial, gonococcal and

chlamydial conjunctivitis

(objective)
The study did not report bacterial, gonococcal, or chlamydial conjunctivitis as outcomes, therefore there was no assessment of bias for this category with these outcomes.

\section{Selective reporting (re- Unclear risk} porting bias)
COMMENT: The study appeared to only report clinical conjunctivitis cases. There was no mention in the methods or results section of the translated paper of any plan to culture conjunctivitis cases. The methods are not explicit on prespecified outcomes. There is no access to the study protocol. There is insufficient information to permit a judgement of low risk or high risk of bias.

COMMENT: The study did not follow neonates for 28 days.

Other bias Unclear risk

COMMENT: We ranked this study as at unclear risk of bias as there is a significant amount of information not reported in the study. Consequently, there is insufficient information to assess whether an important risk of bias exists.

Graf 1994

\section{Study characteristics}

Parallel-group, single-centre RCT.
Paper states "randomly" allocated, no additional detail available.
Unit of randomisation: neonate.
Number randomised: 40.
Exclusions after allocation: none specified and not addressed in paper.
Losses to follow-up: none specified and not addressed in paper.
Missing data: no comment on missing data.
No reported power calculation.
Unusual study design: none identified.

Participants

Setting: not described, according to translation; authors' address in Germany.

Number allocated: 40 neonates:

- silver nitrate: 20 ;

- no prophylaxis: 20.

Age: neonates.

Sex: M:F not described, according to translation. 
Graf 1994 (Continued)

\section{Inclusion criteria:}

1. vaginally delivered neonates;

2. parental consent.

Exclusion criteria: none, according to translation.

No comment on equivalence of baseline characteristics.

Interventions

Number of interventions: 2 .

- Intervention 1: silver nitrate $1 \%, 1$ drop in each eye $(n=20)$.

- Intervention 2: no prophylaxis $(n=20)$.

Time to intervention: not described.

Pre-intervention manoeuvres: none identified.

Postintervention manoeuvres: none identified.

1. eyelid - graded as having the presence or absence of the following:
a. hyperaemia;
b. oedema;
c. haematoma;
d. other.

2. conjunctiva tarsi or bulbi - graded according to the degree of hyperaemia:

a. mild: mild redness with hyperaemic vessels;

b. moderate: more pronounced redness, significantly hyperaemic conjunctiva;

c. severe: pronounced redness and thickening, significant conjunctival injection.

3. cornea - graded according to the presence or absence of the following:
a. epithelial irregularity;
b. staining with fluorescein on Day 2 .

Follow-up: daily examination for 5 days postpartum; fluorescein staining of the cornea performed on Day 2.

Definition of conjunctivitis or ophthalmia neonatorum: none identified.

Notes

Date study conducted not specified.

No source of funding specified.

No declaration of interest specified in study.

No known subgroup analysis.

German language article translated to English.

Authors written to for clarifications on unclear items, no reply received.

\section{Risk of bias}

\begin{tabular}{lll} 
Bias & Authors' judgement & Support for judgement \\
\hline $\begin{array}{l}\text { Random sequence genera- } \\
\text { tion (selection bias) }\end{array}$ & Unclear risk & QUOTE: “...randomly distributed into two groups” \\
& $\begin{array}{l}\text { COMMENT: No information is provided on the method chosen to determine } \\
\text { method of randomisation. }\end{array}$
\end{tabular}




\title{
Graf 1994 (Continued)
}

\section{Allocation concealment (selection bias)}

Blinding of study participants (mothers of infants) Clinical conjunctivitis (subjective)
Unclear risk

COMMENT: No information is provided.
Unclear risk
COMMENT: The study does address masking of mothers. The 2 interventions differ. There was no placebo in the allocation group that received no prophylaxis. Silver nitrate is a clear solution. Also, silver nitrate sometimes causes a chemical conjunctivitis that can last up to 72 hours, and silver nitrate causes lid stains that can last 30 to 48 hours. Therefore, it would be apparent which neonate was given silver nitrate and which neonate was given no prophylaxis. The parents of neonates with lid stains may systematically handle the eyes of the infant differently than parents of neonates without lid stains, potentially affecting the outcome of conjunctivitis.

\begin{abstract}
Blinding of study partici-
Unclear risk

The study did not report bacterial, gonococcal, or chlamydial conjunctivitis pants (mothers of infants) as outcomes, therefore there was no assessment of bias for this category with Bacterial, gonococcal and these outcomes.
\end{abstract} chlamydial conjunctivitis (objective)

Blinding of caregiver who administered medication Clinical conjunctivitis (subjective)
Unclear risk

COMMENT: The study does not mention whether the person administering the medication was masked as to which medication was used. However, no placebo was used, enabling the person administering the medication to be readily aware of which neonate received silver nitrate prophylaxis. The eyes of neonates with silver nitrate prophylaxis were handled by the person administering the medication, whereas the eyes of neonates in the no-prophylaxis group were not touched by the person dispensing medication. It is unclear how this could influence the outcome of conjunctivitis.

\begin{tabular}{|c|c|c|}
\hline $\begin{array}{l}\text { Blinding of caregiver who } \\
\text { administered medication } \\
\text { Bacterial, gonococcal and } \\
\text { chlamydial conjunctivitis } \\
\text { (objective) }\end{array}$ & Unclear risk & $\begin{array}{l}\text { The study did not report bacterial, gonococcal, or chlamydial conjunctivitis } \\
\text { as outcomes, therefore there was no assessment of bias for this category with } \\
\text { these outcomes. }\end{array}$ \\
\hline $\begin{array}{l}\text { Blinding of persons in- } \\
\text { volved in postnatal care } \\
\text { Clinical conjunctivitis } \\
\text { (subjective) }\end{array}$ & Unclear risk & $\begin{array}{l}\text { COMMENT: Since silver nitrate causes lid stains that can last up to } 30 \text { to } 48 \\
\text { hours, those involved in postnatal care were possibly not masked to the med- } \\
\text { ication used as prophylaxis until this time period. Furthermore, there is no } \\
\text { indication in the paper that an attempt was made to mask those involved in } \\
\text { postnatal care. The role of those involved in postnatal care on the outcome of } \\
\text { conjunctivitis measured is unclear. }\end{array}$ \\
\hline
\end{tabular}

\begin{tabular}{|c|c|c|}
\hline $\begin{array}{l}\text { Blinding of persons in- } \\
\text { volved in postnatal care } \\
\text { Bacterial, gonococcal and }\end{array}$ & Unclear risk & $\begin{array}{l}\text { The study did not report bacterial, gonococcal, or chlamydial conjunctivitis } \\
\text { as outcomes, therefore there was no assessment of bias for this category with } \\
\text { these outcomes. }\end{array}$ \\
\hline
\end{tabular}

Bacterial, gonococcal and

chlamydial conjunctivitis (objective) gory with these outcomes.

Blinding of outcome as-
sessment (detection bias)
Clinical conjunctivitis
(subjective)

COMMENT: The study states that the examiner was unaware of the treatment status of the neonate. As silver nitrate creates lid stains that last 30 to 48 hours, outcome assessments during this time were likely not masked. Furthermore, silver nitrate may induce a chemical conjunctivitis that can last up to 72 hours, further leading to loss of masking. This is an issue in any trial that uses silver nitrate, but in this trial follow-up was only 5 days. The study does not address these factors, i.e. lid stains and chemical conjunctivitis, which could affect masking, and subjective determination of clinical conjunctivitis.

$\begin{array}{lll}\begin{array}{l}\text { Blinding of outcome as- } \\ \text { sessment (detection bias) }\end{array} \quad \text { Unclear risk } & \begin{array}{l}\text { The study did not report bacterial, gonococcal, or chlamydial conjunctivitis } \\ \text { as outcomes, therefore there was no assessment of bias for this category with } \\ \text { these outcomes. }\end{array}\end{array}$


Graf 1994 (Continued)

Bacterial, gonococcal and

chlamydial conjunctivitis

(objective)

$\begin{array}{ll}\begin{array}{l}\text { Incomplete outcome data } \\ \text { (attrition bias) }\end{array} & \text { COM risk } \\ \begin{array}{l}\text { Clinical conjunctivitis } \\ \text { (subjective) }\end{array} & \begin{array}{l}\text { days in hospital. The neonates were discharged on Day } 6 . \text { It is therefore unlike- } \\ \text { ly that there was loss to follow-up, although this is not explicitly mentioned in } \\ \text { the study. }\end{array}\end{array}$

Incomplete outcome data Unclear risk

(attrition bias)

Unclear risk the study.

Bacterial, gonococcal and

The study did not report bacterial, gonococcal, or chlamydial conjunctivitis chlamydial conjunctivitis as outcomes, therefore there was no assessment of bias for this category with (objective) these outcomes.

\begin{tabular}{lll}
\hline $\begin{array}{l}\text { Selective reporting (re- } \\
\text { porting bias) }\end{array}$ & Low risk & $\begin{array}{l}\text { COMMENT: The study protocol was not available, but it appears that all ex- } \\
\text { pected outcomes were reported. }\end{array}$ \\
\hline Other bias & Unclear risk & Insufficient information to assess whether an important risk of bias exists \\
\hline
\end{tabular}

\section{Study characteristics}

Methods

Parallel-group, multicentre RCT.

Method of allocation: "randomly"; no other details on method of allocation provided.

Unit of randomisation: neonate.

Exclusions after allocation: none directly specified; possibly up to 5 as there were 5 "perinatal" deaths.

Losses to follow-up: none directly specified; 12 women who did not deliver at the University Hospital were likely lost pre-allocation. Also, for 7 chlamydia-positive women, there is no follow-up data, for reasons unknown (67 chlamydia-positive women, but follow-up data only on 60 neonates born to chlamydia-positive women).

Missing data handled by available-case analysis.

No reported power calculation.

Unusual study design: only a subgroup of infants born to 60 mothers who were chlamydia-positive were followed up.

\section{Participants}

Setting: University Hospital and satellite clinic, Seattle, WA, USA (satellite clinic serves a high-risk, inner-city population).

Number allocated: 559 to 564; 5 "perinatal" deaths - unclear whether this was before or after randomisation; only a subgroup of 60 neonates born to chlamydia-positive women were followed up.

- Silver nitrate: number allocated $=317$; subgroup of 36 born to chlamydia-positive mothers followed up.

- Erythromycin: number allocated $=242$; subgroup of 24 born to chlamydia-positive mothers followed up.

Age: neonates.

Sex: M:F unknown.

Inclusion criteria: 
Hammerschlag 1980 (Continued)

1. pregnant women first seen for prenatal care at the University Hospital, Seattle and those seen at a satellite maternal and infant care clinic that serves a high-risk, inner-city population;

2. written informed consent.

Exclusion criteria: none specified a priori.

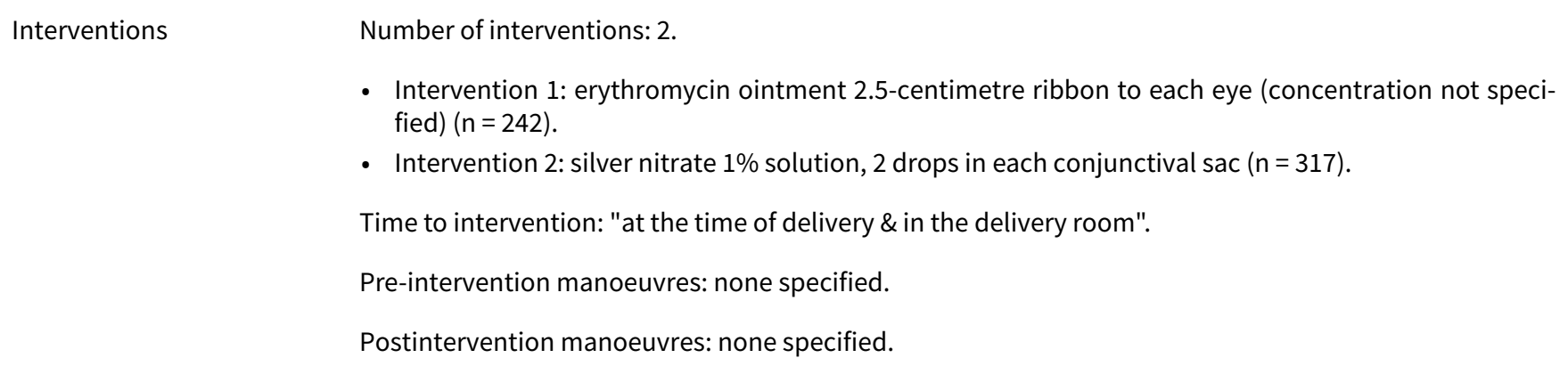

Outcomes Infants with chlamydial conjunctivitis.
2. Infants with positive nasopharyngeal cultures for chlamydia.
3. Infants with chlamydial pneumonia.
4. Infants with gonococcal ophthalmia.
Follow-up: 3 months; infants examined at 2 weeks, 6 weeks, and 3 months, or more frequently if clini-
cally indicated; follow-up data only available for infants born to chlamydia-positive mothers.
Definition of chlamydial conjunctivitis: "the presence of symptoms of conjunctivitis (discharge, erythe-
ma, swelling) associated with positive conjunctival cultures".
Adverse events: study reported rates of chlamydial pneumonia and rates of nasopharyngeal infection.
These are not adverse events.

Study funded by Dista Pharmaceuticals, which supplied erythromycin ointment, and the National Institute of Allergy and Infectious Diseases.

No declaration of interest specified.

Authors have been contacted for clarifications on unclear items, no reply received to date.

Subgroup analysis: amongst the 559 to 564 allocated, only a subgroup of 60 neonates born to chlamydia-positive women were followed up.

\section{Risk of bias}

Bias Authors' judgement Support for judgement

Random sequence genera- Unclear risk tion (selection bias)
QUOTE: "At the time of delivery, infants born to these women, randomly received either $1 \%$ silver nitrate drops or erythromycin ointment...".

COMMENT: No other information provided.

\begin{tabular}{lll}
\hline $\begin{array}{l}\text { Allocation concealment } \\
\text { (selection bias) }\end{array}$ & Unclear risk & $\begin{array}{l}\text { QUOTE: "At the time of delivery, infants born to these women, randomly re- } \\
\text { ceived either } 1 \% \text { silver nitrate drops or erythromycin ointment...". } \\
\text { COMMENT: No other information provided. }\end{array}$ \\
\hline $\begin{array}{l}\text { Blinding of study partici- } \\
\text { pants (mothers of infants) }\end{array}$ & Unclear risk & $\begin{array}{l}\text { All cases of clinical conjunctivitis were not reported as outcomes in this study, } \\
\text { only chlamydial and gonococcal conjunctivitis. Therefore, there was no as- } \\
\text { sessment of bias for this category for the outcome of clinical conjunctivitis. }\end{array}$
\end{tabular}


Hammerschlag 1980 (Continued)

Clinical conjunctivitis

(subjective)

Blinding of study participants (mothers of infants) Bacterial, gonococcal and chlamydial conjunctivitis (objective)
Unclear risk

COMMENT: Masking of mothers of the intervention was not addressed in this study. Furthermore, the 2 interventions differ in colour or consistency, or both. Erythromycin is a translucent ointment that may leave a residue in the eyes that can last for hours. Silver nitrate is a clear solution. Silver nitrate sometimes causes a chemical conjunctivitis that can last up to 72 hours, and lid stains that can last 30 to 48 hours. The mothers of the neonates with noticeable ocular residual ointment or silver nitrate stains may handle the eyes of the infant differently. This could lead to differential introduction of Chlamydia trachomatis bacteria into the eyes of these neonates in the case of poor hygiene. Alternatively, it could lead to contamination with other bacteria, subsequent conjunctivitis, and identification of chlamydia carriers instead.
Blinding of caregiver who administered medication Clinical conjunctivitis (subjective)
Unclear risk

All cases of clinical conjunctivitis were not reported as outcomes in this study, only chlamydial and gonococcal conjunctivitis. Therefore, there was no assessment of bias for this category for the outcome of clinical conjunctivitis.
Blinding of caregiver who administered medication Bacterial, gonococcal and chlamydial conjunctivitis (objective)
High risk

COMMENT: Masking of the person who administers the medication was not addressed in this study. As mentioned, the 2 interventions differ in colour or consistency, or both, making them readily identifiable. Any bias on the part of the person who administers the medication could affect adherence or compliance with method of application of the medication, which, in turn, could affect the medication's prophylactic effect against chlamydial conjunctivitis.

\section{Blinding of persons in-} volved in postnatal care Clinical conjunctivitis (subjective)
Unclear risk

All cases of clinical conjunctivitis were not reported as outcomes in this study, only chlamydial and gonococcal conjunctivitis. Therefore, there was no assessment of bias for this category for the outcome of clinical conjunctivitis.

\section{Blinding of persons in- \\ Unclear risk} volved in postnatal care Bacterial, gonococcal and chlamydial conjunctivitis (objective)
COMMENT: Masking of those involved in postnatal care was not addressed in this study. As mentioned, silver nitrate sometimes causes a chemical conjunctivitis that can last up to 24 hours, and lid stains that can last 30 to 48 hours. Erythromycin ointment may leave a residue in the eyes that can last for hours.

In this study, it is uncertain if there were cases of chlamydial conjunctivitis identified in the time period when masking would be affected. It is uncertain if those involved in postnatal care were also involved in identification of cases of conjunctivitis. If they were, and they were unmasked, this could influence decisions to identify and refer clinical conjunctivitis cases for culture. The definition of conjunctivitis used in this study is discharge, erythema swelling. It is uncertain if all signs had to be present or some or 1 , which would affect rates of diagnostic ambiguity. Although chlamydial conjunctivitis tends to be more severe, evidence shows that there is a clinical spectrum of presentation, and any bias, however minor, could have been significant considering the very low event rates in this trial.

\begin{tabular}{|c|c|c|}
\hline $\begin{array}{l}\text { Blinding of outcome as- } \\
\text { sessment (detection bias) } \\
\text { Clinical conjunctivitis } \\
\text { (subjective) }\end{array}$ & Unclear risk & $\begin{array}{l}\text { All cases of clinical conjunctivitis were not reported as outcomes in this study, } \\
\text { only chlamydial and gonococcal conjunctivitis. Therefore, there was no as- } \\
\text { sessment of bias for this category for the outcome of clinical conjunctivitis. }\end{array}$ \\
\hline $\begin{array}{l}\text { Blinding of outcome as- } \\
\text { sessment (detection bias) } \\
\text { Bacterial, gonococcal and } \\
\text { chlamydial conjunctivitis } \\
\text { (objective) }\end{array}$ & Unclear risk & $\begin{array}{l}\text { COMMENT: Masking of the person involved in outcome assessment was not } \\
\text { addressed in this paper. As mentioned, silver nitrate causes lid stains that can } \\
\text { last } 30 \text { to } 48 \text { hours, and erythromycin ointment leaves an ocular residue. Fur- } \\
\text { thermore, chlamydial conjunctivitis presents with a variable clinical spectrum. } \\
\text { Therefore, lack of masking, in cases of diagnostic ambiguity, may affect which } \\
\text { cases get referred for culture to identify chlamydial conjunctivitis. Under-refer- }\end{array}$ \\
\hline
\end{tabular}


ral for culture may be caused by the erroneous perception that conjunctivitis is chemical from silver nitrate, thereby missing chlamydial conjunctivitis cases. Over-referral may be caused by knowledge of which infants received silver nitrate, or bias, and lead to identifying cases of chemical conjunctivitis with chlamydia carrier, rather than conjunctivitis caused by $C$ trachomatis.

Incomplete outcome data Unclear risk (attrition bias)

Clinical conjunctivitis

(subjective)
All cases of clinical conjunctivitis were not reported as outcomes in this study, only chlamydial and gonococcal conjunctivitis. Therefore, there was no assessment of bias for this category for the outcome of clinical conjunctivitis.
Incomplete outcome data High risk (attrition bias)

Bacterial, gonococcal and

chlamydial conjunctivitis (objective)
QUOTE: "Chlamydia trachomatis was isolated from 67 (12\%) of the 572 women enrolled in the study.."

QUOTE: "Of the total 572 women enrolled in the study, 17 mother-infant pairs were excluded: 12 did not deliver at the University Hospital, and there were five perinatal deaths."

QUOTE: " ... a total of 559 infants were born to the remaining 555 women, including 60 infants born to Chlamydia-positive women."

QUOTE: "The infants were randomly assigned to ocular prophylaxis regimens: $317(57 \%)$ received silver nitrate, and $242(43 \%)$ received erythromycin after delivery"

QUOTE: "To assess the effect of silver nitrate or erythromycin on the subsequent development of chlamydial infection, we prospectively followed up 60 infants born to women with positive chlamydial culture"

COMMENT: The authors only followed up a subset of the 559 infants allocated to prophylaxis, i.e. the subset born to chlamydia-positive women.

COMMENT: There were 67 chlamydia-positive women, but outcome data on only 60 neonates. We are uncertain of the allocation group distribution of the neonates born to the missing 7 chlamydia-positive women. We are also uncertain of the proportion of the 7 missing neonates who eventually developed chlamydial conjunctivitis. These 2 pieces of information could be quite significant considering the low event rates: 12 of the neonates in the silver nitrate group developed chlamydial conjunctivitis, and zero in the erythromycin group developed chlamydial conjunctivitis. For instance, if the majority of the 7 missing neonates were in the erythromycin group, and the majority developed chlamydial conjunctivitis, this could significantly alter the differences between the 2 groups.

COMMENT: The allocation group of 17 excluded mother-infant pairs is unclear. As above, this is again significant considering the low event rates.

Selective reporting (re- High risk
porting bias)
QUOTE: "The infants were examined at two weeks, six weeks, and three months, or more frequently if clinically indicated. At each visit, cultures for $\mathrm{C}$. trachomatis were obtained from the conjunctivae and nasopharynx"

COMMENT: The study authors do not report cases of non-chlamydial conjunctivitis. They also do not report any cases of asymptomatic infants with positive chlamydial conjunctival cultures, which could identify chlamydia carriers. This may be important, as silver nitrate was used in this trial, and there could be chemical conjunctivitis with chlamydia carrier in the first 72 hours, as opposed to true chlamydial conjunctivitis. Finally, over 559 infants received prophylaxis during this study, but only a subgroup of infants born to 60 chlamydia-positive mothers were included in the study.
Other bias
Unclear risk
In any trial with silver nitrate, there could be diagnostic bias. 
Silver nitrate causes a chemical conjunctivitis in the first 72 hours. As a result, in the first 72 hours, more neonates in the silver nitrate allocation group could be referred for culture in the first 72 hours. Finding bacteria in the culture does not necessarily prove that the bacteria caused the conjunctivitis. The conjunctivitis could be chemical, but growing normal flora of the eye. Alternatively, the conjunctivitis could be chemical with a chlamydial carrier. Finally, the conjunctivitis could very well be caused by the bacteria or chlamydia. Consideration of incubation periods, assessing for carriers and normal flora with asymptomatic cases could assist with differential diagnosis.

Method of allocation: rotated monthly.

Unit of randomisation: neonate.

Exclusions after allocation: yes; not specified by allocation group: amongst 279 infants, 49 infants were excluded postallocation for the following reasons:

- 14 born by caesarean section with no rupture of membranes;

- 15 women received antibiotic therapy effective against Chlamydia trachomatis before delivery;

- 2 infants delivered at home;

- 2 infants received unknown prophylaxis;

- 16 infants received therapy against $C$ trachomatis before being seen by investigators.

Losses to follow-up: not specified by allocation group; 54 women lost to follow-up postenrolment, but likely pre-allocation.

Missing data were handled by available-case analysis.

No reported power calculation.

Unusual study design: 12,431 infants received prophylaxis, but only infants born to chlamydia-positive mothers were followed up.

\section{Participants}

Setting: Kings County Hospital Medical Center, Brooklyn, NY, USA.

Ethnicity: "Clinic serves a high-risk inner-city population of patients that is 90 percent black."

Number allocated: 12,431 infants received prophylaxis, but only 279 infants born to chlamydia-positive mothers were followed up.

Subroup followed up in each group:

- silver nitrate: 76 ;

- erythromycin: 92;

- tetracycline: 62 .

Age: neonates.

Sex: M:F unknown.

Inclusion criteria:

1. pregnant women presenting to prenatal clinic at Kings County Hospital Medical Center;

2. women screened for chlamydia infection;

3. women providing informed consent; 
Hammerschlag 1989 (Continued)

\author{
4. chlamydia-positive women.
}

Exclusion criteria: see exclusions after allocation above. No exclusion criteria were specified in the methods a priori.

No comment on equivalence of baseline characteristics.

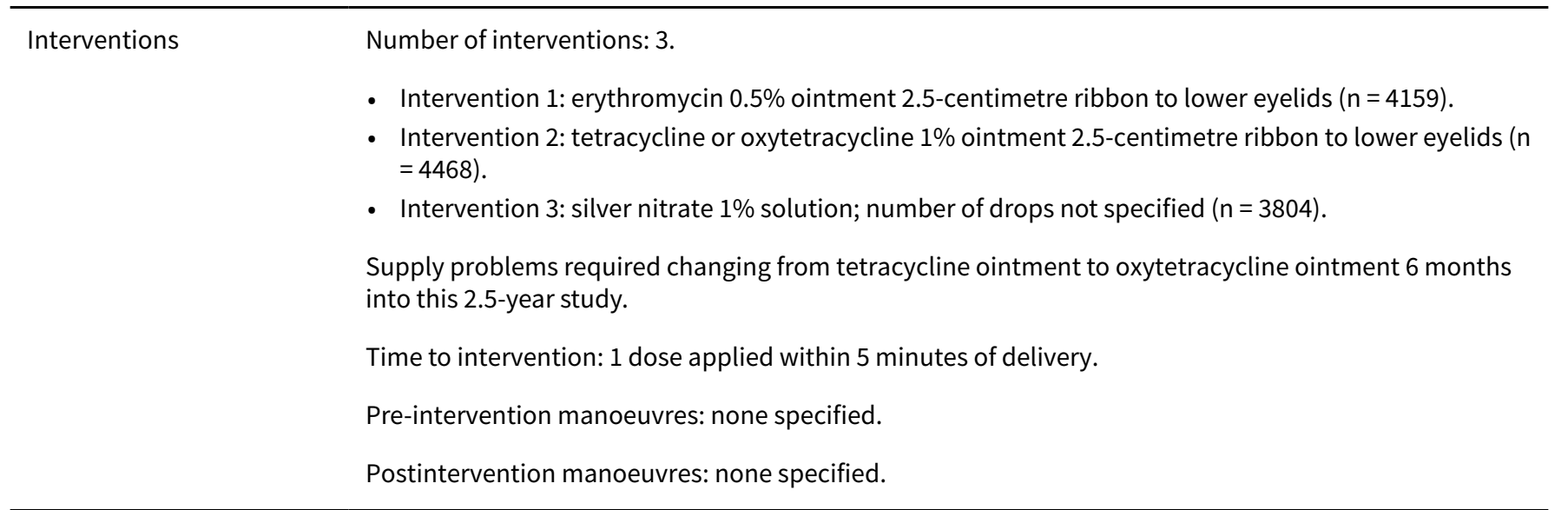

\begin{tabular}{ll}
\hline Outcomes & 1. Infants with chlamydial conjunctivitis. \\
2. Infants with nasopharyngeal infection. \\
3. Infants with chlamydial pneumonia. \\
Follow-up: from 2 to 19 weeks of age.
\end{tabular}

Most infants were seen at least 2 times from 2 to 19 weeks of age; the rest were seen 1 time between 2 and 16 weeks of age.

Definition of chlamydial conjunctivitis: presence of the symptoms of discharge, erythema, and swelling associated with positive chlamydial cultures.

No adverse events reported.

Authors have been contacted for clarifications on masking, no reply received.

Source of funding: supported by a grant from the Division of Maternal and Child Health, Bureau of Health Care Delivery Assistance.

No declaration of interest made in paper.

Subgroup analysis: 12,431 infants received prophylaxis, but only 279 infants born to chlamydia-positive mothers were followed up.

\title{
Risk of bias
}

\begin{tabular}{lll}
\hline Bias & Authors' judgement & Support for judgement \\
\hline $\begin{array}{l}\text { Random sequence genera- } \\
\text { tion (selection bias) }\end{array}$ & High risk & $\begin{array}{l}\text { QUOTE: "The prophylactic preparations ... were rotated monthly". } \\
\text { COMMENT: Sequence generated by odd or even date of birth. }\end{array}$ \\
\hline $\begin{array}{l}\text { Allocation concealment } \\
\text { (selection bias) }\end{array}$ & High risk & QUOTE: "The prophylactic preparations ... were rotated monthly". \\
& $\begin{array}{l}\text { COMMENT: Participants or investigators enrolling participants could possibly } \\
\text { foresee assignments and thus introduce selection bias based on date of birth. }\end{array}$
\end{tabular}


Hammerschlag 1989 (Continued) pants (mothers of infants) Clinical conjunctivitis (subjective)
Unclear risk

All cases of clinical conjunctivitis were not reported as outcomes in this study, only chlamydial and gonococcal conjunctivitis. Therefore, there was no assessment of bias for this category for the outcome of clinical conjunctivitis.
Blinding of study participants (mothers of infants) Bacterial, gonococcal and chlamydial conjunctivitis (objective)
Unclear risk

COMMENT: Masking of mothers of the intervention was not addressed in this study. Furthermore, the 3 interventions differ in colour or consistency, or both. Erythromycin is a translucent ointment, and tetracycline is a light-yellow ointment. Both ointments may leave a residue in the eyes that can last for hours. Silver nitrate is a clear solution. Silver nitrate sometimes causes a chemical conjunctivitis that can last up to 72 hours, and lid stains that can last 30 to 48 hours. Although it may be difficult to distinguish erythromycin from tetracycline, silver nitrate could be readily distinguished from the two ointments. The mothers of neonates with noticeable ocular residual ointment or silver nitrate stains may handle the eyes of the infant differently. This could lead to differential introduction of $C$ trachomatis bacteria into the eyes of these neonates in the case of poor hygiene. Alternatively, it could lead to contamination with other bacteria, subsequent conjunctivitis, and identification of chlamydia carriers instead. There is no evidence of Neisseria gonorrhoeae having a carrier status, but the chemical conjunctivitis and lid stains may lead to systematic differential handling of the eyes, which in cases of poor hygiene could bias the outcome of gonococcal conjunctivitis.
Blinding of caregiver who administered medication Clinical conjunctivitis (subjective)
Unclear risk

All cases of clinical conjunctivitis were not reported as outcomes in this study, only chlamydial and gonococcal conjunctivitis. Therefore, there was no assessment of bias for this category for the outcome of clinical conjunctivitis.
Blinding of caregiver who administered medication Bacterial, gonococcal and chlamydial conjunctivitis (objective)
High risk

COMMENT: Masking of the person who administers the medication was not addressed in this study. As mentioned, the 3 interventions differ in colour or consistency, or both, making them readily identifiable. Any bias on the part of the person who administers the medication could affect adherence or method of application of the medication, which, in turn, could affect the medication's prophylactic effect against chlamydial or gonococcal conjunctivitis. Allocation by month and the low event rates of chlamydial conjunctivitis magnify any potential bias.

\begin{tabular}{|c|c|c|}
\hline $\begin{array}{l}\text { Blinding of persons in- } \\
\text { volved in postnatal care } \\
\text { Clinical conjunctivitis }\end{array}$ & Unclear risk & $\begin{array}{l}\text { All cases of clinical conjunctivitis were not reported as outcomes in this study, } \\
\text { only chlamydial and gonococcal conjunctivitis. Therefore, there was no as- } \\
\text { sessment of bias for this category for the outcome of clinical conjunctivitis. }\end{array}$ \\
\hline
\end{tabular}

(subjective)

Blinding of persons involved in postnatal care Bacterial, gonococcal and chlamydial conjunctivitis (objective)
Unclear risk

COMMENT: Masking of those involved in postnatal care was not addressed in this study. As mentioned, silver nitrate sometimes causes a chemical conjunctivitis that can last up to 24 hours, and lid stains that can last 30 to 48 hours. Both of the ointments may leave a residue in the eyes that can last for hours.

In this study, the mean onset of chlamydial conjunctivitis was $15+/-14$ days with a range of 1 to 61 days, and median of 11.5 days. Consequently, there were cases of chlamydial conjunctivitis identified in the period of time when masking would be affected. The days of onset of gonococcal conjunctivitis were not identified in the study. It is uncertain if those involved in postnatal care were also involved in identification of cases of conjunctivitis. If they were, and they were unmasked, this could influence decisions to identify and refer clinical conjunctivitis cases for culture. The definition of conjunctivitis used is more robust and includes erythema, swelling AND discharge; and chlamydial and gonococcal conjunctivitis tends to be more severe, thereby reducing diagnostic ambiguity. However, evidence shows that there is a clinical spectrum of 

nor, could have been significant considering low event rates.

\begin{tabular}{|c|c|c|}
\hline $\begin{array}{l}\text { Blinding of outcome as- } \\
\text { sessment (detection bias) } \\
\text { Clinical conjunctivitis } \\
\text { (subjective) }\end{array}$ & Unclear risk & $\begin{array}{l}\text { All cases of clinical conjunctivitis were not reported as outcomes in this study, } \\
\text { only chlamydial and gonococcal conjunctivitis. Therefore, there was no as- } \\
\text { sessment of bias for this category for the outcome of clinical conjunctivitis. }\end{array}$ \\
\hline
\end{tabular}

\section{Blinding of outcome as- Unclear risk} sessment (detection bias) Bacterial, gonococcal and chlamydial conjunctivitis (objective)

\begin{abstract}
COMMENT: Masking of the person involved in outcome assessment was not addressed in this paper. As mentioned, silver nitrate causes lid stains that can last 30 to 48 hours, and the mean onset of chlamydial conjunctivitis in this study was $15+/-14$ days with a range of 1 to 61 days, and median of 11.5 days. In fact, chlamydial conjunctivitis developed in $74 \%$ of the infants before they were 14 days of age. Furthermore, chlamydial conjunctivitis presents with a variable clinical spectrum. Therefore, lack of masking, in cases of diagnostic ambiguity, may affect which cases get referred for culture to identify chlamydial conjunctivitis. Under-referral for culture may be caused by the erroneous perception that conjunctivitis is chemical from silver nitrate, thereby missing chlamydial conjunctivitis cases. Over-referral may be caused by knowledge of which infants received silver nitrate, or bias, and lead to identifying cases of chemical conjunctivitis with chlamydia carrier, rather than conjunctivitis caused by $\mathrm{C}$ trachomatis.
\end{abstract}

This study does not report the mean age of onset of gonococcal conjunctivitis, therefore, the effect of silver nitrate lid stains on blinding of the outcome assessment is uncertain.

All cases of clinical conjunctivitis were not reported as outcomes in this study, only chlamydial and gonococcal conjunctivitis. Therefore, there was no assessment of bias for this category for the outcome of clinical conjunctivitis.

\author{
Incomplete outcome data Unclear risk \\ (attrition bias) \\ Clinical conjunctivitis \\ (subjective)
}

\section{Incomplete outcome data High risk} (attrition bias)

Bacterial, gonococcal and chlamydial conjunctivitis (objective)
QUOTE: "The prophylactic preparations...were rotated monthly, from January 1986 through June 1988, and all newborns received them."

QUOTE: "A total of 4357 women were screened, 341 (8 percent) were found to have chlamydial infection."

QUOTE: "Of the 341 pregnant women with chlamydial infection..."

QUOTE: "The remaining 230 infants born to women with chlamydial infection form the population studied for chlamydial conjunctivitis."

COMMENT: The denominator used in this study is the subset of 230 infants born to a subset of mothers with chlamydia, amongst the 4357 women enrolled in the study. The subset of women with chlamydia infection was actually 341 , but was reduced to 230 after exclusions and loss to follow-up. The distribution of women excluded or lost to follow-up by allocation group is unclear. The number of women out of the 4357 that were allocated to each intervention is also unclear.

COMMENT: Postenrolment, 54 out of 339 chlamydia-positive enrolled women were lost to follow-up. It is unclear whether this was pre-allocation or postallocation of prophylaxis. 8 women out of 339 enrolled had a miscarriage or stillbirth. It is unclear how these women were distributed across the allocation groups.

COMMENT: Amongst 279 infants allocated and given prophylaxis, 49 were excluded from the analysis. However, their distribution across intervention groups was not reported. Conjunctivitis developed in 1 of the excluded infants and was treated in the emergency room, but no chlamydial cultures were conducted; the paper did not report the allocation group of this infant. 
COMMENT: Amongst the 49 excluded infants, 14 were born by caesarean section with no rupture of membranes; 15 received antibiotic therapy effective against $C$ trachomatis before delivery; 2 infants were delivered at home with some delay before application of prophylaxis; 2 infants received unknown prophylaxis; and 16 infants received therapy effective against $C$ trachomatis before being assessed. We were unable to impute outcomes for these data in sensitivity analyses or intention-to-treat analysis, as the intervention groups were not specified.

QUOTE: "None of the other excluded infants had an illness compatible with chlamydial infection or had positive chlamydial cultures"

COMMENT: It is unclear if this statement in the study pertains to all the 49 mother-infant pairs excluded from analysis.

COMMENT: The ratio of participants with missing data to participants with events was minimum 49/35 (1.4) or maximum 93/35 (2.7), if we include the 54 women lost to follow-up. Either ratio suggests a high risk of bias, in relation to low event rates, especially considering that the allocation method was monthly rotation.
Selective reporting (reporting bias)
High risk

QUOTE: "Infants born to mothers with chlamydial infection were seen at two weeks, six weeks and three months of age, or more frequently if clinically indicated. A cohort of infants born to mothers without chlamydial infection was also followed at the same intervals...Specimens for chlamydial culture were obtained from the conjunctivae and nasopharynx at each visit, whether or not the infant was symptomatic."

COMMENT: The study authors do not report cases of non-chlamydial conjunctivitis. They also do not report any cases of asymptomatic infants with positive chlamydial conjunctival cultures, which could identify chlamydia carriers. This may be important as silver nitrate was used in this trial, and there could be chemical conjunctivitis with chlamydia carrier in the first 72 hours, as opposed to true chlamydial conjunctivitis. Finally, over 12,431 infants received prophylaxis during this study, but only a subgroup of infants born to 341 chlamydia-positive mothers were included in the study, and only 230 were followed up.

QUOTE: "During the 30-month period from January 1986 through June 1988, gonococcal ophthalmia developed in 8 of 12, 431 infants born at Kings County Hospital. Seven of the mothers of these infants had received no prenatal care."

COMMENT: These mothers and infants entered the trial through a different route, as they had no prenatal screening.

Other bias Unclear risk

In any trial with silver nitrate, there could be diagnostic bias.

Silver nitrate causes a chemical conjunctivitis in the first 72 hours. As a result, in the first 72 hours, more neonates in the silver nitrate allocation group could be referred for culture in the first 72 hours. Finding bacteria in the culture does not necessarily prove that the bacteria caused the conjunctivitis. The conjunctivitis could be chemical, but growing normal flora of the eye. Alternatively, the conjunctivitis could be chemical with a chlamydial carrier. Finally, the conjunctivitis could very well be caused by the bacteria or chlamydia. Consideration of incubation periods, and assessing for carriers and normal flora with asymptomatic cases, could assist with differential diagnosis. 
Harris 1957

\section{Study characteristics}

Methods

Parallel-group, single-centre trial.

Method of allocation: alternate neonates.

Unit of randomisation: neonate.

Exclusions after allocation: none specified and not addressed in paper.

Losses to follow-up: none specified and not addressed in paper.

Missing data: no comment on how missing data were handled.

No reported power calculation.

Unusual study design: outcome definition was changed part way through the study.

Participants Setting: Beyer Memorial Hospital, Ypsilanti, MI, USA.

Number allocated: 2424 neonates;

- penicillin: 1219

- silver nitrate: 1205.

Age: neonates.

Sex: M:F not specified.

Inclusion criteria: all infants born 1 March 1955 to 29 February 1956 at Beyer Memorial Hospital.

Exclusion criteria: none specified.

No comment in study on equivalence of baseline characteristics.

Interventions Number of interventions: 2 .

- Intervention 1: penicillin G 1\% ophthalmic ointment $(n=1219)$.

- Intervention 2: silver nitrate $1 \%$ solution $(n=1205)$.

Time to intervention: not specified.

Pre-intervention manoeuvres: none specified.

Postintervention manoeuvres: none specified.

Outcomes

Used 2 classification schemes. 1 was changed to the other during the study:

Initial classification scheme:

0 - no conjunctival reaction;

1 - mild erythema of conjunctiva without discharge;

2 - moderate erythema of conjunctiva without discharge;

3 - moderate erythema of conjunctiva with discharge;

4 - severe erythema of conjunctiva with discharge.

Subsequent classification scheme:

1 - no conjunctivitis;

2 - conjunctivitis without discharge;

3 - conjunctivitis with discharge.

All conjunctivitis with discharge had smears and cultures made of the discharge.

The results reported total "reactions" and subdivided each "reaction" as either: 
Harris 1957 (Continued)

1. mild without discharge; or

2. severe with discharge.

Follow-up: newborn nursery nurses examined infants on the 2 nd and 5 th days postpartum.

Definition of conjunctivitis: not explicitly defined.

Notes on outcomes:

1. Apparently, about one-half of two-thirds of the conjunctivitis cases followed were identified as congenitally obstructed tear ducts, but these results were not reported by intervention group. It is unknown if these were subtracted from the total. It is unknown what criteria were used to make the diagnosis of obstructed tear ducts.

2. There was 1 case of gram-negative diplococcus on smear only, but not on culture; allocation group is unknown.

Notes

Date study conducted: 1 March 1955 to 29 February 1956.

No source of funding specified.

No declaration of interest statement made.

No reported subgroup analysis.

Trial investigators were not contacted.

\section{Risk of bias}

\begin{tabular}{lll}
\hline Bias & Authors' judgement & Support for judgement \\
\hline $\begin{array}{l}\text { Random sequence genera- } \\
\text { tion (selection bias) }\end{array}$ & High risk & $\begin{array}{l}\text { QUOTE: "Alternate babies had one per cent penicillin G. ophthalmic ointment } \\
\text { supplied by Abbott Laboratories used in their eyes in the delivery room." }\end{array}$
\end{tabular}

Allocation concealment $\quad$ High risk
(selection bias)

QUOTE: "Alternate babies had one per cent penicillin G. ophthalmic ointment supplied by Abbott Laboratories used in their eyes in the delivery room."

COMMENT: Allocation was based on alternation, therefore participants or investigators enrolling participants could possibly have foreseen assignments, thus introducing selection bias.

\begin{tabular}{|c|c|c|}
\hline $\begin{array}{l}\text { Blinding of study partici- } \\
\text { pants (mothers of infants) } \\
\text { Elinical conjunctivitis }\end{array}$ & Unclear risk & $\begin{array}{l}\text { COMMENT: The study does not mention whether mothers were masked to the } \\
\text { intervention. The } 2 \text { interventions differ in colour and consistency. Silver nitrate } \\
\text { is a clear solution, and penicillin is a clear or white ointment. }\end{array}$ \\
\hline
\end{tabular}

(subjective)

Furthermore, silver nitrate sometimes causes eyelid stain that can last for 30 to 48 hours and a chemical conjunctivitis that can last up to 72 hours. Penicillin is an ointment that can leave residue for hours and be noticed in the infant by the mother.

\begin{tabular}{|c|c|c|}
\hline $\begin{array}{l}\text { Blinding of study partici- } \\
\text { pants (mothers of infants) } \\
\text { Bacterial, gonococcal and } \\
\text { chlamydial conjunctivitis } \\
\text { (objective) }\end{array}$ & Unclear risk & $\begin{array}{l}\text { The study did not report bacterial, gonococcal, or chlamydial conjunctivitis } \\
\text { as outcomes, therefore there was no assessment of bias for this category with } \\
\text { these outcomes. }\end{array}$ \\
\hline $\begin{array}{l}\text { Blinding of caregiver who } \\
\text { administered medication } \\
\text { Clinical conjunctivitis } \\
\text { (subjective) }\end{array}$ & High risk & $\begin{array}{l}\text { COMMENT: The study does not address masking of the person who adminis- } \\
\text { ters the medication. The } 2 \text { interventions differ in colour and consistency. Silve } \\
\text { nitrate is a clear solution, and penicillin is a clear or white ointment. } \\
\text { Lack of masking by the person who administers the medication could influ- } \\
\text { ence the outcome of clinical conjunctivitis. There could be differences in ad- }\end{array}$ \\
\hline
\end{tabular}


herence to method of dispensing based on any bias by the person applying the ocular prophylaxis.
Blinding of caregiver who administered medication Bacterial, gonococcal and chlamydial conjunctivitis (objective)
Unclear risk

The study did not report bacterial, gonococcal, or chlamydial conjunctivitis as outcomes, therefore there was no assessment of bias for this category with these outcomes.

\section{Blinding of persons in- Unclear risk} volved in postnatal care Clinical conjunctivitis (subjective)

\begin{abstract}
QUOTE: "Memoranda of which (silver nitrate or penicillin) was used were sent directly to the record room so that the personnel of the newborn nursery would not know which had been used."
\end{abstract}

QUOTE: "Nurses in the newborn nursery examined all babies' eyes on the second and fifth days postpartum."

COMMENT: Silver nitrate is a clear solution, and penicillin is an ointment. Furthermore, silver nitrate sometimes causes eyelid stain that can last for 30 to 48 hours and a chemical conjunctivitis that can last up to 72 hours. This could affect masking of those involved in postnatal care. Apparently the nurses involved in the newborn nursery were involved in outcome assessment also. Even though there were efforts to mask the nurses to intervention, follow-up time in this study was only 5 days.

\begin{tabular}{|c|c|c|}
\hline $\begin{array}{l}\text { Blinding of persons in- } \\
\text { volved in postnatal care } \\
\text { Bacterial, gonococcal and } \\
\text { chlamydial conjunctivitis } \\
\text { (objective) }\end{array}$ & Unclear risk & $\begin{array}{l}\text { The study did not report bacterial, gonococcal, or chlamydial conjunctivitis } \\
\text { as outcomes, therefore there was no assessment of bias for this category with } \\
\text { these outcomes. }\end{array}$ \\
\hline $\begin{array}{l}\text { Blinding of outcome as- } \\
\text { sessment (detection bias) } \\
\text { Clinical conjunctivitis } \\
\text { (subjective) }\end{array}$ & Unclear risk & $\begin{array}{l}\text { QUOTE: "Memoranda of which (silver nitrate or penicillin) was used were } \\
\text { sent directly to the record room so that the personnel of the newborn nursery } \\
\text { would not know which had been used." } \\
\text { QUOTE: "Nurses in the newborn nursery examined all babies' eyes on the sec- } \\
\text { ond and fifth days postpartum." } \\
\text { COMMENT: Silver nitrate is a clear solution, and penicillin is an ointment. Fur- } \\
\text { thermore, silver nitrate sometimes causes eyelid stain that can last for } 30 \text { to } 48 \\
\text { hours and a chemical conjunctivitis that can last up to } 72 \text { hours. This could af- } \\
\text { fect masking of those involved in outcome assessments. Apparently the nurses } \\
\text { involved in the newborn nursery were involved in outcome assessment. Even } \\
\text { though there were efforts to mask the nurses to intervention, follow-up time in } \\
\text { this study was only } 5 \text { days. }\end{array}$ \\
\hline
\end{tabular}

Blinding of outcome as- Unclear risk The study did not report bacterial, gonococcal, or chlamydial conjunctivitis sessment (detection bias) Bacterial, gonococcal and chlamydial conjunctivitis (objective) as outcomes, therefore there was no assessment of bias for this category with these outcomes.

\footnotetext{
Incomplete outcome data Unclear risk (attrition bias)

COMMENT: The study did not comment on exclusions or loss to follow-up. This Clinical conjunctivitis (subjective) was likely low, as the follow-up time was only 5 days.

Incomplete outcome data Unclear risk (attrition bias)

The study did not report bacterial, gonococcal, or chlamydial conjunctivitis as outcomes, therefore there was no assessment of bias for this category with these outcomes.
} 
Harris 1957 (Continued)

Bacterial, gonococcal and chlamydial conjunctivitis (objective)

Selective reporting (re- High risk
porting bias)

QUOTE: "Nurses in the newborn nursery examined all babies' eyes on the second and fifth days postpartum and marked the chart as follows:...It was soon found that such differentiation was not practical and three classifications has to be used."

COMMENT: The classification of categorising ocular signs was modified during the study.

QUOTE: "Only one case showed bacteria on smear and in this case nothing grew on culture."

Other bias Unclear risk

COMMENT: In any trial with silver nitrate, there could be diagnostic bias.

Silver nitrate causes a chemical conjunctivitis in the first 72 hours. As a result, in the first 72 hours, more neonates in the silver nitrate allocation group could be referred for culture in the first 72 hours. Finding bacteria in the culture does not necessarily prove that the bacteria caused the conjunctivitis. The conjunctivitis could be chemical, but growing normal flora of the eye. Alternatively, the conjunctivitis could be chemical with a chlamydial carrier. Finally, the conjunctivitis could very well be caused by the bacteria or chlamydia. Consideration of incubation periods, and assessing for carriers and normal flora with asymptomatic cases, could assist with differential diagnosis.

\section{Hick 1985}

\section{Study characteristics}

Methods

Parallel-group, single-centre RCT.

Method of allocation: randomisation by a pre-randomised ledger.

Unit of randomisation: neonate.

Exclusions after allocation:

1. infants with a history of treated eye infection at the 2-week check-up;

2. infants with evidence of conjunctival inflammation at the time of the 2-week check-up.

Losses to follow-up: yes; complete follow-up data available for 145 of 496 neonates.

Missing data handled by available-case analysis.

No reported power calculation.

Unusual study design: none

Participants

Setting: Rochester Methodist Hospital, Rochester, MN, USA.

Number allocated: 496; unknown how many allocated to each group:

- tetracycline: only 69 followed up;

- silver nitrate: only 76 followed up.

Age: neonates.

Sex: M:F unknown. 
Hick 1985 (Continued)

Inclusion criteria: newborns of consenting parents at Rochester Methodist Hospital.

Exclusion criteria: none specified.

No comment on equivalence of baseline characteristics.

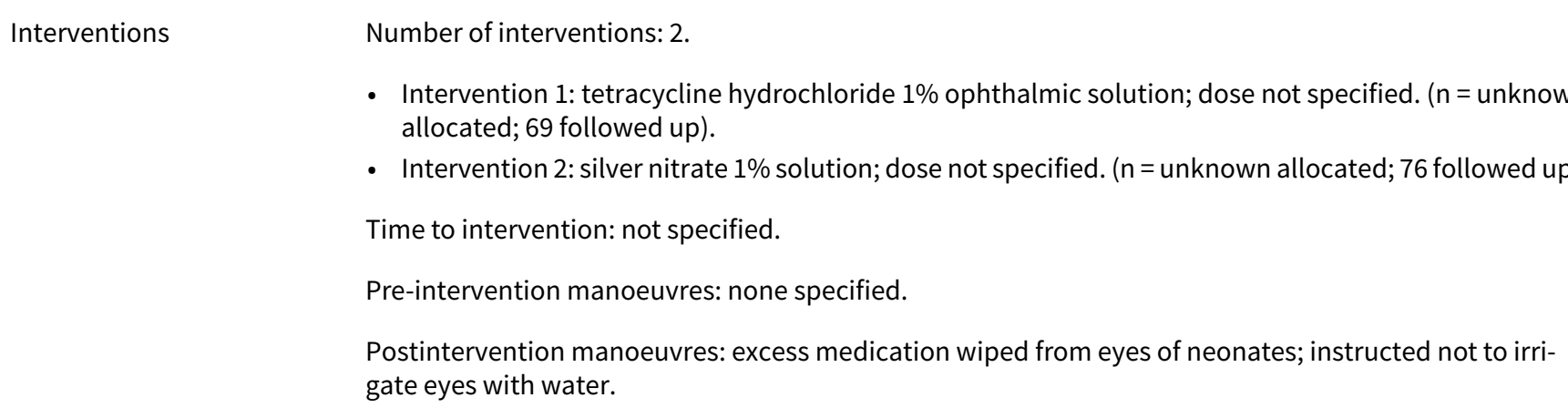

Outcomes

1. Nasolacrimal duct obstruction.

Follow-up: 2 months. Parents of children questioned at the time of 2-week and 2-month well-child examinations.

Definition of nasolacrimal duct obstruction: chronic eye discharge at 2-week visit that persisted to 2month visit in the absence of signs of conjunctival inflammation.

No other outcomes or adverse events reported. Nasolacrimal duct obstruction is considered to be an adverse event of silver nitrate.

Notes

Date study conducted: not specified.

No source of funding identified.

No declaration of interest made.

No reported subgroup analysis.

Trial investigators were contacted and reply received.

\section{Risk of bias}

Bias Authors' judgement Support for judgement
tion (selection bias)
Random sequence genera- Low risk

QUOTE: "All newborns of consenting parents at Rochester Methodist Hospital were randomized to one of two ophthalmic prophylaxis treatment groups,..."

QUOTE: "Randomization was accomplished through the use of a pre-randomized ledger".

COMMENT: Insufficient information about the sequence generation process to permit judgement.

$\begin{array}{ll}\begin{array}{l}\text { Allocation concealment } \\ \text { (selection bias) }\end{array} & \text { COW risk } \\ & \begin{array}{l}\text { letter from author states that sequence of allocation of participants to groups } \\ \text { was concealed until after treatments had been allocated. Method was not de- } \\ \text { scribed. }\end{array}\end{array}$

Blinding of study partici- Unclear risk

pants (mothers of infants)

Total number of all cases of clinical conjunctivitis was not reported as an outcome in this study. Consequently, there was no assessment of bias for this catClinical conjunctivitis egory for the outcome of clinical conjunctivitis. 
Hick 1985 (Continued)

Blinding of study participants (mothers of infants) Bacterial, gonococcal and chlamydial conjunctivitis (objective)
Unclear risk The study did not report bacterial, gonococcal, or chlamydial conjunctivitis as outcomes, therefore there was no assessment of bias for this category with these outcomes.
Blinding of caregiver who administered medication Clinical conjunctivitis (subjective)
Unclear risk

Total number of all cases of clinical conjunctivitis was not reported as an outcome in this study. Consequently, there was no assessment of bias for this category for the outcome of clinical conjunctivitis.
Blinding of caregiver who administered medication Bacterial, gonococcal and chlamydial conjunctivitis (objective)
Unclear risk

The study did not report bacterial, gonococcal, or chlamydial conjunctivitis as outcomes, therefore there was no assessment of bias for this category with these outcomes.

\begin{tabular}{ll} 
Blinding of persons in- & Unclear risk \\
volved in postnatal care & \\
Clinical conjunctivitis & \\
(subjective) & \\
\hline Blinding of persons in- & Unclear risk \\
volved in postnatal care & \\
Bacterial, gonococcal and & \\
chlamydial conjunctivitis & \\
(objective) &
\end{tabular}

Total number of all cases of clinical conjunctivitis was not reported as an outcome in this study. Consequently, there was no assessment of bias for this category for the outcome of clinical conjunctivitis.

\begin{tabular}{|c|c|c|}
\hline $\begin{array}{l}\text { Blinding of outcome as- } \\
\text { sessment (detection bias) } \\
\text { Clinical conjunctivitis } \\
\text { (subjective) }\end{array}$ & Unclear risk & $\begin{array}{l}\text { Total number of all cases of clinical conjunctivitis was not reported as an out- } \\
\text { come in this study. Consequently, there was no assessment of bias for this cat- } \\
\text { egory for the outcome of clinical conjunctivitis. }\end{array}$ \\
\hline
\end{tabular}

The study did not report bacterial, gonococcal, or chlamydial conjunctivitis as outcomes, therefore there was no assessment of bias for this category with these outcomes.
Blinding of outcome as- sessment (detection bias) Bacterial, gonococcal and chlamydial conjunctivitis (objective)

Unclear risk The study did not report bacterial, gonococcal, or chlamydial conjunctivitis as outcomes, therefore there was no assessment of bias for this category with these outcomes.

\begin{tabular}{|c|c|c|}
\hline $\begin{array}{l}\text { Incomplete outcome data } \\
\text { (attrition bias) } \\
\text { Clinical conjunctivitis } \\
\text { (subjective) }\end{array}$ & Unclear risk & $\begin{array}{l}\text { Total number of all cases of clinical conjunctivitis was not reported as an out- } \\
\text { come in this study. Consequently, there was no assessment of bias for this cat- } \\
\text { egory for the outcome of clinical conjunctivitis. }\end{array}$ \\
\hline
\end{tabular}

\begin{tabular}{|c|c|c|}
\hline $\begin{array}{l}\text { Incomplete outcome data } \\
\text { (attrition bias) } \\
\text { Bacterial, gonococcal and } \\
\text { chlamydial conjunctivitis } \\
\text { (objective) }\end{array}$ & Unclear risk & $\begin{array}{l}\text { The study did not report bacterial, gonococcal, or chlamydial conjunctivitis } \\
\text { as outcomes, therefore there was no assessment of bias for this category with } \\
\text { these outcomes. }\end{array}$ \\
\hline \multirow[t]{3}{*}{$\begin{array}{l}\text { Selective reporting (re- } \\
\text { porting bias) }\end{array}$} & Unclear risk & $\begin{array}{l}\text { QUOTE: "Infants with a history of treated eye infection and those with evi- } \\
\text { dence of conjunctival inflammation at the time of the two-week check-up were } \\
\text { excluded from the study." }\end{array}$ \\
\hline & & $\begin{array}{l}\text { COMMENT: Number of infants with a history of conjunctivitis would have been } \\
\text { collected, but this was not reported in the study. }\end{array}$ \\
\hline & & $\begin{array}{l}\text { QUOTE: "Physical signs of inflammation were not found significantly more in } \\
\text { one group than the other at two weeks of age." }\end{array}$ \\
\hline
\end{tabular}


Hick 1985 (Continued)
COMMENT: This outcome was collected but data were not reported.

QUOTE: "The overall incidence of persistent eye discharge at two months of age $13 \%$."

COMMENT: This number of $13 \%$ cannot be re-calculated from the data provided in the paper. The number calculated from the data was $11.7 \%$.

COMMENT: Despite missing data above, the purpose of the study was to look for the relationship between silver nitrate and nasolacrimal duct obstruction. The study protocol was not available. Consequently, there is insufficient information to permit judgement.

Other bias Unclear risk In any trial with silver nitrate, there could be diagnostic bias.

Silver nitrate causes a chemical conjunctivitis in the first 72 hours. As a result, in the first 72 hours, more neonates in the silver nitrate allocation group could be referred for treatment of eye infection, thereby excluding them from the study. This does not appear to have occurred in this study, based on the fact that the groups seem evenly balanced. However, we do not have the numbers originally allocated to intervention or the number lost to follow-up by allocation group.

\section{Study characteristics}

Parallel-group, single-centre trial.
Method of allocation: alternation by week of birth.
Unit of randomisation: neonate.
Exclusions after randomisation: not addressed in this paper.
Losses to follow-up: parents were advised to return to hospital only if their child had conjunctivitis. Un-
able to determine how many infants had conjunctivitis but did not return.
Number randomised: 3117.
Missing data: unknown how many losses to follow-up; available-case analysis.
No reported power calculation.
Unusual study design: none.

Participants

Setting: Presbyterian Church Hospital, Kikuya, Kenya.

Number allocated: 3117:

- povidone-iodine: 1076 ;

- erythromycin: $1112^{\prime}$

- silver nitrate: 929 .

Age: neonates.

Sex: M:F 1452 (53\%):1665 (47\%).

Inclusion criteria: babies born from March 1991 to August 1993 at the Prebyterian Church Hospital.

Exclusion criteria: 
Isenberg 1995 (Continued)

1. infants with ocular malformations;

2. mothers who had received antibiotics during the last month of pregnancy;

3. mothers who were unable to bring infant back to hospital if conjunctivitis developed.

Equivalence of baseline characteristics: yes.

Interventions

Number of interventions: 3 .

- Intervention 1: povidone-iodine, 1 drop $2.5 \%$ solution in each eye $(n=1076)$.

- Intervention 2: erythromycin, 1-centimetre strip of $0.5 \%$ ointment in each eye $(n=1112)$.

- Intervention 3: silver nitrate, 1 drop of $1 \%$ solution in each eye $(n=929)$.

Time to intervention: 1 dose within 20 minutes of birth.

Pre-intervention manoeuvres: eyes and face cleaned.

Postintervention manoeuvres: none specified.

Outcomes

Infants with any conjunctivitis were only determined from mothers who self- reported their infants to hospital.

1. Infants with conjunctivitis.

2. Infants with non-infectious conjunctivitis.

3. Infants with infectious conjunctivitis.

4. Infants with chlamydial conjunctivitis.

5. Infants with gonococcal conjunctivitis.

Follow-up: within a month of birth.

Definition of conjunctivitis: mother shown picture of inflamed eyes and advised to return if eye had discharge or became red. Once infant returned, ophthalmologist assessed for infection clinically, and microbiological analysis was performed.

No adverse events reported.

Notes Date conducted: March 1991 to August 1993.

Source of funding: Karl Kirchgessner Foundation.

No declaration of interest statement made.

No reported subgroup analysis.

Trial investigators contacted and reply received.

\section{Risk of bias}

\section{Bias \\ Authors' judgement Support for judgement}

Random sequence genera- High risk tion (selection bias)
QUOTE: "The three medications were rotated after each was used for a week in the maternity unit. Thus, each infant was assigned to receive a drug according to the week of birth"

Allocation concealment High risk
(selection bias)

QUOTE: "The three medications were rotated after each was used..."

QUOTE: “...each infant was assigned to receive a drug according to the week of birth.”

COMMENT: Allocation could have been foreseen in advance of or during enrolment by using a particular medication for 1 week. Therefore, allocation was not adequately concealed. 
Isenberg 1995 (Continued)

Blinding of study participants (mothers of infants) Clinical conjunctivitis (subjective)
Unclear risk from author)

COMMENT: The 3 interventions differ in colour and consistency. Povidone-iodine is an orange-red solution that leads to transient residual staining of the eye, skin, and lids that can last minutes to hours, which the mother may notice. Erythromycin is a translucent ointment that can last hours and could have been noticed by the mother. Silver nitrate is a clear solution that sometimes causes a chemical conjunctivitis that can last up to 72 hours, and also causes lid stains that can last 30 to 48 hours. It is unknown how this could affect performance bias.

QUOTE: “...the parents, they were unaware of the medication given.” (letter from author)

Blinding of study partici-

Unclear risk

Bacterial, gonococcal and chlamydial conjunctivitis (objective)

COMMENT: The 3 interventions differ in colour and consistency. Povidone-iodine is an orange-red solution that leads to transient residual staining of the eye, skin, and lids that can last minutes to hours, and that the mother may notice. Erythromycin is a translucent ointment that can last hours and could have been noticed by the mother. Silver nitrate is a clear solution that sometimes causes a chemical conjunctivitis that can last up to 72 hours, and also causes lid stains that can last 30 to 48 hours. It is unknown how this could affect performance bias.

The mothers may differentially handle the eyes of neonates based on the visible signs of prophylaxis. This could lead to differential introduction of pathogenic bacteria into the eyes of these neonates. Therefore, lack of masking of medication appearance could lead to bias in bacterial conjunctivitis cases. This bias would be less likely for chlamydial and gonococcal conjunctivitis cases, depending on hygiene measures. However, considering the low event rates of gonococcal conjunctivitis and possible carrier state of Chlamydia trachomatis, this could introduce important bias. It is unknown how many neonates developed conjunctivitis in the time period when the medication could be identified.

\footnotetext{
Blinding of caregiver who administered medication Clinical conjunctivitis (subjective)
}

High risk

QUOTE "The only person who knew and recorded which medication was given was the Ophthalmology Research Nurse. At times, she also administered the eyedrops at birth." (letter from author)

COMMENT: The 3 interventions differ in colour and consistency: povidone-iodine is an orange-red solution, erythromycin is a translucent ointment, and silver nitrate is a clear solution. All 3 medications are readily identifiable to anyone administering the medication. The author states that at times the person administering the prophylaxis was not masked and knew the medication given.

QUOTE "The only person who knew and recorded which medication was given was the Ophthalmology Research Nurse. At times, she also administered the eyedrops at birth." (letter from author)

COMMENT: The 3 interventions differ in colour and consistency: povidone-iodine is an orange-red solution, erythromycin is a translucent ointment, and silver nitrate is a clear solution. All 3 medications are readily identifiable to anyone administering the medication. The author states that at times the person administering the prophylaxis was not masked and knew the medication given. With lack of masking, the person administering the medication could have dispensed the medications differently or there could be differential adherence problems, thereby altering the bactericidal effect. We have seen this in 1 study where 1 of the medications was ointment, and tended to be "skipped" more than solution, owing to difficulty in handling the ointment. 
Isenberg 1995 (Continued)

Blinding of persons involved in postnatal care Clinical conjunctivitis (subjective)
High risk

QUOTE: "The only individual who knew precisely what prophylaxis was given to the child was the research nurse and nurses on duty in the nursery. No physician or other healthcare worker was aware of the medication used." (letter from author)

COMMENT: The author states that nurses on duty in the nursery were not masked as to the study. The 3 interventions differ in colour and consistency. Povidone-iodine is an orange-red solution that leads to transient residual staining of the eye, skin, and lids that can last minutes to hours, depending on whether the surrounding periorbital skin is cleaned or not. Erythromycin is a translucent ointment that can last hours. Silver nitrate is a clear solution that sometimes causes a chemical conjunctivitis that can last up to 72 hours, and also causes lid stains that can last 30 to 48 hours.

It is unclear how long the neonates were in the nursery, and how many cases of conjunctivitis were identified at this time. It is also unclear if the nurses on duty in the nursery were also involved in identifying cases of conjunctivitis, and to what extent.

The nurses in the nursery may have differentially handled the eyes of neonates based on visible signs of prophylaxis. This could lead to differential introduction of pathogenic bacteria into the eyes of these neonates.
Blinding of persons involved in postnatal care Bacterial, gonococcal and chlamydial conjunctivitis (objective)

High risk

QUOTE: "The only individual who knew precisely what prophylaxis was given to the child was the research nurse and nurses on duty in the nursery. No physician or other healthcare worker was aware of the medication used." (letter from author)

COMMENT: The author states that nurses on duty in the nursery were not masked as to the study. The 3 interventions differ in colour and consistency. Povidone-iodine is an orange-red solution that leads to transient residual staining of the eye, skin, and lids that can last minutes to hours, depending on whether the surrounding periorbital skin is cleaned or not. Erythromycin is a translucent ointment that can last hours. Silver nitrate is a clear solution that sometimes causes a chemical conjunctivitis that can last up to 72 hours, and also causes lid stains that can last 30 to 48 hours.

It is unclear how long the neonates were in the nursery, and how many cases of conjunctivitis were identified at this time. It is also unclear if the nurses on duty in the nursery were also involved in identifying cases of conjunctivitis, and to what extent.

Any bias in identification of cases of conjunctivitis cases could influence cases referred for swabbing for bacteria, chlamydia, and gonorrhoea. For instance, the nurse was aware that neonates were given silver nitrate, and were aware of the concomitant chemical conjunctivitis; in ambiguous cases, the nurse may erroneously have ignored cases of 'true' bacterial conjunctivitis that happen to have presented outside the chemical conjunctivitis window.

The incubation period of gonococcal conjunctivitis and chlamydial conjunctivitis is likely outside the time period at which the nurses in the nursery would be influencing identification and care. Furthermore, these cases are likely more clinically severe, eliminating ambiguity. Therefore, the lack of masking of the nurses in the nursery will likely have introduced less bias for the outcomes of gonococcal and chlamydial conjunctivitis. Still, the possible carrier state of chlamydia and the low event rate of gonococcal conjunctivitis could make minor bias important and clinically significant.

Blinding of outcome assessment (detection bias)
Unclear risk

QUOTE: "Infants returning with conjunctivitis were taken to the clinical laboratory of the Nairobi Hospital for microbiologic analysis of the inflamed eyes." (from journal article) 
Isenberg 1995 (Continued) Clinical conjunctivitis (subjective)
QUOTE: “...the outcome assessors (were) totally masked as to the medication used. The clinical outcome assessors (were) primarily the microbiologist who determined the source of infection and the ophthalmologist who determined if the infection or other form of conjunctivitis was present." (letter from author)

QUOTE: "Outcome assessments were determined by the parents who detected an infection and the microbiologist who determined the organism. Both were unaware of the medications used." (letter from author)

COMMENT: The 3 interventions differ in colour and consistency. Povidone-iodine is an orange-red solution that leads to transient residual staining of the eye, skin, and lids that can last minutes to hours, depending on whether the periorbital skin is cleaned. Erythromycin is a translucent ointment that can last hours. Silver nitrate is a clear solution that sometimes causes a chemical conjunctivitis that can last up to 72 hours, and also causes lid stains that can last 30 to 48 hours.

It is unknown how many cases of conjunctivitis were identified during the time when these stains remained.

\section{Blinding of outcome as- Low risk} sessment (detection bias) Bacterial, gonococcal and chlamydial conjunctivitis (objective)

QUOTE: "Infants returning with conjunctivitis were taken to the clinical laboratory of the Nairobi Hospital for microbiologic analysis of the inflamed eyes." (from journal article)

QUOTE: “...the outcome assessors (were) totally masked as to the medication used. The clinical outcome assessors (were) primarily the microbiologist who determined the source of infection and the ophthalmologist who determined if the infection or other form of conjunctivitis was present." (letter from author)

QUOTE: "Outcome assessments were determined by the parents who detected an infection and the microbiologist who determined the organism. Both were unaware of the medications used." (letter from author)

COMMENT: The 3 interventions differ in colour and consistency. Povidone-iodine is an orange-red solution that leads to transient residual staining of the eye, skin, and lids that can last minutes to hours, depending on if the periorbital skin is cleaned. Erythromycin is a translucent ointment that can last hours. Silver nitrate is a clear solution that sometimes causes a chemical conjunctivitis that can last up to 72 hours, and also causes lid stains that can last 30 to 48 hours.

It is unknown how many cases of conjunctivitis were identified during the time when these stains remained. This could affect bias in the decision to swab by the ophthalmologist, thereby altering bacterial conjunctivitis cases, and falsely identifying chemical conjunctivitis cases with normal flora bacteria.

The incubation period of gonococcal conjunctivitis and chlamydial conjunctivitis is likely outside the time period at which these stains would remain. Furthermore, these cases are likely more clinically severe, reducing ambiguity in diagnosis. In the silver nitrate arm, any cases presenting within the chemical conjunctivitis period, but before the incubation period of chlamydial conjunctivitis, could simply be cases of chemical conjunctivitis with chlamydial carriers. It is unclear how authors handled these cases. This bias is likely low in this trial considering the higher event rate of chlamydial conjunctivitis. Although gonococcal conjunctivitis has a lower event rate, there is no reported carrier state, and the clinical cases are more severe, further reducing bias.

\begin{tabular}{|c|c|c|}
\hline $\begin{array}{l}\text { Incomplete outcome data } \\
\text { (attrition bias) } \\
\text { Clinical conjunctivitis }\end{array}$ & Unclear risk & $\begin{array}{l}\text { QUOTE: "Each mother was shown pictures of inflamed eyes and instructed } \\
\text { to return to the hospital with her infant if the child's eye began to have a dis- } \\
\text { charge or became red within a month of birth. " (from journal article) }\end{array}$ \\
\hline
\end{tabular}


COMMENT: The mother first had to make decision as to whether the eyes required follow-up. The definition of conjunctivitis included discharge OR inflamed eyes, adding some ambiguity. It is unknown how many mothers did not return for follow-up at all or for follow-up to the prescribed hospital. It is unclear if loss to follow-up varied by intervention group, or the relationship to event rates. Furthermore, considering allocation was by week of birth, we do not know if allocation groups were equivalent, and if there was correlation with 1 allocation group, and say, distance from hospital, thereby differentially affecting rates of follow-up in allocation groups.

\section{Incomplete outcome data Unclear risk} (attrition bias)

Bacterial, gonococcal and chlamydial conjunctivitis (objective)

\section{QUOTE: "Each mother was shown pictures of inflamed eyes and instructed to return to the hospital with her infant if the child's eye began to have a dis- charge or became red within a month of birth. " (from journal article) \\ QUOTE: "Attrition is not an issue since we reported only babies returning with conjunctivitis" (letter from author)}

COMMENT: It is unknown how many mothers did not return for follow-up at all or for follow-up to the prescribed hospital. It is unclear if loss to follow-up varied by intervention group, or the relationship to event rates. It is also unclear how many infants identified with conjunctivitis, if any, failed to follow up at the clinical laboratory for microbiologic analysis of the inflamed eyes.

\begin{tabular}{|c|c|c|}
\hline $\begin{array}{l}\text { Selective reporting (re- } \\
\text { porting bias) }\end{array}$ & Low risk & COMMENT: There is no evidence of selective outcome reporting. \\
\hline Other bias & Unclear risk & $\begin{array}{l}\text { In any trial with silver nitrate, there could be diagnostic bias. } \\
\text { Silver nitrate causes a chemical conjunctivitis in the first } 72 \text { hours. As a result, } \\
\text { in the first } 72 \text { hours, more neonates in the silver nitrate allocation group could } \\
\text { be referred for culture in the first } 72 \text { hours. Finding bacteria in the culture does } \\
\text { not necessarily prove that the bacteria caused the conjunctivitis. The conjunc- } \\
\text { tivitis could be chemical, but growing normal flora of the eye. Alternatively, } \\
\text { the conjunctivitis could be chemical with a chlamydial carrier. Finally, the con- } \\
\text { junctivitis could very well be caused by the bacteria or chlamydia. Consider- } \\
\text { ation of incubation periods, and assessing for carriers and normal flora with } \\
\text { asymptomatic cases, could assist with differential diagnosis. }\end{array}$ \\
\hline
\end{tabular}

Isenberg 2003

\section{Study characteristics}

Parallel-group, single-centre trial.
Unit of randomisation: neonate.
Method of allocation: alternation by week of birth.
Exclusions after allocation: neonates who returned with conjunctivitis within 48 hours of birth were ex-
cluded; number not specified.
Losses to follow-up: parents were advised to return to hospital only if their children had conjunctivitis;
unable to determine how many infants had conjunctivitis but did not return.
Number randomised: 719.
Missing data: unknown how many were lost to follow-up; available-case analysis.


Unusual study design: none.

Participants
Setting: Presbyterian Church of East Africa Hospital, Kikuya, Kenya.

Number allocated: 719 neonates:

- single-dose povidone-iodine: 402 ;

- double-dose povidone-iodine: 317 .

Age: neonates.

Sex: M:F:

- $\quad$ single-dose group: $49.8 \%: 50.2 \%$;

- double-dose group: 53\%:47\%.

Inclusion criteria: all babies born from January 2000 to October 2001 at the Presbyterian Church of East Africa Hospital.

Exclusion criteria:

1. infants with ocular malformations;

2. mothers who had received antibiotics during the last month of pregnancy;

3. mothers who were unable to bring infant back to hospital if conjunctivitis developed;

4. infants born by caesarean section.

Equivalence of baseline characteristics: yes.
Interventions
Number of interventions: 2 .

- Intervention 1: 1 drop 2.5\% povidone-iodine solution in each eye within 20 minutes of birth $(n=402)$.

- Intervention 2: 1 drop 2.5\% povidone-iodine solution in each eye within 20 minutes of birth AND 1 drop $2.5 \%$ povidone-iodine solution at hospital discharge or 24 hours after delivery, whichever was first. Time to intervention: 1 dose within 20 minutes of birth; second dose at hospital discharge or 24 hours after delivery, whichever was first $(n=317)$.

Pre-intervention manoeuvres: eyes and face cleaned.

Postintervention manoeuvres: none specified.

Outcomes eye had a discharge or became red.

1. Infants returning had each of the following eye signs graded with a score from 0 to 3 (0-none, 1-mild, 2-moderate, 3-severe):

a. eyelid swelling;

b. conjunctival redness;

c. conjunctival swelling;

d. conjunctival discharge.

2. Infants with positive culture results of eye specimens, described by type.

3. Infants with positive Gram stain results of eye specimens, described by type.

4. Cases of Chylamdia trachomatis diagnosed by Giemsa stain, Gram stain, and/or direct fluorescent antibody assay.

5. No cases on gonococcal conjunctivitis found.

Follow-up: within a month of birth, self-report by parent. 
Definition of conjunctivitis or ophthalmia neonatorum: no explicit definition. Mother shown picture of inflamed eyes and advised to return if eye had discharge or became red. Amongst neonates returning for assessment, it was unclear how many neonates actually had red eyes or eye discharge.

No adverse events reported.

Notes Date study conducted: January 2000 to October 2001.

Source of funding: Ronald McDonald House Charities, The Karl Kirchgessner Foundation, Research to Prevent Blindness Senior Scientific Investigator Award.

No declaration of interest statement made in paper.

No reported subgroup analysis.

Trial investigators were not contacted.

\section{Risk of bias}

\begin{tabular}{lll}
\hline Bias & Authors' judgement & Support for judgement \\
\hline $\begin{array}{l}\text { Random sequence genera- } \\
\text { tion (selection bias) }\end{array}$ & High risk & $\begin{array}{l}\text { QUOTE: "Each infant was assigned a dosing schedule according to the week of } \\
\text { birth" }\end{array}$ \\
\hline $\begin{array}{l}\text { Allocation concealment } \\
\text { (selection bias) }\end{array}$ & High risk & $\begin{array}{l}\text { QUOTE: "Each infant was assigned a dosing schedule according to the week of } \\
\text { birth" }\end{array}$ \\
$\begin{array}{l}\text { COMMENT: Allocation could have been foresen in advance or during enrol- } \\
\text { ment by using a particular medication for } 1 \text { week. Therefore, allocation was } \\
\text { not adequately concealed. }\end{array}$
\end{tabular}

Blinding of study partici- Unclear risk pants (mothers of infants) Clinical conjunctivitis (subjective)
COMMENT: The study did not address this outcome. The 2 interventions were the same, except that 1 allocation group received the intervention once at birth, and the other allocation group received the intervention twice, once at birth and the second time within $24 \mathrm{~h}$. Povidone-iodine is an orange-red solution that leads to transient residual staining of the eye, skin, and lids that can last minutes to hours, depending on whether the surrounding periorbital skin is cleaned or not. It is likely that the mothers of neonates knew if their child had received the intervention twice. It is uncertain how possible lack of masking could influence the outcome of clinical conjunctivitis.
Blinding of study partici- Unclear risk pants (mothers of infants) Bacterial, gonococcal and chlamydial conjunctivitis (objective)
COMMENT: The study did not address this outcome. The 2 interventions were the same, except that 1 allocation group received the intervention once at birth, and the other allocation group received the intervention twice, once at birth and the second time within $24 \mathrm{~h}$. Povidone-iodine is an orange-red solution that leads to transient residual staining of the eye, skin, and lids that can last minutes to hours, depending on whether or not the surrounding periorbital skin is cleaned.

The mothers may differentially handle the eyes of neonates based on the visible signs of prophylaxis, at birth vs within 24 hours. This could lead to differential introduction of pathogenic bacteria into the eyes of these neonates with more time in the hospital. Therefore, lack of masking in the single- vs double-application allocation group may lead to bias in bacterial conjunctivitis cases. This bias would be less likely for chlamydial and gonococcal conjunctivitis cases, depending on hygiene measures. However, considering the zero event rates of gonococcal conjunctivitis and possible carrier state of $C$ trachomatis, this could introduce important bias.
Blinding of caregiver who administered medication
Unclear risk

COMMENT: The study did not address this outcome. The 2 interventions were the same, except that 1 allocation group received the intervention once at 
Isenberg 2003 (Continued) Clinical conjunctivitis (subjective)

birth, and the other allocation group received the intervention twice, once at birth and the second time within $24 \mathrm{~h}$. Povidone-iodine is an orange-red solution that leads to transient residual staining of the eye, skin, and lids that can last minutes to hours, depending on whether the surrounding periorbital skin is cleaned or not. Masking would be difficult, but once again it is uncertain how lack of masking would influence outcome of clinical conjunctivitis.

It appears that no placebo was used.

If the person administering the medication knew that the infant was in the double-application group, there could possibly be lack of compliance knowing the neonate was obtaining another application later. This could affect rates of conjunctivitis, as it has been shown that delay in prophylaxis can lead to increased risk of conjunctivitis.

There could be a reporting bias of conjunctivitis in the double-application group, as the eyes of the neonates would have been more closely examined in the double-application group to dispense the second drop of prophylaxis. There could also be more reinforcement of follow-up instructions in the double-application group, which could also lead to reporting bias.

Any reporting bias differentially in 1 arm vs the other could introduce bias in rates of conjunctivitis cases.

Blinding of caregiver who administered medication Bacterial, gonococcal and chlamydial conjunctivitis (objective)
Unclear risk

COMMENT: The study did not address this outcome. The 2 interventions were the same, except that 1 allocation group received the intervention once at birth, and the other allocation group received the intervention twice, once at birth and the second time within $24 \mathrm{~h}$. Povidone-iodine is an orange-red solution that leads to transient residual staining of the eye, skin, and lids that can last minutes to hours, depending on whether the surrounding periorbital skin is cleaned or not. It appears that no placebo was used. It is likely that those involved in postnatal care were not masked as to which neonates were in the double-application group, but it is uncertain how this would affect outcome.

If the person administering the medication knew that the infant was in the double-application group, there could possibly be lack of compliance knowing the neonate was obtaining another application later. This could affect rates of conjunctivitis, as it has been shown that delay in prophylaxis can lead to increased risk of conjunctivitis.

There could be a reporting bias in the double-application group, as the eyes of the neonates would have been more closely examined in the double-application group to dispense the second drop of prophylaxis. There could also be more reinforcement of follow-up instructions in the double-application group, which could also lead to reporting bias.

Any bias in identification of cases of conjunctivitis cases could influence cases referred for swabbing for bacteria, chlamydia, and gonorrhoea, and subsequent rates.
Blinding of persons involved in postnatal care Clinical conjunctivitis (subjective)
Unclear risk

COMMENT: The study did not address this outcome. The 2 interventions were the same, except that 1 allocation group received the intervention once at birth, and the other allocation group received the intervention twice, once at birth and the second time within $24 \mathrm{~h}$. Povidone-iodine is an orange-red solution that leads to transient residual staining of the eye, skin, and lids that can last minutes to hours, depending on whether the surrounding periorbital skin is cleaned or not.

It is likely that those involved in postnatal care were not masked as to which neonates were in the double-application group, but it is uncertain how this would affect outcome. 
Isenberg 2003 (Continued)

Blinding of persons involved in postnatal care Bacterial, gonococcal and chlamydial conjunctivitis (objective)
Unclear risk

COMMENT: The study did not address this outcome. The 2 interventions were the same, except that 1 allocation group received the intervention once at birth, and the other allocation group received the intervention twice, once at birth and the second time within $24 \mathrm{~h}$. Povidone-iodine is an orange-red solution that leads to transient residual staining of the eye, skin, and lids that can last minutes to hours, depending on whether the surrounding periorbital skin is cleaned or not.

It is likely that those involved in postnatal care were not masked as to which neonates were in the double-application group, but it is uncertain how this would affect outcome.

\section{Blinding of outcome as- Unclear risk sessment (detection bias) \\ QUOTE: "The laboratory assessors had no knowledge of which prophylactic ocular medication dose was given to any infant"}

Clinical conjunctivitis

(subjective)

COMMENT: Parents returned infants for clinical assessment before being sent to the laboratory. It is unknown if the clinical assessors were aware of allocation group.

\begin{tabular}{|c|c|c|}
\hline $\begin{array}{l}\text { Blinding of outcome as- } \\
\text { sessment (detection bias) }\end{array}$ & Unclear risk & $\begin{array}{l}\text { QUOTE: "The laboratory assessors had no knowledge of which prophylactic } \\
\text { ocular medication dose was given to any infant" }\end{array}$ \\
\hline
\end{tabular}

Bacterial, gonococcal and chlamydial conjunctivitis (objective)

COMMENT: Parents returned infants for clinical assessment before being sent to the laboratory. It is unknown if the clinical assessors were aware of allocation group. If there was lack of masking, then it could bias referrals for further ocular bacteriological analysis, thereby biasing bacterial, chlamydial, and gonococcal event rates.

$\begin{array}{ll}\begin{array}{l}\text { Incomplete outcome data } \\ \text { (attrition bias) }\end{array} & \text { Unclear risk } \\ \text { Clinical conjunctivitis } & \begin{array}{l}\text { QUOTE: "Each mother was shown pictures of inflamed eyes and instructed } \\ \text { to return to the hospital with her infant if the child's eye began to have a dis- } \\ \text { charge or became red within a month of birth. " (from journal article) }\end{array}\end{array}$

(subjective)

COMMENT: The mother first had to make a decision as to whether the eyes required follow-up. The definition of conjunctivitis included discharge OR inflamed eyes, adding some ambiguity. It is unknown how many mothers did not return for follow-up at all or for follow-up to the prescribed hospital. It is unclear if loss to follow-up varied by intervention group, or the relationship to event rates. Furthermore, considering that allocation was by week of birth, it is unclear if allocation groups were equivalent, and if there was a correlation with 1 allocation group and, say, distance from hospital, thereby differentially affecting rates of follow-up in allocation groups.

\section{Incomplete outcome data Unclear risk} (attrition bias)

Bacterial, gonococcal and chlamydial conjunctivitis (objective)
QUOTE: "Each mother was shown pictures of inflamed eyes and instructed to return to the hospital with her infant if the child's eye began to have a discharge or became red within a month of birth. " (from journal article)

COMMENT: The mother first had to make a decision as to whether the eyes required follow-up. The definition of conjunctivitis included discharge OR inflamed eyes, adding some ambiguity. It is unknown how many mothers did not return for follow-up at all or for follow-up to the prescribed hospital. It is unclear if loss to follow-up varied by intervention group, or the relationship to event rates. Furthermore, considering that allocation was by week of birth, it is unclear if allocation groups were equivalent, and if there was a correlation with 1 allocation group and, say, distance from hospital, thereby differentially affecting rates of follow-up in allocation groups.

Reporting bias by allocation group would affect clinical conjunctivitis cases, and similarly rates of bacterial, chlamydial, and gonococcal conjunctivitis. 
Isenberg 2003 (Continued)

Selective reporting (re- Low risk COMMENT: There is no evidence of selective outcome reporting. porting bias)

Other bias

Low risk

COMMENT: No other sources of bias identified.

Kaivonen 1965a

\section{Study characteristics}

\section{Methods}

Parallel-group, single-centre trial.

Method of allocation: daily alternation.

Unit of randomisation: neonate.

Exclusions after allocation: none specified.

Losses to follow-up: none specified.

Number allocated: 231.

Missing data handling: not specified.

No reported power calculation.

Unusual study design:

1. samples for bacteriologic studies were taken from the conjunctiva of both eyes, irrespective of inflammation, usually a day before child went home;

2. for those infants found by questionnaire to have conjunctivitis after discharge from hospital, cultures were actually taken from infants before discharge from hospital.

Participants

Setting: University Women's Hospital, Helsinki, Finland.

Number allocated: 231 neonates:

- silver nitrate: 115 ;

- "Biosept" (cetyl-pyridinium chloride): 116.

Age: neonates.

Sex: M:F unknown.

Inclusion criteria: all children born 6 November to 20 December 1957 at the obstetric wards of University Women's Hospital and who were not moved from the hospital before the bacterial sample was taken.

Exclusion criteria: none specified.

No comment on equivalence of baseline characteristics.

- Intervention 1: "Biosept" (cetyl-pyridinium chloride) $0.1 \%$ solution, 1 drop into each eye $(n=116)$.

- Intervention 2: silver nitrate $1 \%$ solution, 1 drop into each eye $(n=115)$.

Time to intervention: 1 dose at the time of delivery.

Pre-intervention manoeuvres: ocular region cleaned with dry, sterile gauze. 
Kaivonen 1965a (Continued)

Postintervention manoeuvres: eyes cleaned with dry, sterile cotton every 3 to 4 hours.

Outcomes

1. Total number of infants with inflammation - see definition of inflammation below.

$=[($ Infants with purulent discharge on Day 1 and possibly Day

2 postpartum $)+$ (infants with purulent discharge on Day 1 and continuing after Day 2 postpartum)

+ (infants with purulent discharge beginning after the first day postpartum) + (infants with purulent discharge first seen at home).

2. Infants with culture-positive inflammation - see definition of inflammation below.

$=[$ Infants with culture-positive purulent discharge on Day

1 and possibly Day 2 postpartum) + (infants with culture-positive purulent discharge on Day 1 and continuing after Day 2 postpartum) + (infants with culture-positive purulent discharge beginning after the first day postpartum) + (infants with culture-positive purulent discharge first seen at home)].

3. Infants with ophthalmia neonatorum - see definition of ophthalmia neonatorum below.

4. Infants with culture-positive ophthalmia neonatorum - see definition of ophthalmia neonatorum below.

Follow-up: 2 weeks: in hospital, usually 4 to 6 days postpartum, infants examined once/day, 3 to 4 hours after the child's eyes had been cleaned last. Children's eyes opened daily by the author to search for secretion and hyperaemia of the conjunctiva. On discharge, mothers were given a questionnaire that asked if there had been redness, oedema of the lids, watery or purulent discharge in the child's eyes at home, and were asked to return form 2 weeks postpartum. In the questionnaire, only purulent discharge was regarded as a sign of inflammation.

Definition of conjunctivitis/inflammation in paper: infants with purulent eye discharge, classified as scanty, moderate, profuse.

Definition of ophthalmia neonatorum in paper:

1. eyes with profuse purulent discharge only; or

2. eyes with moderate discharge, but combined with other signs of inflammation, such as oedema of lids or hyperaemia of the conjunctiva.

Bacterial culture: cultures were generally taken from the conjunctiva of the left eye of all children a day before the infant went home. This was done irrespective of presence or absence of inflammation. If the child developed purulent discharge whilst in the hospital, the sample was taken from the eye with a heavier discharge, usually 1 to 2 days after its appearance.

Source of funding: not specified.

No statement of declaration of interest.

Subgroup analysis conducted by day of conjunctival signs and symptoms (inflammation, conjunctivitis or ophthalmia neonatorum).

Trial investigators were not contacted.

\section{Risk of bias}

\section{Bias}

Authors' judgement Support for judgement

Random sequence genera- High risk tion (selection bias)
QUOTE: “...silver nitrate and Biosept were administered to roughly every other child..”

QUOTE: "A blank form with a running number on which no entry was made concerning the prophylaxis employed was attached to the case report of every newborn. The type of the prophylaxis was recorded on another sheet and attached to the blank form later. Thus preconceived ideas on the part of the investigator could not affect the appraisal of the clinical symptoms." 
Allocation concealment High risk

(selection bias)
QUOTE: “...silver nitrate and Biosept were administered to roughly every other child.."

QUOTE: "A blank form with a running number on which no entry was made concerning the prophylaxis employed was attached to the case report of every newborn. The type of the prophylaxis was recorded on another sheet and attached to the blank form later. Thus preconceived ideas on the part of the investigator could not affect the appraisal of the clinical symptoms."

COMMENT: Participants or investigators enrolling participants could possibly have foreseen assignments, thus introducing selection bias

$\begin{array}{lll}\begin{array}{l}\text { Blinding of study partici- } \\ \text { pants (mothers of infants) }\end{array} & \text { Unclear risk } & \begin{array}{l}\text { COMMENT: The study does not mention whether mothers were masked to the } \\ \text { intervention. Silver nitrate sometimes causes a chemical conjunctivitis that }\end{array} \\ \text { Clinical conjunctivitis } & \text { can last up to } 72 \text { hours, and silver nitrate also causes lid stains that can last } 30 \\ \text { (subjective) } & \text { to } 48 \text { hours. The mother may have been able to identify the medication; the } \\ \text { impact on performance bias is unknown. }\end{array}$

Blinding of study partici- Unclear risk pants (mothers of infants) Bacterial, gonococcal and chlamydial conjunctivitis (objective)

bacterial conjunctivitis, depending on hygiene measures.

COMMENT: The study does not mention whether mothers were masked to the intervention. Silver nitrate sometimes causes a chemical conjunctivitis that can last up to 72 hours, and silver nitrate also causes lid stains that can last 30 to 48 hours. The mothers of neonates with noticeable medication of the eyes may handle the eyes of the infant more, potentially affecting the outcome of bacterial conjunctivitis, depending on hygiene measures.

\section{Blinding of caregiver who Unclear risk} administered medication Clinical conjunctivitis (subjective)
COMMENT: The study does not comment on whether or not the person administering the medication was masked. It is uncertain if silver nitrate and "Biosept" (cetyl-pyridinium chloride) appeared different or if they were dispensed from labelled vials. They are both solutions.

COMMENT: Knowledge of the medication being dispensed, along with any concomitant bias, could affect adherence and differential application of prophylaxis, which, in turn, could affect preventive effect of the development of conjunctivitis.

\begin{abstract}
Blinding of caregiver who administered medication Bacterial, gonococcal and chlamydial conjunctivitis

Unclear risk

COMMENT: The study does not comment on whether or not the person administering the medication was masked. It is uncertain if silver nitrate and "Biosept" (cetyl-pyridinium chloride) appeared different or if they were dispensed from labelled vials. They are both solutions.
\end{abstract} (objective)
COMMENT: Knowledge of the medication being dispensed, along with any concomitant bias, could affect adherence and differential application of prophylaxis, which, in turn, could affect preventive effect of the development of bacterial conjunctivitis.
Blinding of persons involved in postnatal care Clinical conjunctivitis (subjective)
Unclear risk

QUOTE: "The children's nurses cleaned the eyes with dry sterile cotton at feeding time at intervals of 3-4 hours."

COMMENT: The study does not comment on whether those involved in postnatal case were masked to intervention. Silver nitrate sometimes causes a chemical conjunctivitis that can last up to 72 hours, and silver nitrate causes lid stains that can last 30 to 48 hours. The children were in hospital usually 4 to 6 days postpartum. Furthermore, the study identifies the day of identification of conjunctivitis, and a significant number of cases of conjunctivitis have been identified in the first 3 days of birth. Therefore, those involved in postnatal care may not be masked to the medication used as prophylaxis during this initial time period. Lack of masking could lead to differential treatment of the neonates' eyes, resulting in differential cases of conjunctivitis. 
Kaivonen 1965a (Continued)

Blinding of persons involved in postnatal care Bacterial, gonococcal and chlamydial conjunctivitis (objective)
Unclear risk ing time at intervals of 3-4 hours."

COMMENT: The study does not comment on whether those involved in postnatal case were masked to intervention. Silver nitrate sometimes causes a chemical conjunctivitis that can last up to 72 hours, and silver nitrate causes lid stains that can last 30 to 48 hours. Therefore, those involved in postnatal care may not be masked as to the medication used as prophylaxis during this initial time period. Lack of masking could lead to differential treatment of the neonates' eyes, resulting in differential cases of conjunctivitis. Note that the neonates were in hospital usually 4 to 6 days postpartum. Furthermore, the study identifies the day of identification of conjunctivitis, and a significant number of cases of bacterial conjunctivitis have been identified in the first 3 days of birth.
Blinding of outcome as- Unclear risk sessment (detection bias) Clinical conjunctivitis (subjective)
QUOTE: "The type of prophylaxis was recorded on another sheet and attached to the blank for later. Thus, preconceived ideas on the part of the investigator could not affect the appraisal of clinical symptoms."

QUOTE: “In series I of the earlier investigation, the children's eyes were opened daily by the author with the fingers to search for secretion and hyperemia of the conjunctiva."

COMMENT: As silver nitrate creates lid stains that last 30 to 48 hours, outcome assessments during this time may not have been masked. Considering that neonates were only followed up 2 weeks postpartum and were in hospital 4 to 6 days postpartum, there is greater potential for bias on outcome assessments.
Blinding of outcome as- Unclear risk sessment (detection bias) Bacterial, gonococcal and chlamydial conjunctivitis (objective)
QUOTE: "The type of prophylaxis was recorded on another sheet and attached to the blank for later. Thus, preconceived ideas on the part of the investigator could not affect the appraisal of clinical symptoms."

QUOTE: “In series I of the earlier investigation, the children's eyes were opened daily by the author with the fingers to search for secretion and hyperemia of the conjunctiva."

COMMENT: As silver nitrate creates lid stains that last 30 to 48 hours, outcome assessments during this time may not have been masked. Considering that neonates were only followed up 2 weeks postpartum and were in hospital 4 to 6 days postpartum, there is greater potential for bias on outcome assessments.

Furthermore, there were a significant number of bacterial conjunctivitis cases identified in the first 3 postpartum days.

In ambiguous cases of clinical conjunctivitis, there may be differential assessor behaviour to include or exclude cases of clinical conjunctivitis, thereby increasing or decreasing likelihood of swabbing the neonate's eye for bacterial conjunctivitis. Presence of bacteria on a swab does not necessarily prove that the bacteria caused the conjunctivitis, as the bacteria could be a carrier but the conjunctivitis caused by chemical conjunctivitis. This possible bias was reduced by the fact that the swabs for culture were reserved those conjunctivitis cases with purulent discharge, as specified in the methods.

COMMENT: The study authors do not provide any information on the presence or absence of incomplete outcome data. Neonates were in hospital 4 to 6 days postpartum, and follow-up time in this study was 2 weeks. (attrition bias)

Clinical conjunctivitis

(subjective)
Unclear risk 
Kaivonen 1965a (Continued)

Incomplete outcome data (attrition bias)

Bacterial, gonococcal and chlamydial conjunctivitis (objective)
Unclear risk

COMMENT: The study authors do not provide any information on the presence or absence of incomplete outcome data. Neonates were in hospital 4 to 6 days postpartum, and follow-up time in this study was 2 weeks.
Selective reporting (re- High risk porting bias)
QUOTE: "The growth of gonococcus with the method employed is uncertain. A special method for the systematic culture of gonococcus was not regarded as necessary as the incidence of gonorrhea in the material would in all probability have been low"

COMMENT: Gonococcal conjunctivitis is of interest to this review, and the microbiological methods to identify Neisseria gonorrhoeae were "uncertain". There were, predictably, no cases identified in the study.

Other bias High risk

COMMENT: In any trial with silver nitrate, there could be differential diagnostic activity. This could lead to increased diagnosis of true but harmless cases of disease. For example, silver nitrate induces a chemical conjunctivitis. This chemical conjunctivitis could lead to increased selective bacterial cultures of infants' eyes in the silver nitrate intervention group. A positive bacterial culture found from a swab of conjunctivitis due to chemical conjunctivitis does not necessarily mean that the bacteria caused the conjunctivitis. The bacteria could be part of the normal flora of the eye, with an associated chemical conjunctivitis, or the bacteria could be the causal agent of the conjunctivitis.

QUOTE: "For series I, the lids of the children were opened daily with the fingers...Careful washing of the hands after every examination was very difficult in practice since over 100 children were examined every day in a relatively short time. The risk of cross infection via the investigator's fingers from one child to another was thus considerable during the study of series I in 1957."

COMMENT: For those infants found by questionnaire to have conjunctivitis after discharge from hospital, cultures were actually taken from infants before discharge from hospital, not on the day the conjunctivitis was identified after discharge.

Kaivonen 1965b

\section{Study characteristics}

\section{Methods}

Parallel-group, single-centre trial.

Method of allocation: daily alternation.

Unit of randomisation: neonate.

Exclusions after allocation: none specified.

Losses to follow-up: none specified.

No comment on missing data in paper and how handled.

No reported power calculation.

Unusual study design:

1. samples for bacteriologic studies were taken from the conjunctiva of from one eye of the neonate, irrespective of inflammation, usually a day before child went home; 
Kaivonen 1965b (Continued)

2. for those infants found by questionnaire to have conjunctivitis after discharge from hospital, cultures were actually taken from infants before discharge from hospital.

\section{Participants}

Setting: University Women's Hospital, Helsinki, Finland.

Number allocated: 368 neonates:

- silver nitrate: 183 ;

- "Biosept" (cetyl-pyridinium chloride): 185.

Age: neonates.

Sex: M:F unknown.

Inclusion criteria: all children born 6 November to 20 December 1957 at the obstetric wards of University Women's Hospital and who were not moved from the hospital before the bacterial sample was taken.

Exclusion criteria: none specified.

No comment on equivalence of baseline characteristics.

- Intervention 1: "Biosept" (cetyl-pyridinium chloride) $0.05 \%$ solution, 1 drop into each eye $(n=185)$.

- Intervention 2: silver nitrate $1 \%$ solution, 1 drop into each eye $(n=183)$.

Time to intervention: 1 dose at the time of delivery.

Pre-intervention manoeuvres: ocular region cleaned with dry, sterile gauze.

Postintervention manoeuvres: eyes cleaned with dry, sterile cotton every 3 to 4 hours.

Outcomes

1. Total number of infants with inflammation - see definition of inflammation below

$=[$ (Infants with purulent discharge on Day 1 and possibly Day 2 postpartum $)+($ infants with purulent discharge on Day 1 and continuing after Day 2 postpartum) + (infants with purulent discharge beginning after the first day postpartum) + (infants with purulent discharge first seen at home)].

2. Infants with culture-positive inflammation - see definition of inflammation below

$=[($ Infants with culture-positive purulent discharge on Day

1 and possibly Day 2 postpartum) + (infants with culture-positive purulent discharge on Day 1 and continuing after Day 2 postpartum) + (infants with culture-positive purulent discharge beginning after the first day postpartum) + (infants with culture-positive purulent discharge first seen at home)].

3. Infants with ophthalmia neonatorum - see definition of ophthalmia neonatorum below.

4. Infants with culture-positive ophthalmia neonatorum - see definition of ophthalmia neonatorum below.

Follow-up: 2 weeks:

In hospital, usually 4 to 6 days postpartum, infants examined once/day, 3 to 4 hours after the child's eyes had been cleaned last. Eyes only opened on the first day postpartum. Otherwise eyes were not opened by the investigator as was done in series.

1. Discharge was noted at the roots of the lashes and in the corner of the eye. Hyperaemia was noted if the eyes were opened spontaneously. On discharge, mothers were given a questionnaire that asked if there had been redness, oedema of the lids, watery or purulent discharge in the child's eyes at home, and were asked to return form 2 weeks postpartum. In the questionnaire, only purulent discharge was regarded as a sign of inflammation.

Definition of conjunctivitis/inflammation in paper: infants with purulent eye discharge, classified as scanty, moderate, profuse.

Definition of ophthalmia neonatorum in paper: 
- eyes with profuse purulent discharge only; or

- eyes with moderate discharge, but combined with other signs of inflammation, such as oedema of lids or hyperaemia of the conjunctiva.

Bacterial culture: cultures were generally taken from the conjunctiva of the left eye of all children a day before going home. This was done irrespective of presence or absence of inflammation. If the child developed purulent discharge whilst in the hospital, the sample was taken from the eye with a heavier discharge, usually 1 to 2 days after its appearance.

Notes

Date study conducted: 6 November to 20 December 1957.

Source of funding: not specified.

No statement of declaration of interest.

Subgroup analysis conducted by day of conjunctival signs and symptoms (inflammation, conjunctivitis or ophthalmia neonatorum).

Trial investigators were not contacted.

\section{Risk of bias}

\begin{tabular}{lll}
\hline Bias & Authors' judgement & Support for judgement \\
\hline $\begin{array}{l}\text { Random sequence genera- } \\
\text { tion (selection bias) }\end{array}$ & High risk & $\begin{array}{l}\text { QUOTE: “....silver nitrate and Biosept were administered to roughly every other } \\
\text { child.” }\end{array}$ \\
& $\begin{array}{l}\text { QUOTE: "A blank form with a running number on which no entry was made } \\
\text { concerning the prophylaxis employed was attached to the case report of every } \\
\text { newborn. The type of the prophylaxis was recorded on another sheet and at- } \\
\text { tached to the blank form later. Thus preconceived ideas on the part of the in- } \\
\text { vestigator could not affect the appraisal of the clinical symptoms." }\end{array}$
\end{tabular}

COMMENT: Non-random process in the sequence generation by alternation.

$\begin{aligned} & \text { Allocation concealment } \\ & \text { (selection bias) }\end{aligned}$
$\begin{array}{ll}\text { QUOTE: “....silver nitrate and Biosept were administered to roughly every other } \\ \text { child.” }\end{array}$

QUOTE: "A blank form with a running number on which no entry was made concerning the prophylaxis employed was attached to the case report of every newborn. The type of the prophylaxis was recorded on another sheet and attached to the blank form later. Thus preconceived ideas on the part of the investigator could not affect the appraisal of the clinical symptoms."

COMMENT: Participants or investigators enrolling participants could possibly have foreseen assignments, thus introducing selection bias.

\begin{tabular}{|c|c|c|}
\hline $\begin{array}{l}\text { Blinding of study partici- } \\
\text { pants (mothers of infants) } \\
\text { Clinical conjunctivitis } \\
\text { (subjective) }\end{array}$ & Unclear risk & $\begin{array}{l}\text { COMMENT: The study does not mention whether mothers were masked to the } \\
\text { intervention. Silver nitrate sometimes causes a chemical conjunctivitis that } \\
\text { can last up to } 72 \text { hours, and silver nitrate also causes lid stains that can last } 30 \\
\text { to } 48 \text { hours. The mother may be able to identify the medication; the impact on } \\
\text { performance bias is unknown. }\end{array}$ \\
\hline
\end{tabular}

Blinding of study participants (mothers of infants) Bacterial, gonococcal and chlamydial conjunctivitis (objective)
Unclear risk

COMMENT: The study does not mention whether mothers were masked to the intervention. Silver nitrate sometimes causes a chemical conjunctivitis that can last up to 72 hours, and silver nitrate also causes lid stains that can last 30 to 48 hours. The mothers of neonates with noticeable medication of the eyes may handle the eyes of the infant more, potentially affecting the outcome of bacterial conjunctivitis, depending on hygiene measures. 
Kaivonen 1965b (Continued)

Blinding of caregiver who administered medication Clinical conjunctivitis (subjective)
Unclear risk

COMMENT: The study does not comment on whether or not the person administering the medication was masked. It is uncertain if silver nitrate and "Biosept" (cetyl-pyridinium chloride) appeared different or if they were dispensed from labelled vials. They are both solutions.

COMMENT: Knowledge of the medication being dispensed, along with any concomitant bias, could affect adherence and differential application of prophylaxis, which, in turn, could affect preventive effect of the development of conjunctivitis.
Blinding of caregiver who administered medication Bacterial, gonococcal and chlamydial conjunctivitis (objective)
Unclear risk

COMMENT: The study does not comment on whether or not the person administering the medication was masked. It is uncertain if silver nitrate and "Biosept" (cetyl-pyridinium chloride) appeared different or if they were dispensed from labelled vials. They are both solutions.

COMMENT: Knowledge of the medication being dispensed, along with any concomitant bias, could affect adherence and differential application of prophylaxis, which, in turn, could affect preventive effect of the development of bacterial conjunctivitis.
Blinding of persons in- Unclear risk QUOTE: "The children's nurses cleaned the eyes with dry sterile cotton at feedvolved in postnatal care Clinical conjunctivitis (subjective) ing time at intervals of 3-4 hours."

COMMENT: The study does not comment on whether those involved in postnatal care were masked to intervention. Silver nitrate sometimes causes a chemical conjunctivitis that can last up to 72 hours, and silver nitrate causes lid stains that can last 30 to 48 hours. The children were in hospital usually 4 to 6 days postpartum. Furthermore, the study identifies the day of identification of conjunctivitis, and a significant number of cases of conjunctivitis were identified in the first 3 days of birth. Therefore, those involved in postnatal care may not be masked to the medication used as prophylaxis during this initial time period. Lack of masking could lead to differential treatment of the eyes of these neonates, resulting in differential cases of conjunctivitis.

\section{Blinding of persons in- Unclear risk} volved in postnatal care Bacterial, gonococcal and chlamydial conjunctivitis (objective)
QUOTE: "The children's nurses cleaned the eyes with dry sterile cotton at feeding time at intervals of 3-4 hours."

COMMENT: The study does not comment on whether those involved in postnatal care were masked to intervention. Silver nitrate sometimes causes a chemical conjunctivitis that can last up to 72 hours, and silver nitrate causes lid stains that can last 30 to 48 hours. Therefore, those involved in postnatal care may not be masked to the medication used as prophylaxis during this initial time period. Lack of masking could lead to differential treatment of the eyes of these neonates, resulting in differential cases of conjunctivitis. Note that the neonates were in hospital usually 4 to 6 days postpartum. Furthermore, the study mentions the day of identification of conjunctivitis, and a significant number of cases of bacterial conjunctivitis were identified in the first 3 days of birth.

QUOTE: "The type of prophylaxis was recorded on another sheet and attached to the blank for later. Thus, preconceived ideas on the part of the investigator could not affect the appraisal of clinical symptoms."

QUOTE: "The method was therefore changed for series II. The eyes were no longer opened with the fingers except on the first day after birth, and to take the bacterial sample."

COMMENT: As silver nitrate creates lid stains that last 30 to 48 hours, outcome assessments during this time may not have been masked. Considering that neonates were only followed up 2 weeks postpartum and were in hospital 4 
ments.

\section{Blinding of outcome as- Unclear risk} sessment (detection bias) Bacterial, gonococcal and chlamydial conjunctivitis (objective)
QUOTE: "The type of prophylaxis was recorded on another sheet and attached to the blank for later. Thus, preconceived ideas on the part of the investigator could not affect the appraisal of clinical symptoms."

QUOTE: "The method was therefore changed for series II. The eyes were no longer opened with the fingers except on the first day after birth, and to take the bacterial sample."

COMMENT: As silver nitrate creates lid stains that last 30 to 48 hours, outcome assessments during this time may not have been masked. Considering that neonates were only followed up 2 weeks postpartum and were in hospital 4 to 6 days postpartum, there is greater potential for bias on outcome assessments.

Furthermore, a significant number of bacterial conjunctivitis cases were identified in the first 3 postpartum days.

In ambiguous cases of clinical conjunctivitis, there may be differential assessor behaviour to include or exclude cases of clinical conjunctivitis, thereby increasing or decreasing the likelihood of swabbing the neonate's eye for bacterial conjunctivitis. Presence of bacteria on a swab does not necessarily prove that the bacteria caused the conjunctivitis, as the bacteria could be a carrier but the conjunctivitis caused by chemical conjunctivitis. This possible bias was reduced by the fact that the swabs for culture were reserved for those conjunctivitis cases with purulent discharge, as specified in the methods.

$\begin{array}{ll}\begin{array}{l}\text { Incomplete outcome data } \\ \text { (attrition bias) }\end{array} & \begin{array}{l}\text { COMMENT: The study authors do not provide any information on the presence } \\ \text { or absence of incomplete outcome data. The neonates were in hospital } 4 \text { to } 6 \\ \text { Clinical conjunctivitis }\end{array}\end{array}$

(subjective) or absence of incomplete outcome data. The neonates were in hospital 4 to 6 .

Incomplete outcome data Unclear risk (attrition bias)

COMMENT: The study authors do not provide any information on the presence

Bacterial, gonococcal and chlamydial conjunctivitis (objective) days postpartum, and follow-up time in this study was 2 weeks.
Selective reporting (re- High risk porting bias)
QUOTE: "The growth of gonococcus with the method employed is uncertain. A special method for the systematic culture of gonococcus was not regarded as necessary as the incidence of gonorrhea in the material would in all probability have been low"

COMMENT: Gonococcal conjunctivitis is of interest to this review, and the microbiological methods to identify Neisseria gonorrhoeae were "uncertain". There were, predictably, no cases identified in the study.

Other bias Unclear risk

COMMENT: In any trial with silver nitrate, there could be differential diagnostic activity. This could lead to increased diagnosis of true but harmless cases of disease. For example, silver nitrate induces a chemical conjunctivitis. This chemical conjunctivitis could lead to increased selective bacterial cultures of infants' eyes in the silver nitrate intervention group. A positive bacterial culture found from a swab of conjunctivitis due to chemical conjunctivitis does not necessarily mean that the bacteria caused the conjunctivitis. The bacteria could part of the normal flora of the eye, with an associated chemical conjunctivitis, or the bacteria could be the causal agent of the conjunctivitis.

QUOTE: "Touching of the eye region with the fingers was avoided as much as possible with series II of the earlier material... The possibility of cross infec- 
tion and of the transfer of bacteria from the lids to the conjunctiva was thus reduced considerably."

COMMENT: For those infants found by questionnaire to have conjunctivitis after discharge from hospital, cultures were actually taken from infants before discharge from hospital, not on the day the conjunctivitis was identified after discharge.

\section{Study characteristics}

Methods

Parallel-group, single-centre trial.

Method of allocation: alternation by week.

Unit of randomisation: neonate.

Exclusions after allocation: none specified.

Losses to follow-up: yes:

- Day 7: silver nitrate $28 \%$ loss; tetracycline $32 \%$ loss;

- Day 28: silver nitrate 54\% loss; tetracycline 59\% loss.

Missing data handled by imputation (assumed losses to follow-up did not have outcome).

Number allocated: 2732.

Statistical power: "Statistical power was calculated with the method of Pocock".

Unusual study design: none identified.

Participants

Setting: Nairobi City Council maternity hospital, Nairobi, Kenya.

Number allocated: 2732:

- silver nitrate: 1233 ;

- tetracycline: 1499.

Age: neonates.

Sex: M:F unknown.

Inclusion criteria:

- 10 women/day in established labour at the Nairobi City Council maternity hospital from February 1985 to April 1986;

- resided in a certain area;

- verbal consent.

Exclusion criteria: none specified.

Equivalence of limited baseline characteristics.

Interventions

Number of interventions: 2.

- Intervention 1: tetracycline $1 \%$ ointment, dose not specified $(n=1499)$.

- Intervention 2: silver nitrate $1 \%$ solution, from single-dose ampoules, dose not specified $(n=1233)$.

Time to intervention: immediately after birth, no later than 30 minutes after birth. 
Laga 1988 (Continued)

Pre-intervention manoeuvres: babies' eyes wiped dry with cotton.

Postintervention manoeuvres: none specified.

Outcomes Infants with conjunctivitis.
2. Infants with gonococcal conjunctivitis.
3. Infants with chlamydial conjunctivitis.
4. Infants with gonococcal and chlamydial conjunctivitis.
5. Infants with non-gonococcal, non-chlamydial conjunctivitis.
6. Infants with gonococcal ophthalmia among newborns exposed to maternal Neisseria gonorrhoeae.
7. Infants with chlamydial ophthalmia among newborns exposed to Chlamydia trachomatis.

Follow-up: 28 days postpartum; infants eyes examined and history taken at 24 h, 7 days, and 28 days postpartum.

Definition of ophthalmia neonatorum: abnormal discharge from 1 or both eyes and at least 1 PMN leukocyte per 1000x field on a Gram stained smear of discharge. If signs of inflammation were present, the infant's eyes and throat were swabbed for $N$ gonorrhoeae and $C$ trachomatis.

No adverse events reported.

Notes Date study conducted: February 1985 to April 1986.

Source of funding: grants from International Development Research Center, Ottawa, Canada; and Subprogram Science and Technology for Development, Commission of European Communities (Brussels, Belgium).

No declaration of interest statement made.

Subgroup analysis of neonates born to $N$ gonorrhoeae-positive mothers and C trachomatis-positive mothers.

Authors have been contacted for clarifications on masking, no reply received.

\section{Risk of bias}

Bias Authors' judgement Support for judgement

Random sequence genera- High risk tion (selection bias)

QUOTE: "Silver nitrate and tetracycline were used during alternate weeks."

QUOTE: "Over a 15 month period, 10 women in labor were enrolled in the study every day but specially trained midwives at a Nairobi City Council maternity hospital".

QUOTE: "More infants were given tetracycline because of a two interruption in randomization due to the unavailability of silver nitrate"

COMMENT: The paper does not state how these 10 women were selected.

Allocation concealment High risk
(selection bias)

QUOTE: "Silver nitrate and tetracycline were used during alternate weeks."

COMMENT: Allocation concealment was not addressed in this study, however the allocation method itself, alternation, allowed for investigators enrolling participants to foresee assignments, thus introducing selection bias.

\begin{tabular}{|c|c|c|}
\hline $\begin{array}{l}\text { Blinding of study partici- } \\
\text { pants (mothers of infants) } \\
\text { Clinical conjunctivitis } \\
\text { (subjective) }\end{array}$ & Unclear risk & $\begin{array}{l}\text { COMMENT: Masking of mothers to the intervention was not addressed in this } \\
\text { study. Furthermore, the } 2 \text { interventions differ in colour and consistency. Sil- } \\
\text { ver nitrate is a clear solution. Also, silver nitrate sometimes causes a chemical } \\
\text { conjunctivitis that can last up to } 72 \text { hours, and lid stains that can last } 30 \text { to } 48 \\
\text { hours. Tetracycline is a light-yellow ointment that is readily distinguished from }\end{array}$ \\
\hline
\end{tabular}



this could affect performance bias.

Blinding of study participants (mothers of infants) Bacterial, gonococcal and chlamydial conjunctivitis (objective)
Unclear risk

COMMENT: Masking of mothers to the intervention was not addressed in this study. Furthermore, the 2 interventions differ in colour and consistency. Silver nitrate is a clear solution. Also, silver nitrate sometimes causes a chemical conjunctivitis that can last up to 72 hours, and lid stains that can last 30 to 48 hours. Tetracycline is a light-yellow ointment that is readily distinguished from silver nitrate, and that may leave an ocular residue for hours that the mother could notice. It is unknown how this could affect performance bias.

The mothers may differentially handle the eyes of neonates based on the visible signs of prophylaxis. This could lead to differential introduction of pathogenic bacteria into the eyes of these neonates. Therefore, lack of masking of medication appearance may lead to bias in chlamydial and gonococcal conjunctivitis cases, depending on hygiene measures. However, considering the low event rates of gonococcal conjunctivitis and possible carrier state of $C$ trachomatis, this could introduce important bias. It is unknown how many neonates developed conjunctivitis in the time period when the medication could be identified.
Blinding of caregiver who administered medication Clinical conjunctivitis (subjective)
High risk

QUOTE: "Single-dose ampules were used to administer the silver nitrate...The tetracycline ointment was administered from multidose tubes, which were used for one day and then discarded."

COMMENT: Masking of the person who administers the medication was not addressed in this study. As mentioned, the 2 interventions differ in colour and consistency. Tetracycline is a light-yellow ointment, and silver nitrate is a clear solution. Both medications are readily identifiable to anyone administering the medication.
Blinding of caregiver who administered medication Bacterial, gonococcal and chlamydial conjunctivitis (objective)

\section{High risk}

QUOTE: "Single-dose ampules were used to administer the silver nitrate...The tetracycline ointment was administered from multidose tubes, which were used for one day and then discarded."

COMMENT: Masking of the person who administers the medication was not addressed in this study. As mentioned, the 2 interventions differ in colour and consistency.

Tetracycline is a light-yellow ointment, and silver nitrate is a clear solution. Both medications are readily identifiable to anyone administering the medication.

With lack of masking, the person administering the medication could dispense the medications differently, or there could be differential adherence, thereby altering the bactericidal effect. We have seen this in 1 study in Africa where 1 of the medications was ointment, and tended to be "skipped" more than solution, owing to difficulty in handling the ointment.

QUOTE: "The eyes of infants were examined 24 hours after delivery, and the mother..."

COMMENT: Masking of those involved in postnatal care has not been addressed in this study. As mentioned, silver nitrate sometimes causes a chemical conjunctivitis that can last up to 72 hours, and lid stains that can last 30 to 48 hours. Tetracycline is an ointment that leaves a residue that can last for hours. The eyes of infants were examined 24 hours after delivery, therefore it is possible that the prophylaxis could be determined at this time.

\section{COMMENT:}


It is unclear how long the neonates were in hospital, and how many cases of conjunctivitis were identified at this time. It is also unclear if those involved in postnatal care were also involved in identifying cases of conjunctivitis, and to what extent.

Those involved in postnatal care may differentially handle the eyes of neonates based on the visible signs of prophylaxis. This could lead to differential introduction of pathogenic bacteria into the eyes of these neonates.

\author{
Blinding of persons in- \\ volved in postnatal care \\ Bacterial, gonococcal and \\ chlamydial conjunctivitis \\ (objective)
}

Unclear risk QUOTE: "The eyes of infants were examined 24 hours after delivery, and the mother..."

COMMENT: Masking of those involved in postnatal care has not been addressed in this study. As mentioned, silver nitrate sometimes causes a chemical conjunctivitis that can last up to 72 hours, and lid stains that can last 30 to 48 hours. Tetracycline is an ointment that leaves a residue that can last for hours. The eyes of infants were examined 24 hours after delivery, therefore it is possible that prophylaxis could be determined at this time.

COMMENT: It is unclear how long the neonates were in hospital, and how many cases of conjunctivitis were identified at this time. It is also unclear if those involved in postnatal care were also involved in identifying cases of conjunctivitis, and to what extent. Those involved in postnatal care may differentially handle the eyes of neonates based on the visible signs of prophylaxis. This could lead to differential introduction of pathogenic bacteria into the eyes of these neonates.

Any bias in identification of cases of conjunctivitis cases could influence cases referred for swabbing for chlamydia and gonorrhoea. For instance, if the nurse was aware that neonates were given silver nitrate, and were aware of the concomitant chemical conjunctivitis, in ambiguous cases the nurse may erroneously ignore cases of 'true' bacterial conjunctivitis that happen to present outside the chemical conjunctivitis window.

The incubation period of gonococcal conjunctivitis and chlamydial conjunctivitis is likely outside the time period at which the nurses in the nursery would be influencing identification and care. Furthermore, these cases are likely to be more clinically severe, eliminating ambiguity. Therefore, the lack of masking of the nurses in the nursery will likely introduce less bias for the outcomes of gonococcal and chlamydial conjunctivitis. Still, the possible carrier state of chlamydia and the low event rate of chlamydial and gonococcal conjunctivitis in the study could make minor bias important and clinically significant.

\section{Blinding of outcome as- Unclear risk} sessment (detection bias) Clinical conjunctivitis (subjective)
QUOTE: "The eyes of the infants were examined 24 hours after delivery, and the mother and baby were requested to return to one of three postnatal clinics 7 and 30 days post partum. On day 7 , the medical history of the infants was taken, and their eyes were examined for evidence of conjunctival inflammation"

COMMENT: Masking of outcome assessors was not addressed in this study.

Silver nitrate sometimes causes a chemical conjunctivitis that can last up to 72 hours, and lid stains that can last 30 to 48 hours. Tetracycline may leave an ocular residue for hours. It appears that the infants were examined 24 hours after delivery, when the prophylaxis could be identified. It is unknown how many cases of conjunctivitis were identified during the time when these stains remained.

The definition of conjunctivitis includes discharge and microscopic presentation of at least 1 polymorphonuclear leukocyte per oil-immersion field on a Gram stained smear of the discharge, which adds more objectivity. 
Laga 1988 (Continued)

Blinding of outcome assessment (detection bias) Bacterial, gonococcal and chlamydial conjunctivitis (objective)
Unclear risk

QUOTE: "The eyes of the infants were examined 24 hours after delivery, and the mother and baby were requested to return to one of three postnatal clinics 7 and 30 days post partum. On day 7 , the medical history of the infants was taken, and their eyes were examined for evidence of conjunctival inflammation"

QUOTE: "However, an evaluation of a subgroup of exposed newborns in the present study population suggests that in at least $25 \%$ of these infants, asymptomatic ocular infection with C. trachomatis did develop and was usually diagnosed after the first month of life" (Datta P et al; unpublished data)

COMMENT: Silver nitrate sometimes causes a chemical conjunctivitis that can last up to 72 hours, and lid stains that can last 30 to 48 hours. Tetracycline is a light-yellow ointment that may leave an ocular residue for hours.

It is unknown how many cases of conjunctivitis were identified during the time when these stains remained. This could affect bias in the decision to swab the eyes for microbiological analysis, thereby altering chlamydial and gonococcal conjunctivitis cases. Any minor bias could be significant as event rates in this study were low.

The incubation period of gonococcal conjunctivitis and chlamydial conjunctivitis is likely outside the time period at which these stains would remain. Furthermore, gonococcal and chlamydial conjunctivitis are usually more severe, with less diagnostic ambiguity than other forms of conjunctivitis. In the case of chlamydial conjunctivitis, however, it can present with a variable clinical spectrum. $C$ trachomatis may also asymptomatically colonise the eye. Therefore, lack of masking, in cases of diagnostic ambiguity, may affect which cases get referred for culture to identify chlamydial conjunctivitis. Lack of masking may lead to under-referral of neonates for culture due to the erroneous perception that conjunctivitis is chemical from silver nitrate, thereby missing chlamydial conjunctivitis cases. Over-referral may be caused by knowledge of which infants received silver nitrate, or bias, and lead to identifying cases of chemical conjunctivitis with chlamydia carrier, rather than conjunctivitis truly caused by C trachomatis.
Incomplete outcome data High risk (attrition bias)

Clinical conjunctivitis

(subjective)

COMMENT: On Day 7, 351/1233 neonates were missing from the silver nitrate group, and 474/1499 neonates were missing from the tetracycline group.

COMMENT: On Day 28, 665/1233 neonates were missing from the silver nitrate group, and $888 / 1499$ were missing from the tetracycline group.

COMMENT: Although there were no major asymmetries in loss to follow-up, the loss to follow-up in relation to event rates was high.

\section{Incomplete outcome data High risk} (attrition bias)

Bacterial, gonococcal and chlamydial conjunctivitis (objective)

COMMENT: Among the 82 newborns born to mothers with $N$ gonorrhoeae, 11 did not return for follow-up in the silver nitrate group, and 27/99 did not return for follow-up in the tetracycline group.

COMMENT: $16 / 115$ infants born to mothers with $C$ trachomatis infection in the silver nitrate arm and 18/129 exposed infants in the tetracycline arm were not followed up.

COMMENT: Although there were no major asymmetries in loss to follow-up, the loss to follow-up in relation to event rates of gonococcal and chlamydial conjunctivitis was high.

Selective reporting (re- Unclear risk

QUOTE: "However, an evaluation of a subgroup of exposed newborns in the present study population suggests that in at least $25 \%$ of these infants, asymptomatic ocular infection with C. trachomatis did develop and was usually diagnosed after the first month of life" (Datta P et al; unpublished data) 
COMMENT: It is unknown if asymptomatic ocular infection with $C$ trachomatis was prespecified in the protocol.

COMMENT: The study did not report cases of other bacterial conjunctivitis, or conjunctivitis that led to no growth on culture. There is a category of nongonococcal and non-chlamydial conjunctivitis reported in the study, but this category does not distinguish bacterial conjunctivitis from no-culture growth conjunctivitis. It is unknown if these outcomes were prespecified in the study protocol. However, these 2 outcomes of other bacterial conjunctivitis and nogrowth conjunctivitis are outcomes of interest to this review, and cannot be entered in the meta-analysis.

Other bias Unclear risk In any trial with silver nitrate, there could be diagnostic bias.

Silver nitrate causes a chemical conjunctivitis in the first 72 hours. As a result, in the first 72 hours, more neonates in the silver nitrate allocation group could be referred for culture. Finding bacteria in the culture does not necessarily prove that the bacteria caused the conjunctivitis. The conjunctivitis could be chemical, with a chlamydial carrier. This study did note the presence of asymptomatic chlamydial infection. Finally, the conjunctivitis could very well be caused by chlamydia. Consideration of incubation periods, and assessing for carriers with asymptomatic cases, could assist with differential diagnosis.

\section{Study characteristics}

\section{Methods}

Parallel-group RCT.

Method of allocation: randomisation into 2 groups by blocked randomisation with a fixed block size of 4.

Unit of randomisation: neonate.

Exclusions after randomisation: mothers were included in the study, but 72 neonates were excluded after delivery, before randomisation, and before application of prophylaxis, for the following reasons:

1. low weight;

2. respiratory distress;

3. death;

4. transfer of the mother to a more specialised centre for dystocic delivery.

Losses to follow-up: 229 out of 245 were lost to follow-up, which is $93 \%$ loss to follow-up.

Number randomised: 245 neonates.

Missing data were handled by available-case analysis in the study.

Power calculation was done: sample size of 334 newborns with power of $80 \%$.

Unusual study design: follow-up time only 7 to 10 days with $93 \%$ loss to follow-up.

Participants

Country: Luanda, Angola.

Setting: General Augusto N'Gangula Specialized Hospital and the Health Center of Samba.

Ethnic group: not specified; maternal data were collected on race but not reported in study.

Total number of participants: 245 . 
Pastor 2015 (Continued)

Sex: M:F 118:123 (4 unknown as not registered).

Average age and age range: average age of neonates not available; gestational age range 36 to 40 weeks.

Inclusion criteria:

1. healthy children weighing at least $2.3 \mathrm{~kg}$;

2. gestation period of at least 37 weeks.

Exclusion criteria:

1. respiratory distress at birth;

2. mother diagnosed with thyroid disease.

No comment was made in the study on equivalence of baseline characteristics.

Interventions

Number of interventions: 2

- Intervention 1: povidone-iodine 2.5\%; 1 drop of povidone-iodine in the lower sac of each eye from a new bottle for each newborn ( $\mathrm{n}=115$ neonates).

- Intervention 2: no intervention; no placebo was administered ( $n=130$ neonates).

Time to intervention: immediately after a basic eye examination and the collection of conjunctival smears within 3 hours of birth.

Pre-intervention manoeuvres: basic eye exam and collection of conjunctival smears by vigorous swabbing across the interior tarsal conjunctiva.

Postintervention manoeuvres: none specified.

Outcomes

1. Presence or absence of Chylamdia trachomatis, Neisseria gonorrhoeae, or Mycoplasma genitalium in mother endocervical samples or neonate conjunctival smears.

2. Conjunctivitis in neonates.

3. Ophthalmia neonatorum in neonates.

No comment was made on adverse events.

Follow-up:

1. up to 10 days;

2. planned follow-up: between the 5 th day and 7 th day postpartum phone calls were made to mothers to bring their infants for observation, especially if they had signs of ophthalmia neonatorum;

3. actual follow-up: attempts were made to have every mother perform a follow-up visit within 7 to 10 days.

Notes

Date conducted: 7 December 2011 to 22 November 2012.

Sources of funding: AECID: Spanish Agency for International Cooperation and Development.

Declaration of interest: the authors declared that there were no conflict of interests regarding the publication of the paper.

No reported subgroup analysis.

Trial investigators were contacted.

No data could be extracted from this study in spite of contacting the authors.

\section{Risk of bias}


Pastor 2015 (Continued)

Random sequence genera- Low risk QUOTE: "Neonates were randomly distributed into two groups, A and B, by tion (selection bias) blocked randomization with a fixed block size of $4 . "$

Allocation concealment Unclear risk (selection bias)

Blinding of study partici- Unclear risk pants (mothers of infants)

Clinical conjunctivitis

(subjective)
COMMENT: No information is provided on allocation concealment.

QUOTE: "An interventional, randomized, and prospective study with a blinded, randomized control group was designed."

COMMENT: Povidone-iodine is an orange-red solution that leads to transient residual staining of the eye, and possible periocular stains that can last minutes to hours. The mother could have noticed these stains.

The control group did not receive placebo, but received no prophylaxis.

The mothers may handle the eyes of neonates with periocular stains differently than the neonates with no stains.

\begin{tabular}{|c|c|c|}
\hline $\begin{array}{l}\text { Blinding of study partici- } \\
\text { pants (mothers of infants) } \\
\text { Bacterial, gonococcal and } \\
\text { chlamydial conjunctivitis } \\
\text { (objective) }\end{array}$ & Unclear risk & $\begin{array}{l}\text { The study did not report bacterial, gonococcal, or chlamydial conjunctivitis } \\
\text { as outcomes, therefore there was no assessment of bias for this category with } \\
\text { these outcomes. }\end{array}$ \\
\hline $\begin{array}{l}\text { Blinding of caregiver who } \\
\text { administered medication } \\
\text { Clinical conjunctivitis } \\
\text { (subjective) }\end{array}$ & High risk & $\begin{array}{l}\text { QUOTE: "The ophthalmologist responsible for the study (IA) administered the } \\
\text { P-I eyedrops." } \\
\text { COMMENT: The ophthalmologist responsible for the study also administered } \\
\text { the prophylaxis, which is readily identifiable. Furthermore, povidone-iodine is } \\
\text { an orange-red solution that leads to transient residual staining of the eye, and } \\
\text { possible periocular stains that can last minutes to hours. The control group did } \\
\text { not receive placebo, but received no prophylaxis, therefore masking has been } \\
\text { compromised. }\end{array}$ \\
\hline
\end{tabular}

\begin{tabular}{|c|c|c|}
\hline $\begin{array}{l}\text { Blinding of caregiver who } \\
\text { administered medication } \\
\text { Bacterial, gonococcal and }\end{array}$ & Unclear risk & $\begin{array}{l}\text { did not report bacterial, gonococcal, or chlamydial conjunctivitis } \\
\text { nes, therefore there was no assessment of bias for this category with } \\
\text { comes. }\end{array}$ \\
\hline
\end{tabular}

chlamydial conjunctivitis

(objective)
Blinding of persons in- Unclear risk volved in postnatal care Clinical conjunctivitis (subjective)
COMMENT: Povidone-iodine is an orange-red solution that leads to transient residual staining of the eye, and possible periocular stains that can last minutes to hours. The control group did not receive placebo, but received no prophylaxis. A significant number of cases of conjunctivitis were apparently diagnosed at delivery. It is unclear who diagnosed conjunctivitis, and whether those involved in postnatal care were involved in identifying and referring possible conjunctivitis cases. Nonetheless, it remains possible that diagnosis of conjunctivitis was made when the prophylaxis administered could be readily identified.

\begin{tabular}{|c|c|c|}
\hline $\begin{array}{l}\text { Blinding of persons in- } \\
\text { volved in postnatal care } \\
\text { Bacterial, gonococcal and } \\
\text { chlamydial conjunctivitis } \\
\text { (objective) }\end{array}$ & Unclear risk & $\begin{array}{l}\text { The study did not report bacterial, gonococcal, or chlamydial conjunctivitis } \\
\text { as outcomes, therefore there was no assessment of bias for this category with } \\
\text { these outcomes. }\end{array}$ \\
\hline $\begin{array}{l}\text { Blinding of outcome as- } \\
\text { sessment (detection bias) } \\
\text { Clinical conjunctivitis } \\
\text { (subjective) }\end{array}$ & Unclear risk & $\begin{array}{l}\text { QUOTE: "The ophthalmologist responsible for the study (IA) administered the } \\
\text { P-I eyedrops." }\end{array}$ \\
\hline
\end{tabular}


COMMENT: The paper does not comment on who conducted the outcome assessments of conjunctivitis. We do not know if the ophthalmologist responsible for administering the povidone-iodine drops was involved. If so, this would affect masking. Separate from this issue, povidone-iodine is an orange-red solution that leads to transient residual staining of the eye, and possible periocular stains that can last minutes to hours. The control group did not receive any placebo, but received no prophylaxis. Considering that a significant number of acute conjunctivitis cases were diagnosed at delivery, it remains possible that outcome assessors were not masked for some outcome assessments.

\begin{tabular}{|c|c|c|}
\hline $\begin{array}{l}\text { Blinding of outcome as- } \\
\text { sessment (detection bias) } \\
\text { Bacterial, gonococcal and }\end{array}$ & Unclear risk & $\begin{array}{l}\text { The study did not report bacterial, gonococcal, or chlamydial conjunctivitis } \\
\text { as outcomes, therefore there was no assessment of bias for this category with } \\
\text { these outcomes. }\end{array}$ \\
\hline
\end{tabular}
these outcomes. chlamydial conjunctivitis (objective)

$\begin{array}{ll}\begin{array}{l}\text { Incomplete outcome data } \\ \text { (attrition bias) }\end{array} & \text { High risk } \\ \begin{array}{l}\text { Clinical conjunctivitis } \\ \text { (subjective) }\end{array} & \begin{array}{l}\text { COMMENT: Only 5\% of the total study participants were followed up. The pro- } \\ \text { portion of missing outcomes compared with the observed event risk is highly } \\ \text { likely to induce clinically relevant bias in the intervention effect estimate. }\end{array}\end{array}$

Unclear risk Incomplete outcome dat (attrition bias)

Bacterial, gonococcal and chlamydial conjunctivitis (objective)
The study did not report bacterial, gonococcal, or chlamydial conjunctivitis as outcomes, therefore there was no assessment of bias for this category with these outcomes.

\begin{tabular}{ll}
\hline $\begin{array}{l}\text { Selective reporting (re- } \\
\text { porting bias) }\end{array}$ & High risk \\
& $\begin{array}{l}\text { COMMENT: The study protocol is not available. Furthermore, considerable } \\
\text { communication was required with the study author to clarify actual numbers } \\
\text { of conjunctivitis cases by allocation group in the small number of cases that } \\
\text { were followed up. Questions remain. }\end{array}$ \\
\hline
\end{tabular}

Other bias Unclear risk Insufficient information to assess whether an important risk of bias exists

Posner 1959

\title{
Study characteristics
}

Methods

\author{
Parallel-group, single-centre trial.
}

Method of allocation: alternation by date of birth (odd days allocated to 1 group and even days to another).

Unit of randomisation: neonate.

Exclusions after allocation: none specified and not addressed in paper.

Losses to follow-up: none specified and not addressed in paper.

Number allocated: 3355 neonates.

No comment on how missing data handled.

No reported power calculation.

Unusual study design: none identified

Participants Setting: Harlem Hospital, New York, NY, USA.


Number allocated: 3355 neonates:

- bacitracin: 1719 ;

- "mechanical cleansing": 1636.

Age: neonates.

Sex: M:F not specified.

Inclusion criteria: all infants born 1 July 1957 to 30 June 1958 at Harlem Hospital.

Exclusion criteria: none specified.

Equivalence of baseline characteristics not addressed.

Interventions

Number of interventions: 2 .

- Intervention 1: "mechanical cleansing" + bacitracin-phenacaine ophthalmic ointment, 500 units bacitracin/gram and $2 \%$ phenacaine hydrochloride $(n=1719)$.

- Intervention 2: "mechanical cleansing" only; eyes swabbed with distilled water and wiped dry ( $\mathrm{n}=$ 1636).

Time to intervention: not specified.

Pre-intervention manoeuvres: none specified.

Postintervention manoeuvres: none specified (unclear if mechanical cleansing was before or after bacitracin administration).

\begin{tabular}{ll}
\hline Outcomes & 1. Infants with non-specific conjunctivitis. \\
2. Infants with gonorrhoeal ophthalmia. \\
Follow-up: not specified. \\
Definition of non-specific conjunctivitis: not defined. \\
Definition of gonorrhoeal ophthalmia: not defined. \\
Date conducted: 1 July 1957 to 30 June 1958. \\
Source of funding: Upjohn Company, Kalamazoo, MI, which supplied the bacitracin-phenacaine oint- \\
ment. \\
No declaration of interest statement made. \\
No reported subgroup analysis. \\
Trial investigators were not contacted.
\end{tabular}

\section{Risk of bias}

Bias Authors' judgement Support for judgement

Random sequence genera- High risk tion (selection bias)
QUOTE: "The infants born on the numerically even days of the calendar received no medication for ophthalmia neonatorum, the eyes being swabbed with distilled water, and wiped dry. For those delivered on the odd days, in addition to mechanical cleansing of the eyes, we used the bacitracin-phenacine ointment."

COMMENT: Sequence generated by odd or even date of birth. 
Posner 1959 (Continued)

$\begin{array}{ll}\begin{array}{l}\text { Allocation concealment } \\ \text { (selection bias) }\end{array} & \text { High risk }\end{array} \quad \begin{aligned} & \text { Participants or investigators enrolling participants could possibly have fore- } \\ & \text { seen assignments, thus introducing selection bias based on date of birth. }\end{aligned}$

$\begin{array}{lll}\begin{array}{l}\text { Blinding of study partici- } \\ \text { pants (mothers of infants) }\end{array} & \text { Unclear risk } & \begin{array}{l}\text { All cases of clinical conjunctivitis were not reported as outcomes in this study, } \\ \text { Clinical conjunctivitis }\end{array} \\ \text { (subjective) } & \begin{array}{l}\text { or total clinical conjunctivitis cases could not be extracted from this study. } \\ \text { come of clinical conjunctivitis. }\end{array}\end{array}$

Blinding of study participants (mothers of infants) Bacterial, gonococcal and chlamydial conjunctivitis (objective)
Unclear risk

COMMENT: Masking of the mother was not addressed in this paper.

Bacitracin is an ointment that would initially be noticed in the infant by the mother. There was no placebo in the allocation group that received no prophylaxis. The mothers of neonates with noticeable residual ointment or colostrum of the eyes may handle the eyes of the infant more than mothers of neonates with no prophylaxis. This could lead to differential introduction of Neisseria gonorrhoeae bacteria into the eyes of these neonates in the case of poor hygiene. This would be less likely for $\mathrm{N}$ gonorrhoeae than for other bacteria.
Unclear risk
All cases of clinical conjunctivitis were not reported as outcomes in this study, or total clinical conjunctivitis cases could not be extracted from this study. Consequently, there was no assessment of bias for this category for the out- come of clinical conjunctivitis.
Blinding of caregiver who Unclear risk Clinical conjunctivitis (subjective)

Unclear risk

COMMENT: Masking of the person who administers medication was not addressed in this paper. Bacitracin is an ointment. There was no placebo in the allocation group that received no prophylaxis. The person who administers the medication would handle the eyes of neonates with bacitracin, but not neonates with no prophylaxis as there was no placebo. This could lead to differential introduction of $\mathrm{N}$ gonorrhoeae bacteria into the eyes of these neonates, if the person administering the medication was potentially involved in the delivery.

Blinding of caregiver who administered medication chlamydial conjunctivitis (objective)

\begin{tabular}{|c|c|c|}
\hline $\begin{array}{l}\text { Blinding of persons in- } \\
\text { volved in postnatal care } \\
\text { Clinical conjunctivitis } \\
\text { (subjective) }\end{array}$ & Unclear risk & $\begin{array}{l}\text { All cases of clinical conjunctivitis were not reported as outcomes in this study, } \\
\text { or total clinical conjunctivitis cases could not be extracted from this study. } \\
\text { Consequently, there was no assessment of bias for this category for the out- } \\
\text { come of clinical conjunctivitis. }\end{array}$ \\
\hline
\end{tabular}
Blinding of persons in-
volved in postnatal care Bacterial, gonococcal and chlamydial conjunctivitis (objective)
Unclear risk

COMMENT: Masking of the person who was involved in postnatal care was not addressed in this paper.

Bacitracin is a translucent ointment that can be noticed in the eyes for hours. There was no placebo in the allocation group that received no prophylaxis.

\begin{tabular}{lll}
\hline Blinding of outcome as- & Unclear risk & All cases of clinical conjunctivitis were not reported as outcomes in this study, \\
sessment (detection bias) & or total clinical conjunctivitis cases could not be extracted from this study. \\
Clinical conjunctivitis & Consequently, there was no assessment of bias for this category for the out- \\
(subjective) & come of clinical conjunctivitis.
\end{tabular}

Blinding of outcome assessment (detection bias) Bacterial, gonococcal and chlamydial conjunctivitis (objective)
Unclear risk
COMMENT: Masking of the person who was involved in outcome assessment was not addressed in this paper.

Bacitracin is an ointment that can be noticed in the eyes for hours. It is unclear how many cases of conjunctivitis were diagnosed during this early period of time. There was no placebo in the no-prophylaxis group. In ambiguous cases of clinical conjunctivitis, whether identified by personnel, mother, or outcome assessor, there may be differential group behaviour to include or exclude cases of clinical conjunctivitis referred for culture with lack of masking. Although gonococcal conjunctivitis usually presents with high clinical severity, reducing ambiguity, there is a clinical spectrum of its presentation, and event rates are 


$\begin{array}{ll}\begin{array}{l}\text { Incomplete outcome data } \\ \text { (attrition bias) }\end{array} & \text { Unclear risk } \\ \begin{array}{l}\text { Clinical conjunctivitis } \\ \text { (subjective) }\end{array} & \begin{array}{l}\text { All cases of clinical conjunctivitis were not reported as outcomes in this study, } \\ \text { or total clinical conjunctivitis cases could not be extracted from this study. } \\ \text { Consequently, there was no assessment of bias for this category for the out- } \\ \text { come of clinical conjunctivitis. }\end{array}\end{array}$

\begin{tabular}{|c|c|c|}
\hline $\begin{array}{l}\text { Incomplete outcome data } \\
\text { (attrition bias) } \\
\text { Bacterial, gonococcal and } \\
\text { chlamydial conjunctivitis } \\
\text { (objective) }\end{array}$ & Unclear risk & $\begin{array}{l}\text { COMMENT: The study authors do not provide any information on the presence } \\
\text { or absence of incomplete outcome data. Attritions and exclusions are not re- } \\
\text { ported. No statement is provided on how long neonates were followed up. }\end{array}$ \\
\hline $\begin{array}{l}\text { Selective reporting (re- } \\
\text { porting bias) }\end{array}$ & High risk & $\begin{array}{l}\text { COMMENT: The only outcome that could be extracted was rates of gonococ- } \\
\text { cal conjunctivitis. At the very least, rates of clinical conjunctivitis would be of } \\
\text { interest, but they are reported unclearly so that they cannot be entered into a } \\
\text { meta-analysis. The paper reports rates of "nonspecific" conjunctivitis; it is un- } \\
\text { clear whether this is clinical, bacterial, or inclusion conjunctivitis. }\end{array}$ \\
\hline
\end{tabular}

Other bias Unclear risk Insufficient information to assess whether an important risk of bias exists

\section{Ramirez-Ortiz 2007}

\section{Study characteristics}

Methods $\quad$ Parallel-group, multicentre RCT.

Method of allocation: neonates "randomly assigned". "Randomisation assignments were allocated centrally in a weekly fashion by the coordinating centre in a 1: 1 ratio". No further information on method of allocation provided in paper.

Unit of randomisation: neonate.

Number allocated: 2004.

Handling of missing data: unclear; review authors used imputation, assuming neonates lost to follow-up had no conjunctivitis.

Exclusions after allocation: 22 neonates were excluded after enrolment but before randomisation.

Losses to follow-up:

Chloramphenicol group

1. First eye examination in the first 24 to 48 hours: 0 out of 972 lost to follow-up.

2. Second eye examination between Days 10 and 15: 310 out of 972 lost to follow-up.

3. Third eye examination between Day 16 and Day 30: 502 out of 972 lost to follow-up.

Povidone-iodine group

1. First eye examination in the first 24 to 48 hours: 0 out of 1032 lost to follow-up.

2. Second eye examination between Days 10 and 15: 348 out of 1032 lost to follow-up.

3. Third eye examination between Day 16 and Day 30: 572 out of 1032 lost to follow-up.

Reported power calculation: yes; power 0.80 ; sample size of 660 per allocation group calculated. "Allowing for an estimated loss of $35 \%$ of cases after the start of the study, 270 additional infants were recruited." 
Ramirez-Ortiz 2007 (Continued)

Unusual study design: none.

Participants

Setting: 3 hospitals in the highlands of Chiapas, Mexico:

1. rural - San Felipe Ecatepec;

2. general - San Cristo bal de las Casas;

3. rural - Ocosingo.

Number allocated: 2004

Age: neonates.

Sex: M:F 51.7\%:48.4\%.

Inclusion criteria: all neonates born by vaginal or caesarean section.

Exclusion criteria:

1. eyelid malformations that prevented appropriate conjunctival evaluation;

2. death in the first month of life.

Equivalence of baseline characteristics: yes.

No statistically significant difference in the following characteristics:

1. birthweight;

2. M:F ratio;

3. mode of delivery;

4. sociodemographic characteristics;

5. cases by reference hospital;

6. there were statistically more cases of premature rupture of membranes in the chloramphenicol group.

Interventions

Number of interventions: 2 .

- Intervention 1: chloramphenicol eye drops; dose not specified $(\mathrm{n}=972)$.

- Intervention 2: 2.5\% povidone-iodine eye drops; dose not specified $(n=1032)$.

Time to intervention: within 20 minutes of birth.

Pre-intervention manoeuvres: none specified.

Postintervention manoeuvres: eyelids wiped immediately after prophylaxis.

Outcomes

1. "Incidence density per 1000 neonate days of bacteria isolated from conjunctival specimens by treatment group."

2. Paper reports cases of chlamydial conjunctivitis by treatment group.

3. No cases of gonococcal conjunctivitis reported in study.

Based on the data as currently reported, we are unable to determine total number of cases of conjunctivitis and total number of cases of bacterial conjunctivitis per treatment group.

Length of follow-up: 30 days postdelivery.

Intervals at which outcomes assessed:

1. first interval: 24 to 48 hours in postnatal ward;

2. second interval: between Day 10 and Day 15;

3. third interval: between Day 16 and Day 30 .

Definition of conjunctivitis or ophthalmia neonatorum: "Neonatal conjunctivitis was defined clinically by a yellow or greenish discharge in the conjunctival cul-de-sac or involving the eyelids and eyelashes, or both." 
Ramirez-Ortiz 2007 (Continued)

Non-infectious conjunctivitis, described as side effect of prophylactic treatment, was defined as "conjunctival hyperaemia, chemosis, and eyelid swelling without greenish or yellowish discharge". It is unclear if clinical "non-infectious conjunctivitis" had swabs taken for bacterial cultures.

Adverse events reported as "non-infectious conjunctivitis", bronchospasm, or death.

Notes Date study conducted not specified.

Sources of funding: Hospital Infantil de Mexico Board of Trustees Grant HIM/ 2002/024.

Declaration of interest: "Competing interests: None declared."

Subgroup analysis reported by method of delivery (vaginal versus caesarean).

Trial investigators were contacted on unclear items; reply received.

\section{Risk of bias}

\begin{tabular}{lll} 
Bias & Authors' judgement & Support for judgement \\
\hline $\begin{array}{ll}\text { Random sequence genera- } \\
\text { tion (selection bias) }\end{array}$ & Low risk & $\begin{array}{l}\text { Paper states neonates "randomly assigned". } \\
\text { "Randomisation assignments were allocated centrally in a weekly fashion by } \\
\text { the coordinating centre in a } 1: 1 \text { ratio". }\end{array}$
\end{tabular}

\begin{tabular}{ll}
\hline $\begin{array}{l}\text { Allocation concealment } \\
\text { (selection bias) }\end{array}$ & Paper states neonates "randomly assigned". \\
& "Randomisation assignments were allocated centrally in a weekly fashion by \\
& the coordinating centre in a 1: 1 ratio".
\end{tabular}

\begin{tabular}{|c|c|c|}
\hline $\begin{array}{l}\text { Blinding of study partici- } \\
\text { pants (mothers of infants) } \\
\text { Clinical conjunctivitis } \\
\text { (subjective) }\end{array}$ & Unclear risk & $\begin{array}{l}\text { All cases of clinical conjunctivitis were not reported as outcomes in this study } \\
\text { or total clinical conjunctivitis cases could not be extracted from this study. } \\
\text { Consequently, there was no assessment of bias for this category for the out- } \\
\text { come of clinical conjunctivitis. }\end{array}$ \\
\hline
\end{tabular}

Blinding of study partici- Unclear risk COMMENT: Masking of mothers of the intervention was not addressed in this pants (mothers of infants) study. Furthermore, the 2 interventions differ in colour. Povidone-iodine is Bacterial, gonococcal and an orange-red solution that may lead to periocular stains that last minutes to chlamydial conjunctivitis hours. Chloramphenicol is a clear, colourless to slightly yellow solution. (objective)

The mothers of neonates with periocular stains may handle the eyes of the infant differently than mothers of neonates with no stains. The paper does state that eyelids were wiped immediately after birth, but it is uncertain if this eliminated all periocular stains. This could lead to differential introduction of Chlamydia trachomatis bacteria into the eyes of these neonates in the case of poor hygiene. Alternatively, it could lead to contamination with other bacteria, subsequent conjunctivitis, and identification of chlamydia carriers instead.

\begin{tabular}{|c|c|c|}
\hline $\begin{array}{l}\text { Blinding of caregiver who } \\
\text { administered medication } \\
\text { Clinical conjunctivitis } \\
\text { (subjective) }\end{array}$ & Unclear risk & $\begin{array}{l}\text { All cases of clinical conjunctivitis were not reported as outcomes in this study, } \\
\text { or total clinical conjunctivitis cases could not be extracted from this study. } \\
\text { Consequently, there was no assessment of bias for this category for the out- } \\
\text { come of clinical conjunctivitis. }\end{array}$ \\
\hline $\begin{array}{l}\text { Blinding of caregiver who } \\
\text { administered medication } \\
\text { Bacterial, gonococcal and } \\
\text { chlamydial conjunctivitis } \\
\text { (objective) }\end{array}$ & High risk & $\begin{array}{l}\text { COMMENT: Masking of the person who administers the medication was not ad- } \\
\text { dressed in this study. The } 2 \text { interventions differ in colour. Povidone-iodine is } \\
\text { an orange-red solution that may lead to periocular stains that last minutes to } \\
\text { hours. Chloramphenicol is a clear, colourless to slightly yellow solution. The in- } \\
\text { terventions are therefore readily identifiable. Any bias on the part of the per- } \\
\text { son who administers the medication could affect adherence or compliance } \\
\text { with method of application of the medication, which, in turn, could affect the } \\
\text { prophylactic effect of the medication against chlamydial conjunctivitis. }\end{array}$ \\
\hline
\end{tabular}


Ramirez-Ortiz 2007 (Continued)
Blinding of persons in-
Unclear risk
All cases of clinical conjunctivitis were not reported as outcomes in this study, volved in postnatal care or total clinical conjunctivitis cases could not be extracted from this study. Clinical conjunctivitis (subjective) Consequently, there was no assessment of bias for this category for the out- come of clinical conjunctivitis.

\section{Blinding of persons in- volved in postnatal care Bacterial, gonococcal and chlamydial conjunctivitis (objective)}

Unclear risk

COMMENT: Masking of those involved in postnatal care was not addressed in this study. The 2 interventions differ in colour. Povidone-iodine is an orange-red solution that may lead to periocular stains that last minutes to hours. Chloramphenicol is a clear, colourless to slightly yellow solution. The paper does state that eyelids were wiped immediately after birth, but it is uncertain if this eliminated all periocular stains.

In this study, it is uncertain if there were cases of chlamydial conjunctivitis identified in the time period when masking would be affected. It is unclear if those involved in postnatal care were also involved in identification of cases of conjunctivitis. If they were, and they were unmasked, this may have influenced decisions to identify and refer clinical conjunctivitis cases for culture. The definition of conjunctivitis used in this study included discharge, which reduced diagnostic ambiguity. Although chlamydial conjunctivitis tends to be more severe, evidence shows there is a clinical spectrum of presentation, and any bias, however minor, can be significant considering the very low event rates in this trial.

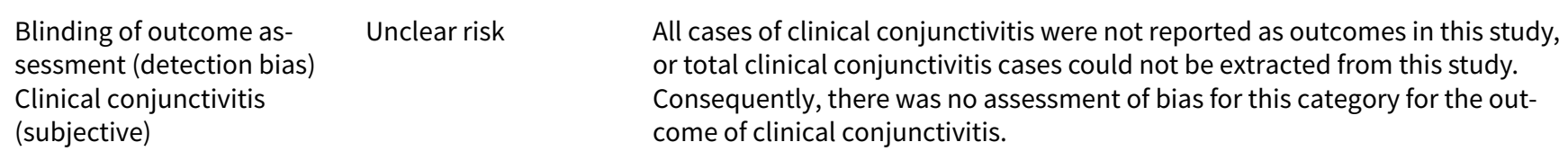

(subjective)

Blinding of outcome assessment (detection bias) Bacterial, gonococcal and chlamydial conjunctivitis (objective)
Unclear risk

COMMENT: Masking of the person involved in outcome assessment was not addressed in this paper. The 2 interventions differ in colour. Povidone-iodine is an orange-red solution that may lead to periocular stains that last minutes to hours. Chloramphenicol is a clear, colourless to slightly yellow solution. The paper does state that eyelids were wiped immediately after birth, but it is uncertain if this eliminated all periocular stains.

Chlamydial conjunctivitis presents with a variable clinical spectrum. Therefore, lack of masking, in cases of diagnostic ambiguity, may affect which cases get referred for culture to identify chlamydial conjunctivitis. $C$ trachomatis can be a carrier or it can lead to chlamydial conjunctivitis.
Incomplete outcome data Unclear risk
(attrition bias)
All cases of clinical conjunctivitis were not reported as outcomes in this study,
Clinical conjunctivitis
(subjective) or total clinical conjunctivitis cases could not be extracted from this study. Consequently, there was no assessment of bias for this category for the out- come of clinical conjunctivitis.

\section{Incomplete outcome data High risk}

(attrition bias)

COMMENT: Loss to follow-up was greater than $50 \%$ in each intervention group

Bacterial, gonococcal and

chlamydial conjunctivitis

(objective)

by Day 30 . Whilst it is possible that missing outcome data may be unrelated to true outcome, and they are balanced across intervention groups, the proportion of missing outcomes compared with observed event risk was high enough to induce clinically relevant bias in the intervention effect estimate.

Selective reporting (re- High risk
porting bias)

COMMENT: Based on the data as currently reported, we are unable to determine the total number of cases of conjunctivitis and total number of cases of bacterial conjunctivitis per treatment group.

Other bias Low risk No other sources of bias identified.


Richter 2006

\section{Study characteristics}

Methods
Parallel-group single-centre randomised trial.

Method of allocation: "randomized".

Unit of randomisation: neonate.

Exclusions after allocation: at least $n=4$, delayed urination greater than 12 hours postpartum.

Losses to follow-up: not specified, likely minimal as follow-up time until Day 5, and whilst neonate was in hospital.

Number randomised: at least 73.

Number analysed: 69 .

There was no comment on how missing data were handled.

Reported power calculation: yes. Sample size of 32 newborns per group to detect statistical power of $90 \%$.
Country: Germany.

Total number of participants: 69

Sex: M:F 37 (54\%):32 (46\%).

Average age and range: neonates; specific weeks of age not specified, but neonates greater than 37 weeks included.

Inclusion criteria: newborns at the obstetrics department of the University of Griefswald and the Demmim Community Hospital.

Exclusion criteria:

1. newborns of mothers with thyroid disease or additional intake of iodine during pregnancy;

2. newborns with gestational age of less than 37 weeks;

3. refusal to participate;

4. delayed urination greater than 12 hours postpartum.

Setting: obstetrics department of the University of Greifswald and the Demmim Community Hospital, a teaching hospital of the University of Greifswald.

Ethnic group: not specified.

Equivalence of baseline characteristics: yes.

- Intervention 1: $1.25 \%$ povidone-iodine into each conjunctival sac $(n=36)$.

- Intervention 2: $1 \%$ silver nitrate into each conjunctival sac $(n=33)$.

Time to intervention: within 60 minutes of delivery.

Pre-intervention manoeuvres: cleansing.

Postintervention manoeuvres: none specified.

Outcomes

1. Blood thyroid-stimulating hormone concentrations on Day 1 and Day 5 (day of discharge).

2. Urinary iodide concentrations on Days 1,2 , and 5 of life.

3. "Irritations" (not specified in methods).

4. "Infections" (not specified in methods). 
Richter 2006 (Continued)

5. "Pain reactions" (not specified in methods).

6. Chemical conjunctivitis categorised as mild hyperaemia, purulent discharge, oedema (not specified in methods).

The paper was a study to look for adverse events of povidone-iodine that specifically influence thyroid function.

Length of follow-up: appears to be 5 days although not explicit.

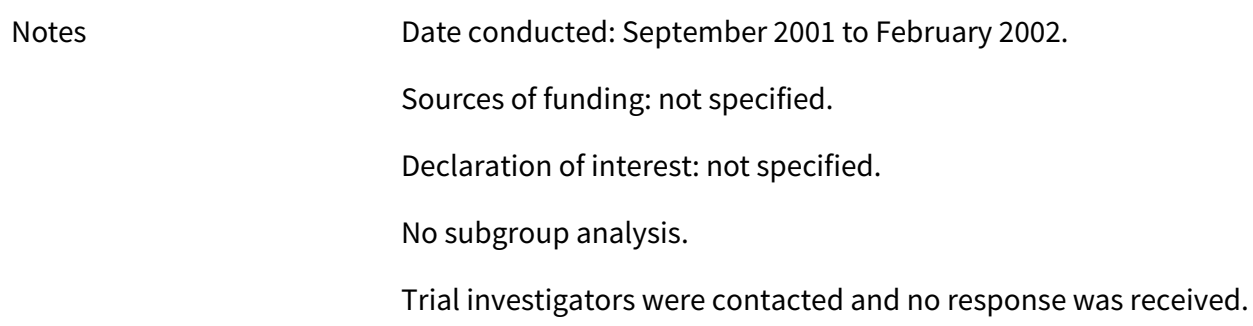

\section{Risk of bias}

\begin{tabular}{lll}
\hline Bias & Authors' judgement & Support for judgement \\
\hline $\begin{array}{l}\text { Random sequence genera- } \\
\text { tion (selection bias) }\end{array}$ & Unclear risk & $\begin{array}{l}\text { COMMENT: Reported that study was "randomized". No other information was } \\
\text { provided on the sequence generation process. }\end{array}$ \\
\hline $\begin{array}{l}\text { Allocation concealment } \\
\text { (selection bias) }\end{array}$ & Unclear risk & $\begin{array}{l}\text { COMMENT: Reported that study was "randomized". No other information was } \\
\text { provided on allocation concealment. }\end{array}$ \\
\hline $\begin{array}{l}\text { Blinding of study partici- } \\
\text { pants (mothers of infants) } \\
\begin{array}{l}\text { Clinical conjunctivitis } \\
\text { (subjective) }\end{array}\end{array}$ & Unclear risk & $\begin{array}{l}\text { COMMENT: The } 2 \text { interventions differ in colour. Povidone-iodine is an or- } \\
\text { ange-red solution that leads to transient residual staining of the eye, skin, and } \\
\text { lids that can last minutes to hours, and that the mother may notice. Silver ni- } \\
\text { trate is a clear solution that sometimes causes a chemical conjunctivitis that } \\
\text { can last up to } 72 \text { hours, and also causes lid stains that can last } 30 \text { to } 48 \text { hours. It } \\
\text { is unknown how this could affect performance bias. }\end{array}$
\end{tabular}

\begin{tabular}{|c|c|c|}
\hline $\begin{array}{l}\text { Blinding of study partici- } \\
\text { pants (mothers of infants) } \\
\text { Bacterial, gonococcal and }\end{array}$ & Unclear risk & $\begin{array}{l}\text { The study did not report bacterial, gonococcal, or chlamydial conjunctivitis } \\
\text { as outcomes, therefore there was no assessment of bias for this category with } \\
\text { these outcomes. }\end{array}$ \\
\hline
\end{tabular}
chlamydial conjunctivitis (objective)

Blinding of caregiver who Unclear risk administered medication Clinical conjunctivitis (subjective)

\begin{abstract}
QUOTE "The 1\% SN (silver nitrate)...and 1.25\% PVP-I (povidone-iodine) eye drops....were prepared by the university pharmacy in single ready-for-use vials. The test vials were labeled by code and completely covered. Laboratory sam-
\end{abstract} ples were decoded at the end of the investigation."

COMMENT: The 2 interventions differ in colour: povidone-iodine is an orange-red solution, and silver nitrate is a clear solution. The 2 medications are readily identifiable to the person administering the medication once it is dispensed into the eyes. There was no comment on whether the person administering the medication was specifically masked to the medication, even though the vials were covered.

Unclear risk

The study did not report bacterial, gonococcal, or chlamydial conjunctivitis as outcomes, therefore there was no assessment of bias for this category with these outcomes. administered medication

Bacterial, gonococcal and

chlamydial conjunctivitis

(objective) 
Richter 2006 (Continued)

Blinding of persons involved in postnatal care Clinical conjunctivitis (subjective)
Unclear risk

COMMENT: The 2 interventions differ in colour. Povidone-iodine is an orange-red solution that leads to transient residual staining of the eye, skin, and lids that can last minutes to hours, depending on whether the surrounding periorbital skin is cleaned or not. Silver nitrate is a clear solution that sometimes causes a chemical conjunctivitis that can last up to 72 hours, and also causes lid stains that can last 30 to 48 hours.

It is clear that follow-up was at least 5 days, but it is unclear how many cases of conjunctivitis were diagnosed in the first 72 hours. The nurses in the nursery may differentially handle the eyes of neonates based on the type of visible signs of prophylaxis. This could lead to differential introduction of pathogenic bacteria into the eyes of these neonates.
Blinding of persons involved in postnatal care Bacterial, gonococcal and chlamydial conjunctivitis (objective)
Unclear risk

The study did not report bacterial, gonococcal, or chlamydial conjunctivitis as outcomes, therefore there was no assessment of bias for this category with these outcomes.

\section{Blinding of outcome as- Unclear risk}

Clinical conjunctivitis (subjective) sessment (detection bias)

\section{COMMENT: The 2 interventions differ in colour. Povidone-iodine is an or-} ange-red solution that leads to transient residual staining of the eye, skin, and lids that can last minutes to hours, depending on whether the periorbital skin is cleaned. Silver nitrate is a clear solution that sometimes causes a chemical conjunctivitis that can last up to 72 hours, and also causes lid stains that can last 30 to 48 hours.

It is unknown how many cases of conjunctivitis were identified during the time when these stains remained.

\begin{tabular}{|c|c|c|}
\hline $\begin{array}{l}\text { Blinding of outcome as- } \\
\text { sessment (detection bias) } \\
\text { Bacterial, gonococcal and } \\
\text { chlamydial conjunctivitis } \\
\text { (objective) }\end{array}$ & Unclear risk & $\begin{array}{l}\text { The study did not report bacterial, gonococcal, or chlamydial conjunctivitis } \\
\text { as outcomes, therefore there was no assessment of bias for this category with } \\
\text { these outcomes. }\end{array}$ \\
\hline $\begin{array}{l}\text { Incomplete outcome data } \\
\text { (attrition bias) } \\
\text { Clinical conjunctivitis } \\
\text { (subjective) }\end{array}$ & Unclear risk & $\begin{array}{l}\text { COMMENT: There is no specific comment on incomplete outcome data in the } \\
\text { paper, however it is unlikely that there were incomplete data as neonates were } \\
\text { followed up whilst in hospital to Day } 5 \text {. }\end{array}$ \\
\hline $\begin{array}{l}\text { Incomplete outcome data } \\
\text { (attrition bias) } \\
\text { Bacterial, gonococcal and } \\
\text { chlamydial conjunctivitis } \\
\text { (objective) }\end{array}$ & Unclear risk & $\begin{array}{l}\text { The study did not report bacterial, gonococcal, or chlamydial conjunctivitis } \\
\text { as outcomes, therefore there was no assessment of bias for this category with } \\
\text { these outcomes. }\end{array}$ \\
\hline $\begin{array}{l}\text { Selective reporting (re- } \\
\text { porting bias) }\end{array}$ & High risk & $\begin{array}{l}\text { Reported outcomes of conjunctivitis were not prespecified and were reported } \\
\text { incompletely. }\end{array}$ \\
\hline Other bias & Unclear risk & Insufficient information to assess whether an important risk of bias exists \\
\hline
\end{tabular}

\section{Siegel 1982}

\section{Study characteristics}

Methods Parallel-group, single-centre trial.


Method of allocation: alternate weeks.

Unit of randomisation: neonate.

Exclusions after allocation: none specified.

Losses to follow-up: none specified.

Number allocated: 32,058 .

No comment on how missing data were handled.

No reported power calculation.

Unusual study design: none identified.

Participants

Setting: Parkland Memorial Hospital, Dallas, TX, USA.

Number allocated: 32,058:

- penicillin IM: 16,082;

- tetracycline: 15,976 .

Age: neonates.

Sex: M:F unknown.

Inclusion criteria: infants born at Parkland Memorial Hospital from 4 December 1977 to 31 December 1979.

Exclusion criteria: none specified.

No comment on equivalence of baseline characteristics.

- Intervention 1: penicillin G IM injection (50,000 units, greater than $2000 \mathrm{~g}$ birthweight; 25,000 units, less than $2000 \mathrm{~g}$ birthweight) $(\mathrm{n}=16,082)$.

- Intervention 2: tetracycline $1 \%$ ointment; dose not specified $(n=15,976)$.

Time to intervention: penicillin G IM within 60 minutes of delivery; unknown when tetracycline was administered.

Pre-intervention manoeuvres: none specified.

Postintervention manoeuvres: none specified.

Outcomes

1. Infants with gonococcal ophthalmia.

2. Infants with chlamydial ophthalmia.

3. Infants with systemic group B streptococcal infections.

4. Mortality.

Follow-up: unclear; likely 31 days. Interval of follow-up not specified.

Definition of conjunctivitis: method of outcome assessment not specified; clinical criteria for conjunctivitis not specified in paper; chlamydial conjunctivitis diagnosed by growth in tissue culture.

Adverse events reported: "No hypersensitivity reactions to penicillin were observed."

Notes

Date study conducted: 4 December 1977 to 31 May 1981.

No source of funding specified.

No declaration of interest made. 
Siegel 1982 (Continued)

No reported subgroup analysis.

Authors have been contacted for clarifications on unclear information, no reply received to date.

\section{Risk of bias}

Bias Authors' judgement Support for judgement

Random sequence genera- High risk tion (selection bias)

Allocation concealment $\quad$ High risk
(selection bias)

Allocation concealment High risk
(selection bias)

(selection bias)

QUOTE: "Newborn infants were assigned to one of two prophylactic regimens, according to week of birth."

COMMENT: Non-random component in the sequence generation process.
QUOTE: "Newborn infants were assigned to one of two prophylactic regimens, according to week of birth."

COMMENT: Participants or investigators enrolling participants could possibly have foreseen assignments.
All cases of clinical conjunctivitis were not reported as outcomes in this study, only chlamydial and gonococcal conjunctivitis. Therefore, there was no assessment of bias for this category for the outcome of clinical conjunctivitis. (mothers ofints)

(subjective)

Unclear risk

Unclear risk

Blinding of study participants (mothers of infants) Bacterial, gonococcal and chlamydial conjunctivitis (objective)

COMMENT: Masking of mothers to the intervention was not addressed in this study. Furthermore, the 2 interventions differ in method of application. Tetracycline is a light-yellow ointment that may leave a residue in the eyes that can last for hours. Penicillin G IM is an injection that may leave a needle mark on the neonate. There could be differential handling of the eyes by the mother, by allocation group. This could lead to differential introduction of Chlamydia trachomatis bacteria into the eyes of these neonates in the case of poor hygiene. Alternatively, it could lead to contamination with other bacteria, subsequent conjunctivitis, and identification of chlamydia carriers instead.

\begin{tabular}{|c|c|c|}
\hline $\begin{array}{l}\text { Blinding of caregiver who } \\
\text { administered medication } \\
\text { Clinical conjunctivitis } \\
\text { (subjective) }\end{array}$ & Unclear risk & $\begin{array}{l}\text { All cases of clinical conjunctivitis were not reported as outcomes in this study, } \\
\text { only chlamydial and gonococcal conjunctivitis. Therefore, there was no as- } \\
\text { sessment of bias for this category for the outcome of clinical conjunctivitis. }\end{array}$ \\
\hline $\begin{array}{l}\text { Blinding of caregiver who } \\
\text { administered medication } \\
\text { Bacterial, gonococcal and } \\
\text { chlamydial conjunctivitis } \\
\text { (objective) }\end{array}$ & High risk & $\begin{array}{l}\text { COMMENT: Masking of the person who administers the medication was not } \\
\text { addressed in this study. Furthermore, the } 2 \text { interventions differ in method of } \\
\text { application. Tetracycline is a light-yellow ointment applied to the eyes. Peni- } \\
\text { cillin G IM is an injection. No placebo was used. Any lack of masking and con- } \\
\text { comitant bias on the part of the person administering the medication could af- } \\
\text { fect adherence or compliance with method of application of the medication, } \\
\text { which, in turn, could affect the prophylactic effect of the medication against } \\
\text { chlamydial conjunctivitis. }\end{array}$ \\
\hline
\end{tabular}

\begin{tabular}{|c|c|c|}
\hline $\begin{array}{l}\text { Blinding of persons in- } \\
\text { volved in postnatal care } \\
\text { Clinical conjunctivitis } \\
\text { (subjective) }\end{array}$ & Unclear risk & $\begin{array}{l}\text { All cases of clinical conjunctivitis were not reported as outcomes in this study, } \\
\text { only chlamydial and gonococcal conjunctivitis. Therefore, there was no as- } \\
\text { sessment of bias for this category for the outcome of clinical conjunctivitis. }\end{array}$ \\
\hline
\end{tabular}

(subjective)

Blinding of persons involved in postnatal care Bacterial, gonococcal and chlamydial conjunctivitis (objective)
Unclear risk

COMMENT: Masking of those involved in postnatal care was not addressed in this study. Tetracycline is a light-yellow ointment that may leave a residue in the eyes that can last for hours. Penicillin G IM is an injection that may leave a needle mark on the neonate.

In this study, it is uncertain if there were cases of chlamydial conjunctivitis identified in the time period when masking would be affected. It is unclear if 
those involved in postnatal care were also involved in identification of cases of conjunctivitis. If they were, and they were unmasked, this could influence decisions to identify and refer clinical conjunctivitis cases for culture. The definition of conjunctivitis used in this study was not specified. Although chlamydial conjunctivitis tends to be more severe, evidence shows there is a clinical spectrum of presentation, and any bias, however minor, can be significant considering the low event rates in this trial.

$\begin{array}{lll}\begin{array}{l}\text { Blinding of outcome as- } \\ \text { sessment (detection bias) }\end{array} & \text { Unclear risk } & \begin{array}{l}\text { All cases of clinical conjunctivitis were not reported as outcomes in this study, } \\ \text { only chlamydial and gonococcal conjunctivitis. Therefore, there was no as- } \\ \text { Clinical conjunctivitis }\end{array} \\ \text { sessment of bias for this category for the outcome of clinical conjunctivitis. }\end{array}$

(subjective) sessment of bias for this category for the outcome of clinical conjunctivitis.

Blinding of outcome as- Unclear risk sessment (detection bias) Bacterial, gonococcal and chlamydial conjunctivitis (objective)

\begin{abstract}
QUOTE: "Systemic bacterial infections were identified by daily review of the microbiology laboratory records and hospital charts of infants regarded as infected on the basis of positive culture results or clinical course."
\end{abstract}

COMMENT: Masking of those involved in postnatal care was not addressed in this study. Tetracycline is a light-yellow ointment that may leave a residue in the eyes that can last for hours. Penicillin G IM is an injection that may leave a needle mark on the neonate.

We do not know the follow-up time in this study.

Chlamydial conjunctivitis presents with a variable clinical spectrum. Therefore, lack of masking, in cases of diagnostic ambiguity, may affect which cases get referred for culture to identify chlamydial conjunctivitis.

$\begin{array}{ll}\begin{array}{l}\text { Incomplete outcome data } \\ \text { (attrition bias) }\end{array} & \begin{array}{l}\text { All cases of clinical conjunctivitis were not reported as outcomes in this study, } \\ \text { only chlamydial and gonococcal conjunctivitis. Therefore, there was no as- } \\ \text { Clinical conjunctivitis }\end{array} \\ \text { sessment of bias for this category for the outcome of clinical conjunctivitis. }\end{array}$

only chlamydial and gonococcal conjunctivitis. Therefore, there was no as-

COMMENT: Follow-up time is not explicitly stated in the paper. Also, losses to follow-up are not addressed in the paper. There is no mention of attrition. Ongitis. 1 case was in the penicillin group and the other was in the tetracycline group. In a trial where 16,082 neonates were allocated to the penicillin group and 15,976 to the tetracycline group, it is likely that there was attrition and exclusions from the analysis. chlamydial conjunctivitis

(objective) ly 2 exclusions were mentioned in the study. These 2 infants suffered menin-

Unclear risk

QUOTE: "Systemic bacterial infections were identified by daily review of the microbiology laboratory records and hospital charts of infants regarded as infected on the basis of positive culture results or clinical course."

COMMENT: The study authors report no cases of gonococcal ophthalmia and 79 cases of chlamydial conjunctivitis, 34 in the penicillin group and 45 in the tetracycline group. It is likely that there were other cases of conjunctivitis that were due to pathogens other than C trachomatis and Neisseria gonorrhoeae, but these have not been reported.
Selective reporting (re- Unclear risk porting bias)
Insufficient information to assess whether an important risk of bias exists

\section{Study characteristics}


Wahlberg 1982 (Continued)

Methods
Parallel-group RCT: there was not equal amounts of each prophylactic intervention: $40 \%$ of the total prophylaxis bottles contained Hexarginum; $20 \%$ of the total prophylaxis bottles contained silver nitrate; and $40 \%$ of the total prophylaxis bottles contained physiological saline.

Method of allocation: "randomized"; no further details on method of randomisation.

Unit of randomisation: neonate.

Exclusions after randomisation: unclear. The study reports 1027 deliveries during the study period, of which $86 \%$ were reported to be vaginal (calculated to be 883 ) and $14 \%$ were reported to be caesarean sections (calculated to be 144). Only vaginal deliveries were allocated to prophylaxis. However, only 544 of the 883 vaginal deliveries were allocated to prophylaxis for reasons unexplained in the study.

Losses to follow-up: not reported in study, but it appears that only subgroups of those allocated prophylaxis were followed up. It is unclear if these are losses to follow-up or selective follow-up.

Number randomised: unclear; possibly all vaginal deliveries, which is 883 . However, the study later states that only 544 were allocated to prophylaxis, with no explanation for the discrepancy.

Missing data appeared to be handled by available-case analysis.

Power calculation: no reported power calculation in study.

Unusual study design: the study reports 1027 deliveries during the study period, of which $86 \%$ were reported to be vaginal (calculated to be 883 ) and $14 \%$ were reported to be caesarean sections (calculated to be 144). Only vaginal deliveries were allocated to prophylaxis. However, only 544 of the 883 vaginal deliveries were allocated to prophylaxis for reasons that were unexplained in the study.

Participants

Country: Stockholm, Sweden.

Setting: Karolinska Hospital.

Ethnic group: not specified.

Total number of participants: 1027 mother-infant pairs.

Sex: M:F not specified.

Average age and age range: not specified.

Inclusion criteria:

1. mothers not suspected of having gonorrhoea;

2. informed consent.

Exclusion criteria:

1. mother suspected of having gonorrhoea.

Equivalence of baseline characteristics: there did not appear to be equivalence in some of the baseline characteristics of the mothers. $40 \%$ of the total prophylaxis bottles contained Hexarginum; $20 \%$ of the prophylaxis bottles contained silver nitrate; and $40 \%$ of the bottles contained physiological saline.
Number of interventions: 3.

- Intervention 1: silver nitrate $1 \%(n=105)$.

- Intervention 2: Hexarginum 10\% (1 g silver nitrate + 36 g methylamine dissolved in $63 \mathrm{~g}$ of sterile water) $(n=225)$.

- Intervention 3: physiological saline $(n=214)$.

Time to intervention: approximately 2 hours postpartum.

Pre-intervention manoeuvres: 
Wahlberg 1982 (Continued)

1. neonate placed on the mother's abdomen and left there for 20 to 30 minutes;

2. neonate then taken away for weighing, measuring, and dressing;

3. neonate left alone with mother and father for 2 hours.

Postintervention manoeuvres: not specified.

Outcomes

1. Daily inspection of eye irritation in a subset of 627 neonates, for the following:

a. swelling of the eyelids classified as none, slight, moderate, considerable;

b. redness of the conjunctiva classified as none, slight, moderate, considerable;

c. secretion classified as none, serous, mucous or purulent.

2. Neonates with bacterial conjunctivitis in a subset of 156 neonates with conjunctival secretion.

3. Neonates with gonococcal conjunctivitis among the 156 neonates with conjunctival secretion.

4. Neonates with chlamydial conjunctivitis among the 156 neonates with conjunctival secretion.

5. Neonates without conjunctivitis, cultured for chlamydia. Done in a subset of 250 neonates, Days 5 to 7 postpartum.

6. Pain reaction to prophylaxis in a subset of 810 neonates, looking at 2 variables:
a. cry;
b. averting movements of the head and extremities.

7. Visual alertness in a subset of 39 neonates via score from 0 to 5 .

8. Mother-infant relationship in 65 mothers measured by the following:

a. two 6- to 10-minute observation sessions on Days 4 to 5 postpartum followed by an interview on Day 5;

b. follow-up telephone interview 6 to 8 weeks later.

9. Long-term effects on conjunctival secretion, infant behaviour, breastfeeding, and maternal feelings for the baby, and experiences of the care system in a subset of 136 mothers, via:

a. interview 3 to 3.5 months postpartum;

b. questionnaire 6 months postpartum.

Length of follow-up for conjunctivitis:

1. daily inspection of eye irritation in 544 neonates until Day 6;

2. a small subset of 15 neonates with conjunctivitis were followed until Day 15 and cultured for chlamydia.

Adverse events: many of the outcomes listed above can be considered adverse events.

Notes

Dates conducted:

1. May 1978 to May 1979 for conjunctivitis;

2. "Spring 1979" for outcome of mother-infant relationship.

Sources of funding: "Aided by the Swedish Delegation for Social Research; Medical Research Council (grant no. 21x-5433) and Radda Barnen -the Swedish Save the Children Foundation; Stockholm, the SSSH Foundation, Lund; The Swedish Midwives' Association; Medical Faculty, Karolinska Institute, Stockholm."

Declaration of interest: none made.

Multiple subgroup analyses.

Trial investigators were contacted.

\section{Risk of bias}

Bias Authors' judgement Support for judgement

Random sequence genera- Unclear risk tion (selection bias)
QUOTE: "One thousand such bottles were prepared and numbered in a random series. We elected to make the proportion of infants receiving silver nitrate less than the two other comparison groups, based on the investigators' 
Wahlberg 1982 (Continued)

previous experiences with the irritating quality of this agent. Out of the bottles $20 \%$ contained silver nitrate, $40 \%$ contained Hexarginum, and $40 \%$ contained physiological saline."

COMMENT: Sequence generation method unclear.

$\begin{array}{ll}\begin{array}{l}\text { Allocation concealment } \\ \text { (selection bias) }\end{array} & \text { QUOTE: "One thousand such bottles were prepared and numbered in a ran- } \\ & \text { dom series. We elected to make the proportion of infants receiving silver ni- } \\ & \text { trate less than the two other comparison groups, based on the investigators' } \\ \text { previous experiences with the irritating quality of this agent. Out of the bottles } & 20 \% \text { contained silver nitrate, } 40 \% \text { contained Hexarginum, and } 40 \% \text { contained } \\ \text { physiological saline." }\end{array}$

QUOTE: "The study was performed as a double-blind randomized test with $1 \%$ silver nitrate...10\% Hexarginum... and physiological saline... administered from dark brown, non-returnable bottles."

COMMENT: Method of concealment not described in detail.

Blinding of study partici- Unclear risk pants (mothers of infants)

Clinical conjunctivitis

(subjective)
QUOTE: "The study was performed as a double-blind, randomized test with $1 \%$ silver nitrate (AgNO3), 10\% Hexargiunum ( a less irritating compound consisting of $1 \mathrm{~g} \mathrm{AgNO} 3+36 \mathrm{~g} \mathrm{CH} 3 \mathrm{NH} 2$ dissolved in $63 \mathrm{~g}$ sterile water) and physiological saline ( $\mathrm{NaCl}$ as placebo) administered from dark-brown, non-returnable bottles."

COMMENT: Silver nitrate is a clear solution that sometimes causes a chemical conjunctivitis that can last up to 72 hours, and also causes lid stains that can last 30 to 48 hours. Physiological saline is a clear solution that does not cause lid stains. It is unknown if Hexarginum is a clear solution, but it is possible that it is. Both Hexarginum and silver nitrate have the same concentration of silver nitrate in solution. It is unknown if Hexarginum causes lid stains. According to the study Hexarginum causes significantly less chemical conjunctivitis than silver nitrate. It is unknown if the mother is able to differentiate the prophylactic agents on the basis of lid stains.

\begin{tabular}{|c|c|c|}
\hline $\begin{array}{l}\text { Blinding of study partici- } \\
\text { pants (mothers of infants) } \\
\text { Bacterial, gonococcal and } \\
\text { chlamydial conjunctivitis }\end{array}$ & Unclear risk & $\begin{array}{l}\text { We were unable to extract bacterial, gonococcal, and chlamydial conjunctivi- } \\
\text { tis cases from the reported data, therefore there was no assessment of bias for } \\
\text { this category with these outcomes. }\end{array}$ \\
\hline
\end{tabular}
chlamydial conjunctivitis (objective)

Blinding of caregiver who Unclear risk administered medication

QUOTE: "The study was performed as a double-blind, randomized test with $1 \%$ silver nitrate (AgNO3), 10\% Hexargiunum ( a less irritating compound consisting of $1 \mathrm{~g} \mathrm{AgNO} 3+36 \mathrm{~g} \mathrm{CH} 3 \mathrm{NH} 2$ dissolved in $63 \mathrm{~g}$ sterile water) and physiological saline ( $\mathrm{NaCl}$ as placebo) administered from dark-brown, non-returnable bottles."

(subjective)
COMMENT: The prophylactic agents were administered from dark-brown, nonreturnable bottles. This may have concealed any colour differences between the solutions. However, it is unknown if all 3 solutions appeared the same when dispensed from the bottle. Silver nitrate is a clear solution that sometimes causes a chemical conjunctivitis that can last up to 72 hours, and also causes lid stains that can last 30 to 48 hours. Physiological saline is a clear solution. It is unknown if Hexarginum is a clear solution, but it is possible that it is. Both Hexarginum and silver nitrate have the same concentration of silver nitrate in solution, but it is unknown if Hexarginum causes lid stains. According to the study Hexarginum causes significantly less chemical conjunctivitis than silver nitrate. Depending on the time of onset of chemical conjunctivitis, chemical conjunctivitis may affect the masking of the person administering the medication. For example, if chemical conjunctivitis is almost immediate, 
Blinding of caregiver who administered medication Bacterial, gonococcal and chlamydial conjunctivitis (objective)
Unclear risk

We were unable to extract bacterial, gonococcal, and chlamydial conjunctivitis cases from the reported data, therefore there was no assessment of bias for this category with these outcomes.

\section{Blinding of persons in- Unclear risk} volved in postnatal care Clinical conjunctivitis (subjective)

\begin{abstract}
QUOTE: "Two observers made all daily inspections of the infants' eyes. The observers did not know to which prophylaxis group the infants belonged."
\end{abstract}

COMMENT: The role of those involved in postnatal care is unknown in this study. Silver nitrate is a clear solution that sometimes causes a chemical conjunctivitis that can last up to 72 hours, and also causes lid stains that can last 30 to 48 hours. Physiological saline is a clear solution that does not cause lid stains. Both Hexarginum and silver nitrate solution have the same concentration of silver nitrate, but it is unknown if Hexarginum causes lid stains. Certainly the presence of lid stains with silver nitrate, and the absence of lid stains with physiological saline, would permit such neonates to be distinguished, at least up to 72 hours. Conjunctivitis outcomes were assessed on Days 1 to 3 , in the first 72 hours.

\author{
Blinding of persons in- \\ volved in postnatal care \\ Bacterial, gonococcal and \\ chlamydial conjunctivitis \\ (objective) \\ Unclear risk
}

Unclear risk

We were unable to extract bacterial, gonococcal, and chlamydial conjunctivitis cases from the reported data, therefore there was no assessment of bias for this category with these outcomes.

\section{Blinding of outcome as- Unclear risk} sessment (detection bias)

Clinical conjunctivitis

(subjective) QUOTE: "Two observers made all daily inspections of the infants' eyes. The ob-
servers did not know to which prophylaxis group the infants belonged."

COMMENT: Silver nitrate is a clear solution that sometimes causes a chemical conjunctivitis that can last up to 72 hours, and also causes lid stains that can last 30 to 48 hours. Physiological saline is a clear solution that does not cause lid stains. Both Hexarginum and silver nitrate solution have the same concentration of silver nitrate, but it is unknown if Hexarginum causes lid stains. Certainly the presence of lid stains with silver nitrate, and the absence of lid stains with physiological saline, would permit such neonates to be distinguished, at least up to 72 hours. The study reports that conjunctivitis outcomes were assessed on Days 1 to 3 , in the first 72 hours, thereby affecting masking. Cultures were taken from a subset of neonates with purulent conjunctivitis. A significant proportion of purulent conjunctivitis developed in the first 48 hours, when lid stains would be present, and masking would be affected.

We were unable to extract bacterial, gonococcal, and chlamydial conjunctivitis cases from the reported data, therefore there was no assessment of bias for this category with these outcomes.

\begin{abstract}
Blinding of outcome assessment (detection bias) Bacterial, gonococcal and chlamydial conjunctivitis (objective)
\end{abstract}

Unclear risk

\section{Incomplete outcome data High risk} (attrition bias)

Clinical conjunctivitis

(subjective)

\begin{abstract}
QUOTE: "In the investigation, there was a main study group of 1027 mother-infant pairs.... There were also six subsamples...The second subsample comprised the first 810 infants entered into the study...observed for pain reaction to eye drop prophylaxis. The third subsample came from this group of $810 \mathrm{in}$ fants, among whom the first 627 were observed for symptoms of eye irritation. The fourth subsample came from the group of 627 infants, namely 156 infants with purulent conjunctivitis and from whom bacterial cultures were obtained...Finally, a small group of 15 subjects from the 627 observed for eye irri-
\end{abstract}


tation who displayed prolonged conjunctivitis were also cultured for Chlamydia."

QUOTE: "One thousand such bottles were prepared and numbered in a random series. We elected to make the proportion of infants receiving silver nitrate less than the two other comparison groups, based on the investigators' previous experiences with the irritating quality of this agent. Out of the bottles $20 \%$ contained silver nitrate, $40 \%$ contained Hexarginum, and $40 \%$ contained physiological saline."

COMMENT: As the above quotes validate, significant numbers of neonates were not followed up. Furthermore, despite having 1000 bottles, only 544 neonates were allocated to prophylaxis. The remaining 83 of the 627 neonates were born via caesarean section and did not receive any prophylaxis.

\section{Incomplete outcome data Unclear risk} (attrition bias)

Bacterial, gonococcal and chlamydial conjunctivitis (objective)
We were unable to extract bacterial, gonococcal, and chlamydial conjunctivitis cases from the reported data, therefore there was no assessment of bias for this category with these outcomes.

\section{Selective reporting (re- High risk} porting bias)
COMMENT: The study protocol is not available. Conjunctivitis outcomes are reported incompletely so that they cannot be entered into a meta-analysis. The study reports percentages in a bar graph only and by time periods, making it impossible to determine absolute numbers for extraction into a meta-analysis.

\section{Zbojan 2004}

\section{Study characteristics}

$\begin{array}{ll}\text { Methods } & \text { Parallel-group, single-centre RCT. } \\ \text { Unit of randomisation: neonate (not eye). } \\ \text { No losses to follow-up specified. } \\ \text { Number randomised: } 100 . \\ \text { No exclusions after randomisation specified. } \\ \text { No comment on missing data or how handled. } \\ \text { No reported power calculation. } \\ \text { Setting: hospital in Slovenia. } \\ \text { Number allocated: } 100 . \\ \text { Age: neonates. } \\ \text { Sex: M:F not specified. } \\ \text { Inclusion criteria: } \\ \text { 1. neonates. } \\ \text { Exclusion criteria: not specified. }\end{array}$


Zbojan 2004 (Continued)

There was no comment on equivalence of baseline characteristics.

\begin{tabular}{l}
\hline Outcomes Infants with clinical conjunctivitis classified as: \\
a. suppurative; \\
b. mucosal secretion; \\
C. chemical conjunctivitis. \\
2. Average concentration of thyroid stimulating hormone on Day 5 of birth. \\
Follow-up: 4 weeks. \\
Intervals at which outcomes assessed: \\
- 8 hours; \\
- 5 days; 4 weeks. \\
Notes on definition of conjunctivitis: defined erythema or discharge. \\
No comments on adverse events. \\
Study report was translated. \\
Date recruited neonates: study published in 2004; otherwise not specified. \\
No sources of funding specified. \\
No declarations of interest specified among researchers. \\
Trial investigators were not contacted.
\end{tabular}

\section{Risk of bias}

Bias Authors' judgement Support for judgement

\begin{tabular}{lll}
\hline $\begin{array}{l}\text { Random sequence genera- } \\
\text { tion (selection bias) }\end{array}$ & Unclear risk & $\begin{array}{l}\text { COMMENT: Translation of study states that the study was randomised. No oth- } \\
\text { er information was provided on the sequence generation process. }\end{array}$
\end{tabular}

\begin{tabular}{lll}
\hline $\begin{array}{l}\text { Allocation concealment } \\
\text { (selection bias) }\end{array}$ & Unclear risk & Insufficient information to permit judgement \\
\hline $\begin{array}{l}\text { Blinding of study partici- } \\
\text { pants (mothers of infants) } \\
\text { Clinical conjunctivitis } \\
\text { (subjective) }\end{array}$ & Unclear risk & COMMENT: Masking of the mother was not addressed in this paper. \\
& $\begin{array}{l}\text { Povidone-iodine is an orange-red solution that may lead to periocular stains } \\
\text { that last minutes to hours. The colour of C-bromide is unknown. The mothers } \\
\text { of neonates }\end{array}$ \\
$\begin{array}{l}\text { with noticeable medication of the eyes may handle the eyes of the infant dif- } \\
\text { ferently than mothers of neonates with no stains. This could lead to differen- }\end{array}$
\end{tabular}


tial introduction of pathogenic bacteria into the eyes of these neonates, depending on hygiene measures.

\begin{tabular}{|c|c|c|}
\hline $\begin{array}{l}\text { Blinding of study partici- } \\
\text { pants (mothers of infants) } \\
\text { Bacterial, gonococcal and }\end{array}$ & Unclear risk & $\begin{array}{l}\text { The study did not report bacterial, gonococcal, or chlamydial conjunctivitis } \\
\text { as outcomes, therefore there was no assessment of bias for this category with } \\
\text { these outcomes. }\end{array}$ \\
\hline
\end{tabular}
chlamydial conjunctivitis (objective)

\section{Blinding of caregiver who High risk} administered medication Clinical conjunctivitis (subjective) COMMENT: Masking of the person who administers the medication was not ad-
dressed in this study.

Povidone-iodine is an orange-red solution that may lead to periocular stains that last minutes to hours. The colour of C-bromide is unknown.

These medications may be readily distinguishable. Lack of masking and any bias on the part of the person administering the medication could affect adherence or compliance with method of application of the medication, which, in turn, could affect the prophylactic effect of the medication against conjunctivitis.

\begin{tabular}{|c|c|c|}
\hline $\begin{array}{l}\text { Blinding of caregiver who } \\
\text { administered medication } \\
\text { Bacterial, gonococcal and }\end{array}$ & Unclear risk & $\begin{array}{l}\text { The study did not report bacterial, gonococcal, or chlamydial conjunctivitis } \\
\text { as outcomes, therefore there was no assessment of bias for this category with } \\
\text { these outcomes. }\end{array}$ \\
\hline
\end{tabular}

chlamydial conjunctivitis (objective)
COMMENT: Masking of the person who was involved in postnatal care was not addressed in this paper. Povidone-iodine is an orange-red solution that may lead to periocular stains that last minutes to hours. The colour of C-bromide is unknown.

In this study, it is uncertain if there were cases of conjunctivitis identified in the time period when masking would be affected. It is unclear if those involved in postnatal care were also involved in identification of cases of conjunctivitis. If they were, and they were unmasked, this may influence decisions to identify clinical conjunctivitis cases. Considering the low event rates in this small trial, this may be significant.

\author{
Blinding of persons in- \\ volved in postnatal care \\ Bacterial, gonococcal and \\ chlamydial conjunctivitis \\ (objective) \\ Unclear risk
}

The study did not report bacterial, gonococcal, or chlamydial conjunctivitis as outcomes, therefore there was no assessment of bias for this category with these outcomes.

Blinding of outcome as-
sessment (detection bias)
Clinical conjunctivitis
(subjective)

\section{COMMENT: The study does not mention masking of outcome assessors.}

In the first hours after application, there may be residual periocular povidone-iodine skin stains that can last minutes to hours. The colour of C-bromide is unknown. Therefore, there may be lack of masking of outcome assessors during this time, and there may be recall of the prophylaxis applied after this time. Where there is ambiguity in the diagnosis of conjunctivitis, and there is concomitant lack of masking, bias could result in clinical diagnosis. This bias could be significant considering the low event rates.

The study did not report bacterial, gonococcal, or chlamydial conjunctivitis as outcomes, therefore there was no assessment of bias for this category with

\footnotetext{
Blinding of outcome assessment (detection bias) Bacterial, gonococcal and chlamydial conjunctivitis (objective)
} these outcomes.

Unclear risk 
Zbojan 2004 (Continued)
Incomplete outcome data
Unclear risk
COMMENT: The study did not address incomplete outcome data.

(attrition bias)

Clinical conjunctivitis

(subjective)

\section{Incomplete outcome data Unclear risk} (attrition bias)

Bacterial, gonococcal and

chlamydial conjunctivitis

(objective)
The study did not report bacterial, gonococcal, or chlamydial conjunctivitis as outcomes, therefore there was no assessment of bias for this category with these outcomes.

Selective reporting (re- Unclear risk
porting bias)

COMMENT: The study appeared to only report clinical conjunctivitis cases. There was no mention in the methods or results section of the translated paper of any plan to culture conjunctivitis cases. The methods are not explicit on prespecified outcomes. There is no access to the study protocol. There is insufficient information to permit a judgement of low risk or high risk of bias.

Other bias Unclear risk

COMMENT: We assessed this study as at unclear risk of bias, as a significant amount of information is not reported in the study. Consequently, there is insufficient information to assess whether an important risk of bias exists.

IM: intramuscular ; PCR: polymerase chain reaction; PMN: polymorphonuclear; RCT: randomised controlled trial

Characteristics of excluded studies [ordered by study ID]

\begin{tabular}{|c|c|}
\hline Study & Reason for exclusion \\
\hline Assadian 2002 & Not an RCT or a quasi-RCT \\
\hline Bailey 1993 & $\begin{array}{l}\text { Intervention for treatment, not prophylaxis of conjunctivitis, and population not restricted to } \\
\text { neonates }\end{array}$ \\
\hline Baptista 1968 & Unable to determine method of allocation \\
\hline Baveja 1997 & Not an RCT or a quasi-RCT of ophthalmia neonatorum prophylaxis \\
\hline Bobo 1997 & Not an RCT or a quasi-RCT of ophthalmia neonatorum prophylaxis \\
\hline Brady 1997 & Not a randomised or quasi-randomised prophylaxis trial; case report \\
\hline Burr 2017 & Unit of randomisation is expecting mothers, not neonates. \\
\hline Candano 1951 & Not an RCT or a quasi-RCT \\
\hline CDC 1998 & Not a randomised or quasi-randomised prophylaxis trial; outbreak report \\
\hline Clark 1951 & $\begin{array}{l}\text { Method of allocation to groups not directly addressed in paper; likely not an RCT or a quasi-RCT } \\
\text { based on description in methods section. }\end{array}$ \\
\hline Cohen 1990 & Intervention for treatment of conjunctivitis, not prophylaxis; population not neonates \\
\hline Darling 2010 & Not an RCT or a quasi-RCT; this is a meta-analysis \\
\hline Darougar 1977 & Intervention for treatment of conjunctivitis, not prophylaxis; population not neonates \\
\hline
\end{tabular}




\begin{tabular}{|c|c|}
\hline Study & Reason for exclusion \\
\hline Dhir 1967 & Population not neonates \\
\hline Drago 1998 & Intervention for treatment, not prophylaxis; population not neonates \\
\hline Fok 1995 & $\begin{array}{l}\text { Intervention not topical, systemic or, combination medication, and not for prophylaxis of conjunc- } \\
\text { tivitis }\end{array}$ \\
\hline Fransen 1984 & Intervention for treatment of ophthalmia neonatorum, not prophylaxis \\
\hline Gross 1997 & Intervention for treatment of conjunctivitis, not prophylaxis \\
\hline Hammerschlag 1997 & Not a randomised or quasi-randomised prophylaxis trial \\
\hline Hammerschlag 1998 & Intervention for treatment, not prophylaxis \\
\hline Heggie 1985 & Intervention for treatment of ophthalmia neonatorum, not prophylaxis \\
\hline Horven 1993 & Intervention for treatment, not prophylaxis of conjunctivitis, and not restricted to neonates \\
\hline Iroha 1998 & Intervention for treatment, not prophylaxis \\
\hline Isenberg 1994 & $\begin{array}{l}\text { Unit of randomisation is eyes as opposed to neonates; unable to transform results reported in pa- } \\
\text { per into clinically relevant outcomes. Dr Isenberg was contacted } 25 \text { March 2002, and a response } \\
\text { was received } 8 \text { May } 2002 \text {. }\end{array}$ \\
\hline Jacobson 1988 & Intervention for treatment of conjunctivitis, not prophylaxis, and population not neonates \\
\hline Keenan 2010 & Cost-analysis letter \\
\hline Khan 2016 & Unit of randomisation is eye. \\
\hline Klein 1997 & Not a randomised or quasi-randomised prophylaxis trial \\
\hline Kramer 1997 & Not a randomised or quasi-randomised prophylaxis trial; review article \\
\hline Laga 1986 & Intervention for treatment of conjunctivitis, not prophylaxis \\
\hline Lietman 1998 & Review article, not a trial \\
\hline Maharajan 1997 & Not an RCT or a quasi-RCT of ophthalmia neonatorum prophylaxis \\
\hline Mani 1997 & Retrospective study \\
\hline Mann 1954 & Not an RCT or a quasi-RCT \\
\hline Margileth 1957 & Not an RCT or a quasi-RCT \\
\hline Markham 1994 & Not an RCT or a quasi-RCT of prophylaxis of ophthalmia neonatorum \\
\hline McAuley 1994 & Intervention not for prophylaxis of ophthalmia neonatorum \\
\hline Mets 1997 & Not an RCT or a quasi-RCT prophylaxis trial of neonates \\
\hline Meyer 1997 & Not a trial of ophthalmia neonatorum prophylaxis; letter \\
\hline
\end{tabular}




\begin{tabular}{|c|c|}
\hline Study & Reason for exclusion \\
\hline Nakagawa 1997 & Not a randomised or quasi-randomised prophylaxis trial \\
\hline Nichols 1966 & $\begin{array}{l}\text { Intervention is trachoma vaccine; population was not exclusively neonates and included children } \\
\text { up to } 3 \text { years of age. }\end{array}$ \\
\hline Normann 2002 & Intervention is for treatment of ophthalmia neonatorum, not prophylaxis. \\
\hline Peters 1992 & Not a trial of ophthalmia neonatorum prophylaxis; review article \\
\hline Ratelle 1997 & Retrospective cohort study \\
\hline Reimer 1997 & Review article; no trial identified \\
\hline Rivlin 1997 & Not a randomised or quasi-randomised prophylaxis trial \\
\hline Rosenman 2003 & Not an RCT or a quasi-RCT; decision analysis \\
\hline Schaller 1997 & Not a randomised or quasi-randomised prophylaxis trial \\
\hline Seiga 1993 & $\begin{array}{l}\text { Unable to determine method of allocation from translated paper; communication from author } \\
\text { states alternation }\end{array}$ \\
\hline Silva 2008 & $\begin{array}{l}\text { Study did not measure clinical conjunctivitis in the methods, only eye cultures of all neonates with- } \\
\text { in the first } 2 \text { hours of life and } 1 \text { week later; study did not report any clinical conjunctivitis cases in } \\
\text { the outcomes. Study author was contacted via email } 1 \text { August 2016, no response received. }\end{array}$ \\
\hline Sorsby 1949 & Intervention for treatment of conjunctivitis, not prophylaxis \\
\hline Stenberg 1991 & Intervention for treatment of conjunctivitis, not prophylaxis \\
\hline Sud 1995 & Intervention for treatment of conjunctivitis, not prophylaxis \\
\hline Sung 1998 & Not a randomised or quasi-randomised prophylaxis trial \\
\hline van Bogaert 1997 & Not a randomised or quasi-randomised prophylaxis trial; letter \\
\hline Wallace 1998 & Review article; no trial identified \\
\hline West 1995 & Intervention for treatment of conjunctivitis, not prophylaxis. Population not neonates \\
\hline Winceslaus 1987 & Not an RCT or a quasi-RCT \\
\hline Woolridge 1967 & Intervention is trachoma vaccine; population included preschool-aged children. \\
\hline Wu 2003 & Not an RCT or a quasi-RCT \\
\hline Yasunaga 1977 & $\begin{array}{l}\text { Interventions in trial were different irrigation solutions with silver nitrate; outcome was chemical } \\
\text { conjunctivitis; follow-up time was } 2 \text { days. }\end{array}$ \\
\hline Yetman 1997 & Not a trial of ophthalmia neonatorum prophylaxis; clinical practice guideline \\
\hline Zanoni 1992 & Not an RCT or a quasi-RCT \\
\hline
\end{tabular}

$\mathrm{RCT}$ : randomised controlled trial 
Characteristics of studies awaiting classification [ordered by study ID]

Matinzadeh 2007

Methods
Method of allocation uncertain:

- "In this random clinical case-control study..."

- "These infants were classified into three groups. Selection of newborn for each group was done regardless of sex, kind of birth and exclusively done randomly."

- "Type of study was clinical trial, and sampling method was census form."

Number allocated: 1002.

No comment on exclusions after allocation.

No comment on losses to follow-up, however the study states that 1002 newborns were included, but the numbers in the 3 allocation groups add up to 992, leaving 10 neonates unaccounted for with no explanation.

No comment on missing data.

No reported power calculation.
Country: Tehran, Iran.

Setting: Najmieh Hospital.

Ethnic group: not specified.

Sex: M:F 523 (52\%):479 (48\%).

Average age and age range: not specified.

Inclusion criteria:

1. healthy term infants;

2. weight greater than $2500 \mathrm{~g}$;

3. gestation age more than 37 weeks.

Exclusion criteria: none specified

No comment on equivalence of baseline characteristics.
Number of interventions: 3.

Intervention 1: erythromycin ointment $0.5 \%$ used for both eyes $(n=320)$.

Intervention 2: sterile normal saline 1 drop into each eye $(n=337)$.

Intervention 3: no treatment used for both eyes $(n=335)$.

Time to intervention: "immediately".

Pre-intervention manoeuvres: none specified.

Postintervention manoeuvres: none specified.

\section{Outcomes}

1. Conjunctivitis.

2. Culture-positive conjunctivitis, but not by allocation group.

3. Severe sight-threatening disease.

4. Systemic disease.

5. Gonococcal conjunctivitis.

Follow-up length: unknown: self-report by mother, follow-up limit unknown. 
"Tenth day visit was mandatory to diagnose or rule out conjunctivitis.

"There was no case of severe sight-threatening or systemic disease in our cases on tenth-day visit and afterwards."

Adverse events: no specific comment on adverse events.

Sources of funding: not specified.

Declaration of interest: not specified.

No reported subgroup analysis.

Trial investigators were contacted in August 2016 and again in February 2017. No response was received.

DATA AND ANALYSES

\section{Comparison 1. Any prophylaxis versus no prophylaxis}

\begin{tabular}{lllll}
\hline Outcome or subgroup title & $\begin{array}{l}\text { No. of } \\
\text { studies }\end{array}$ & $\begin{array}{l}\text { No. of } \\
\text { partici- } \\
\text { pants }\end{array}$ & Statistical method & Effect size \\
\hline 1.1 Chlamydial conjunctivitis & 2 & 4874 & Risk Ratio (IV, Fixed, 95\% Cl) & $0.96[0.57,1.61]$ \\
\hline 1.2 Bacterial conjunctivitis & 2 & 3685 & Risk Ratio (IV, Fixed, 95\% Cl) & $0.84[0.37,1.93]$ \\
\hline $\begin{array}{l}1.3 \text { Any conjunctivitis of any aetiol- } \\
\text { ogy }\end{array}$ & 8 & 9666 & Risk Ratio (M-H, Random, 95\% Cl) & $0.65[0.54,0.78]$ \\
\hline $\begin{array}{l}1.4 \text { Conjunctivitis of unknown aeti- } \\
\text { ology }\end{array}$ & 1 & & Risk Ratio (IV, Fixed, 95\% Cl) & Totals not selected \\
\hline $\begin{array}{l}1.5 \text { Nasolacrimal duct obstruction } \\
1.6 \text { Keratitis }\end{array}$ & 1 & & Risk Ratio (IV, Fixed, 95\% Cl) & Totals not selected \\
\hline
\end{tabular}


Analysis 1.1. Comparison 1: Any prophylaxis versus no prophylaxis, Outcome 1: Chlamydial conjunctivitis

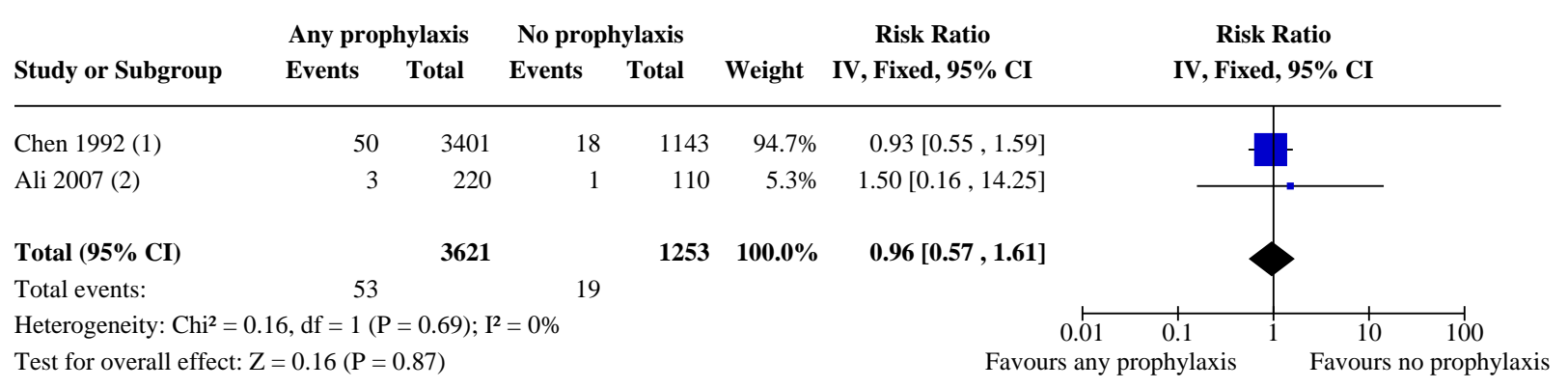

Footnotes

(1) Three intervention arms: erythromycin $0.5 \%$, tetracycline $1 \%$, silver nitrate $1 \%$

(2) Two intervention arms: povidine-iodine $2.5 \%$, erythromycin $0.5 \%$

\section{Analysis 1.2. Comparison 1: Any prophylaxis versus no prophylaxis, Outcome 2: Bacterial conjunctivitis}

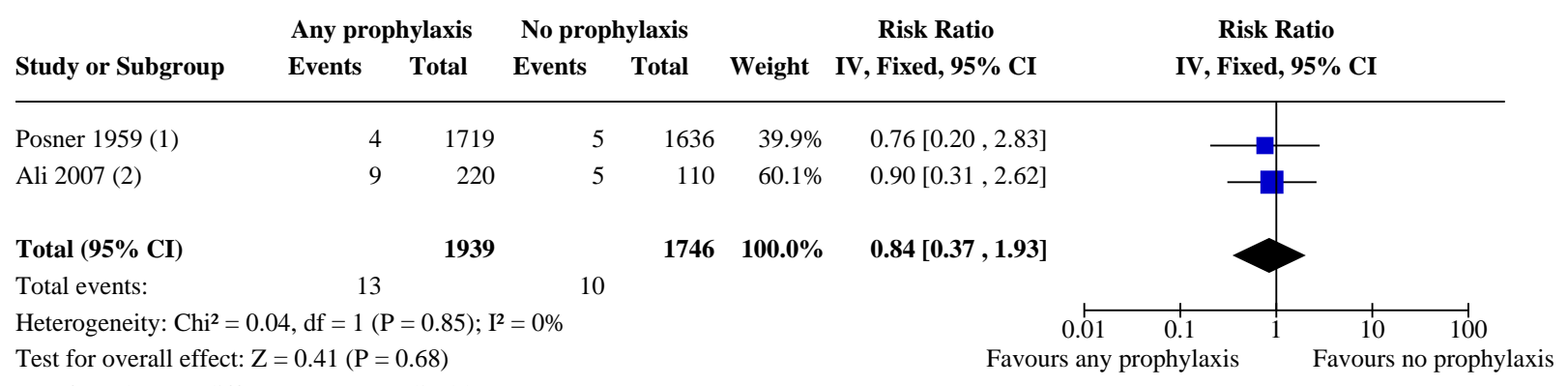

(1) Bacitracin-phenacaine ointment (both intervention and control received mechanical cleansing)

(2) Two intervention arms: povidine-iodine $2.5 \%$, erythromycin $0.5 \%$ 
Analysis 1.3. Comparison 1: Any prophylaxis versus no prophylaxis, Outcome 3: Any conjunctivitis of any aetiology

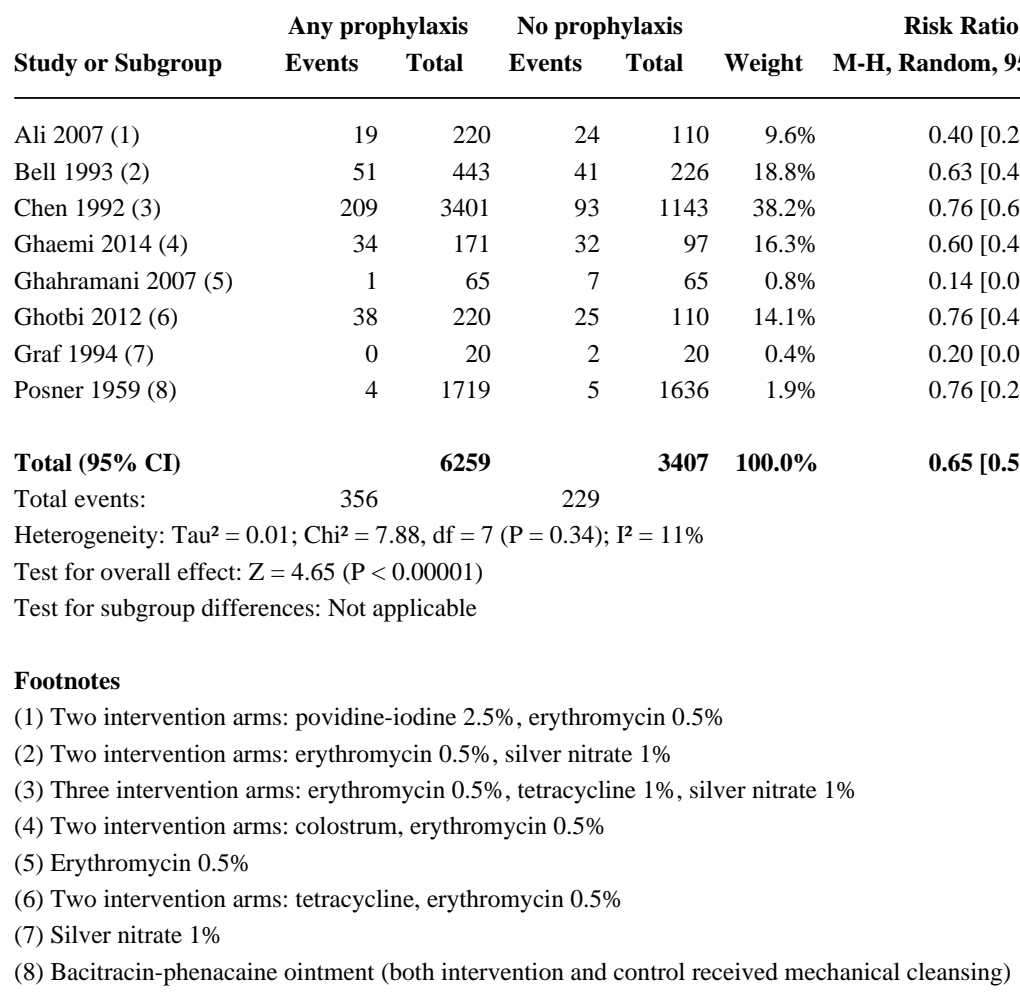

Analysis 1.4. Comparison 1: Any prophylaxis versus no prophylaxis, Outcome 4: Conjunctivitis of unknown aetiology

\begin{tabular}{|c|c|c|c|c|}
\hline & Any prophylaxis & No prophylaxis & Risk Ratio & Risk Ratio \\
\hline Study or Subgroup & Events & Events & IV, Fixed, 95\% CI & IV, Fixed, 95\% CI \\
\hline
\end{tabular}

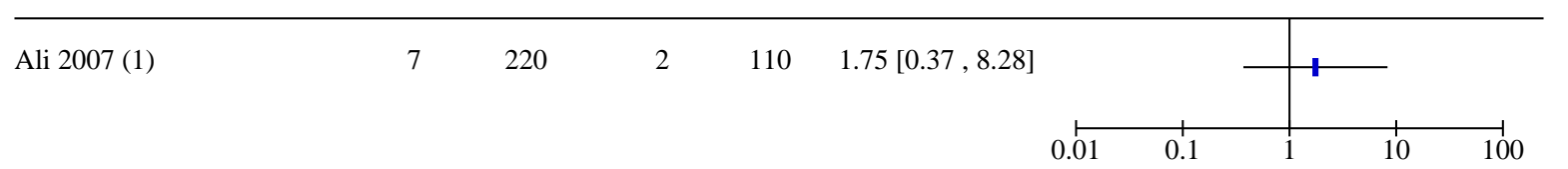

Footnotes

Risk Ratio

M-H, Random, 95\% CI

(1) Two intervention arms: povidine-iodine $2.5 \%$, erythromycin $0.5 \%$

\section{Analysis 1.5. Comparison 1: Any prophylaxis versus no prophylaxis, Outcome 5: Nasolacrimal duct obstruction}

\begin{tabular}{|c|c|c|c|c|}
\hline & Any prophylaxis & No prophylaxis & Risk Ratio & Risk Ratio \\
\hline Study or Subgroup & Events & Events & IV, Fixed, 95\% CI & IV, Fixed, 95\% CI \\
\hline
\end{tabular}

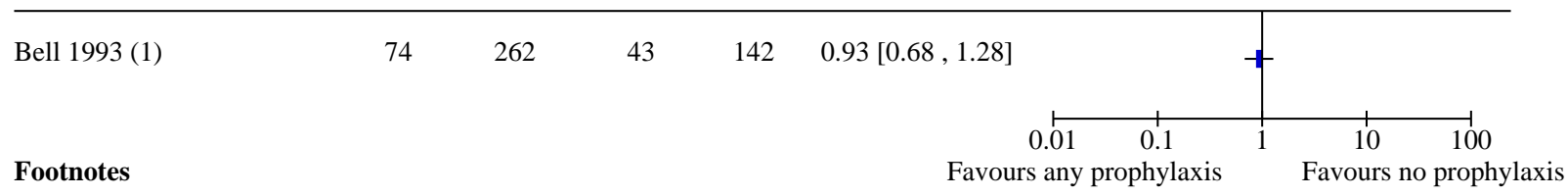

(1) Two intervention arms: erythromycin $0.5 \%$, silver nitrate $1 \%$ 
Analysis 1.6. Comparison 1: Any prophylaxis versus no prophylaxis, Outcome 6: Keratitis

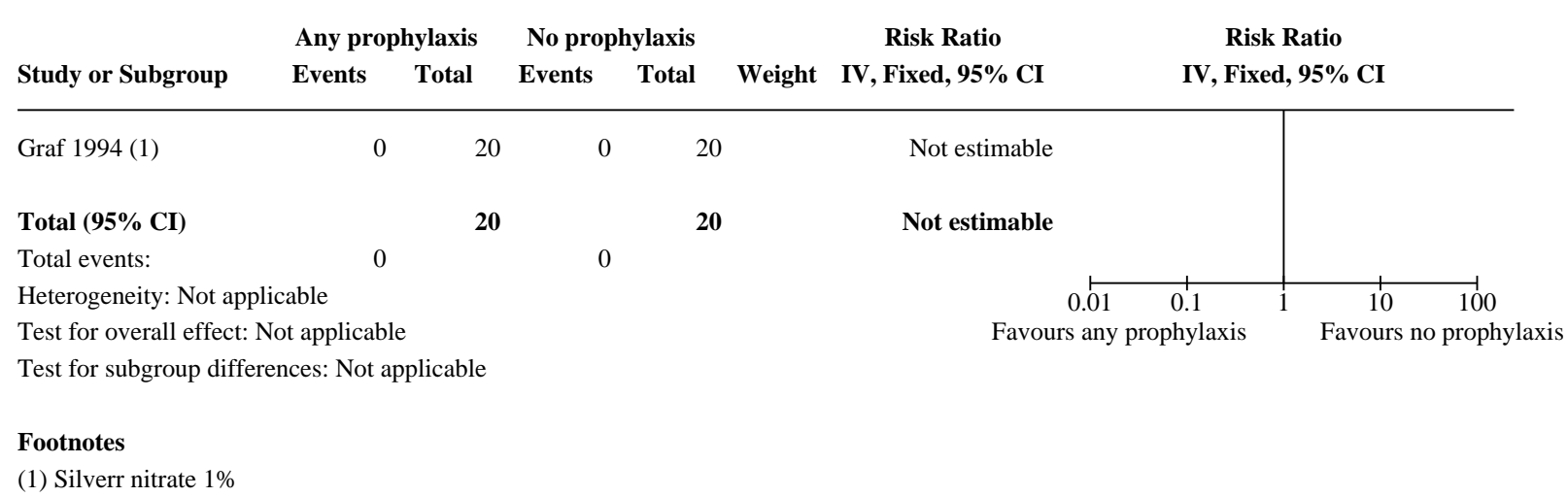

Comparison 2. Silver nitrate versus no prophylaxis

\begin{tabular}{lllll}
\hline Outcome or subgroup title & $\begin{array}{l}\text { No. of } \\
\text { studies }\end{array}$ & $\begin{array}{l}\text { No. of } \\
\text { partici- } \\
\text { pants }\end{array}$ & Statistical method & Effect size \\
\hline 2.1 Gonococcal conjunctivitis & 1 & 2225 & Risk Ratio (M-H, Fixed, 95\% Cl) & Not estimable \\
\hline 2.2 Chlamydial conjunctivitis & 1 & Risk Ratio (IV, Fixed, 95\% Cl) & Totals not selected \\
\hline $\begin{array}{l}2.3 \text { Any conjunctivitis of any aetiolo- } \\
\text { gy }\end{array}$ & 3 & 2713 & Risk Ratio (M-H, Random, 95\% Cl) & $0.67[0.52,0.87]$ \\
\hline 2.4 Corneal abrasion & 1 & 40 & Risk Ratio (IV, Fixed, 95\% Cl) & Not estimable \\
\hline 2.5 Nasolacrimal duct obstruction & 1 & & Risk Ratio (IV, Fixed, 95\% Cl) & Totals not selected \\
\hline
\end{tabular}

\section{Analysis 2.1. Comparison 2: Silver nitrate versus no prophylaxis, Outcome 1: Gonococcal conjunctivitis}

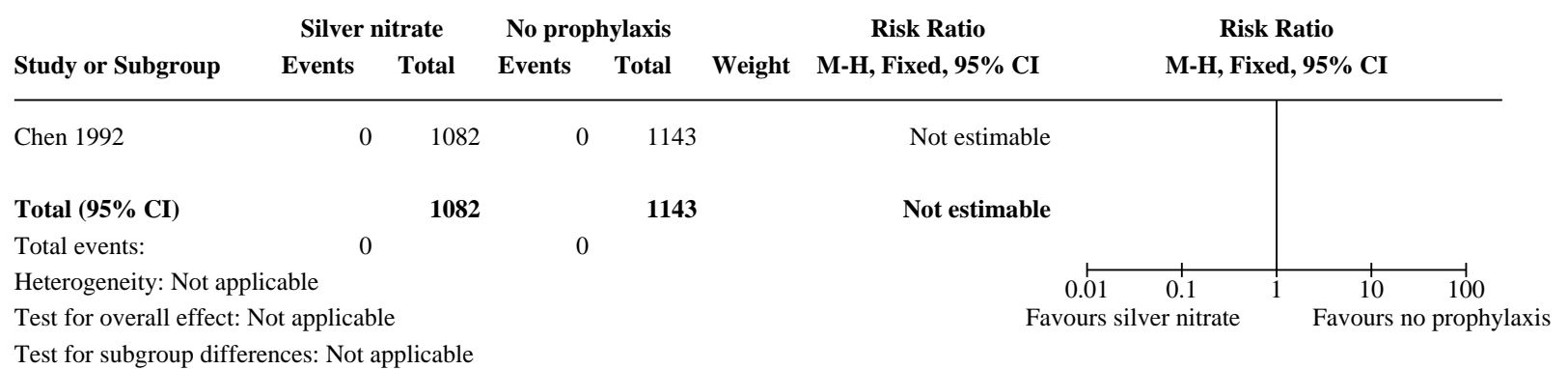


Analysis 2.2. Comparison 2: Silver nitrate versus no prophylaxis, Outcome 2: Chlamydial conjunctivitis

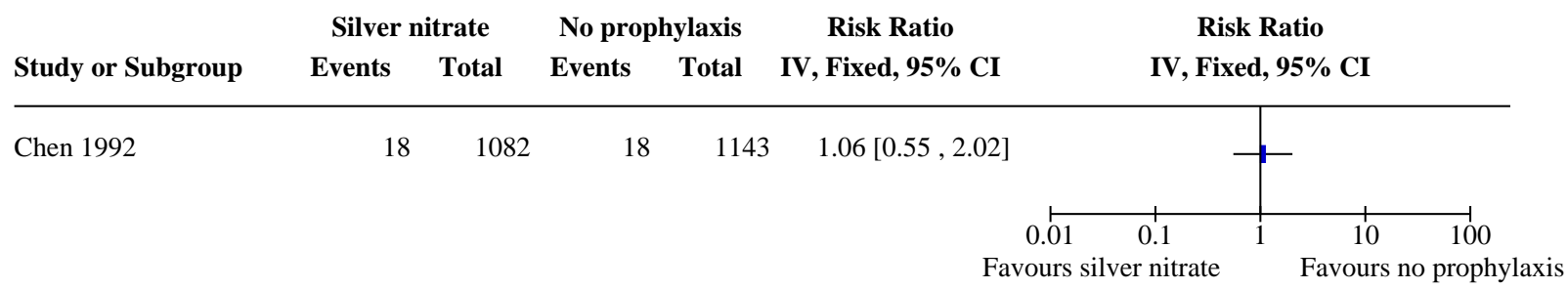

Analysis 2.3. Comparison 2: Silver nitrate versus no prophylaxis, Outcome 3: Any conjunctivitis of any aetiology

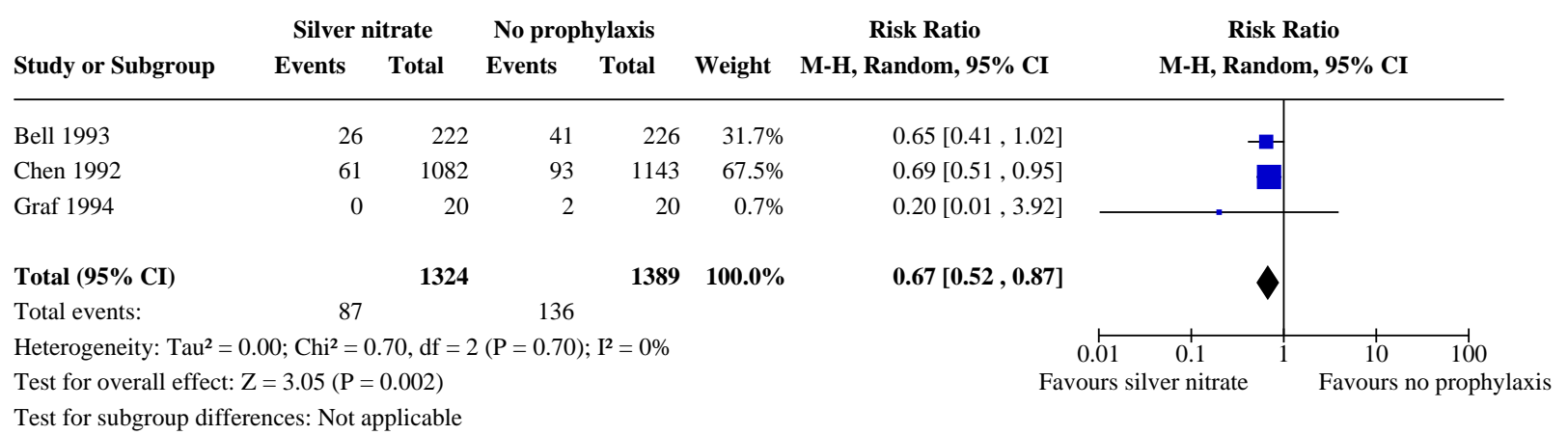

\section{Analysis 2.4. Comparison 2: Silver nitrate versus no prophylaxis, Outcome 4: Corneal abrasion}

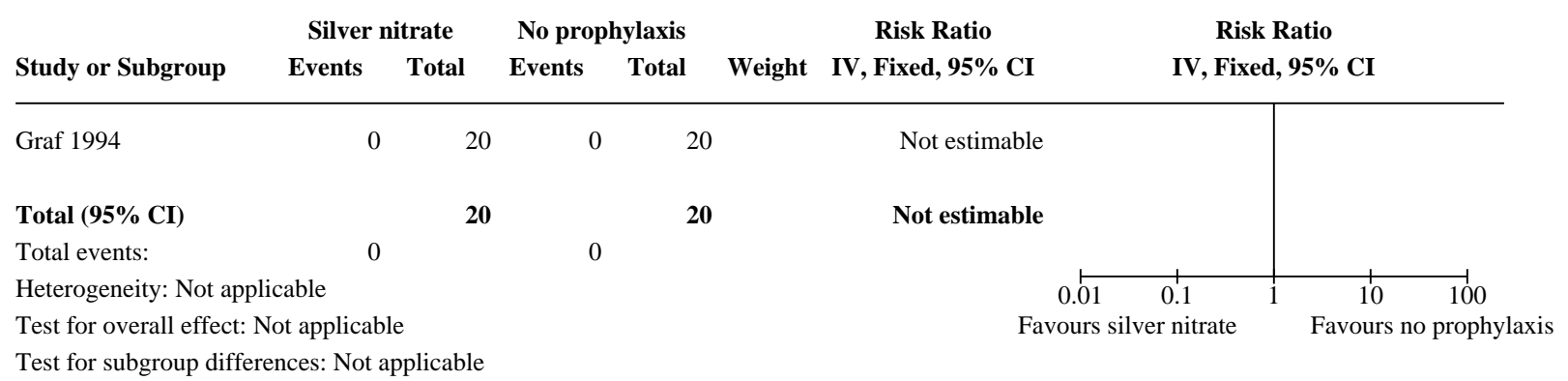

Analysis 2.5. Comparison 2: Silver nitrate versus no prophylaxis, Outcome 5: Nasolacrimal duct obstruction

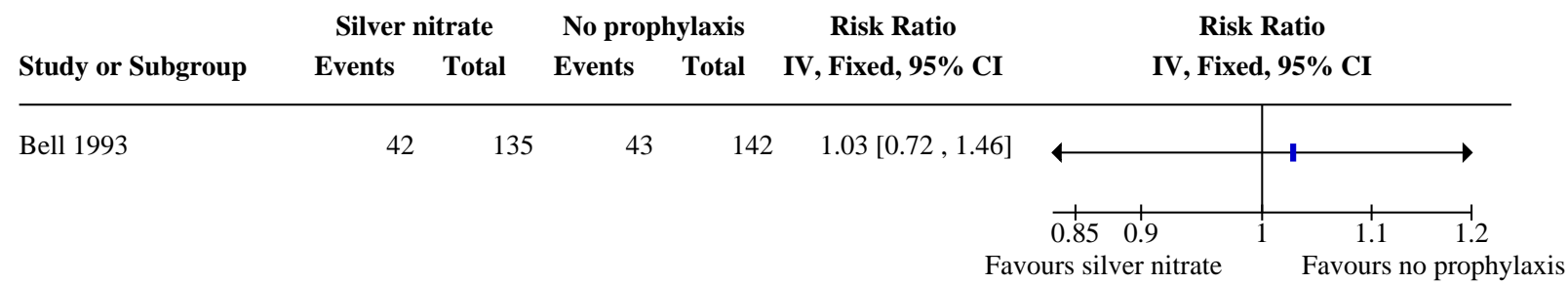


Comparison 3. Erythromycin versus no prophylaxis

\begin{tabular}{|c|c|c|c|c|}
\hline Outcome or subgroup title & $\begin{array}{l}\text { No. of } \\
\text { studies }\end{array}$ & $\begin{array}{l}\text { No. of } \\
\text { partici- } \\
\text { pants }\end{array}$ & Statistical method & Effect size \\
\hline 3.1 Gonococcal conjunctivitis & 2 & 2526 & Risk Ratio (IV, Fixed, 95\% CI) & Not estimable \\
\hline 3.2 Chlamydial conjunctivitis & 2 & 2526 & Risk Ratio (IV, Fixed, 95\% CI) & $0.93[0.49,1.77]$ \\
\hline 3.3 Bacterial conjunctivitis & 1 & & Risk Ratio (IV, Fixed, 95\% CI) & Totals not selected \\
\hline $\begin{array}{l}\text { 3.4 Any conjunctivitis of any aetiolo- } \\
\text { gy }\end{array}$ & 6 & 3509 & Risk Ratio (IV, Random, 95\% CI) & $0.68[0.51,0.89]$ \\
\hline $\begin{array}{l}\text { 3.5 Conjunctivitis of unknown aeti- } \\
\text { ology }\end{array}$ & 1 & & Risk Ratio (IV, Fixed, 95\% CI) & Totals not selected \\
\hline 3.6 Nasolacrimal duct obstruction & 1 & & Risk Ratio (IV, Fixed, 95\% CI) & Totals not selected \\
\hline
\end{tabular}

Analysis 3.1. Comparison 3: Erythromycin versus no prophylaxis, Outcome 1: Gonococcal conjunctivitis

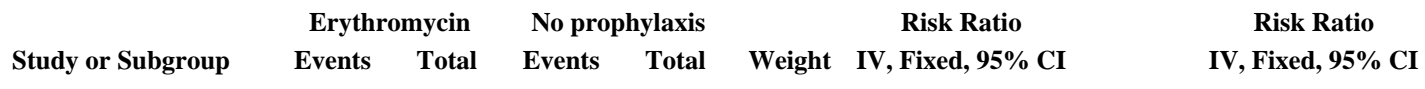

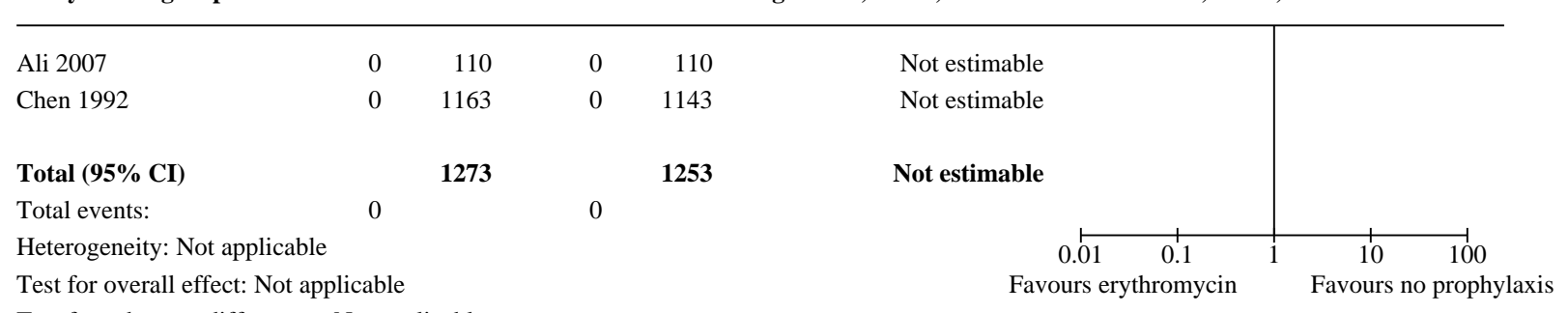

Test for subgroup differences: Not applicable

\section{Analysis 3.2. Comparison 3: Erythromycin versus no prophylaxis, Outcome 2: Chlamydial conjunctivitis}

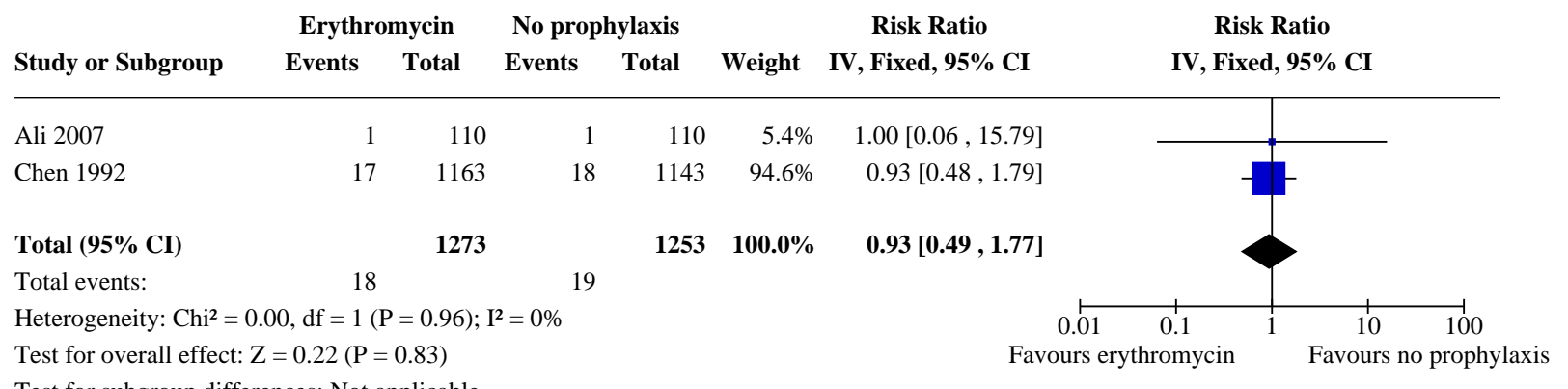


Analysis 3.3. Comparison 3: Erythromycin versus no prophylaxis, Outcome 3: Bacterial conjunctivitis

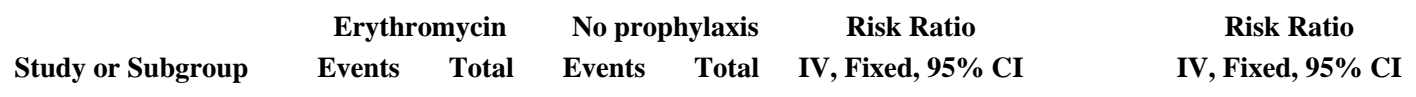

$\begin{array}{llllll}\text { Ali } 2007 & 4 & 110 & 5 & 110 & 0.80[0.22,2.90]\end{array}$

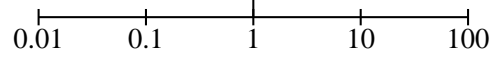

Favours erythromycin Favours no prophylaxis

Analysis 3.4. Comparison 3: Erythromycin versus no prophylaxis, Outcome 4: Any conjunctivitis of any aetiology

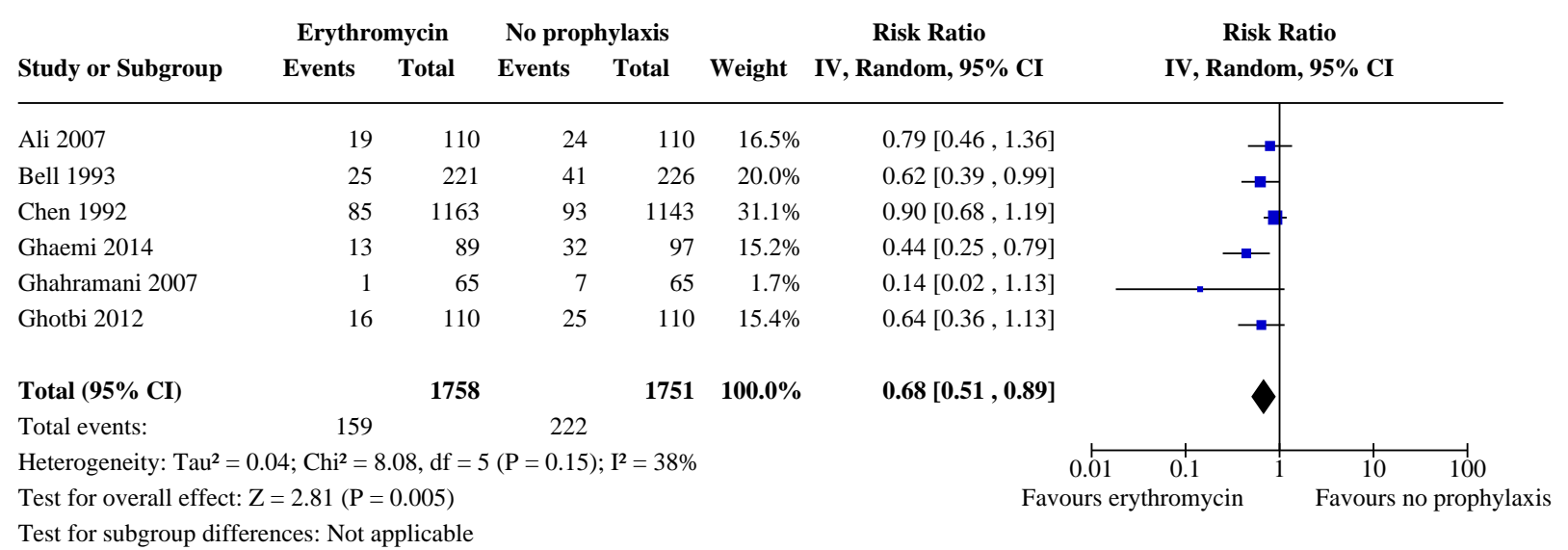

Analysis 3.5. Comparison 3: Erythromycin versus no prophylaxis, Outcome 5: Conjunctivitis of unknown aetiology

\begin{tabular}{|c|c|c|c|c|c|c|}
\hline & Eryth & nycin & No pre & ylaxis & Risk Ratio & Risk Ratio \\
\hline Study or Subgroup & Events & Total & Events & Total & IV, Fixed, 95\% CI & IV, Fixed, 95\% CI \\
\hline
\end{tabular}

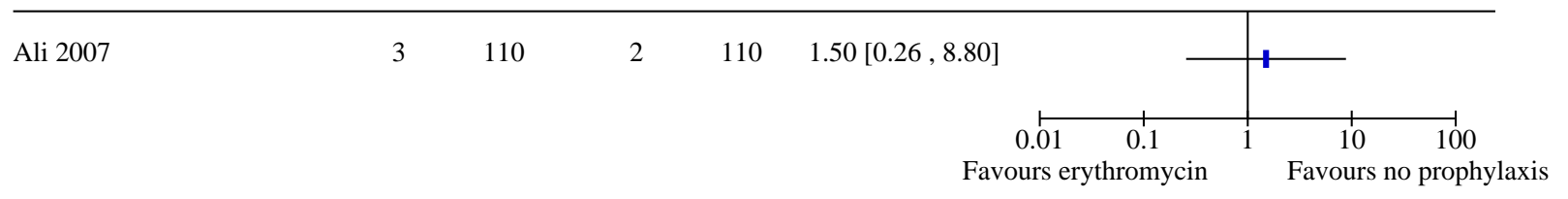

Analysis 3.6. Comparison 3: Erythromycin versus no prophylaxis, Outcome 6: Nasolacrimal duct obstruction

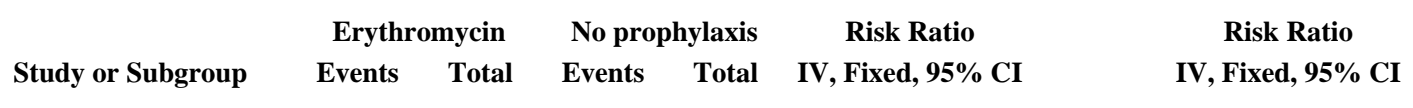

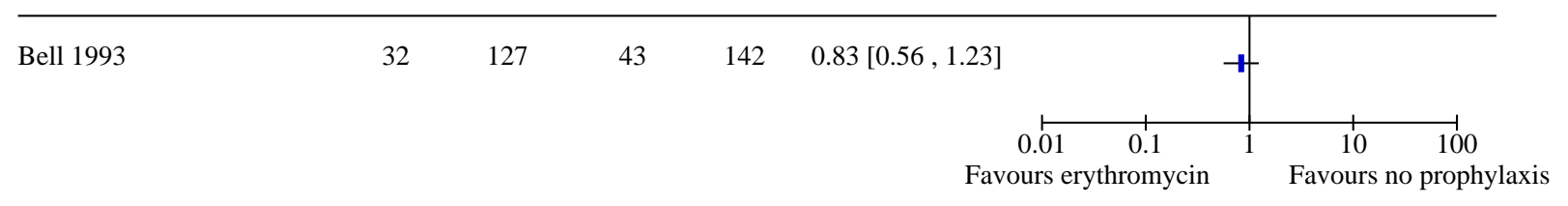


Comparison 4. Tetracycline versus no prophylaxis

\begin{tabular}{lllll}
\hline Outcome or subgroup title & $\begin{array}{l}\text { No. of } \\
\text { studies }\end{array}$ & $\begin{array}{l}\text { No. of } \\
\text { partici- } \\
\text { pants }\end{array}$ & Statistical method & Effect size \\
\hline 4.1 Gonococcal conjunctivitis & 1 & 2299 & Risk Ratio (M-H, Fixed, 95\% Cl) & Not estimable \\
\hline 4.2 Chlamydial conjunctivitis & 1 & Risk Ratio (IV, Fixed, 95\% Cl) & Totals not selected \\
\hline 4.3 Any conjunctivitis of any aetiology & 2 & 2519 & Risk Ratio (IV, Fixed, $95 \% \mathrm{Cl})$ & $0.72[0.55,0.94]$ \\
\hline
\end{tabular}

Analysis 4.1. Comparison 4: Tetracycline versus no prophylaxis, Outcome 1: Gonococcal conjunctivitis

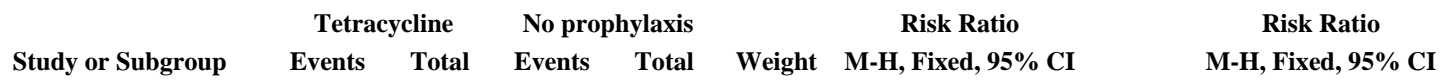

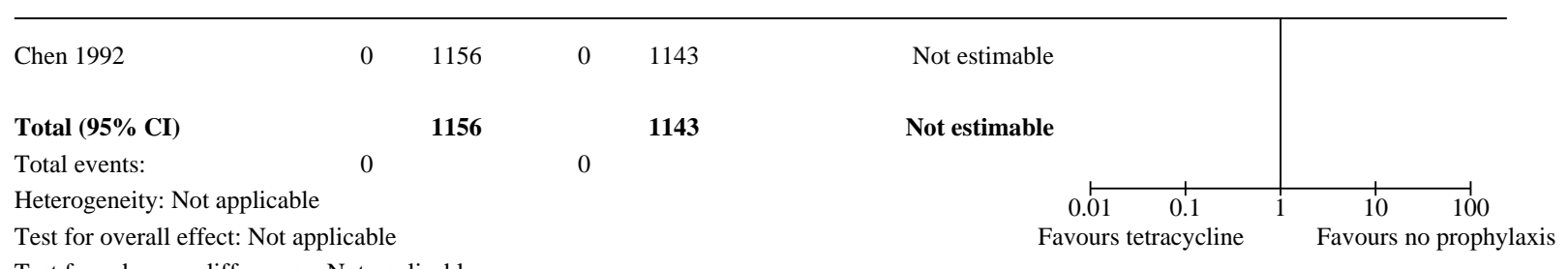

Test for subgroup differences: Not applicable

\section{Analysis 4.2. Comparison 4: Tetracycline versus no prophylaxis, Outcome 2: Chlamydial conjunctivitis}

\begin{tabular}{|c|c|c|}
\hline & & \\
\hline udy or Subgroup & Events & Tota \\
\hline
\end{tabular}

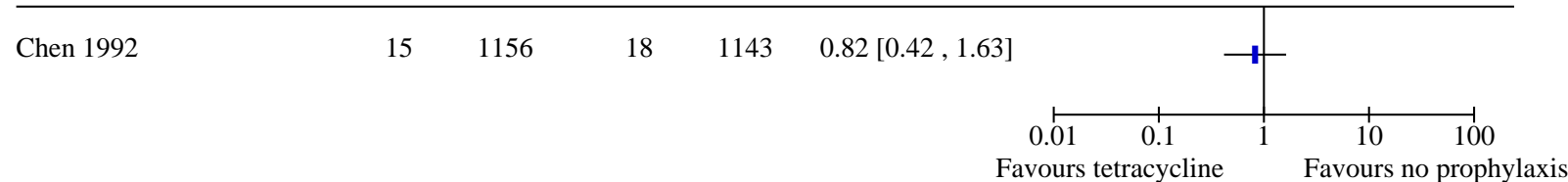

\section{Analysis 4.3. Comparison 4: Tetracycline versus no prophylaxis, Outcome 3: Any conjunctivitis of any aetiology}

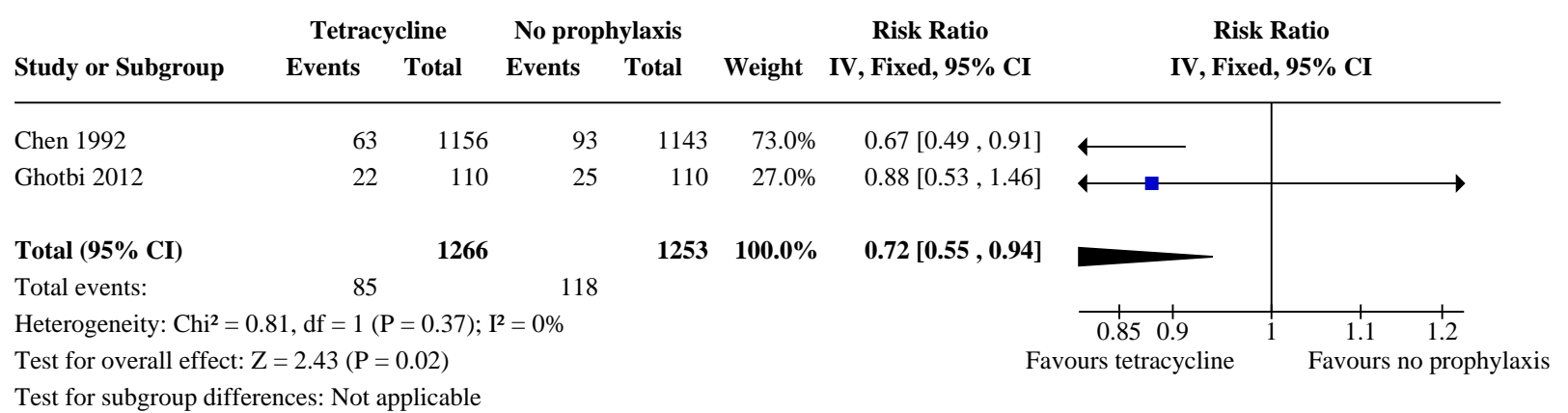


Comparison 5. Povidone-iodine versus no prophylaxis

\begin{tabular}{lllll}
\hline Outcome or subgroup title & $\begin{array}{l}\text { No. of } \\
\text { studies }\end{array}$ & $\begin{array}{l}\text { No. of } \\
\text { partici- } \\
\text { pants }\end{array}$ & Statistical method & Effect size \\
\hline 5.1 Gonococcal conjunctivitis & 1 & 220 & Risk Ratio (M-H, Fixed, 95\% Cl) & Not estimable \\
\hline 5.2 Chlamydial conjunctivitis & 1 & Risk Ratio (IV, Fixed, 95\% Cl) & Totals not selected \\
\hline 5.3 Bacterial conjunctivitis & 1 & Risk Ratio (IV, Fixed, 95\% Cl) & Totals not selected \\
\hline $\begin{array}{l}5.4 \text { Any conjunctivitis of any aetiolo- } \\
\text { gy }\end{array}$ & 1 & Risk Ratio (IV, Fixed, 95\% Cl) & Totals not selected \\
\hline $\begin{array}{l}5.5 \text { Conjunctivitis of unknown aeti- } \\
\text { ology }\end{array}$ & 1 & Risk Ratio (IV, Fixed, 95\% Cl) & Totals not selected \\
\hline
\end{tabular}

Analysis 5.1. Comparison 5: Povidone-iodine versus no prophylaxis, Outcome 1: Gonococcal conjunctivitis

\begin{tabular}{lccccccc} 
& \multicolumn{2}{c}{ Povidone-iodine } & \multicolumn{2}{c}{ No prophylaxis } & & Risk Ratio & Risk Ratio \\
Study or Subgroup & Events & Total & Events & Total & Weight & M-H, Fixed, 95\% CI & M-H, Fixed, 95\% CI
\end{tabular}

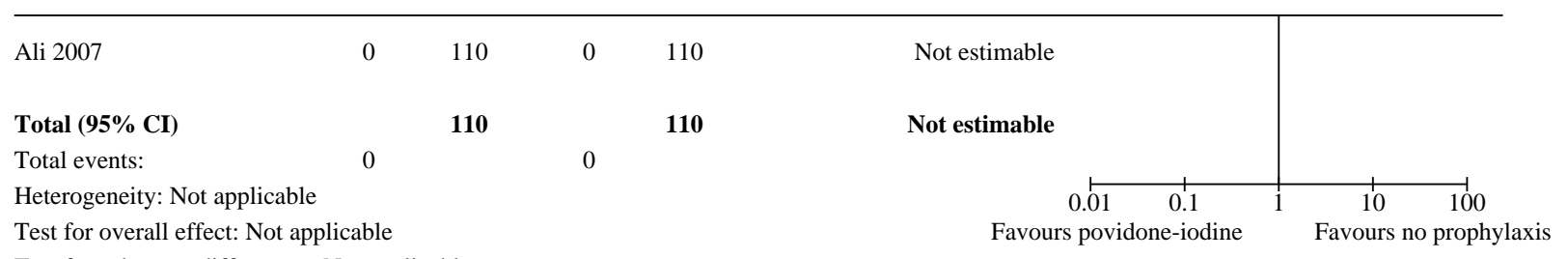

Test for subgroup differences: Not applicable

Analysis 5.2. Comparison 5: Povidone-iodine versus no prophylaxis, Outcome 2: Chlamydial conjunctivitis

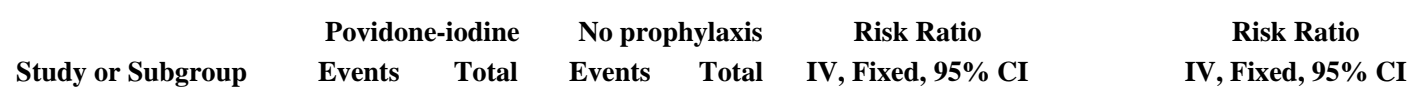

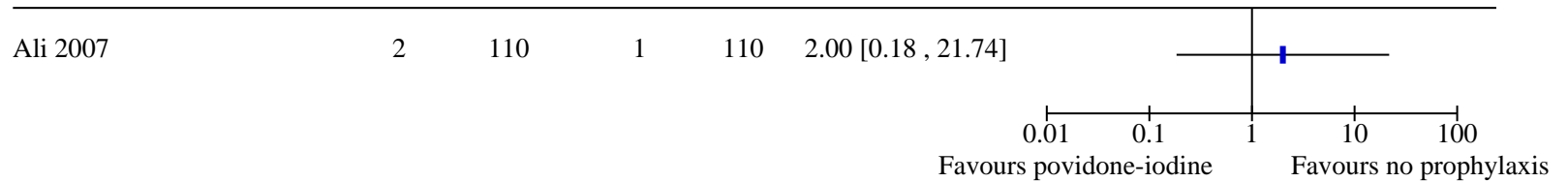

Analysis 5.3. Comparison 5: Povidone-iodine versus no prophylaxis, Outcome 3: Bacterial conjunctivitis

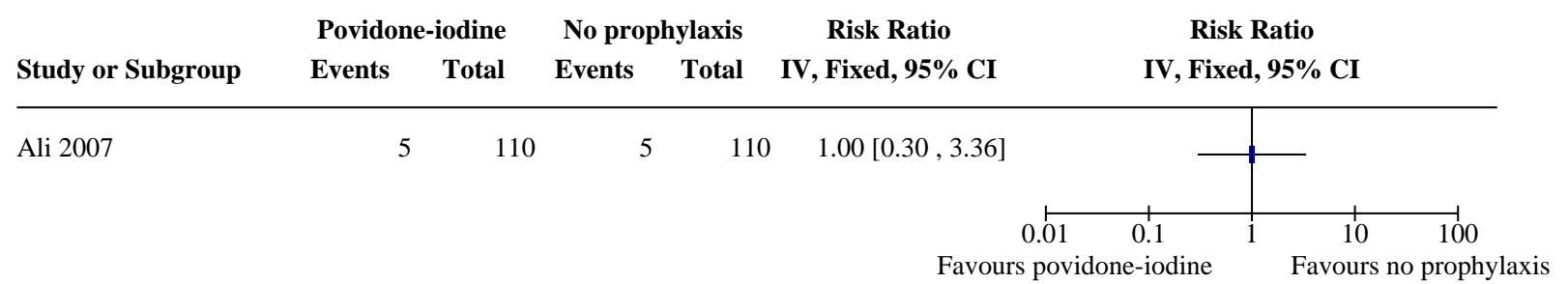


Analysis 5.4. Comparison 5: Povidone-iodine versus no prophylaxis, Outcome 4: Any conjunctivitis of any aetiology

\begin{tabular}{|c|c|c|c|c|c|c|c|}
\hline \multirow[b]{2}{*}{ Study or Subgroup } & \multicolumn{2}{|c|}{ Povidone-iodine } & \multicolumn{2}{|c|}{ No prophylaxis } & \multirow{2}{*}{$\begin{array}{c}\text { Risk Ratio } \\
\text { IV, Fixed, 95\% CI }\end{array}$} & \multirow{2}{*}{\multicolumn{2}{|c|}{$\begin{array}{c}\text { Risk Ratio } \\
\text { IV, Fixed, 95\% CI }\end{array}$}} \\
\hline & Events & Total & Events & Total & & & \\
\hline Ali 2007 & 9 & 110 & 24 & 110 & $0.38[0.18,0.77]$ & † & \\
\hline
\end{tabular}

Analysis 5.5. Comparison 5: Povidone-iodine versus no prophylaxis, Outcome 5: Conjunctivitis of unknown aetiology

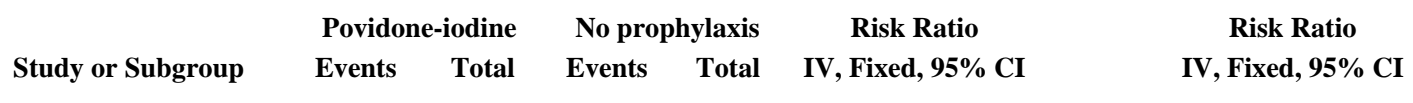

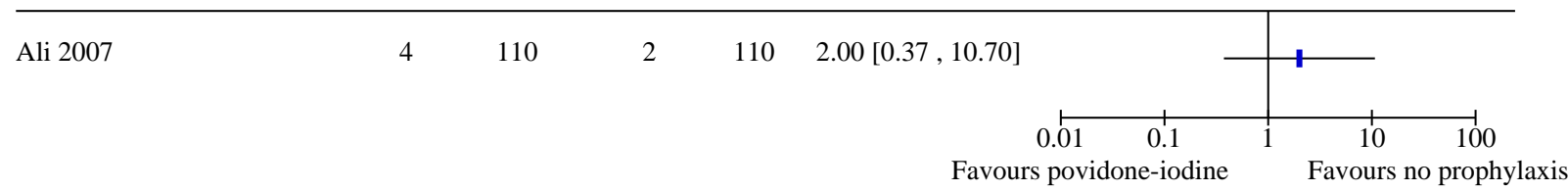

Comparison 6. Bacitracin-phenacaine versus no prophylaxis

\begin{tabular}{lllll}
\hline Outcome or subgroup title & $\begin{array}{l}\text { No. of } \\
\text { studies }\end{array}$ & $\begin{array}{l}\text { No. of par- } \\
\text { ticipants }\end{array}$ & Statistical method & Effect size \\
\hline 6.1 Gonococcal conjunctivitis & 1 & Risk Ratio (IV, Fixed, 95\% Cl) & Totals not selected \\
\hline
\end{tabular}

Analysis 6.1. Comparison 6: Bacitracin-phenacaine versus no prophylaxis, Outcome 1: Gonococcal conjunctivitis

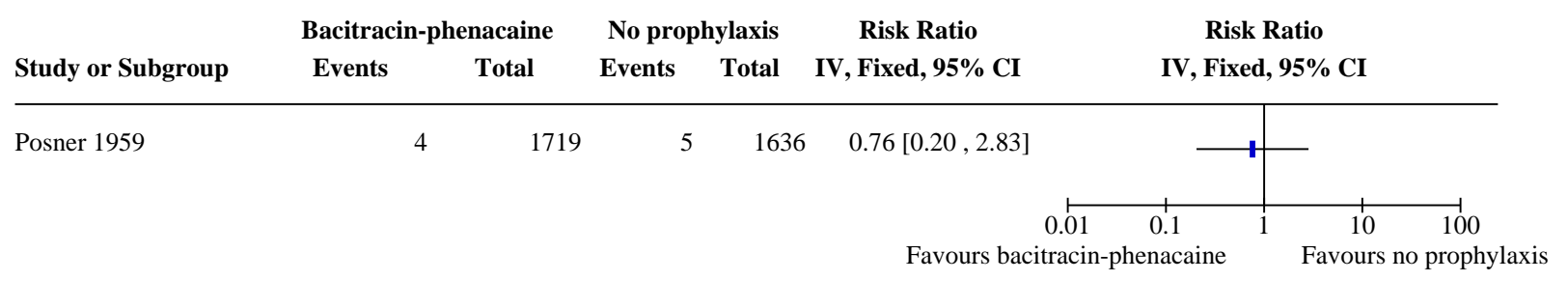

Comparison 7. Colostrum versus no prophylaxis

\begin{tabular}{lllll}
\hline Outcome or subgroup title & $\begin{array}{l}\text { No. of } \\
\text { studies }\end{array}$ & $\begin{array}{l}\text { No. of par- } \\
\text { ticipants }\end{array}$ & Statistical method & Effect size \\
\hline 7.1 Any conjunctivitis of any aetiology & 1 & Risk Ratio (IV, Fixed, 95\% Cl) & Totals not selected \\
\hline
\end{tabular}


Analysis 7.1. Comparison 7: Colostrum versus no prophylaxis, Outcome 1: Any conjunctivitis of any aetiology

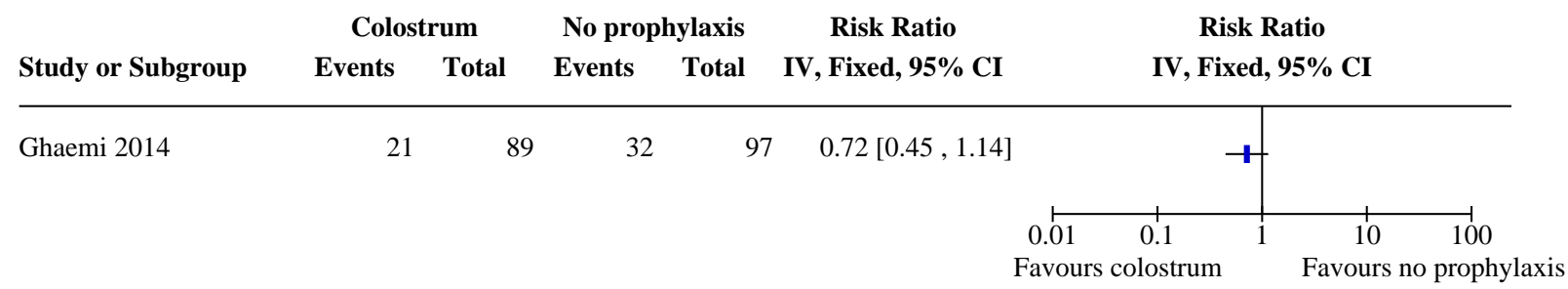

Comparison 8. Erythromycin versus silver nitrate

\begin{tabular}{lllll}
\hline Outcome or subgroup title & $\begin{array}{l}\text { No. of } \\
\text { studies }\end{array}$ & $\begin{array}{l}\text { No. of } \\
\text { partici- } \\
\text { pants }\end{array}$ & Statistical method & Effect size \\
\hline 8.1 Chlamydial conjunctivitis & 4 & 13472 & Risk Ratio (M-H, Random, 95\% Cl) & $0.75[0.51,1.09]$ \\
\hline 8.2 Bacterial conjunctivitis & 2 & 6333 & Risk Ratio (IV, Fixed, 95\% Cl) & $0.83[0.69,1.01]$ \\
\hline 8.3 Any conjunctivitis of any aetiology & 3 & 4729 & Risk Ratio (IV, Random, 95\% Cl) & $1.02[0.80,1.30]$ \\
\hline $\begin{array}{l}8.4 \text { Conjunctivitis of unknown aetiolo- } \\
\text { gy }\end{array}$ & 1 & & Risk Ratio (IV, Fixed, 95\% Cl) & Totals not selected \\
\hline 8.5 Nasolacrimal duct obstruction & 1 & & Risk Ratio (IV, Fixed, 95\% Cl) & Totals not selected \\
\hline
\end{tabular}

Analysis 8.1. Comparison 8: Erythromycin versus silver nitrate, Outcome 1: Chlamydial conjunctivitis

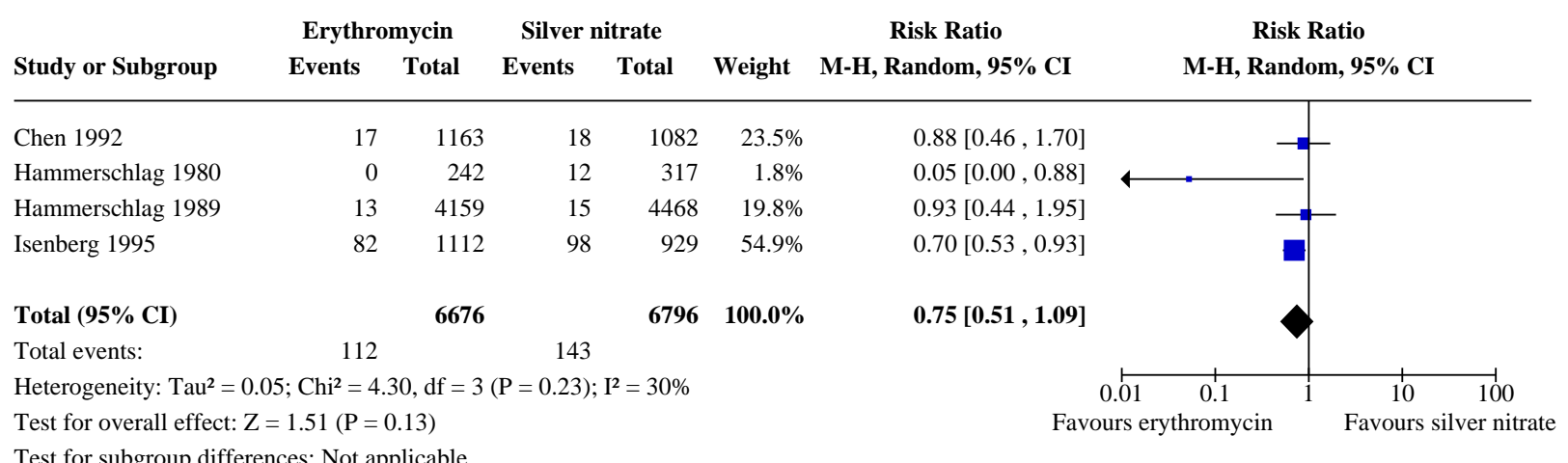


Analysis 8.2. Comparison 8: Erythromycin versus silver nitrate, Outcome 2: Bacterial conjunctivitis

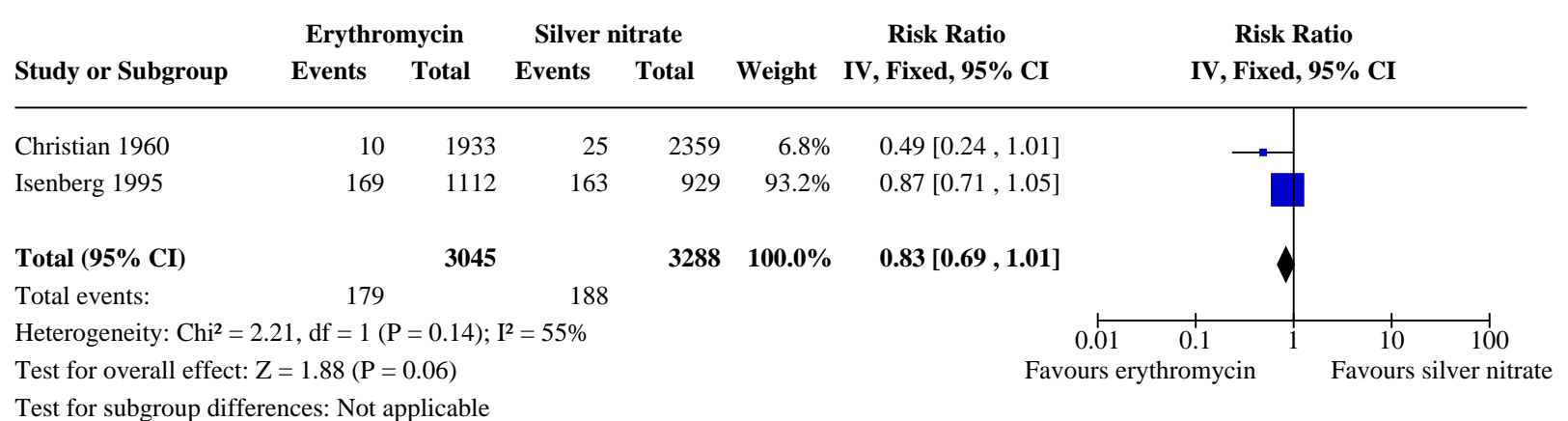

Analysis 8.3. Comparison 8: Erythromycin versus silver nitrate, Outcome 3: Any conjunctivitis of any aetiology

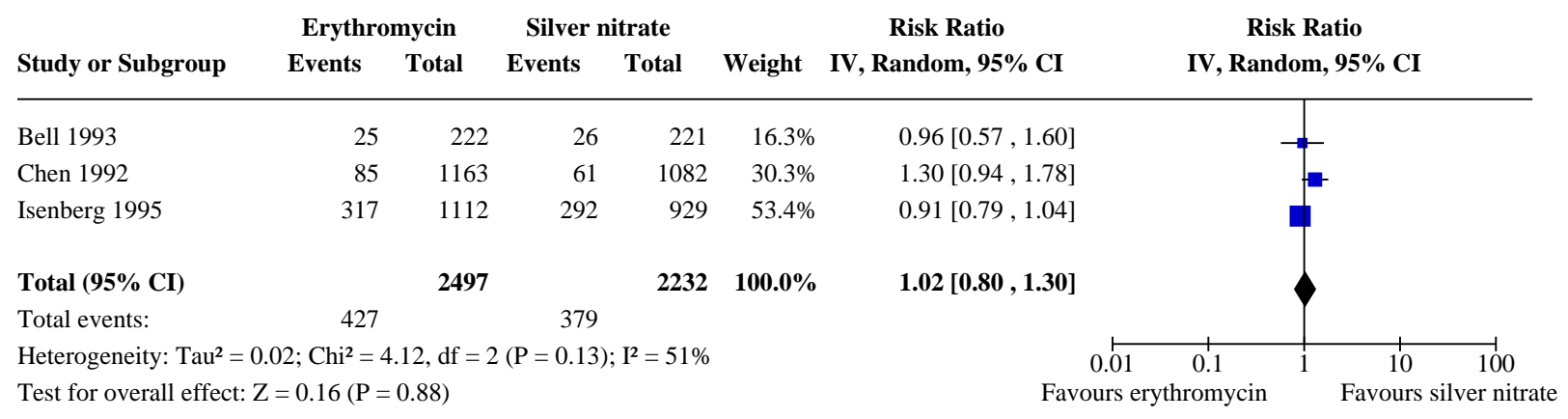

Test for subgroup differences: Not applicable

Analysis 8.4. Comparison 8: Erythromycin versus silver nitrate, Outcome 4: Conjunctivitis of unknown aetiology

\begin{tabular}{|c|c|c|c|c|}
\hline & Erythromycin & Silver nitrate & Risk Ratio & Risk Ratio \\
\hline Study or Subgroup & Events & Events & IV, Fixed, 95\% CI & IV, Fixed, $95 \%$ CI \\
\hline
\end{tabular}

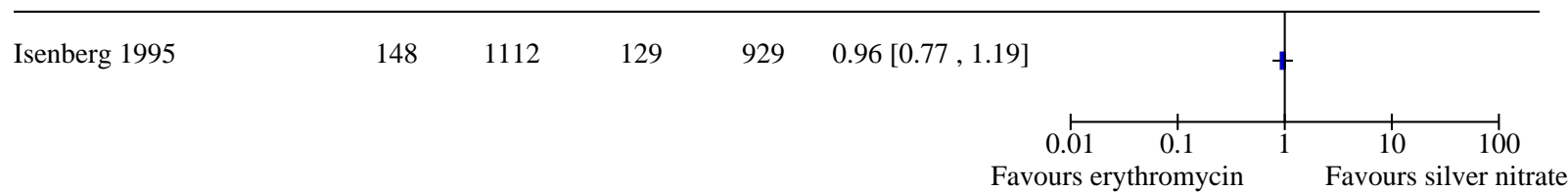

Analysis 8.5. Comparison 8: Erythromycin versus silver nitrate, Outcome 5: Nasolacrimal duct obstruction

\begin{tabular}{|c|c|c|c|c|}
\hline & Erythromycin & Silver nitrate & Risk Ratio & Risk Ratio \\
\hline Study or Subgroup & Events & Events & IV, Fixed, 95\% CI & IV, Fixed, 95\% CI \\
\hline
\end{tabular}

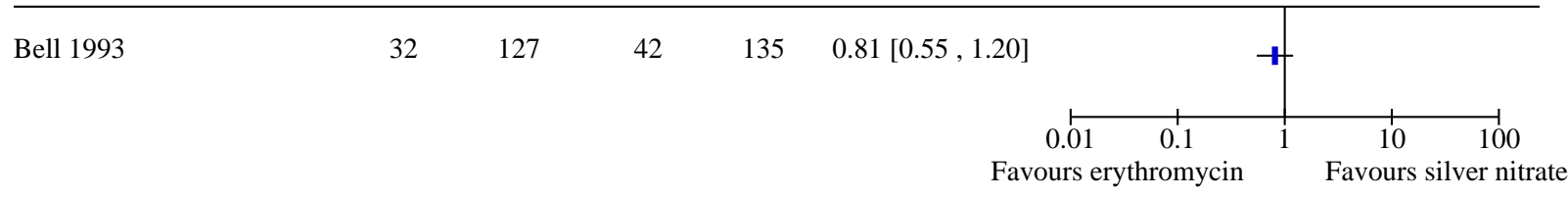


Comparison 9. Tetracycline versus silver nitrate

\begin{tabular}{lllll}
\hline Outcome or subgroup title & $\begin{array}{l}\text { No. of } \\
\text { studies }\end{array}$ & $\begin{array}{l}\text { No. of } \\
\text { partici- } \\
\text { pants }\end{array}$ & Statistical method & Effect size \\
\hline 9.1 Chlamydial conjunctivitis & 4 & 14142 & Risk Ratio (IV, Random, 95\% Cl) & 0.64 [0.40, 1.02] \\
\hline 9.2 Nasolacrimal duct obstruction & 1 & & Risk Ratio (IV, Fixed, 95\% Cl) & Totals not selected \\
\hline
\end{tabular}

\section{Analysis 9.1. Comparison 9: Tetracycline versus silver nitrate, Outcome 1: Chlamydial conjunctivitis}

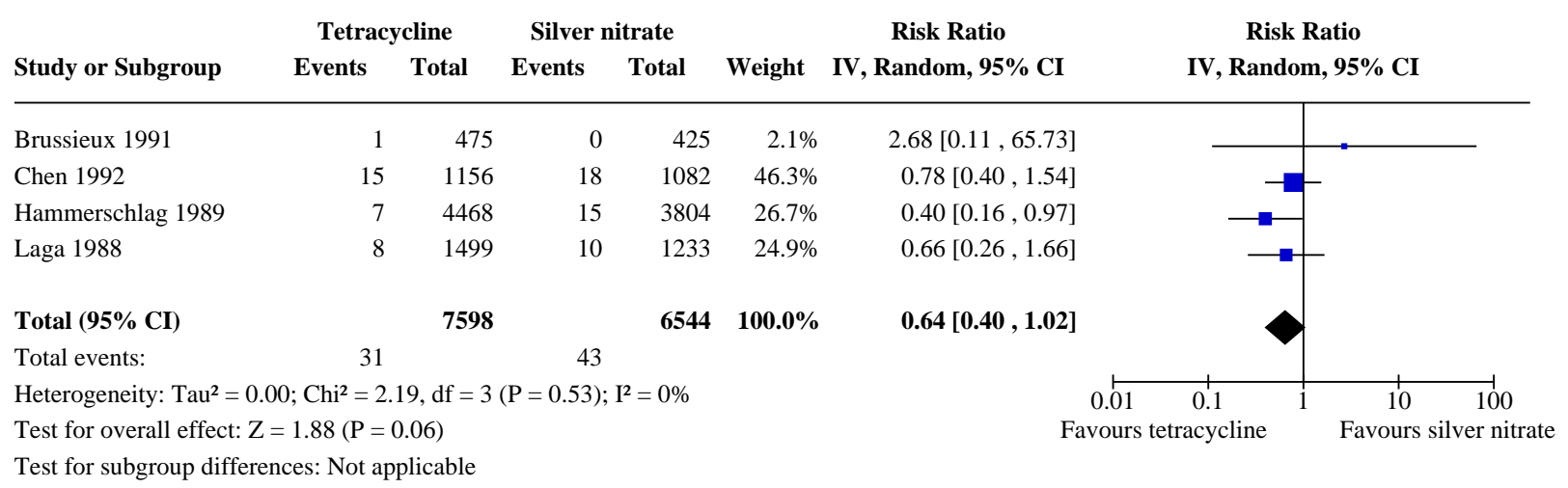

\section{Analysis 9.2. Comparison 9: Tetracycline versus silver nitrate, Outcome 2: Nasolacrimal duct obstruction}

\begin{tabular}{|c|c|c|c|c|c|c|}
\hline \multirow[b]{2}{*}{ Stu } & \multicolumn{2}{|c|}{ Tetracycline } & \multicolumn{2}{|c|}{ Silver nitrate } & Risk Ratio & Risk Ratio \\
\hline & Events & Total & Events & Total & IV, Fixed, 95\% CI & IV, Fixed, 95\% CI \\
\hline
\end{tabular}

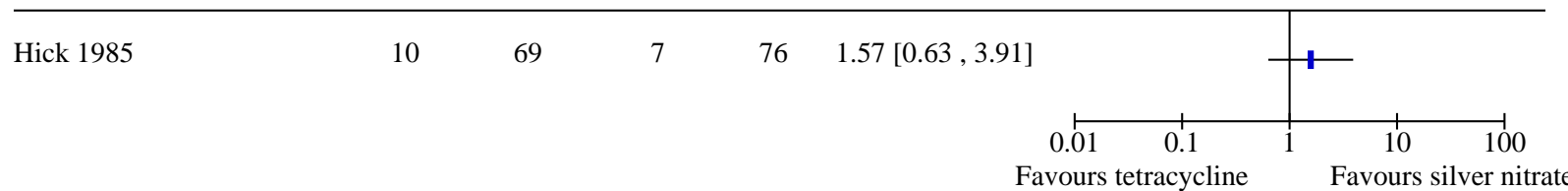

\section{Comparison 10. Sulfacetamide versus silver nitrate}

\begin{tabular}{lllll}
\hline Outcome or subgroup title & $\begin{array}{l}\text { No. of } \\
\text { studies }\end{array}$ & $\begin{array}{l}\text { No. of } \\
\text { partici- } \\
\text { pants }\end{array}$ & Statistical method & Effect size \\
\hline 10.1 Gonococcal conjunctivitis & 1 & 640 & Risk Ratio (M-H, Fixed, 95\% Cl) & Not estimable \\
\hline 10.2 Bacterial conjunctivitis & 1 & Risk Ratio (IV, Fixed, 95\% Cl) & Totals not selected \\
\hline 10.3 Any conjunctivitis of any aetiology & 1 & Risk Ratio (IV, Fixed, $95 \% \mathrm{Cl})$ & Totals not selected \\
\hline $\begin{array}{l}10.4 \text { Conjunctivitis of unknown aetiolo- } \\
\text { gy }\end{array}$ & 1 & Risk Ratio (IV, Fixed, $95 \% \mathrm{Cl})$ & Totals not selected \\
\hline
\end{tabular}


Analysis 10.1. Comparison 10: Sulfacetamide versus silver nitrate, Outcome 1: Gonococcal conjunctivitis

\begin{tabular}{lccccccc} 
& \multicolumn{2}{c}{ Sulphacetimide } & \multicolumn{2}{c}{ Silver nitrate } & & Risk Ratio & Risk Ratio \\
Study or Subgroup & Events & Total & Events & Total & Weight & M-H, Fixed, 95\% CI & M-H, Fixed, 95\% CI
\end{tabular}

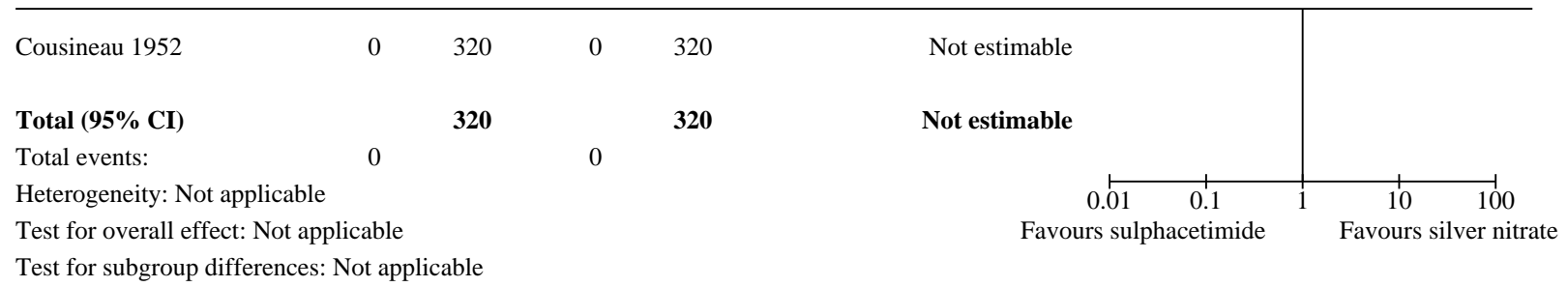

Analysis 10.2. Comparison 10: Sulfacetamide versus silver nitrate, Outcome 2: Bacterial conjunctivitis

\begin{tabular}{|c|c|c|c|c|}
\hline & Sulphacetimide & Silver nitrate & Risk Ratio & Risk Ratio \\
\hline Study or Subgroup & Events & Events & IV, Fixed, 95\% CI & IV, Fixed, 95\% CI \\
\hline
\end{tabular}

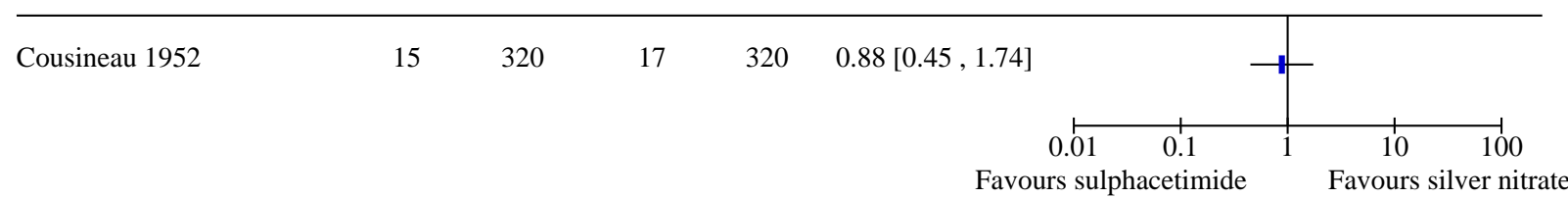

Analysis 10.3. Comparison 10: Sulfacetamide versus silver nitrate, Outcome 3: Any conjunctivitis of any aetiology

\begin{tabular}{|c|c|c|c|c|}
\hline & Sulphacetimide & Silver nitrate & Risk Ratio & Risk Ratio \\
\hline Study or Subgroup & Events & Events & IV, Fixed, 95\% CI & IV, Fixed, 95\% CI \\
\hline
\end{tabular}

\begin{tabular}{llllll|l}
\hline Cousineau 1952 & 21 & 320 & 39 & 320 & $0.54[0.32,0.89]$ & $\rightarrow-1$ \\
& & $0.01 \quad 0.1$ & 1 & 10 & 100 \\
& & Favours sulphacetimide & Favours silver nitrate
\end{tabular}

Analysis 10.4. Comparison 10: Sulfacetamide versus silver nitrate, Outcome 4: Conjunctivitis of unknown aetiology

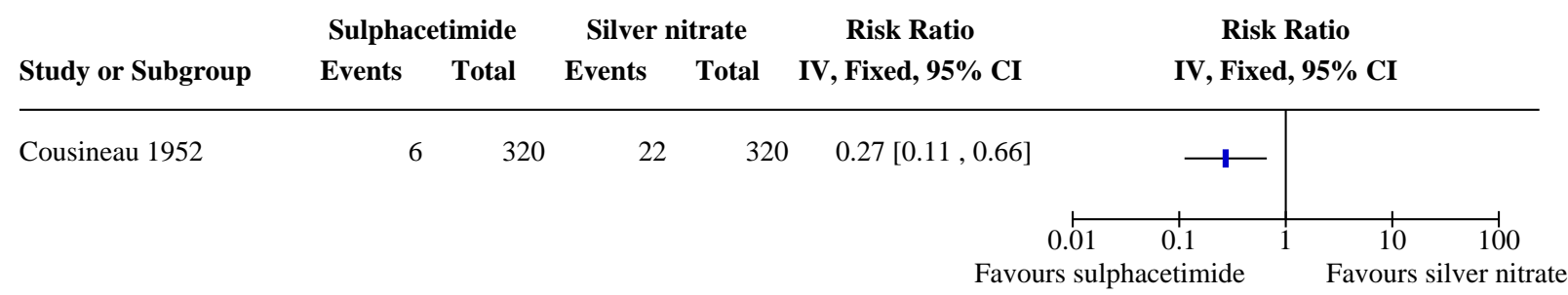


Comparison 11. Cetyl-pyridinium chloride versus silver nitrate

\begin{tabular}{lllll}
\hline Outcome or subgroup title & $\begin{array}{l}\text { No. of } \\
\text { studies }\end{array}$ & $\begin{array}{l}\text { No. of } \\
\text { partici- } \\
\text { pants }\end{array}$ & Statistical method & Effect size \\
\hline 11.1 Bacterial conjunctivitis & 2 & 599 & Risk Ratio (IV, Fixed, 95\% Cl) & $1.79[0.59,5.41]$ \\
\hline 11.2 Any conjunctivitis of any aetiology & 2 & 599 & Risk Ratio (IV, Fixed, 95\% Cl) & $1.08[0.40,2.90]$ \\
\hline 11.3 Conjunctivitis of unknown aetiology & 2 & 599 & Risk Ratio (IV, Fixed, 95\% Cl) & $0.14[0.01,2.71]$ \\
\hline
\end{tabular}

\section{Analysis 11.1. Comparison 11: Cetyl-pyridinium chloride versus silver nitrate, Outcome 1: Bacterial conjunctivitis}

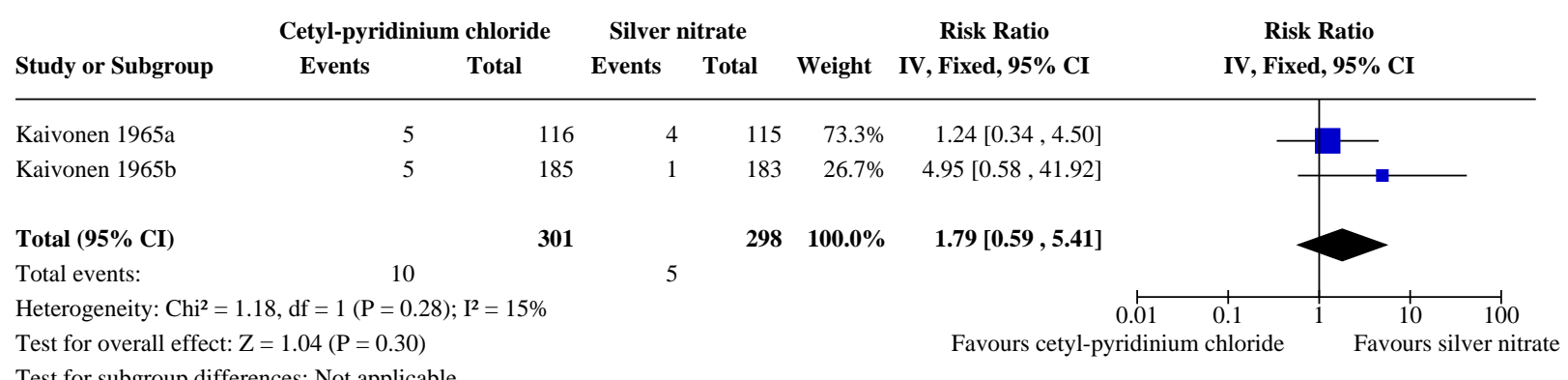

Test for subgroup differences: Not applicable

\section{Analysis 11.2. Comparison 11: Cetyl-pyridinium chloride versus} silver nitrate, Outcome 2: Any conjunctivitis of any aetiology

\begin{tabular}{|c|c|c|c|c|}
\hline & Cetyl-pyridinium chloride & Silver nitrate & Risk Ratio & Risk Ratio \\
\hline Study or Subgroup & Events & Events & Weight IV, Fixed, 95\% CI & IV, Fixed, 95\% CI \\
\hline
\end{tabular}

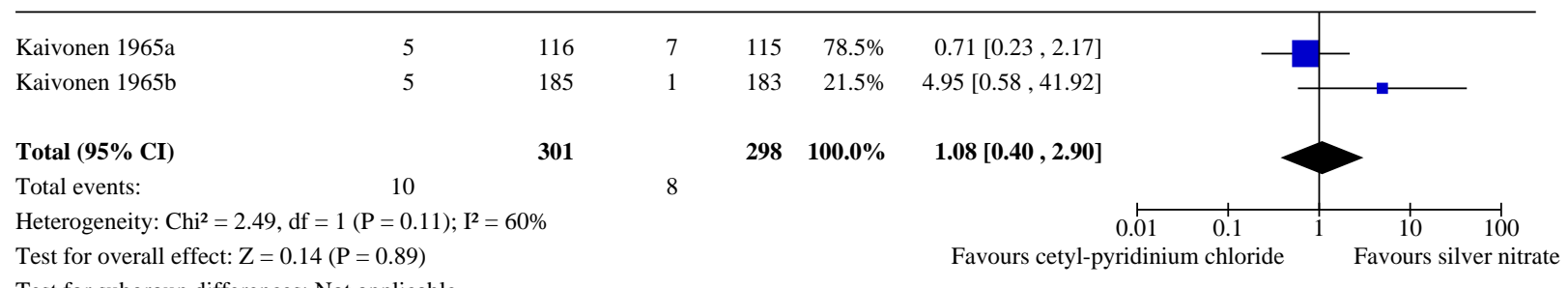


Analysis 11.3. Comparison 11: Cetyl-pyridinium chloride versus silver nitrate, Outcome 3: Conjunctivitis of unknown aetiology

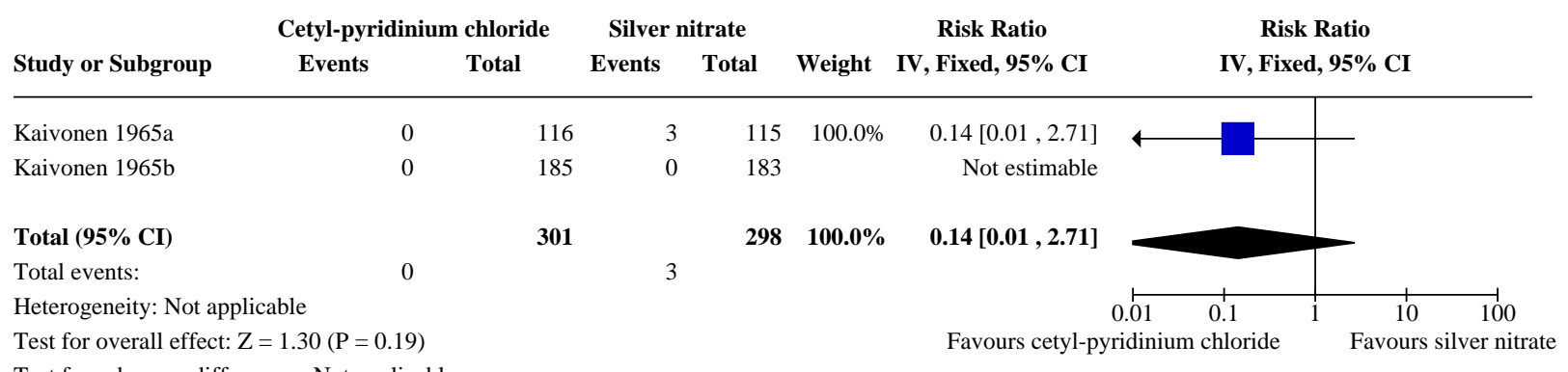

Comparison 12. Penicillin versus silver nitrate

\begin{tabular}{lllll}
\hline Outcome or subgroup title & $\begin{array}{l}\text { No. of } \\
\text { studies }\end{array}$ & $\begin{array}{l}\text { No. of } \\
\text { partici- } \\
\text { pants }\end{array}$ & Statistical method & Effect size \\
\hline 12.1 Gonococcal conjunctivitis & 1 & 2804 & Risk Ratio (M-H, Fixed, 95\% Cl) & Not estimable \\
\hline 12.2 Bacterial conjunctivitis & 1 & Risk Ratio (IV, Fixed, $95 \% \mathrm{Cl})$ & Totals not selected \\
\hline $\begin{array}{l}12.3 \text { Any conjunctivitis of any aetiology } \\
\text { 12.4 Conjunctivitis of unknown aetiolo- } \\
\text { gy }\end{array}$ & 1 & Risk Ratio (IV, Fixed, $95 \% \mathrm{Cl})$ & Totals not selected \\
\hline
\end{tabular}

Analysis 12.1. Comparison 12: Penicillin versus silver nitrate, Outcome 1: Gonococcal conjunctivitis

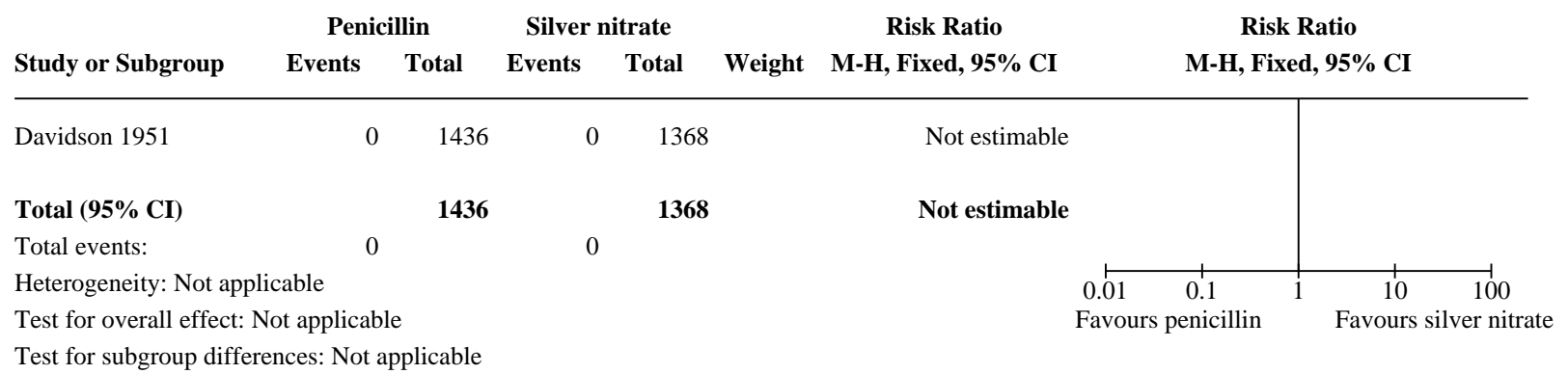

Analysis 12.2. Comparison 12: Penicillin versus silver nitrate, Outcome 2: Bacterial conjunctivitis

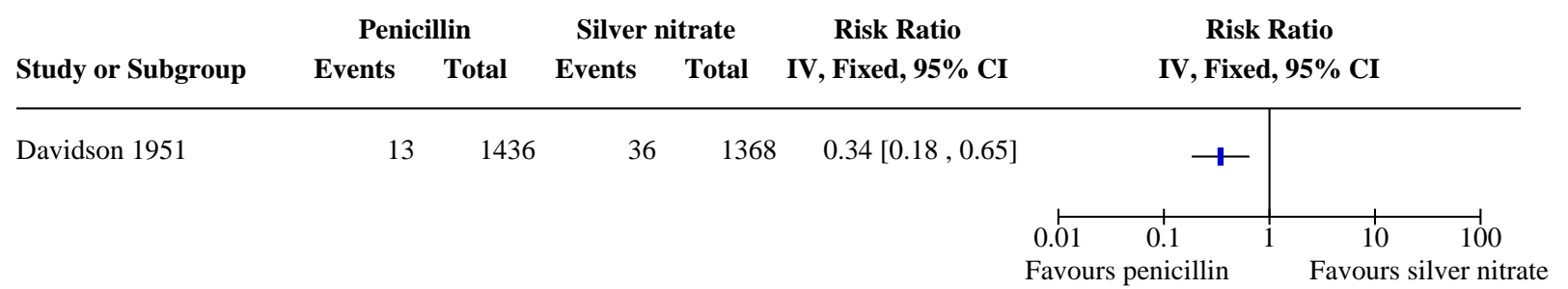


Analysis 12.3. Comparison 12: Penicillin versus silver nitrate, Outcome 3: Any conjunctivitis of any aetiology

\begin{tabular}{|c|c|c|c|c|}
\hline & Penicillin & Silver nitrate & Risk Ratio & Risk Ratio \\
\hline Study or Subgroup & Events & Events & IV, Fixed, 95\% CI & IV, Fixed, $95 \%$ CI \\
\hline
\end{tabular}

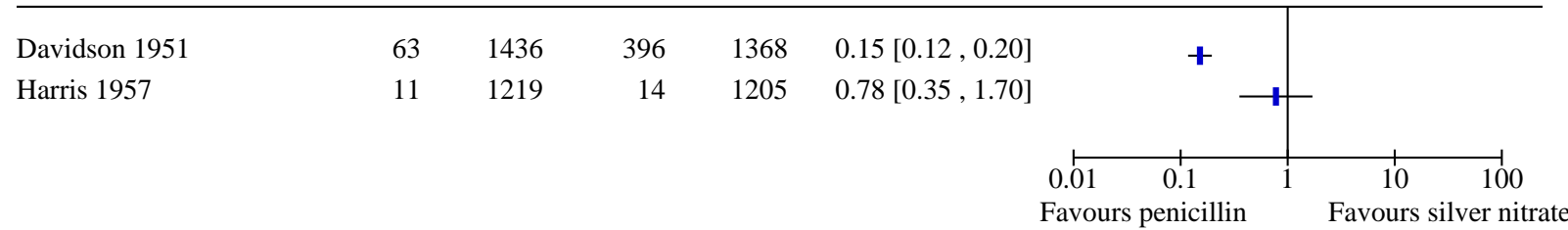

Analysis 12.4. Comparison 12: Penicillin versus silver nitrate, Outcome 4: Conjunctivitis of unknown aetiology

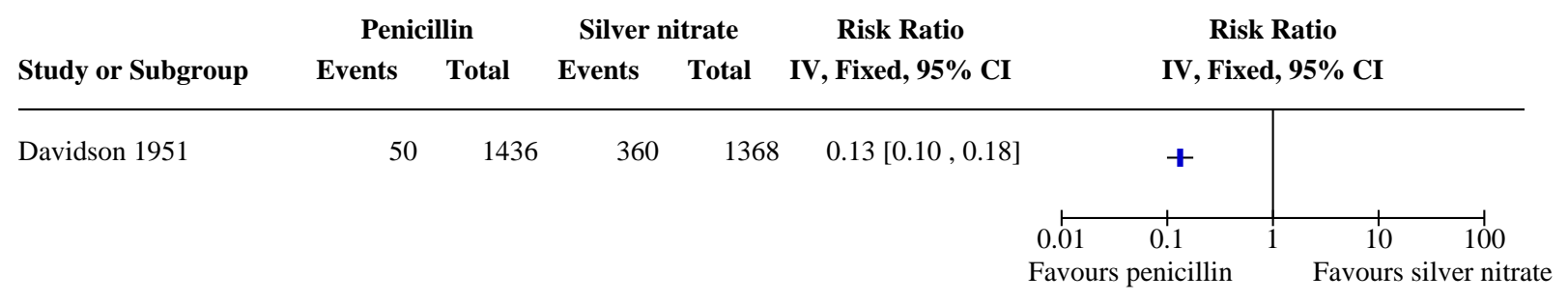

Comparison 13. Penicillin IM versus silver nitrate

\begin{tabular}{lllll}
\hline Outcome or subgroup title & $\begin{array}{l}\text { No. of } \\
\text { studies }\end{array}$ & $\begin{array}{l}\text { No. of } \\
\text { partici- } \\
\text { pants }\end{array}$ & Statistical method & Effect size \\
\hline 13.1 Gonococcal conjunctivitis & 1 & 2727 & Risk Ratio (M-H, Fixed, $95 \% \mathrm{Cl})$ & Not estimable \\
\hline 13.2 Bacterial conjunctivitis & 1 & Risk Ratio (IV, Fixed, $95 \% \mathrm{Cl})$ & Totals not selected \\
\hline 13.3 Any conjunctivitis of any aetiology & 1 & Risk Ratio (IV, Fixed, $95 \% \mathrm{Cl})$ & Totals not selected \\
\hline $\begin{array}{l}13.4 \text { Conjunctivitis of unknown aetiolo- } \\
\text { gy }\end{array}$ & 1 & Risk Ratio (IV, Fixed, $95 \% \mathrm{Cl})$ & Totals not selected \\
\hline
\end{tabular}

Analysis 13.1. Comparison 13: Penicillin IM versus silver nitrate, Outcome 1: Gonococcal conjunctivitis

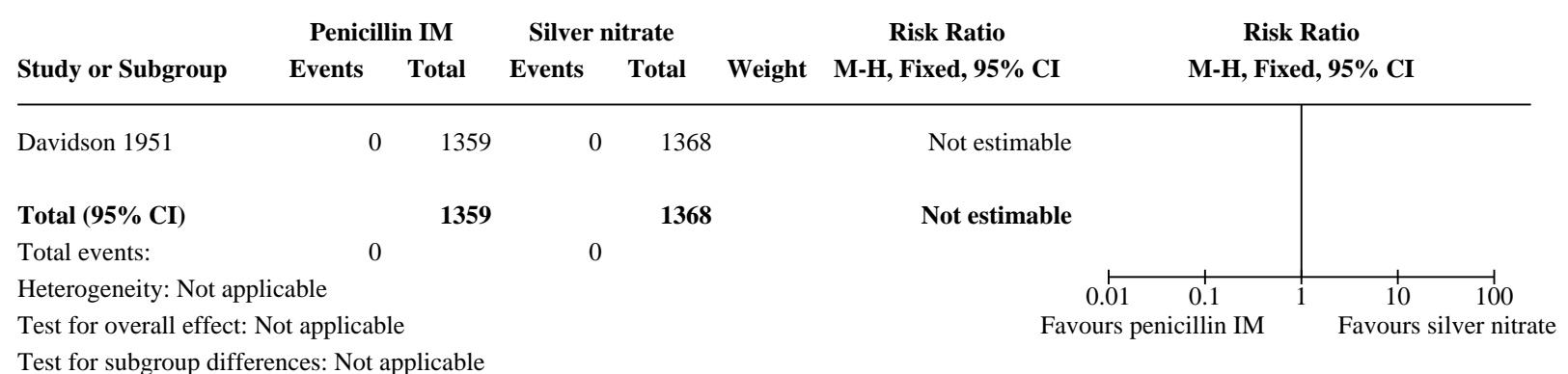


Analysis 13.2. Comparison 13: Penicillin IM versus silver nitrate, Outcome 2: Bacterial conjunctivitis

\begin{tabular}{lccccc|c} 
& \multicolumn{2}{c}{ Penicillin IM } & Silver nitrate & Risk Ratio & \multicolumn{2}{c}{ Risk Ratio } \\
Study or Subgroup & Events & Total & Events & Total & IV, Fixed, 95\% CI & \multicolumn{2}{c}{ IV, Fixed, 95\% CI } \\
\hline
\end{tabular}

\begin{tabular}{llllll}
\hline Davidson 1951 & 27 & 1359 & 36 & 1368 & $0.75[0.46,1.24$
\end{tabular}

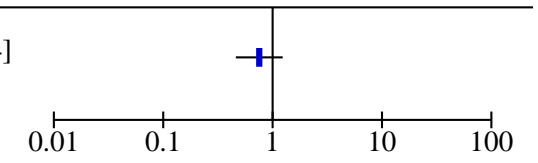

Favours penicillin IM Favours silver nitrate

Analysis 13.3. Comparison 13: Penicillin IM versus silver nitrate, Outcome 3: Any conjunctivitis of any aetiology

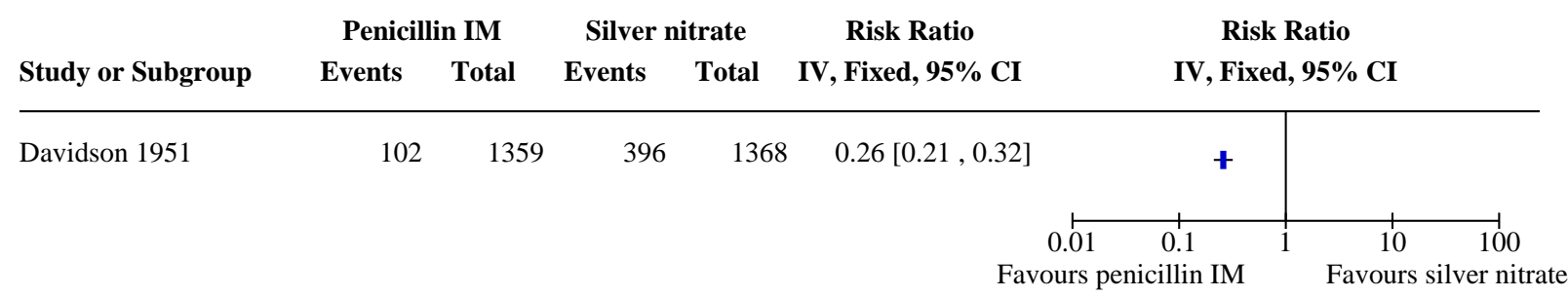

Analysis 13.4. Comparison 13: Penicillin IM versus silver nitrate, Outcome 4: Conjunctivitis of unknown aetiology

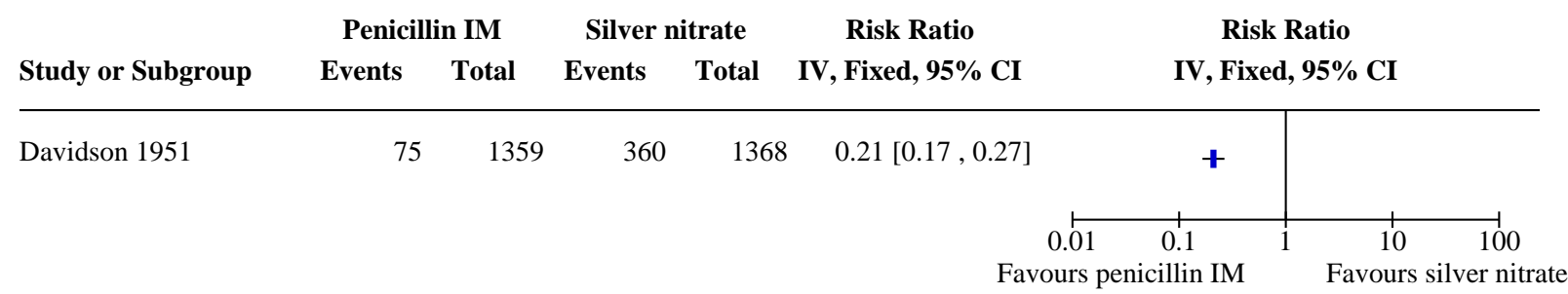

Comparison 14. Povidone-iodine versus silver nitrate

\begin{tabular}{lllll}
\hline Outcome or subgroup title & $\begin{array}{l}\text { No. of } \\
\text { studies }\end{array}$ & $\begin{array}{l}\text { No. of } \\
\text { partici- } \\
\text { pants }\end{array}$ & Statistical method & Effect size \\
\hline 14.1 Gonococcal conjunctivitis & 1 & Risk Ratio (IV, Fixed, 95\% Cl) & Totals not selected \\
\hline 14.2 Chlamydial conjunctivitis & 1 & Risk Ratio (IV, Fixed, 95\% Cl) & Totals not selected \\
\hline 14.3 Bacterial conjunctivitis & 1 & Risk Ratio (IV, Fixed, 95\% Cl) & Totals not selected \\
\hline $\begin{array}{l}14.4 \text { Any conjunctivitis of any aetiol- } \\
\text { ogy }\end{array}$ & 1 & Risk Ratio (IV, Fixed, 95\% Cl) & Totals not selected \\
\hline $\begin{array}{l}14.5 \text { Conjunctivitis of unknown aeti- } \\
\text { ology }\end{array}$ & 1 & Risk Ratio (IV, Fixed, 95\% Cl) & Totals not selected \\
\hline
\end{tabular}


Analysis 14.1. Comparison 14: Povidone-iodine versus silver nitrate, Outcome 1: Gonococcal conjunctivitis

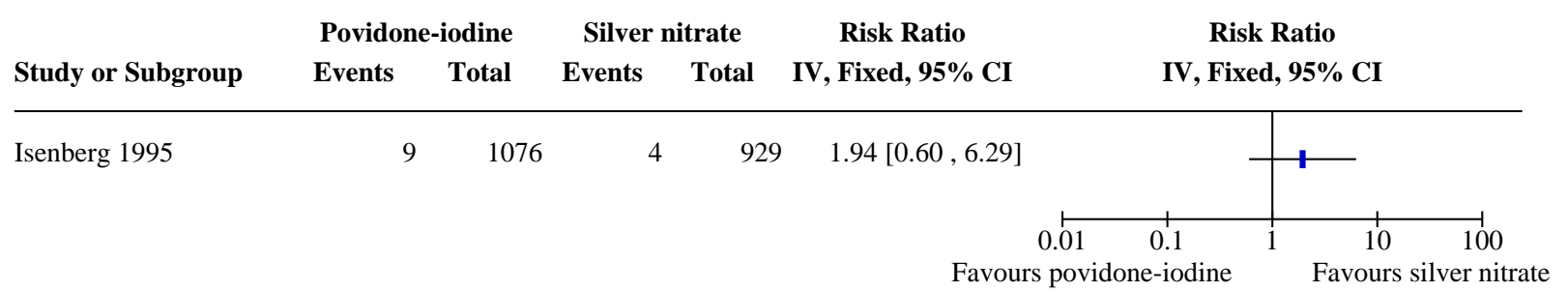

Analysis 14.2. Comparison 14: Povidone-iodine versus silver nitrate, Outcome 2: Chlamydial conjunctivitis

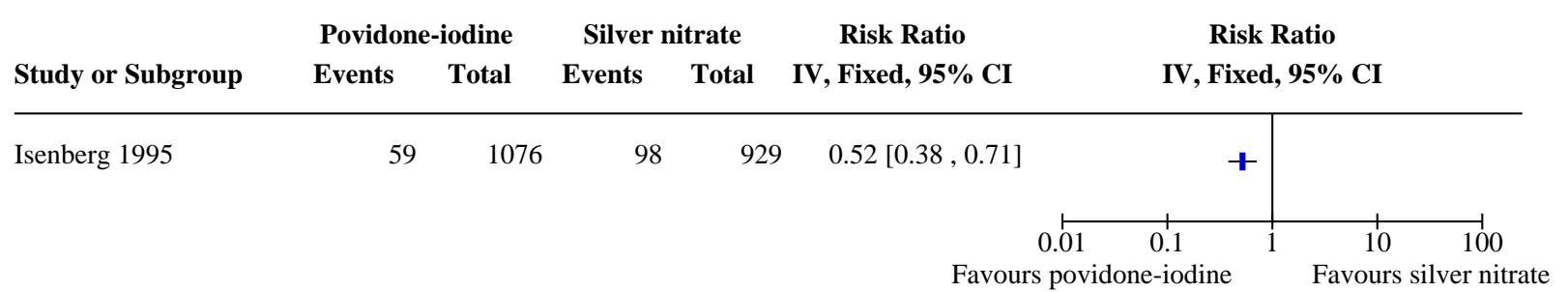

Analysis 14.3. Comparison 14: Povidone-iodine versus silver nitrate, Outcome 3: Bacterial conjunctivitis

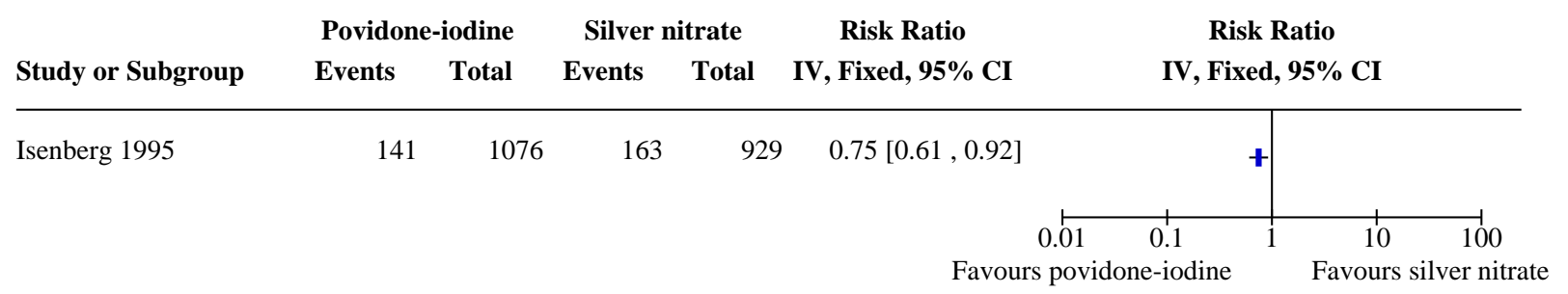

Analysis 14.4. Comparison 14: Povidone-iodine versus silver nitrate, Outcome 4: Any conjunctivitis of any aetiology

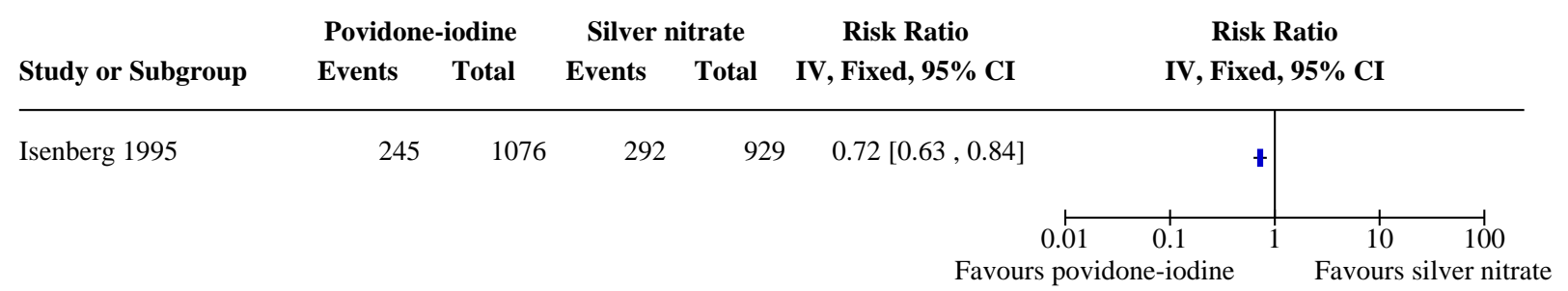


Analysis 14.5. Comparison 14: Povidone-iodine versus silver nitrate, Outcome 5: Conjunctivitis of unknown aetiology

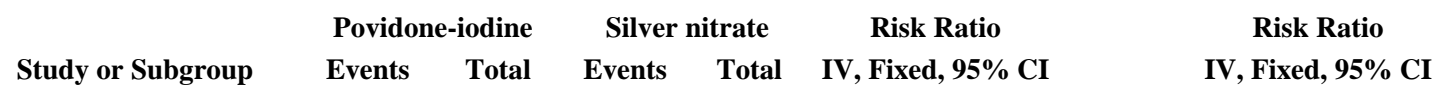

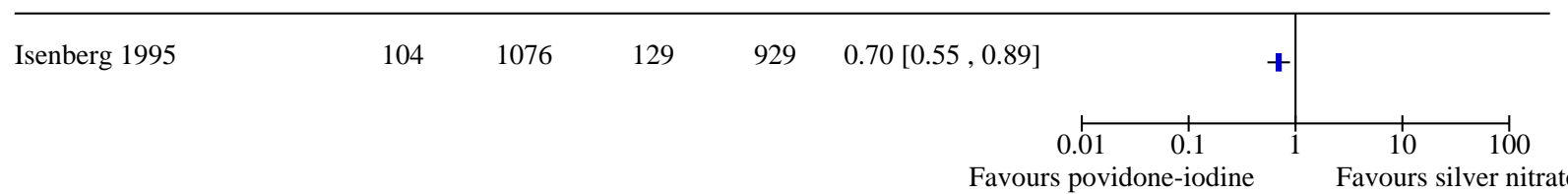

Comparison 15. Tetracycline versus erythromycin

\begin{tabular}{lllll}
\hline Outcome or subgroup title & $\begin{array}{l}\text { No. of } \\
\text { studies }\end{array}$ & $\begin{array}{l}\text { No. of } \\
\text { partici- } \\
\text { pants }\end{array}$ & Statistical method & Effect size \\
\hline 15.1 Chlamydial conjunctivitis & 2 & 10946 & Risk Ratio (IV, Fixed, 95\% Cl) & $0.72[0.42,1.25]$ \\
\hline 15.2 Any conjunctivitis of any aetiology & 2 & Risk Ratio (IV, Fixed, 95\% Cl) & Totals not selected \\
\hline
\end{tabular}

Analysis 15.1. Comparison 15: Tetracycline versus erythromycin, Outcome 1: Chlamydial conjunctivitis

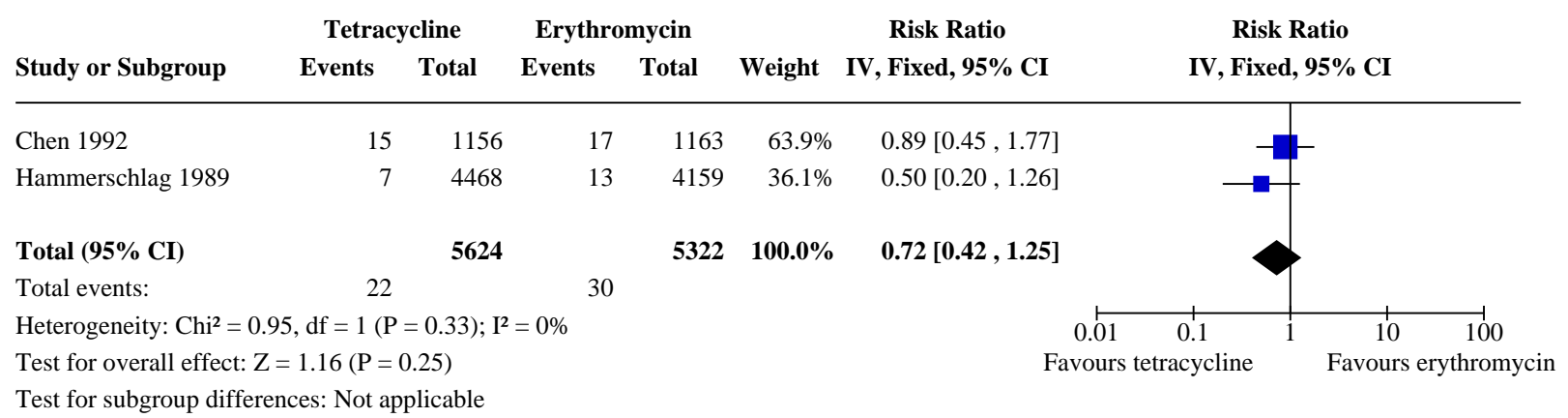

Analysis 15.2. Comparison 15: Tetracycline versus erythromycin, Outcome 2: Any conjunctivitis of any aetiology

\begin{tabular}{|c|c|c|c|c|c|}
\hline & Tetr: & cline & Erythromycin & Risk Ratio & Risk Ratio \\
\hline Study or Subgroup & Events & Total & Events & IV, Fixed, $95 \%$ CI & IV, Fixed, $95 \%$ CI \\
\hline
\end{tabular}

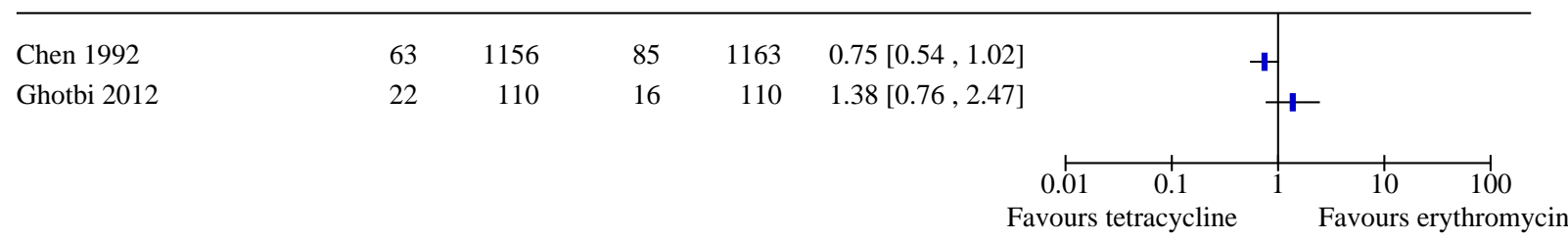


Comparison 16. Colostrum versus erythromycin

\begin{tabular}{lllll}
\hline Outcome or subgroup title & $\begin{array}{l}\text { No. of } \\
\text { studies }\end{array}$ & $\begin{array}{l}\text { No. of par- } \\
\text { ticipants }\end{array}$ & Statistical method & Effect size \\
\hline 16.1 Any conjunctivitis of any aetiology & 1 & Risk Ratio (IV, Fixed, 95\% CI) & Totals not selected \\
\hline
\end{tabular}

Analysis 16.1. Comparison 16: Colostrum versus erythromycin, Outcome 1: Any conjunctivitis of any aetiology

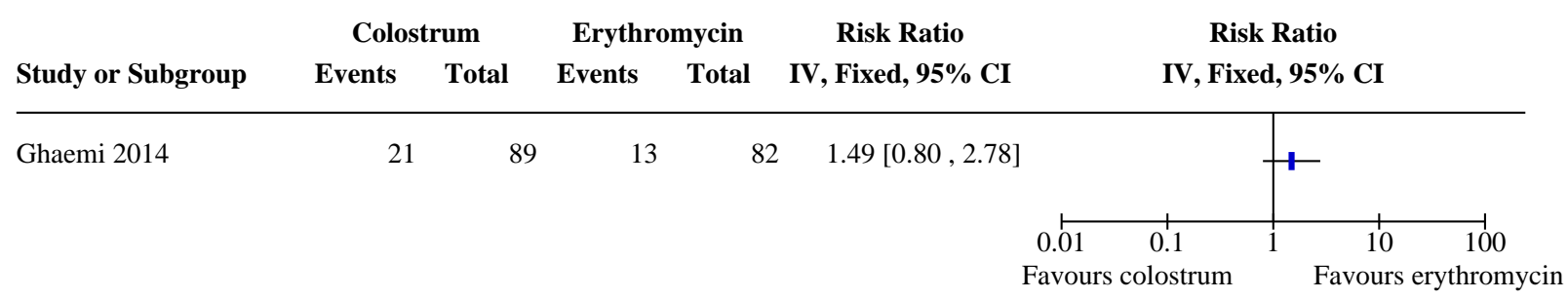

Comparison 17. Povidone-iodine versus erythromycin

\begin{tabular}{lllll}
\hline Outcome or subgroup title & $\begin{array}{l}\text { No. of } \\
\text { studies }\end{array}$ & $\begin{array}{l}\text { No. of } \\
\text { partici- } \\
\text { pants }\end{array}$ & Statistical method & Effect size \\
\hline 17.1 Chlamydial conjunctivitis & 2 & 2408 & Risk Ratio (IV, Fixed, 95\% Cl) & 0.74 [0.54, 1.02] \\
\hline 17.2 Bacterial conjunctivitis & 2 & 2408 & Risk Ratio (IV, Fixed, 95\% Cl) & $0.87[0.71,1.07]$ \\
\hline 17.3 Any conjunctivitis of any aetiology & 2 & 2408 & Risk Ratio (IV, Fixed, 95\% Cl) & $0.78[0.68,0.90]$ \\
\hline $\begin{array}{l}17.4 \text { Conjunctivitis of unknown aetiolo- } \\
\text { gy }\end{array}$ & 2 & 2408 & Risk Ratio (IV, Fixed, 95\% Cl) & 0.74 [0.58, 0.93] \\
\hline
\end{tabular}

Analysis 17.1. Comparison 17: Povidone-iodine versus erythromycin, Outcome 1: Chlamydial conjunctivitis

\begin{tabular}{lccccccc} 
& \multicolumn{2}{c}{ Povidone-iodine } & \multicolumn{2}{c}{ Erythromycin } & & Risk Ratio & Risk Ratio \\
Study or Subgroup & Events & Total & Events & Total & Weight & IV, Fixed, 95\% CI & IV, Fixed, 95\% CI
\end{tabular}

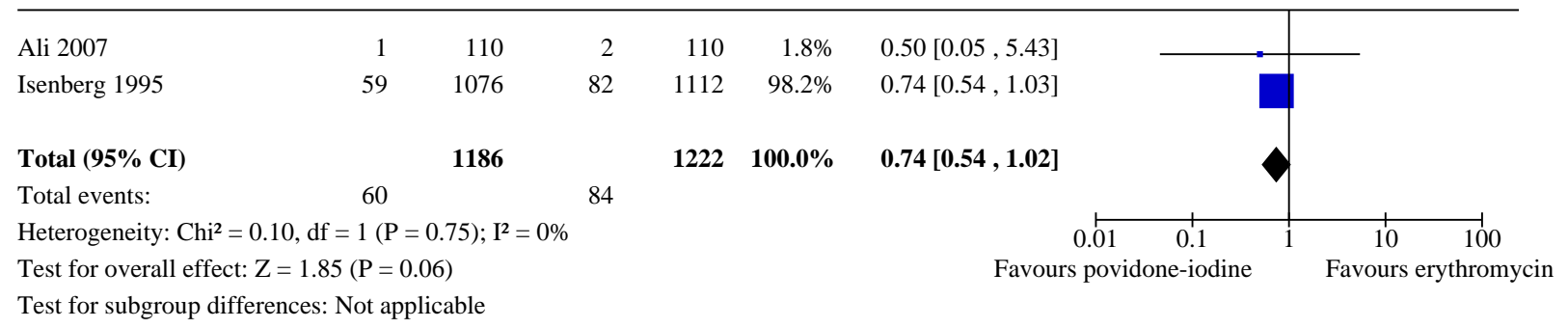


Analysis 17.2. Comparison 17: Povidone-iodine versus erythromycin, Outcome 2: Bacterial conjunctivitis

\begin{tabular}{|c|c|c|c|c|c|}
\hline & Povid & iodine & Erythromycin & Risk Ratio & Risk Ratio \\
\hline Study or Subgroup & Events & Total & Events & Weight IV, Fixed, 95\% CI & IV, Fixed, 95\% CI \\
\hline
\end{tabular}

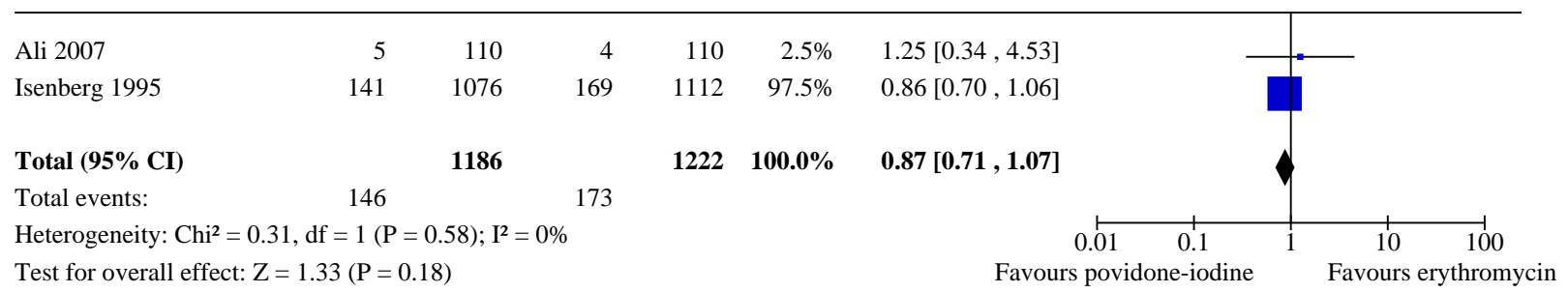

Test for subgroup differences: Not applicable

Analysis 17.3. Comparison 17: Povidone-iodine versus erythromycin, Outcome 3: Any conjunctivitis of any aetiology

\begin{tabular}{lccccccc} 
& \multicolumn{2}{c}{ Povidone-iodine } & \multicolumn{2}{c}{ Erythromycin } & & Risk Ratio & Risk Ratio \\
Study or Subgroup & Events & Total & Events & Total & Weight & IV, Fixed, 95\% CI & IV, Fixed, 95\% CI
\end{tabular}

\begin{tabular}{lrrrrrr}
\hline Ali 2007 & 9 & 110 & 19 & 110 & $3.6 \%$ & $0.47[0.22,1.00]$ \\
Isenberg 1995 & 245 & 1076 & 317 & 1112 & $96.4 \%$ & $0.80[0.69,0.92]$ \\
& & & & & & \\
Total (95\% CI) & & $\mathbf{1 1 8 6}$ & & $\mathbf{1 2 2 2}$ & $\mathbf{1 0 0 . 0 \%}$ & $\mathbf{0 . 7 8}[\mathbf{0 . 6 8 , \mathbf { 0 . 9 0 } ]}$ \\
Total events: & 254 & & 336 & & &
\end{tabular}

Heterogeneity: $\mathrm{Chi}^{2}=1.81, \mathrm{df}=1(\mathrm{P}=0.18) ; \mathrm{I}^{2}=45 \%$

Test for overall effect: $\mathrm{Z}=3.37(\mathrm{P}=0.0007)$

Test for subgroup differences: Not applicable

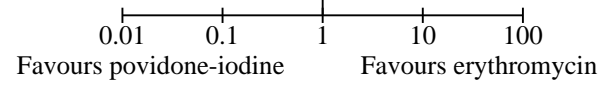

Analysis 17.4. Comparison 17: Povidone-iodine versus erythromycin, Outcome 4: Conjunctivitis of unknown aetiology

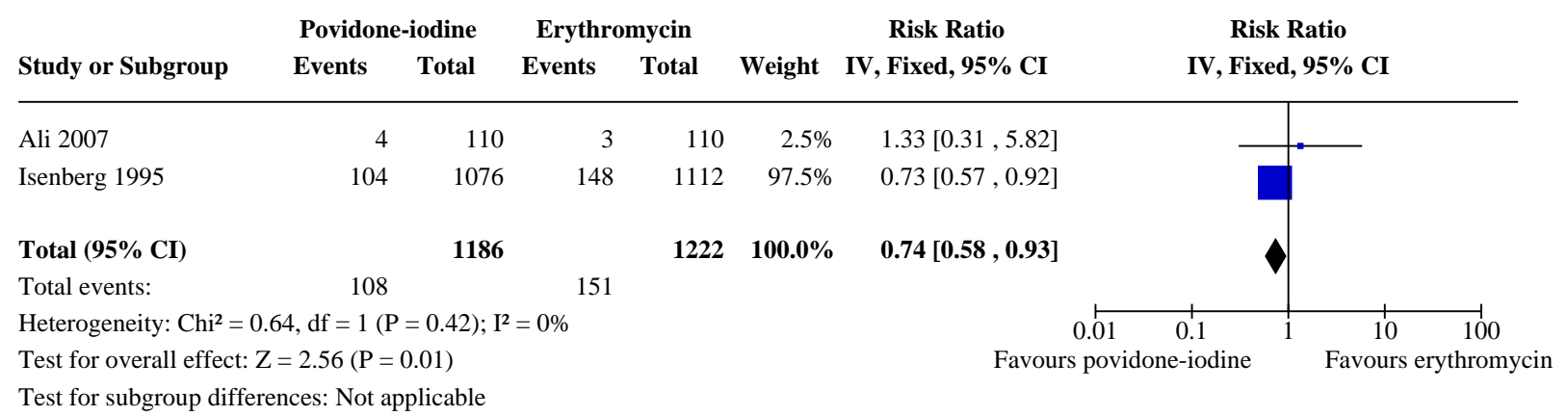

\section{Comparison 18. Pencillin IM versus tetracycline}

\begin{tabular}{lllll}
\hline Outcome or subgroup title & $\begin{array}{l}\text { No. of } \\
\text { studies }\end{array}$ & $\begin{array}{l}\text { No. of } \\
\text { partici- } \\
\text { pants }\end{array}$ & Statistical method & Effect size \\
\hline 18.1 Gonococcal conjunctivitis & 1 & 32058 & Risk Ratio (IV, Fixed, 95\% Cl) & Not estimable \\
\hline 18.2 Chlamydial conjunctivitis & 1 & Risk Ratio (IV, Fixed, $95 \% \mathrm{Cl})$ & Totals not selected \\
\hline
\end{tabular}


Analysis 18.1. Comparison 18: Pencillin IM versus tetracycline, Outcome 1: Gonococcal conjunctivitis

\begin{tabular}{|c|c|c|c|c|c|c|c|}
\hline \multirow[b]{2}{*}{ Study or Subgroup } & \multicolumn{2}{|c|}{ Penicillin IM } & \multicolumn{2}{|c|}{ Tetracycline } & \multicolumn{2}{|r|}{ Risk Ratio } & Risk Ratio \\
\hline & Events & Total & Events & Total & Weight & IV, Fixed, $95 \%$ CI & IV, Fixed, 95\% CI \\
\hline
\end{tabular}

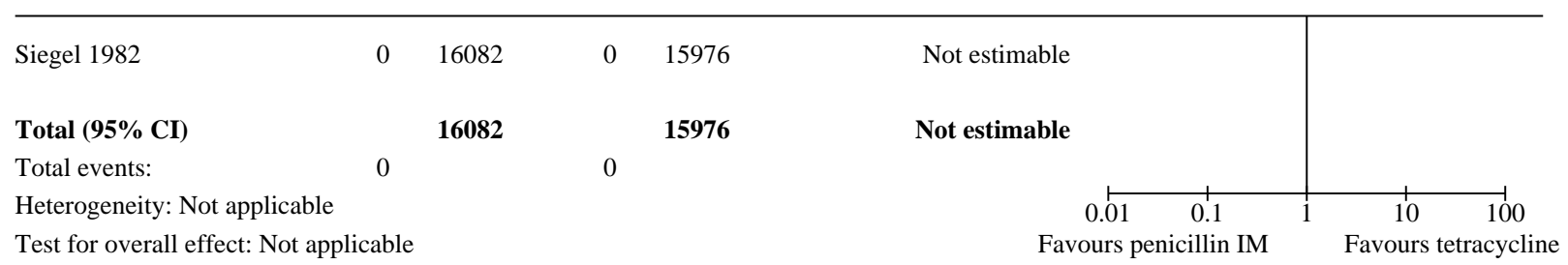

Test for subgroup differences: Not applicable

\section{Analysis 18.2. Comparison 18: Pencillin IM versus tetracycline, Outcome 2: Chlamydial conjunctivitis}

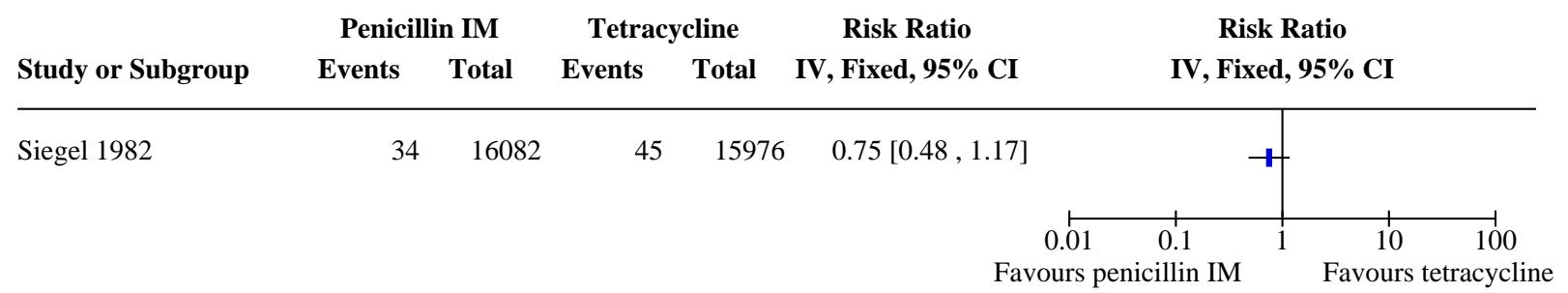

Comparison 19. Povidone-iodine versus tetracycline

\begin{tabular}{lllll}
\hline Outcome or subgroup title & $\begin{array}{l}\text { No. of } \\
\text { studies }\end{array}$ & $\begin{array}{l}\text { No. of } \\
\text { partici- } \\
\text { pants }\end{array}$ & Statistical method & Effect size \\
\hline 19.1 Gonococcal conjunctivitis & 1 & 410 & Risk Ratio (IV, Fixed, 95\% Cl) & Not estimable \\
\hline 19.2 Chlamydial conjunctivitis & 1 & 410 & Risk Ratio (IV, Fixed, 95\% Cl) & Not estimable \\
\hline 19.3 Bacterial conjunctivitis & 1 & Risk Ratio (IV, Fixed, $95 \% \mathrm{Cl})$ & Totals not selected \\
\hline $\begin{array}{l}19.4 \text { Any conjunctivitis of any aetiolo- } \\
\text { gy }\end{array}$ & 1 & Risk Ratio (IV, Fixed, $95 \% \mathrm{Cl})$ & Totals not selected \\
\hline $\begin{array}{l}19.5 \text { Conjunctivitis of unknown aeti- } \\
\text { ology }\end{array}$ & 1 & Risk Ratio (IV, Fixed, $95 \% \mathrm{Cl})$ & Totals not selected \\
\hline
\end{tabular}


Analysis 19.1. Comparison 19: Povidone-iodine versus tetracycline, Outcome 1: Gonococcal conjunctivitis

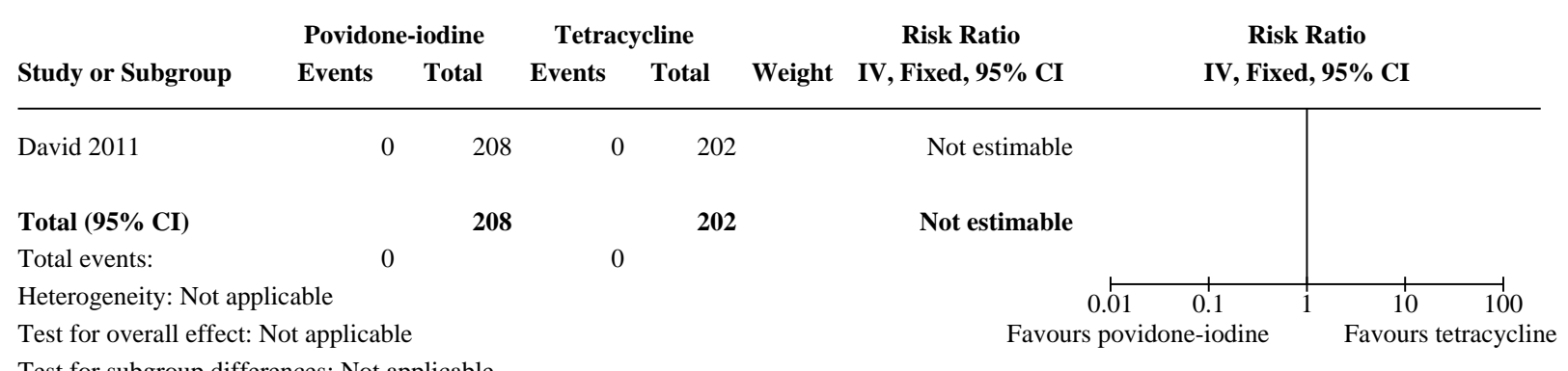

\section{Analysis 19.2. Comparison 19: Povidone-iodine versus tetracycline, Outcome 2: Chlamydial conjunctivitis}

\begin{tabular}{|c|c|c|c|c|c|}
\hline & & ne & \multicolumn{2}{|c|}{ Tetracycline } & \multirow{2}{*}{$\begin{array}{c}\text { Risk Ratio } \\
\text { IV, Fixed, 95\% C }\end{array}$} \\
\hline & Events & Total & Events & Tota & \\
\hline
\end{tabular}

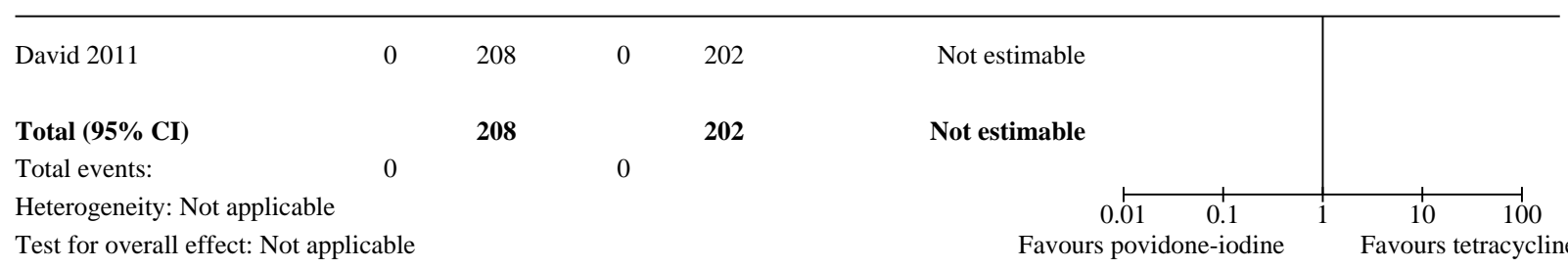

Test for subgroup differences: Not applicable

Analysis 19.3. Comparison 19: Povidone-iodine versus tetracycline, Outcome 3: Bacterial conjunctivitis

\begin{tabular}{|c|c|c|c|c|c|c|}
\hline \multirow[b]{2}{*}{ Subgroup } & \multicolumn{2}{|c|}{ Povidone-iodine } & \multicolumn{2}{|c|}{ Tetracycline } & Risk Ratio & \multirow{2}{*}{$\begin{array}{c}\text { Risk Ratio } \\
\text { IV, Fixed, 95\% CI }\end{array}$} \\
\hline & Events & Total & Events & Total & IV, Fixed, 95\% CI & \\
\hline
\end{tabular}

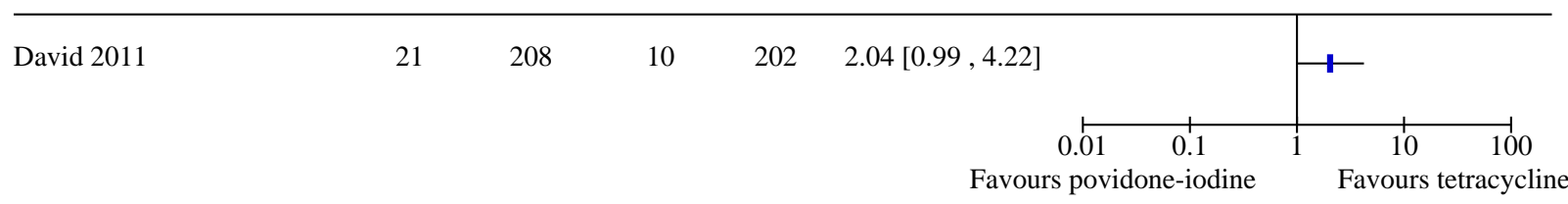

Analysis 19.4. Comparison 19: Povidone-iodine versus tetracycline, Outcome 4: Any conjunctivitis of any aetiology

\begin{tabular}{|c|c|c|c|c|c|c|}
\hline & Povido & iodine & Tetr: & cline & Risk Ratio & Risk Ratio \\
\hline Study or Subgroup & Events & Total & Events & Total & IV, Fixed, 95\% CI & IV, Fixed, 95\% CI \\
\hline
\end{tabular}

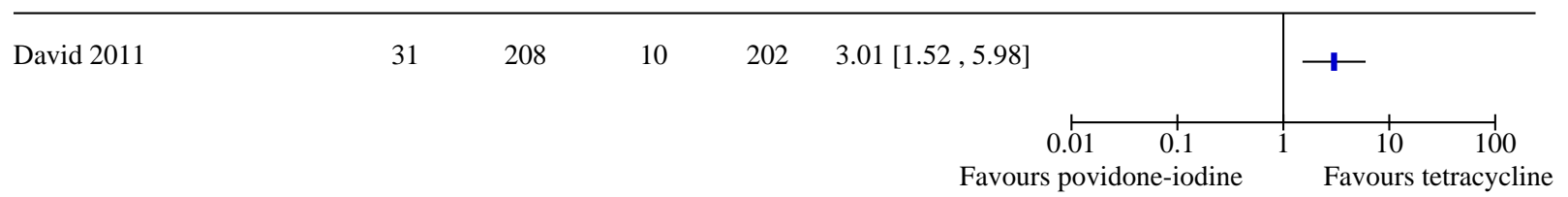


Analysis 19.5. Comparison 19: Povidone-iodine versus tetracycline, Outcome 5: Conjunctivitis of unknown aetiology

\begin{tabular}{|c|c|c|c|c|c|c|}
\hline & Povido & iodine & Tetr: & cline & Risk Ratio & Risk Ratio \\
\hline Study or Subgroup & Events & Total & Events & Total & IV, Fixed, 95\% CI & IV, Fixed, $95 \%$ CI \\
\hline
\end{tabular}

\begin{tabular}{llllll}
\hline David 2011 & 10 & 208 & 0 & 202 & $20.40[1.20,345.80$
\end{tabular}

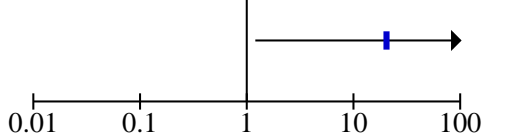

Favours povidone-iodine Favours tetracycline

Comparison 20. Povidone-iodine versus chloramphenicol

\begin{tabular}{lllll}
\hline Outcome or subgroup title & $\begin{array}{l}\text { No. of } \\
\text { studies }\end{array}$ & $\begin{array}{l}\text { No. of } \\
\text { partici- } \\
\text { pants }\end{array}$ & Statistical method & Effect size \\
\hline 20.1 Gonococcal conjunctivitis & 1 & 2004 & Risk Ratio (IV, Fixed, 95\% Cl) & Not estimable \\
\hline 20.2 Chlamydial conjunctivitis & 1 & Risk Ratio (IV, Fixed, 95\% Cl) & Totals not selected \\
\hline
\end{tabular}

Analysis 20.1. Comparison 20: Povidone-iodine versus chloramphenicol, Outcome 1: Gonococcal conjunctivitis

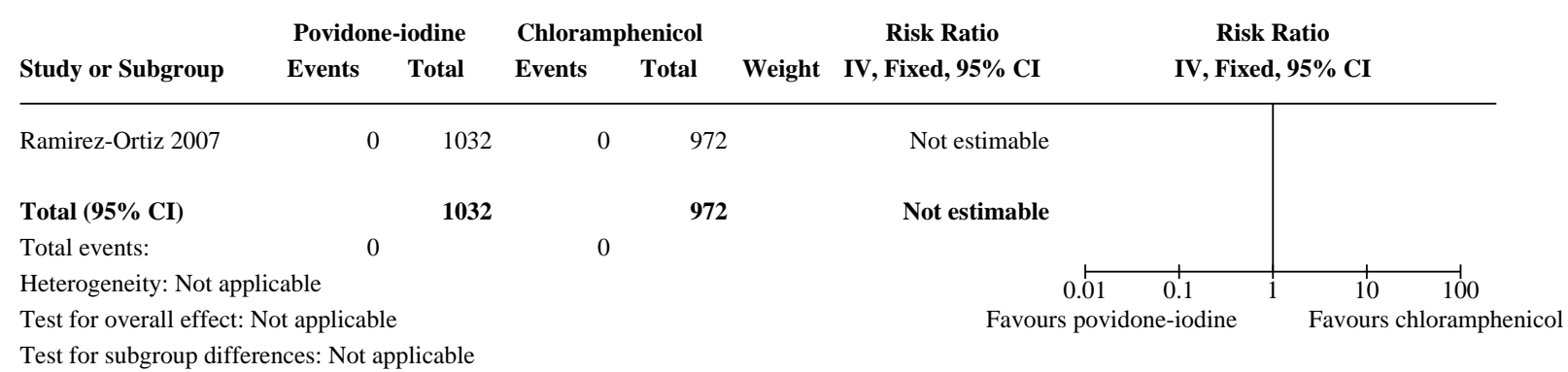

\section{Analysis 20.2. Comparison 20: Povidone-iodine versus chloramphenicol, Outcome 2: Chlamydial conjunctivitis}

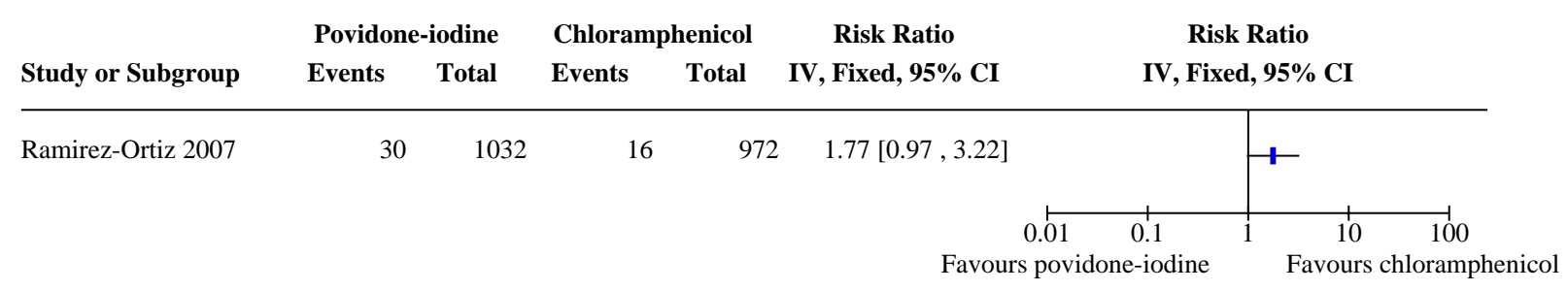

Comparison 21. Povidone-iodine versus carbethopendecinium bromide

\begin{tabular}{lllll}
\hline Outcome or subgroup title & $\begin{array}{l}\text { No. of } \\
\text { studies }\end{array}$ & $\begin{array}{l}\text { No. of par- } \\
\text { ticipants }\end{array}$ & Statistical method & Effect size \\
\hline 21.1 Any conjunctivitis of any aetiology & 1 & Risk Ratio (IV, Fixed, 95\% CI) & Totals not selected \\
\hline
\end{tabular}




\section{Analysis 21.1. Comparison 21: Povidone-iodine versus carbethopendecinium bromide, Outcome 1: Any conjunctivitis of any aetiology}
Povidone-iodine
C-Bromide
Risk Ratio
Risk Ratio
Events Total
Events Total
IV, Fixed, 95\% CI
IV, Fixed, 95\% CI

Study or Subgroup

Zbojan 2004

$4 \quad 50$

9

50

$0.44[0.15,1.35]$

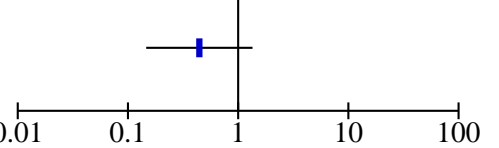

Favours povidone-iodine

Favours C-Bromide

Comparison 22. Povidone-iodine twice versus povidone-iodine once

\begin{tabular}{lllll}
\hline Outcome or subgroup title & $\begin{array}{l}\text { No. of } \\
\text { studies }\end{array}$ & $\begin{array}{l}\text { No. of } \\
\text { partici- } \\
\text { pants }\end{array}$ & Statistical method & Effect size \\
\hline 22.1 Gonococcal conjunctivitis & 1 & 719 & Risk Ratio (IV, Fixed, 95\% Cl) & Not estimable \\
\hline 22.2 Chlamydial conjunctivitis & 1 & Risk Ratio (IV, Fixed, 95\% Cl) & Totals not selected \\
\hline 22.3 Bacterial conjunctivitis & 1 & Risk Ratio (IV, Fixed, 95\% Cl) & Totals not selected \\
\hline $\begin{array}{l}22.4 \text { Any conjunctivitis of any aetiolo- } \\
\text { gy }\end{array}$ & 1 & Risk Ratio (IV, Fixed, 95\% Cl) & Totals not selected \\
\hline $\begin{array}{l}22.5 \text { Conjunctivitis of unknown aeti- } \\
\text { ology }\end{array}$ & 1 & Risk Ratio (IV, Fixed, 95\% CI) & Totals not selected
\end{tabular}

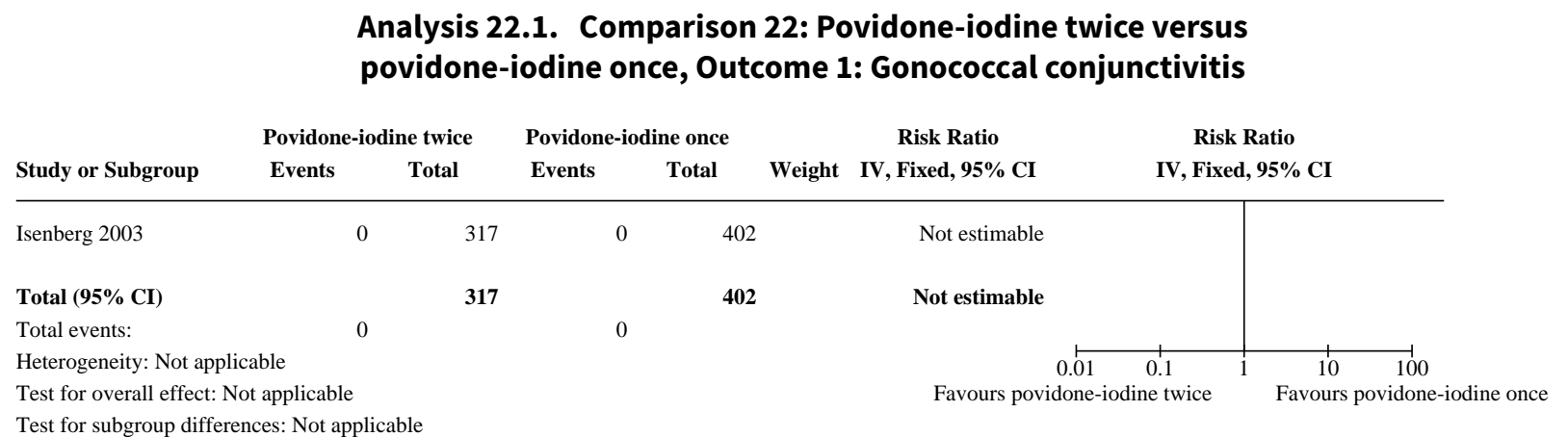


Analysis 22.2. Comparison 22: Povidone-iodine twice versus povidone-iodine once, Outcome 2: Chlamydial conjunctivitis

\begin{tabular}{|c|c|c|c|c|c|c|}
\hline \multirow[b]{2}{*}{ Study or Subgroup } & \multicolumn{2}{|c|}{ Povidone-iodine twice } & \multicolumn{2}{|c|}{ Povidone-iodine once } & Risk Ratio & \multirow{2}{*}{$\begin{array}{c}\text { Risk Ratio } \\
\text { IV, Fixed, 95\% CI }\end{array}$} \\
\hline & Events & Total & Events & Total & IV, Fixed, 95\% CI & \\
\hline
\end{tabular}

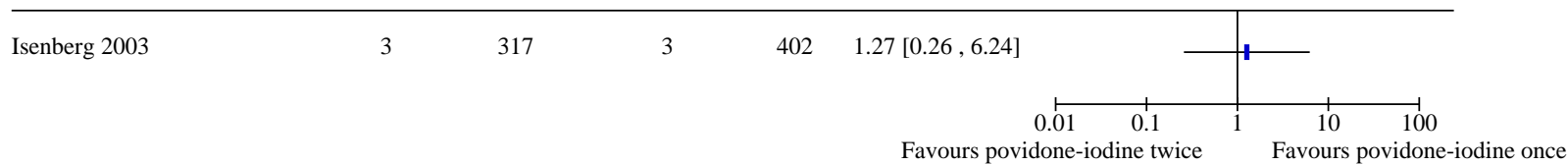

Analysis 22.3. Comparison 22: Povidone-iodine twice versus povidone-iodine once, Outcome 3: Bacterial conjunctivitis

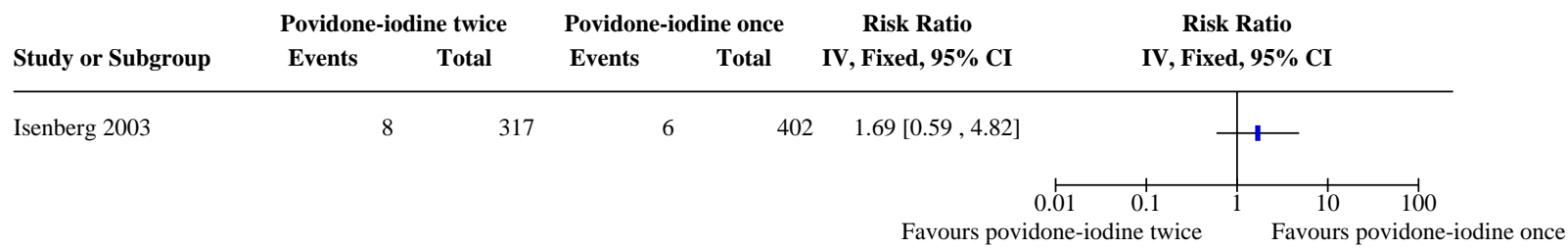

Analysis 22.4. Comparison 22: Povidone-iodine twice versus povidone-iodine once, Outcome 4: Any conjunctivitis of any aetiology

\begin{tabular}{ccccccc} 
& \multicolumn{2}{c}{ Povidone-iodine twice } & \multicolumn{2}{c}{ Povidone-iodine once } & Risk Ratio & Risk Ratio \\
Study or Subgroup & Events & Total & Events & Total & IV, Fixed, 95\% CI & IV, Fixed, 95\% CI
\end{tabular}

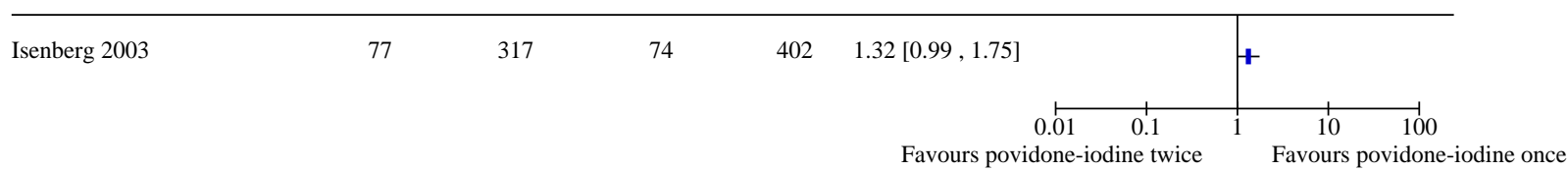

Analysis 22.5. Comparison 22: Povidone-iodine twice versus povidoneiodine once, Outcome 5: Conjunctivitis of unknown aetiology

\begin{tabular}{llccccc} 
& \multicolumn{2}{c}{ Povidone-iodine twice } & \multicolumn{2}{c}{ Povidone-iodine once } & Risk Ratio & Risk Ratio \\
Study or Subgroup & Events & Total & Events & Total & IV, Fixed, 95\% CI & IV, Fixed, 95\% CI \\
\hline
\end{tabular}

$\begin{array}{llllll}\text { Isenberg } 2003 & 69 & 317 & 68 & 402 & 1.29[0.95,1.74]\end{array}$

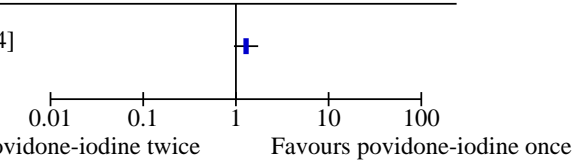

Comparison 23. Penicillin IM versus topical penicillin

\begin{tabular}{lllll}
\hline Outcome or subgroup title & $\begin{array}{l}\text { No. of } \\
\text { studies }\end{array}$ & $\begin{array}{l}\text { No. of } \\
\text { partici- } \\
\text { pants }\end{array}$ & Statistical method & Effect size \\
\hline 23.1 Gonococcal conjunctivitis & 1 & 2795 & Risk Ratio (M-H, Fixed, 95\% Cl) & Not estimable \\
\hline 23.2 Bacterial conjunctivitis & 1 & & Risk Ratio (IV, Fixed, 95\% Cl) & Subtotals only \\
\hline
\end{tabular}




\begin{tabular}{lllll}
\hline Outcome or subgroup title & $\begin{array}{l}\text { No. of } \\
\text { studies }\end{array}$ & $\begin{array}{l}\text { No. of } \\
\text { partici- } \\
\text { pants }\end{array}$ & Statistical method & Effect size \\
\hline 23.3 Any conjunctivitis of any aetiology & 1 & & Risk Ratio (IV, Fixed, 95\% Cl) & Subtotals only \\
\hline 23.4 Conjunctivitis of unknown aetiology & 1 & Risk Ratio (IV, Fixed, $95 \% \mathrm{Cl})$ & Subtotals only \\
\hline
\end{tabular}

Analysis 23.1. Comparison 23: Penicillin IM versus topical penicillin, Outcome 1: Gonococcal conjunctivitis

\begin{tabular}{|c|c|c|c|c|c|c|c|}
\hline & Penici & n IM & Peni & & & Risk Ratio & Risk Ratio \\
\hline Study or Subgroup & Events & Total & Events & Total & Weight & M-H, Fixed, 95\% CI & M-H, Fixed, 95\% CI \\
\hline
\end{tabular}

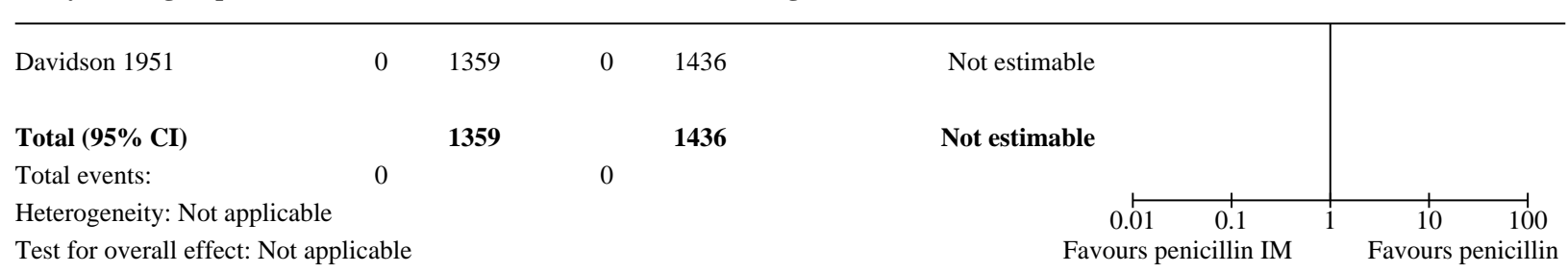

Test for subgroup differences: Not applicable

\section{Analysis 23.2. Comparison 23: Penicillin IM versus topical penicillin, Outcome 2: Bacterial conjunctivitis}

\begin{tabular}{|c|c|c|c|c|}
\hline & Penicillin IM & Penicillin & Risk Ratio & Risk Ratio \\
\hline Study or Subgroup & Events & Events & IV, Fixed, 95\% CI & IV, Fixed, 95\% CI \\
\hline
\end{tabular}

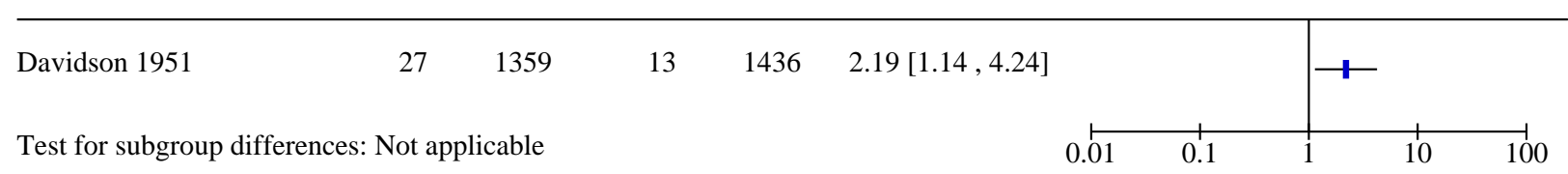

Analysis 23.3. Comparison 23: Penicillin IM versus topical penicillin, Outcome 3: Any conjunctivitis of any aetiology

\begin{tabular}{|c|c|c|c|c|}
\hline & Penicillin IM & Penicillin & Risk Ratio & Risk Ratio \\
\hline Study or Subgroup & Events & Events & IV, Fixed, 95\% CI & IV, Fixed, 95\% CI \\
\hline
\end{tabular}

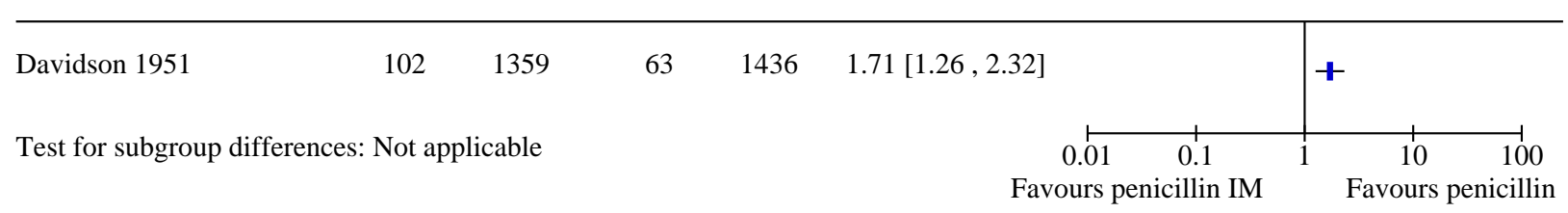




\section{Analysis 23.4. Comparison 23: Penicillin IM versus topical} penicillin, Outcome 4: Conjunctivitis of unknown aetiology

\begin{tabular}{|c|c|c|c|c|c|c|}
\hline & Penic & n IM & Per & llin & Risk Ratio & Risk Ratio \\
\hline Study or Subgroup & Events & Total & Events & Total & IV, Fixed, 95\% CI & IV, Fixed, $95 \%$ CI \\
\hline
\end{tabular}

\begin{tabular}{llllll}
\hline Davidson 1951 & 75 & 1359 & 50 & 1436 & $1.58[1.12,2.25]$
\end{tabular}

Test for subgroup differences: Not applicable

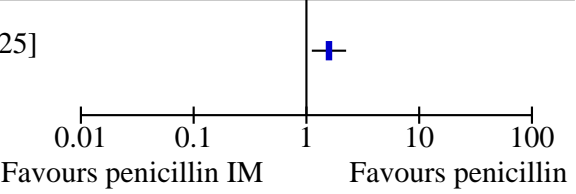

\section{ADDITIONAL TABLES}

Table 1. Physical characteristics of interventions studied in included trials

\begin{tabular}{|c|c|}
\hline $\begin{array}{l}\text { Interventions studied in included tri- } \\
\text { als }\end{array}$ & Physical characteristics/method of delivery \\
\hline Silver nitrate solution & Clear solution, topical instillation \\
\hline Erythromycin ointment & Translucent/white ointment, topical application \\
\hline Tetracycline ointment & Light-yellow ointment, topical application \\
\hline Oxytetracycline hydrochlorate solution & Unknown colour/consistency, aqueous solution, topical instillation \\
\hline Povidone-iodine solution & Orange-red, clear solution, topical instillation \\
\hline Hexarginum solution & $\begin{array}{l}\text { Colour unknown, solution with } 1 \mathrm{~g} \text { silver nitrate }+36 \mathrm{~g} \text { methylamine dissolved in } 63 \mathrm{~g} \text { ster- } \\
\text { ile water, topical application }\end{array}$ \\
\hline Penicillin G ointment & Clear/white ointment, topical application \\
\hline Penicillin G intramuscular injection & Clear, aqueous solution, intramuscular injection \\
\hline Cetyl-pyridinium chloride solution & Solution of unknown colour/consistency, topical instillation \\
\hline Bacitracin-phenacaine ointment & Ointment of unknown colour, topical application \\
\hline Sulfacetamide ointment & Ointment of unknown colour, topical application \\
\hline Chloramphenicol solution & Clear, colourless to slightly yellow solution, topical instillation \\
\hline Chloramphenicol ointment & Colourless ointment; topical instillation \\
\hline Colostrum & Yellowish, white or clear liquid, topical instillation \\
\hline Carbethopendecinium bromide solution & Yellowish to white powder in solution, solution colour unknown, topical instillation \\
\hline Mechanical cleansing & Eyes swabbed with clear, distilled water and wiped dry \\
\hline
\end{tabular}


Table 2. Summary results

\begin{tabular}{|c|c|c|c|c|c|c|c|c|c|c|}
\hline & \multicolumn{2}{|c|}{ Gonococcal conjunctivitis } & \multicolumn{2}{|c|}{$\begin{array}{l}\text { Chlamydial conjunc- } \\
\text { tivitis }\end{array}$} & \multicolumn{2}{|c|}{ Bacterial conjunctivitis } & \multicolumn{2}{|c|}{$\begin{array}{l}\text { Any conjunctivitis of any ae- } \\
\text { tiology }\end{array}$} & \multicolumn{2}{|c|}{$\begin{array}{l}\text { Conjunctivitis of un- } \\
\text { known aetiology }\end{array}$} \\
\hline & $\begin{array}{l}\text { Number } \\
\text { of trials }\end{array}$ & $\begin{array}{l}\text { Risk ratio ( } 95 \% \text { con- } \\
\text { fidence interval) }\end{array}$ & $\begin{array}{l}\text { Number } \\
\text { of trials }\end{array}$ & $\begin{array}{l}\text { Risk ratio } \\
\text { (95\% con- } \\
\text { fidence in- } \\
\text { terval) }\end{array}$ & $\begin{array}{l}\text { Number } \\
\text { of trials }\end{array}$ & $\begin{array}{l}\text { Risk ratio } \\
\text { (95\% con- } \\
\text { fidence in- } \\
\text { terval) }\end{array}$ & $\begin{array}{l}\text { Number } \\
\text { of trials }\end{array}$ & $\begin{array}{l}\text { Risk ratio ( } 95 \% \\
\text { confidence in- } \\
\text { terval) }\end{array}$ & $\begin{array}{l}\text { Number } \\
\text { of trials }\end{array}$ & $\begin{array}{l}\text { Risk ratio } \\
\text { (95\% confi- } \\
\text { dence inter- } \\
\text { val) }\end{array}$ \\
\hline $\begin{array}{l}\text { Any prophylax- } \\
\text { is vs no prophy- } \\
\text { laxis }\end{array}$ & 3 & 0.79 (0.24 to 2.65$)$ & 2 & $\begin{array}{l}0.96(0.57 \text { to } \\
1.61)\end{array}$ & 2 & $\begin{array}{l}0.84(0.37 \text { to } \\
1.93)\end{array}$ & 8 & 0.65 (0.54 to 0.78$)$ & 1 & $\begin{array}{l}1.75(0.37 \text { to } \\
8.28)\end{array}$ \\
\hline $\begin{array}{l}\text { Silver nitrate vs } \\
\text { no prophylaxis }\end{array}$ & 1 & $\begin{array}{l}\text { No events reported } \\
\text { in } 1 \text { or both arms. }\end{array}$ & 1 & $\begin{array}{l}1.06(0.55 \text { to } \\
2.02)\end{array}$ & \multicolumn{2}{|c|}{ No data were available. } & 3 & 0.67 (0.52 to 0.87$)$ & \multicolumn{2}{|c|}{ No data were available. } \\
\hline $\begin{array}{l}\text { Erythromycin vs } \\
\text { no prophylaxis }\end{array}$ & 2 & $\begin{array}{l}\text { No events reported } \\
\text { in } 1 \text { or both arms. }\end{array}$ & 2 & $\begin{array}{l}0.93 \text { (0.49 to } \\
1.77)\end{array}$ & 1 & $\begin{array}{l}0.80(0.22 \text { to } \\
2.90)\end{array}$ & 6 & 0.68 (0.51 to 0.89$)$ & 1 & $\begin{array}{l}1.50(0.26 \text { to } \\
8.80)\end{array}$ \\
\hline $\begin{array}{l}\text { Tetracycline vs } \\
\text { no prophylaxis }\end{array}$ & 1 & $\begin{array}{l}\text { No events reported } \\
\text { in } 1 \text { or both arms. }\end{array}$ & 1 & $\begin{array}{l}0.82(0.42 \text { to } \\
1.63)\end{array}$ & \multicolumn{2}{|c|}{ No data were available. } & 2 & $0.72(0.55$ to 0.94$)$ & \multicolumn{2}{|c|}{ No data were available. } \\
\hline $\begin{array}{l}\text { Povidone-io- } \\
\text { dine vs no pro- }\end{array}$ & 1 & $\begin{array}{l}\text { No events reported } \\
\text { in } 1 \text { or both arms. }\end{array}$ & 1 & $\begin{array}{l}2.00(0.18 \text { to } \\
21.74)\end{array}$ & 1 & $\begin{array}{l}1.00(0.30 \text { to } \\
3.36)\end{array}$ & 1 & 0.38 (0.18 to 0.77$)$ & 1 & $\begin{array}{l}2.00(0.37 \text { to } \\
10.70)\end{array}$ \\
\hline
\end{tabular}

phylaxis

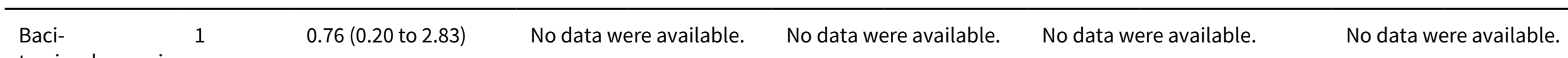

tracin-phenacaine

vs no prophy-

laxis

$\begin{array}{lllllll}\text { Colostrum vs no } & \text { No data were available. } & \text { No data were available. } & \text { No data were available. } & 1 & 0.72(0.45 \text { to } 1.14) & \text { No data were available. }\end{array}$ prophylaxis

\begin{tabular}{lllllllll}
\hline $\begin{array}{l}\text { Erythromycin vs } \\
\text { silver nitrate }\end{array}$ & 4 & 2.28 (0.88 to 5.90) & 4 & $\begin{array}{l}0.75(0.51 \text { to } \\
1.09)\end{array}$ & $\begin{array}{l}0.83 \text { (0.69 to } \\
1.01)\end{array}$ & No data were available. \\
\hline $\begin{array}{l}\text { Tetracycline vs } \\
\text { silver nitrate }\end{array}$ & 5 & $0.66(0.21$ to 2.05$)$ & 4 & $\begin{array}{l}0.64(0.40 \text { to } \\
1.02)\end{array}$ & No data were available. & 4 & 0.80 (0.66 to 0.98$)$ & No data were available. \\
\hline $\begin{array}{l}\text { Sulfacetamide } \\
\text { vs silver nitrate }\end{array}$ & 1 & $\begin{array}{l}\text { No events reported } \\
\text { in } 1 \text { or both arms. }\end{array}$ & No data were available. & 1 & $\begin{array}{l}0.88(0.45 \text { to } \\
1.74)\end{array}$ & 1 & $0.54(0.32$ to 0.89$)$ & 1
\end{tabular}




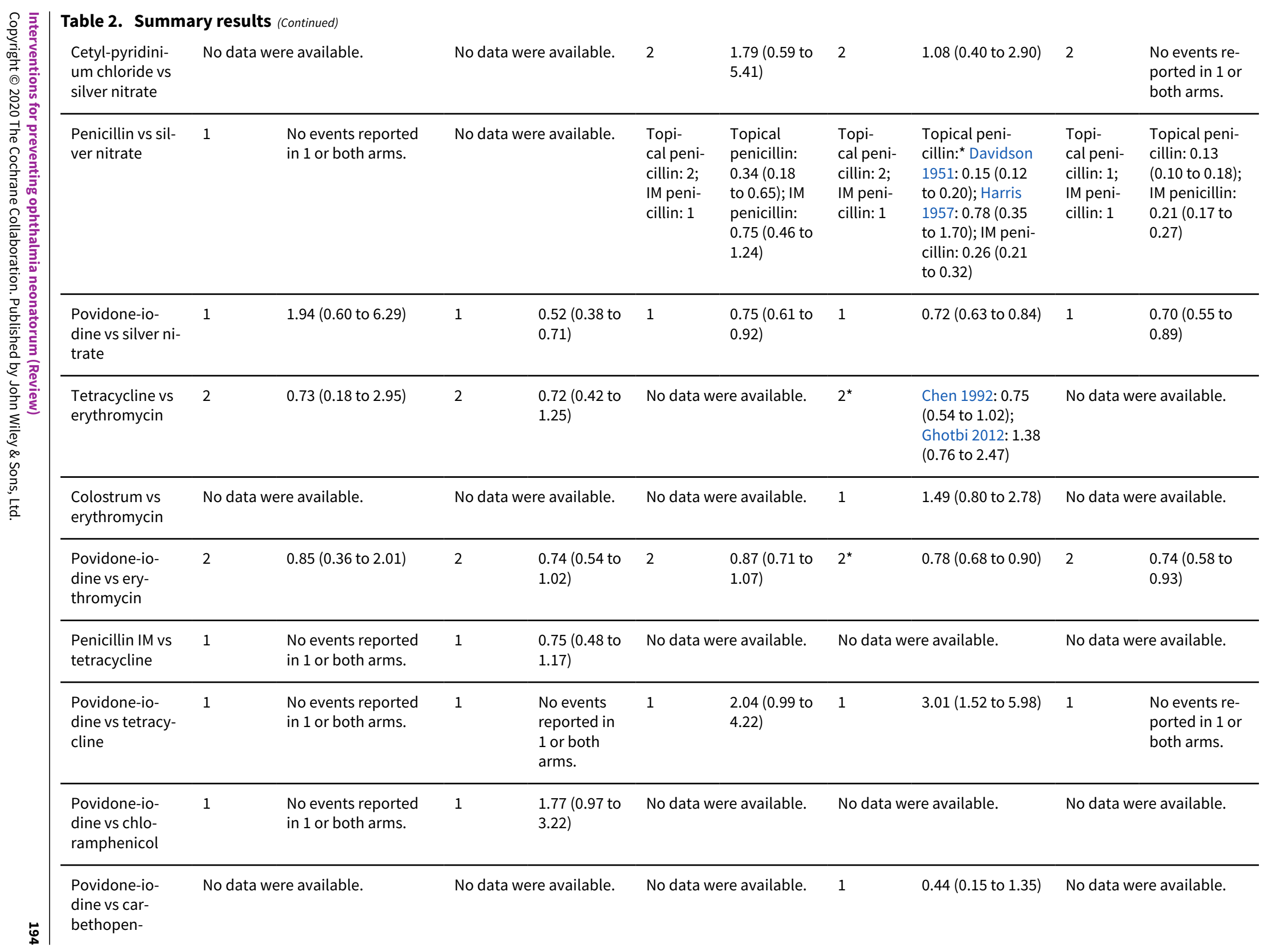




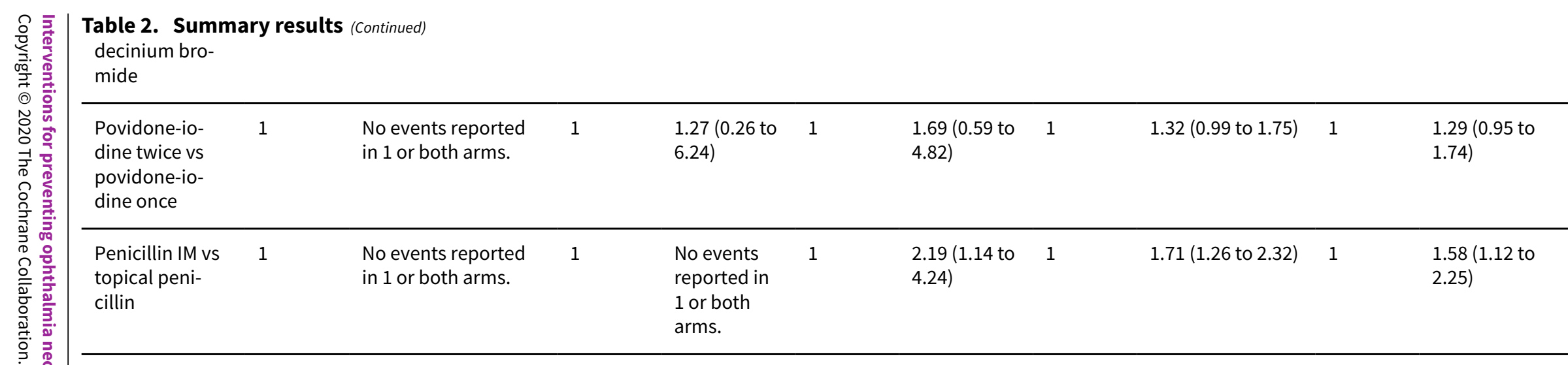

IM: intramuscular

*Indicates statistically significant heterogeneity precluding a meta-analysis. 
Table 3. Silver nitrate compared to no prophylaxis for prevention of ophthalmia neonatorum in newborn children Silver nitrate compared to no prophylaxis for prevention of ophthalmia neonatorum in newborn children

Patient or population: newborn children

Setting: any maternity setting

Intervention: silver nitrate

Comparison: no prophylaxis

\begin{tabular}{|c|c|c|c|c|c|c|}
\hline \multirow[t]{2}{*}{ Outcomes } & \multicolumn{2}{|c|}{$\begin{array}{l}\text { Anticipated absolute effects }{ }^{\star}(95 \% \\
\mathrm{Cl})\end{array}$} & \multirow[t]{2}{*}{$\begin{array}{l}\text { Relative ef- } \\
\text { fect } \\
(95 \% \mathrm{CI})\end{array}$} & \multirow[t]{2}{*}{$\begin{array}{l}\text { № of partici- } \\
\text { pants } \\
\text { (studies) }\end{array}$} & \multirow{2}{*}{$\begin{array}{l}\text { Certain- } \\
\text { ty of } \\
\text { the evi- } \\
\text { dence } \\
\text { (GRADE) }\end{array}$} & \multirow[t]{2}{*}{$\begin{array}{l}\text { Com- } \\
\text { ments }\end{array}$} \\
\hline & $\begin{array}{l}\text { Risk with no } \\
\text { prophylaxis }\end{array}$ & $\begin{array}{l}\text { Risk with silver } \\
\text { nitrate }\end{array}$ & & & & \\
\hline
\end{tabular}

Blindness (visual acuity less than

No studies reported this outcome.

20/200)

follow-up: 12 months

Any adverse visual outcome No studies reported this outcome.

follow-up: 12 months

Conococcalconjunctivitis

Gonococcal conjunctivitis assessed with: Neisseria gonorrhoeae-positive culture

follow-up: 1 month

\section{See comment}

(1)

rang

\begin{tabular}{|c|c|c|c|c|}
\hline & & $\begin{array}{l}2225 \\
(1 \mathrm{RCT})\end{array}$ & $\begin{array}{l}\oplus \Theta \Theta \Theta \\
\text { VERY } \\
\text { LOW } 1,2\end{array}$ & $\begin{array}{l}\text { No } \\
\text { cas- } \\
\text { es of } \\
\text { gono- } \\
\text { coccal } \\
\text { con- } \\
\text { junc- } \\
\text { tivitis } \\
\text { were } \\
\text { re- } \\
\text { port- } \\
\text { ed in } \\
\text { this } \\
\text { study. }\end{array}$ \\
\hline & \multirow[t]{3}{*}{$\begin{array}{l}\text { RR } 1.06 \\
\text { (0.55 to } 2.02 \text { ) }\end{array}$} & \multirow[t]{3}{*}{$\begin{array}{l}2225 \\
(1 \mathrm{RCT})\end{array}$} & \multirow[t]{3}{*}{$\begin{array}{l}\oplus \oplus \Theta \Theta \\
\text { LOW } 1,3\end{array}$} & \\
\hline $\begin{array}{l}5 \text { per } 1000 \\
(3 \text { to } 10)\end{array}$ & & & & \\
\hline $\begin{array}{l}106 \text { per } 1000 \\
(55 \text { to } 202)\end{array}$ & & & & \\
\hline
\end{tabular}

\begin{tabular}{|c|c|c|c|c|c|}
\hline \multirow{4}{*}{$\begin{array}{l}\text { Chlamydial conjunctivitis } \\
\text { assessed with: Chlamydia trachoma- } \\
\text { tis culture, PCR, or direct fluores- } \\
\text { cent monoclonal antibody stain } \\
\text { follow-up: } 1 \text { month }\end{array}$} & \multicolumn{2}{|l|}{ Low risk } & \multirow{4}{*}{$\begin{array}{l}\text { RR } 1.06 \\
\text { (0.55 to } 2.02)\end{array}$} & \multirow{4}{*}{$\begin{array}{l}2225 \\
(1 \mathrm{RCT})\end{array}$} & \multirow{4}{*}{$\begin{array}{l}\oplus \oplus \Theta \Theta \\
\operatorname{LOW} 1,3\end{array}$} \\
\hline & 5 per 1000 & $\begin{array}{l}5 \text { per } 1000 \\
(3 \text { to } 10)\end{array}$ & & & \\
\hline & \multicolumn{2}{|l|}{ High risk } & & & \\
\hline & 100 per 1000 & $\begin{array}{l}106 \text { per } 1000 \\
(55 \text { to } 202)\end{array}$ & & & \\
\hline
\end{tabular}

Bacterial conjunctivitis assessed with: any bacteria-positive culture, smear, or Gram stain follow-up: 1 month
2 of the 3 studies did not measure or report bacterial conjunctivitis (Bell 1993; Graf 1994). 1 study measured bacterial conjunctivitis but did not report this outcome by study arm (Chen 1992).

\begin{tabular}{llll}
\hline $\begin{array}{lll}\text { Any conjunctivitis of any aetiology } \\
\text { assessed with: clinical assessment }\end{array}$ & Low risk & RR 0.67 & 2713 \\
(0.52 to 0.87) & (3 RCTs) & (0.67 & \\
\hline
\end{tabular}


Table 3. Silver nitrate compared to no prophylaxis for prevention of ophthalmia neonatorum in newborn

childwenpednemoetth

\begin{tabular}{ll}
3 per 1000 & $\begin{array}{l}2 \text { per } 1000 \\
\text { (2 to } 3)\end{array}$ \\
\hline High risk & \\
\hline 300 per 1000 & $\begin{array}{l}201 \text { per } 1000 \\
\text { (156 to } 261)\end{array}$
\end{tabular}

Conjunctivitis of unknown aetiology assessed with: culture negative follow-up: 1 month
2 of the 3 studies did not measure did not measure or report conjunctivitis of unknown aetiology (Bell 1993; Graf 1994). 1 study measured conjunctivitis of unknown aetiology but did not report this outcome by trial arm (Chen 1992).

Adverse effects

\begin{abstract}
In a single study (Bell 1993), silver nitrate prophylaxis appeared to be associated with an increased risk of nasolacrimal duct obstruction compared with no prophylaxis, but the variance in the estimate was imprecise with wide confidence intervals (RR $1.28,95 \% \mathrm{Cl} 0.40$ to 4.02 ).

A single study comparing silver nitrate with control reported that no events of keratitis were observed in the prophylaxis and no-prophylaxis groups (Graf 1994).
\end{abstract}

\begin{abstract}
*The risk in the intervention group (and its 95\% confidence interval) is based on the assumed risk in the comparison group and the relative effect of the intervention (and its $95 \% \mathrm{Cl}$ ). The assumed risks (low and high) in the comparison group were estimated from relevant prevalence studies (Appendix 8).
\end{abstract}

Cl: confidence interval; PCR: polymerase chain reaction; $\mathbf{R C T}$ : randomised controlled trial; RR: risk ratio

\title{
GRADE Working Group grades of evidence
}

High-certainty: We are very confident that the true effect lies close to that of the estimate of the effect.

Moderate-certainty: We are moderately confident in the effect estimate: the true effect is likely to be close to the estimate of the effect, but there is a possibility that it is substantially different.

Low-certainty: Our confidence in the effect estimate is limited: the true effect may be substantially different from the estimate of the effect.

Very low-certainty: We have very little confidence in the effect estimate: the true effect is likely to be substantially different from the estimate of effect.

${ }^{1}$ Downgraded for risk of bias (-1): single study with high risk of selection bias, unclear and high risk of performance bias, unclear risk of detection bias.

2Downgraded for imprecision (-2): no events in either arm of trial; study underpowered to assess relative effects of treatment on this outcome.

3Downgraded for imprecision (-1): 95\% confidence interval includes no effect.

${ }^{4}$ Downgraded for risk of bias (-1): largest trial has high or unclear risk of bias; second-largest trial has low risk of selection bias, but higher risk of performance or detection bias.

5Downgraded (-2) for imprecision: sparse data.

Table 4. Erythromycin compared to no prophylaxis for the prevention of ophthalmia neonatorum in newborn children

Erythromycin compared to no prophylaxis for the prevention of ophthalmia neonatorum in newborn children

Patient or population: newborn children

Setting: any maternity setting

Intervention: erythromycin 
Table 4. Erythromycin compared to no prophylaxis for the prevention of ophthalmia neonatorum in newborn children (Continued)

Comparison: no prophylaxis

\begin{tabular}{|c|c|c|c|c|c|c|}
\hline \multirow[t]{2}{*}{ Outcomes } & \multicolumn{2}{|c|}{$\begin{array}{l}\text { Anticipated absolute effects }{ }^{*} \\
(95 \% \mathrm{CI})\end{array}$} & \multirow{2}{*}{$\begin{array}{l}\text { Relative } \\
\text { effect } \\
(95 \% \mathrm{Cl})\end{array}$} & \multirow{2}{*}{$\begin{array}{l}\text { № of } \\
\text { partici- } \\
\text { pants } \\
\text { (stud- } \\
\text { ies) }\end{array}$} & \multirow{2}{*}{$\begin{array}{l}\text { Cer- } \\
\text { tain- } \\
\text { ty of } \\
\text { the ev- } \\
\text { idence } \\
\text { (GRADE) }\end{array}$} & \multirow[t]{2}{*}{$\begin{array}{l}\text { Com- } \\
\text { ments }\end{array}$} \\
\hline & $\begin{array}{l}\text { Risk with no } \\
\text { prophylaxis }\end{array}$ & $\begin{array}{l}\text { Risk with ery- } \\
\text { thromycin }\end{array}$ & & & & \\
\hline
\end{tabular}

Blindness (visual acuity less than 20/200)

No studies reported this outcome.

follow-up: 12 months

No studies reported this outcome.

Any adverse visual outcome

follow-up: 12 months

\begin{tabular}{|c|c|c|c|c|c|c|}
\hline $\begin{array}{l}\text { Gonococcal conjunctivitis } \\
\text { assessed with: Neisseria gonorrhoeae-positive } \\
\text { culture } \\
\text { follow-up: } 1 \text { month }\end{array}$ & See commen & & & $\begin{array}{l}2526 \\
(2 \\
\text { RCTs })\end{array}$ & $\begin{array}{l}\oplus \ominus \ominus \ominus \\
\text { VERY } \\
\text { LOW } 1,2\end{array}$ & $\begin{array}{l}\text { No } \\
\text { cas- } \\
\text { es of } \\
\text { gono- } \\
\text { coccal } \\
\text { con- } \\
\text { junc- } \\
\text { tivitis } \\
\text { were } \\
\text { re- } \\
\text { port- } \\
\text { ed in } \\
\text { these } \\
\text { stud- } \\
\text { ies. }\end{array}$ \\
\hline \multirow{4}{*}{$\begin{array}{l}\text { Chlamydial conjunctivitis } \\
\text { assessed with: Chlamydia trachomatis culture, } \\
\text { PCR, or direct fluorescent monoclonal antibody } \\
\text { stain } \\
\text { follow-up: } 1 \text { month }\end{array}$} & \multicolumn{2}{|l|}{ Low risk } & \multirow{4}{*}{$\begin{array}{l}\text { RR } 0.93 \\
(0.49 \text { to } \\
1.77)\end{array}$} & \multirow{4}{*}{$\begin{array}{l}2526 \\
(2 \\
\text { RCTs })\end{array}$} & \multirow{4}{*}{$\begin{array}{l}\oplus \oplus \Theta \Theta \\
\text { LOW } 1,3\end{array}$} & \\
\hline & 5 per 1000 & $\begin{array}{l}5 \text { per } 1000 \\
(2 \text { to } 9)\end{array}$ & & & & \\
\hline & \multicolumn{2}{|l|}{ High risk } & & & & \\
\hline & 100 per 1000 & $\begin{array}{l}93 \text { per } 1000 \\
\text { (49 to } 177 \text { ) }\end{array}$ & & & & \\
\hline \multirow{4}{*}{$\begin{array}{l}\text { Bacterial conjunctivitis } \\
\text { assessed with: any bacteria-positive culture, } \\
\text { smear, or Gram stain. } \\
\text { follow-up: } 1 \text { month }\end{array}$} & Low risk & & \multirow{4}{*}{$\begin{array}{l}\text { RR } 0.80 \\
(0.22 \text { to } \\
2.90)\end{array}$} & \multirow{4}{*}{$\begin{array}{l}220 \\
(1 \mathrm{RCT})\end{array}$} & \multirow{4}{*}{$\begin{array}{l}\oplus \oplus \odot \odot \\
\operatorname{LOW}^{3,4}\end{array}$} & \\
\hline & 3 per 1000 & $\begin{array}{l}2 \text { per } 1000 \\
(1 \text { to } 9)\end{array}$ & & & & \\
\hline & \multicolumn{2}{|l|}{ High risk } & & & & \\
\hline & 50 per 1000 & $\begin{array}{l}40 \text { per } 1000 \\
\text { (11 to } 145)\end{array}$ & & & & \\
\hline
\end{tabular}


Table 4. Erythromycin compared to no prophylaxis for the prevention of ophthalmia neonatorum in newborn children (Continued)

Any conjunctivitis of any aetiology assessed with: clinical assessment follow-up: 1 month

$\begin{array}{lllll}\text { Low risk } & & \text { RR } 0.68 & 3509 & \oplus \oplus \oplus \odot \\ 3 \text { per } 1000 & \begin{array}{l}\text { 2 per } 1000 \\ \text { (2 to 3) }\end{array} & 0.51 \text { to } & (6 & \text { MOD- } \\ & & & \text { RCTs }) & \text { ERATE } \\ & & & 1\end{array}$

High risk

300 per 1000204 per 1000

(153 to 267$)$

\begin{tabular}{|c|c|c|c|c|c|}
\hline \multirow{2}{*}{$\begin{array}{l}\text { Conjunctivitis of unknown aetiology assessed } \\
\text { with: culture negative } \\
\text { follow-up: } 1 \text { month }\end{array}$} & \multicolumn{2}{|c|}{ Study population } & \multirow{2}{*}{$\begin{array}{l}\text { RR } 1.50 \\
(0.26 \text { to } \\
8.80)\end{array}$} & \multirow{2}{*}{$\begin{array}{l}220 \\
(1 \mathrm{RCT})\end{array}$} & $\oplus \odot \odot \odot$ \\
\hline & 18 per 1000 & $\begin{array}{l}27 \text { per } 1000 \\
(5 \text { to } 160)\end{array}$ & & & LOW \\
\hline
\end{tabular}

Adverse effects

In a single study of 269 people, there was no clear relation-

$\oplus \odot \odot \ominus$

ship between erythromycin and nasolacrimal duct obstruc- VERY tion (RR $0.83,95 \% \mathrm{Cl} 0.56$ to 1.23 ).

\begin{abstract}
*The risk in the intervention group (and its $95 \%$ confidence interval) is based on the assumed risk in the comparison group and the relative effect of the intervention (and its $95 \% \mathrm{Cl}$ ). The assumed risks (low and high) in the comparison group were estimated from relevant prevalence studies (Appendix 8).
\end{abstract}

Cl: confidence interval; PCR: polymerase chain reaction; $\mathbf{R C T}$ : randomised controlled trial; RR: risk ratio

\title{
GRADE Working Group grades of evidence
}

High-certainty: We are very confident that the true effect lies close to that of the estimate of the effect.

Moderate-certainty: We are moderately confident in the effect estimate: the true effect is likely to be close to the estimate of the effect, but there is a possibility that it is substantially different.

Low-certainty: Our confidence in the effect estimate is limited: the true effect may be substantially different from the estimate of the effect.

Very low-certainty: We have very little confidence in the effect estimate: the true effect is likely to be substantially different from the estimate of effect.

1Downgraded for risk of bias (-1): studies were at high risk or unclear risk of bias. High loss to follow-up in one trial, and unclear if followup time is two weeks or one month in same trial. No placebo used in either trial.

2Downgraded for imprecision (-2): no events in either arm of trial.

3Downgraded for imprecision (-1): 95\% confidence interval includes no effect; very few events; one study; small sample size.

${ }^{4}$ Downgraded for risk of bias (-1): study has high or unclear risk of bias with high loss to follow-up. No placebo used.

5Downgraded for imprecision (-2): very wide confidence intervals.

Table 5. Tetracycline compared to no prophylaxis for the prevention of ophthalmia neonatorum in newborn children

Tetracycline compared to no prophylaxis for the prevention of ophthalmia neonatorum in newborn children

Patient or population: newborn children

Setting: any maternity setting

Intervention: tetracycline

Comparison: no prophylaxis

\begin{tabular}{|c|c|c|c|c|c|}
\hline Outcomes & $\begin{array}{l}\text { Anticipated absolute effects }{ }^{*} \\
(95 \% \mathrm{Cl})\end{array}$ & $\begin{array}{l}\text { Relative } \\
\text { effect } \\
(95 \% \mathrm{CI})\end{array}$ & $\begin{array}{l}\text { № of } \\
\text { partici- } \\
\text { pants }\end{array}$ & $\begin{array}{l}\text { Certain- } \\
\text { ty of } \\
\text { the evi- } \\
\text { dence }\end{array}$ & $\begin{array}{l}\text { Com- } \\
\text { ments }\end{array}$ \\
\hline
\end{tabular}


Table 5. Tetracycline compared to no prophylaxis for the prevention of ophthalmia neonatorum in newborn children (Continued)

$\begin{array}{lll}\text { Risk with } & \text { Risk with } & \text { (stud- } \\ \text { no prophy- } & \text { tetracycline } & \text { ies) } \\ \text { laxis } & & \end{array}$

(stud-

(GRADE)

laxis

Blindness (visual acuity less than 20/200)

No studies reported this outcome.

follow-up: 12 months

No studies reported this outcome.

Any adverse visual outcome

follow-up: 12 months

See comment

Gonococcal conjunctivitis

assessed with: Neisseria gonorrhoeae-positive cul-

ture

follow-up: 1 month

2299

$(1 \mathrm{RCT})$

$\oplus \ominus \ominus \ominus$

VERY

No cases

of gono-

LOW 1

coccal

conjunc-

tivitis

were re-

ported

in this

study.

\begin{tabular}{|c|c|c|c|c|c|}
\hline \multirow{4}{*}{$\begin{array}{l}\text { Chlamydial conjunctivitis } \\
\text { assessed with: Chlamydia trachomatis culture, } \\
\text { PCR, or direct fluorescent monoclonal antibody } \\
\text { stain } \\
\text { follow-up: } 1 \text { month }\end{array}$} & \multicolumn{2}{|l|}{ Low risk } & \multirow{4}{*}{$\begin{array}{l}\text { RR } 0.82 \\
(0.42 \text { to } \\
1.63)\end{array}$} & \multirow{4}{*}{$\begin{array}{l}2299 \\
\text { (1 RCT) }\end{array}$} & \multirow{4}{*}{$\begin{array}{l}\oplus \oplus \Theta \Theta \\
\text { LOW 2,3 }\end{array}$} \\
\hline & 5 per 1000 & $\begin{array}{l}4 \text { per } 1000 \\
(7 \text { to } 26)\end{array}$ & & & \\
\hline & \multicolumn{2}{|l|}{ High risk } & & & \\
\hline & $\begin{array}{l}100 \text { per } \\
1000\end{array}$ & $\begin{array}{l}82 \text { per } 1000 \\
\text { ( } 42 \text { to } 163 \text { ) }\end{array}$ & & & \\
\hline
\end{tabular}

\section{Bacterial conjunctivitis}

assessed with: any bacteria-positive culture, smear, or Gram stain

follow-up: 1 month
1 study did not measure or report bacterial conjunctivitis (Ghotbi 2012), whilst the other study measured bacterial conjunctivitis but did not report by allocation group (Chen 1992).
Any conjunctivitis of any aetiology assessed with: clinical assessment follow-up: 1 month

\begin{tabular}{|c|c|c|c|c|}
\hline \multicolumn{2}{|l|}{ Low risk } & \multirow{4}{*}{$\begin{array}{l}\text { RR } 0.72 \\
(0.55 \text { to } \\
0.94)\end{array}$} & \multirow{4}{*}{$\begin{array}{l}2519 \\
\text { (2 RCTs) }\end{array}$} & \multirow{4}{*}{$\begin{array}{l}\oplus \oplus \ominus \ominus \\
\text { LOW }^{3,4}\end{array}$} \\
\hline 3 per 1000 & $\begin{array}{l}2 \text { per } 1000 \\
(2 \text { to } 3)\end{array}$ & & & \\
\hline \multicolumn{2}{|l|}{ High risk } & & & \\
\hline $\begin{array}{l}300 \text { per } \\
1000\end{array}$ & $\begin{array}{l}216 \text { per } 1000 \\
\text { (1165 to } 282 \text { ) }\end{array}$ & & & \\
\hline
\end{tabular}


Table 5. Tetracycline compared to no prophylaxis for the prevention of ophthalmia neonatorum in newborn children (Continued)

Conjunctivitis of unknown aetiology assessed with: culture negative

follow-up: 1 month
1 study did not measure or report conjunctivitis of unknown aetiology (CUE) (Ghotbi 2012), whilst the other study measured CUE but did not report by allocation group (Chen 1992).

Adverse effects

No studies reported this outcome.

\begin{abstract}
*The risk in the intervention group (and its 95\% confidence interval) is based on the assumed risk in the comparison group and the relative effect of the intervention (and its $95 \% \mathrm{Cl}$ ). The assumed risks (low and high) in the comparison group were estimated from relevant prevalence studies (Appendix 8).
\end{abstract}

Cl: confidence interval; PCR: polymerase chain reaction; $\mathbf{R C T}$ : randomised controlled trial; RR: risk ratio

\title{
GRADE Working Group grades of evidence
}

High-certainty: We are very confident that the true effect lies close to that of the estimate of the effect.

Moderate-certainty: We are moderately confident in the effect estimate: the true effect is likely to be close to the estimate of the effect, but there is a possibility that it is substantially different.

Low-certainty: Our confidence in the effect estimate is limited: the true effect may be substantially different from the estimate of the effect.

Very low-certainty: We have very little confidence in the effect estimate: the true effect is likely to be substantially different from the estimate of effect.

1Downgraded for imprecision (-3): no events in this trial.

2Downgraded for risk of bias (-1): single trial with high risk of bias for sequence generation, allocation concealment, and masking, and unclear risk of bias for detection and attrition bias, which could affect outcomes considering the low event rates.

3Downgraded for imprecision (-1): optimal information size not met, and confidence interval overlaps no effect in this single study.

${ }^{4}$ Downgraded for risk of bias (-1): studies at high or unclear risk of bias.

Table 6. Povidone-iodine compared to no prophylaxis for the prevention of ophthalmia neonatorum in newborn children

Povidone-iodine compared to no prophylaxis for the prevention of ophthalmia neonatorum in newborn children

Patient or population: newborn children

Setting: any maternity setting

Intervention: povidone-iodine

Comparison: no prophylaxis

\begin{tabular}{|c|c|c|c|c|c|c|}
\hline \multirow[t]{2}{*}{ Outcomes } & \multicolumn{2}{|c|}{$\begin{array}{l}\text { Anticipated absolute effects }{ }^{*} \\
(95 \% \mathrm{Cl})\end{array}$} & \multirow{2}{*}{$\begin{array}{l}\text { Relative } \\
\text { effect } \\
(95 \% \mathrm{CI})\end{array}$} & \multirow{2}{*}{$\begin{array}{l}\text { № of } \\
\text { par- } \\
\text { tici- } \\
\text { pants } \\
\text { (stud- } \\
\text { ies) }\end{array}$} & \multirow{2}{*}{$\begin{array}{l}\text { Cer- } \\
\text { tain- } \\
\text { ty of } \\
\text { the ev- } \\
\text { idence } \\
\text { (GRADE) }\end{array}$} & \multirow[t]{2}{*}{$\begin{array}{l}\text { Com- } \\
\text { ments }\end{array}$} \\
\hline & $\begin{array}{l}\text { Risk with } \\
\text { no prophy- } \\
\text { laxis }\end{array}$ & $\begin{array}{l}\text { Risk with povi- } \\
\text { done-iodine }\end{array}$ & & & & \\
\hline
\end{tabular}

Blindness (visual acuity less than 20/200)

No studies reported this outcome.

follow-up: 12 months 
Table 6. Povidone-iodine compared to no prophylaxis for the prevention of ophthalmia neonatorum in newborn children (Continued)

Any adverse visual outcome $\quad$ No studies reported this outcome.
follow-up: 12 months

follow-up: 12 months

\begin{tabular}{|c|c|c|c|c|}
\hline $\begin{array}{l}\text { Gonococcal conjunctivitis } \\
\text { assessed with: Neisseria gonorrhoeae-positive cul- } \\
\text { ture } \\
\text { follow-up: } 1 \text { month }\end{array}$ & See comment & $\begin{array}{l}220 \\
(1 \\
\mathrm{RCT})\end{array}$ & $\begin{array}{l}\oplus \odot \Theta \odot \\
\text { VERY } \\
\text { LOW } 1\end{array}$ & $\begin{array}{l}\text { No } \\
\text { cas- } \\
\text { es of } \\
\text { gono- } \\
\text { coccal } \\
\text { con- } \\
\text { junc- } \\
\text { tivitis } \\
\text { were } \\
\text { re- } \\
\text { port- } \\
\text { ed in } \\
\text { this } \\
\text { study. }\end{array}$ \\
\hline
\end{tabular}

\section{Chlamydial conjunctivitis}

assessed with: Chlamydia trachomatis culture, PCR,

or direct fluorescent monoclonal antibody stain

follow-up: 1 month

\begin{tabular}{ll} 
Low risk & \\
\hline 5 per 1000 & $\begin{array}{l}10 \text { per } 1000 \\
(1 \text { to } 109)\end{array}$ \\
\hline High risk & \\
\hline 100 per & 200 per 1000 \\
1000 & (18 to 1000)
\end{tabular}

$\begin{array}{lll}\text { RR } 2.00 & 220 & \oplus \oplus \odot \odot \\ (0.18 \text { to } & (1 & \text { LOW } 2,3 \\ 21.7) & \text { RCT }) & \\ & & \end{array}$

\begin{tabular}{|c|c|c|c|c|c|}
\hline \multirow{4}{*}{$\begin{array}{l}\text { Bacterial conjunctivitis } \\
\text { assessed with: any bacteria-positive culture, smear, } \\
\text { or Gram stain. } \\
\text { follow-up: } 1 \text { month }\end{array}$} & \multicolumn{2}{|l|}{ Low risk } & \multirow{4}{*}{$\begin{array}{l}\text { RR } 1.00 \\
(0.30 \text { to } \\
3.36)\end{array}$} & \multirow{4}{*}{$\begin{array}{l}220 \\
(1 \\
\mathrm{RCT})\end{array}$} & \multirow{4}{*}{$\begin{array}{l}\oplus \oplus \Theta \Theta \\
\text { LOW 2,3 }\end{array}$} \\
\hline & 3 per 1000 & $\begin{array}{l}3 \text { per } 1000 \\
(1 \text { to } 10)\end{array}$ & & & \\
\hline & \multicolumn{2}{|l|}{ High risk } & & & \\
\hline & 50 per 1000 & $\begin{array}{l}50 \text { per } 1000 \\
(15 \text { to } 168)\end{array}$ & & & \\
\hline \multirow{4}{*}{$\begin{array}{l}\text { Any conjunctivitis of any aetiology assessed with: } \\
\text { clinical assessment } \\
\text { follow-up: } 1 \text { month }\end{array}$} & Low risk & & \multirow{4}{*}{$\begin{array}{l}\text { RR } 0.38 \\
(0.18 \text { to } \\
0.77)\end{array}$} & \multirow{4}{*}{$\begin{array}{l}220 \\
(1 \\
\mathrm{RCT})\end{array}$} & \multirow{4}{*}{$\begin{array}{l}\oplus \oplus \oplus \ominus \\
\text { MOD- } \\
\text { ERATE } \\
2\end{array}$} \\
\hline & 3 per 1000 & $\begin{array}{l}1 \text { per } 1000 \\
(1 \text { to } 2)\end{array}$ & & & \\
\hline & \multicolumn{2}{|l|}{ High risk } & & & \\
\hline & $\begin{array}{l}300 \text { per } \\
1000\end{array}$ & $\begin{array}{l}114 \text { per } 1000 \\
(54 \text { to } 231)\end{array}$ & & & \\
\hline $\begin{array}{l}\text { Conjunctivitis of unknown aetiology assessed with: } \\
\text { culture negative }\end{array}$ & \multicolumn{2}{|c|}{ Study population } & RR 2.00 & 220 & $\oplus \ominus \odot \odot$ \\
\hline
\end{tabular}


Table 6. Povidone-iodine compared to no prophylaxis for the prevention of ophthalmia neonatorum in newborn children (Continued)

follow-up: 1 month

$$
18 \text { per } 1000 \quad 36 \text { per } 1000
$$

No studies reported this outcome.

Adverse effects

*The risk in the intervention group (and its $95 \%$ confidence interval) is based on the assumed risk in the comparison group and the relative effect of the intervention (and its $95 \% \mathrm{Cl}$ ). The assumed risks (low and high) in the comparison group were estimated from relevant prevalence studies (Appendix 8).

Cl: confidence interval; PCR: polymerase chain reaction; $\mathbf{R C T}$ : randomised controlled trial; RR: risk ratio

\section{GRADE Working Group grades of evidence}

High-certainty: We are very confident that the true effect lies close to that of the estimate of the effect.

Moderate-certainty: We are moderately confident in the effect estimate: the true effect is likely to be close to the estimate of the ef-

fect, but there is a possibility that it is substantially different.

Low-certainty: Our confidence in the effect estimate is limited: the true effect may be substantially different from the estimate of the effect.

Very low-certainty: We have very little confidence in the effect estimate: the true effect is likely to be substantially different from the estimate of effect.

1Downgraded for imprecision (-3): no events in either arm of this study.

2Downgraded for risk of bias (-1): the study is unclear regarding sequence generation and allocation concealment. Masking is a concern as there was no placebo. Very high losses to follow-up.

3Downgraded for imprecision (-1): optimal information size criteria not met, and 95\% confidence interval includes no effect.

${ }^{4}$ Downgraded for imprecision (-2): very wide confidence intervals.

Table 7. Bacitracin-phenacaine compared to no prophylaxis for the prevention of ophthalmia neonatorum in newborn children

Bacitracin-phenacaine compared to no prophylaxis for the prevention of ophthalmia neonatorum in newborn children

Patient or population: newborn children

Setting: any maternity setting

Intervention: bacitracin-phenacaine

Comparison: no prophylaxis

\begin{tabular}{llll}
\hline Outcomes & $\begin{array}{l}\text { Anticipated absolute effects* } \\
(95 \% \mathrm{Cl})\end{array}$ & $\begin{array}{l}\text { Relative ef- } \\
\text { fect }\end{array}$ & $\begin{array}{l}\text { No of partici- } \\
\text { pants } \\
\text { (studies) }\end{array}$
\end{tabular}

Blindness (visual acuity less than 20/200)

No studies reported this outcome.

follow-up: 12 months 
Table 7. Bacitracin-phenacaine compared to no prophylaxis for the prevention of ophthalmia neonatorum in newborn children (Continued)

\begin{tabular}{|c|c|c|c|c|c|}
\hline \multirow{4}{*}{$\begin{array}{l}\text { Gonococcal conjunctivitis } \\
\text { assessed with: Neisseria gonorrhoeae-positive } \\
\text { culture } \\
\text { follow-up: } 1 \text { month }\end{array}$} & \multicolumn{2}{|l|}{ Low risk } & \multirow{4}{*}{$\begin{array}{l}\text { RR } 0.76 \\
\text { (0.20 to } 2.83 \text { ) }\end{array}$} & \multirow{4}{*}{$\begin{array}{l}3355 \\
(1 \mathrm{RCT})\end{array}$} & \multirow{4}{*}{$\begin{array}{l}\oplus \odot \odot \odot \\
\text { VERY LOW 1,2 }\end{array}$} \\
\hline & 1 per 1000 & $\begin{array}{l}1 \text { per } 1000 \\
(0 \text { to } 3)\end{array}$ & & & \\
\hline & \multicolumn{2}{|l|}{ High risk } & & & \\
\hline & 100 per 1000 & $\begin{array}{l}76 \text { per } 1000 \\
(20 \text { to } 283 \text { per } \\
1000)\end{array}$ & & & \\
\hline
\end{tabular}

Chlamydial conjunctivitis

assessed with: Chlamydia trachomatis cul-

ture, PCR, or direct fluorescent monoclonal

antibody stain

follow-up: 1 month
The 1 study does not seem to have measured or reported cases of chlamydial conjunctivitis (Posner 1959). Diagnostic methods for chlamydia would not have been readily available in 1959 .
Bacterial conjunctivitis assessed with: any bacteria-positive culture follow-up: 1 month
It is unknown if bacterial conjunctivitis was measured in the 1 study (Posner 1959), as it categorises conjunctivitis only as "nonspecific conjunctivitis" and "gonorrhoeal ophthalmia".
Any conjunctivitis of any aetiology assessed with: clinical assessment follow-up: 1 month
It is unknown if all conjunctivitis were measured in the 1 study (Posner 1959), as it categorises conjunctivitis only as "nonspecific conjunctivitis" and "gonorrhoeal ophthalmia".
Conjunctivitis of unknown aetiology assessed with: culture negative follow-up: 1 month
The category of "nonspecific conjunctivitis" reported in the 1 study is undefined and may or may not also include bacterial conjunctivitis cases (Posner 1959).

Adverse effects No studies reported this outcome.

* The risk in the intervention group (and its $95 \%$ confidence interval) is based on the assumed risk in the comparison group and the relative effect of the intervention (and its $95 \% \mathrm{Cl}$ ). The assumed risks (low and high) in the comparison group were estimated from relevant prevalence studies (Appendix 8).

CI: confidence interval; PCR: polymerase chain reaction; $\mathbf{R C T}$ : randomised controlled trial; RR: risk ratio

\section{GRADE Working Group grades of evidence}

High-certainty: We are very confident that the true effect lies close to that of the estimate of the effect.

Moderate-certainty: We are moderately confident in the effect estimate: the true effect is likely to be close to the estimate of the effect, but there is a possibility that it is substantially different.

Low-certainty: Our confidence in the effect estimate is limited: the true effect may be substantially different from the estimate of the effect.

Very low-certainty: We have very little confidence in the effect estimate: the true effect is likely to be substantially different from the estimate of effect. 
1Downgraded for risk of bias (-1): single trial with high risk of selection bias, unclear risk of performance and detection bias, high risk of selective outcome reporting.

2Downgraded for imprecision (-2): very wide confidence intervals, which include no effect.

Table 8. Colostrum compared to no prophylaxis for the prevention of ophthalmia neonatorum in newborn children

\title{
Colostrum compared to no prophylaxis for the prevention of ophthalmia neonatorum in newborn children
}

\author{
Patient or population: newborn children \\ Setting: any maternity setting \\ Intervention: colostrum \\ Comparison: no prophylaxis
}

\begin{tabular}{|c|c|c|c|c|c|}
\hline \multirow[t]{2}{*}{ Outcomes } & \multicolumn{2}{|c|}{$\begin{array}{l}\text { Anticipated absolute effects }{ }^{*} \\
(95 \% \mathrm{Cl})\end{array}$} & \multirow{2}{*}{$\begin{array}{l}\text { Relative ef- } \\
\text { fect } \\
(95 \% \mathrm{Cl})\end{array}$} & \multirow{2}{*}{$\begin{array}{l}\text { № of par- } \\
\text { ticipants } \\
\text { (studies) }\end{array}$} & \multirow{2}{*}{$\begin{array}{l}\text { Certainty } \\
\text { of the evi- } \\
\text { dence } \\
\text { (GRADE) }\end{array}$} \\
\hline & $\begin{array}{l}\text { Risk with no } \\
\text { prophylaxis }\end{array}$ & $\begin{array}{l}\text { Risk with } \\
\text { colostrum }\end{array}$ & & & \\
\hline
\end{tabular}

Blindness (visual acuity less than 20/200)

No studies reported this outcome.

follow-up: 12 months
Any adverse visual outcome
No studies reported this outcome.
follow-up: 12 months

\section{Gonococcal conjunctivitis}

assessed with: Neisseria gonorrhoeae-positive

culture

follow-up: 1 month
No discussion of gonococcal conjunctivitis in this study (Ghaemi 2014). The trial excluded culture-positive neonates before application of prophylaxis.
Chlamydial conjunctivitis

assessed with: Chlamydia trachomatis culture, $\mathrm{PCR}$, or direct fluorescent monoclonal antibody

stain

follow-up: 1 month

Bacterial conjunctivitis

assessed with: any bacteria-positive culture follow-up: 1 month
Any conjunctivitis of any aetiology assessed with: clinical assessment follow-up: 1 month
No discussion of chlamydial conjunctivitis in this 1 study (Ghaemi 2014). It is unknown if it was measured. Chlamydial conjunctivitis cases were not reported.
The 1 trial trial does not report bacterial conjunctivitis cases as distinguished from all conjunctivitis cases (Ghaemi 2014). It is unknown if bacterial conjunctivitis was measured.

\begin{tabular}{lllll} 
Low risk & $\begin{array}{l}\text { RR 0.72 } \\
\text { (0.45 to 1.14) }\end{array}$ & $\begin{array}{l}186 \\
\text { (1 RCT) }\end{array}$ & $\begin{array}{l}\oplus \oplus \odot \odot \\
\text { LOW } 1,2\end{array}$ \\
\hline 3 per 1000 & $\begin{array}{l}\text { 2 per } 1000 \\
\text { (1 to 3) }\end{array}$ & & \\
\hline
\end{tabular}

\begin{tabular}{ll}
\hline High risk \\
\hline 300 per $1000 \quad 216$ per 1000
\end{tabular}

(135 to 342$)$

Conjunctivitis of unknown aetiology assessed with: culture negative follow-up: 1 month
The 1 trial only reports all conjunctivitis cases, and does not distinguish bacterial conjunctivitis cases from cases of conjunctivitis of unknown aetiology (Ghaemi 2014).

Adverse effects

No studies reported this outcome. 
Table 9. Erythromycin compared to silver nitrate for the prevention of ophthalmia neonatorum in newborn

children (Continued)

assessed with: Chlamydia trachomatis culture, PCR, or direct fluorescent monoclonal antibody stain

21 per $\quad 16$ per 1000

follow-up: 1 month

$1000 \quad$ (11 to 23 )

\begin{tabular}{|c|c|c|c|c|c|c|}
\hline \multirow{2}{*}{$\begin{array}{l}\text { Bacterial conjunctivitis } \\
\text { assessed with: any bacteria-positive } \\
\text { culture } \\
\text { follow-up: } 1 \text { month }\end{array}$} & \multicolumn{2}{|c|}{ Study population } & \multirow{2}{*}{$\begin{array}{l}\text { RR } 0.83 \\
(0.69 \text { to } \\
1.01)\end{array}$} & \multirow{2}{*}{$\begin{array}{l}6333 \\
(2 \\
\text { RCTs })\end{array}$} & \multirow{2}{*}{$\begin{array}{l}\oplus \oplus \ominus \ominus \\
\mathrm{LOW} \\
1,3\end{array}$} & \multirow{2}{*}{$\begin{array}{l}\text { Follow-up: range } 9 \text { days to } 60 \\
\text { days }\end{array}$} \\
\hline & $\begin{array}{l}57 \text { per } \\
1000\end{array}$ & $\begin{array}{l}47 \text { per } 1000 \\
(39 \text { to } 58)\end{array}$ & & & & \\
\hline
\end{tabular}

Any conjunctivitis of any aetiology as- See comment

$9021 \quad \oplus \odot \odot \odot \quad$ A meta-analysis was not con-

sessed with: clinical assessment

(4) VERY ducted considering the consid-

follow-up: 1 month

RCTs) LOW erable statistical heterogene-

$1,3,5 \quad$ ity $\left(I^{2}=90 \%\right)$. Therefore, there was no pooled effect.

Follow-up: range 9 days to 60 days

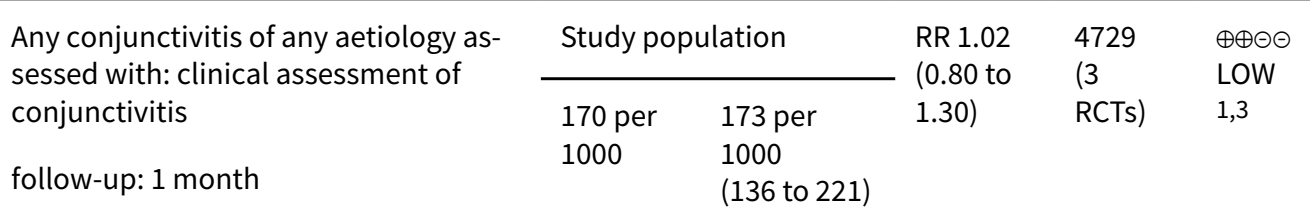

After excluding 1 study (Christian 1960), I2 declined to 51\%, but was still moderate. A pooled effect was then presented for these 3 remaining studies.

Follow-up: range 9 days to 60 days

\begin{tabular}{|c|c|c|c|c|c|}
\hline \multirow{2}{*}{$\begin{array}{l}\text { Conjunctivitis of unknown aetiology } \\
\text { assessed with: culture negative } \\
\text { follow-up: } 1 \text { month }\end{array}$} & \multicolumn{2}{|c|}{ Study population } & \multirow{2}{*}{$\begin{array}{l}\text { RR } 0.96 \\
(0.77 \text { to } \\
1.19)\end{array}$} & \multirow{2}{*}{$\begin{array}{l}2041 \\
(1 \mathrm{RCT})\end{array}$} & \multirow{2}{*}{$\begin{array}{l}\oplus \oplus \oplus \Theta \\
\mathrm{LOW} \\
1,3\end{array}$} \\
\hline & $\begin{array}{l}139 \text { per } \\
1000\end{array}$ & $\begin{array}{l}133 \text { per } \\
1000 \\
(107 \text { to } 165)\end{array}$ & & & \\
\hline Adverse effects & \multicolumn{4}{|c|}{$\begin{array}{l}\text { In } 1 \text { study, erythromycin was associated with a } \\
\text { reduced risk of nasolacrimal duct obstruction } \\
\text { compared with silver nitrate. This association } \\
\text { was not statistically significant (RR } 0.81,95 \% \mathrm{Cl} \\
0.55 \text { to } 1.20 \text { ). }\end{array}$} & $\begin{array}{l}\oplus \oplus \Theta \Theta \\
\mathrm{LOW} \\
1,3\end{array}$ \\
\hline
\end{tabular}

\footnotetext{
* The risk in the intervention group (and its $95 \%$ confidence interval) is based on the assumed risk in the comparison group and the relative effect of the intervention (and its $95 \% \mathrm{Cl}$ ). The assumed risk is taken from the study population in the included studies.

Cl: confidence interval; PCR: polymerase chain reaction; $\mathbf{R C T}$ : randomised controlled trial; RR: risk ratio

\section{GRADE Working Group grades of evidence}

High-certainty: We are very confident that the true effect lies close to that of the estimate of the effect.

Moderate-certainty: We are moderately confident in the effect estimate: the true effect is likely to be close to the estimate of the effect, but there is a possibility that it is substantially different.

Low-certainty: Our confidence in the effect estimate is limited: the true effect may be substantially different from the estimate of the effect.

Very low-certainty: We have very little confidence in the effect estimate: the true effect is likely to be substantially different from the estimate of effect.
}

1Downgraded for risk of bias (-1): studies at high or unclear risk of bias.

2Downgraded for imprecision (-2): very wide 95\% confidence intervals including no effect. 
3Downgraded for imprecision (-1): 95\% confidence intervals include no effect.

4Downgraded for risk of bias (-1): one of the four studies, the smallest one (Hammerschlag 1980), was funded by the pharmaceutical company that produces erythromycin (Dista Pharmaceuticals). That trial favoured erythromycin, and was the only trial amongst four trials with no chlamydial conjunctivitis in the erythromycin arm (12 cases in the silver nitrate arm). There were 36 infants in the silver nitrate arm and only 24 in the erythromycin arm. Furthermore, 7 of 67 chlamydia-positive mothers remained unaccounted for. Their distribution in the allocation arms is unknown. With a transmission rate of about 30\%, low event rates, and small sample size, these missing mothers could have a significant effect on the results.

${ }^{5}$ Downgraded for inconsistency (-1): confidence intervals do not overlap; point estimates vary widely and on either side of no effect; 12 is $90 \%$; and $\mathrm{Chi}^{2} \mathrm{P}<0.001$. Heterogeneity could be explained by one older trial that did not define conjunctivitis, and review authors applied definition to eye reaction classifications.

Table 10. Tetracycline compared to silver nitrate for the prevention of ophthalmia neonatorum in newborn children

\section{Tetracycline compared to silver nitrate for the prevention of ophthalmia neonatorum in newborn children}

Patient or population: newborn children

Setting: any maternity setting

Intervention: tetracycline

Comparison: silver nitrate

\begin{tabular}{|c|c|c|c|c|c|c|}
\hline \multirow[t]{2}{*}{ Outcomes } & \multicolumn{2}{|c|}{$\begin{array}{l}\text { Anticipated absolute effects* } \\
(95 \% \mathrm{CI})\end{array}$} & \multirow{2}{*}{$\begin{array}{l}\text { Relative } \\
\text { effect } \\
(95 \% \mathrm{Cl})\end{array}$} & \multirow{2}{*}{$\begin{array}{l}\text { № of par- } \\
\text { ticipants } \\
\text { (studies) }\end{array}$} & \multirow{2}{*}{$\begin{array}{l}\text { Certain- } \\
\text { ty of } \\
\text { the evi- } \\
\text { dence } \\
\text { (GRADE) }\end{array}$} & \multirow[t]{2}{*}{ Comments } \\
\hline & $\begin{array}{l}\text { Risk with } \\
\text { silver ni- } \\
\text { trate }\end{array}$ & $\begin{array}{l}\text { Risk with } \\
\text { tetracycline }\end{array}$ & & & & \\
\hline
\end{tabular}

Blindness (visual acuity less than 20/200) No studies reported this outcome.

follow-up: 12 months

Any adverse visual outcome

No studies reported this outcome.

follow-up: 12 months

\begin{tabular}{|c|c|c|c|c|c|c|}
\hline \multirow{3}{*}{$\begin{array}{l}\text { Gonococcal conjunctivitis } \\
\text { assessed with: Neisseria gonorrhoeae-posi- } \\
\text { tive culture } \\
\text { follow-up: } 1 \text { month }\end{array}$} & \multicolumn{2}{|c|}{ Study population } & \multirow{3}{*}{$\begin{array}{l}\text { RR } 0.66 \\
(0.21 \text { to } \\
2.05)\end{array}$} & \multirow{3}{*}{$\begin{array}{l}14,501 \\
\text { (5 RCTs) }\end{array}$} & \multirow{3}{*}{$\begin{array}{l}\oplus \odot \Theta \odot \\
\text { VERY } \\
\text { LOW } 1,2,3\end{array}$} & \multirow{3}{*}{$\begin{array}{l}\text { Amongst } \\
\text { the } 5 \text { in- } \\
\text { cluded tri- } \\
\text { als, } 3 \text { had } \\
\text { no events } \\
\text { in either } \\
\text { study arm. } \\
\text { Range } 4 \\
\text { weeks to } 19 \\
\text { weeks }\end{array}$} \\
\hline & 1 per 1000 & $\begin{array}{l}1 \text { per } 1000 \\
(0 \text { to } 2)\end{array}$ & & & & \\
\hline & & & & & & \\
\hline \multirow{2}{*}{$\begin{array}{l}\text { Chlamydial conjunctivitis } \\
\text { assessed with: Chlamydia trachomatis cul- } \\
\text { ture, PCR, or direct fluorescent monoclonal } \\
\text { antibody stain } \\
\text { follow-up: } 1 \text { month }\end{array}$} & \multicolumn{2}{|c|}{ Study population } & \multirow{2}{*}{$\begin{array}{l}\text { RR } 0.64 \\
(0.40 \text { to } \\
1.02)\end{array}$} & \multirow{2}{*}{$\begin{array}{l}14,142 \\
\text { (4 RCTs) }\end{array}$} & \multirow{2}{*}{$\begin{array}{l}\oplus \oplus \Theta \Theta \\
\text { LOW } 1,4\end{array}$} & \multirow{2}{*}{$\begin{array}{l}\text { Range } 4 \\
\text { weeks to } 19 \\
\text { weeks }\end{array}$} \\
\hline & 7 per 1000 & $\begin{array}{l}4 \text { per } 1000 \\
(3 \text { to } 7)\end{array}$ & & & & \\
\hline
\end{tabular}


Table 10. Tetracycline compared to silver nitrate for the prevention of ophthalmia neonatorum in newborn children (Continued)

Bacterial conjunctivitis assessed with: any bacteria-positive culture

follow-up: 1 month
Amongst 5 studies, 4 measured bacterial conjunctivitis but did not report the results (2) or the data could not be extracted (2). 1 study did not measure or report bacterial conjunctivitis.

\begin{tabular}{|c|c|c|c|c|c|}
\hline \multirow{2}{*}{$\begin{array}{l}\text { Any conjunctivitis of any aetiology as- } \\
\text { sessed with: clinical assessment } \\
\text { follow-up: } 1 \text { month }\end{array}$} & \multicolumn{2}{|c|}{ Study population } & \multirow{2}{*}{$\begin{array}{l}\text { RR } 0.80 \\
(0.66 \text { to } \\
0.98)\end{array}$} & \multirow{2}{*}{$\begin{array}{l}6229 \\
\text { (4 RCTs) }\end{array}$} & \multirow{2}{*}{$\begin{array}{l}\oplus \oplus \oplus \ominus \\
\text { MODER } \\
\text { ATE }^{1}\end{array}$} \\
\hline & 64 per 1000 & $\begin{array}{l}51 \text { per } 1000 \\
(42 \text { to } 63)\end{array}$ & & & \\
\hline
\end{tabular}

Conjunctivitis of unknown aetiology assessed with: culture negative follow-up: 1 month
Amongst 5 trials, 4 measured conjunctivitis of unknown aetiology (CUE) but did not report the results (2) or the data could not be extracted (2). 1 trial did not measure or report CUE.

$\begin{array}{lll}\text { Adverse effects } & \text { In } 1 \text { trial, tetracycline appeared to be associated with a } & \oplus \oplus \ominus \ominus \\ & \text { higher risk of nasolacrimal duct obstruction, but the esti- } & \text { LOW 1,5 } \\ \text { mate was imprecise and included no effect (RR } 1.57,95 \% \mathrm{Cl} & \\ & 0.63 \text { to 3.91). }\end{array}$

${ }^{\star}$ The risk in the intervention group (and its $95 \%$ confidence interval) is based on the assumed risk in the comparison group and the
relative effect of the intervention (and its $95 \% \mathrm{Cl}$ ). The assumed risk is taken from the study population in the included studies.

CI: confidence interval; PCR: polymerase chain reaction; $\mathbf{R C T}$ : randomised controlled trial; $\mathbf{R R}$ : risk ratio

\section{GRADE Working Group grades of evidence}

High-certainty: We are very confident that the true effect lies close to that of the estimate of the effect.

Moderate-certainty: We are moderately confident in the effect estimate: the true effect is likely to be close to the estimate of the effect, but there is a possibility that it is substantially different.

Low-certainty: Our confidence in the effect estimate is limited: the true effect may be substantially different from the estimate of the effect.

Very low-certainty: We have very little confidence in the effect estimate: the true effect is likely to be substantially different from the estimate of effect.

1Downgraded for risk of bias (-1): studies at high or unclear risk of bias.

2Downgraded for inconsistency (-1): $\mathrm{I}^{2}$ is $60 \%, \mathrm{Chi}^{2} \mathrm{P}$ value is 0.11 . Two trials had no events in either arm. Downgraded due to variable methods and length of follow-up.

3Downgraded for imprecision (-1): optimal information size is not met, but large sample size (7721 neonates in tetracycline group and 6780 neonates in silver nitrate group) with low baseline risk; however, confidence intervals are quite wide around relative effects, but around absolute effects relatively narrow. Outcome of gonococcal conjunctivitis is of critical significance and can affect vision. Therefore, downgraded one level for imprecision.

4Downgraded for imprecision (-1): optimal information size is not met, but large sample size (7598 neonates in tetracycline group and 6544 neonates in silver nitrate group) with low baseline risk; confidence intervals are wide around relative effects, but around absolute effects relatively narrow. Confidence interval overlaps no effect. RR of $60 \%$ with tetracycline versus RR increase of $2 \%$ with tetracycline. Confidence interval fails to exclude important benefit. Chlamydial conjunctivitis outcome is important. Downgraded for imprecision.

5Downgraded for imprecision (-1): confidence intervals include benefit and harm 
Table 11. Sulfacetamide compared to silver nitrate for the prevention of ophthalmia neonatorum in newborn children

Sulfacetamide compared to silver nitrate for the prevention of ophthalmia neonatorum in newborn children

Patient or population: newborn children

Setting: any maternity setting

Intervention: sulfacetamide

Comparison: silver nitrate

\begin{tabular}{|c|c|c|c|c|c|c|}
\hline \multirow[t]{2}{*}{ Outcomes } & \multicolumn{2}{|c|}{$\begin{array}{l}\text { Anticipated absolute ef- } \\
\text { fects }^{\star}(95 \% \mathrm{Cl})\end{array}$} & \multirow{2}{*}{$\begin{array}{l}\text { Relative } \\
\text { effect } \\
(95 \% \mathrm{CI})\end{array}$} & \multirow{2}{*}{$\begin{array}{l}\text { № of } \\
\text { partici- } \\
\text { pants } \\
\text { (stud- } \\
\text { ies) }\end{array}$} & \multirow{2}{*}{$\begin{array}{l}\text { Certain- } \\
\text { ty of } \\
\text { the evi- } \\
\text { dence } \\
\text { (GRADE) }\end{array}$} & \multirow[t]{2}{*}{ Comments } \\
\hline & $\begin{array}{l}\text { Risk with } \\
\text { silver ni- } \\
\text { trate }\end{array}$ & $\begin{array}{l}\text { Risk with } \\
\text { sulfac- } \\
\text { etamide }\end{array}$ & & & & \\
\hline
\end{tabular}

Blindness (visual acuity less than 20/200)

No studies reported this outcome.

follow-up: 12 months

No studies reported this outcome.

Any adverse visual outcome

follow-up: 12 months

\begin{tabular}{|c|c|c|c|c|}
\hline $\begin{array}{l}\text { Gonococcal conjunctivitis } \\
\text { assessed with: Neisseria gonorrhoeae-positive } \\
\text { culture } \\
\text { follow-up: } 1 \text { month }\end{array}$ & See comment & $\begin{array}{l}640 \\
(1 \mathrm{RCT})\end{array}$ & $\begin{array}{l}\oplus \Theta \Theta \odot \\
\text { VERY } \\
\text { LOW } 1,2\end{array}$ & $\begin{array}{l}\text { The } 1 \text { study re- } \\
\text { ported no cas- } \\
\text { es of gonococ- } \\
\text { cal conjunctivi- } \\
\text { tis (Cousineau } \\
\text { 1952). } \\
\text { follow-up: } 3 \text { to } \\
9 \text { days }\end{array}$ \\
\hline
\end{tabular}

Chlamydial conjunctivitis

assessed with: Chlamydia trachomatis culture, $\mathrm{PCR}$, or direct fluorescent monoclonal antibody

stain

follow-up: 1 month
It is likely that chlamydial conjunctivitis was measured in this 1952 study but not reported in this trial. It is described in other observational studies in the same paper ("virus inclusion bodies").

\begin{tabular}{|c|c|c|c|c|c|c|}
\hline \multirow{2}{*}{$\begin{array}{l}\text { Bacterial conjunctivitis } \\
\text { assessed with: any bacteria-positive culture } \\
\text { follow-up: } 1 \text { month }\end{array}$} & \multicolumn{2}{|c|}{ Study population } & \multirow{2}{*}{$\begin{array}{l}\text { RR } 0.88 \\
(0.45 \text { to } \\
1.74)\end{array}$} & \multirow{2}{*}{$\begin{array}{l}640 \\
(1 \mathrm{RCT})\end{array}$} & \multirow{2}{*}{$\begin{array}{l}\oplus \oplus \odot \odot \\
\mathrm{LOW} 1,3\end{array}$} & \multirow{2}{*}{$\begin{array}{l}\text { Follow-up: } 3 \text { to } \\
9 \text { days }\end{array}$} \\
\hline & $\begin{array}{l}53 \text { per } \\
1000\end{array}$ & $\begin{array}{l}47 \text { per } 1000 \\
\text { ( } 24 \text { to } 92 \text { ) }\end{array}$ & & & & \\
\hline \multirow{2}{*}{$\begin{array}{l}\text { Any conjunctivitis of any aetiology assessed } \\
\text { with: clinical assessment } \\
\text { follow-up: } 1 \text { month }\end{array}$} & \multicolumn{2}{|c|}{ Study population } & \multirow{2}{*}{$\begin{array}{l}\text { RR } 0.54 \\
(0.32 \text { to } \\
0.89)\end{array}$} & \multirow{2}{*}{$\begin{array}{l}640 \\
(1 \mathrm{RCT})\end{array}$} & \multirow{2}{*}{$\begin{array}{l}\oplus \oplus \oplus \odot \\
\text { MODER- } \\
\text { ATE }^{1}\end{array}$} & \multirow{2}{*}{$\begin{array}{l}\text { Follow-up: } 3 \text { to } \\
9 \text { days }\end{array}$} \\
\hline & $\begin{array}{l}122 \text { per } \\
1000\end{array}$ & $\begin{array}{l}66 \text { per } 1000 \\
\text { (39 to } 109)\end{array}$ & & & & \\
\hline $\begin{array}{l}\text { Conjunctivitis of unknown aetiology assessed } \\
\text { with: culture negative } \\
\text { follow-up: } 1 \text { month }\end{array}$ & \multicolumn{2}{|c|}{ Study population } & $\begin{array}{l}\text { RR } 0.27 \\
(0.11 \text { to } \\
0.66)\end{array}$ & $\begin{array}{l}640 \\
(1 \mathrm{RCT})\end{array}$ & $\begin{array}{l}\oplus \oplus \oplus \ominus \\
\text { MODER- } \\
\text { ATE }^{1}\end{array}$ & $\begin{array}{l}\text { Follow-up: } 3 \text { to } \\
9 \text { days }\end{array}$ \\
\hline
\end{tabular}


Table 11. Sulfacetamide compared to silver nitrate for the prevention of ophthalmia neonatorum in newborn children (Continued)

$\begin{array}{ll}69 \text { per } & 19 \text { per } 1000 \\ 1000 & \text { (8 to } 46)\end{array}$

Adverse effects

No studies reported this outcome.

\begin{abstract}
${ }^{\star}$ The risk in the intervention group (and its 95\% confidence interval) is based on the assumed risk in the comparison group and the relative effect of the intervention (and its $95 \% \mathrm{Cl}$ ). The assumed risk is taken from the study population in the included studies.

CI: confidence interval; PCR: polymerase chain reaction; $\mathbf{R C T}$ : randomised controlled trial; RR: risk ratio

\section{GRADE Working Group grades of evidence}

High-certainty: We are very confident that the true effect lies close to that of the estimate of the effect.

Moderate-certainty: We are moderately confident in the effect estimate: the true effect is likely to be close to the estimate of the effect, but there is a possibility that it is substantially different.

Low-certainty: Our confidence in the effect estimate is limited: the true effect may be substantially different from the estimate of the effect.

Very low-certainty: We have very little confidence in the effect estimate: the true effect is likely to be substantially different from the estimate of effect.
\end{abstract}

1Downgraded for risk of bias (-1): single study with high risk of selection bias, high risk of performance bias, unclear risk of detection bias. 2Downgraded for imprecision (-2): no events in either arm of trial; study was underpowered to assess this outcome.

3Downgraded for imprecision (-1): optimal information size criteria not met, and 95\% confidence interval overlaps no effect.

Table 12. Cetyl-pyridinium chloride compared to silver nitrate for the prevention of ophthalmia neonatorum in newborn children

Cetyl-pyridinium chloride compared to silver nitrate for the prevention of ophthalmia neonatorum in newborn children

Patient or population: newborn children

Setting: any maternity setting

Intervention: cetyl-pyridinium chloride

Comparison: silver nitrate

\begin{tabular}{|c|c|c|c|c|c|c|}
\hline \multirow[t]{2}{*}{ Outcomes } & \multicolumn{2}{|c|}{$\begin{array}{l}\text { Anticipated absolute ef- } \\
\text { fects }^{\star}(95 \% \mathrm{Cl})\end{array}$} & \multirow{2}{*}{$\begin{array}{l}\text { Relative } \\
\text { effect } \\
(95 \% \mathrm{CI})\end{array}$} & \multirow{2}{*}{$\begin{array}{l}\text { № of } \\
\text { partici- } \\
\text { pants } \\
\text { (stud- } \\
\text { ies) }\end{array}$} & \multirow{2}{*}{$\begin{array}{l}\text { Certain- } \\
\text { ty of } \\
\text { the evi- } \\
\text { dence } \\
\text { (GRADE) }\end{array}$} & \multirow[t]{2}{*}{$\begin{array}{l}\text { Com- } \\
\text { ments }\end{array}$} \\
\hline & $\begin{array}{l}\text { Risk with } \\
\text { silver ni- } \\
\text { trate }\end{array}$ & $\begin{array}{l}\text { Risk with } \\
\text { cetyl-pyri- } \\
\text { dinium chlo- } \\
\text { ride }\end{array}$ & & & & \\
\hline
\end{tabular}

Blindness (visual acuity less than 20/200)

No studies reported this outcome.

follow-up: 12 months 
Table 12. Cetyl-pyridinium chloride compared to silver nitrate for the prevention of ophthalmia neonatorum in newborn children (Continued)

Gonococcal conjunctivitis

assessed with: Neisseria gonorrhoeae-positive culture

follow-up: 1 month
Gonococcal conjunctivitis not measured and not reported. Study explicitly reported that there was no culture method used to detect Neisseria gonorrhoeae.

\section{Chlamydial conjunctivitis}

assessed with: Chlamydia trachomatis culture, $\mathrm{PCR}$, or direct fluorescent monoclonal antibody stain

follow-up: 1 month
None of the studies reported the diagnosis of chlamydial conjunctivitis. None of the studies specified if chlamydia was measured, which was unlikely considering publication date of 1965 .

\section{Bacterial conjunctivitis assessed with: any bacteria-positive culture follow-up: 1 month}

\begin{tabular}{lll}
\multicolumn{2}{l}{ Study population } & $\begin{array}{l}\text { RR } 1.79 \\
(0.59 \text { to }\end{array}$ \\
\cline { 1 - 2 } 17 per 1000 & $\begin{array}{l}30 \text { per } 1000 \\
(10 \text { to } 92)\end{array}$ & \\
&
\end{tabular}

$\begin{array}{lll}599 & \oplus \oplus \Theta \odot & \text { Fol- } \\ (2 \text { RCTs }) & \text { LOW 1,2 } & \text { low-up: } \\ & & \text { 2 weeks }\end{array}$

\begin{tabular}{|c|c|c|c|c|c|c|}
\hline \multirow{2}{*}{$\begin{array}{l}\text { Any conjunctivitis of any aetiology assessed with: } \\
\text { clinical assessment } \\
\text { follow-up: } 1 \text { month }\end{array}$} & \multicolumn{2}{|c|}{ Study population } & \multirow{2}{*}{$\begin{array}{l}\text { RR } 1.08 \\
(0.40 \text { to } \\
2.90)\end{array}$} & \multirow{2}{*}{$\begin{array}{l}599 \\
(2 \text { RCTs) }\end{array}$} & \multirow{2}{*}{$\begin{array}{l}\oplus \odot \Theta \odot \\
\text { VERY } \\
\text { LOW } 1,2,3\end{array}$} & \multirow{2}{*}{$\begin{array}{l}\text { Fol- } \\
\text { low-up: } \\
2 \text { weeks }\end{array}$} \\
\hline & 27 per 1000 & $\begin{array}{l}29 \text { per } 1000 \\
(11 \text { to } 78)\end{array}$ & & & & \\
\hline \multirow{2}{*}{$\begin{array}{l}\text { Conjunctivitis of unknown aetiology assessed } \\
\text { with: culture negative } \\
\text { follow-up: } 1 \text { month }\end{array}$} & \multicolumn{2}{|c|}{ Study population } & RR 0.14 & & $\oplus \oplus \ominus \ominus$ & Fol- \\
\hline & 10 per 1000 & $\begin{array}{l}1 \text { per } 1000 \\
(0 \text { to } 27)\end{array}$ & 2.71) & & & 2 weeks \\
\hline
\end{tabular}

Adverse effects

No studies reported this outcome.

\begin{abstract}
${ }^{\star}$ The risk in the intervention group (and its 95\% confidence interval) is based on the assumed risk in the comparison group and the relative effect of the intervention (and its $95 \% \mathrm{Cl}$ ). The assumed risk is taken from the study population in the included studies.

CI: confidence interval; PCR: polymerase chain reaction; $\mathbf{R C T}$ : randomised controlled trial; RR: risk ratio

\section{GRADE Working Group grades of evidence}

High-certainty: We are very confident that the true effect lies close to that of the estimate of the effect.

Moderate-certainty: We are moderately confident in the effect estimate: the true effect is likely to be close to the estimate of the effect, but there is a possibility that it is substantially different.

Low-certainty: Our confidence in the effect estimate is limited: the true effect may be substantially different from the estimate of the effect.

Very low-certainty: We have very little confidence in the effect estimate: the true effect is likely to be substantially different from the estimate of effect.
\end{abstract}

1Downgraded for risk of bias (-1): studies at high risk of bias.

2Downgraded for imprecision (-1): optimal information size criteria not met, and 95\% confidence interval overlaps no effect. Confidence intervals very wide.

${ }^{3}$ Downgraded for inconsistency (-1): point estimates on opposite sides of no effect. $\mathrm{I}^{2}$ is $60 \%$, but confidence intervals overlap and and Chi ${ }^{2}$ $P$ value is 0.11 . Borderline. Downgraded.

\title{
Table 13. Penicillin compared to silver nitrate for the prevention of ophthalmia neonatorum in newborn children
}

Penicillin compared to silver nitrate for the prevention of ophthalmia neonatorum in newborn children

Patient or population: newborn children

Setting: any maternity setting 
Table 13. Penicillin compared to silver nitrate for the prevention of ophthalmia neonatorum in newborn clitdreentionmpenicillin

Comparison: silver nitrate

\begin{tabular}{|c|c|c|c|c|c|c|}
\hline \multirow[t]{2}{*}{ Outcomes } & \multicolumn{2}{|c|}{$\begin{array}{l}\text { Anticipated absolute } \\
\text { effects }^{*}(95 \% \mathrm{Cl})\end{array}$} & \multirow{2}{*}{$\begin{array}{l}\text { Rela- } \\
\text { tive } \\
\text { effect } \\
(95 \% \\
\text { CI) }\end{array}$} & \multirow{2}{*}{$\begin{array}{l}\text { № of } \\
\text { par- } \\
\text { tici- } \\
\text { pants } \\
\text { (stud- } \\
\text { ies) }\end{array}$} & \multirow{2}{*}{$\begin{array}{l}\text { Cer- } \\
\text { tain- } \\
\text { ty of } \\
\text { the ev- } \\
\text { idence } \\
\text { (GRADE) }\end{array}$} & \multirow[t]{2}{*}{ Comments } \\
\hline & $\begin{array}{l}\text { Risk } \\
\text { with sil- } \\
\text { ver ni- } \\
\text { trate }\end{array}$ & $\begin{array}{l}\text { Risk with } \\
\text { penicillin }\end{array}$ & & & & \\
\hline
\end{tabular}

Blindness (visual acuity less than 20/200)

No studies reported this outcome.

follow-up: 12 months

No studies reported this outcome.

Any adverse visual outcome

follow-up: 12 months

\begin{tabular}{|c|c|c|c|c|}
\hline $\begin{array}{l}\text { Gonococcal conjunctivitis } \\
\text { assessed with: Neisseria gonorrhoeae-posi- } \\
\text { tive culture } \\
\text { follow-up: } 1 \text { month }\end{array}$ & See comment & $\begin{array}{l}2804 \\
(1 \\
\mathrm{RCT})\end{array}$ & $\begin{array}{l}\oplus \Theta \Theta \odot \\
\text { VERY } \\
\text { LOW } 1,2\end{array}$ & $\begin{array}{l}\text { The } 1 \text { study with this com- } \\
\text { parison measured gonococ- } \\
\text { cal conjunctivitis but found } \\
\text { no cases of gonococcal con- } \\
\text { junctivitis in either study arm } \\
\text { (Davidson 1951). follow-up: } 10 \\
\text { days }\end{array}$ \\
\hline
\end{tabular}

Chlamydial conjunctivitis

assessed with: Chlamydia trachomatis cul-

ture, PCR, or direct fluorescent monoclonal

antibody stain

follow-up: 1 month
Studies comparing penicillin to silver nitrate do not report the diagnosis of chlamydial conjunctivitis. They do not specify if chlamydia was measured, which is understandable considering 1950s publication dates.

\begin{tabular}{|c|c|c|c|c|c|c|}
\hline \multirow{2}{*}{$\begin{array}{l}\text { Bacterial conjunctivitis } \\
\text { assessed with: any bacteria-positive cul- } \\
\text { ture } \\
\text { follow-up: } 1 \text { month }\end{array}$} & \multicolumn{2}{|c|}{ Study population } & \multirow{2}{*}{$\begin{array}{l}\text { RR } \\
0.34 \\
(0.18 \\
\text { to } \\
0.65)\end{array}$} & \multirow{2}{*}{$\begin{array}{l}2804 \\
(1 \\
\text { RCT })\end{array}$} & \multirow{2}{*}{$\begin{array}{l}\oplus \oplus \oplus \ominus \\
\text { MOD- } \\
\text { ERATE1 }\end{array}$} & \multirow{2}{*}{$\begin{array}{l}\text { Davidson } 1951 \\
\text { follow-up: } 10 \text { days }\end{array}$} \\
\hline & $\begin{array}{l}26 \text { per } \\
1000\end{array}$ & $\begin{array}{l}9 \text { per } 1000 \\
(5 \text { to } 17)\end{array}$ & & & & \\
\hline \multirow{2}{*}{$\begin{array}{l}\text { Any conjunctivitis of any aetiology as- } \\
\text { sessed with: clinical assessment } \\
\text { follow-up: } 1 \text { month }\end{array}$} & \multicolumn{2}{|c|}{ Study population } & \multirow[t]{2}{*}{-} & \multirow{2}{*}{$\begin{array}{l}5228 \\
(2 \\
\text { RCTs })\end{array}$} & \multirow{2}{*}{$\begin{array}{l}\oplus \odot \Theta \odot \\
\text { VERY } \\
\text { LOW } 1,2\end{array}$} & \multirow{2}{*}{$\begin{array}{l}\text { Significant statistical hetero- } \\
\text { geneity, therefore meta-analy- } \\
\text { sis not conducted. Hetero- } \\
\text { geneity may be explained by } \\
\text { differing definitions of con- } \\
\text { junctivitis between trials. } \\
\text { follow-up: } 10 \text { days }\end{array}$} \\
\hline & $\begin{array}{l}\text { See } \\
\text { com- } \\
\text { ment }\end{array}$ & $\begin{array}{l}\text { See com- } \\
\text { ment }\end{array}$ & & & & \\
\hline \multirow{2}{*}{$\begin{array}{l}\text { Conjunctivitis of unknown aetiology as- } \\
\text { sessed with: culture negative } \\
\text { follow-up: } 1 \text { month }\end{array}$} & \multicolumn{2}{|c|}{ Study population } & $\mathrm{RR}$ & 2804 & $\oplus \oplus \ominus \ominus$ & Follow-up: 10 days \\
\hline & $\begin{array}{l}263 \text { per } \\
1000\end{array}$ & $\begin{array}{l}34 \text { per } \\
1000 \\
(26 \text { to } 47)\end{array}$ & $\begin{array}{l}(0.10 \\
\text { to } \\
0.18)\end{array}$ & $\mathrm{RCT})$ & & \\
\hline
\end{tabular}


Table 13. Penicillin compared to silver nitrate for the prevention of ophthalmia neonatorum in newborn children (fentinued)

*The risk in the intervention group (and its $95 \%$ confidence interval) is based on the assumed risk in the comparison group and the relative effect of the intervention (and its $95 \% \mathrm{Cl}$ ). The assumed risk is taken from the study population in the included studies.

Cl: confidence interval; PCR: polymerase chain reaction; $\mathbf{R C T}$ : randomised controlled trial; RR: risk ratio

\section{GRADE Working Group grades of evidence}

High-certainty: We are very confident that the true effect lies close to that of the estimate of the effect.

Moderate-certainty: We are moderately confident in the effect estimate: the true effect is likely to be close to the estimate of the effect, but there is a possibility that it is substantially different.

Low-certainty: Our confidence in the effect estimate is limited: the true effect may be substantially different from the estimate of the effect.

Very low-certainty: We have very little confidence in the effect estimate: the true effect is likely to be substantially different from the estimate of effect.

1Downgraded for risk of bias (-1): single trial with high risk of selection bias, unclear and high risk of performance bias, and high risk of attrition bias.

2Downgraded for imprecision (-2): no events in either penicillin or silver nitrate arms of trial. Study was underpowered to assess this outcome.

${ }^{3}$ Downgraded for inconsistency (-1): point estimates vary; confidence intervals do not overlap; 12 is 93\%; Chi² $\mathrm{P}<0.001$.

Table 14. Penicillin IM compared to silver nitrate for the prevention of ophthalmia neonatorum in newborn children

Penicillin IM compared to silver nitrate for the prevention of ophthalmia neonatorum in newborn children

Patient or population: newborn children

Setting: any maternity setting

Intervention: penicillin IM

Comparison: silver nitrate

\begin{tabular}{|c|c|c|c|c|c|c|}
\hline \multirow[t]{2}{*}{ Outcomes } & \multicolumn{2}{|c|}{$\begin{array}{l}\text { Anticipated absolute } \\
\text { effects }^{\star}(95 \% \mathrm{Cl})\end{array}$} & \multirow{2}{*}{$\begin{array}{l}\text { Relative } \\
\text { effect } \\
(95 \% \mathrm{CI})\end{array}$} & \multirow{2}{*}{$\begin{array}{l}\text { № of } \\
\text { partici- } \\
\text { pants } \\
\text { (stud- } \\
\text { ies) }\end{array}$} & \multirow{2}{*}{$\begin{array}{l}\text { Certain- } \\
\text { ty of } \\
\text { the evi- } \\
\text { dence } \\
\text { (GRADE) }\end{array}$} & \multirow[t]{2}{*}{ Comments } \\
\hline & $\begin{array}{l}\text { Risk } \\
\text { with sil- } \\
\text { ver ni- } \\
\text { trate }\end{array}$ & $\begin{array}{l}\text { Risk with } \\
\text { penicillin } \\
\text { IM }\end{array}$ & & & & \\
\hline
\end{tabular}

Blindness (visual acuity less than 20/200)

No studies reported this outcome.

follow-up: 12 months 
Table 14. Penicillin IM compared to silver nitrate for the prevention of ophthalmia neonatorum in newborn children (Continued)

Gonococcal conjunctivitis

assessed with: Neisseria gonorrhoeae-positive culture

follow-up: 1 month
See comment

2727

(1 RCT)

$\oplus \ominus \odot \odot$

VERY

LOW 1,2
The 1 study with follow-up: 10 days measured gonococcal conjunctivitis but found no cases of gonococcal conjunctivitis in either study arm (Davidson 1951).
Chlamydial conjunctivitis assessed with: Chlamydia trachomatis culture, $\mathrm{PCR}$, or direct fluorescent monoclonal antibody stain

follow-up: 1 month
1 study comparing penicillin IM to silver nitrate does not report chlamydial conjunctivitis and does not specify if chlamydia was measured, which is understandable considering the study was published in 1951.

\begin{tabular}{|c|c|c|c|c|c|c|}
\hline \multirow{2}{*}{$\begin{array}{l}\text { Bacterial conjunctivitis } \\
\text { assessed with: any bacteria-positive culture } \\
\text { follow-up: } 1 \text { month }\end{array}$} & \multicolumn{2}{|c|}{ Study population } & \multirow{2}{*}{$\begin{array}{l}\text { RR } 0.75 \\
(0.46 \text { to } \\
1.24)\end{array}$} & \multirow{2}{*}{$\begin{array}{l}2727 \\
(1 \mathrm{RCT})\end{array}$} & \multirow{2}{*}{$\begin{array}{l}\oplus \odot \ominus \ominus \\
\text { VERY } \\
\text { LOW } 1,3\end{array}$} & \multirow{2}{*}{$\begin{array}{l}\text { Follow-up } 10 \text { days } \\
\text { (Davidson 1951). }\end{array}$} \\
\hline & $\begin{array}{l}26 \text { per } \\
1000\end{array}$ & $\begin{array}{l}20 \text { per } 1000 \\
(12 \text { to } 32)\end{array}$ & & & & \\
\hline \multirow{2}{*}{$\begin{array}{l}\text { Any conjunctivitis of any aetiology assessed } \\
\text { with: clinical assessment } \\
\text { follow-up: } 1 \text { month }\end{array}$} & \multicolumn{2}{|c|}{ Study population } & \multirow{2}{*}{$\begin{array}{l}\text { RR } 0.26 \\
(0.21 \text { to } \\
0.32)\end{array}$} & \multirow{2}{*}{$\begin{array}{l}2727 \\
(1 \mathrm{RCT})\end{array}$} & \multirow{2}{*}{$\begin{array}{l}\oplus \oplus \oplus \odot \\
\text { MODER- } \\
\text { ATE1 }^{1}\end{array}$} & \multirow[t]{2}{*}{ Follow-up: 10 days } \\
\hline & $\begin{array}{l}289 \text { per } \\
1000\end{array}$ & $\begin{array}{l}75 \text { per } 1000 \\
\text { ( } 61 \text { to } 93)\end{array}$ & & & & \\
\hline \multirow{2}{*}{$\begin{array}{l}\text { Conjunctivitis of unknown aetiology assessed } \\
\text { with: culture negative } \\
\text { follow-up: } 1 \text { month }\end{array}$} & \multicolumn{2}{|c|}{ Study population } & \multirow{2}{*}{$\begin{array}{l}\text { RR } 0.21 \\
(0.17 \text { to } \\
0.27)\end{array}$} & \multirow{2}{*}{$\begin{array}{l}2727 \\
(1 \mathrm{RCT})\end{array}$} & \multirow{2}{*}{$\begin{array}{l}\oplus \oplus \oplus \odot \\
\text { MODER- } \\
\text { ATE }^{1}\end{array}$} & \multirow[t]{2}{*}{ Follow-up: 10 days } \\
\hline & $\begin{array}{l}263 \text { per } \\
1000\end{array}$ & $\begin{array}{l}55 \text { per } 1000 \\
(45 \text { to } 71)\end{array}$ & & & & \\
\hline
\end{tabular}

Adverse effects

No studies reported this outcome.

\footnotetext{
*The risk in the intervention group (and its 95\% confidence interval) is based on the assumed risk in the comparison group and the relative effect of the intervention (and its $95 \% \mathrm{Cl}$ ). The assumed risk is taken from the study population in the included studies.

CI: confidence interval; IM: intramuscular; PCR: polymerase chain reaction; RCT: randomised controlled trial; RR: risk ratio

\section{GRADE Working Group grades of evidence}

High-certainty: We are very confident that the true effect lies close to that of the estimate of the effect.

Moderate-certainty: We are moderately confident in the effect estimate: the true effect is likely to be close to the estimate of the effect, but there is a possibility that it is substantially different.

Low-certainty: Our confidence in the effect estimate is limited: the true effect may be substantially different from the estimate of the effect.

Very low-certainty: We have very little confidence in the effect estimate: the true effect is likely to be substantially different from the estimate of effect.
}

1Downgraded for risk of bias: single trial with high risk of selection bias, unclear to high risk of performance bias, and unclear to high risk of attrition bias.

2Downgraded for imprecision (-2): no events in either arm, likely not meeting optimal information size criteria.

3Downgraded for imprecision (-1): optimal information size criteria not met; confidence interval wide and crosses null effect. 
Table 15. Povidone-iodine compared to silver nitrate for the prevention of ophthalmia neonatorum in newborn children

\section{Povidone-iodine compared to silver nitrate for the prevention of ophthalmia neonatorum in newborn children}

Patient or population: newborn children

Setting: any maternity setting

Intervention: povidone-iodine

Comparison: silver nitrate

\begin{tabular}{|c|c|c|c|c|c|c|}
\hline \multirow[t]{2}{*}{ Outcomes } & \multicolumn{2}{|c|}{$\begin{array}{l}\text { Anticipated absolute effects }{ }^{*} \\
(95 \% \mathrm{CI})\end{array}$} & \multirow{2}{*}{$\begin{array}{l}\text { Relative } \\
\text { effect } \\
(95 \% \mathrm{Cl})\end{array}$} & \multirow{2}{*}{$\begin{array}{l}\text { № of } \\
\text { par- } \\
\text { tici- } \\
\text { pants } \\
\text { (stud- } \\
\text { ies) }\end{array}$} & \multirow{2}{*}{$\begin{array}{l}\text { Cer- } \\
\text { tain- } \\
\text { ty of } \\
\text { the ev- } \\
\text { idence } \\
\text { (GRADE) }\end{array}$} & \multirow[t]{2}{*}{$\begin{array}{l}\text { Com- } \\
\text { ments }\end{array}$} \\
\hline & $\begin{array}{l}\text { Risk with } \\
\text { silver ni- } \\
\text { trate }\end{array}$ & $\begin{array}{l}\text { Risk with povi- } \\
\text { done-iodine }\end{array}$ & & & & \\
\hline
\end{tabular}

Blindness (visual acuity less than 20/200)

No studies reported this outcome.

follow-up: 12 months

Any adverse visual outcome
follow-up: 12 months

follow-up: 12 months

\begin{tabular}{|c|c|c|c|c|c|c|}
\hline \multirow{2}{*}{$\begin{array}{l}\text { Gonococcal conjunctivitis } \\
\text { assessed with: Neisseria gonorrhoeae-positive culture } \\
\text { follow-up: } 1 \text { month }\end{array}$} & \multicolumn{2}{|c|}{ Study population } & \multirow{2}{*}{$\begin{array}{l}\text { RR } 1.94 \\
(0.60 \text { to } \\
6.29)\end{array}$} & \multirow{2}{*}{$\begin{array}{l}2005 \\
(1 \\
\mathrm{RCT})\end{array}$} & \multirow{2}{*}{$\begin{array}{l}\oplus \oplus \Theta \odot \\
\operatorname{LOW} 1,2\end{array}$} & \multirow{2}{*}{$\begin{array}{l}\text { Isen- } \\
\text { berg } \\
1995\end{array}$} \\
\hline & 4 per 1000 & $\begin{array}{l}8 \text { per } 1000 \\
(3 \text { to } 25)\end{array}$ & & & & \\
\hline \multirow{2}{*}{$\begin{array}{l}\text { Chlamydial conjunctivitis } \\
\text { assessed with: Chlamydia trachomatis culture, PCR, or } \\
\text { direct fluorescent monoclonal antibody stain } \\
\text { follow-up: } 1 \text { month }\end{array}$} & \multicolumn{2}{|c|}{ Study population } & \multirow{2}{*}{$\begin{array}{l}\text { RR } 0.52 \\
(0.38 \text { to } \\
0.71)\end{array}$} & \multirow{2}{*}{$\begin{array}{l}2005 \\
(1 \\
\text { RCT })\end{array}$} & \multirow{2}{*}{$\begin{array}{l}\oplus \oplus \oplus \Theta \\
\text { MOD- } \\
\text { ERATE}^{1}\end{array}$} & \multirow{2}{*}{$\begin{array}{l}\text { Isen- } \\
\text { berg } \\
1995\end{array}$} \\
\hline & $\begin{array}{l}105 \text { per } \\
1000\end{array}$ & $\begin{array}{l}55 \text { per } 1000 \\
(40 \text { to } 75)\end{array}$ & & & & \\
\hline \multirow{2}{*}{$\begin{array}{l}\text { Bacterial conjunctivitis } \\
\text { assessed with: any bacteria-positive culture } \\
\text { follow-up: } 1 \text { month }\end{array}$} & \multicolumn{2}{|c|}{ Study population } & \multirow{2}{*}{$\begin{array}{l}\text { RR } 0.75 \\
(0.61 \text { to } \\
0.92)\end{array}$} & \multirow{2}{*}{$\begin{array}{l}2005 \\
(1 \\
\mathrm{RCT})\end{array}$} & \multirow{2}{*}{$\begin{array}{l}\oplus \oplus \oplus \ominus \\
\text { MOD- } \\
\text { ERATE1 }\end{array}$} & \multirow{2}{*}{$\begin{array}{l}\text { Isen- } \\
\text { berg } \\
1995\end{array}$} \\
\hline & $\begin{array}{l}175 \text { per } \\
1000\end{array}$ & $\begin{array}{l}132 \text { per } 1000 \\
(107 \text { to } 161)\end{array}$ & & & & \\
\hline \multirow{2}{*}{$\begin{array}{l}\text { Any conjunctivitis of any aetiology assessed with: clin- } \\
\text { ical assessment } \\
\text { follow-up: } 1 \text { month }\end{array}$} & \multicolumn{2}{|c|}{ Study population } & \multirow{2}{*}{$\begin{array}{l}\text { RR } 0.72 \\
(0.63 \text { to } \\
0.84)\end{array}$} & \multirow{2}{*}{$\begin{array}{l}2005 \\
(1 \\
\mathrm{RCT})\end{array}$} & \multirow{2}{*}{$\begin{array}{l}\oplus \oplus \oplus \ominus \\
\text { MOD- } \\
\text { ERATE}^{1}\end{array}$} & \multirow{2}{*}{$\begin{array}{l}\text { Isen- } \\
\text { berg } \\
1995\end{array}$} \\
\hline & $\begin{array}{l}314 \text { per } \\
1000\end{array}$ & $\begin{array}{l}226 \text { per } 1000 \\
\text { (198 to } 264)\end{array}$ & & & & \\
\hline \multirow{2}{*}{$\begin{array}{l}\text { Conjunctivitis of unknown aetiology assessed with: } \\
\text { culture negative } \\
\text { follow-up: } 1 \text { month }\end{array}$} & \multicolumn{2}{|c|}{ Study population } & \multirow{2}{*}{$\begin{array}{l}\text { RR } 0.70 \\
(0.55 \text { to } \\
0.89)\end{array}$} & \multirow{2}{*}{$\begin{array}{l}2005 \\
(1 \\
\mathrm{RCT})\end{array}$} & \multirow{2}{*}{$\begin{array}{l}\oplus \oplus \oplus \ominus \\
\text { MOD- } \\
\text { ERATE}^{1}\end{array}$} & \multirow{2}{*}{$\begin{array}{l}\text { Isen- } \\
\text { berg } \\
1995\end{array}$} \\
\hline & $\begin{array}{l}139 \text { per } \\
1000\end{array}$ & $\begin{array}{l}97 \text { per } 1000 \\
\text { (76 to } 124)\end{array}$ & & & & \\
\hline
\end{tabular}

Adverse effects

No studies reported this outcome.

${ }^{*}$ The risk in the intervention group (and its 95\% confidence interval) is based on the assumed risk in the comparison group and the relative effect of the intervention (and its $95 \% \mathrm{Cl}$ ). The assumed risk is taken from the study population in the included studies.

CI: confidence interval; PCR: polymerase chain reaction; $\mathbf{R C T}$ : randomised controlled trial; RR: risk ratio

High-certainty: We are very confident that the true effect lies close to that of the estimate of the effect. 
Table 15. Povidone-iodine compared to silver nitrate for the prevention of ophthalmia neonatorum in newborn children (Continued)

Moderate-certainty: We are moderately confident in the effect estimate: the true effect is likely to be close to the estimate of the effect, but there is a possibility that it is substantially different.

Low-certainty: Our confidence in the effect estimate is limited: the true effect may be substantially different from the estimate of the effect.

Very low-certainty: We have very little confidence in the effect estimate: the true effect is likely to be substantially different from the estimate of effect.

1Downgraded for risk of bias (-1): single trial has high or unclear risk of selection bias, performance bias, and attrition bias.

2Downgraded for imprecision (-1): optimal information size criteria not met, and 95\% confidence interval includes no effect.

Table 16. Tetracycline compared to erythromycin for the prevention of ophthalmia neonatorum in newborn children

\section{Tetracycline compared to erythromycin for the prevention of ophthalmia neonatorum in newborn children}

Patient or population: newborn children

Setting: any maternity setting

Intervention: tetracycline

Comparison: erythromycin

\begin{tabular}{|c|c|c|c|c|c|c|}
\hline \multirow[t]{2}{*}{ Outcomes } & \multicolumn{2}{|c|}{$\begin{array}{l}\text { Anticipated absolute ef- } \\
\text { fects }^{\star}(95 \% \mathrm{CI})\end{array}$} & \multirow{2}{*}{$\begin{array}{l}\text { Relative } \\
\text { effect } \\
(95 \% \mathrm{CI})\end{array}$} & \multirow{2}{*}{$\begin{array}{l}\text { № of } \\
\text { partici- } \\
\text { pants } \\
\text { (stud- } \\
\text { ies) }\end{array}$} & \multirow{2}{*}{$\begin{array}{l}\text { Certain- } \\
\text { ty of } \\
\text { the evi- } \\
\text { dence } \\
\text { (GRADE) }\end{array}$} & \multirow[t]{2}{*}{ Comments } \\
\hline & $\begin{array}{l}\text { Risk } \\
\text { with ery- } \\
\text { thromycin }\end{array}$ & $\begin{array}{l}\text { Risk with } \\
\text { tetracy- } \\
\text { cline }\end{array}$ & & & & \\
\hline
\end{tabular}

Blindness (visual acuity less than 20/200)

No studies reported this outcome.

follow-up: 12 months

No studies reported this outcome.

Any adverse visual outcome

follow-up: 12 months

\begin{tabular}{|c|c|c|c|c|c|c|}
\hline \multirow{2}{*}{$\begin{array}{l}\text { Gonococcal conjunctivitis } \\
\text { assessed with: Neisseria gonorrhoeae-posi- } \\
\text { tive culture } \\
\text { follow-up: } 1 \text { month }\end{array}$} & \multicolumn{2}{|c|}{ Study population } & \multirow{2}{*}{$\begin{array}{l}\text { RR } 0.73 \\
(0.18 \text { to } \\
2.95)\end{array}$} & \multirow{2}{*}{$\begin{array}{l}10,946 \\
\text { (2 RCTs) }\end{array}$} & \multirow{2}{*}{$\begin{array}{l}\oplus \oplus \Theta \Theta \\
\text { LOW } 1,2\end{array}$} & \multirow{2}{*}{$\begin{array}{l}1 \text { of } 2 \text { included } \\
\text { studies did not } \\
\text { identify any cases } \\
\text { of gonococcal con- } \\
\text { junctivitis (Chen } \\
\text { 1992). }\end{array}$} \\
\hline & 1 per 1000 & $\begin{array}{l}1 \text { per } 1000 \\
\text { (0 to } 3)\end{array}$ & & & & \\
\hline
\end{tabular}

\begin{tabular}{|c|c|c|c|c|c|}
\hline \multirow{2}{*}{$\begin{array}{l}\text { Chlamydial conjunctivitis } \\
\text { assessed with: Chlamydia trachomatis cul- } \\
\text { ture, PCR, or direct fluorescent monoclonal } \\
\text { antibody stain } \\
\text { follow-up: } 1 \text { month }\end{array}$} & \multicolumn{2}{|c|}{ Study population } & \multirow{2}{*}{$\begin{array}{l}\text { RR } 0.72 \\
(0.42 \text { to } \\
1.25)\end{array}$} & \multirow{2}{*}{$\begin{array}{l}10,946 \\
\text { (2 RCTs) }\end{array}$} & \multirow{2}{*}{$\begin{array}{l}\oplus \oplus \ominus \ominus \\
\text { LOW } 1,2\end{array}$} \\
\hline & 6 per 1000 & $\begin{array}{l}4 \text { per } 1000 \\
(3 \text { to } 8)\end{array}$ & & & \\
\hline
\end{tabular}

Bacterial conjunctivitis

assessed with: any bacteria-positive culture

follow-up: 1 month
Amongst the 3 studies comparing these interventions, Chen 1992 measured and reported bacterial conjunctivitis, but data could not be extracted, and Hammerschlag 1989 and Ghotbi 2012 did not report and outcome was unlikely measured.

\begin{tabular}{|c|c|c|c|c|}
\hline \multicolumn{2}{|c|}{ Study population } & \multirow{2}{*}{$\begin{array}{l}2539 \\
\text { (2 RCTs) }\end{array}$} & \multirow{2}{*}{$\begin{array}{l}\oplus \odot \Theta \odot \\
\text { VERY } \\
\text { LOW 1,2,3 }\end{array}$} & \multirow{2}{*}{$\begin{array}{l}\text { Data not pooled be- } \\
\text { cause of significant } \\
\text { heterogeneity. }\left.\right|^{2} \text { is } \\
69 \% \text { and point esti- } \\
\text { mates on opposite }\end{array}$} \\
\hline $\begin{array}{l}\text { See com- } \\
\text { ment }\end{array}$ & $\begin{array}{l}\text { See com- } \\
\text { ment }\end{array}$ & & & \\
\hline
\end{tabular}


Table 16. Tetracycline compared to erythromycin for the prevention of ophthalmia neonatorum in newborn children (Continued)

side of line of no effect.

Conjunctivitis of unknown aetiology assessed with: culture negative

follow-up: 1 month
Amongst the 3 studies comparing these interventions, Chen 1992 measured and reported conjunctivitis of unknown aetiology, but data could not be extracted, and Hammerschlag 1989 and Ghotbi 2012 did not report and outcome was unlikely measured.

Adverse effects

No studies reported this outcome.

*The risk in the intervention group (and its $95 \%$ confidence interval) is based on the assumed risk in the comparison group and the relative effect of the intervention (and its $95 \% \mathrm{Cl}$ ). The assumed risk is taken from the study population in the included studies.

CI: confidence interval; PCR: polymerase chain reaction; $\mathbf{R C T}$ : randomised controlled trial; RR: risk ratio

\section{GRADE Working Group grades of evidence}

High-certainty: We are very confident that the true effect lies close to that of the estimate of the effect.

Moderate-certainty: We are moderately confident in the effect estimate: the true effect is likely to be close to the estimate of the ef-

fect, but there is a possibility that it is substantially different.

Low-certainty: Our confidence in the effect estimate is limited: the true effect may be substantially different from the estimate of the effect.

Very low-certainty: We have very little confidence in the effect estimate: the true effect is likely to be substantially different from the estimate of effect.

1Downgraded for risk of bias (-1): studies at unclear or high risk of bias.

2Downgraded for imprecision (-1): optimal information size criteria not met, but sample size is large at 5624 in tetracycline group and 5322 in erythromycin group. However, confidence interval includes no effect, confidence intervals wide, and one trial has no events.

${ }^{3}$ Downgraded for inconsistency (-1): significant statistical heterogeneity. Point estimates on either side of line of no effect. 12 is $69 \%$.

Table 17. Colostrum compared to erythromycin for the prevention of ophthalmia neonatorum in newborn children

Colostrum compared to erythromycin for the prevention of ophthalmia neonatorum in newborn children

Patient or population: newborn children

Setting: any maternity setting

Intervention: colostrum

Comparison: erythromycin

\begin{tabular}{|c|c|c|c|c|c|c|}
\hline \multirow[t]{2}{*}{ Outcomes } & \multicolumn{2}{|c|}{$\begin{array}{l}\text { Anticipated absolute ef- } \\
\text { fects }^{\star}(95 \% \mathrm{Cl})\end{array}$} & \multirow{2}{*}{$\begin{array}{l}\text { Relative } \\
\text { effect } \\
(95 \% \mathrm{CI})\end{array}$} & \multirow{2}{*}{$\begin{array}{l}\text { № of par- } \\
\text { ticipants } \\
\text { (studies) }\end{array}$} & \multirow{2}{*}{$\begin{array}{l}\text { Certainty } \\
\text { of the evi- } \\
\text { dence } \\
\text { (GRADE) }\end{array}$} & \multirow[t]{2}{*}{$\begin{array}{l}\text { Com- } \\
\text { ments }\end{array}$} \\
\hline & $\begin{array}{l}\text { Risk } \\
\text { with ery- } \\
\text { thromycin }\end{array}$ & $\begin{array}{l}\text { Risk with } \\
\text { colostrum }\end{array}$ & & & & \\
\hline
\end{tabular}

Blindness (visual acuity less than 20/200)

No studies reported this outcome.

follow-up: 12 months

Any adverse visual outcome

No studies reported this outcome.

follow-up: 12 months

Gonococcal conjunctivitis

assessed with: Neisseria gonorrhoeae-positive culture

follow-up: 1 month
The 1 study excluded neonates with any positive cultures before administration of prophylaxis. Gonococcal conjunctivitis was not reported, and there is no evidence that it was measured. 
Table 17. Colostrum compared to erythromycin for the prevention of ophthalmia neonatorum in newborn

children (çntinued)

assessed with: Chlamydia trachomatis culture, PCR, or direct fluorescent monoclonal antibody stain

follow-up: 1 month
The 1 study excluded neonates with any positive cultures before administration of prophylaxis. Chlamydial conjunctivitis was not reported, and there is no evidence that it was measured.
Bacterial conjunctivitis assessed with: any bacteria-positive culture follow-up: 1 month
The 1 study excluded neonates with any positive cultures before administration of prophylaxis. Bacterial conjunctivitis was reported, but data could not be extracted.

\begin{tabular}{|c|c|c|c|c|c|}
\hline \multirow{2}{*}{$\begin{array}{l}\text { Any conjunctivitis of any aetiology assessed } \\
\text { with: clinical assessment } \\
\text { follow-up: } 1 \text { month }\end{array}$} & Study popula & & \multirow{2}{*}{$\begin{array}{l}\text { RR } 1.49 \\
(0.80 \text { to } \\
2.78)\end{array}$} & \multirow{2}{*}{$\begin{array}{l}171 \\
(1 \mathrm{RCT})\end{array}$} & \multirow{2}{*}{$\begin{array}{l}\oplus \oplus \ominus \odot \\
\text { LOW } 1,2\end{array}$} \\
\hline & 159 per 1000 & $\begin{array}{l}236 \text { per } 1000 \\
\text { (127 to } 442 \text { ) }\end{array}$ & & & \\
\hline
\end{tabular}

Conjunctivitis of unknown aetiology assessed with: culture negative

follow-up: 1 month
The 1 study excluded neonates with any positive cultures before administration of prophylaxis. Conjunctivitis of unknown aetiology was not reported, and there was no evidence that it was measured.

Adverse effects No studies reported this outcome.

*The risk in the intervention group (and its $95 \%$ confidence interval) is based on the assumed risk in the comparison group and the relative effect of the intervention (and its $95 \% \mathrm{Cl}$ ). The assumed risk is taken from the study population in the included studies.

CI: confidence interval; PCR: polymerase chain reaction; $\mathbf{R C T}$ : randomised controlled trial; RR: risk ratio

\section{GRADE Working Group grades of evidence}

High-certainty: We are very confident that the true effect lies close to that of the estimate of the effect.

Moderate-certainty: We are moderately confident in the effect estimate: the true effect is likely to be close to the estimate of the effect, but there is a possibility that it is substantially different.

Low-certainty: Our confidence in the effect estimate is limited: the true effect may be substantially different from the estimate of the effect.

Very low-certainty: We have very little confidence in the effect estimate: the true effect is likely to be substantially different from the estimate of effect.

1Downgraded for risk of bias (-1): single trial at unclear or high risk of performance bias and attrition bias.

2Downgraded for imprecision (-1): optimal information size criteria not met, and 95\% confidence interval includes no effect.

Table 18. Povidone-iodine compared to erythromycin for the prevention of ophthalmia neonatorum in newborn children

Povidone-iodine compared to erythromycin for the prevention of ophthalmia neonatorum in newborn children

Patient or population: newborn children

Setting: any maternity setting

Intervention: povidone-iodine

Comparison: erythromycin

\begin{tabular}{|c|c|c|c|c|c|c|}
\hline \multirow[t]{2}{*}{ Outcomes } & \multicolumn{2}{|c|}{$\begin{array}{l}\text { Anticipated absolute ef- } \\
\text { fects }^{*}(95 \% \mathrm{CI})\end{array}$} & \multirow{2}{*}{$\begin{array}{l}\text { Relative } \\
\text { effect } \\
(95 \% \mathrm{CI})\end{array}$} & \multirow{2}{*}{$\begin{array}{l}\text { № of } \\
\text { par- } \\
\text { tici- } \\
\text { pants } \\
\text { (stud- } \\
\text { ies) }\end{array}$} & \multirow{2}{*}{$\begin{array}{l}\text { Cer- } \\
\text { tain- } \\
\text { ty of } \\
\text { the ev- } \\
\text { idence } \\
\text { (GRADE) }\end{array}$} & \multirow[t]{2}{*}{$\begin{array}{l}\text { Com- } \\
\text { ments }\end{array}$} \\
\hline & $\begin{array}{l}\text { Risk } \\
\text { with ery- } \\
\text { thromycin }\end{array}$ & $\begin{array}{l}\text { Risk with povi- } \\
\text { done-iodine }\end{array}$ & & & & \\
\hline
\end{tabular}

Blindness (visual acuity less than 20/200)

No studies reported this outcome. 
Table 18. Povidone-iodine compared to erythromycin for the prevention of ophthalmia neonatorum in newborn children (Continued)

follow-up: 12 months

Any adverse visual outcome No studies reported this outcome.
follow-up: 12 months

follow-up: 12 months

Gonococcal conjunctivitis
assessed with: Neisseria gonorrhoeae-positive culture
follow-up: 1 month

follow-up: 1 month

\begin{tabular}{lll} 
Study population & $\begin{array}{l}\text { RR } 0.85 \\
(0.36 \text { to }\end{array}$ \\
\hline 9 per 1000 & $\begin{array}{l}8 \text { per } 1000 \\
\text { (3 to } 18)\end{array}$ &
\end{tabular}

2408
$(2$
RCTs $)$

$\oplus \oplus \ominus \ominus \quad 1$ of the

LOW 1,2 2 stud-

(3 to 18$)$ 
3Downgraded for imprecision (-1): optimal information size criterion not met.

Table 19. Penicillin IM compared to tetracycline for the prevention of ophthalmia neonatorum in newborn children

\section{Penicillin IM compared to tetracycline for the prevention of ophthalmia neonatorum in newborn children}

Patient or population: newborn children

Setting: any maternity setting

Intervention: penicillin IM

Comparison: tetracycline

\begin{tabular}{|c|c|c|c|c|c|c|}
\hline \multirow[t]{2}{*}{ Outcomes } & \multicolumn{2}{|c|}{$\begin{array}{l}\text { Anticipated absolute } \\
\text { effects }^{\star}(95 \% \mathrm{Cl})\end{array}$} & \multirow{2}{*}{$\begin{array}{l}\text { Relative } \\
\text { effect } \\
(95 \% \mathrm{Cl})\end{array}$} & \multirow{2}{*}{$\begin{array}{l}\text { № of } \\
\text { partici- } \\
\text { pants } \\
\text { (stud- } \\
\text { ies) }\end{array}$} & \multirow{2}{*}{$\begin{array}{l}\text { Certainty } \\
\text { of the ev- } \\
\text { idence } \\
\text { (GRADE) }\end{array}$} & \multirow[t]{2}{*}{ Comments } \\
\hline & $\begin{array}{l}\text { Risk } \\
\text { with } \\
\text { tetracy- } \\
\text { cline }\end{array}$ & $\begin{array}{l}\text { Risk with } \\
\text { penicillin } \\
\text { IM }\end{array}$ & & & & \\
\hline
\end{tabular}

Blindness (visual acuity less than 20/200)

No studies reported this outcome.

follow-up: 12 months

Any adverse visual outcome

follow-up: 12 months
No studies reported this outcome.

32,058

$(1 \mathrm{RCT})$

See comment

The 1 study with

this comparison

measured gonococcal conjunctivitis but reported no cases in either study arm (Siegel 1982).

follow-up: 41 months

\begin{tabular}{|c|c|c|c|c|c|c|}
\hline \multirow{2}{*}{$\begin{array}{l}\text { Chlamydial conjunctivitis } \\
\text { assessed with: Chlamydia trachomatis cul- } \\
\text { ture, PCR, or direct fluorescent monoclonal } \\
\text { antibody stain }\end{array}$} & \multicolumn{2}{|c|}{ Study population } & \multirow{2}{*}{$\begin{array}{l}\text { RR } 0.75 \\
(0.48 \text { to } \\
1.17)\end{array}$} & \multirow{2}{*}{$\begin{array}{l}32,058 \\
(1 \mathrm{RCT})\end{array}$} & \multirow{2}{*}{$\begin{array}{l}\oplus \oplus \oplus \ominus \\
\text { MODER- } \\
\text { ATE }^{1,2}\end{array}$} & \multirow{2}{*}{$\begin{array}{l}\text { Siegel } 1982 \\
\text { follow-up: } 41 \\
\text { months }\end{array}$} \\
\hline & $\begin{array}{l}3 \text { per } \\
1000\end{array}$ & $\begin{array}{l}2 \text { per } 1000 \\
(1 \text { to } 4)\end{array}$ & & & & \\
\hline
\end{tabular}

follow-up: 1 month

In the 1 study with these 2 interventions (Siegel 1982), it is uncertain whether all cases of bacterial conjunctivitis were measured. Bacterial conjunctivitis was not reported in this study.

assessed with: any bacteria-positive culture follow-up: 1 month

Any conjunctivitis of any aetiology assessed with: clinical assessment follow-up: 1 month
Total conjunctivitis cases of any aetiology (ACAE) was not reported in the 1 study with this comparison (Siegel 1982). It is uncertain whether ACAE was measured.

\section{Conjunctivitis of unknown aetiology assessed In the 1 study comparing these interventions (Siegel 1982), conjunctivitis of un- with: culture negative known aetiology was not reported, and it is uncertain if it was measured.}

follow-up: 1 month

Adverse effects

No studies reported this outcome.

\footnotetext{
${ }^{\star}$ The risk in the intervention group (and its 95\% confidence interval) is based on the assumed risk in the comparison group and the relative effect of the intervention (and its $95 \% \mathrm{Cl}$ ). The assumed risk taken from the study population in the included studies.
} 
Table 20. Povidone-iodine compared to tetracycline for the prevention of ophthalmia neonatorum in newborn children (Continued)

\begin{tabular}{|c|c|c|c|c|c|c|}
\hline & & $\begin{array}{l}\text { (49 to } \\
209)\end{array}$ & $\begin{array}{l}(0.99 \\
\text { to } \\
4.22)\end{array}$ & & & \\
\hline \multirow{2}{*}{$\begin{array}{l}\text { Any conjunctivitis of any aetiology assessed } \\
\text { with: clinical assessment } \\
\text { follow-up: } 1 \text { month }\end{array}$} & \multicolumn{2}{|c|}{ Study population } & \multirow{2}{*}{$\begin{array}{l}\text { RR } \\
3.01 \\
(1.52 \\
\text { to } \\
5.98)\end{array}$} & \multirow{2}{*}{$\begin{array}{l}410 \\
(1 \\
\mathrm{RCT})\end{array}$} & \multirow{2}{*}{$\begin{array}{l}\oplus \oplus \Theta \Theta \\
\mathrm{LOW} \\
1,5\end{array}$} & \\
\hline & $\begin{array}{l}50 \text { per } \\
1000\end{array}$ & $\begin{array}{l}149 \text { per } \\
1000 \\
(75 \text { to } \\
296)\end{array}$ & & & & \\
\hline \multirow{2}{*}{$\begin{array}{l}\text { Conjunctivitis of unknown aetiology as- } \\
\text { sessed with: culture negative } \\
\text { follow-up: } 1 \text { months }\end{array}$} & \multicolumn{2}{|c|}{ Study population } & \multirow{2}{*}{$\begin{array}{l}\mathrm{RR} \\
20.4 \\
(1.2 \text { to } \\
345.8)\end{array}$} & \multirow{2}{*}{$\begin{array}{l}410 \\
(1 \\
\mathrm{RCT})\end{array}$} & \multirow{2}{*}{$\begin{array}{l}\oplus \oplus \Theta \odot \\
\mathrm{LOW} \\
1,6\end{array}$} & \multirow{2}{*}{$\begin{array}{l}\text { There were no cases of conjunc- } \\
\text { tivitis in the comparator group. } \\
\text { The value of } 1 \text { per } 1000 \text { is the risk } \\
\text { for tetracycline for illustrative pur } \\
\text { poses only. }\end{array}$} \\
\hline & $\begin{array}{l}1 \text { per } \\
1000\end{array}$ & $\begin{array}{l}20 \text { per } \\
1000 \\
(1 \text { to } \\
346)\end{array}$ & & & & \\
\hline
\end{tabular}

${ }^{*}$ The risk in the intervention group (and its 95\% confidence interval) is based on the assumed risk in the comparison group and the relative effect of the intervention (and its $95 \% \mathrm{Cl}$ ). The assumed risk is taken from the study population in the included studies.

CI: confidence interval; PCR: polymerase chain reaction; $\mathbf{R C T}$ : randomised controlled trial; RR: risk ratio

\section{GRADE Working Group grades of evidence}

High-certainty: We are very confident that the true effect lies close to that of the estimate of the effect.

Moderate-certainty: We are moderately confident in the effect estimate: the true effect is likely to be close to the estimate of the effect, but there is a possibility that it is substantially different.

Low-certainty: Our confidence in the effect estimate is limited: the true effect may be substantially different from the estimate of the effect.

Very low-certainty: We have very little confidence in the effect estimate: the true effect is likely to be substantially different from the estimate of effect.

1Downgraded for risk of bias (-1): study has unclear or high risk of bias, which could influence outcome.

2Downgraded for imprecision (-1): small trial size and lack of detection of any gonorrhoea cases.

3Downgraded for imprecision (-1): small trial size and lack of detection of any chlamydia cases.

4Downgraded for imprecision (-1): optimal information size criteria not met; confidence interval overlaps no effect; small trial size.

5Downgraded for imprecision (-1): optimal information size criteria met, but one trial, wide confidence interval, low event rates, small sample size.

${ }^{6}$ Downgraded for imprecision (-1): optimal information size criteria possibly met, but one trial, wide confidence interval, low event rates, small sample size.

Table 21. Povidone-iodine compared to chloramphenicol for the prevention of ophthalmia neonatorum in newborn children

Povidone-iodine compared to chloramphenicol for the prevention of ophthalmia neonatorum in newborn children

Patient or population: newborn children

Setting: any maternity setting

Intervention: povidone-iodine

Comparison: chloramphenicol

\begin{tabular}{llll}
\hline Outcomes & $\begin{array}{l}\text { Anticipated absolute } \\
\text { effects }{ }^{*}(95 \% \mathrm{Cl})\end{array}$ & $\begin{array}{l}\text { Rela- } \\
\text { tive ef- } \begin{array}{l}\text { No of } \\
\text { partici- } \\
\text { fect }\end{array}\end{array}$ & $\begin{array}{l}\text { Certain- Comments } \\
\text { ty of }\end{array}$
\end{tabular}


Table 21. Povidone-iodine compared to chloramphenicol for the prevention of ophthalmia neonatorum in newborn children (Continued)

\begin{tabular}{|c|c|c|c|c|c|}
\hline (1) & $\begin{array}{l}\text { Risk } \\
\text { with } \\
\text { chlo- } \\
\text { ram- } \\
\text { phenicol }\end{array}$ & $\begin{array}{l}\text { Risk with } \\
\text { povi- } \\
\text { done-io- } \\
\text { dine }\end{array}$ & $\begin{array}{l}(95 \% \\
\mathrm{CI})\end{array}$ & $\begin{array}{l}\text { (stud- } \\
\text { ies) }\end{array}$ & $\begin{array}{l}\text { the evi- } \\
\text { dence } \\
\text { (GRADE) }\end{array}$ \\
\hline
\end{tabular}

Blindness (visual acuity less than 20/200)

follow-up: 12 months
No studies reported this outcome.

No studies reported this outcome.
Any adverse visual outcome

follow-up: 12 months

\section{Gonococcal conjunctivitis} assessed with: Neisseria gonorrhoeae-positive culture follow-up: 1 month

\section{See comment}

$\begin{array}{ll}2004 & \oplus \oplus \odot \ominus \\ (1 \mathrm{RCT}) & \text { LOW } 1,2\end{array}$

1 included

study mea-

sured gonococcal conjunctivitis but did not find any cases in either study arm (RamirezOrtiz 2007).

\begin{tabular}{|c|c|c|c|c|c|c|}
\hline \multirow{2}{*}{$\begin{array}{l}\text { Chlamydial conjunctivitis } \\
\text { assessed with: Chlamydia trachomatis culture, PCR, or } \\
\text { direct fluorescent monoclonal antibody stain } \\
\text { follow-up: } 1 \text { month }\end{array}$} & \multicolumn{2}{|c|}{ Study population } & \multirow{2}{*}{$\begin{array}{l}\text { RR } 1.77 \\
(0.97 \text { to } \\
3.22)\end{array}$} & \multirow{2}{*}{$\begin{array}{l}2004 \\
(1 \mathrm{RCT})\end{array}$} & \multirow{2}{*}{$\begin{array}{l}\oplus \oplus \odot \odot \\
\text { LOW } 1,3\end{array}$} & \multirow{2}{*}{$\begin{array}{l}\text { Ramirez-Ortiz } \\
2007\end{array}$} \\
\hline & $\begin{array}{l}16 \text { per } \\
1000\end{array}$ & $\begin{array}{l}28 \text { per } \\
1000 \\
(16 \text { to } 52)\end{array}$ & & & & \\
\hline
\end{tabular}

Bacterial conjunctivitis assessed with: any bacteria-positive culture follow-up: 1 month
The 1 study measured and reported cases of bacterial conjunctivitis, but data could not be extracted (Ramirez-Ortiz 2007).
Any conjunctivitis of any aetiology assessed with: clinical assessment follow-up: 1 month
The 1 study measured and reported cases of any conjunctivitis of any aetiology, but data could not be extracted (Ramirez-Ortiz 2007).

The 1 study likely measured conjunctivitis of unknown aetiology, but data could not be extracted (Ramirez-Ortiz 2007).

Adverse effects No studies reported this outcome.

*The risk in the intervention group (and its $95 \%$ confidence interval) is based on the assumed risk in the comparison group and the relative effect of the intervention (and its $95 \% \mathrm{Cl}$ ). The assumed risk is taken from the study population in the included studies.

Cl: confidence interval; PCR: polymerase chain reaction; RCT: randomised controlled trial; RR: risk ratio

\section{GRADE Working Group grades of evidence}

High-certainty: We are very confident that the true effect lies close to that of the estimate of the effect.

Moderate-certainty: We are moderately confident in the effect estimate: the true effect is likely to be close to the estimate of the effect, but there is a possibility that it is substantially different.

Low-certainty: Our confidence in the effect estimate is limited: the true effect may be substantially different from the estimate of the effect.

Very low-certainty: We have very little confidence in the effect estimate: the true effect is likely to be substantially different from the estimate of effect. 
2Downgraded for imprecision (-1): zero event rates, sample size small, and unable to determine relative effects; single trial.

3Downgraded for imprecision (-1): optimal information size not met, and confidence interval overlaps no effect; single trial.

Table 22. Povidone-iodine compared to carbethopendecinium bromide for the prevention of ophthalmia neonatorum in newborn children

Povidone-iodine compared to carbethopendecinium bromide for the prevention of ophthalmia neonatorum in newborn children

Patient or population: newborn children

Setting: any maternity setting

Intervention: povidone-iodine

Comparison: carbethopendecinium bromide

\begin{tabular}{|c|c|c|c|c|c|c|}
\hline \multirow[t]{2}{*}{ Outcomes } & \multicolumn{2}{|c|}{$\begin{array}{l}\text { Anticipated absolute ef- } \\
\text { fects }^{\star}(95 \% \mathrm{Cl})\end{array}$} & \multirow{2}{*}{$\begin{array}{l}\text { Relative } \\
\text { effect } \\
(95 \% \mathrm{CI})\end{array}$} & \multirow{2}{*}{$\begin{array}{l}\text { № of par- } \\
\text { ticipants } \\
\text { (studies) }\end{array}$} & \multirow{2}{*}{$\begin{array}{l}\text { Certainty } \\
\text { of the ev- } \\
\text { idence } \\
\text { (GRADE) }\end{array}$} & \multirow[t]{2}{*}{$\begin{array}{l}\text { Com- } \\
\text { ments }\end{array}$} \\
\hline & $\begin{array}{l}\text { Risk } \\
\text { with car- } \\
\text { bethopen- } \\
\text { decinium } \\
\text { bromide }\end{array}$ & $\begin{array}{l}\text { Risk with } \\
\text { povi- } \\
\text { done-iodine }\end{array}$ & & & & \\
\hline
\end{tabular}

Blindness (visual acuity less than 20/200)

No studies reported this outcome.

follow-up: 12 months

Any adverse visual outcome No studies reported this outcome.

follow-up: 12 months

Gonococcal conjunctivitis

assessed with: Neisseria gonorrhoeae-positive culture

follow-up: 1 month
Translation of the 1 study does not make any reference to gonococcal conjunctivitis (GC). There is no reference to culturing. It is unknown if GC was measured. GC cases were not reported.
Chlamydial conjunctivitis assessed with: Chlamydia trachomatis culture, PCR, or direct fluorescent monoclonal antibody stain

follow-up: 1 month
Translation of the 1 study does not make any reference to chlamydial conjunctivitis (CC). It unknown if CC was measured. No reference to culturing. No CC cases were reported.

\section{Bacterial conjunctivitis}

assessed with: any bacteria-positive culture follow-up: 1 month
Translation of the 1 study does not make reference to bacterial conjunctivitis (BC). It unknown if $B C$ was measured. No reference to culturing. No BC cases reported.

\begin{tabular}{|c|c|c|c|c|c|c|}
\hline \multirow{2}{*}{$\begin{array}{l}\text { Any conjunctivitis of any aetiology assessed } \\
\text { with: clinical assessment } \\
\text { follow-up: } 1 \text { month }\end{array}$} & \multicolumn{2}{|c|}{ Study population } & \multirow{2}{*}{$\begin{array}{l}\text { RR } 0.44 \\
(0.15 \text { to } \\
1.35)\end{array}$} & \multirow{2}{*}{$\begin{array}{l}100 \\
(1 \mathrm{RCT})\end{array}$} & \multirow{2}{*}{$\begin{array}{l}\oplus \oplus \ominus \ominus \\
\text { LOW } 1,2\end{array}$} & \multirow{2}{*}{$\begin{array}{l}1 \text { trial on- } \\
\text { ly (Zbojan } \\
2004) \text {. }\end{array}$} \\
\hline & 180 per 1000 & $\begin{array}{l}79 \text { per } 1000 \\
\text { (27 to } 243 \text { ) }\end{array}$ & & & & \\
\hline
\end{tabular}

Conjunctivitis of unknown aetiology assessed with: culture negative follow-up: 1 month
Translation of the 1 study does not make reference to conjunctivitis of unknown aetiology (CUE). It unknown if CUE was measured. No CUE cases were reported and CUE could not be calculated.

Adverse effects No studies reported this outcome.

*The risk in the intervention group (and its 95\% confidence interval) is based on the assumed risk in the comparison group and the relative effect of the intervention (and its $95 \% \mathrm{Cl}$ ). The assumed risk is taken from the study population in the included studies.

Cl: confidence interval; PCR: polymerase chain reaction; $\mathbf{R C T}$ : randomised controlled trial; RR: risk ratio 
Table 22. Povidone-iodine compared to carbethopendecinium bromide for the prevention of ophthalmia neonatorum in newborn children (Continued)

GRADE Working Group grades of evidence

High-certainty: We are very confident that the true effect lies close to that of the estimate of the effect.

Moderate-certainty: We are moderately confident in the effect estimate: the true effect is likely to be close to the estimate of the ef-

fect, but there is a possibility that it is substantially different.

Low-certainty: Our confidence in the effect estimate is limited: the true effect may be substantially different from the estimate of the effect.

Very low-certainty: We have very little confidence in the effect estimate: the true effect is likely to be substantially different from the estimate of effect.

1Downgraded for risk of bias (-1): single trial with unclear risk of selection bias, performance bias, detection bias, and attrition bias.

2Downgraded for imprecision (-1): optimal information size criteria not met, and 95\% confidence interval includes no effect; small trial.

Table 23. Double application of povidone-iodine compared to single application of povidone-iodine for the prevention of ophthalmia neonatorum in newborn children

Double application of povidone-iodine compared to single application of povidone-iodine for the prevention of ophthalmia neonatorum in newborn children

Patient or population: newborn children

Setting: any maternity setting

Intervention: double application of povidone-iodine

Comparison: single application of povidone-iodine

\begin{tabular}{|c|c|c|c|c|c|c|}
\hline \multirow[t]{2}{*}{ Outcomes } & \multicolumn{2}{|c|}{$\begin{array}{l}\text { Anticipated absolute effects }{ }^{*} \\
(95 \% \mathrm{CI})\end{array}$} & \multirow{2}{*}{$\begin{array}{l}\text { Relative } \\
\text { effect } \\
(95 \% \mathrm{CI})\end{array}$} & \multirow{2}{*}{$\begin{array}{l}\text { № of } \\
\text { par- } \\
\text { tici- } \\
\text { pants } \\
\text { (stud- } \\
\text { ies) }\end{array}$} & \multirow{2}{*}{$\begin{array}{l}\text { Cer- } \\
\text { tain- } \\
\text { ty of } \\
\text { the ev- } \\
\text { idence } \\
\text { (GRADE) }\end{array}$} & \multirow[t]{2}{*}{$\begin{array}{l}\text { Com- } \\
\text { ments }\end{array}$} \\
\hline & $\begin{array}{l}\text { Risk with } \\
\text { single ap- } \\
\text { plication } \\
\text { of povi- } \\
\text { done-io- } \\
\text { dine }\end{array}$ & $\begin{array}{l}\text { Risk with dou- } \\
\text { ble applica- } \\
\text { tion of povi- } \\
\text { done-iodine }\end{array}$ & & & & \\
\hline
\end{tabular}

Blindness (visual acuity less than 20/200)

No studies reported this outcome.

follow-up: 12 months

Any adverse visual outcome $\quad$ No studies reported this outcome.
follow-up: 12 months

follow-up: 12 months

Gonococcal conjunctivitis See comment

assessed with: Neisseria gonorrhoeae-positive cul-

ture

follow-up: 1 month

$\begin{array}{lll}719 & \oplus \ominus \odot \odot & \text { The 1 } \\ (1 & \text { VERY } & \text { study } \\ \text { RCT) } & \text { LOW 1,2 } & \text { measured } \\ & & \text { cases of } \\ & \text { gonococ- } \\ & \text { cal con- } \\ & \text { junctivi- } \\ & \text { tis, but } \\ & \text { no cas- } \\ & \text { es were } \\ & \text { found } \\ & \text { in either } \\ & \text { study arm } \\ & \text { (Isenberg } \\ & \text { 2003). }\end{array}$


Table 23. Double application of povidone-iodine compared to single application of povidone-iodine for the prevention of ophthalmia neonatorum in newborn children (Continued)

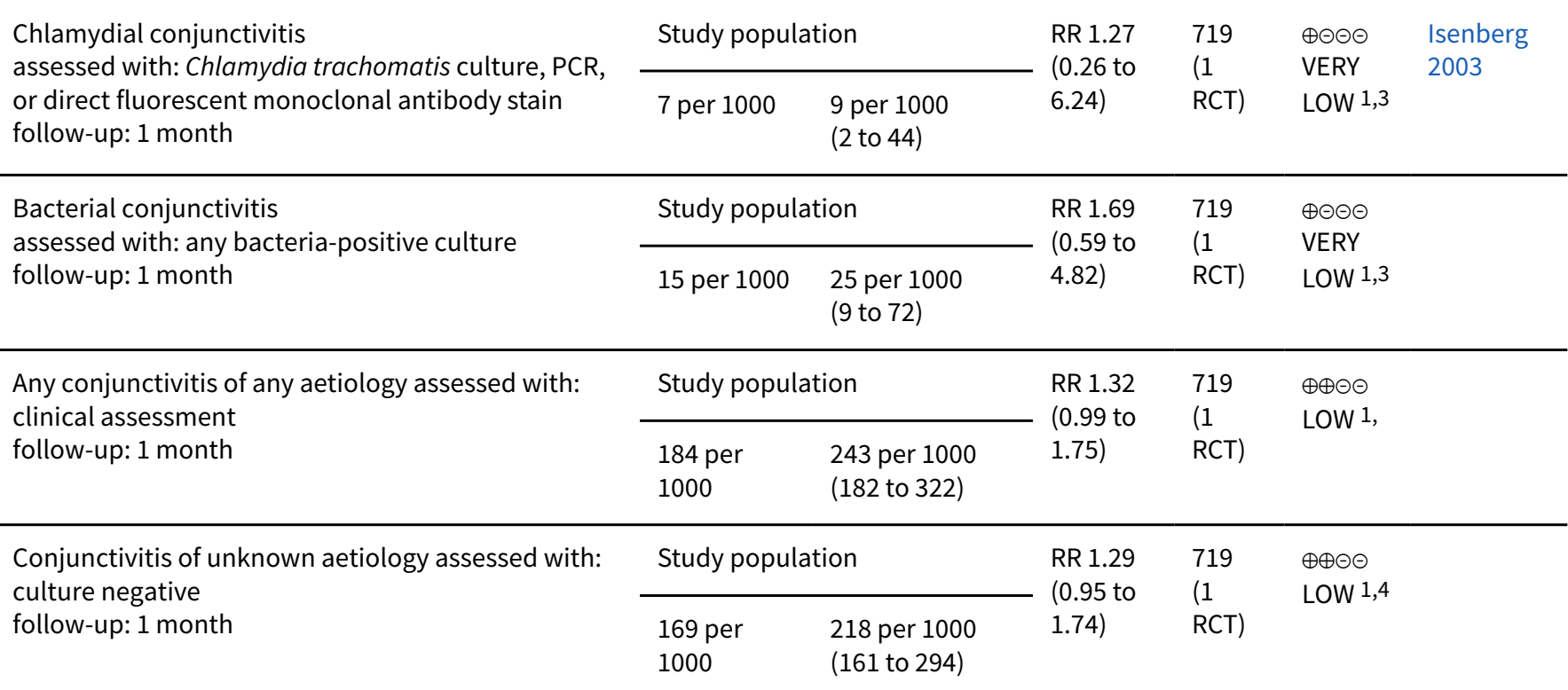

Adverse effects

No studies reported this outcome.

* The risk in the intervention group (and its $95 \%$ confidence interval) is based on the assumed risk in the comparison group and the relative effect of the intervention (and its $95 \% \mathrm{Cl}$ ). The assumed risk is taken from the study population in the included studies.

CI: confidence interval; PCR: polymerase chain reaction; $\mathbf{R C T}$ : randomised controlled trial; RR: risk ratio

\section{GRADE Working Group grades of evidence}

High-certainty: We are very confident that the true effect lies close to that of the estimate of the effect.

Moderate-certainty: We are moderately confident in the effect estimate: the true effect is likely to be close to the estimate of the effect, but there is a possibility that it is substantially different.

Low-certainty: Our confidence in the effect estimate is limited: the true effect may be substantially different from the estimate of the effect.

Very low-certainty: We have very little confidence in the effect estimate: the true effect is likely to be substantially different from the estimate of effect.

1Downgraded for risk of bias (-1): single trial has high risk of selection bias, unclear risk of performance bias, and unclear risk of attrition bias.

2Downgraded for imprecision (-2): no events in either arm.

3Downgraded for imprecision (-2): limited number of events in each arm, very wide confidence intervals.

${ }^{4}$ Downgraded for imprecision (-1): optimal information size criteria not met, and 95\% confidence interval includes no effect.

Table 24. Pencillin IM compared to topical penicillin ointment for the prevention of ophthalmia neonatorum in newborn children

Pencillin IM compared to topical penicillin ointment for the prevention of ophthalmia neonatorum in newborn children

Patient or population: newborn children

Setting: any maternity setting

Intervention: penicillin IM

Comparison: topical penicillin ointment

\section{Outcomes}

Anticipated absolute ef-
fects $^{*}(95 \% \mathrm{Cl})$

$\begin{array}{llll}\begin{array}{l}\text { Relative } \\ \text { effect }\end{array} & \begin{array}{l}\text { № of } \\ \text { partici- } \\ \text { pants }\end{array} & \begin{array}{l}\text { Certain- } \\ \text { ty of }\end{array} & \text { Comments } \\ (95 \% \mathrm{Cl}) & & \end{array}$


Table 24. Pencillin IM compared to topical penicillin ointment for the prevention of ophthalmia neonatorum in newborn children (Continued)

\begin{tabular}{|c|c|c|c|c|}
\hline & $\begin{array}{ll}\text { Risk with } & \text { Risk with } \\
\text { topical } & \text { penicillin IM } \\
\text { penicillin } & \\
\text { ointment } & \end{array}$ & $\begin{array}{l}\text { (stud- } \\
\text { ies) }\end{array}$ & $\begin{array}{l}\text { the evi- } \\
\text { dence } \\
\text { (GRADE) }\end{array}$ & \\
\hline $\begin{array}{l}\text { Blindness (visual acuity less than } 20 / 200 \text { ) } \\
\text { follow-up: } 12 \text { months }\end{array}$ & No studies reported this outcome. & & & \\
\hline $\begin{array}{l}\text { Any adverse visual outcome } \\
\text { follow-up: } 12 \text { months }\end{array}$ & No studies reported this outcome. & & & \\
\hline $\begin{array}{l}\text { Gonococcal conjunctivitis } \\
\text { assessed with: Neisseria gonorrhoeae-positive cul- } \\
\text { ture } \\
\text { follow-up: } 1 \text { month }\end{array}$ & See comment & $\begin{array}{l}2795 \\
(1 \mathrm{RCT})\end{array}$ & $\begin{array}{l}\oplus \Theta \Theta \odot \\
\text { VERY } \\
\text { LOW } 1,2\end{array}$ & $\begin{array}{l}\text { The } 1 \text { study } \\
\text { measured } \\
\text { cases of } \\
\text { gonococcal } \\
\text { conjunctivi- } \\
\text { tis, but no } \\
\text { cases were } \\
\text { found in ei- } \\
\text { ther study } \\
\text { arm (David- } \\
\text { son 1951). }\end{array}$ \\
\hline
\end{tabular}

Chlamydial conjunctivitis assessed with: Chlamydia trachomatis culture, PCR, or direct fluorescent monoclonal antibody stain follow-up: 1 month
This 1 study from 1951 did not report the diagnosis of chlamydial conjunctivitis, nor did the authors specify if chlamydia was measured. It is unlikely that techniques to detect chlamydia were used in this time period.

\begin{tabular}{|c|c|c|c|c|c|}
\hline \multirow{2}{*}{$\begin{array}{l}\text { Bacterial conjunctivitis } \\
\text { assessed with: any bacteria-positive culture } \\
\text { follow-up: } 1 \text { month }\end{array}$} & \multicolumn{2}{|c|}{ Study population } & \multirow{2}{*}{$\begin{array}{l}\text { RR } 2.19 \\
(1.14 \text { to } \\
4.24)\end{array}$} & \multirow{2}{*}{$\begin{array}{l}2795 \\
(1 \mathrm{RCT})\end{array}$} & \multirow{2}{*}{$\begin{array}{l}\oplus \oplus \odot \ominus \\
\text { LOW } 1,3\end{array}$} \\
\hline & 9 per 1000 & $\begin{array}{l}20 \text { per } 1000 \\
(10 \text { to } 38)\end{array}$ & & & \\
\hline \multirow{2}{*}{$\begin{array}{l}\text { Any conjunctivitis of any aetiology assessed with: } \\
\text { clinical assessment } \\
\text { follow-up: } 1 \text { month }\end{array}$} & \multicolumn{2}{|c|}{ Study population } & \multirow{2}{*}{$\begin{array}{l}\text { RR } 1.71 \\
(1.26 \text { to } \\
2.32)\end{array}$} & \multirow{2}{*}{$\begin{array}{l}2795 \\
(1 \mathrm{RCT})\end{array}$} & \multirow{2}{*}{$\begin{array}{l}\oplus \oplus \ominus \ominus \\
\operatorname{LOW} 1,3\end{array}$} \\
\hline & 44 per 1000 & $\begin{array}{l}75 \text { per } 1000 \\
(55 \text { to } 102)\end{array}$ & & & \\
\hline \multirow{2}{*}{$\begin{array}{l}\text { Conjunctivitis of unknown aetiology assessed with: } \\
\text { culture negative } \\
\text { follow-up: } 1 \text { month }\end{array}$} & \multicolumn{2}{|c|}{ Study population } & \multirow{2}{*}{$\begin{array}{l}\text { RR } 1.58 \\
(1.12 \text { to } \\
2.25)\end{array}$} & \multirow{2}{*}{$\begin{array}{l}2795 \\
(1 \mathrm{RCT})\end{array}$} & \multirow{2}{*}{$\begin{array}{l}\oplus \oplus \oplus \ominus \\
\operatorname{LOW} 1,3\end{array}$} \\
\hline & 35 per 1000 & $\begin{array}{l}55 \text { per } 1000 \\
(39 \text { to } 79)\end{array}$ & & & \\
\hline
\end{tabular}

Adverse effects

No studies reported this outcome.

${ }^{*}$ The risk in the intervention group (and its 95\% confidence interval) is based on the assumed risk in the comparison group and the relative effect of the intervention (and its $95 \% \mathrm{Cl}$ ). The assumed risk is taken from the study population in the included studies.

Cl: confidence interval; IM: intramuscular; PCR: polymerase chain reaction; RCT: randomised controlled trial; RR: risk ratio

\section{GRADE Working Group grades of evidence}

High-certainty: We are very confident that the true effect lies close to that of the estimate of the effect.

Moderate-certainty: We are moderately confident in the effect estimate: the true effect is likely to be close to the estimate of the effect, but there is a possibility that it is substantially different.

Low-certainty: Our confidence in the effect estimate is limited: the true effect may be substantially different from the estimate of the effect. 
Table 24. Pencillin IM compared to topical penicillin ointment for the prevention of ophthalmia neonatorum in newborn children (Continued)

Very low-certainty: We have very little confidence in the effect estimate: the true effect is likely to be substantially different from the estimate of effect.

1Downgraded for risk of bias (-1): single study at high risk or unclear risk of bias.

2Downgraded for imprecision (-2): optimal information size criteria likely not met; no events.

3Downgraded for imprecision (-1): optimal information size criteria not met. However, confidence interval excludes no effect.

\section{APPEN DICES}

\section{Appendix 1. CENTRAL search strategy}

\#1 MeSH descriptor: [Gonorrhea] explode all trees

\#2 MeSH descriptor: [Neisseria gonorrhoeae] explode all trees

\#3 gonorr*

\#4 MeSH descriptor: [Chlamydia] explode all trees

\#5 MeSH descriptor: [Chlamydia Infections] explode all trees

\#6 chlamyd*

\#7 MeSH descriptor: [Streptococcus] this term only

\#8 MeSH descriptor: [Staphylococcus aureus] this term only \#9 MeSH descriptor: [Staphylococcal Infections] this term only

\#10 MeSH descriptor: [Haemophilus] this term only

\#11 MeSH descriptor: [Vaginal Diseases] this term only

$\# 12 \# 1$ or \#2 or \#3 or \#4 or \#5 or \#6 or \#7 or \#8 or \#9 or \#10 or \#11

\#13 MeSH descriptor: [Conjunctivitis] explode all trees

\#14 conjunctiv ${ }^{\star}$

\#15 MeSH descriptor: [Eye Infections] explode all trees

\#16 (eye* or ocular) near/3 infection ${ }^{\star}$

\#17 \#13 or \#14 or \#15 or \#16

\#18 MeSH descriptor: [Infant, Newborn] explode all trees

\#19 infan* or newborn or new-born* or neonat* or neo-nat*

\#20 \#18 or \#19

$\# 21 \# 12$ and \#17 and \#20

\#22 MeSH descriptor: [Ophthalmia Neonatorum] explode all trees

\#23 ophthalmia near/2 neonat*

\#24 ophthalmia near/2 newborn*

\#25(neonatal or ophthalmia or gonococcal or Chlamydia) near/4 conjunctivit*

\#26 \#22 or \#23 or \#24 or \#25

\#27 \#21 or \#26

\section{Appendix 2. MEDLINE Ovid search strategy}

1. randomized controlled trial.pt.

2. (randomized or randomised).ab,ti.

3. placebo.ab,ti.

4. dt.fs.

5. randomly.ab,ti.

6. trial.ab,ti.

7. groups.ab,ti.

8. or/1-7

9. $\exp$ animals/

10. exp humans/

11.9 not (9 and 10$)$

12. 8 not 11

13. exp gonorrhea/

14. exp neisseria gonorrhoeae/

15. gonorr\$.tw.

16. exp chlamydia/

17. exp chlamydia infections/

Interventions for preventing ophthalmia neonatorum (Review) 
18. chlamyd\$.tw.

19. Streptococcus/

20. Staphylococcus aureus/

21. Staphylococcal Infections/

22. Haemophilus/

23. Vaginal Diseases/

24. or/13-23

25. exp conjunctivitis/

26. conjunctiv\$.tw.

27. exp Eye Infections/

28. ((eye or ocular) adj3 infection\$).tw.

29. or $/ 25-28$

30. exp infant newborn/

31. (infan\$ or newborn or neonat\$).tw.

32. (new adj1 born\$).tw.

33. (neo adj1 nat\$).tw.

34. or/30-33

35. exp ophthalmia neonatorum/

36. (ophthalmia adj2 neonat\$).tw.

37. (ophthalmia adj2 newborn\$).tw.

38. ((neonatal or ophthalmia or gonococcal or Chlamydia) adj4 conjunctivit\$).tw.

39. or $/ 35-38$

40.24 and 29 and 34

41.39 or 40

42. 12 and 41

The search filter for trials at the beginning of the MEDLINE strategy is from the published paper by Glanville 2006.

\section{Appendix 3. Embase Ovid search strategy}

1. exp randomized controlled trial/

2. exp randomization/

3. exp double blind procedure/

4. exp single blind procedure/

5. random\$.tw.

6. or/1-5

7. (animal or animal experiment).sh.

8. human.sh.

9.7 and 8

10. 7 not 9

11.6 not 10

12. exp clinical trial/

13. (clin\$ adj3 trial\$).tw.

14. ((singl\$ or doubl\$ or trebl\$ or tripl\$) adj3 (blind\$ or mask\$)).tw.

15. exp placebo/

16. placebo\$.tw.

17. random\$.tw.

18. exp experimental design/

19. exp crossover procedure/

20. exp control group/

21. exp latin square design/

22. or $/ 12-21$

23. 22 not 10

24. 23 not 11

25. exp comparative study/

26. exp evaluation/

27. exp prospective study/

28. (control\$ or prospectiv\$ or volunteer\$).tw.

29. or $/ 25-28$

30. 29 not 10

31.30 not ( 11 or 23 )

32. 11 or 24 or 31 
33. exp gonorrhea/

34. exp neisseria gonorrhoeae/

35. gonorr\$.tw.

36. exp chlamydia/

37. exp chlamydia trachomatis/

38. chlamyd\$.tw.

39. Streptococcus/

40. Staphylococcus aureus/

41. Staphylococcus infection/

42. Haemophilus/

43. vagina disease/

44. or/33-43

45. exp conjunctivitis/

46. conjunctiv\$.tw.

47. eye infection/

48. ((eye or ocular) adj3 infection\$).tw.

49. or/45-48

50. exp infant newborn/

51. (infan\$ or newborn or neonat\$).tw.

52. (new adj1 born\$).tw.

53. (neo adj1 nat\$).tw.

54. or/50-53

55. exp newborn ophthalmia/

56. (ophthalmia adj2 neonat\$).tw.

57. (ophthalmia adj2 newborn\$).tw.

58. ((neonatal or ophthalmia or gonococcal or Chlamydia) adj4 conjunctivit\$).tw.

59. or $/ 55-58$

60.44 and 49 and 54

61.59 or 60

62.32 and 61

\section{Appendix 4. LILACS search strategy}

ophthalmia or conjunctivitis and neonatorum or newborn or neonatal or gonococcal or Chlamydia

\section{Appendix 5. ISRCTN search strategy}

(ophthalmia OR conjunctivitis) AND (neonatorum OR newborn OR neonatal OR gonococcal OR Chlamydia)

\section{Appendix 6. ClinicalTrials.gov search strategy}

(ophthalmia OR conjunctivitis) AND (neonatorum OR newborn OR neonatal OR gonococcal OR Chlamydia)

\section{Appendix 7. ICTRP search strategy}

(ophthalmia OR conjunctivitis) AND (neonatorum OR newborn OR neonatal OR gonococcal OR Chlamydia)

Appendix 8. Estimating assumed risks in the comparator group (for no-prophylaxis comparisons)

\begin{tabular}{|c|c|c|c|c|}
\hline Outcome & Low risk & Source & High risk & Source \\
\hline $\begin{array}{l}\text { Gonococcal conjunctivitis } \\
\text { assessed with: Neisseria gonor- } \\
\text { rhoeae-positive culture } \\
\text { follow-up: } 1 \text { month }\end{array}$ & 1 per 1000 & $\begin{array}{l}\text { Prevalence of gonorrhoea in preg- } \\
\text { nant women estimated at 0.275\% } \\
\text { in } 1 \text { study in the USA (Blatt 2012). } \\
\text { Assumed gonococcal ophthalmia } \\
\text { neonatorum occurs in 30\% to 50\% } \\
\text { of infants born to infected mothers } \\
\text { (Mullick 2005). }\end{array}$ & $\begin{array}{l}50 \text { per } \\
1000\end{array}$ & $\begin{array}{l}\text { Mullick 2005: The incidence } \\
\text { of gonococcal ophthalmia } \\
\text { neonatorum was } 3.6 \text { per } 100 \\
\text { live births in Nairobi and } 2.1 \% \\
\text { in The Gambia. }\end{array}$ \\
\hline Chlamydial conjunctivitis & 5 per 1000 & $\begin{array}{l}\text { Studies from China have estimated } \\
\text { that chlamydial conjunctivitis occurs } \\
\text { in } 4 \text { per } 1000 \text { live births (Adachi 2016). }\end{array}$ & $\begin{array}{l}100 \text { per } \\
1000\end{array}$ & $\begin{array}{l}\text { Considerable heterogeneity of } \\
\text { prevalence in different stud- } \\
\text { ies in sub-Saharan Africa and }\end{array}$ \\
\hline
\end{tabular}


(Continued)

assessed with: Chlamydia tra-

chomatis polymerase chain re-

action (PCR) or culture

follow-up: 1 month
Asia, range from $0 \%$ to $44 \%$

Unclear exactly what the risk

of conjunctivitis is in babies of infected women, but likely to be in the region of $30 \%$ to $50 \%$ (Adachi 2016).

\begin{tabular}{|c|c|c|c|c|}
\hline $\begin{array}{l}\text { Bacterial conjunctivitis } \\
\text { assessed with: any bacte- } \\
\text { ria-positive culture } \\
\text { follow-up: } 1 \text { month }\end{array}$ & 3 per 1000 & $\begin{array}{l}\text { Estimate taken from risk in the con- } \\
\text { trol group of included study Posner } \\
\text { 1959. }\end{array}$ & $\begin{array}{l}50 \text { per } \\
1000\end{array}$ & $\begin{array}{l}\text { Estimate taken from risk in } \\
\text { the control group of included } \\
\text { study Ali } 2007 \text {. }\end{array}$ \\
\hline $\begin{array}{l}\text { Any conjunctivitis of any aeti- } \\
\text { ology assessed with: clinical } \\
\text { assessment } \\
\text { follow-up: } 1 \text { month }\end{array}$ & $\begin{array}{l}10 \text { per } \\
1000\end{array}$ & $\begin{array}{l}\text { Estimate taken from risk in the con- } \\
\text { trol group of included study Posner } \\
1959 .\end{array}$ & $\begin{array}{l}300 \text { per } \\
1000\end{array}$ & $\begin{array}{l}\text { Estimate taken from risk in } \\
\text { the control group of included } \\
\text { study Ghaemi } 2014 \text {. }\end{array}$ \\
\hline
\end{tabular}

\section{H I S T O R Y}

Protocol first published: Issue 9, 2016

Review first published: Issue 9, 2020

\section{CONTRIBUTIONSOF AUTHORS}

Designing the review: VK, SSV

Screening search results: VK, SSV

Organising retrieval of papers: CEV Information Specialist, VK

Screening retrieved papers against inclusion criteria: VK, SSV

Appraising risk of bias: VK, SSV

Extracting data from included studies: VK, SSV

Writing to authors of included trials for additional information: VK

Managing data for the review: SSV, VK

Entering data into Review Manager 5: VK, SSV

Analysis of data: SSV, VK

Interpretation of data

- Providing a methodological perspective: SSV, JE

- Providing a clinical perspective: VK

Writing the review: VK, JE, SSV

Edits in response to peer review and copy edit comments: VK, JE, SSV

\section{DECLARATIONS OF INTEREST}

VK was funded by a three-month Pharmaceutical Manufacturers' Association of Canada/IWK-Grace Scholarship for part of the time spent on this systematic review. However, neither Dr Kapoor nor anyone else involved with this review has ever had any financial links with the Pharmaceutical Manufacturers' Association of Canada.

JE has no known interests to declare.

SSV's earlier effort on this review was supported by the Cochrane Eyes and Vision Group US Project with funding through Contract N-01EY-2-1003 with the National Eye Institute, National Institutes of Health, USA. The Cochrane Eyes and Vision Group US Project is now supported by co-operative agreement 1 U01 EY020522, National Eye Institute, National Institutes of Health, USA. 


\section{SOURCES OF SUPPORT}

\section{Internal sources}

- Pharmaceutical Manufacturers' Association of Canada / IWK-Grace Studentship, Canada

- George Mattar Research Scholarship, Dalhousie University, Canada

\section{External sources}

- National Institute for Health Research (NIHR), UK

* Richard Wormald, Co-ordinating Editor for Cochrane Eyes and Vision (CEV) acknowledges financial support for his CEV research sessions from the Department of Health through the award made by the National Institute for Health Research to Moorfields Eye Hospital NHS Foundation Trust and UCL Institute of Ophthalmology for a Specialist Biomedical Research Centre for Ophthalmology.

* This review was supported by the NIHR, via Cochrane Infrastructure funding to the CEV UK editorial base where JE works.

The views and opinions expressed therein are those of the authors and do not necessarily reflect those of the Systematic Reviews Programme, NIHR, NHS or the Department of Health.

- Cochrane Eyes and Vision Group US Project, USA

SV worked with the Cochrane Eyes and Vision Group US Project, which is supported by Contract N01-EY-002, National Eye Institute, National Institutes of Health, USA and Grant 1 U01 EY020522-01, National Eye Institute, National Institutes of Health, USA.

- Department of Epidemiology, Johns Hopkins Bloomberg School of Public Health, USA

\section{DIFFERENCES BETWEEN PROTOCOLANDREVIEW}

We used a treatment arm continuity correction approach to include trials with zero events in meta-analyses (Sweeting 2004). 